\title{
Evolving Distributed Algorithms with Genetic Programming
}

\author{
Dissertation \\ in partial fulfillment of the requirements for the degree \\ Doktor-Ingenieur (Dr.-Ing.) \\ for submission to the \\ Department of Electrical Engineering and Computer Science \\ University of Kassel, Germany \\ May 2009 \\ Dipl.-Inf. Thomas Weise

\footnotetext{
$\mathbf{1}^{\text {st }}$ Reviewer: Prof. Dr. Kurt Geihs (University of Kassel)

$2^{\text {nd }}$ Reviewer: Prof. Dr. Christian Tschudin (University of Basel)

Date of Defense: 2009-05-04
} \\ Committee: Prof. Geihs, Prof. Tschudin, Prof. Vogel-Heuser, Prof. Zündorf
}




\section{Abstract}

Distributed systems are one of the most vital components of the economy. The most prominent example is probably the internet, a constituent element of our knowledge society. During the recent years, the number of novel network types has steadily increased. Amongst others, sensor networks, distributed systems composed of tiny computational devices with scarce resources, have emerged. The further development and heterogeneous connection of such systems imposes new requirements on the software development process. Mobile and wireless networks, for instance, have to organize themselves autonomously and must be able to react to changes in the environment and to failing nodes alike. Researching new approaches for the design of distributed algorithms may lead to methods with which these requirements can be met efficiently. In this thesis, one such method is developed, tested, and discussed in respect of its practical utility.

Our new design approach for distributed algorithms is based on Genetic Programming, a member of the family of evolutionary algorithms. Evolutionary algorithms are metaheuristic optimization methods which copy principles from natural evolution. They use a population of solution candidates which they try to refine step by step in order to attain optimal values for predefined objective functions.

The synthesis of an algorithm with our approach starts with an analysis step in which the wanted global behavior of the distributed system is specified. From this specification, objective functions are derived which steer a Genetic Programming process where the solution candidates are distributed programs. The objective functions rate how close these programs approximate the goal behavior in multiple randomized network simulations. The evolutionary process step by step selects the most promising solution candidates and modifies and combines them with mutation and crossover operators. This way, a description of the global behavior of a distributed system is translated automatically to programs which, if executed locally on the nodes of the system, exhibit this behavior.

In our work, we test six different ways for representing distributed programs, comprising adaptations and extensions of well-known Genetic Programming methods (SGP, eSGP, and LGP), one bio-inspired approach (Fraglets), and two new program representations called Rule-based Genetic Programming (RBGP, eRBGP) designed by us. We breed programs in these representations for three well-known example problems in distributed systems: election algorithms, the distributed mutual exclusion at a critical section, and the distributed computation of the greatest common divisor of a set of numbers.

Synthesizing distributed programs the evolutionary way does not necessarily lead to the envisaged results. In a detailed analysis, we discuss the problematic features which make this form of Genetic Programming particularly hard. The two Rule-based Genetic Programming approaches have been developed especially in order to mitigate these difficulties. In our experiments, at least one of them (eRBGP) turned out to be a very efficient approach and in most cases, was superior to the other representations. 


\section{Zusammenfassung}

Verteilte Systeme stellen eine der wichtigsten Komponenten der Wirtschaft dar. Das wohl bekannteste solche System, das Internet, ist zentraler Bestandteil unserer Wissensgesellschaft. In den letzten Jahren hat die Anzahl neuartiger Netzwerke stetig zugenommen. So entstanden unter Anderem mit Sensornetzen auch verteilte Systeme, die aus kleinen, in ihren Ressourcen stark begrenzten, Knoten bestehen. Die stetige Weiterentwicklung und heterogene Vermaschung solcher Systeme stellt neue Anforderungen an die Softwarearchitekten. Mobile und kabellose Netze müssen sich beispielsweise selbstständig organisieren und autonom auf ausfallende Knoten oder sich ändernde Umgebungsvariablen reagieren. Um diesen Anforderungen entgegenzutreten ist es daher notwendig, neue Methoden für den Entwurf verteilter Systeme verstärkt zu erforschen. Ziel dieser Arbeit ist es, einen solchen neuen Ansatz zu entwickeln, zu erproben und im Hinblick auf seine praktische Anwendbarkeit zu diskutieren.

Unser neuer Ansatz zur Synthese verteilter Algorithmen basiert auf der Genetischen Programmierung, einem Mitglied der Familie der evolutionären Algorithmen. Evolutionäre Algorithmen sind der natürlichen Evolution nachempfundene, metaheuristische Optimierungsverfahren. Sie verwenden eine Population von vielen Lösungskandidaten und versuchen, diese schrittweise immer weiter anzupassen, um optimale Werte für vorher definierte Zielfunktionen zu erreichen.

Die Erzeugung eines verteilten Algorithmus mit unserem Ansatz beginnt mit einer Analysephase, in der das gewünschte globale Verhalten eines Systems spezifiziert wird. Aus dieser Spezifikation werden Zielfunktionen abgeleitet, die den Prozess der Genetischen Programmierung steuern, bei dem die Lösungskandidaten verteilte Programme sind. Die Zielfunktionen bewerten, wie weit diese Programme das spezifizierte Verhalten in mehreren zufallsbasierten Netzwerksimulation annähern. Schritt für Schritt wählt der evolutionäre Prozess die vielversprechendsten Lösungskandidaten aus und verändert und kombiniert sie mit Hilfe von Mutations- und Crossover-Operatoren. Auf diese Weise wird die Beschreibung des globalen Verhaltens eines verteilten Systems vollautomatisch in Programme umgerechnet, die dieses Verhalten erreichen, wenn sie lokal auf seinen Knoten ausgeführt werden.

In unserer Arbeit testen wir sechs verschiedene Darstellungsformen verteilter Programme auf ihre Tauglichkeit zu diesem Zweck. Drei davon sind Anpassungen und Erweiterungen bekannter Ansätze (SGP, eSGP, LGP), eine stammt aus der biologisch-inspirierten Forschung (Fraglets) und zwei neue Methoden, genannt Regelbasierte Genetische Programmierung, wurden von uns selbst entwickelt (RBGP, eRBGP). In unseren Experimenten züchten wir mit diesen Darstellungsformen Programme für drei bekannte Beispielprobleme in verteilten Systemen: Wahlalgorithmen, den verteilte wechselseitige Ausschluss am kritischen Abschnitt und die verteilte Berechnung des größten gemeinsamen Teilers einer Menge von natürlichen Zahlen.

Die evolutionäre Synthese von verteilten Programmen führt nicht immer zum gewünschten Ziel. In einer ausführlichen Analyse legen wir die Eigenschaften dar, welche diese Form der Genetischen Programmierung besonders schwer machen. Die beiden regelbasierten Ansätze wurden speziell auf Basis dieser Analyse entworfen. In den Experimenten stellte sich einer der beiden (die erweiterte regelbasierte Genetische Programmierung eRBGP) als besonders effizient heraus.

Eine ausführliche deutsche Zusammenfassung dieser Dissertation ist in Chapter E auf Seite 207 enthalten. 


\section{Acknowledgements}

The first person I wish to thank is Winfried Kalfa from the Chemnitz, University of Technology. He encouraged me to pursue a scientific career and also recommended me for a position at the Distributed Systems Group of the University of Kassel.

I am very grateful to my doctoral advisor Kurt Geihs who gave me the chance to be a member of this wonderful group. When I was not sure whether I would be able to bring my research to a successful end, he encouraged me to continue. I furthermore want to thank Kurt for introducing me to scientific writing.

The Distributed Systems Group offered me a great working environment. Colleagues like Michael Zapf, Hendrik Skubch, and Jochen Fromm showed me that scientific work is more than programming and that literature research is not just a requirement but can open up one's horizon for many previously unfamiliar aspects. With Philipp Baer and Roland Reichle, I found friends of a similar dedication to bring a project forward. Their commitment to their robotic soccer team was a constant inspiration for me. With Steffen Bleul I enjoyed struggling side by side in both, the Web Service Challenge and the fitness studio. Diana Comes, Mohammad "Titu" Khan, and Michael Wagner were always there for a pleasant chat. I count myself lucky that I was able to share my fascination for evolutionary algorithms with the excellent students Stefan Niemczyk and Alexander Podlich. Last but not least, a good infrastructure was provided and maintained by Iris Roßbach and Thomas Kleppe.

Besides acting as external reviewer of this thesis, Christian Tschudin gave me the opportunity to present my work in a great environment at his chair in Basel. He, Lidia Yamamoto, and Thomas Meyer enriched my research with valuable tips and fruitful discussions on the topic of Genetic Programming and bio-inspired networking.

Finally, I wish to apologize to my family and friends. Because of my delving into research, I have shamefully neglected visiting you and we spent way too little time with each other. I hope to make up for that this summer. 


\section{Erklärung}

Hiermit versichere ich, dass ich die vorliegende Dissertation selbständig und ohne unerlaubte Hilfe angefertigt und andere als die in der Dissertation angegebenen Hilfsmittel nicht benutzt habe. Alle Stellen, die wörtlich oder sinngemäß aus veröffentlichten oder unveröffentlichten Schriften entnommen sind, habe ich als solche kenntlich gemacht. Kein Teil dieser Arbeit ist in einem anderen Promotions- oder Habilitationsverfahren verwendet worden.

Kassel, 2009-05-07

Dipl.-Inf. Thomas Weise 



\section{Contents}

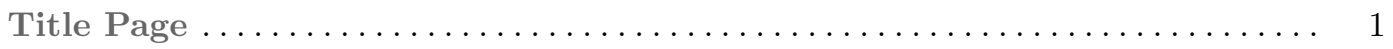

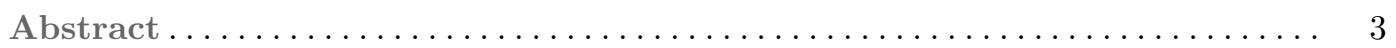

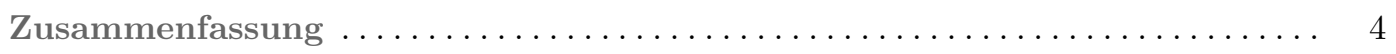

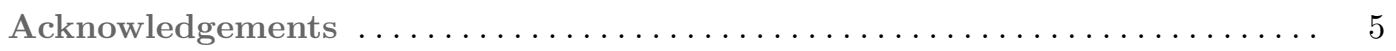

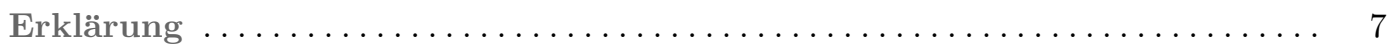

Contents.............................................. 9

Part I Foundations

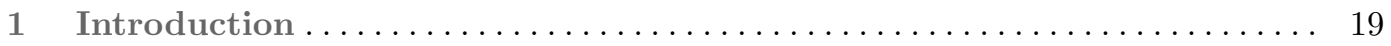

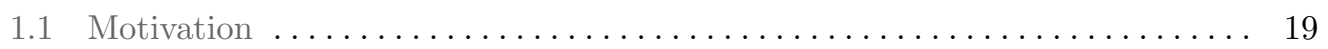

1.1.1 Challenges in Distributed Systems . . . . . . . . . . . . . . . 19

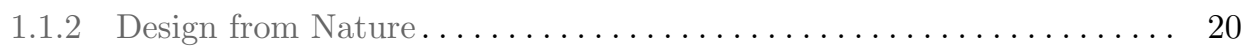

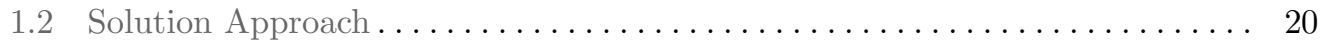

1.3 Major Results.................................... 21

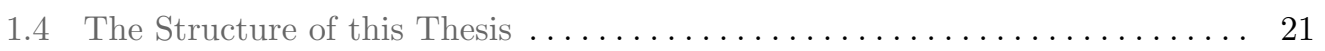

$2 \quad$ Metaheuristic Optimization............................ 23

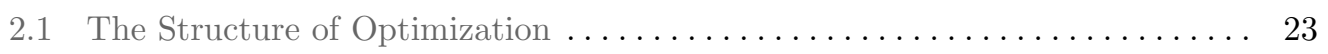

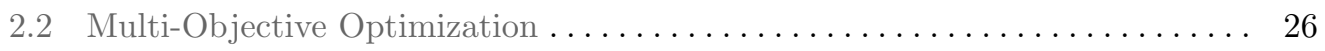

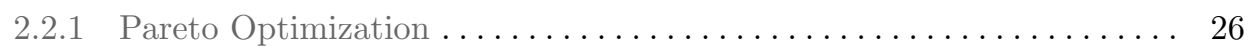

2.2 .2 Pareto Ranking ................................ 27

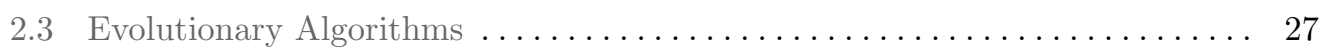

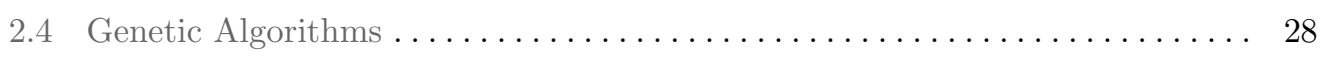

2.4.1 Mutation: Unary Reproduction $\ldots \ldots \ldots \ldots \ldots \ldots \ldots \ldots \ldots \ldots$

2.4.2 Crossover: Binary Reproduction ....................... 29

2.5 Genetic Programming . . . . . . . . . . . . . . . . . . . . . . . 29

2.5.1 Mutation: Unary Reproduction $\ldots \ldots \ldots \ldots \ldots \ldots \ldots \ldots \ldots \ldots . \ldots \ldots$ 
2.5.2 Recombination: Binary Reproduction ................. 30

2.5.3 Automatically Defined Functions ................... 30

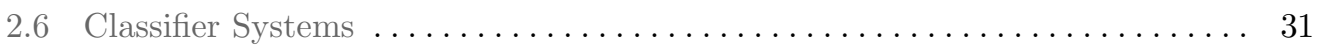

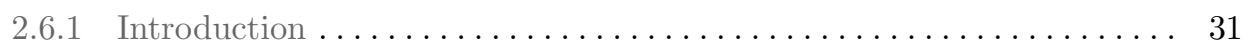

2.6 .2 A Small Example............................ 32

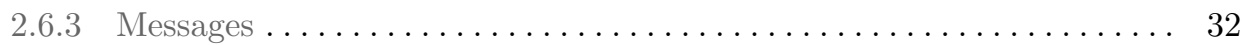

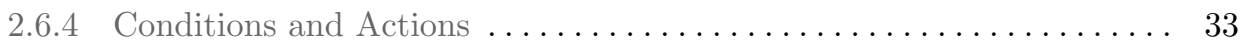

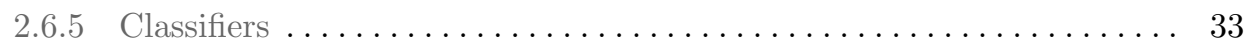

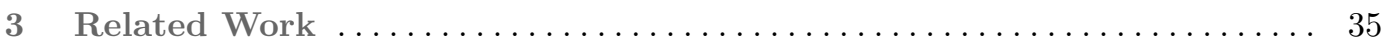

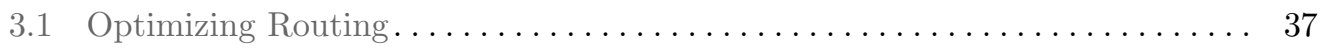

3.1.1 Evolving Fault-tolerant Routing Rules . . . . . . . . . . . . . . 37

3.1.2 Genetic Programming of Broadcasting Algorithms . . . . . . . . . . . . 38

3.1 .3 Optimizing Collective Communication ................. 39

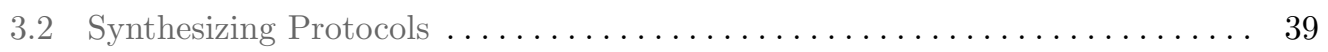

3.2.1 Transformation of Finite State Machine-based Specifications . . . . . . 40

3.2.2 Evolving Fraglet-based Protocols ..................... . 42

3.2.3 Online Protocol Adaptation and Evolution with Fraglets . . . . . . . . 42

Part II Genetic Programming of Distributed Algorithms

4 Features which make Genetic Programming Hard . . . . . . . . . . . . 47

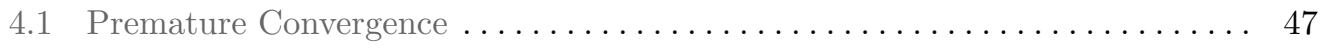

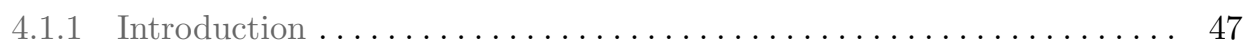

4.1 .2 The Problem ............................ 49

4.1 .3 One Cause: Loss of Diversity . . . . . . . . . . . . . . . . . 49

4.1 .4 Countermeasures ......................... 50

4.2 Ruggedness and Weak Causality.......................... 51

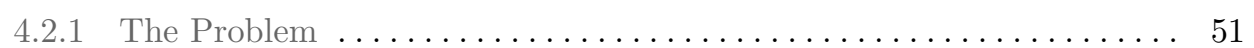

4.2 .2 One Cause: Weak Causality ........................ 51

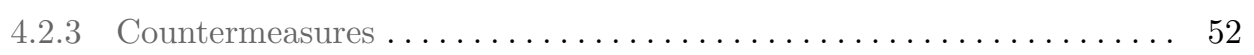

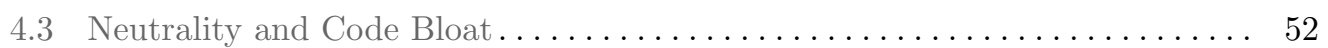

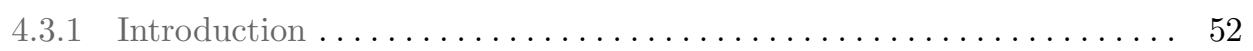

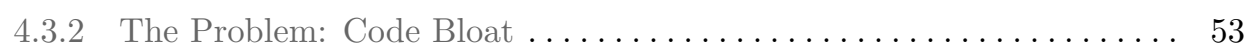

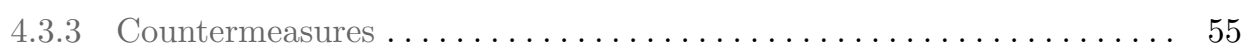

4.4 Needle-In-A-Haystack Problems . . . . . . . . . . . . . . . . . . . . 56

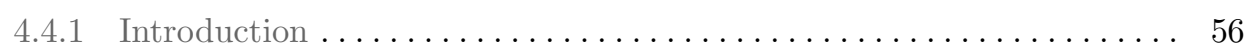

4.4.2 The Problem: All-Or-Nothing? .................... 56

4.4 .3 Countermeasures ......................... 56

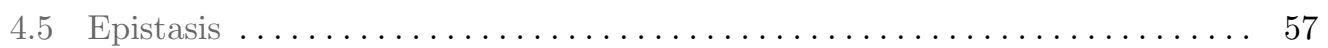

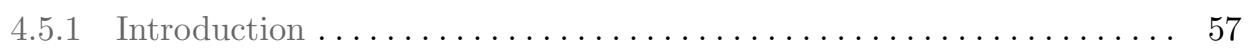

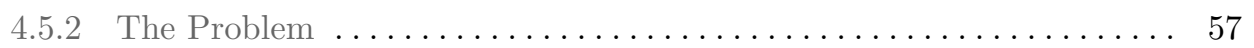

4.5 .3 Countermeasures ........................ 60 


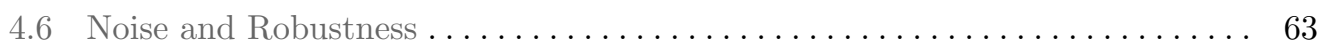

4.6 .1 Introduction - Noise $\ldots \ldots \ldots \ldots \ldots \ldots \ldots \ldots \ldots \ldots \ldots \ldots \ldots \ldots \ldots \ldots \ldots \ldots \ldots$

4.6.2 The Problem: Need for Robustness .................... 63

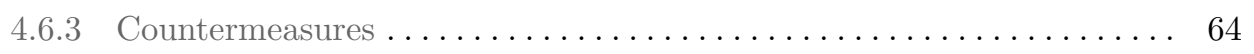

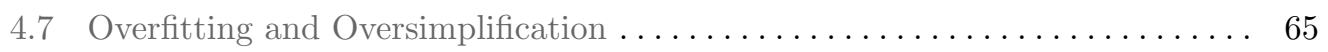

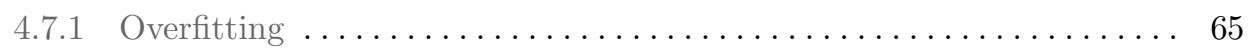

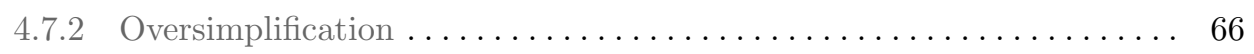

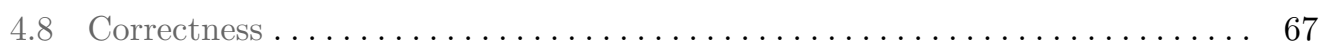

4.8.1 The Problems................................ 67

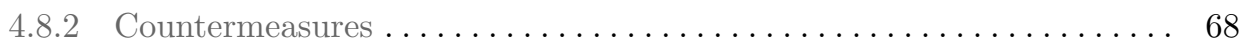

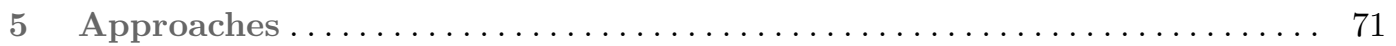

5.1 Standard Genetic Programming with Memory $[\mathrm{SGP}] \ldots \ldots \ldots \ldots \ldots \ldots \ldots 71$

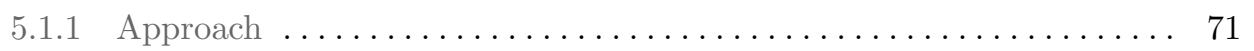

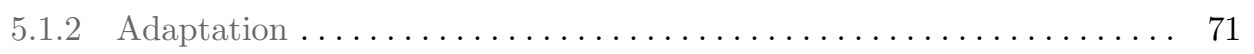

5.2 Extended Standard Genetic Programming with Indexed Memory [eSGP] . . 74

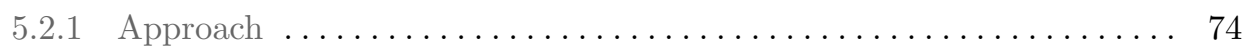

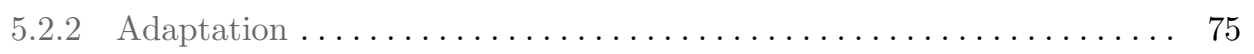

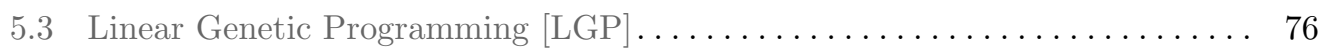

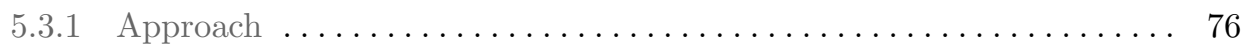

5.3 .2 Adaptation ................................... 77

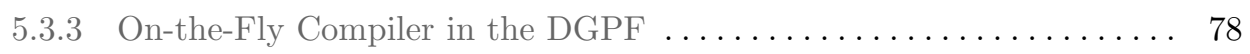

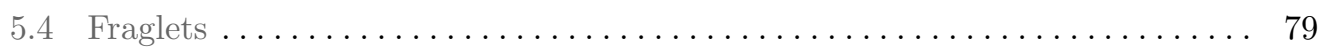

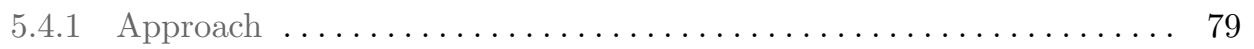

5.4 .2 Adaptation .................................... 80

5.5 Rule-based Genetic Programming $[\mathrm{RBGP}] \ldots \ldots \ldots \ldots \ldots \ldots \ldots \ldots \ldots \ldots$

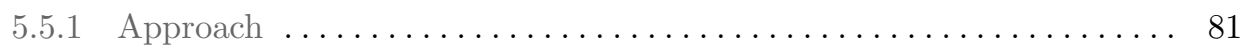

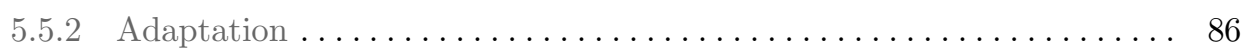

5.6 Extended Rule-based Genetic Programming $[\mathrm{eRBGP}] \ldots \ldots \ldots \ldots \ldots \ldots$

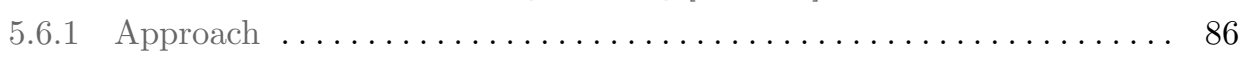

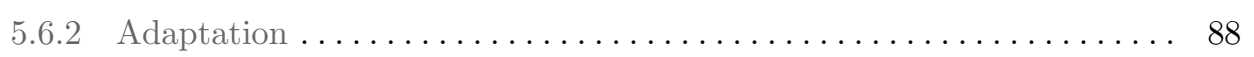

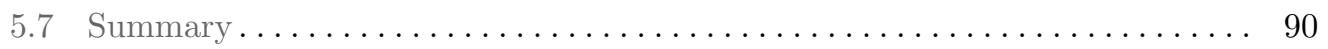

6 Distributed System Model and Simulations . . . . . . . . . . . . 91

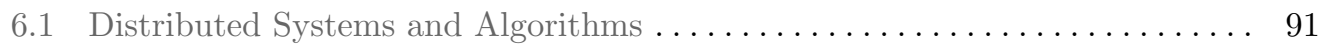

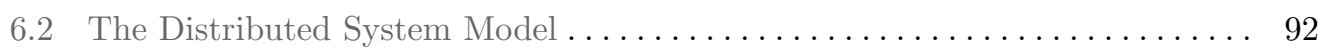

6.2.1 The Node Model ... . . . . . . . . . . . . . . . . . . . . . . . . 92

6.2 .2 The Parallelism Model ......................... 94

6.2.3 The Topology and Messaging Model .................... 95

6.2 .4 The Message Latency Model ........................... 96

6.3 The Structure of the Simulations . . . . . . . . . . . . . . . . . 97

6.4 The System Architecture . . . . . . . . . . . . . . . . . . . . . 99

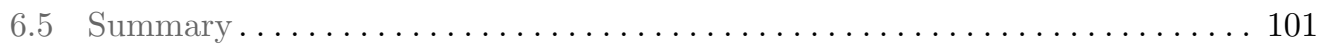


$7 \quad$ Evolving Election Algorithms .............................. 103

7.1 Specification of the Global Behavior $\ldots \ldots \ldots \ldots \ldots \ldots \ldots \ldots \ldots \ldots \ldots \ldots \ldots$

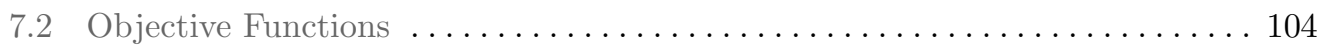

7.2 .1 Functional Objective Functions . . . . . . . . . . . . . . . . . 104

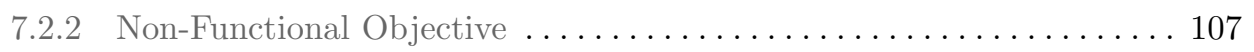

7.3 Experimental Settings ................................ 107

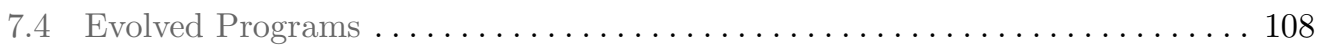

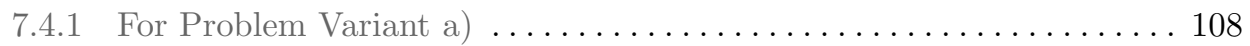

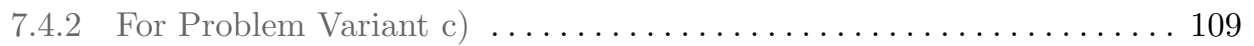

7.4.3 For Problem Variant b) and d) ....................... 111

7.5 Results and Evaluation.................................... 112

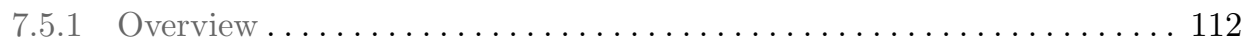

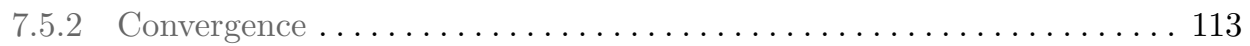

7.5.3 Comparison of the GP Approaches ..................... 116

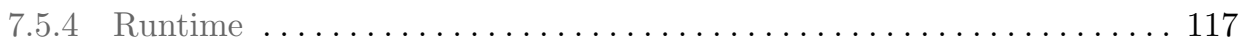

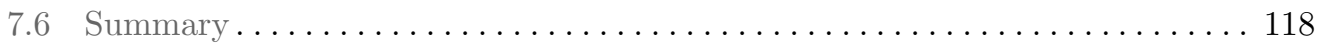

8 Evolving Mutual Exclusion for Critical Sections .................. 121

8.1 Specification of the Global Behavior $\ldots \ldots \ldots \ldots \ldots \ldots \ldots \ldots \ldots \ldots \ldots \ldots \ldots \ldots \ldots$

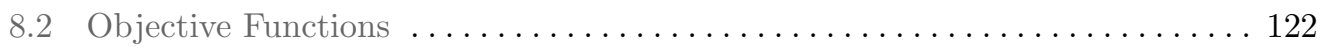

8.2.1 Protection of the Critical Section ........................ 122

8.2.2 Utilization of the Critical Section ..................... 123

8.3 Experimental Settings................................. 125

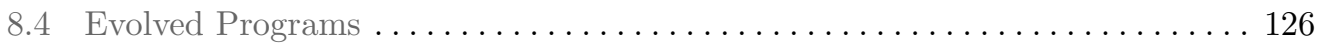

8.5 Results and Evaluation ............................... 131

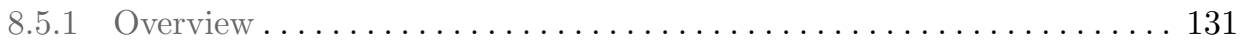

8.5 .2 Pareto Frontiers . . . . . . . . . . . . . . . . . . . . . . 132

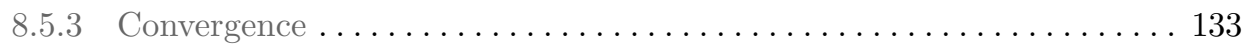

8.5.4 Comparison of the GP Approaches ...................... 135

8.5.5 Comparison of the Scenarios . . . . . . . . . . . . . . . . . 136

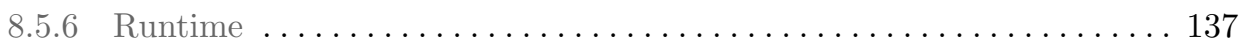

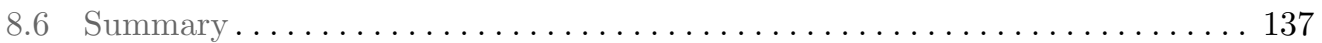

9 Evolving Algorithms for the Distributed Greatest Common Divisor

Problem ........................................... 139

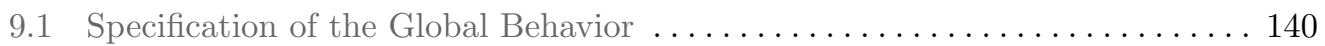

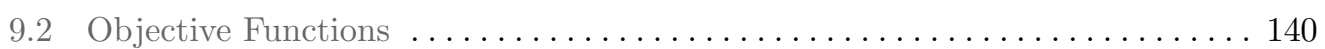

9.3 Experimental Settings......................................... 142

9.4 Evolved Programs ................................... 142

9.5 Results and Evaluation ................................... 145

9.5 .1 Overview ..................................... 145

9.5 .2 Convergence ..................................... 145

9.5.3 Comparison of the GP Approaches .................... 147

9.5.4 Runtime .................................... 148 


\section{Part III Conclusions}

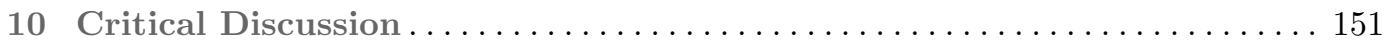

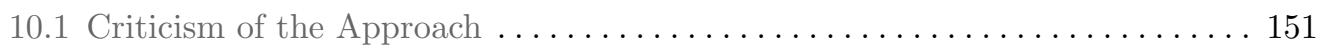

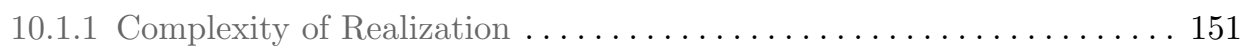

10.1.2 The Evolved Programs . . . . . . . . . . . . . . . . . . . . . 152

10.2 Significance of our Experiments . . . . . . . . . . . . . . . . 153

10.2 .1 Settings . . . . . . . . . . . . . . . . . . . . . . . 153

10.2.2 Why Evolutionary Algorithms? . . . . . . . . . . . . . . . . . 153

10.2.3 Genetic Programming Approaches . . . . . . . . . . . . . . . . . . . 154

10.2.4 Adaptation of the Genetic Programming Approaches . . . . . . . . . . 154

10.2.5 Choice of Scenarios . . . . . . . . . . . . . . . . . . . . . 154

10.3 Quality GP Approach Features . . . . . . . . . . . . . . . . . . . 155

10.3.1 Is Rule-based Genetic Programming Good? ...................... 155

10.3.2 Is Indexed Memory Good? . . . . . . . . . . . . . . . . . . . . 155

11 Other Projects . . . . . . . . . . . . . . . . . . . . . . . . 157

11.1 The Evolution of Aggregation Protocols . . . . . . . . . . . . . . . 157

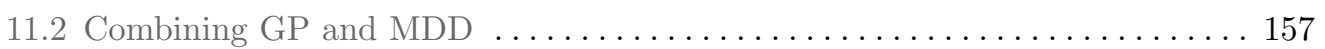

11.3 Offline Emergence Engineering with GP . . . . . . . . . . . . . . 157

11.4 Tunable Model for Problematic Fitness Landscapes . . . . . . . . . . . . . . . . 158

11.5 GAs for Semantic Web Service Composition . . . . . . . . . . . . . . . 158

11.6 The Electronic Book on Global Optimization . . . . . . . . . . . . . 158

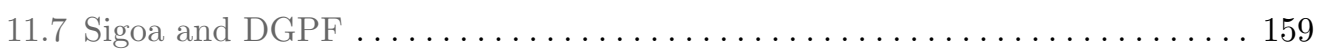

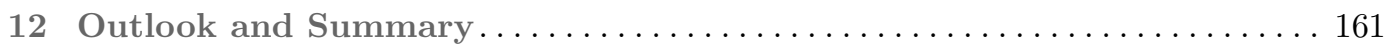

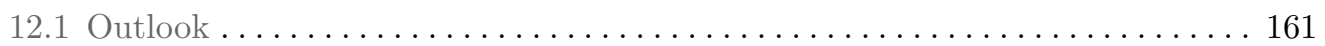

12.1.1 From Theory to Practice ...................... 161

12.1.2 Investigating the Optimization of Non-Functional Features . . . . . . 161

12.1.3 Combining Temporary Memory with other GP Approaches . . . . . . . 162

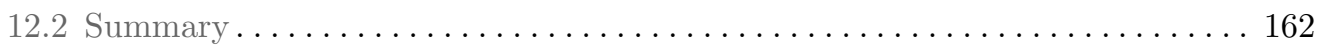

\section{Appendices}

A The Election Experiments $\ldots \ldots \ldots \ldots \ldots \ldots \ldots \ldots \ldots \ldots \ldots \ldots \ldots$

A.1 Precise Settings . . . . . . . . . . . . . . . . . . . . . . . . . 167

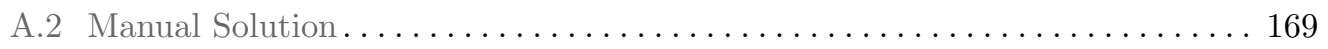

A.3 Further Results . . . . . . . . . . . . . . . . . . . . . . . . 170

A.3.1 Convergence Diagrams ......................... 170

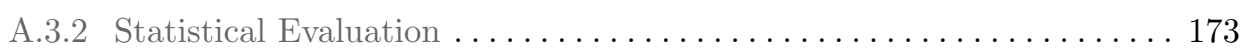

B The Critical Section Experiments...................... 175 


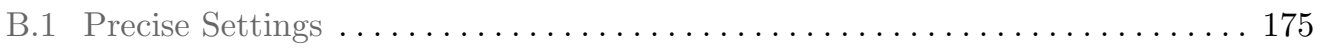

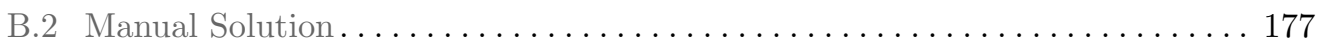

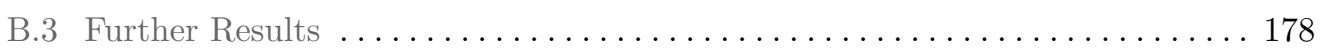

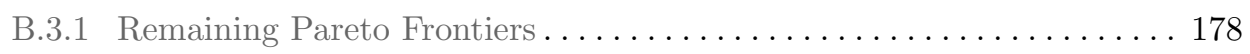

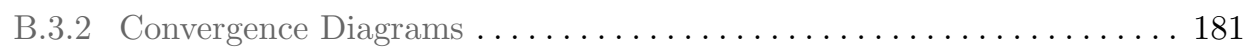

B.3.3 Statistical Evaluation $\ldots \ldots \ldots \ldots \ldots \ldots \ldots \ldots \ldots \ldots \ldots \ldots \ldots \ldots \ldots \ldots \ldots \ldots \ldots \ldots$

C The Distributed Greatest Common Divisor Experiments . . . . . . . . . 189

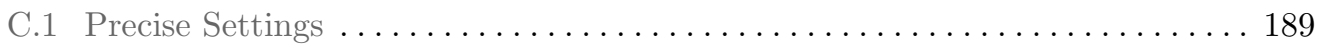

C.2 Manual Solution.................................. 190

C.3 Further Results . . . . . . . . . . . . . . . . . . . . . . . 191

C.3.1 Convergence Diagrams . . . . . . . . . . . . . . . . . . . . . 191

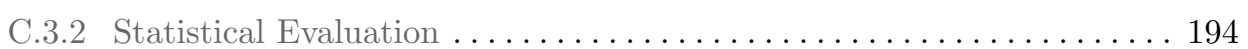

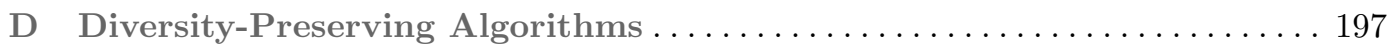

D.1 Fitness Assignment Methods . . . . . . . . . . . . . . . . . . . . . 197

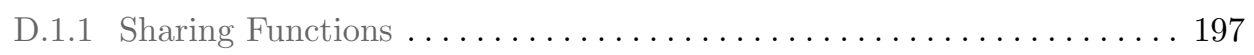

D.1.2 Variety Preserving Ranking . . . . . . . . . . . . . . . . 197

D.2 Clearing and Simple Convergence Prevention (SCP) . . . . . . . . . . 203

E Ausführliche Deutsche Zusammenfassung ................. 207

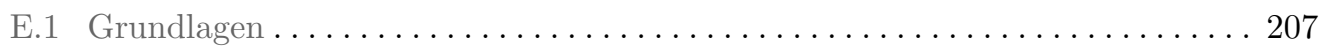

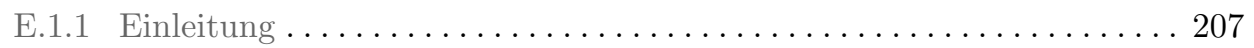

E.2 Die verschiedenen Ansätze der Genetischen Programmierung . . . . . . . . . 210

E.2.1 Standard Genetic Programming $[\mathrm{SGP}] \ldots \ldots \ldots \ldots \ldots \ldots \ldots \ldots \ldots$

E.2.2 Erweitertes Standard GP $[\mathrm{eSGP}] \ldots \ldots \ldots \ldots \ldots \ldots \ldots \ldots \ldots \ldots \ldots \ldots$

E.2.3 Lineare Genetische Programmierung $[\mathrm{LGP}] \ldots \ldots \ldots \ldots \ldots \ldots \ldots \ldots . \ldots \ldots 2$

E.2.4 Fraglets .............................. 213

E.2.5 Regelbasierte Genetische Programmierung $[\mathrm{RBGP}] \ldots \ldots \ldots \ldots \ldots \ldots 213$

E.2.6 Erweiterte Regelbasierte Genetische Programmierung [eRBGP] . . . . 215

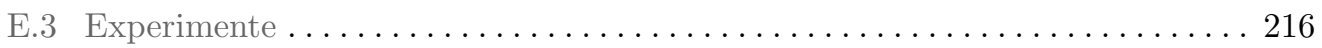

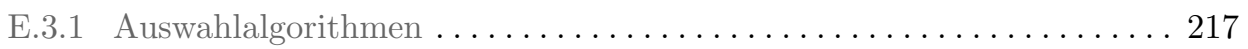

E.3.2 Wechselseitiger Ausschluss am Kritischen Abschnitt . . . . . . . . . . 217

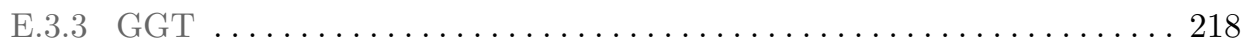

E.4 Kritische Diskussion ... . . . . . . . . . . . . . . . . . . . . . . 219

E.4.1 Kritik am Ansatz........................... 219

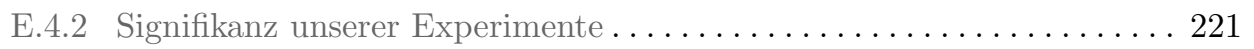

E.4.3 Qualität der Eigenschaften der GP-Ansätze ................. 223

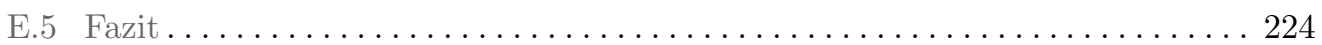

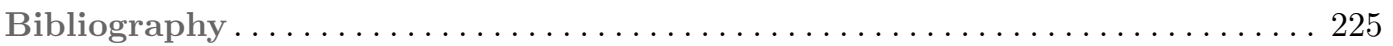

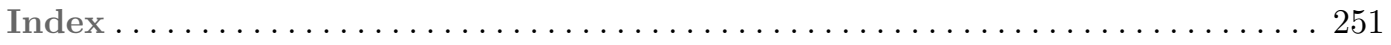

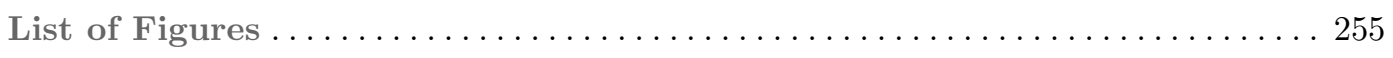




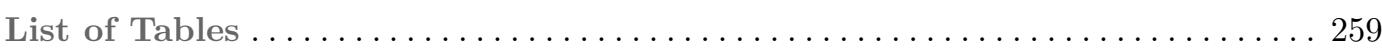

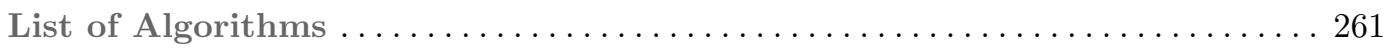

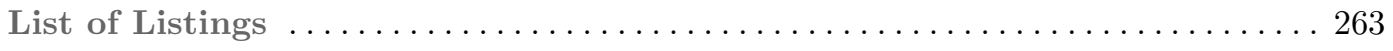



Part I

Foundations 



\section{Introduction}

\subsection{Motivation}

\subsubsection{Challenges in Distributed Systems}

Distributed systems are one of the most vital components of the economy. They provide communication infrastructures, both world-wide with the internet as well as locally by connecting different modules of car electronics or as body area networks. Virtually every computer systems today is either already part of a distributed system or may become one in future [273].

While many internet technologies, protocols, and applications grew into maturity and have widely been researched, new forms of networks and distributed computing have emerged during the recent years. Amongst them, we can find wireless networks, sensor networks [139], wireless sensor networks [42], Smart Home environments, Ubiquitous Computing [82], and experimental ideas like Amorphous Computing [2].

The devices interacting in such networks often have scarce resources, such as small memory, low processing power, and a limited battery capacity. Additionally to these restrictions, new requirements such as self-adaptation [102] and self-organization [101] arise. A software architect designing a sensor network, for instance, must not only be concerned with functional aspects like data aggregation but also consider non-functional criteria such as energy consumption throughout the complete software engineering process.

It may be too bold of a statement to say that these challenges ask for new programming paradigms. Nobody will, however, argue that the development of applications and algorithms for such novel classes of distributed systems is surely to become more complicated than for traditional networks. Hence, exploring the utility of new programming methodologies is a demand of the current situation. Since algorithms are the backbone of every computer application, this also involves researching new representations for distributed algorithms.

Definition 1.1 (Design of a Distributed Algorithm). The design of a distributed algorithm is the transformation of a specification of the behavior of a network as a whole to a program that, if executed locally on each of the nodes of the network, achieves this behavior.

In this work, we will provide some first steps into the direction of automating the process described by Definition 1.1. 

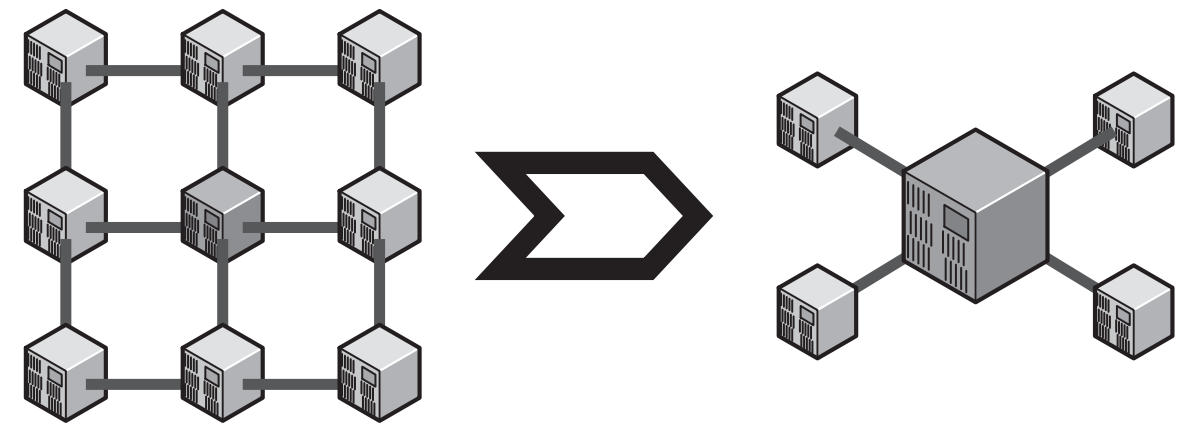

Figure 1.1: Design of distributed algorithms.

\subsubsection{Design from Nature}

The transformation of a global system behavior to local rules as depicted in Figure 1.1 is no process specific only to distributed algorithm design. Instead, a widely studied example for it are swarming behaviors [242] in nature, which have evolved over millions of years.

By travelling together in a configuration which has a good volume/surface-ratio, individuals of a species can strengthen their defense against predators, increase the chance of finding mating partners, enhance foraging success, and improve hydro- or aerodynamics. Nature has evolved many efficient swarming behaviors such as the shoaling of fish (sketched in Figure 1.2), flocking of birds, and swarming of locusts [219].

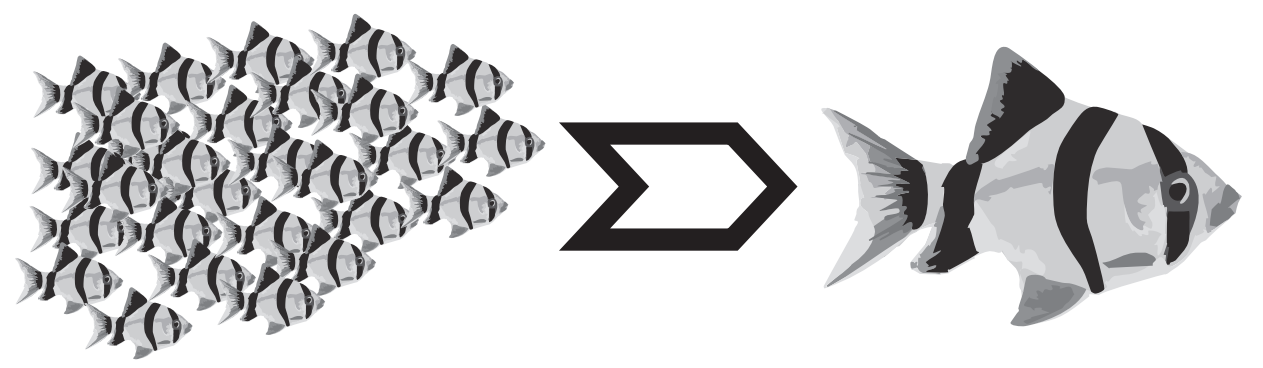

Figure 1.2: Evolutionary behavior design.

\subsection{Solution Approach}

Evolutionary algorithms copy the natural evolution for solving complex optimization problems [9, 293]. In this work, we will utilize Genetic Programming [232, 293], one class of evolutionary algorithms, for breeding distributed algorithms - in other words - for transforming descriptions of global behaviors to locally running programs.

Figure 1.3 illustrates this new design process for distributed algorithms. First, the wanted system behavior is specified. Objective functions which rate "how close" the behavior of an evolved program comes to the target behavior are derived manually from the specification. In order to approximate the quality of the evolved programs, we execute them on simulated nodes in a simulated network. We apply multi-objective Genetic Programming since it 


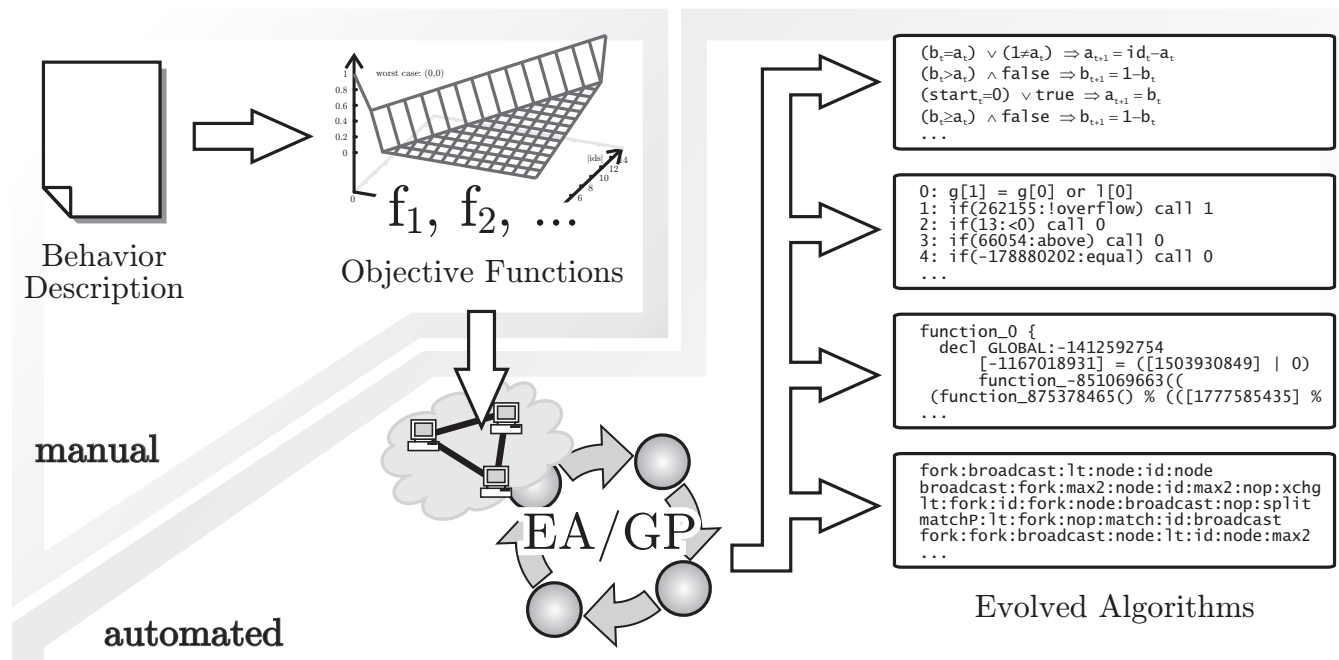

Genetic Programming

Figure 1.3: The general solution approach pursued by this work.

allows us to optimize the algorithms also for non-functional aspects such as program size, the economical use of resources, and minimizing communication.

As part of our research work, a general optimization framework (Sigoa $\left.{ }^{1}\right)$ has been implemented and an extension library for Genetic Programming ( GPF $^{2}$ ) has been placed on top of it. The core of this thesis is the evaluation of the approach just discussed with statistically significant experiments using these two software packages.

\subsection{Major Results}

The research work documented with this thesis led to four major results:

1. A new approach for designing programs for distributed systems has been defined on the basis of evolutionary algorithms.

2. Six different methods (program representations) which can be used for realizing this approach have been tested on three different problems.

3. The efficiency of our approach is challenged by several facets which make the evolution of (distributed) programs difficult. We discuss these facets in-depth and also provide possible countermeasures against them.

4. Two of the six program representations (RBGP, eRBGP) have been designed especially with these problematic features of GP in mind. In a good share of the experiments, one of the two (eRBGP) had a remarkably better performance when compared to the other techniques.

\subsection{The Structure of this Thesis}

This thesis is structured into three parts. The foundations of the subject area are discussed in the remainder of this first part. This includes the basics of metaheuristic optimization

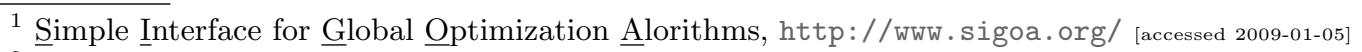

2 Distributed Genetic Programming Framework, also available at http://www.sigoa.org/
} 
(Chapter 2) and related work on its application to distributed systems (Chapter 3). From the analysis of the related work, it becomes clear that very little research directly targeting the evolution of distributed algorithms has been conducted yet, especially in the area of asynchronous systems.

The main contributions of this thesis are included in Part II. In Chapter 4, we investigate the reasons which speak against using Genetic Programming to synthesize distributed algorithms: a set of challenging features which make the evolution of algorithms hard. One of the objectives of our research is to find program representations which are unaffected by these problems or can mitigate them. We therefore analyze six different Genetic Programming techniques:

1. Standard, tree-based Genetic Programming (SGP, Section 5.1),

2. extended Standard Genetic Programming (eSGP, Section 5.2),

3. linear Genetic Programming (LGP, Section 5.3),

4. Fraglets (Section 5.4),

5. Rule-based Genetic Programming (RBGP, Section 5.5), and

6. Extended Rule-based Genetic Programming (eRBGP, Section 5.6).

SGP and LGP are based on common standard methods enhanced with three forms of memory and adapted to the evolution of distributed algorithms. eSGP is the extension of SGP with indexed memory. The Fraglets approach stems from bio-inspired research. RBGP and eRBGP have been explicitly designed by us in the hope to reduce epistasis, one of the problematic features outlined in Chapter 4.

The model for distributed systems used in this research is outlined in Chapter 6 . The Genetic Programming approaches are then applied to three different example problems: distributed election (Chapter 7), mutual exclusion (Chapter 8), and the distributed computation of the greatest common divisor (Chapter 9).

In Part III we draw conclusions from these experiments, discuss the utility of our approach, and give pointers to future work. Finally, the appendices starting at page 167 comprise more detailed listings of the experimental settings and results, definitions of the specialized selection and fitness assignment algorithms utilized in our Genetic Programming system, and a comprehensive bibliography. 


\section{Metaheuristic Optimization}

A metaheuristic optimization algorithm is an optimization method for solving very general classes of problems. It combines objective functions or heuristics in an abstract and hopefully efficient way, usually without utilizing deeper insight into their structure, i. e., by treating them as black-box-procedures [105, 293]. We first give an introduction into the general structure of such optimization processes in the next section and discuss their multi-objective variant in Section 2.2. Evolutionary algorithms (Section 2.3) are a family of metaheuristics which all proceed according to the same scheme. Two members of this family which we have utilized in the context of this work are genetic algorithms (Section 2.4) and Genetic Programming (Section 2.5). Classifier systems are discussed in Section 2.6 since they gave inspiration for our new Rule-based Genetic Programming approaches which are one of the main contributions of this work.

\subsection{The Structure of Optimization}

Before going any more into detail about evolutionary algorithms and Genetic Programming, we will define some of the terminology used throughout this work and put it into the context of the general structure common to all metaheuristic optimization processes. This structure comprises a number of well-defined spaces and sets as well as the mappings and relations between them. It is illustrated in Figure 2.1 by using a genetic algorithm which encodes the coordinates of points in a plane into bit strings as example.

Whenever an optimization problem is approached, the first step is to define the type of the possible solutions. If the root of a mathematical function is sought, the real numbers would be proper solution candidates and when synthesizing distributed algorithms, some sort of programming language would be used.

Definition 2.1 (Problem Space). The problem space (phenome) $\mathbb{X}$ of an optimization problem is the set containing all elements $x$ which could be its solution.

Usually, more than one problem space can be defined for a given optimization problem. In this work, different program representations are utilized for evolving distributed algorithms, for example.

Definition 2.2 (Phenotype). A solution candidate (phenotype) $x$ is an element of the problem space $\mathbb{X}$ of a certain optimization problem.

Definition 2.3 (Search Space). The search space (genome) $\mathbb{G}$ of an optimization problem is the set of all elements $g$ which can be processed by the search operations. 
Fitness and heuristic values (normally) have only a meaning in the context of a population or a set of solution candidates.

Fitness Space $\mathbb{R}^{+}$

Fitness Assignment Process

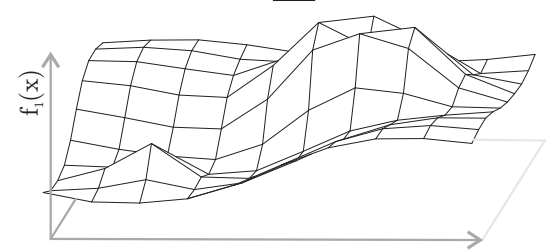

Objective Space

$\mathbb{R}^{\mathrm{n}}$

Objective Function(s)

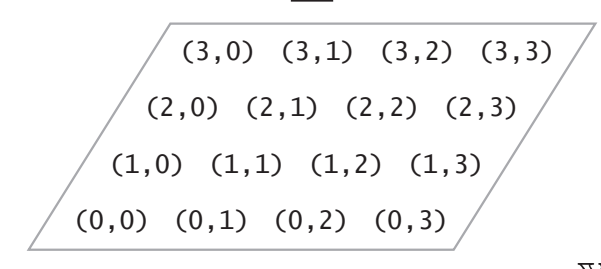

Problem Space

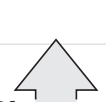

Genotype-Phenotype Mapping

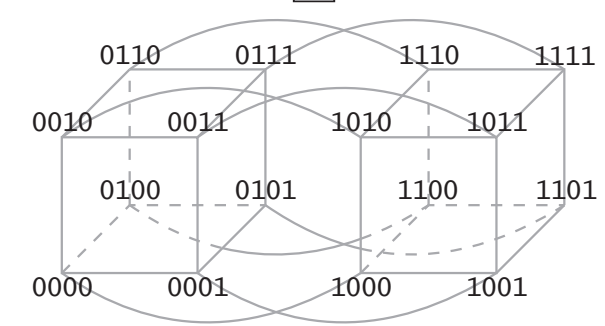

Search Space

\section{The Involved Spaces}

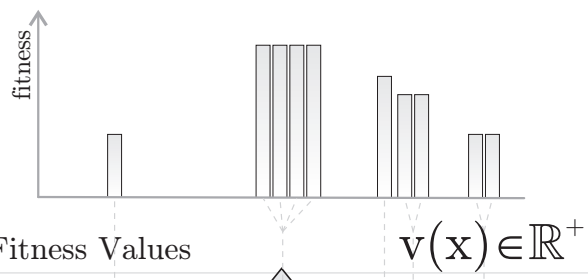

Fitness Values

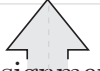

Fitness Assignment Process

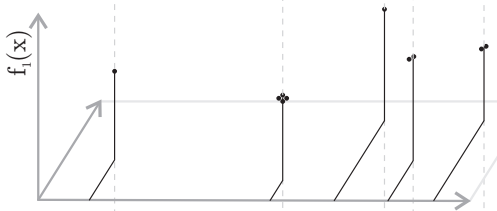

Objective Values $\quad \mathrm{F}(\mathrm{x} \in \mathbb{X}) \in \mathbb{R}^{\mathrm{n}}$

Objective Function(s)

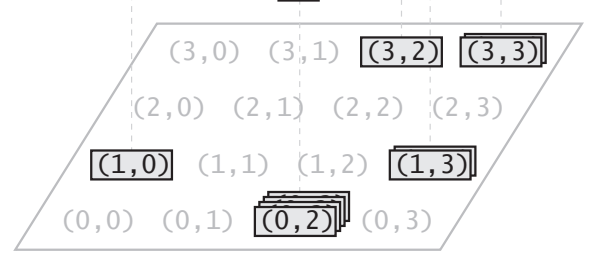

Population (Phenotypes) Pop $\subseteq \mathbb{G} \times \mathbb{X}$

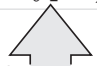

Genotype-Phenotype Mapping

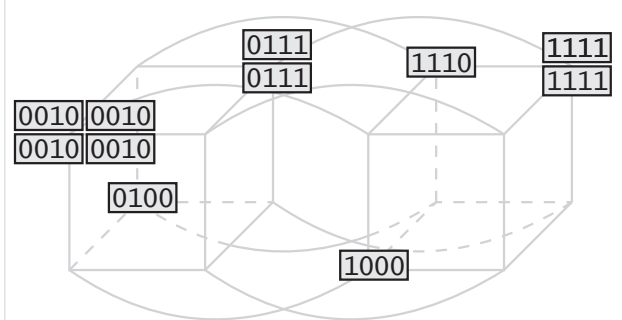

Population (Genotypes) Pop $\subseteq \mathbb{G} \times \mathbb{X}$

The Involved Sets/Elements

Figure 2.1: Spaces, Sets, and Elements involved in an optimization process. 
Especially in the context of evolutionary algorithms, a couple of well-known search spaces and operators exist. Even if the original problem space of an optimization task is none of those, it often makes sense to map them to such a space. This decreases the time spent in development, is usually less error-prone, and helps to consolidate and analyze findings.

Definition 2.4 (Genotype). The elements $g \in \mathbb{G}$ of the search space $\mathbb{G}$ of a given optimization problem are called the genotypes.

The elements of the search space rarely are unstructured aggregations. Instead, they often consist of distinguishable parts, hierarchical units, or well-typed data structures. We refer to the elements of a genotype which are subject to alteration by the search operations as genes and to their possible values as alleles. Figure 2.2 refines the relations of genotypes and phenotypes from the initial example in Figure 2.1 by also marking genes and alleles.

Definition 2.5 (Search Operations). The search operations are used by optimization algorithms in order to explore the search space $\mathbb{G}$.

Search operations can be defined with different arities. searchOp : $\mathbb{G}^{n} \mapsto \mathbb{G}$, for instance, denotes an $n$-ary operator, i. e., one with $n$ arguments. The result of a search operation is one element of the search space. If the search space differs from the problem space, a translation between them is required. In our work, for instance, some higher-level program representations (SGP and eSGP) are mapped to assembler-like code (LGP) in order to ease their execution.

Definition 2.6 (Genotype-Phenotype Mapping). The genotype-phenotype mapping $(\mathrm{GPM}) \mathrm{gpm}: \mathbb{G} \mapsto \mathbb{X}$ is a left-total binary relation which maps the elements the of search space $\mathbb{G}$ to elements in the problem space $\mathbb{X}$.

$$
\forall g \in \mathbb{G} \exists x \in \mathbb{X}: \operatorname{gpm}(g)=x
$$

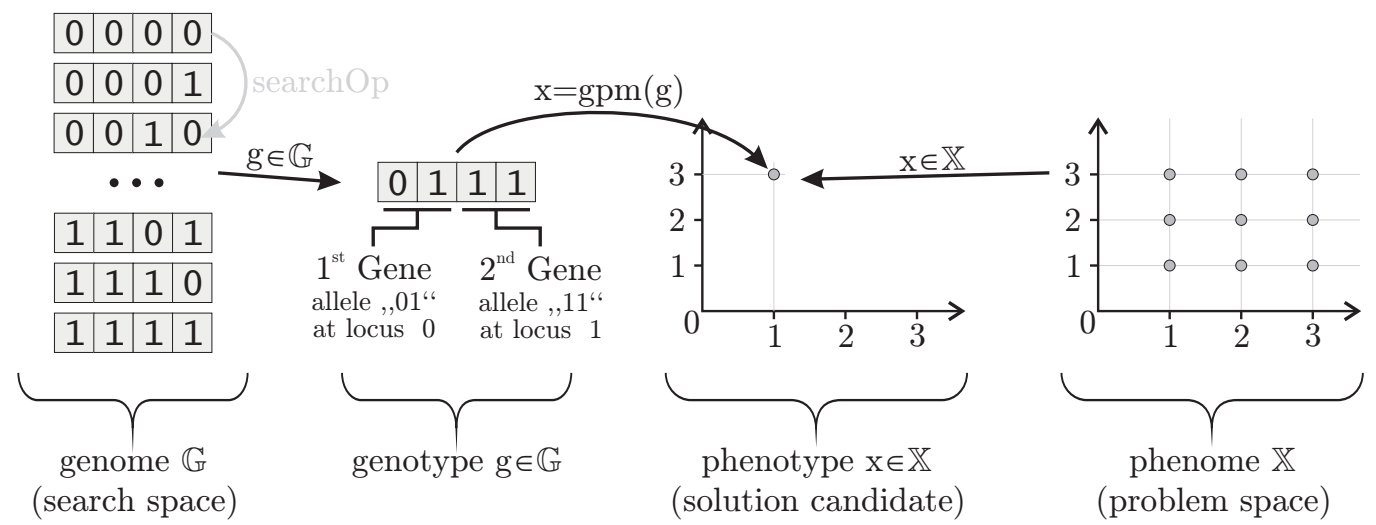

Figure 2.2: The relation of genome, genes, and the problem space.

The main purpose of an optimization algorithm is to find the solution candidates with the best possible evaluation results. This evaluation is performed by the set $F$ of objective functions $f \in F$, each contributing one numerical value describing the characteristics of a phenotype $x$.

Definition 2.7 (Objective Function). An objective function $f: \mathbb{X} \mapsto \mathbb{R}$ is a mathematical function which is subject to optimization. 
In the context of this work, all objective functions are subject to minimization. In order to ease the discussions of different evolutionary algorithms, we furthermore define the data structures individual and population. An individual $p$ is a tuple $(p . g, p . x)$ of an element $p . g$ in the search space $\mathbb{G}$ and the corresponding element $p . x=\operatorname{gpm}(p . g)$ in the problem space $\mathbb{X}$. A population $P o p$ is a list of individuals used during an optimization process.

\subsection{Multi-Objective Optimization}

In this work, we will always perform multi-objective optimization where $n=|F|>1$. For evolving distributed election algorithms, we will use two objective functions $\left(f_{e l}, f_{p s}\right)$ and for synthesizing mutual exclusion methods, the three criteria $f_{c o l}, f_{u s e}$, and $f_{p s}$ are optimized, for instance. Algorithms designed to optimize such sets of objective functions are usually named with the prefix multi-objective. The Genetic Programming approaches in our work were backed by a multi-objective evolutionary algorithm (MOEA). Multi-Objective metaheuristic optimization methods require an additional process that maps the objective values of each solution candidate $x$ to a single real number $v(x)$.

Definition 2.8 (Fitness). The fitness value $v(x) \in \mathbb{R}$ of an element $x$ of the problem space $\mathbb{X}$ corresponds to its utility as solution or its priority in the subsequent steps of the optimization process.

Like objective functions, the fitness is minimized in this work, meaning that smaller values of $v$ are better.

\subsubsection{Pareto Optimization}

Pareto optimality is an important notion in economics, game theory, engineering, and social sciences [81, 311]. It defines the frontier of solutions that can be reached by trading-off conflicting objectives in an optimal manner. From this front, a decision maker (be it a human or an algorithm) can finally choose the configurations that, in his opinion, suit best [92]. The notation of optimal in the Pareto sense is strongly based on the definition of domination:

Definition 2.9 (Domination). An element $x_{1}$ dominates (is preferred to) an element $x_{2}$ $\left(x_{1} \vdash x_{2}\right)$ if $x_{1}$ is better than $x_{2}$ in at least one objective function and not worse with respect to all other objectives. Based on the set $F$ of objective functions $f$, we can write:

$$
\begin{aligned}
x_{1} \vdash x_{2} \Leftrightarrow & \forall i: 0<i \leq n \Rightarrow f_{i}\left(x_{1}\right) \leq f_{i}\left(x_{2}\right) \wedge \\
& \exists j: 0<j \leq n: f_{j}\left(x_{1}\right)<f_{j}\left(x_{2}\right)
\end{aligned}
$$

The Pareto domination relation defines a strict partial order on the space of possible objective values.

Definition 2.10 (Pareto Optimal). An element $\mathbf{x}^{\star} \in \mathbb{X}$ is Pareto optimal (and hence, part of the optimal set $\mathbf{X}^{\star}$ ) if it is not dominated by any other element in the problem space $\mathbb{X}$. In terms of Pareto optimization, $\mathbf{X}^{\star}$ is called the Pareto set or the Pareto Frontier.

$$
\mathbf{x}^{\star} \in \mathbf{X}^{\star} \Leftrightarrow \nexists x \in \mathbb{X}: x \vdash \mathbf{x}^{\star}
$$




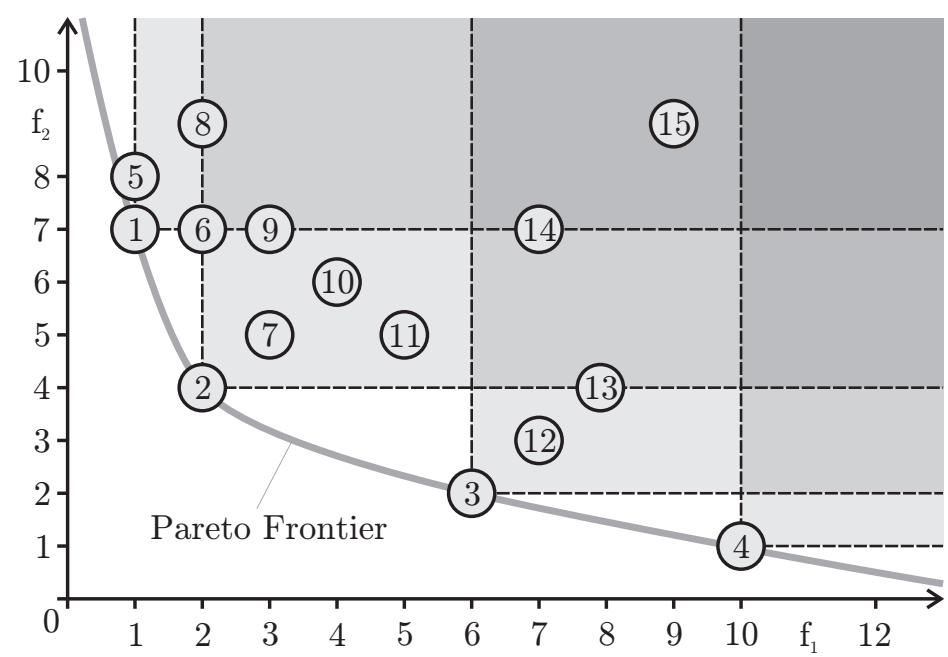

$\mathrm{v}($ (1) $=0$
$\mathrm{v}($ (2) $=0$
$\mathrm{v}($ (3) $=0$
$\mathrm{v}($ (4) $=0$
$\mathrm{v}($ (5) $=1$
$\mathrm{v}($ (6) $=2$
$\mathrm{v}($ (7) $=1$
$\mathrm{v}($ (8) $)=4$
$\mathrm{v}($ (9) $=4$
$\mathrm{v}($ (1) $)=2$

Figure 2.3: An example scenario for Pareto ranking.

\subsubsection{Pareto Ranking}

A simple method for computing fitness values is to let them directly reflect the Pareto domination relation. Figure 2.3 illustrates the Pareto relations in a population of 15 individuals and their corresponding objective values $f_{1}$ and $f_{2}$. The Pareto ranking fitness assignment process has first been proposed by Goldberg [106] and defines the fitness $v$ of a solution candidate as the number of individuals it is dominated by $[184,111]$. The column on the right hand side of Figure 2.3 shows that all four non-dominated individuals have the best possible fitness 0 . This ranking can be performed by first removing all non-dominated individuals from the population and assigning the rank 0 to them. Then, the same is performed with the rest of the population. The individuals only dominated by those on rank 0 (now non-dominated) will be removed and get the rank 1 . This is repeated until all solution candidates have a proper fitness assigned to them.

As already mentioned, the fitness values of all non-dominated elements in our example (Figure 2.3) are equally 0. However, the region around the individuals 1 and 2 has probably already extensively been explored, whereas the surrounding of solution candidate 4 is rather unknown. A better approach of fitness assignment should incorporate such information and put a bit more pressure into the direction of individual 4, in order to investigate this area more thoroughly.

One way for computing more balanced fitness values is using sharing methods (see Section D.1.1) which incorporate density information into the fitness assignment process. In our research, we adopted a fitness assignment algorithm based on Pareto-ranking combined with a sharing variant specified in Section D.1.2 in the appendix.

\subsection{Evolutionary Algorithms}

Evolutionary algorithms (EAs) are population-based metaheuristic optimization algorithms that use biology-inspired mechanisms like mutation, crossover, natural selection, and survival of the fittest in order to refine a set of solution candidates iteratively [10, 293]. Figure 2.4 illustrates the general course of an EA: When the optimization process starts up, there exists no information about which features are good and which are bad. Hence, it initially creates a population $P o p$ of individuals $p$ with randomly configured genotypes. These genotypes 


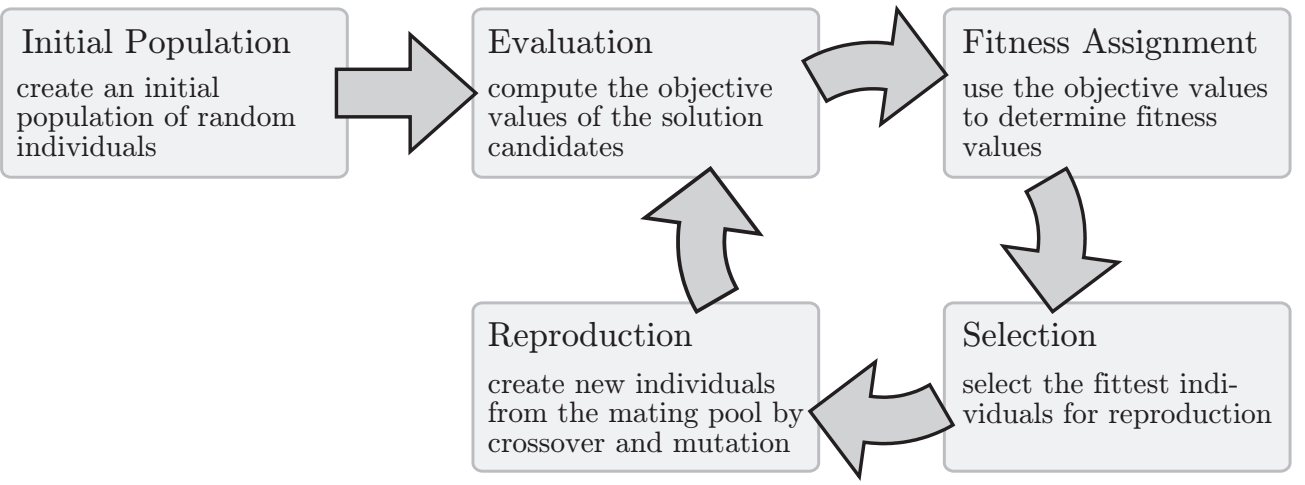

Figure 2.4: The basic cycle of evolutionary algorithms.

$p . g \in \mathbb{G}$ are mapped to corresponding phenotypes $p . x \in \mathbb{X}$, the actual solutions candidates, with the genotype-phenotype mapping $(p \cdot x=\operatorname{gpm}(p . g))$. Subsequently, a vector of objective values $F(p . x)$ is determined for each solution candidate. These vectors form the basis of a fitness assignment process, which attributes a fitness value $v(p . x)$ to every single phenotype describing its relative utility as solution compared to the other members of the population.

A selection algorithm now picks the most interesting individuals (usually in a randomized manner) and places them in into the mating pool. Then, reproduction operations such as mutation and recombination are applied to the genotypes of these individuals in order to create new points in the search space. This cycle is repeated until the termination criterion is met, which may happen when a satisfyingly good solution has been found or a maximum number of generations ${ }^{1}$ has been exhausted, for instance.

\subsection{Genetic Algorithms}

Genetic algorithms (GAs) are a subclass of evolutionary algorithms where the elements of the search space $\mathbb{G}$ are usually binary strings or arrays of integers. Genetic algorithms are often used with genotypes of a fixed length $[129,293]$. In the context of this work, however, genomes which consist of a variable number of genes are utilized as search space for three of the Genetic Programming approaches (LGP, Fraglets, and RBGP). In this section, we will therefore shortly discuss the search operations available for such variable-length strings.

\subsubsection{Mutation: Unary Reproduction}

Mutation is an important method for preserving the diversity of the solution candidates by introducing small, random changes into them. This can be achieved by randomly modifying the value (allele) of a gene, as illustrated in Figure 2.5. More generally, a mutation may change $0<n<\operatorname{len}(g)$ locations in the genotype $g$ at once. In binary coded chromosomes, these genes would be bits which can simply be toggled. Variable-length genomes additionally allow inserting new genes with randomly chosen allelic state into the genotypes (Fig. 2.5.d). This operation can be reversed by deleting gene sequences as sketched in Fig. 2.5.e. Insertion and deletion may also implicitly be performed with crossover operations.

\footnotetext{
${ }^{1}$ Iterations in evolutionary algorithms are usually referred to as generations.
} 


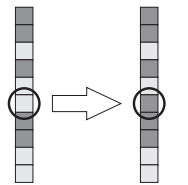

Fig. 2.5.a: Singlegene mutation.

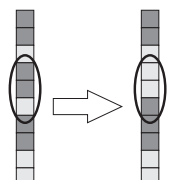

Fig. 2.5.b: Multigene mutation (1).

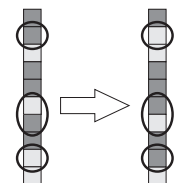

Fig. 2.5.c: Multigene mutation (2).

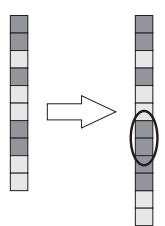

Fig. 2.5.d: In- Fig. 2.5.e: Delesertion of random tion of genes. genes.

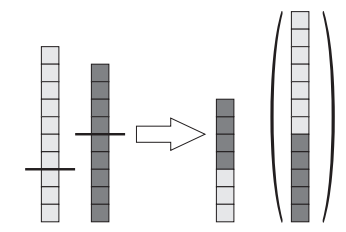

Fig.

Single-Point Crossover 2.5.f:

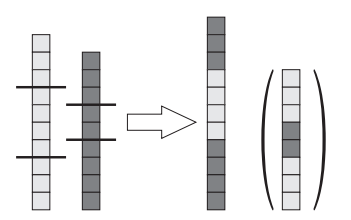

Fig.

Two-Point Crossover

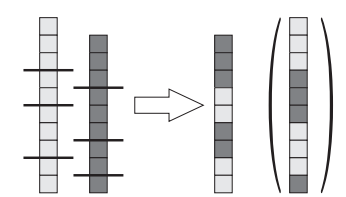

Fig.

Multi-Point Crossover 2.5.h:

Figure 2.5: Mutation and crossover operators for string chromosomes.

\subsubsection{Crossover: Binary Reproduction}

Amongst all evolutionary algorithms, genetic algorithms have the recombination operation which probably comes closest to the natural paragon. Figure 2.5 also illustrates different ways of recombining two string chromosomes by swapping parts of them. The lengths of the new strings resulting from such crossover or cut and splice operations may differ from the lengths of the parents. A special case of string recombination is the homologous crossover, where only genes at the same loci are exchanged. This method is discussed thoroughly in Section 5.3.1 on page 77.

\subsection{Genetic Programming}

The term Genetic Programming (GP) $[232,293]$ has two possible meanings. On one hand, it is often used to subsume all evolutionary algorithms that have tree data structures as genotypes. We include three such approaches (SGP, eSGP, and eRBGP) in our experiments. On the other hand, GP can also be defined as the set of all evolutionary algorithms that breed programs, algorithms, and similar constructs. From this point of view, it comprises all the techniques discussed in this work.

Tree-based Genetic Programming, usually referred to as Standard Genetic Programming (SGP), is the most widespread Genetic Programming variant, both for historical reasons and because of its efficiency in many problem domains.

\subsubsection{Mutation: Unary Reproduction}

Tree genotypes may undergo small variations during the reproduction process in the evolutionary algorithm. Such a mutation is usually defined as the random selection of a node in the tree, removing this node and all of its children, and finally replacing it with another node. From this idea, three operators can be derived:

1. replacement of existing nodes with randomly created ones (Fig. 2.6.a), 
2. insertions of new nodes or small trees (Fig. 2.6.b), and

3. the deletion of nodes, as illustrated in Fig. 2.6.c.

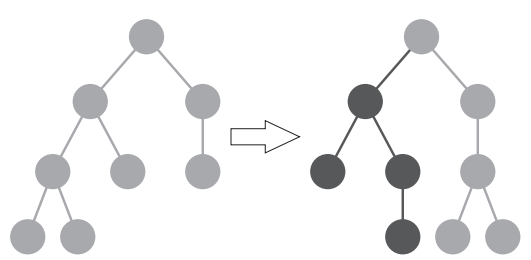

Fig. 2.6.a: Sub-tree replacement.

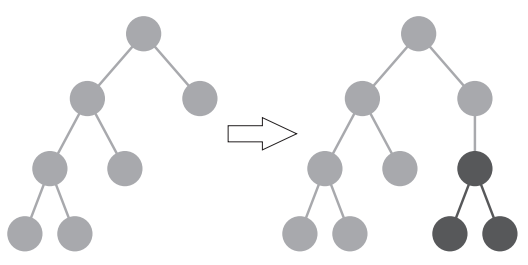

Fig. 2.6.b: Sub-tree insertions.

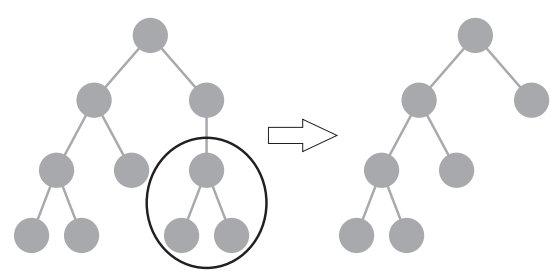

Fig. 2.6.c: Sub-tree deletion.

Figure 2.6: Possible tree mutation operations.

\subsubsection{Recombination: Binary Reproduction}

As illustrated in Figure 2.7, applying the default sub-tree exchange recombination operator to two trees means to swap sub-trees between them. Therefore, one single sub-tree is selected randomly from each of the parents and subsequently cut out and reinserted in the partner genotype. Notice that, like in genetic algorithms, the effects of insertion and deletion operations can also be achieved by recombination.
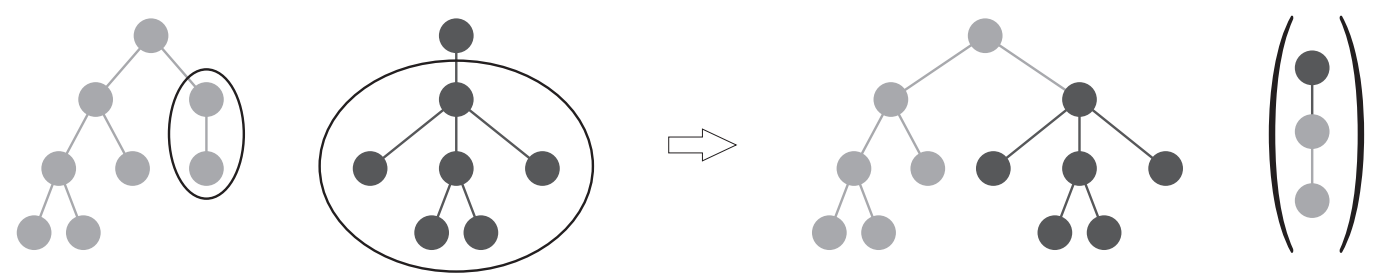

Figure 2.7: Tree crossover by exchanging sub-trees.

\subsubsection{Automatically Defined Functions}

The concept of automatically defined functions (ADFs) introduced by Koza [165] provides some sort of pre-specified modularity for Genetic Programming. Finding a way to evolve modules and reusable building blocks is one of the key issues in using GP to derive higherlevel abstractions and solutions to more complex problems [6]. If ADFs are used, a certain 
structure is defined for the genome. The root of the tree usually loses its functional responsibility and now serves only as glue that holds the individual together and has a fixed number $n$ of children. When evaluating the fitness of an individual, often only the first branch is taken into consideration whereas the root and the other children are ignored. This result-generating branch, however, may use any of the automatically defined functions to produce its output.

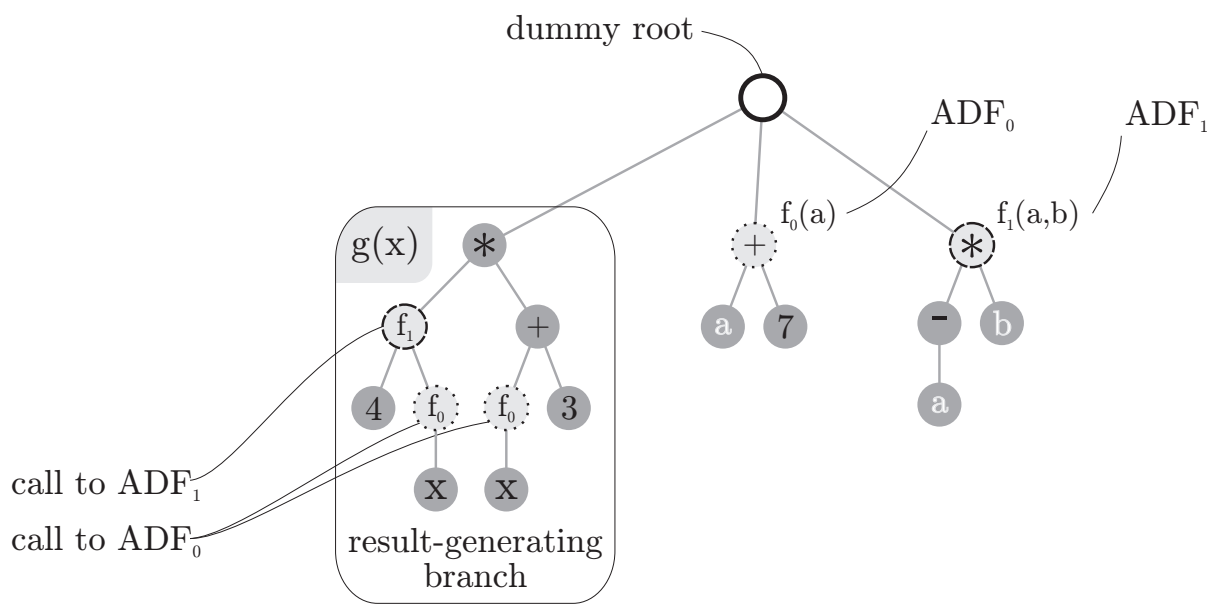

Figure 2.8: A concrete example for automatically defined functions.

When ADFs are employed, typically not only their number must be specified beforehand but also the number of arguments of each of them. How this works can maybe best illustrated by using the example given in Figure 2.8.

Assume that the goal of GP is to approximate a function $g$ with the one parameter $x$ and that a genome is used where two functions $\left(f_{0}\right.$ and $\left.f_{1}\right)$ are automatically defined. $f_{0}$ has a single formal parameter $a$ and $f_{1}$ has two formal parameters $a$ and $b$. The genotype Figure 2.8 encodes the following mathematical functions:

$$
\begin{aligned}
g(x) & =f_{1}\left(4, f_{0}(x)\right) *\left(f_{0}(x)+3\right) \\
f_{0}(a) & =a+7 \\
f_{1}(a, b) & =(-a) * b
\end{aligned}
$$

Hence, $g(x) \equiv((-4) *(x+7)) *((x+7)+3)$. The number of children of the function calls in the result-generating branch must be equal to the number of the parameters of the corresponding ADF.

In this work, two of the tree-based approaches (SGP and eSGP) use ADFs. Instead of defining a fixed total number of functions for these programs, we only specify a lower bound. Thus, the GP process is allowed to acquire additional functions by itself. The first ADF is also used as result-generating branch and the number of parameters of the functions is variable and is determined on the fly.

\subsection{Classifier Systems}

\subsubsection{Introduction}

In the late 1970s, Holland, the father of genetic algorithms, also invented the concept of classifier systems (CS) [132] which consist of four major parts: 
1. a set of interacting production rules, called classifiers,

2. a performance algorithm which directs the actions of the system in the environment,

3. a learning algorithm which keeps track on the success of each classifier and distributes rewards, and

4. a genetic algorithm which modifies the set of classifiers so that variants of good classifiers persist and new, potentially better ones are created in an efficient manner [131].

Here, classifier systems deserve some attention since they provided the inspiration for two of the Genetic Programming approaches studied: RBGP (Section 5.5) and eRBGP (Section 5.6). We will, however, omit any elaborations on their most popular form - the Learning Classifier Systems (LCSs) - since the learning features (points 3 and 4) provided by them are not relevant in the context this work.

\subsubsection{A Small Example}

In order to describe how basic classifier systems are structured in rules and messages, we borrow a simple example from Heitkötter and Beasley [120], a classifier system able to control a frog. The simulated frog likes to eat nutritious flies. Therefore, it can detect small, flying objects and eat them if they are right in front of it. The frog also has a sense of direction and can distinguish between objects which are in front, to the left, or to the right of it and may also turn into any of these directions. It can furthermore distinguish objects with stripes from those without. Flying objects with stripes are most likely bees or wasps, eating of which would probably result in being stung. The frog can also sense large, looming objects far above: birds, which should be avoided by jumping away quickly. The corresponding behavior can be compiled into the form of simple if-then rules which are listed in Table 2.1.

\begin{tabular}{lll}
\hline No. & premise (if-part) & conclusion (then-part) \\
\hline 1 & small, flying object with no stripes to the left & send $a$ \\
2 & small, flying object with no stripes to the right & send $b$ \\
3 & small, flying object with no stripes to the front & send $c$ \\
4 & large, looming object & send $d$ \\
5 & $a$ and not $d$ & turn left \\
6 & $b$ and not $d$ & turn right \\
7 & $c$ and not $d$ & eat \\
8 & $d$ & move away rapidly \\
\hline
\end{tabular}

Table 2.1: if-then rules for frogs.

\subsubsection{Messages}

In Figure 2.9, we demonstrate how the messages in a classifier system that drives such a frog can be encoded. Here, input information as well as action commands (the conclusions of the rules) are compiled in one message type. Also, three bits are assigned for encoding the internal messages $a$ to $d$. Two bits would not suffice, since 00 occurs in all "original" input messages. At the beginning of a classification process, the input messages are written to the message list. They contain information only at the positions reserved for detections and have zeros in the bits for memory or actions. The classifiers transform them to internal messages which normally have only the bits marked as "memory" set. These messages are finally transformed to output messages by setting some action bits. In our frog system, a message is in total $k=12$ bits long. 

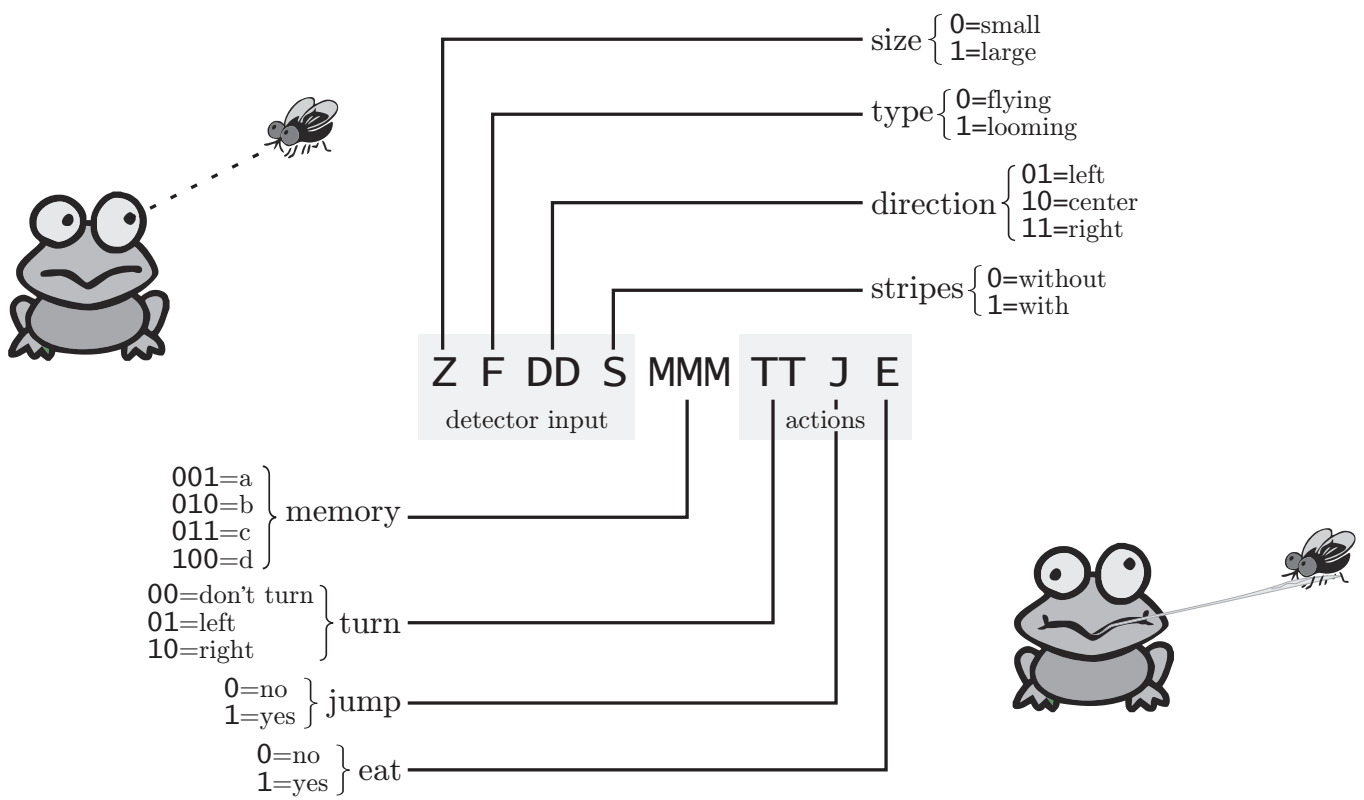

Figure 2.9: One possible encoding of messages for a frog classifier system.

\subsubsection{Conditions and Actions}

Rules in classifier systems consist of a condition part and an action part. The conditions have the same length $k$ as the messages. Instead of being binary encoded strings, a ternary system consisting of the symbols 0,1 , and $*$ is used. In a condition,

1. 0 means that the corresponding bit in the message must be 0 ,

2. 1 means that the corresponding bit in the message must be 1 , and

3. * means don't care, i. e., the corresponding bit in the message may be 0 as well as 1 for the condition to match.

The conditional part of a rule may consist of multiple conditions which are implicitly concatenated with logical and $(\wedge)$. A classifier is satisfied if all its conditions are satisfied by at least one message in the current message list. It is allowed that each of the conditions of a classifier may match to different messages. The action part of a rule has normally the same length as a message and is simple copied to the message list if the classifier is satisfied.

\subsubsection{Classifiers}

A classifier (rule) $x$ consists of a condition part $C(x)$ and an action part $a(x) . \quad C$ is a list of $r \in \mathbb{N}$ conditions $c_{i}$, and we distinguish between representations with $(C=$ $\left.\left(n_{1}, c_{1}, n_{2}, c_{2}, \ldots, n_{r}, c_{r}\right)\right)$ and without negation symbol $\left(C=\left(c_{1}, c_{2}, \ldots, c_{r}\right)\right)$. Based on the encoding scheme defined in Figure 2.9, we can translate Table 2.1 into a set of classifiers for controlling our frog. We therefore compose the condition parts of two conditions $c_{1}$ and $c_{2}$ with the negation symbols $n_{1}$ and $n_{2}$, i. e., $r=2$. Table 2.2 contains the result of this encoding. 


\begin{tabular}{|c|c|c|c|c|c|c|c|c|c|c|c|c|c|c|c|c|c|c|c|c|c|c|c|c|}
\hline No. & $n_{1}$ & & & & & $c_{1}$ & & & & $n_{2}$ & & & & & $c_{2}$ & & & & & & & $a$ & & \\
\hline 1 & 0 & 0 & 00 & & 0 & $* * *$ & $* *$ & $*$ & $*$ & 0 & & * & $*$ & $*$ & $* * *$ & $* *$ & & $*$ & & 000 & 0 & 0010 & 00 & 00 \\
\hline 2 & 0 & 0 & 01 & 11 & 0 & *** & $* *$ & $*$ & $*$ & 0 & * & $*$ & $*$ & $*$ & $* * *$ & $* *$ & $*$ & * & 00 & 000 & 0 & 0100 & 00 & 00 \\
\hline 3 & 0 & 0 & 01 & 10 & 0 & $* * *$ & $* *$ & $*$ & * & 0 & $*$ & * & $*$ & $*$ & $* * *$ & $* *$ & * & $*$ & 00 & 000 & 0 & 0110 & 000 & 00 \\
\hline 4 & 0 & 1 & $1 *$ & $* *$ & * & *** & $* *$ & $*$ & & 0 & * & $*$ & $* *$ & $*$ & $* * *$ & $* *$ & * & * & 00 & 000 & 0 & 1000 & $00 \mathrm{C}$ & 00 \\
\hline 5 & 0 & * & $* *$ & $* *$ & * & 001 & $* *$ & $*$ & * & * & * & * & $* *$ & * & 100 & $* *$ & * & * & 00 & 000 & 0 & 0000 & 01 & 00 \\
\hline 6 & 0 & $*$ & $* *$ & ** & * & 010 & $* *$ & * & * & $*$ & & $*$ & :* & * & 100 & $* *$ & * & * & 00 & 000 & 0 & 0001 & 10 & 00 \\
\hline 7 & 0 & $*$ & $* *$ & ** & * & 011 & $* *$ & * & $*$ & * & & $*$ & ** & & 100 & $* *$ & * & * & 00 & 000 & 0 & 0000 & 00 & 01 \\
\hline 8 & 0 & * & $* *$ & ** & * & 100 & $* *$ & $*$ & * & 0 & * & * & $*$ & * & $* * *$ & $* *$ & $*$ & * & 00 & 000 & 0 & 0000 & 00 & 10 \\
\hline
\end{tabular}

Table 2.2: The encoded form of the if-then rules for frogs from Table 2.1. 


\section{Related Work}

We have introduced optimization algorithms as methods for finding optimal configurations of different features of their solution candidates in Chapter 2. Many aspects of distributed systems are configurable or depend on parameter settings, such as the topology, security, and routing. Hence, there is a huge potential for using metaheuristic optimization algorithms in order to improve them.

And indeed, this potential is widely utilized. The study by Sinclair [256] from 1999 reported that more than 120 papers had been published on work which employed Evolutionary Computation for optimizing network topologies, dimensioning, node placement, routing, and wavelength or frequency allocation. The comprehensive master's thesis by Kampstra from $2005[152,153]$ builds on this aforementioned study and classifies over 400 papers. According to Kampstra, communication networks was the field with the most researchers listed in EvoWeb, the European Network of Excellence in Evolutionary Computing, in 2005. The first workshop on this topic, Evolutionary Telecommunications [257], took place in 1999. In the year 2000 alone, two books ([52] and [223]) have been published on the application of Evolutionary Computation to networking. Further summary papers appeared around the same time [253, 222, 277, 287]. The recent studies from Alba and Chicano [4] and Cortés Achedad et al. [53] as well as the high number of papers published every year show that the interest in applying metaheuristic optimization techniques in this problem domain has by no means decreased.

Most of the mentioned summaries concentrate on giving an overview in form of a more prosaic version of paper listings. In [303], we provide such a listing too, but enrich it with many clear and detailed in-depth discussions of example applications. We therefore studied more than 130 papers from two decades of research in evolutionary telecommunication. Figure 3.1 illustrates how these papers distribute over the time from 1987 to 2008. We classified them according to the area of application, their optimization goals, problem representations, and the optimization algorithms utilized. Figure 3.2 gives an overview of which areas were tackled and which optimization algorithms were applied in the papers we studied. $^{1}$

Here, we will give a small selection from these discussions containing the applications closest related to our work only. In Section 3.1, the evolution of routing protocols will be discussed and different approaches to the synthesis of general protocols with metaheuristic optimization algorithms are summarized in Section 3.2.

\footnotetext{
${ }^{1}$ A paper may deal with multiple applications at once (like routing algorithms which also perform load balancing) and thus may appear in multiple columns.
} 


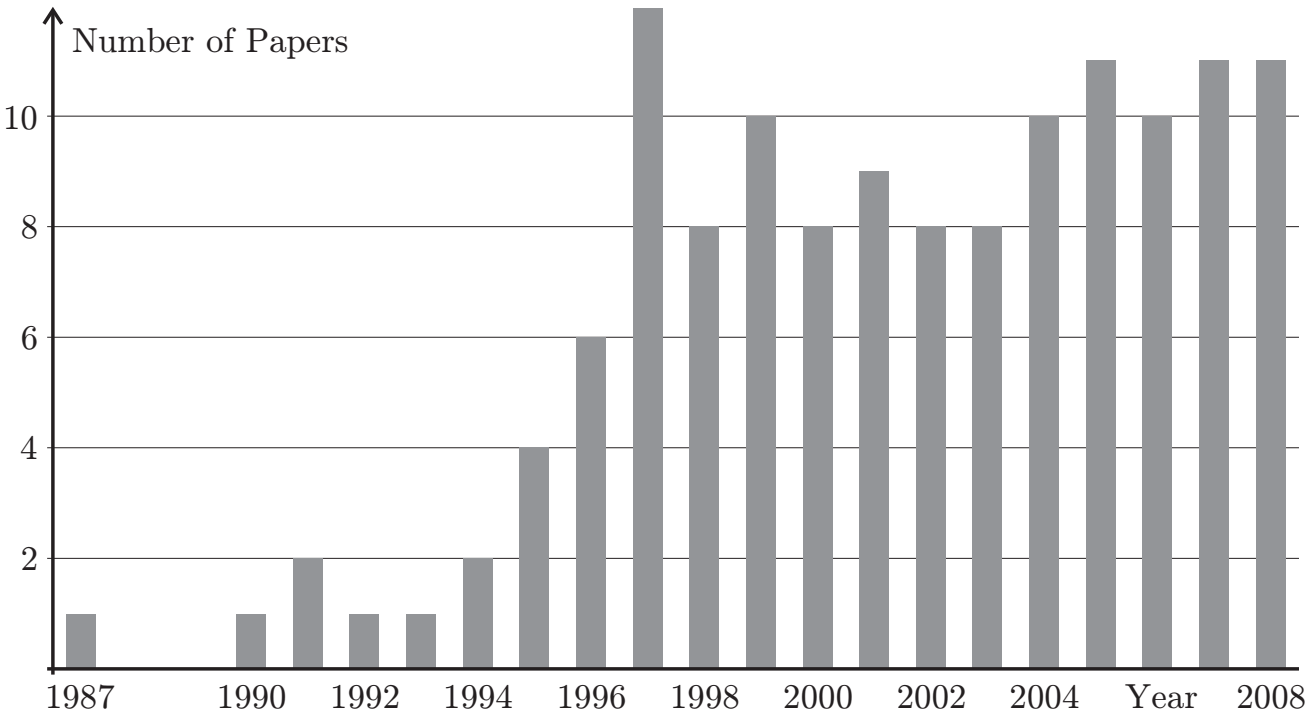

Figure 3.1: The number of papers studied in [303, 293] per year.

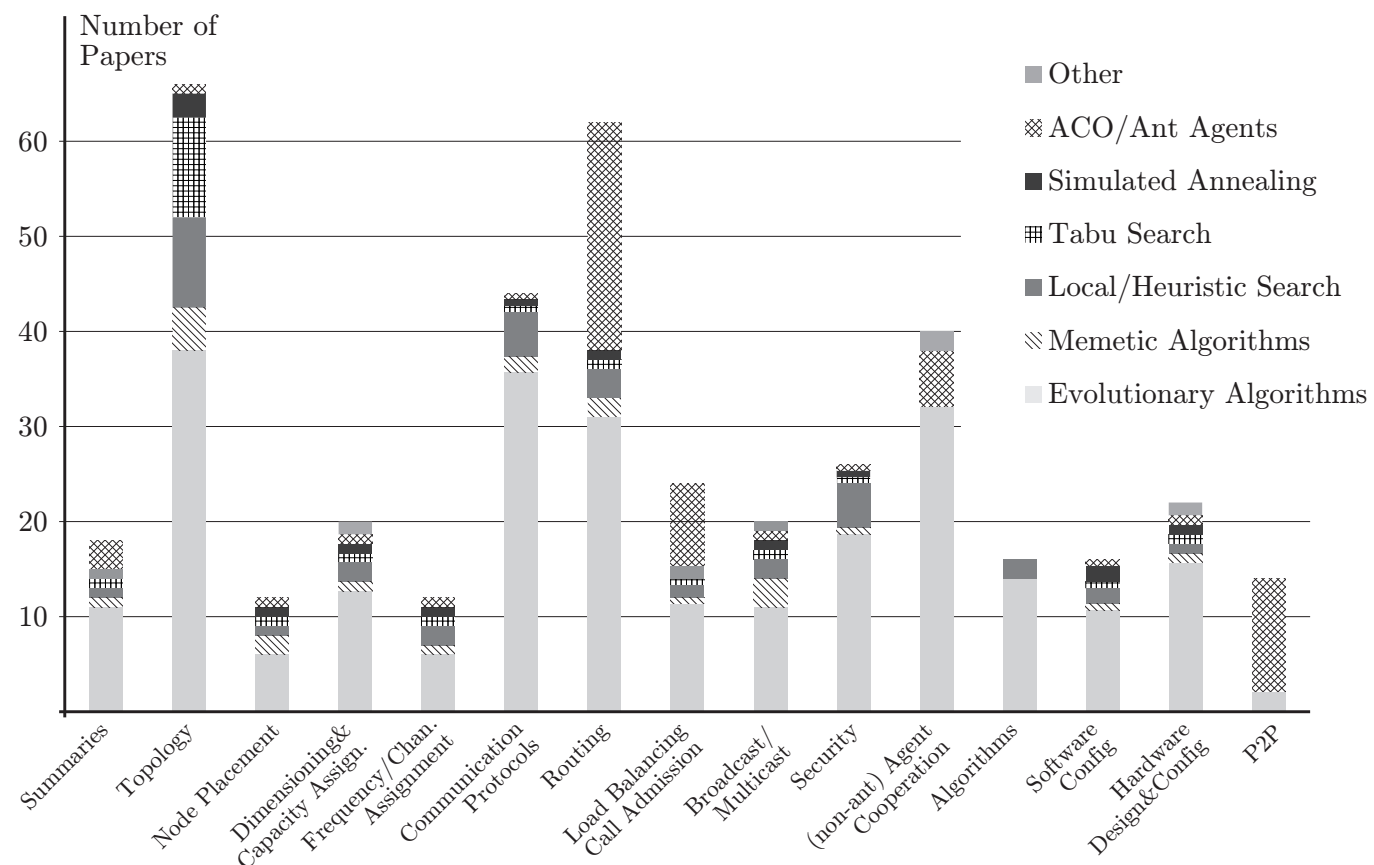

Figure 3.2: The number of papers analyzed, broken down to application area and optimization method [303, 293]. 


\subsection{Optimizing Routing}

Routing is the way in which messages are relayed over multiple nodes in a network in order to reach their destination. Naturally, there often exist multiple different routes from a source to a destination. The objective of a routing algorithm is to guide the messages along the shortest $^{2}$ possible routes. However, a path in the network may get blocked if any of its nodes is congested, i.e., has to process too many messages at once. Hence, routing algorithms have to be robust and should be able to deliver messages even if links fail.

In this section, we will discuss three approaches for improving routing with metaheuristic optimization algorithms, spanning from the evolution of routing rules to the synthesis of broadcast algorithms.

\subsubsection{Evolving Fault-tolerant Routing Rules}

Routing is tightly coupled with the problem of finding the shortest paths from one vertex in a graph to all others. This problem has been solved in 1959 by Dijkstra [70]. About forty years later, Kirkwood et al. [157, 254] used Genetic Programming to breed robust routing rules for networks where links may fail.

Their Genetic Programming system takes the ID of a target node and a graph (containing this node) as input. Each node in the graph has a unique ID and each connection has a certain length. The system then breeds routing rules in form of LISP S-expressions. In the context of this application, these expressions are trees consisting of nested instances of the domain-specific IF-CUR-GO instruction. The four parameters W, X, Y, and Z of this instruction as well as its return value each represent a node ID. On each node reached by a message, the routing rule is evaluated and the message is sent to the node whose ID is returned. IF-CUR-GO W X Y Z evaluates to X if the current node is $\mathrm{W}, \mathrm{X}$ and $\mathrm{W}$ are directly connected, and $\mathrm{X}$ has not yet been visited twice. It returns $\mathrm{Y}$ if one of the latter two conditions is violated and $\mathrm{z}$ if the current node is not $\mathrm{W}$.

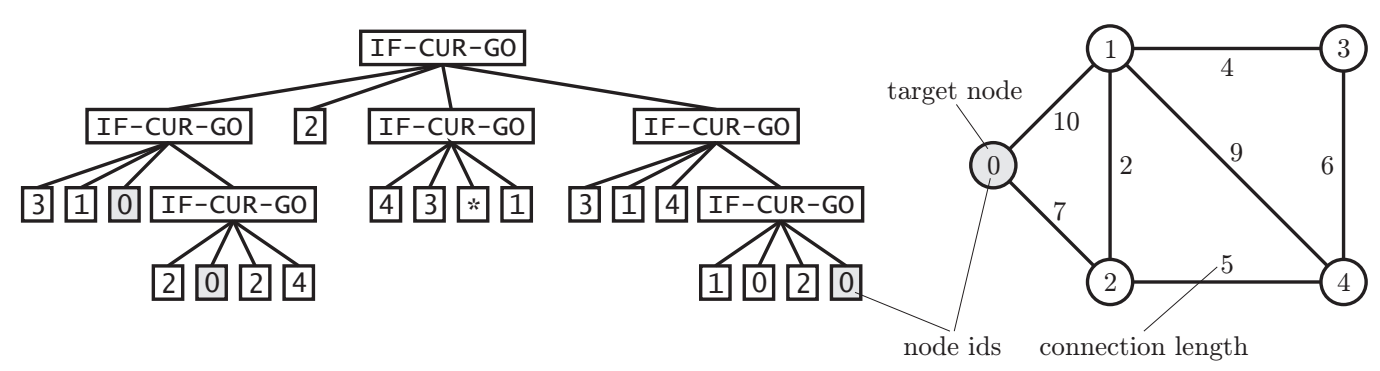

Figure 3.3: An evolved routing rule set (left) with target node 0 for the specified network (right).

As objective function, Kirkwood et al. use the difference in length between the optimal route (computed with Dijkstra's algorithm) and the routes obtained with the evolved scheme on the target network with and without simulated link failures. The results of the evolution were rules that are very robust against connection loss and, for instance, find correct routes in two third of all cases even if $47 \%$ of the connections have broken down. In Figure 3.3, a routing rule set resulting from one of the experiments [157] is displayed on the left. It routes messages through the graph sketched on the right-hand side to node 0 .

\footnotetext{
${ }^{2}$ According to some appropriate metric such as latency.
} 


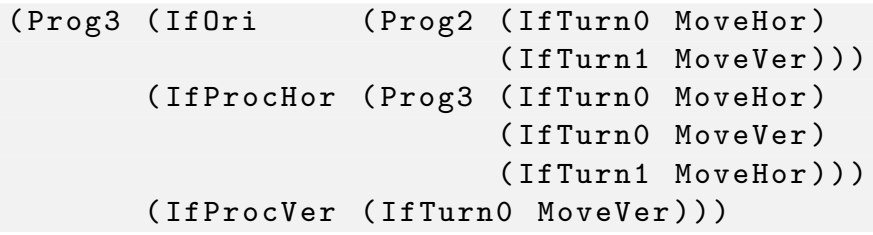

Listing 3.1: The optimal broadcasting scheme evolved for 2-dimensional, directed grids.

\subsubsection{Genetic Programming of Broadcasting Algorithms}

Routing does not necessarily concern the delivery of messages to single destinations only, but may also involve $1: n$ communications, i. e., broadcasts and multicast. Broadcasting in a network (represented as a graph) is the process of spreading information, which is initially known to only one node, to all other nodes. Comellas and Giménez [51] formulated an optimization problem with the following constraints:

1. Only a node which already knows the information can spread it further.

2. A node can only send one message per time step.

3. A node can only send a message over vertexes which are connected to it.

The goal of their work was to find the broadcasting scheme which disseminates the information to all nodes in the shortest possible time. They used Genetic Programming for growing such broadcasting algorithms for two-dimensional directed grids, toroidal grids, hypercubes, cube-connected cycles, and butterfly graphs. For the butterfly graph, a solution even better than the best known upper bound at that time [159] was found, whereas in all other cases, (known) optimal algorithms evolved.

\section{Example: Two-Dimensional Directed Grid}

In their experiments, Comellas and Giménez used Standard Genetic Programming for evolving Lisp S-expressions. The function set for the directed 2-grid example contained the following expressions:

1. IfOri executes its parameter action if the node the program runs on was the one which initially knew the information to be disseminated.

2. IfProcHor and IfProcVer execute their parameter if they received the information from a horizontally or vertically adjacent node, respectively.

3. Proc1, Proc2, Proc3, and Proc4 concatenate 1, 2, 3, or 4 instructions to a sequential block.

4. IfTurn0/IfTurn1 execute their actions only in the first/second time step after the node received the information.

As terminal symbols, MoveHor and MoveVer are available, which send the information to the next horizontally or vertically adjacent node, as well as NULL which does nothing.

For computing the objective values, the algorithms evolved were applied to an example graph. The number of nodes reached was counted and multiplied with a factor $T$. From this value, 2 is subtracted for each condition value or NULL action and 4 for each other action in the tree. The number of the ProgN functions had no influence on fitness. Listing 3.1 illustrates the result delivered by Genetic Programming for two-dimensional directed grids.

In Figure 3.4, we applied this algorithm to a $5 \times 5$ grid. The gray nodes have no knowledge about the information and turn black once they receive it. It is interesting to know that the IfTurno MoveVer in line 4 of Listing 3.1, although seemingly useless [51], is important. 

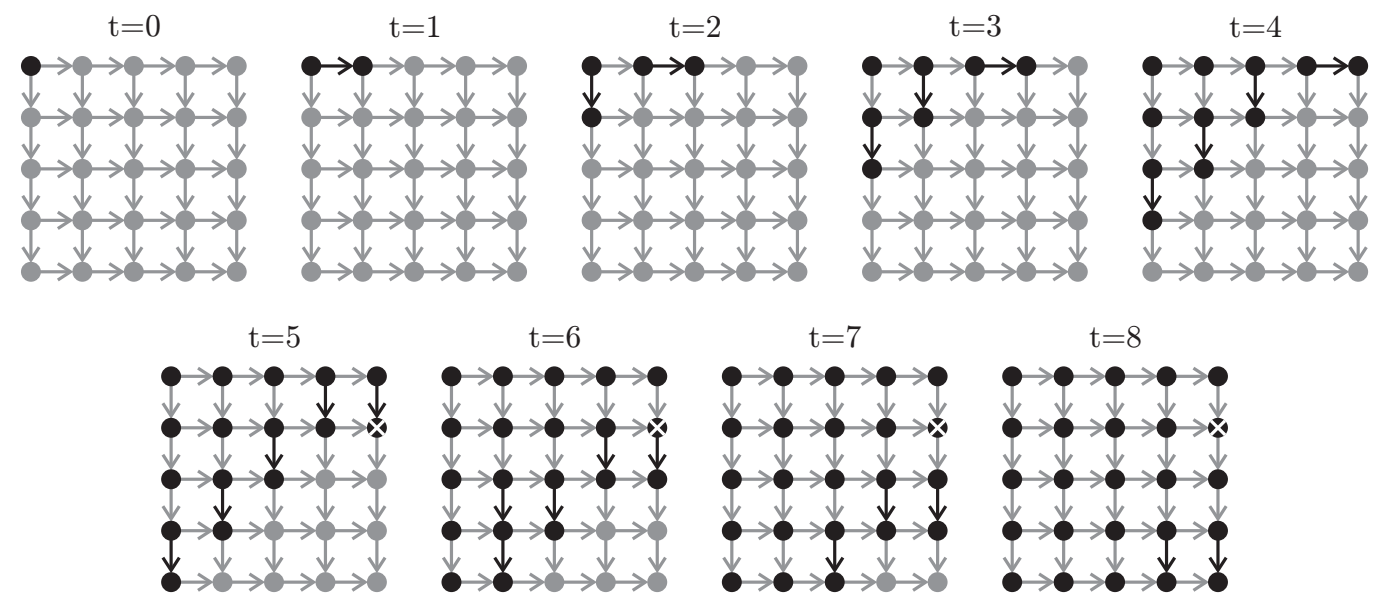

Figure 3.4: The application of Listing 3.1 to a $5 \times 5$ grid.

Without it, the last node in the second line in the network illustrated in Figure 3.4 would not be reached in time step 5 but in step 6 , since the MoveHor action cannot be executed by the last node in the first line. With the MoveVert instruction with the same preconditions, an unnecessary pause is avoided. For the other topologies mentioned, Comellas and Giménez [51] used modified function and terminal sets and obtained optimal results, too.

\subsubsection{Optimizing Collective Communication}

Recently, Jaroš and Dvořák [147] developed a scheduling technique for similar collective communication (CC) tasks on basis of evolutionary algorithms. Their work addresses highperformance multi-processor systems where single CPUs are connected via NoCs (networks on chip). Jaroš and Dvořák experiment with one-to-all broadcast (OAB), all-to-all broadcast (AAB), one-to-all scatter (OAS, a private message to each partner), and all-to-all scatter (AAS) [147].

Each collective communication process is considered as a set of point-to-point communications which can further be partitioned into subsets. The communications in each subset are executed in parallel and the subsets themselves are executed one after the other in a sequence of synchronized steps. The $C C$ scheduling problem is defined as finding a minimal partition without causing conflicts.

Jaroš and Dvořák introduce different encodings for broadcast and scatter communication as well as special reproduction and repairing operators. Their objective function corresponds to the number of conflicts that a schedule introduces, i. e., the number of times that two point-to-point communications share the same link in the same time step. The experimental results showed that this approach is very efficient and even led to an improvement of the theoretical lower bounds of the number of steps needed for AAS communication.

\subsection{Synthesizing Protocols}

Protocols like IP [140] and TCP [45] are the rules for message and information exchange in a network. Depending on the application, protocols can become arbitrarily complex and strongly influence the efficiency and robustness of a distributed system. 


\subsubsection{Transformation of Finite State Machine-based Specifications}

An approach for synthesizing protocol specifications from service specifications has been contributed by de Araújo et al. $[60,61]$. We will use the communication protocol example given in [60] to summarize this method in which all specifications are represented as finite state machines.

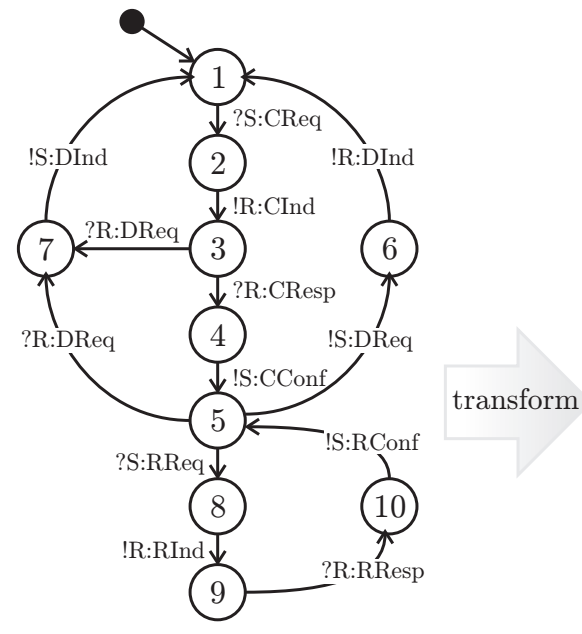

Global Service Specification

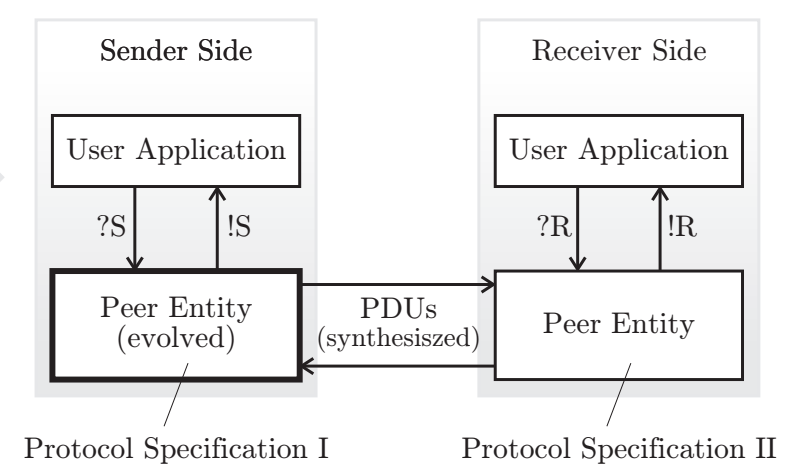

Figure 3.5: The scenario for protocol synthesis as used in [60].

In Figure 3.5, we sketch the starting situation from [60]. At the left side, a global service specification is defined in form of a finite state machine which describes the interactions that take place in a communication protocol between a sender and a receiver. It contains only the interaction primitives between a user (or a user application) and the peer entities, where ?xyz represents an input message xyz from and !xyz stands for the output of xyz to the user. Events on the sender side are marked with $\mathrm{S}$ and those occurring at the receiver are annotated with $\mathrm{R}$.

The finite state machine starts in state 1. On the sender side, the user may then issue a connection request CReq causing a transition to state 2. A window indicating an incoming connection CInd may pop up at the receiver side, for instance, which then can either be confirmed $(3 \rightarrow 4)$ or declined $(3 \rightarrow 7)$. In the latter case, a disconnection indication will ensue on the sender side whereas the former transition leads to a connection confirmation. A connection may be reconnected (states $5,8,9,10)$ or be closed by either the sender $(5 \rightarrow 6)$ or the receiver $(5 \rightarrow 7)$ with a disconnect request DReq, causing a disconnect indication DInd (maybe again in form of a window) to occur on the other end.

The goal is to evolve a finite state machine that governs the behavior of one of the two protocol partners and to synthesize the messages (Protocol Data Units, PDUs) that have to be exchanged between the sender and the receiver. Therefore, de Araújo et al. first create a sequence of interaction primitives by traversing the service specification FSM. In this traversal, all state transitions need to be visited. One such sequence could be (?S:CReq, !R:CInd, ?R:CResp, !S:CConf, ?S:RReq, !R:RInd, ?R:RResp, !S:RConf,!S:DReq, !R:DInd, ?S:CReq, !R:CInd, ?R:DReq, !S:DInd, ?S:CReq, !R:CInd, ?R:CResp, !S:CConf, ?R:DReq, !S:DInd). It is obvious that if one event on the sender side is followed by an event on the receiver side (or vice versa) and the second event is not triggered by the user (?xyz), a message (i. e., a PDU) has to be exchanged between the two sides. These PDUs can either be automatically 
generated or predefined and are then inserted into the sequence, resulting in the list (?S:CReq, pduConReq, !R:CInd, ?R:CResp, pduConAcc, !S:CConf, ?S:RReq, pduReiReq, !R:RInd, ?R:RResp, pduReiAcc, !S:RConf, !S:DReq, pduDisInd, !R:DInd, ?S:CReq, pduConReq, !R:CInd, ?R:DReq, pduDisInd, !S:DInd, ?S:CReq, pduConReq, !R:CInd, ?R:CResp, pduConAcc, !S:CConf, ?R:DReq, pduDisInd, !S:DInd ), for instance.

From this list, the message exchanges are extracted which concern the protocol peer to be evolved. The experiments presented in [60] were focused on the sender side protocol, which here results in the following tuples: ((?S:CReq, pduConAcc), (pduConAcc, !S:CConf), (?S:RReq, pduReiReq), (pduReiAcc, !S:RConf), (!S:DReq, pduDisInd), (?S:CReq, pduConReq), (pduDisInd, !S:DInd), (?S:CReq, pduConReq), (pduConAcc, !S:CConf), and (pduDisInd, !S:DInd)).

In order to allow the synthesized protocol to react to all possible messages in all states, random non-expected inputs are added to the list like ((CReq, pduConAcc), (CReq, NULL), (pduConAcc, CConf),...). Such a list is then encoded and used as test set during the individual evaluation. For the evolution of the protocol FSM, de Araújo et al. use the Genetic Programming Kernel (GPK) of Hörner [135] which allows for variable-length genomes. The objective function compares the behavior of a solution candidate with the expectations and counts the number of correct outputs.

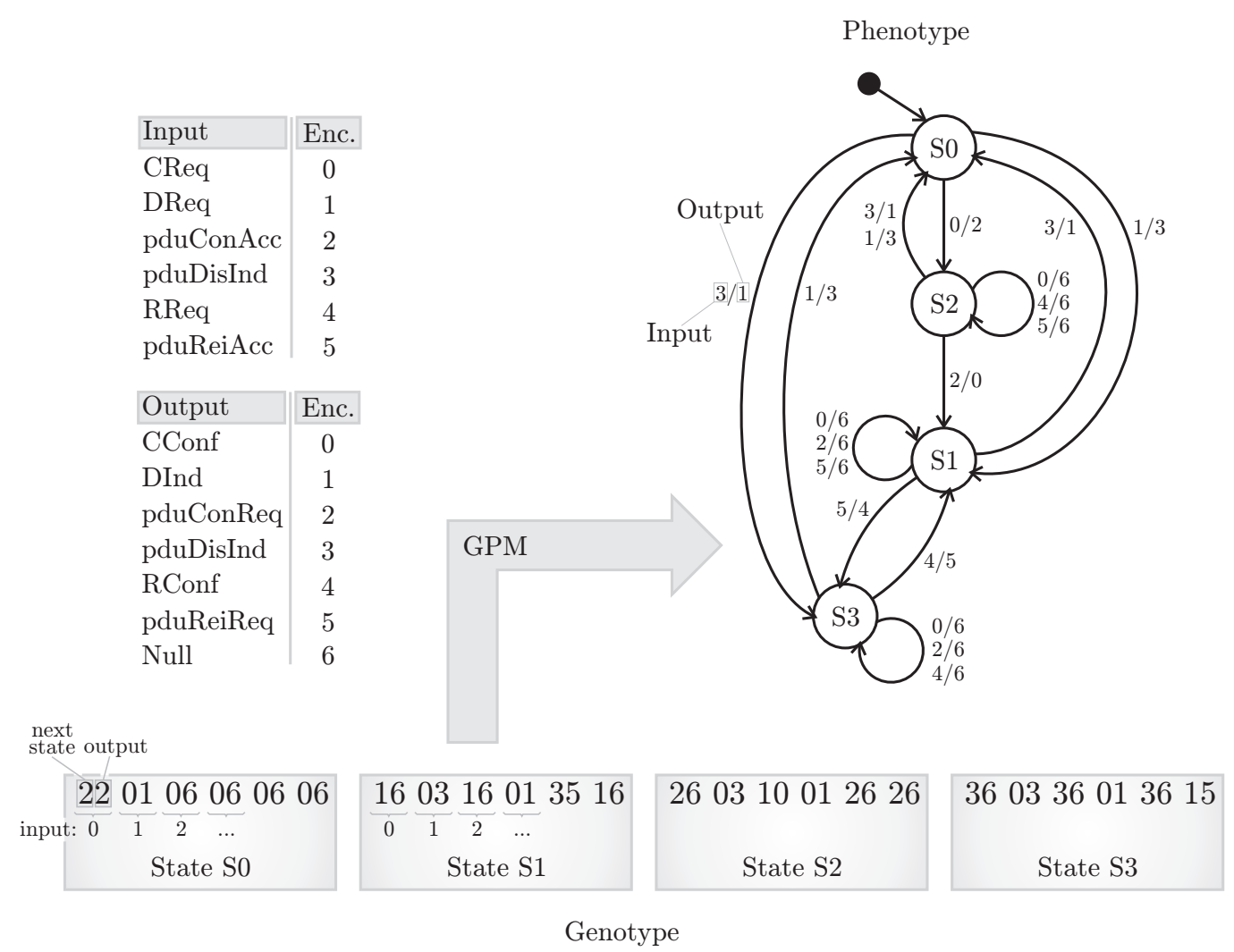

Figure 3.6: The best individual from the experiment of de Araújo et al. [60].

Figure 3.6 illustrates the state-based representation (SBR) used by de Araújo et al. to encode protocol finite state machines. The genotypes are divided into genes and each gene represents one state. The total number of states is variable. For each possible input, every gene contains one tuple of two numbers. The first number in this tuple represents the next state and the second one stands for the output of the corresponding transition. 
The receiver side of the protocol was evolved in the same manner [60] and the results were able to pass an automated equivalence check. The good performance reported further indicates the high utility of this approach for protocol synthesis.

\subsubsection{Evolving Fraglet-based Protocols}

Another interesting piece of related work is the Fraglet language, which we actually utilize in our own experiments and therefore describe in more detail in Section 5.4 on page 79. This form of protocol representation is predestined for evolutionary synthesis: Fraglets have almost no syntactical constraints and can represent complicated protocols in a compact manner. Similar to us, Tschudin investigated the offline evolution of protocols using a genetic algorithm.

In his work, a complete communication system was simulated for a given number of time steps during the evaluation of each Fraglet program. The objective values denote the correlation of the behavior observed during the simulation and the target behavior. Tschudin concluded that evolutionary methods are suitable to optimize existing Fraglet protocols, but also indicated that the evolution of new distributed algorithms is difficult because of a strong tendency to overfitting (see Section 4.7.1) and the all-or-nothing problem known from Genetic Programming (see Section 4.4.2).

Tschudin's approach is tightly related to our envisaged methodology defined in Section 1.2. We even encountered similar problems which are discussed in depth in Chapter 4 where we also outline means to mitigate them. In Section 5.5, we propose a new program representation for this purpose.

\subsubsection{Online Protocol Adaptation and Evolution with Fraglets}

Autonomic networks are networks where manual management is not desired or hard to realize, such as systems of hundreds of gadgets in an e-home, sensor networks, or arbitrary mesh networks with wireless and wired links. Yamamoto and Tschudin [321] pointed out that software in such networks should be self-modifying so as to be able to react to unforeseen network situations. They distinguish two forms of such reactions - adaption and evolution. Adaption is the short-term reconfiguration of existing modules whereas evolution stands for the modification of old and the discovery of new functionality and happens at a larger timescale. Software with these abilities probably cannot predict whether the effects of a modification are positive or negative in advance and therefore, needs to be resilient in order to mitigate faulty code that could evolve. In [282], Tschudin and Yamamoto showed that such resilience can be achieved to a certain degree by introducing redundancy into Fraglet protocols.

Complementing Tschudin's work on offline protocol synthesis and optimization [281], Yamamoto and Tschudin [321] describe online protocol evolution as a continuously ongoing, decentralized, and asynchronous process of constant competition and selection of the most feasible modules. Genetic Programming with mutation and homologous crossover is chosen for accomplishing these features. The fitness measure (subject to maximization) is the performance of the protocols as perceived by the applications running in the network. The score of a solution candidate (i. e., a protocol) is incremented if it behaves correctly and decremented whenever an error is detected. The resource consumption in terms of the memory allocated by the protocols is penalized proportionally.

Yamamoto and Tschudin $[319,320]$ create populations containing a mix of different confirmed delivery and reliable delivery protocols for messages. These populations were then confronted with either reliable or unreliable transmission challenges and were able to adapt to these conditions quickly. If the environment changes afterwards, when a formerly reliable channel becomes unreliable, for example, the degree of re-adaptation was, however, 
unsatisfying. The loss of diversity due to the selection of only highly fit protocols during the adaptation phase could not yet be compensated by mutation in these first experiments.

Further information on approaches for evolutionary online optimization of communication protocols can be found in the report Framework for Distributed On-line Evolution of Protocols and Services, 2nd Edition from the EU-sponsored project BIONETS [200]. 

Part II

Genetic Programming of Distributed Algorithms 



\section{Features which make Genetic Programming Hard}

In Chapter 3, we have given a short overview on some of the ways in which metaheuristics have been applied in order to derive communication protocols. The focus of this work is the use of optimization for synthesizing distributed algorithms. Apparently, there are hardly any contributions to this area except for the work of the author and his colleagues themselves. The research approach which comes the closest to our ideas is the Fraglet-based protocol evolution by Tschudin [281] (Section 3.2.2). Indeed, there are few scientists which use Genetic Programming to derive general, deterministic programs or procedures as we know them from our everyday programming experience.

The question why Genetic Programming is used for many applications but only rarely for the naive interpretation of its name arises. The answer to this question is simple and yet, very unsatisfying: The evolution of "real" algorithms is very hard. So hard, that the optimization process may degenerate to a random walk if no measures are taken.

In this chapter, we will provide an in-depth discussion of the problems which make Genetic Programming of full-fledged algorithms complicated. We will analyze the causes of these problems and point out approaches to mitigate their effects. ${ }^{1}$

Many features of optimization tasks can be illustrated with fitness landscapes. Fitness landscapes are a very powerful metaphor in metaheuristic optimization. Like many other abstractions, they have originally been developed by evolutionary biologists [317, 100]. A fitness landscape is the visualization of the relation between solution candidates (or genotypes) and their corresponding objective values. From its shape, a set of problematic features can directly be recognized. In Figure 4.1, we have sketched a set of different types of fitness landscapes which we are going to discuss. The objective values in the figure are subject to minimization and the small bubbles represent solution candidates under investigation. An arrow from one bubble to another means that the second individual is found by applying one search operation to the first one.

\subsection{Premature Convergence}

\subsubsection{Introduction}

An optimization algorithm has converged if it cannot reach new solution candidates anymore or if it keeps on producing solution candidates from a "small" 2 subset of the problem space. Metaheuristic optimization algorithms will usually converge at some point in time. One of

\footnotetext{
${ }^{1}$ An even more elaborate discussion and analysis can be found in [293]. A general outline of problems in optimization has been given by Weise et al. [306], too.

2 according to a suitable metric like numbers of modifications or mutations which need to be applied to a given solution in order to leave this subset
} 


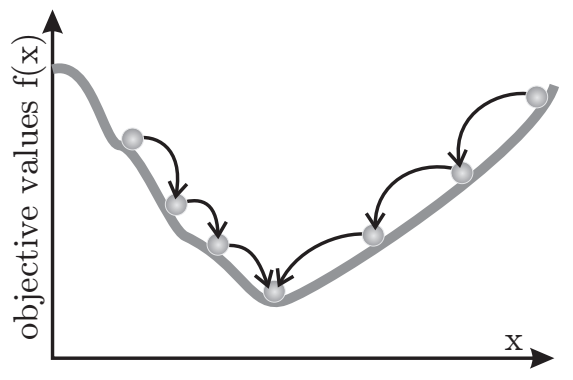

Fig. 4.1.a: Best Case

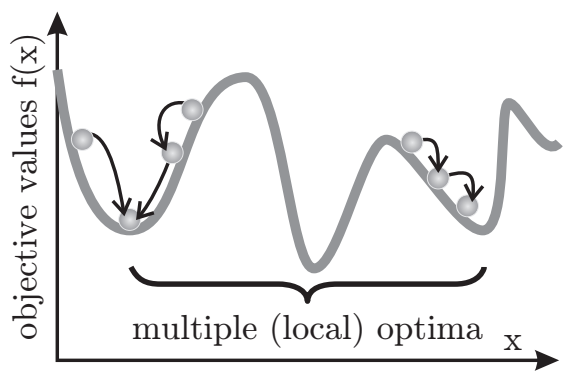

Fig. 4.1.c: Multimodal

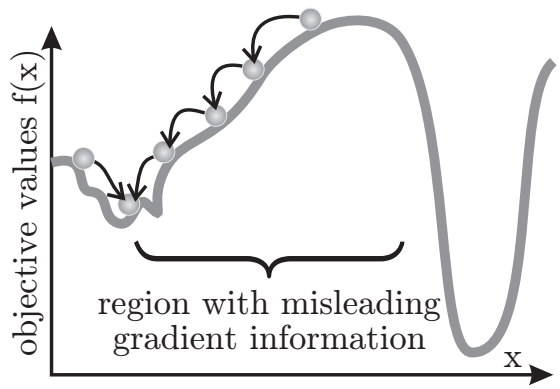

Fig. 4.1.e: Deceptive

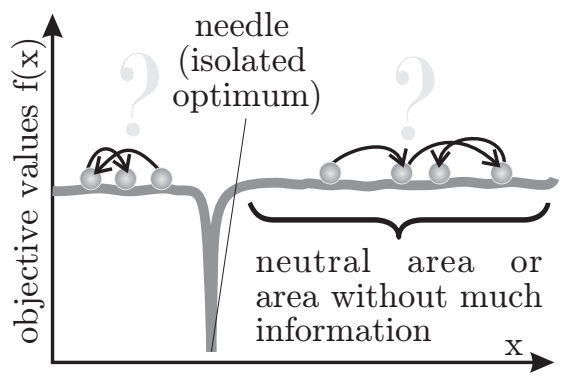

Fig. 4.1.g: Needle-In-A-Haystack

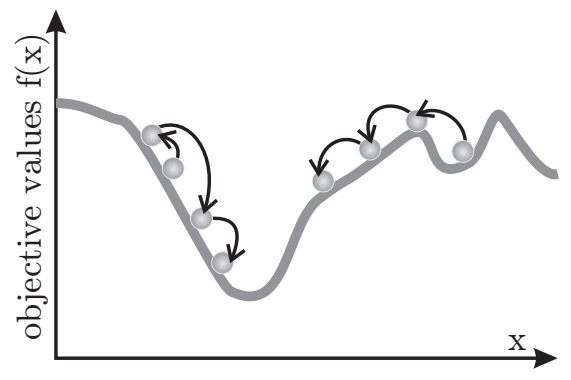

Fig. 4.1.b: Low Variation

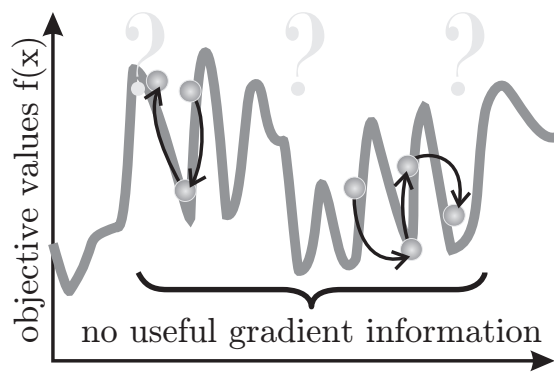

Fig. 4.1.d: Rugged

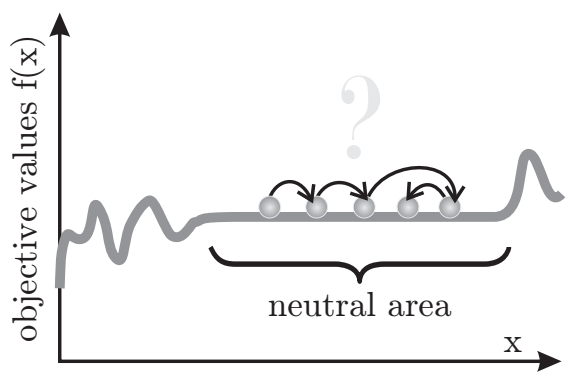

Fig. 4.1.f: Neutral

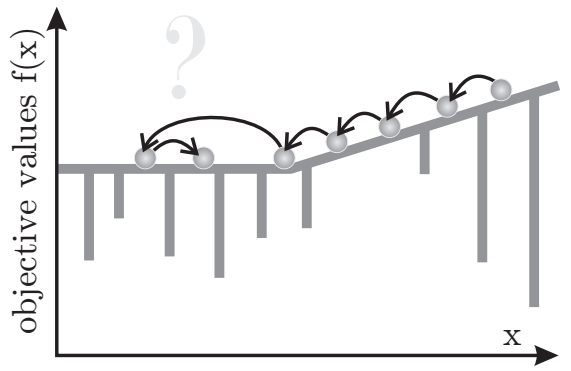

Fig. 4.1.h: Nightmare

Figure 4.1: Different possible properties of fitness landscapes (minimization). 
the problems in optimization is that it is often not possible to determine whether the best solution currently known is situated on a local or a global optimum and thus, if convergence is acceptable. In other words, it is usually not clear whether the optimization process can be stopped, whether it should concentrate on refining the current optimum, or whether it should examine other parts of the search space instead. This can, of course, only become cumbersome if there are multiple (local) optima, i. e., the problem is multimodal as depicted in Fig. 4.1.c.

A mathematical function is multimodal if it has multiple maxima or minima [255]. A set of objective functions (or a vector function) $F$ is multimodal if it has multiple (local or global) optima - depending on the definition of "optimum" in the context of the corresponding optimization problem [293].

\subsubsection{The Problem}

An optimization process has prematurely converged to a local optimum if it is no longer able to explore other parts of the search space than the area currently being examined and there exists another region that contains a superior solution [252].

The existence of multiple global optima itself is not problematic and the discovery of only a subset of them can still be considered as successful in many cases. The occurrence of numerous local optima, however, is more complicated. In Genetic Programming of algorithms, we observe a very rugged fitness landscape and thus, a large number of local optima. Figure 4.2 illustrates examples for prematurely converging optimization processes.

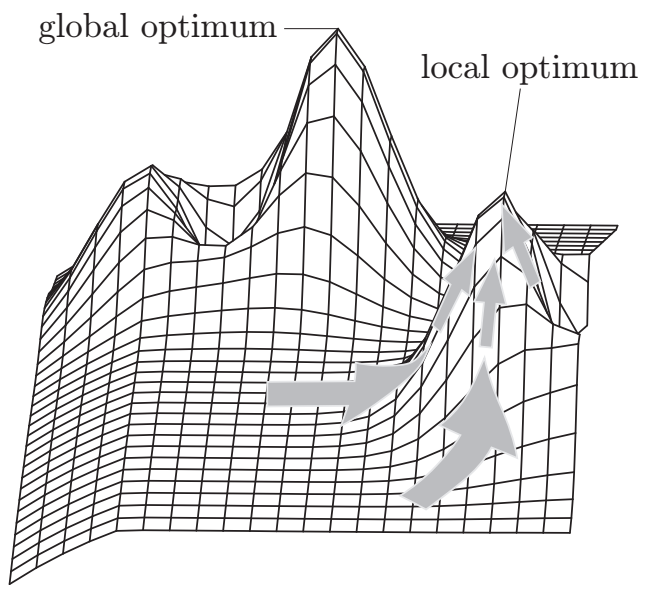

Fig. 4.2.a: Example 1: Maximization

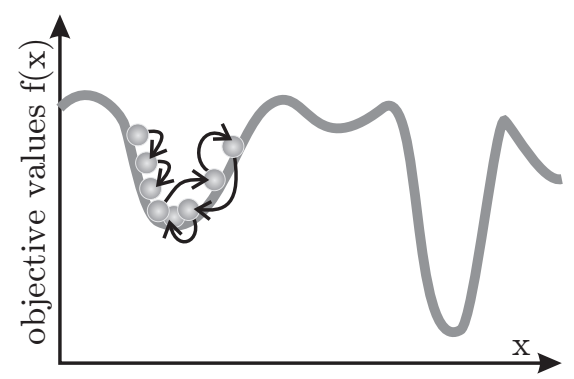

Fig. 4.2.b: Example 2: Minimization

Figure 4.2: Premature convergence in the objective space.

\subsubsection{One Cause: Loss of Diversity}

In biology, diversity is the variety and abundance of organisms at a given place and time $[217,190]$. Much of the beauty and efficiency of natural ecosystems is based on a dazzling array of species interacting in manifold ways. Diversification is also a good investment strategy utilized by investors in the economy in order to increase their profit.

In population-based optimization algorithms, maintaining a set of diverse solution candidates is very important as well (see, for instance, Section 3.2.3). Losing diversity means 
approaching a state where all the solution candidates under investigation are similar to each other. Another term for this state is convergence. Discussions about how diversity can be measured have been provided by Routledge [247], Cousins [55], Magurran [190], Morrison and de Jong [203], and Paenke et al. [217]. Preserving diversity is directly linked with maintaining a good balance between exploitation and exploration [217] and has been studied in the area of Genetic Programming by various researchers [63, 41, 38].

\section{Exploration vs. Exploitation}

The operations which create new solutions from existing ones have a very large impact on the speed of convergence and the diversity of the populations [80, 261]. The step size in Evolution Strategy is a good example of this issue: setting it properly is very important and leads over to the "exploration versus exploitation" problem [125] which can be observed in other areas of optimization as well.

In the context of optimization, exploration means finding new points in areas of the search space which have not been investigated before. Since computers have only limited memory, already evaluated solution candidates usually have to be discarded in order to accommodate the new ones. Exploration is a metaphor for the procedure which allows search operations to find novel and maybe better solution structures. Such operators (like mutation in evolutionary algorithms) have a high chance of creating inferior solutions by destroying good building blocks but also a small chance of finding totally new, superior traits (which, however, is not guaranteed at all).

Exploitation, on the other hand, is the process of improving and combining the traits of the currently known solutions, as done by the crossover operator in evolutionary algorithms, for instance. Exploitation operations often incorporate small changes into already tested individuals leading to new, very similar solution candidates or try to merge building blocks of different, promising individuals. They usually have the disadvantage that other, possibly better, solutions located in distant areas of the problem space will not be discovered.

Almost all components of optimization strategies can either be used for increasing exploitation or in favor of exploration. Unary search operations that improve an existing solution in small steps can often be built, hence being exploitation operators. They can also be implemented in a way that introduces much randomness into the individuals, effectively making them exploration operators. Selection operations in Evolutionary Computation choose a set of the most promising solution candidates which will be investigated in the next iteration of the optimizers. They can either return a small group of best individuals (exploitation) or a wide range of existing solution candidates (exploration).

Optimization algorithms that favor exploitation over exploration have higher convergence speed but run the risk of not finding the optimal solution and may get stuck at a local optimum. Then again, algorithms which perform excessive exploration may never improve their solution candidates well enough to find the global optimum or it may take them very long to discover it "by accident". A good example for this dilemma is the Simulated Annealing algorithm [156]. It is often modified to a form called simulated quenching which focuses on exploitation but loses the guaranteed convergence to the optimum [141]. Generally, optimization algorithms should employ at least one search operation of explorative character and at least one which is able to exploit good solutions further. There exists a vast body of research on the trade-off between exploration and exploitation that optimization algorithms have to face $[129,76,67]$.

\subsubsection{Countermeasures}

There is no general approach which can prevent premature convergence. The probability that an optimization process gets caught in a local optimum depends on the characteristics 
of the problem to be solved and the parameter settings and features of the optimization algorithms applied.

Using low selection pressure in evolutionary algorithms generally decreases the chance of premature convergence but also decreases the speed with which good solutions are exploited. In order to extend the duration of the evolution itself, many methods have been devised for steering the search away from areas which have already been frequently sampled. This can best be achieved by integrating density metrics into the fitness assignment process. In this work, we utilize two complementary approaches of this kind:

1. a fitness assignment process involving niching for increasing the spread of the solution candidates by discouraging tight aggregations (Section D.1.2),

2. a mechanism which increases the reproduction rate of individuals which only exist in small numbers of copies in the population by deleting duplicates with a certain probability (Section D.2).

Especially the latter approach, the simple convergence prevention algorithm $(S C P)$, has shown to be very beneficial in many different applications [293, 228, 305].

\subsection{Ruggedness and Weak Causality}

\subsubsection{The Problem}

Optimization algorithms generally depend on some form of gradient in the objective or fitness space. The objective functions should be continuous and exhibit low total variation, so the optimizer can descend the gradient easily. If the objective functions are unsteady or fluctuating, i. e., going up and down, it becomes more complicated for the optimization process to find the right directions to proceed to. The more rugged a function gets, the harder it becomes to optimize it. From a simplified point of view, ruggedness is multi-modality plus steep ascends and descends in the fitness landscape.

\subsubsection{One Cause: Weak Causality}

During an optimization process, new points in the search space are created by the search operations. Generally we can assume that the genotypes which are the input of the search operations correspond to phenotypes which have previously been selected. Usually, the better or the more promising an individual is, the higher are its chances of being selected for further investigation. Reversing this statement suggests that individuals which are passed to the search operations are likely to have a good fitness. Since the fitness of a solution candidate depends on its properties, it can be assumed that the features of these individuals are not so bad either. It should thus be possible for the optimizer to introduce slight changes to their properties in order to find out whether they can be improved any further. Normally, such exploitive modifications should also lead to small changes in the objective values and hence, in the fitness of the solution candidate.

Definition 4.1 (Strong Causality). Strong causality (locality) means that small changes in the properties of an object also lead to small changes in its behavior [239, 246].

In fitness landscapes with weak (low) causality, small changes in the solution candidates often lead to large changes in the objective values, i. e., ruggedness, as sketched in Fig. 4.1.d on page 48. It then becomes harder to decide which region of the problem space to explore and the optimizer cannot find reliable gradient information to follow. A small modification of a very bad solution candidate may then lead to a new local optimum and the best solution 
candidate currently known may be surrounded by points that are inferior to all other tested individuals.

The lower the causality of an optimization problem, the more rugged its fitness landscape is, which leads to a degeneration of the performance of the optimizer [161]. This does not necessarily mean that it is impossible to find good solutions, but it may take very long to do so. In Genetic Programming of real algorithms, we often experience very weak causality. Even slight changes to good programs (solution candidates) usually render will them infeasible. We can therefore assume that the fitness landscapes of such Genetic Programming applications are rugged.

\subsubsection{Countermeasures}

To the knowledge of the author, no viable method which can directly mitigate the effects of rugged fitness landscapes exists. In population-based approaches, using large population sizes and applying methods to increase the diversity can reduce the influence of ruggedness, but only up to a certain degree.

Weak causality is often a home-made problem because it results to some extent from the choice of the solution representation and search operations. We pointed out that exploration operations are important for lowering the risk of premature convergence. Exploitation operators are as same as important for refining solutions to a certain degree. In order to apply evolutionary algorithms in an efficient manner, it is necessary to find representations which allow for iterative modifications with bounded influence on the objective values, i. e., exploitation. The question whether such an ideal representation can be found for Genetic Programming at all is yet to be answered. In this work we will introduce two new approaches to this issue from which we believe that they represent one step into the right direction.

\subsection{Neutrality and Code Bloat}

\subsubsection{Introduction}

We consider the outcome of the application of a search operation to an element of the search space as neutral if it yields no change in the objective values [240,22]. It is challenging for optimization algorithms if the best solution candidate currently known is situated on a plane of the fitness landscape, i. e., all adjacent solution candidates have the same objective values. As illustrated in Fig. 4.1.f, an optimizer then cannot find any gradient information and thus, no direction in which to proceed in a systematic manner. From its point of view, each search operation will yield identical individuals.

The degree of neutrality $\nu$ is defined as the fraction of neutral results among all possible products of the search operations applied to a specific genotype [22]. We can generalize this measure to areas $G$ in the search space $\mathbb{G}$ by averaging over all their elements. Regions where $\nu$ is close to one are considered as neutral.

$$
\begin{aligned}
& \forall g_{1} \in \mathbb{G} \Rightarrow \nu\left(g_{1}\right)=\frac{\left|\left\{g_{2} \mid P\left(g_{2}=O p\left(g_{1}\right)\right)>0 \wedge F\left(\operatorname{gpm}\left(g_{2}\right)\right)=F\left(\operatorname{gpm}\left(g_{1}\right)\right)\right\}\right|}{\left|\left\{g_{2} \mid P\left(g_{2}=O p\left(g_{1}\right)\right)>0\right\}\right|} \\
& \forall G \subseteq \mathbb{G} \Rightarrow \nu(G)=\frac{1}{|G|} \sum_{g \in G} \nu(g)
\end{aligned}
$$

An interesting metaphor in optimization borrowed from biological systems is evolvability [59]. Wagner [290, 291] points out that this word has two uses in biology: According to Kirschner and Gerhart [158], a biological system is evolvable if it is able to generate heritable, selectable phenotypic variations. Such properties can then be spread by natural selection 
and changed during the course of evolution. In its second sense, a system is evolvable if it can acquire new characteristics via genetic change that help the organism(s) to survive and to reproduce. The idea of evolvability can be adopted for optimization as follows:

Definition 4.2 (Evolvability). The evolvability of an optimization process in its current state defines how likely the search operations will lead to solution candidates with new (and eventually, better) objectives values.

The evolvability of neutral parts of a fitness landscape depends on the optimization algorithm used. It is especially low for hill climbing and similar approaches, since the search operations cannot directly provide improvements or even changes. The optimization process then degenerates to a random walk, as illustrated in Fig. 4.1.f on page 48.

Researchers in molecular evolution, on the other hand, found indications that the majority of mutations in biology have no selective influence $[95,136]$ and that the transformation from genotypes to phenotypes is a many-to-one mapping. Wagner [291] states that neutrality in natural genomes is beneficial if it concerns only a subset of the properties peculiar to the offspring of a solution candidate while allowing meaningful modifications of the others. Toussaint and Igel [280] even go as far as declaring it a necessity for self-adaptation.

The theory of punctuated equilibria in biology, introduced by Eldredge and Gould [79, 78], states that species experience long periods of evolutionary inactivity which are interrupted by sudden, localized, and rapid phenotypic evolutions. It is assumed that the populations explore neutral layers during the time of stasis until, suddenly, a relevant change in a genotype leads to a better adapted phenotype [286] which then reproduces quickly.

The key to differentiating between "good" and "bad" neutrality is its degree $\nu$ in relation to the number of possible solutions maintained by the optimization algorithms. Smith et al. [263] have used illustrative examples similar to Figure 4.3 showing that a certain amount of neutral reproductions can foster the progress of optimization. In Fig. 4.3.a, basically the same scenario of premature convergence as in Fig. 4.2.a on page 49 is depicted. The optimizer is drawn to a local optimum from which it cannot escape anymore. Fig. 4.3.b shows that a little shot of neutrality could form a bridge to the global optimum. The optimizer now has a chance to escape the smaller peak if it is able to find and follow that bridge, i. e., the evolvability of the system has increased. If this bridge gets wider, as sketched in Fig. 4.3.c, the chance of finding the global optimum increases as well. Of course, if the bridge gets too wide, the optimization process may end up in a scenario like in Fig. 4.1.f on page 48 where it cannot find any direction. Furthermore, in this scenario the neutral bridge is expected to lead to somewhere useful, which is not necessarily the case in reality.

Different from ruggedness which is always bad for optimization algorithms, neutrality has aspects that may further as well as hinder the process of finding good solutions. Generally we can state that degrees of neutrality $\nu$ very close to 1 degenerate optimization processes to random walks. Some forms of neutral networks [271, 21, 312] accompanied by low (nonzero) values of $\nu$ can improve the evolvability and hence, increase the chance of finding good solutions.

\subsubsection{The Problem: Code Bloat}

In Genetic Programming, bloat is the uncontrolled growth in size of the individuals during the course of the evolution [165]. The term code bloat is often used in conjunction with code introns, which are regions inside programs that do not contribute to the functional objective values (because they can never be reached, for example). Bloat is an increase of program size without functional effect and thus, an instance of the "negative" form of neutrality. Limiting the code size and increasing the code efficiency by reducing the number of introns is therefore an important task in Genetic Programming since disproportionate program growth has many bad side effects like: 
global optimum

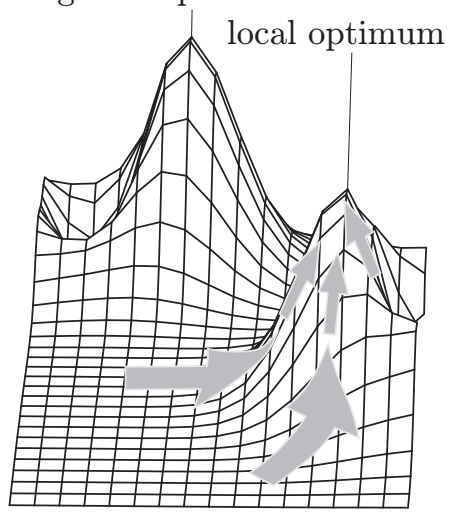

Fig. 4.3.a: Premature Convergence

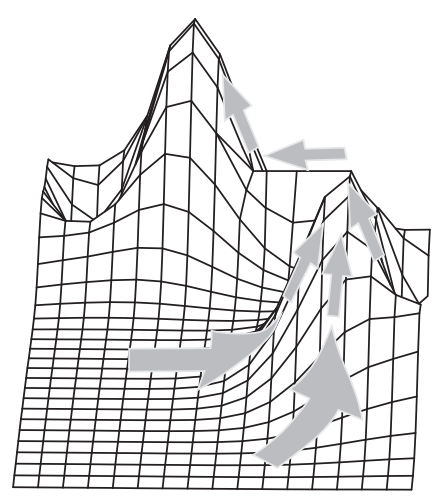

Fig. 4.3.b: Small Neutral Bridge

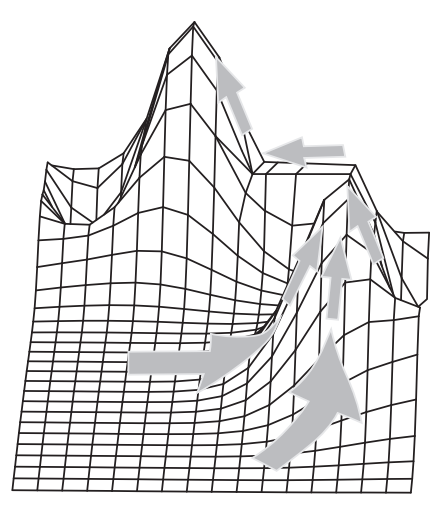

Fig. 4.3.c: Wide Neutral Bridge

Figure 4.3: Possible positive influence of neutrality.

1. The evolving programs become unnecessarily big while elegant solutions should always be as small and simple as possible.

2. Mutation and recombination operators always have to select the point in an individual where they will apply their changes. If there are many points that do not contribute to functionality, the probability of selecting such a point for modification is high. The generated offspring will then have exactly the same functionality as its parents and the genetic operation performed was neutral.

3. Bloat slows down both, the evaluation [117] and the breeding process of new solution candidates.

4. Furthermore, it leads to increased memory consumption of the Genetic Programming system.

There are many theories about how code bloat emerges [185], some of them are:

1. Unnecessary code hitchhikes with good individuals. If it is part of a fit solution candidate that creates many offspring, it is likely to be part of many new individuals. According to Tackett [272], high selection pressure is thus likely to cause code growth. This idea is supported by the research of Langdon and Poli [172], Smith and Harries [260], and Gustafson et al. [117].

2. As already stated, unnecessary code makes it harder for genetic operations to alter the functionality of an individual. In most cases, genetic operators yield offspring with worse fitness than its parents. If a solution candidate has good objective values, unnecessary code can be one defense method against recombination and mutation. If the genetic operators are neutralized, the offspring will have the same fitness as its parent. This idea has been suggested in many sources, such as $[36,19,260]$. From this point of view, introns are a "bad" form of neutrality. By the way, the reduction of the destructive effect of recombination on the fitness may also have positive effects, as pointed out by Nordin et al. [213, 214], since it may lead to a more durable evolution.

3. Luke [185] defines a theory for tree growth based on the fact that recombination is likely to destroy the functionality of an individual. However, the deeper the crossover point is located in the tree, the smaller is its influence because fewer instructions are removed. If only a few instructions are replaced from a functionally adequate program, they are likely to be exchanged by a larger sub-tree. A new offspring that retains the functionality of its parents therefore tends to be larger. 
4. Similar to the last two theories, the idea of removal bias by Soule and Foster [265] states that removing code from an individual will preserve the individual's functionality if the code removed is non-functional. Since the portion of useless code inside a program is finite, there also exists an upper limit of the amount of code that can be removed without altering the functionality of the program. For the size of new sub-trees that could be inserted instead (due to mutation or crossover), no such limit exists. Therefore, programs tend to grow $[265,174]$.

5. According to the diffusion theory of Langdon et al. [174], the number of large programs in the problem space that are functionally adequate is higher than the number of small adequate programs. Thus, code bloat could correspond to the movement of the population into the direction of equilibrium [185].

6. Another theory considers the invalidators that make code unreachable or dysfunctional. In the formula $4+0 *(4-x)$ for example, the multiplication with 0 makes the whole part $(4-x)$ inviable. Luke [185] argues that the influence of invalidators would be higher in large trees than in small trees. If programs grow while the fraction of invalidators remains constant and those inherited from the parents stay in place, their chance to occur proportionally closer to the root increases. Then, the amount of unnecessary instructions would increase too and naturally approach $100 \%$.

7. Instead of being real solutions, programs that grow uncontrolled also tend to be some sort of decision tables. This phenomenon is called overfitting and will be discussed in detail in Section 4.7.1. The problem is that overfitted programs tend to have marvelous good fitness for the training cases/sample data, but are normally useless for any other input.

8. Like Tackett [272], Gustafson et al. [117] link code growth to high selection pressure but also to loss of diversity in general. In populations with less diversity, recombination will frequently be applied to very similar individuals, which often yields slightly larger offspring.

\subsubsection{Countermeasures}

One of the simplest means against code bloat is to define additional objective functions which minimize the program size and to perform a multi-objective optimization. Successful and promising experiments by Bleuler et al. [35], de Jong et al. [63], and Ekárt and Németh [77] showed that this is a viable countermeasure for bloat. We therefore applied this approach in our work.

Another very simple method to limit code bloat is to prohibit the evolution of trees with a depth surpassing a certain limit [186]. Poli [231] furthermore suggests that the fitness of a certain portion of the population with above-average code size should simply be set to the worst possible value. These artificial fitness holes will repel the individuals from becoming too large and hence, reduce the code bloat. Besides bloat, adverse forms of neutrality are often caused by bad design of the search space or genotype-phenotype mapping. Uniform redundancy, i. e., trivial mappings like $n: 1$ voters in GAs, should be avoided where possible and the amount of neutrality in the search space should generally be limited.

In this section, we also pointed out that non-uniform neutrality, provided by pathways through neutral networks [271, 95], for instance, may have positive effect on the evolution. In the hope that it will increase the evolvability of the algorithm synthesis, the Rule-based Genetic Programming approaches introduced in this work were designed to also exhibit a certain degree of this form of neutrality. 


\subsection{Needle-In-A-Haystack Problems}

\subsubsection{Introduction}

One of the worst cases of fitness landscapes is the needle-in-a-haystack (NIAH) problem sketched in Fig. 4.1.g on page 48, where the optimum occurs as isolated spike in a plane. In other words, small instances of extreme ruggedness combine with a general lack of information in the fitness landscape. Such problems are extremely hard to solve and the optimization processes often will converge prematurely or take very long to find the global optimum. An example for such fitness landscapes is the all-or-nothing property often inherent to Genetic Programming of algorithms.

\subsubsection{The Problem: All-Or-Nothing?}

The evolution of algorithms often proves as a special instance of the needle-in-a-haystack problem. From a naïve and, at the same time, mathematically precise point of view, an algorithm computing the greatest common divisor of two numbers, for instance, is either correct or wrong. Approaching this problem straightforwardly leads to the application of a single objective function which can take on only two values, provoking the all-or-nothing problem in Genetic Programming [281]. In such a fitness landscape, a few steep spikes of equal height represent the correct algorithms and are distributed over a large plane of infeasible solution candidates with equally bad fitness.

The negative influence of all-or-nothing problems have been reported from many areas of Genetic Programming, such as the evolution of distributed protocols [281] (see Section 3.2.2), quantum algorithms [268], expression parsers [146], and mathematical algorithms (such as the GCD [293]).

\subsubsection{Countermeasures}

There are two direct countermeasures against the all-or-nothing problem in GP. The first one is to devise objective functions which can take on as many values as possible, i. e., which also reward partial solutions.

The second countermeasure is using as many test cases as possible and applying the objective functions to all of them, setting the final objective values to be the average of the results. Testing with ten training cases will transform a binary objective function to one which (theoretically) can take on eleven values, for instance: 1.0 if all training cases were processed correctly, 0.9 if one training case failed while nine worked out properly, ..., and 0.0 if the evolved algorithm was unable to behave adequately in any of the training cases. Using multiple training cases has, of course, the drawback that the time needed for the objective function evaluation will increase linearly.

Vaguely related to these two measures is another approach, the utilization of the Baldwin effect $[11,123]$ or Lamarckian evolution [148, 308], i. e., incorporating a local search into the optimization process. This may further help to smoothen out the fitness landscape [114] but also tends to increase the runtime. In our experiments reported in [295], an approach similar to Lamarckian evolution was incorporated. Although providing good results, the runtime of the approaches increased to a degree rendering it infeasible for large-scale experiments as done for this thesis. ${ }^{3}$

3 These timing issues were not the subject of the paper [295] and thus, not discussed there. 


\subsection{Epistasis}

\subsubsection{Introduction}

In biology, epistasis is defined as a form of interaction between different genes [227]. The term was coined by Bateson [23] and originally meant that one gene suppresses the phenotypical expression of another gene. In the context of statistical genetics, epistasis was initially called "epistacy" by Fisher [87]. According to Lush [188], the interaction between genes is epistatic if the effect on the fitness of altering one gene depends on the allelic state of other genes. This understanding of epistasis comes very close to another biological expression: Pleiotropy, which means that a single gene influences multiple phenotypic traits [313]. In the area of optimization, such fine-grained distinctions are usually not made and the two terms are often used more or less synonymously.

Definition 4.3 (Epistasis). In optimization, epistasis is the dependency of the contribution of one gene to the value of the objective functions on the allelic state of other genes. [5]

We speak of minimal epistasis when every gene is independent of every other gene. Then, the optimization process equals finding the best value for each gene and can most efficiently be carried out by a simple greedy search [57]. A problem is maximally epistatic when no proper subset of genes is independent of any other gene [266, 206].

\subsubsection{The Problem}

As sketched in Figure 4.4, epistasis has a strong influence on many of the previously discussed problematic features. If one gene can "turn off" or affect the expression of other genes, a modification of this gene will lead to a large change in the features of the phenotype. Hence, the causality will be weakened and ruggedness ensues in the fitness landscape. It also becomes harder to define search operations with exploitive character. Moreover, subsequent changes to the "deactivated" genes may have no influence on the phenotype at all, which would then increase the degree of neutrality in the search space. Epistasis is mainly an aspect of the way in which the genome $\mathbb{G}$ and the genotype-phenotype mapping are defined. It should be avoided where possible.

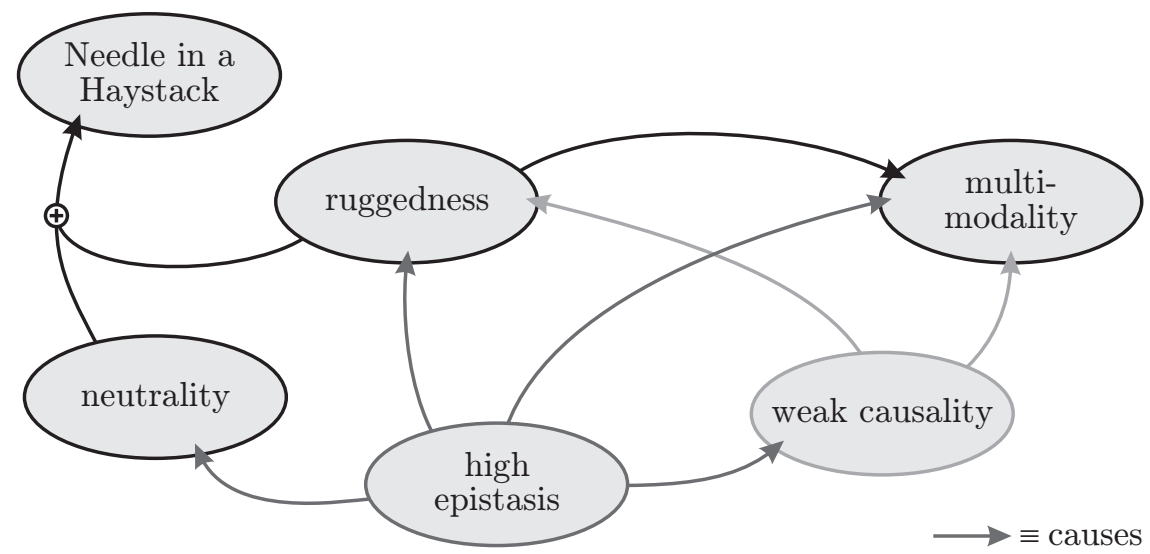

Figure 4.4: The influence of epistasis on the fitness landscape.

Generally, epistasis and conflicting objectives in multi-objective optimization should be distinguished. Epistasis as well as pleiotropy is a property of the influence of the editable 
elements (the genes) of the genotypes on the phenotypes. Objective functions can conflict without the involvement of any of these phenomena. We can, for example, define two objective functions $f_{1}(x)=x$ and $f_{2}(x)=-x$ which are clearly contradicting regardless of whether they both are subject to maximization or minimization. Nevertheless, if the solution candidates $x$ and the genotypes are simple real numbers and the genotype-phenotype mapping is an identity mapping, neither epistatic nor pleiotropic effects can occur.

When we use Genetic Programming to evolve full-fledged algorithms, we often find that the fitness landscape is very rugged. To a good part, this ruggedness is rooted in epistasis. In this section, we want to discuss the different epistatic effects which can be observed in Genetic Programming.

\section{Semantic Epistasis}

In an algorithm, the behavior of each instruction depends on the operations that have been executed before. If an instruction is changed, if the arithmetic operation $a=b+c$ is swapped with $\mathrm{a}=\mathrm{b}-\mathrm{c}$, for instance, its effects on subsequent instructions will change too [274]. This obvious fact fully complies with the definition of epistasis and we will refer to it as semantic epistasis.

\section{Positional Epistasis}

Epistasis also occurs in form of positional interdependencies. In order to clarify the role of this facet in the context of Genetic Programming, we begin with some basic assumptions [298]. Let us consider a program $P$ as some sort of function $P: I \mapsto O$ that connects the possible inputs $I$ of a system to its possible outputs $O$. Two programs $P_{1}$ and $P_{2}$ can be considered as equivalent if $P_{1}(i)=P_{2}(i) \forall i \in I{ }^{4}$

For the sake of simplicity, we further define a program as a sequence of $n$ statements $P=\left(s_{1}, s_{2}, \ldots, s_{n}\right)$. For these statements, there are $n$ ! possible permutations. We argue that the fraction $\theta(P)=\frac{m}{n !}$ of $m$ permutations that lead to programs equivalent to $P$ is one measure of robustness for a given program representation. More precisely, a low value of $\theta$ indicates a high degree of positional epistasis, which means that the loci (the positions) of many different genes in a genome have influence on their functionality. This reduces, for example, the efficiency of reproduction operations like recombination, since they often change the number and order of instructions in a program.

Many of the phenotypic and most genotypic representations in Genetic Programming seem to be rather fragile in terms of insertion and crossover points. One of the causes is that their genomes have high positional epistasis (low $\theta$-measures), as sketched in Figure 4.5.

\section{Embryogenic Epistasis (Problems of String-to-Tree GPMs)}

Grammar-guided Genetic Programming (GGGP) is a family of GP approaches which take a grammar that defines the shape of their phenotypes as input. Comprehensive summaries on these approaches can be found in [293, 194, 232].

Many GGGP methods like Grammatical Evolution (GE, [248]), Christiansen Grammar Evolution [64], and Gads [221] employ a genotype-phenotype mapping between an (integer) string genome and trees that represent sentences in a given grammar. According to Ryan et al. [248], the idea of mapping string genotypes can very well be compared to one of the natural prototypes of genotype-phenotype mappings: the translation of the DNA into

\footnotetext{
${ }^{4}$ In order to cover stateful programs, the input and output sets may also comprise sequences of data.
} 


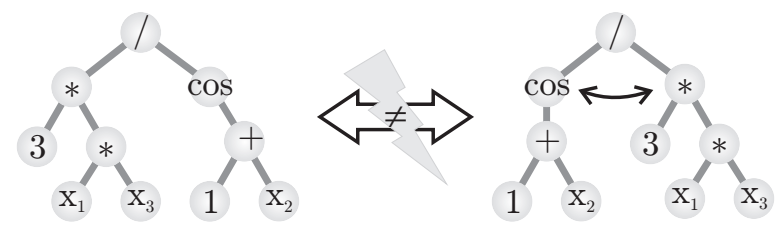

Fig. 4.5.a: in Standard Genetic Programming and symbolic regression

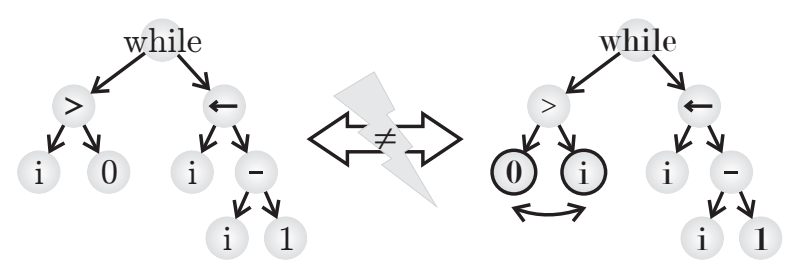

Fig. 4.5.b: in Standard Genetic Programming

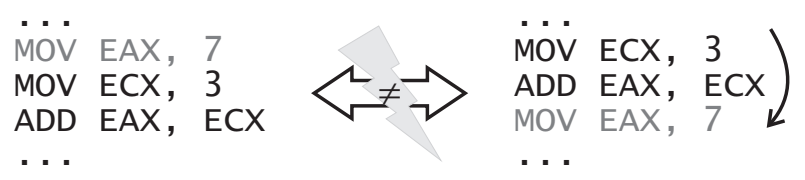

Fig. 4.5.c: in linear Genetic Programming

a3 ff $17 \mathrm{~cd} \mathrm{4c...}$

GPM

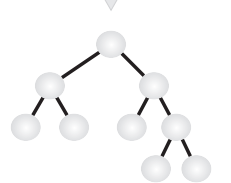

$\curvearrowleft$

a3 $17 \mathrm{ff} \mathrm{cd} 4 \mathrm{c} \ldots$

GPM
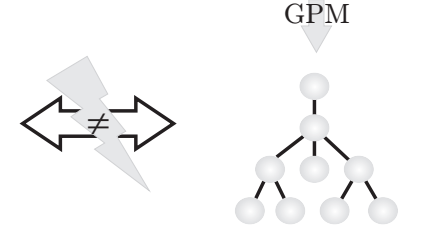

Fig. 4.5.d: in genotype-phenotype mappings, like in Grammatical Evolution-like approaches

Figure 4.5: Positional epistasis in Genetic Programming.

proteins. This process depends very much on the proteins already produced and which are now present around the cellular facilities. If a certain piece of DNA has created a protein $X$ and is transcripted again, a molecule of protein type $Y$ may result because of the presence of $X$.

Although this is a nice analogy, it also bears an important weakness. Search spaces which exhibit such effects usually suffer from weak causality [194]. Different from the aforementioned positional epistasis, this embryogenic episasis interacts with the genotype-phenotype mapping and modifies the phenotypic outcome. In Grammatical Evolution for example, a change in any gene in a genotype will likely also change the meaning of all alleles following after its locus. This means that mutation and crossover will probably have very destructive impacts on the individuals [207]. Hence, even the smallest change in the genotype can modify the whole structure and functionality of the phenotype, i. e., the evolving programs. A valid solution can become infeasible after a single reproduction operation.

Figure 4.6 outlines how the change of a single bit in a genotype (in hexadecimal notation) may lead to a drastic modification in the tree structure when a string-to-tree mapping is applied. The resulting phenotype in the example has more or less nothing in common with its parent except maybe the type of its root node. Furthermore, the efficiency of the 
reproduction operations of the mentioned approaches will likely decrease with a growing set of non-terminal symbols and corresponding productions. This facet of epistasis occurring additionally to the already mentioned effects led us to the decision not to consider Grammarguided Genetic Programming any further in this work.

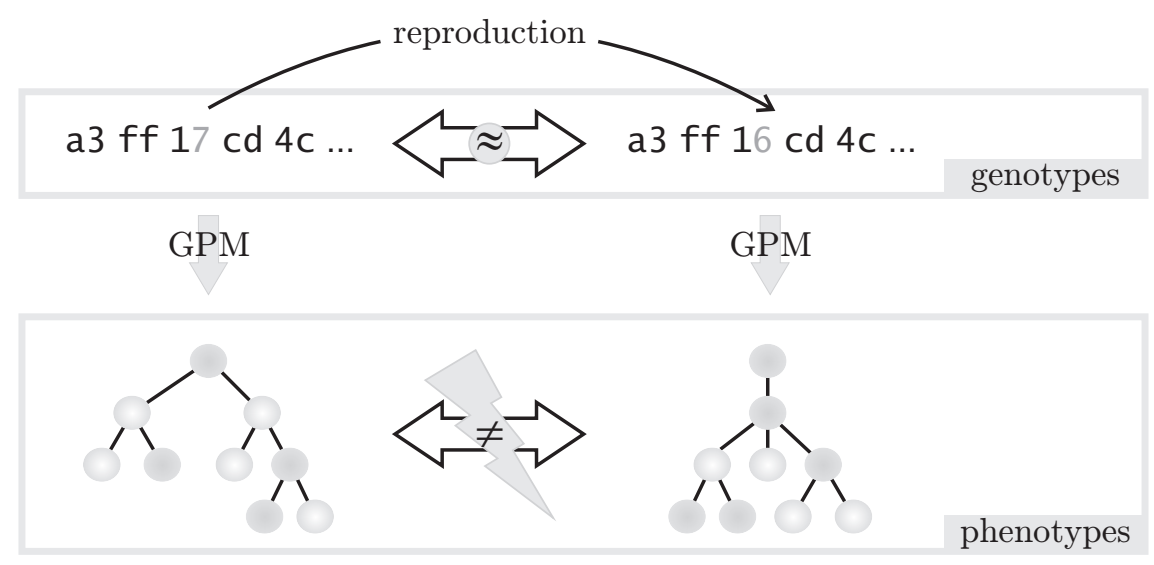

Figure 4.6: Epistasis in a Grammatical Evolution-like approach.

\subsubsection{Countermeasures}

We have shown that epistasis is a root cause for multiple problematic features of optimization tasks. General countermeasures against epistasis can be divided into two groups. The symptoms of epistasis can be mitigated with the same methods which increase the chance of finding good solutions in the presence of ruggedness or neutrality - using larger populations and favoring explorative search operations. Epistasis itself is a feature which results from the choice of the search space structure, the search operations, and the genotype-phenotype mapping. Avoiding epistatic effects should be a major concern during their design. This can lead to a great improvement in the quality of the solutions produced by the optimization process [298]. General advice for good search space design is given in [245, 238], and [293].

In the area of genetic algorithms, much effort has been spent in investigating methods which are able to learn how the genes in the search space depend on each other. Such linkage learning approaches include the messy GA by Goldberg et al. [108, 109] and the Bayesian Optimization Algorithm (BOA) [225, 44]. Transferred to Genetic Programming, linkage learning would mean to find out about the dependencies between the single instructions. Module acquisition [6] may be considered as such an effort.

Generally, the linkage between the primitives in GP is far more complicated than in usual genetic algorithm problems, which is why the author tends to believe that linkage learning will not achieve the same success in GP than it did in the area of genetic algorithms. Instead, we assume that the focus of the research will be on special genetic operators and program representations which minimize epistasis. In the following sections, we will outline two such approaches before introducing our own methods later in Section 5.5.

\section{Algorithmic Chemistry}

Algorithmic Chemistries were first discussed by Fontana [94] on basis of the $\lambda$-calculus. The Algorithmic Chemistry approach by Lasarczyk and Banzhaf $[176,18]$ represents one possible 
method to circumvent the positional epistasis discussed in Section 4.5.2. To put it bluntly, this form of Algorithmic Chemistries is basically a variant of linear Genetic Programming ${ }^{5}$ where the execution order of the single instructions is defined by some random distribution instead of being fixed as in normal programs.

This can probably best be described by using a simple example. Therefore, let us define the set of basic constructs which will make up the programs first. In both, [18] and [177], an assembler-like language is used where each instruction has three parameters: two input and one output register addresses. Registers are the basic data stores, the variables, of this language. Instructions with a behavior depending on a single input only simply ignore their second parameter. In [18], Banzhaf and Lasarczyk use a language comprising the eight instructions $+,-, /, *,-$, and, or, and not. Furthermore, they provided eleven read-only registers with evolved constants and thirty read-write registers, the so-called connection registers.

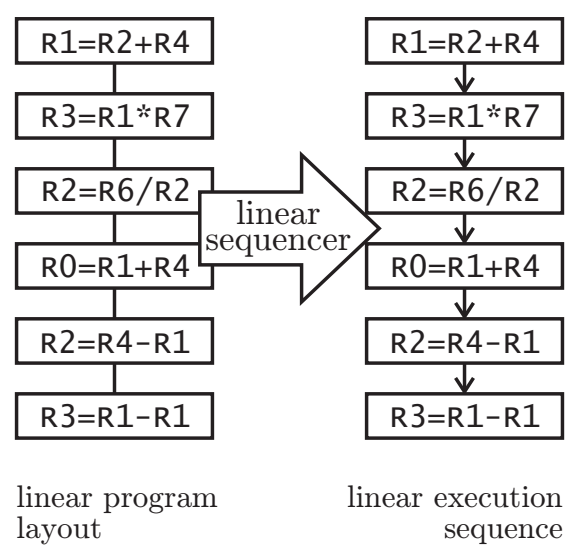

Fig. 4.7.a: Linear Genetic Programming phenotype and execution.

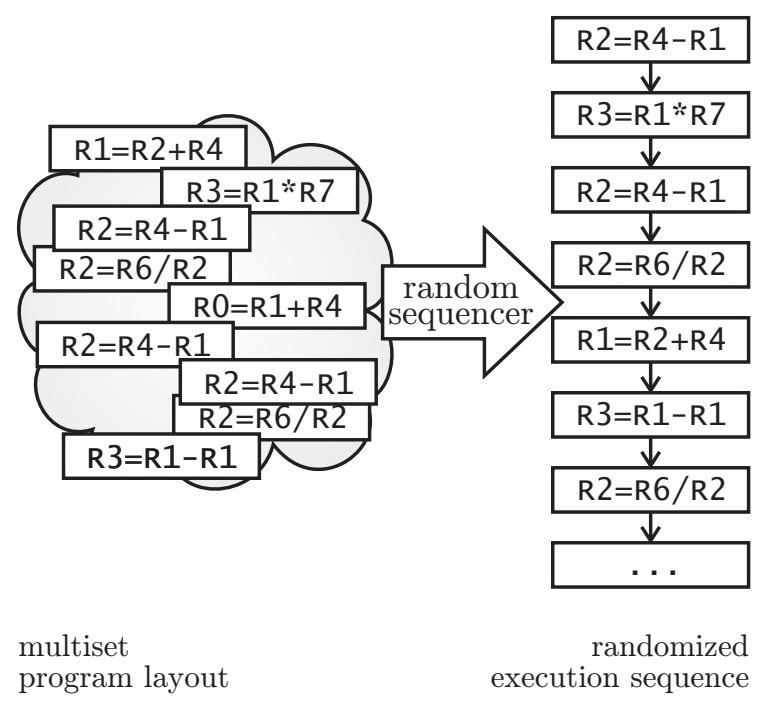

Fig. 4.7.b: Algorithmic Chemistry phenotype and execution.

Figure 4.7: The difference between linear Genetic Programming and Algorithmic Chemistries.

In Fig. 4.7.a we have illustrated the sequential structure of a normal program which might have been evolved in a linear Genetic Programming experiment. Whenever it is executed, be it for determining its fitness or later, as part of an application, the instructions are processed one after another, step by step. This execution scheme is common to all offthe-shelf PCs where the CPU uses an internal register (the instruction pointer) which points to the instruction to be executed next and which is incremented in this process.

Programs in the Algorithmic Chemistry representation can evolve essentially in the same way as programs in linear Genetic Programming do. As genotypes, exactly the same sequences of instructions can be used. This similarity, however, stops at the phenotypic level ${ }^{6}$. Here, the programs are considered as multisets which do not define any order on their el-

${ }^{5}$ a form of GP where the solution candidates are sequences of instructions (see Section 5.3)

${ }^{6}$ Although we distinguish between genotype and phenotype, no genotype-phenotype mapping is needed since the randomized execution can perfectly be performed on an array of instructions. 
ements (the instructions), as sketched in Fig. 4.7.b. When such a program is executed, a random sequencer draws one element from this set in each time step and executes it. ${ }^{7}$

This approach clearly leads to a $\theta$-value of zero, since all positional dependencies amongst the instructions have vanished. As trade-off, however, there are a number of interesting side effects. Since programs no longer are sequences, there is, for example, no last instruction anymore. Thus, the Algorithmic Chemistry programs also have no clear "end". Therefore, the randomized execution step is performed for a fixed number of iterations - five times the number of instructions in [176]. As pictured in Fig. 4.7.b, a certain instruction may occur multiple times in a program, which increases its probability of being picked for execution.

The biggest drawback of this approach is that the programs are no longer deterministic and their behavior and results may vary between two consecutive executions. Therefore, multiple independent runs should always be performed and the median or mean return value of them should be considered as the true result. Stripping the instructions of their order also will make it harder for higher-level constructs like alternatives or loops to evolve, let alone modules or functions. On the positive side, it also creates a large potential for parallelization and distribution which could be beneficial especially in multi-processor systems. Then again, because of its indeterminism, Algorithmic Chemistries did not lent themselves as suitable representation for our research work.

\section{Soft Assignment}

Another approach for reducing the epistasis is the soft assignment method (memory with memory) by McPhee and Poli [195]. It implicitly targets semantic epistasis by weakening the way values are assigned to variables.

In traditional programs, instructions like $\mathrm{x}=\mathrm{y}$ or mov $\mathrm{x}, \mathrm{y}$ will completely overwrite the value of $\mathrm{x}$ with the value of $\mathrm{y}$. McPhee and Poli replace this strict assignment semantic with

$$
\mathrm{x}_{t+1}=\mathrm{y}_{t} \equiv \mathrm{x}_{t+1} \longleftarrow \gamma \mathrm{y}_{t}+(1-\gamma) \mathrm{x}_{t}
$$

where $\mathrm{x}_{t+1}$ is the value that the variable $\mathrm{x}$ will have after and $\mathrm{x}_{t}$ its value before the assignment. $\mathrm{y}_{t}$ is the value of an arbitrary expression which is to be stored in $\mathrm{x}$. The parameter $\gamma$ is "a constant that indicates the assignment hardness" [195]. For $\gamma=1$, the assignments are completely overwriting as in normal programming and for $\gamma=0$, the values of the variables cannot be changed. McPhee and Poli [195] report that $\gamma=0.7$ performed best (better than $\gamma=1$ ) when applied to different symbolic regression problems.

For mathematical or approximation problems, this approach is very beneficial. The drawback of programs using soft assignment is that, although they are deterministic, there are situations where precise values are required which they may not be able to compute. Assume, for instance, that an algorithm is to be evolved which returns the largest element max from an input list 1 . This program could contain an instruction like if 1 [i] $>\max$ then $\max =1[i]$. If a $\gamma$-value smaller than one is applied, the final value in max will most likely be no element of the list.

In this thesis, we evolve algorithms where exactly this type of problem would occur if soft assignments were used. The results of election processes as synthesized in Chapter 7 would, for instance, become incorrect in virtually all scenarios. Therefore, we cannot utilize soft assignments here.

In this work, we will provide a new, third approach for reducing epistasis. We will introduce and discuss it in detail in Section 5.5 on page 81.

\footnotetext{
${ }^{7}$ Banzhaf and Lasarczyk [18] use the same approach for introducing a new recombination operator which creates an offspring by drawing instructions randomly from both parents.
} 


\subsection{Noise and Robustness}

\subsubsection{Introduction - Noise}

In the context of optimization, three types of noise can be distinguished. The first form is noise in the training data used as basis for learning ( $i$ ). In many applications of machine learning or optimization where a model for a given system is to be learned, data samples including the input of the system and its measured response are used for training. Since no measurement device is $100 \%$ accurate and there are always random errors, noise is present in such optimization problems.

Besides inexactnesses and fluctuations in the input data of the optimization process, perturbations are also likely to occur during the application of its results. This is the reason why robust solutions are needed in most practical cases and not just the global optima. This category subsumes the other two types of noise: perturbations that may arise from (ii) inaccuracies in the process of realizing the solutions and (iii) environmentally induced perturbations during the applications of the products.

This issue can be illustrated by using the process of developing the perfect tire for a car as an example. As input for the optimizer, all sorts of material coefficients and geometric constants measured from all known types of wheels and rubber could be available. Since these constants have been measured or calculated from measurements, they include a certain degree of noise and imprecision (i).

The result of the optimization process will be the best tire construction plan discovered during its course and it will likely incorporate different materials and structures. We would hope that the tires created according to the plan will not fall apart if, accidently, an extra $0.0001 \%$ of a specific rubber component is used (ii). During the optimization process, the behavior of many construction plans will be simulated in order to find out about their utility. When actually manufactured, the tires should not behave unexpectedly when used in scenarios different from those simulated (iii) and should instead be applicable in all driving situations likely to occur.

In the context of this work, the inputs of the optimizers are the objective functions and a generator for creating randomized network scenarios which are used for evaluating the evolved programs. The parameters of the scenario generator are based on experience which is a weak form of measurements (i). Type (ii) perturbations would correspond to errors in the translation of the evolved code to programs in a language like $\mathrm{C}$ or Java which can be compiled and actually executed in a real network ${ }^{8}$. Such errors can be ruled out under the condition that no mistakes are made during the translation of the programs. The third class of problems, the occurrence of unforeseen conditions (iii), is very likely since it is not possible to train all network situations.

The effects of noise in optimization have been studied by various researchers; Miller and Goldberg [197, 196], Lee and Wong [179], and Gurin and Rastrigin [115] are some of them. Many optimization algorithms and theoretical results have been proposed which can deal with noise [90, 32, 218].

\subsubsection{The Problem: Need for Robustness}

The goal of global optimization is to find the global optima of the objective functions. While this is fully true from a theoretical point of view, it may not suffice in practice. Optimization problems are normally used to find good parameters or designs for components or plans to be put into action by human beings or machines. As we have already pointed out, there will always be noise and perturbations in practical realizations of the results of optimization.

\footnotetext{
${ }^{8}$ In [299, 300, 307] we have investigated a model-driven approach to such translations, see al-
} so Section 11.2 on page 157 . 
Definition 4.4 (Robustness). A system in engineering or biology is robust if it is able to function properly in the face of genetic or environmental perturbations [290].

Therefore, a local optimum (or even a non-optimal element) for which slight disturbances only lead to gentle performance degenerations is usually favored over a global optimum located in a highly rugged area of the fitness landscape [39]. In other words, local optima in regions of the fitness landscape with strong causality are sometimes better than global optima with weak causality. Of course, the level of this acceptability is application-dependent. Figure 4.8 illustrates the issue of local optima which are robust vs. global optima which are not.

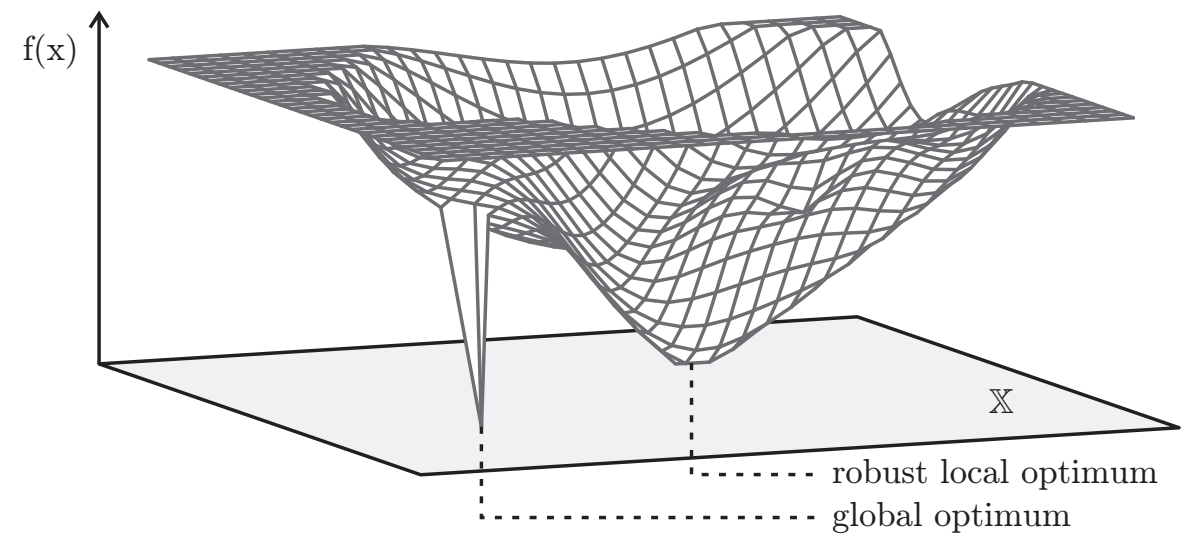

Figure 4.8: A robust local optimum vs. a "unstable" global optimum.

In the context of this work, a distributed algorithm which is not optimal would strongly be favored over a seemingly perfect one which fails in situations not predicted. This, however, would require foreseeing unpredicted situations during the comparison of both algorithms which, unfortunately, is not possible. Therefore, we have to take precautions and apply the countermeasures discussed in the following section in the hope to derive robust algorithms.

\subsubsection{Countermeasures}

For the special case where the phenome is a real vector space $\left(\mathbb{X} \subseteq \mathbb{R}^{n}\right)$, several approaches for dealing with the need for robustness have been developed. In the method suggested by Greiner [112, 113], possible disturbances are represented by a vector $\boldsymbol{\delta}=\left(\delta_{1}, \delta_{2}, . ., \delta_{n}\right)^{T}, \delta_{i} \in$ $\mathbb{R}$. If the distributions and influences of the $\delta_{i}$ are known, the objective function $f(\mathbf{x}): \mathbf{x} \in \mathbb{X}$ can be rewritten as $\tilde{f}(\mathbf{x}, \boldsymbol{\delta})$ [310]. In the special case where $\boldsymbol{\delta}$ is normally distributed, this can be simplified to $\tilde{f}\left(\left(x_{1}+\delta_{1}, x_{2}+\delta_{2}, . ., x_{n}+\delta_{n}\right)^{T}\right)$. It would then make sense to sample the probability distribution of $\boldsymbol{\delta}$ a number of $t$ times and to use the mean values of $\tilde{f}(\mathbf{x}, \boldsymbol{\delta})$ for each objective function evaluation during the optimization process. In cases where the optimal value $y^{\star}$ of the objective function $f$ is known, Equation 4.4 can be minimized. This approach is also used in the work of Wiesmann et al. [309, 310] and basically turns the optimization algorithm into something like a maximum likelihood estimator.

$$
f^{\prime}(\mathbf{x})=\frac{1}{t} \sum_{i=1}^{t}\left(y^{\star}-\tilde{f}\left(\mathbf{x}, \boldsymbol{\delta}_{i}\right)\right)^{2}
$$

This method corresponds to using multiple, different training scenarios during the objective function evaluation in situations where $\mathbb{X} \nsubseteq \mathbb{R}^{n}$, as is in our case. By adding random 
noise and artificial perturbations to the training cases, the chance of obtaining robust solutions which are stable when applied or realized under noisy conditions can be increased. In the context of this work, we therefore use multiple, randomized training scenarios for each objective value evaluation.

\subsection{Overfitting and Oversimplification}

In all scenarios where optimizers evaluate some of the objective values of the solution candidates by using training data, two additional phenomena with negative influence can be observed: overfitting and oversimplification.

\subsubsection{Overfitting}

\section{The Problem}

Overfitting is the emergence of an overly complicated solution candidate in an optimization process resulting from the effort to provide the best results for as much of the available training data as possible $[250,259,69]$. A phenotype $x \in \mathbb{X}$ (a program, in the context of this work) optimized based on a finite set of training data is considered to be overfitted if a less complicated, alternative program $x^{\prime} \in \mathbb{X}$ exists which has a smaller error for the set of all possible (maybe even infinitely many), available, or (theoretically) producible data samples. This solution candidate $x^{\prime}$ may, however, have worse objective values when judged on basis of the training data only.

\section{Countermeasures}

There exist many techniques that can be utilized in order to prevent overfitting to a certain degree. It is most efficient to apply multiple such techniques together in order to achieve best results.

A very simple approach is to restrict the problem space $\mathbb{X}$ in a way that only solutions up to a given maximum complexity can be found. In terms of algorithms or programs, this means limiting the maximum number of instructions and is applied in our experiments.

Furthermore, the functional objective functions which solely concentrate on the error of the solution candidates should be augmented by penalty terms or non-functional objective functions putting pressure in the direction of small and simple models [69, 154].

Large sets of sample data, although slowing down the optimization process, may improve the generalization capabilities of the derived solutions. In the context of this work, this means that the objective values of the evolved programs are to be determined using as many simulation runs as possible. If arbitrarily many training datasets or training scenarios (for simulations, for instance) can be generated, there are two approaches which work against overfitting:

1. The first method is to use a new set of (randomized) scenarios for each evaluation of each solution candidate. The resulting objective values then may differ largely even if the same individual is evaluated twice in a row, introducing incoherence and ruggedness into the fitness landscape.

2. At the beginning of each iteration of the optimizer, a new set of (randomized) scenarios is generated which is used for all individual evaluations during that iteration. This method leads to objective values which can be compared without bias. They can be made even more comparable if the objective functions are always normalized into some fixed interval, say $[0,1]$. 
In both cases it is helpful to use more than one training sample or scenario per evaluation and to set the resulting objective value to the average (or better median) of the outcomes. Otherwise, the fluctuations of the objective values between the iterations will be very large, making it hard for the optimizers to follow a stable gradient for multiple steps.

Another simple method to prevent overfitting is to limit the runtime of the optimizers [250]. It is commonly assumed that learning processes normally first find relatively general solutions which subsequently begin to overfit because the noise "is learned", too. However, it is our experience that programs which are general are rarely overtrained and overfit in the later course of the evolution. In our (earlier) experiments, we have found that they are often either overfitted or general from the start.

Some algorithms allow decreasing the rate at which the solution candidates are modified by time. Such a decay of the learning rate which makes overfitting less likely is, however, hard to realize in Genetic Programming.

If only one finite set of data samples is available for training/optimization, it is common practice to separate it into a set of training data $A_{t}$ and a set of test cases $A_{c}$. During the optimization process, only the training data is used. The resulting solutions are tested with the test cases afterwards. If their behavior is significantly worse when applied to $A_{c}$ than when applied to $A_{t}$, they are probably overfitted.

In this work, we utilize this approach for detecting when the evolution can be stopped by generating different simulation scenarios for testing the evolved programs in order to save runtime. We use much more scenarios for testing than for fitness evaluation.

\subsubsection{Oversimplification}

\section{The Problem}

Oversimplification (also called overgeneralization) is the opposite of overfitting. Whereas overfitting denotes the emergence of overly complicated solution candidates, oversimplified solutions are not complicated enough. Although they represent the training samples used during the optimization process seemingly well, they are rough overgeneralizations which fail to provide good results for cases not part of the training.

A common cause for oversimplification are training sets that only represent a fraction of the set of possible inputs. As this is normally the case (see also Section 4.8.1), one should always be aware that such an incomplete coverage may fail to represent some of the dependencies and characteristics of the data, which then may lead to oversimplified solutions. Another possible reason for oversimplification is that other factors which we will discuss in this chapter, such as ruggedness, deceptiveness, too much neutrality, or high epistasis in the fitness landscape, may lead to premature convergence and prevent the optimizer from surpassing a certain quality of the solution candidates. It then cannot adapt them completely even if the training data perfectly represents the sampled process. A third possible cause is that a problem space could have been chosen which does not include the correct solution.

\section{Countermeasures}

In order to counter oversimplification, its causes have to be mitigated. Generally, it is not possible to have training scenarios which cover the complete input space of the evolved programs. By using multiple scenarios for each individual evaluation, the chance of missing important aspects is decreased. These scenarios can be replaced with new, randomly created ones in each generation, in order to decrease this chance even more. The problem space, i. e., the representation of the solution candidates, should further be chosen in a way which allows constructing a correct solution to the problem defined. Then again, releasing too 
many constraints on the solution structure increases the risk of overfitting and thus, careful proceeding is recommended.

\subsection{Correctness}

Genetic Programming can be utilized to breed programs or algorithms suitable for a given problem class. In order to guide such an evolutionary process, the synthesized programs have to be evaluated, i. e., assessed in terms of functional and non-functional requirements. The functional properties comprise all features regarding how good a program solves the specified problem and the non-functional aspects are concerned with, for example, its size and memory consumption. In this work, a set $F=\left\{f_{1}, . ., f_{n}\right\}$ of objective functions is specified in order to map these attributes to the real numbers.

\subsubsection{The Problems}

In Genetic Programming, some of the non-functional objective values such as the size of the evolved programs can easily be computed. Determining their functional utility, however, cannot be achieved by any arithmetically closed function or algorithm - at least if a Turing-complete representation is chosen - since this would be an instance of the Entscheidungsproblem [58] as well as of the Halting Problem [258].

\section{Entscheidungsproblem}

The Entscheidungsproblem, formulated by David Hilbert [121, 122], asks for an algorithm that, if provided with a description of a formal language and a statement in that language, can decide whether or not the statement holds [311]. In the case of Genetic Programming, the formal language is the language in which the programs are evolved, i. e., the problem space, and the statements are the programs themselves. Church [47, 48] and Turing [283, 284] both proved that an algorithm solving the Entscheidungsproblem cannot exist.

\section{No Exhaustive Testing}

It is not possible to use some kind algorithm in order to determine whether the evolved programs will provide correct results. Thus, training cases must be used as tests to find out whether a program is suitable for the given problem. Software Testing is a very important field in software engineering $[24,86]$. The core problem of testing programs for their functionality and performance is the size of the input space. Assume that we pursued the evolution of a program that takes two integer numbers (32 bit) as input and computes another one as output. For testing this program with all possible inputs, $2^{32} * 2^{32}=2^{64}=18446744073709551616$ single tests would be required. Even if each test run took only $1 \mu \mathrm{s}$, exhaustive testing would take approximately 584542 years. In most practical applications, the input space is much larger. Exhaustive testing of the evolved algorithms is thus not feasible in virtually all cases.

Instead, we can only pick a very small fraction of the possible test scenarios for training and hope that they will provide significant results. The probability that this will happen depends very much on the method with which the training cases are selected.

Most often, one cannot be sure whether evolved behavioral patterns (or algorithms) are perfect and free from errors in all possible situations. Here, nature indeed has the same problem as the scientists who apply Genetic Programming, as the following small analogy ${ }^{9}$ will show.

\footnotetext{
${ }^{9}$ It is hard to find references confirming this story. It occurred in one scene of the movie Animals are
} beautiful people by Uys [285], roughly resembles one of Aesop's fables [3], and is mentioned on the 


\section{The Monkey and the Orange}

Consider a certain scheme in the behavior of monkeys. If a monkey sees or smells something delicious in, for example, a crevice, it sticks its hand in, grabs the item of desire and pulls it out. This simple behavior itself is quite optimal and has served the monkeys well for generations. With the occurrence of homo sapiens, the situation changed. African hunters still use this behavior against the monkeys by creating a situation that was never relevant during its "evolutionary testing period": They slice a coconut in half and put a hole in one side just big enough for a monkey's hand to fit through. Now they place an orange between the two coconut halves, tie them closely together and secure the trap with a rope to a tree. Sooner or later, a monkey will smell the orange, find the coconut with the hole, stick its hand inside and grab the fruit. However, with the orange in its fist, it cannot pull the hand out anymore. The hunter can now easily catch the monkey, to whom it never occurs that it could let go the fruit and save its life.

In other words, although evolutionary algorithms like Genetic Programming may provide good solutions for many problems, their results still need to be analyzed and interpreted by a human being. This implies that the solutions need to be delivered in a human-readable way. In some optimization problems, we may, however, choose a representation for the solution candidates which is very hard to understand for human beings but more suitable for evolution (see the outputs of the eSGP approach for election in Listing 7.2 on page 109, for instance). Hence, it is not always possible to perform a sanity check on the evolved programs by hand.

\section{Halting Problem}

The Halting Problem is basically an instance of the Entscheidungsproblem and asks for an algorithm that decides whether another algorithm will terminate at some point in time or runs forever if provided with a certain, finite input. Again, Turing [283, 284] proved that a general algorithm solving the Halting Problem cannot exist in general. One possible way to show this is to use a simple counter-example: Assume that a correct algorithm doesHalt exists which takes a program as input and determines whether it will terminate or not. It is now possible to specify a program trouble which, in turn, uses doesHalt to determine if it will halt at some point in time. If doesHalt returns true, trouble loops forever. Otherwise it halts immediately. In other words, doesHalt cannot return the correct result for trouble and hence, cannot be applied universally. Thus, it is not possible to solve the Halting Problem algorithmically for Turing-complete programs in a Turing-complete representation. One consequence of this fact is that there are no means to determine when an evolved program will terminate or whether it will do so at all (if its representation allows infinite execution, that is) $[274,316]$. Langdon and Poli $[173]$ have shown that in Turing-complete linear Genetic Programming systems, most synthesized programs loop forever and the fraction of halting programs of size length is proportional to $\sqrt{\text { length }}$, i. e., small.

\subsubsection{Countermeasures}

\section{Against the Entscheidungsproblem}

For general, Turing-complete program representations, neither exhaustive testing nor algorithmic detection of correctness is possible.

Wikipedia [311] page on coconuts from 2007-07-21 (http://en.wikipedia.org/w/index.php? title=Coconut\&oldid=146038518) where they had the same problem and eventually removed the corresponding text. But regardless whether it is just an urban legend or not - it is a nice story. 


\section{Model Checking}

Model checking techniques [50, 205] have made great advance since the 1980s. According to Clarke and Emerson [49], "Model checking is an automated technique that, given a finitestate model of a system and a logical property, systematically checks whether this property holds for (a given initial state in) that model." The result of the checking process is either a confirmation of the correctness of the checked model, a counterexample in which it fails to obey its specification, or failure, i. e., a situation in which no conclusion could be reached.

In the context of Genetic Programming, a model checker can be utilized as a Boolean function $\varphi: \mathbb{X} \mapsto \mathbb{B}$ which maps the evolved programs to correct (三 true) or incorrect (三false). As objective function, $\varphi$ therefore is rather infeasible, since it would lead directly to the all-or-nothing problem discussed in Section 4.4.2. ${ }^{10}$

Still, model checkers can be an interesting way to define termination criteria for the evolution or to verify its results. This may require a reduction of the expressiveness of the Turing-complete GP approaches tested in this work in order to make them compliant with the input languages of the model checkers. Then again, there are very powerful model checkers such as SPIN $[133,26]$, which processes systems written in the Promela (the Process Meta Language) with which asynchronous distributed algorithms can be specified [25]. If such a system was used, no reduction of the expressiveness of the program representation would be needed at all. Nevertheless, a formal transformation of the GP representation to these languages must be provided in any circumstance. Creating such a transformation is complicated and requires a formal proof of correctness - checking a model without having shown the correctness of the model representation first is, basically, nonsense. ${ }^{11}$

The idea of using model checkers like SPIN for our work was very tempting. One important drawback of this method was the unforeseeable runtime of the checking process which spans from almost instantaneous return up to almost half an hour [276]. In the same series of experiments $([276])$, the checking process also failed in a fraction of cases $(\approx 18 \%)$ depending on the problem to be verified. Especially the unpredictable runtime for general problems led us to the decision to not use SPIN in our own experiments yet, since in the worst case, more than one thousand program verifications could be required per generation in the GP system. Still, it is an interesting idea to evolve programs in Promela language and we will reconsider it in our future work and evaluate the utility and applicability of SPIN for the said purposes in detail.

\section{Functional Adequacy}

In the face of this situation where we cannot automatically determine whether an evolved algorithm is correct, overfitted, or oversimplified, a notation for which solutions are acceptable and which are not is required. One definition which fits perfectly in this context is the idea of functional adequacy provided by Gleizes et al. [103, 43]:

Definition 4.5 (Functional Adequacy). When a system has the "right" behavior judged by an external observer knowing the environment - we say that it is functionally adequate [103].

In the context of this work, the external observer is represented by the objective functions which evaluate the behavior of the programs in the simulation environments. According to Gleizes et al., functional adequacy also subsumes non-functional criteria such as memory consumption or response time if they become crucial in a certain environment, i.e., influence the functionality. At the current state of our research, the non-functional aspects are either optimized with additional objective functions (program size) or are fixed constants (memory size).

$\overline{10}$ One approach to circumvent this problem would be to check for several properties separately.

11 Thanks to Hendrik Skubch for discussing this issue with me. 


\section{Against The Halting Problem}

In order to circumvent the Halting Problem, the evolved programs can be executed in simulations which allow limiting their runtime [316, 146]. Programs which have not finished until the time limit has elapsed are terminated automatically. Especially in linear Genetic Programming approaches, it is easy to do so by simply defining an upper bound for the number of instructions being executed. For tree-based representations, this is slightly more complicated.

Teller [274] suggests to apply time-limiting approaches too, but also the use of so-called anytime algorithms, i. e., algorithms that store their best guess of the result in a certain memory cell and update it during their run. Anytime algorithms can be stopped at any time, since the result is always there, although it may have been refined in their future course. We will research the possibility of evolving distributed anytime algorithms for distributed elections in Section 7.2.1 and the distributed GCD problem in Chapter 9.

Another way to deal with this problem is to prohibit the evolution of infinite loops or recursions from the start by restricting the structural elements in the programming language. If there are no loops, there surely cannot be infinite ones either. Imposing such limitations, however, also restricts the programs that can evolve: A representation which does not allow infinite loops cannot be Turing-complete either.

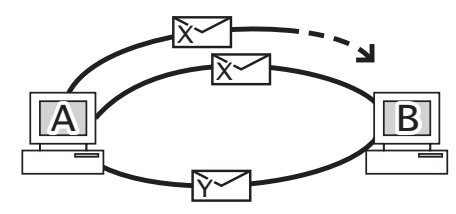

Figure 4.9: A sketch of an infinite message loop.

Furthermore, in the context of this work, it is not sufficient to restrict just the programming language. If the goal is to evolve distributed algorithms, the possible network situations and the reactions to them would also need to be limited. One would need to exclude situations like the one illustrated in Figure 4.9 where

1. node $\mathrm{A}$ sends message $\mathrm{X}$ to node $\mathrm{B}$ which

2. triggers an action there, leading to a response message $\mathrm{Y}$ from $\mathrm{B}$ back to node $\mathrm{A}$ which, in turn,

3. causes an action on $\mathrm{A}$ that includes sending $\mathrm{X}$ to $\mathrm{B}$ again

4. and so on...

Preventing such a situation is even more complicated and will, most likely, also prevent the evolution of useful solutions. 


\section{Approaches}

In this work, six different Genetic Programming approaches were utilized. When choosing and designing them, our intention was to cover a wide range of different program representations and genomes. By doing so, we hoped to obtain a good impression on the utility of GP for evolving distributed algorithms.

\subsection{Standard Genetic Programming with Memory [SGP]}

\subsubsection{Approach}

The baseline approach for Genetic Programming is to use a tree-based genome as discussed in Section 2.5. Koza used such genomes back in his seminal 1992 work Genetic Programming: On the Programming of Computers by Means of Natural Selection [165] in which he formalized Genetic Programming. Therefore, such approaches are usually referred to as Standard Genetic Programming (SGP) methods. In a tree defining a program, the inner nodes stand for functions and operations whereas the leaf nodes represent terminal symbols such as constants or variables. In this section we introduce some modifications to this approach in order to adapt it to the evolution of distributed algorithms.

\subsubsection{Adaptation}

In our GP system, we use the tree-based Standard Genetic Programming method with some alterations in order to facilitate the requirements of cooperative computations based on asynchronous network communication:

1. A program consists of at least two automatically defined functions (ADFs).

2. The first function is called on the startup of the program, similar to the main method in $\mathrm{C}++$ programs.

3. The second function acts as an interrupt service routine (ISR) which is invoked asynchronously whenever a message is received by the node.

4. A program may consist of more than two functions if the evolutionary process chooses to add them. These additional functions are no automatically defined functions.

5. Memory is divided into global memory accessible by all functions and local memory which is private to the scope of the currently running function.

6. All parameters of a function are stored in local memory.

7. The contents of an incoming message are stored in the local memory of the second automatically defined function which is invoked on its arrival. 
8. Functions may be called from within the evolved code with an arbitrary number of parameters. The total number of parameters of a function is automatically determined as the maximum number of parameters of all calls to it. If it is invoked with lesser arguments, the remaining, unused parameters are set to zero.

9. A function may return a value and call instructions can therefore be treated as expressions, i. e., nested in other expressions and function calls.

10. Function parameters are expressions.

11. The send instruction has between one and three parameters which denote the contents of the message to be sent.

\section{The Primitives}

All the primitives of this approach are listed in Table $5.1^{1}$ where expressions are marked with (exp) and instructions are annotated with (instr), elements which can be both, instructions or expressions, are marked with (instr/exp). Instructions and expressions can be nested, but while expressions can be parameters of instructions, instructions are never part of expressions. Furthermore, expressions have no side-effect whereas instructions issue changes to the global state of the nodes they are executed on or to the distributed system containing these nodes. The call construct is an exception to this rule, since it can be used as expression and as instruction and may have side-effects.

Figure 5.1 gives an example for the syntax of this language by sketching both, the internal and the textual representation of a program. The illustrated program does nothing useful except for sending a message consisting of two words - the return value of the function at index one (since $7 \bmod 2=1 \equiv$ func_ $_{-}$) and a constant $\left(00100001_{16}\right)$. func_1 is also the ADF which will be invoked whenever a message arrives, but in the example, it does nothing than performing a small computation and returning a result: If we assume that there are two global and two local memory cells, it returns the value of its first parameter $(14 \bmod 4=2 \equiv$ first local memory cell) minus DOOBAAC1 $1_{16}$, i. e., $20000000_{16}$ when invoked from func_0.

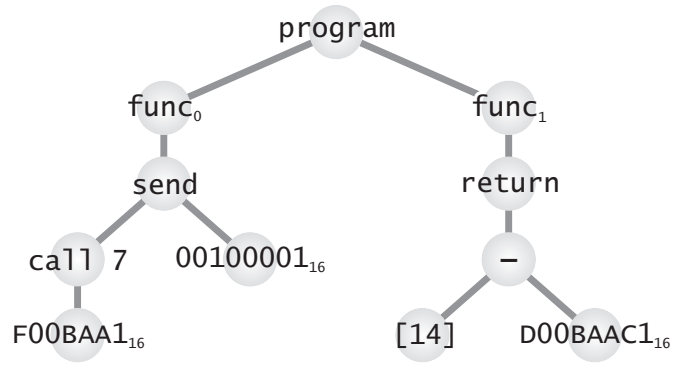

Fig. 5.1.a: Internal tree representation.

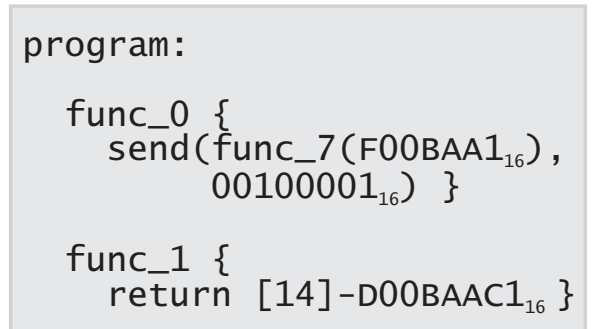

Fig. 5.1.b: Textual representation.

Figure 5.1: Example for the SGP syntax.

It should be mentioned that the readability of the results from such a GP process is usually low since function identifiers in call instructions and memory addresses are always to be taken modulo the total function number or the memory size. Nevertheless, the goal of evolving distributed algorithms is not to evolve human readable but functionally adequate and efficient results. With a subsequent process such as the transformation into UML diagrams (Section 11.2) or the translation to assembler-like code (Section 5.3.3), the readability of the results can be increased significantly.

\footnotetext{
${ }^{1}$ The modulo operator was only available in the GCD experiment (see Chapter 9).
} 


\begin{tabular}{|c|c|}
\hline Primitive & Meaning \\
\hline program & the root node of the program \\
\hline func $\{$ instrs $\}$ & $\begin{array}{l}\text { a new function is introduced which can be called and has the body instrs. } \\
\text { func can only be attached to the root node (program). }\end{array}$ \\
\hline $\operatorname{call}\left(\mathrm{v}\left[, \exp _{1}[, \ldots]\right]\right)$ & $\begin{array}{l}\text { (instr/exp) This expression invokes the function at index } \\
\text { (v mod \#functions) with the parameters } \exp _{1}, \ldots \text { and evaluates } \\
\text { to its return value. }\end{array}$ \\
\hline$\left\{\right.$ inst $_{1}$, instr $\left._{2}, \ldots\right\}$ & $\begin{array}{l}\text { (instr) a grouping primitive which combines instr } \text { instr }_{2}, \ldots \text { to a } \\
\text { block }\end{array}$ \\
\hline if $(\exp )$ instr $_{1}\left[\right.$ instr $\left._{2}\right]$ & $\begin{array}{l}\text { (instr) if exp evaluates to a non-zero value, execute instr }{ }_{1} \text {, otherwise } \\
\text { instr }_{2} \text { (if } \text { instr }_{2} \text { is present) }\end{array}$ \\
\hline while (exp) instr & (instr) execute instr as long as exp evaluates to a non-zero value \\
\hline$[v]=\exp$ & $\begin{array}{l}\text { (instr) set memory cell at address } \mathrm{v} \text { to the value of exp and return this } \\
\text { new value }\end{array}$ \\
\hline return (exp) & (instr) leave the current function and return exp \\
\hline send $\left(\exp _{1}[, \ldots]\right)$ & $\begin{array}{l}\text { (instr) send a message containing the result of at most as many param- } \\
\text { eters as the maximum message size to all nodes in reach }\end{array}$ \\
\hline terminate & (instr) terminate the process \\
\hline $\mathrm{v} \in \mathbb{N}$ & $\begin{array}{l}\text { (exp) a simple constant expression which evaluates to the integer value } \\
\mathrm{v} \text {, can be modified by mutation }\end{array}$ \\
\hline $\exp _{1} \quad \mathrm{cmp} \exp _{2}$ & $\begin{array}{l}(\exp ) \text { Compare } \exp _{1} \text { with } \exp _{2} \text { using the operator } \mathrm{cmp} \in \\
\{=, \leq,<, \neq,>, \geq\} \text { and return }-1 \text { if the comparison holds and } 0 \text { other- } \\
\text { wise. See also Table } 5.3\end{array}$ \\
\hline$[\mathrm{v}]$ & $\begin{array}{l}\text { (exp) access the (v mod } 2 n \text { ) th memory cell; the first } n \text { memory cells are } \\
\text { global, the next } n \text { memory cells are local }\end{array}$ \\
\hline $\exp _{1}+\exp _{2}$ & (exp) add $\exp _{1}$ and $\exp _{2}$ and return the result \\
\hline $\exp _{1}-\exp _{2}$ & (exp) subtract $\exp _{2}$ from $\exp _{1}$ and return the result \\
\hline $\exp _{1} * \exp _{2}$ & (exp) multiply $\exp _{1}$ by $\exp _{2}$ and return the result \\
\hline $\exp _{1} / \exp _{2}$ & (exp) divide $\exp _{1}$ by $\exp _{2}$ and return the result $\left(\exp _{2}=0 \Rightarrow\right.$ error) \\
\hline $\exp _{1} \% \exp _{2}$ & $\begin{array}{l}(\exp ) \text { compute the modulo of the division of } \exp _{1} \text { by } \exp _{2} \text { and return the } \\
\text { result }\left(\exp _{2}=0 \Rightarrow \text { error }\right)\end{array}$ \\
\hline $\exp _{1}$ and $\exp _{2}$ & (exp) binary and of $\exp _{1}$ and $\exp _{2}$ \\
\hline $\exp _{1}$ or $\exp _{2}$ & (exp) binary or of $\exp _{1}$ and $\exp _{2}$ \\
\hline not exp & (exp) binary not of exp (ones complement) \\
\hline
\end{tabular}

Table 5.1: The Standard Genetic Programming primitives.

\section{Memory in the SGP Approach}

As already outlined, we distinguish local and global memory in our SGP approach. The reason for this division is to allow functions to have local variables and parameters private to their scope [316]. This way, the main function and the message handler can perform computations without interfering with each other. Global memory enables sharing of information. Without it, the message processing code could not create persistent results, for instance. Messages are processed by passing their contents as parameters to an asynchronously invoked, ISRlike, automatically defined function. If only local memory was available, there would be no means for this function to store any permanent information.

For each function scope, a private stack is provided. These stacks are only used internally to aggregate parameters for function invocation and return values. The distinction of stack, global, and local memory is completely hidden from the Genetic Programming layer. The addresses of the global and local memory are mapped to a shared address space and the stack is utilized only indirectly by the call, return, and send statements.

Figure 5.2 extends the example from Figure 5.1 for demonstrating the use of the different forms of memory in our Standard Genetic Programming approach. It shows a sequence of 


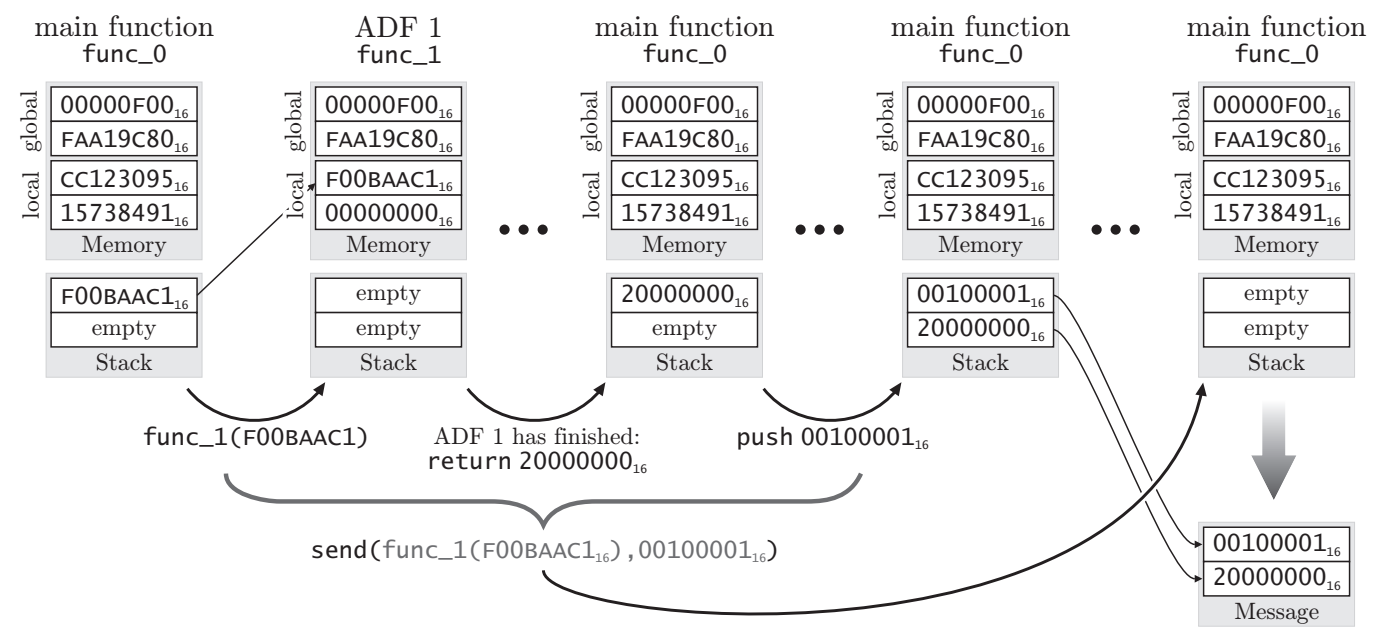

Figure 5.2: An example for the use of memory in the SGP approach based on the program given in Figure 5.1.

operations and their impact on the memory state. When the virtual machine starts up, the main function (func_0) is invoked. In the leftmost picture, we assume that this function already has made some progress and loaded the global and local memory with a set of values. The program is about to call func_1 which would also be invoked when messages arrive.

The expression func_1(FOOBAAC1 $1_{16}$ ) will push the (hexadecimal) value FOOBAAC1 16 onto the stack and then pass control to func_1. In this function, the global memory has the same contents as in the calling instance, but the local memory is initialized with the values from the stack (as illustrated in the second picture).

The third picture shows the memory state at the moment after func_1 has returned the value $20000000_{16}$ where the local memory of the main function has been restored. In the fourth step, the value $00100001_{16}$ is pushed onto the stack - again automatically since this is part of a message sending process. When actually being executed, the send command takes all values from the stack, packs them into a message, and broadcasts it.

The idea of separating memory in three different forms allows expressing very powerful algorithm structures but also sounds rather complicated. After this example it should have become clear that it does not come with an overhead of complexity if subtly integrated into the program representation.

\subsection{Extended Standard Genetic Programming with Indexed Memory [eSGP]}

\subsubsection{Approach}

As we have shown, using different forms of memory made our SGP method quite powerful. Yet, we did not make it Turing-complete. In order to visualize this problem, imagine the restriction that only simple types like integer variables and parameters were allowed was imposed on the Java programming language. Then, no complex types like arrays could be used. In this case, it would become hard to create programs which process data structures like lists, since single variables for each and every of their elements would have to be defined and accessed independently. Writing a method for sorting a list of arbitrary length would even become impossible. 
The same restrictions hold in SGP as introduced in Section 5.5 - the symbols there resemble plain integer variables. Teller [275, 274], Nordin and Banzhaf [212], and Woodward [316] showed that Turing completeness can be achieved by adding indexed memory access to program representations.

The most straightforward method for creating such an extension for SGP is simply to increase the number of address modes. Our Standard Genetic Programming approach provides three modes: access to the local and to the global memory plus an implicit stack. We now introduce addressing of

1. global memory with a value from global memory,

2. global memory with a value from local memory,

3. local memory with a value from global memory, and

4. local memory with a value from local memory.

Such an approach, however, has a slight drawback: In a scenario with two local and two global memory cells, $2 * 6=12$ different addresses can be constructed, three times as many as before. Hence, the chance that information stored by an instruction in a certain memory cell is found by another, randomly created memory access operation decreases to one third of what it was in SGP.

In order to mitigate this degeneration, we introduce the concept of declared variables. Like in most high-level programming languages, only variables which have formerly been declared can be utilized. The declaration of a variable is done with the decl command which can occur at any position in a block and has the effect that a variable becomes available in its whole scope. The first argument of decl identifies the address mode (global, local, global [global], global [local], etc.) and the second one is a base address. Declaring a variable in this extended Standard Genetic Programming form thus differs from declaring a variable in a high-level programming language by enabling GP to explicitly assigning a symbol to a given address.

In the Standard Genetic Programming approach, values from memory were read with the primitive [v] where $\mathrm{v}$ was constant address index. This mechanism is now replaced [var] where var is an index into the list of variables available in the scope of the block the instruction belongs to. The index may exceed the number of variables and is therefore normalized into this interval. ${ }^{2}$ The effect of defining named symbols for memory accesses is that the actual memory size has no influence on the probability of finding formerly stored values anymore. Instead, this probability now only depends on the number of declared variables. Hence, the variables create something like a virtual address space smaller than the physical one. Another advantage of this method is that it allows us to create constructs such as for loops which automatically declare local counter variables for their scope without any need for the GP system to evolve them.

\subsubsection{Adaptation}

The primitives available for our eSGP variant have been derived from those of SGP defined in Table 5.1 and are listed in Table $5.2^{3}$. Again, expressions are annotated with (exp) and instructions with (instr). With the eSGP approach, the readability of the evolved programs suffers even more since now, at least two rather complex steps have to be taken in order to find the correct address in memory belonging to a variable.

\footnotetext{
${ }^{2}$ Here we do not use the modulo operator, but rather a translation based on predefined intervals roughly similar to the approach employed by Paterson [220] in his Gads 2 Grammar-guided Genetic Programming method.

3 The modulo operator was only available in the GCD experiment (see Chapter 9).
} 


\begin{tabular}{|c|c|}
\hline Primitive & Meaning \\
\hline program & the root node of the program \\
\hline func $\{$ instrs $\}$ & same as in Table 5.1 \\
\hline $\operatorname{call}\left(\mathrm{v}\left[, \exp _{1}[, \ldots]\right]\right)$ & (instr/exp) same as in Table 5.1 \\
\hline$\left\{\right.$ instr $_{1}$, instr $\left._{2}, \ldots\right\}$ & (instr) same as in Table 5.1 \\
\hline decl am, addr & $\begin{array}{l}\text { (instr) declare a symbol for the memory cell identified by the address } \\
\text { mode am and address addr (modulo to the total memory size) }\end{array}$ \\
\hline if $(\exp )$ instr $_{1}\left[\right.$ instr $\left._{2}\right]$ & (instr) same as in Table 5.1 \\
\hline while (exp) instr & (instr) same as in Table 5.1 \\
\hline for $\left(c, \exp _{1}, \exp _{2}\right)$ instr & $\begin{array}{l}\text { (instr) execute instr for } i \text { (an automatically declared local variable) } \\
\text { from } \exp _{1} \text { to } \exp _{2} \text { in steps of } 1 \text { or }-1 \text { (depending on } \mathrm{c} \text { ), where } i \text { is an } \\
\text { automatically allocated local variable in the scope of instr }\end{array}$ \\
\hline$[\mathrm{var}]=\exp$ & $\begin{array}{l}\text { (instr) set the memory location identified by the variable at index var } \\
\text { to the value of exp }\end{array}$ \\
\hline return (exp) & (instr) same as in Table 5.1 \\
\hline send $\left(\exp _{1}[, \ldots]\right)$ & (instr) same as in Table 5.1 \\
\hline terminate & (instr) terminate this program \\
\hline $\mathrm{v} \in \mathbb{N}$ & (exp) same as in Table 5.1 \\
\hline $\exp _{1} c m p \exp _{2}$ & (exp) same as in Table 5.1 \\
\hline [var] & (exp) access the varth variable \\
\hline $\exp _{1}+\exp _{2}$ & (exp) same as in Table 5.1 \\
\hline $\exp _{1}-\exp _{2}$ & (exp) same as in Table 5.1 \\
\hline $\exp _{1} * \exp _{2}$ & (exp) same as in Table 5.1 \\
\hline $\exp _{1} / \exp _{2}$ & (exp) same as in Table 5.1 \\
\hline $\exp _{1} \% \exp _{2}$ & (exp) same as in Table 5.1 \\
\hline $\exp _{1}$ and $\exp _{2}$ & (exp) same as in Table 5.1 \\
\hline $\exp _{1}$ or $\exp _{2}$ & (exp) same as in Table 5.1 \\
\hline not $\exp$ & (exp) same as in Table 5.1 \\
\hline
\end{tabular}

Table 5.2: The extended Standard Genetic Programming primitives.

\subsection{Linear Genetic Programming [LGP]}

\subsubsection{Approach}

Trees are not the only way for representing programs - a computer processes them as sequences of instructions instead. These sequences may contain branches in form of jumps to other places in the code. Every possible flowchart describing the behavior of a program can be translated into such a sequence. It is therefore only natural that the first approach to automated program generation developed by Friedberg [97] at the end of the 1950s used a fixed-length instruction sequence genome [97, 98]. The area of Genetic Programming focused on such instruction string representations is called linear Genetic Programming (LGP) [293].

One of the earliest contributions to this field comes from Banzhaf [17], who used a genotype-phenotype mapping with repair mechanisms to translate a bit string into a sequence of simple arithmetic instructions in 1993. The Compiling Genetic Programming System $^{4}$ (CGPS) by Nordin [211], the Automatic Induction of Machine Code with GP system (AIMGP) of Nordin et al. [215], and the analyses by Brameier and Banzhaf [37] are likely the most important works on LGP. Maybe the major advantage shared by all these approaches is the straightforward way in which the programs in linear representations can be evaluated.

${ }^{4}$ Unlike our DGPF, this system works directly on machine code and actually does not involve any compiler. 


\section{Representation}

In our LGP approach, we again distinguish between global and local memory. Additionally, we introduce a private stack for each function scope in order to facilitate return values, function parameters, and arguments for the send instruction. All programs are represented as list of integer strings, where each string stands for one function. The nodes executing the programs are three-address machines, i. e., machines where arithmetic instructions have up to three parameters: the target address and the addresses of two operands. In this, our LGP language is similar to the one used by Lasarczyk and Banzhaf [176] in the Algorithmic Chemistries approach discussed in Section 4.5.3.

Each instruction is represented by four integer values. The first number is the opcode which identifies the instruction and the other three contain the arguments. Value arguments are encoded with three bits standing for the address mode (constant, global memory, local memory, global [global], global [local], local [local], local [global], and the top of stack), while 29 bits are used for a signed value.

\section{Homologous Crossover}

The drawback of linear Genetic Programming is that simply reusing the genetic operators for variable-length string genomes, which randomly insert, delete, or toggle bits, is most often not really feasible. If precautions are taken in order to mitigate these problems, linear Genetic Programming becomes more competitive to Standard Genetic Programming in terms of robustness of the recombination operations. One approach to do so is to create intelligent mutation and crossover operators which preserve the control flow of the program when inserting or deleting instructions. Such operations could, for instance, analyze the program structure and automatically correct jump targets, for instance. Operations which are restricted to have only minimal effect on the control flow from the start can also be introduced.

Although we have tested these methods in the past ${ }^{5}$, in this work we will only utilize homologous crossover together with multi-point crossover. According to Banzhaf et al. [19], natural crossover is very restricted and usually exchanges only genes that express the same functionality and are located at the same positions (loci) on the chromosomes.

Definition 5.1 (Homology). In genetics, homology of protein-coding DNA sequences means that they code for the same protein which may indicate common functionality. Homologous chromosomes are either chromosomes in a biological cell that pair during meiosis or non-identical chromosomes which code for the same functional feature by containing similar genes in different allelic states.

In other words, homologous genetic material is very similar and in nature, only such material is exchanged in sexual reproduction. In linear Genetic Programming with default crossover, it is hard for the evolution to establish a clear structure or a map between locus and functionality. Francone et al. [96, 215] introduce a sticky crossover operator which resembles homology by allowing the exchange of instructions between two genotypes (programs) only if they reside at the same loci. It first chooses a sequence of code in the first genotype and then swaps it with the sequence at exactly the same position in the second parent.

\subsubsection{Adaptation}

The instruction set of this Genetic Programming method is defined in Table 5.3. For the

\footnotetext{
${ }^{5}$ in the experiments in $[295,299,300]$ without explicitly stating this in these papers
} 


\begin{tabular}{|c|c|}
\hline Primitive & Meaning \\
\hline $\operatorname{addr} r_{1}=\operatorname{add} r_{2}+\operatorname{add} r_{3}$ & self-explaining \\
\hline $\operatorname{addr} r_{1}=\operatorname{addr} r_{2}-\operatorname{addr}_{3}$ & self-explaining \\
\hline $\operatorname{addr}_{1}=\operatorname{addr}_{2} * \operatorname{addr}_{3}$ & self-explaining \\
\hline $\operatorname{add} r_{1}=\operatorname{addr} r_{2} / \operatorname{add} r_{3}$ & self-explaining \\
\hline $\operatorname{add} r_{1}=\operatorname{add} r_{2} \%$ add $r_{3}$ & self-explaining \\
\hline $\operatorname{add} r_{1}=$ not add $r_{2}$ & self-explaining, binary not \\
\hline $\operatorname{add} r_{1}=\operatorname{add} r_{2}$ and $\operatorname{add} r_{3}$ & self-explaining, binary and \\
\hline $\operatorname{addr}_{1}=\operatorname{addr}_{2}$ or $\operatorname{addr}_{3}$ & self-explaining, binary or \\
\hline $\operatorname{add} r_{1}=\operatorname{addr}_{2}$ xor $\operatorname{addr}_{3}$ & self-explaining, binary xor \\
\hline $\operatorname{add} r_{1}=\operatorname{add} r_{2}$ & self-explaining \\
\hline $\mathrm{xchg}$ add $r_{1}$, add $r_{2}$ & $t m p=\left[\operatorname{addr}_{1}\right],\left[\operatorname{addr}_{1}\right]=\left[\operatorname{add} r_{1}\right],\left[\operatorname{addr}_{1}\right]=t m p$ \\
\hline $\operatorname{cmp} \operatorname{addr}_{1}, \operatorname{addr}_{2}, \operatorname{addr}_{3}$ & $\begin{array}{l}\text { Compare the values in }\left[\operatorname{addr}_{1}\right] \text { and }\left[\operatorname{addr}_{1}\right] \text { and store the result in } \\
{\left[\operatorname{addr}_{1}\right] \text {. The result is a set of flags similar to the arithmetics-related }} \\
\text { bits in } 80 \times 86 \text { flags register }[142] \text {. }\end{array}$ \\
\hline jmp cond, addr, dst & $\begin{array}{l}\left.\text { cond is a condition which is applied to the value of [addr }{ }_{1}\right] \text { (possibly con- } \\
\text { taining a comparison result): true (jump always), false (jump never), } \\
\mathrm{c} \text { (on carry), nc (no carry), o (overflow), no (no overflow), s (sign), ns } \\
\text { (no sign), } \mathrm{z} \text { (zero), nz (not zero), c or } \mathrm{z}, \mathrm{nc} \text { or } \mathrm{nz}, \mathrm{s} \text { xor o, } \mathrm{s} \text { exor o, } \\
\mathrm{z} \text { or } \mathrm{s} \text { xor } \mathrm{o}, \mathrm{nz} \text { and } \mathrm{s} \text { exor o, null (value is } 0 \text { ), or nnull (value } \neq 0 \text { ). } \\
\text { Flags have the same meanings as in the Intel architecture [142]. Jumps } \\
\text { are relative, their distance is dst. }\end{array}$ \\
\hline call cond, addr, fn & conditional call of function $\mathrm{fn}$; cond has the same semantics as in jmp \\
\hline send cond, addr & $\begin{array}{l}\text { send everything which is on the stack if the stack is not empty and con- } \\
\text { dition cond (same semantics as in jmp) is met }\end{array}$ \\
\hline terminate cond, addr & $\begin{array}{l}\text { conditionally terminate the process; cond has the same semantics as in } \\
\text { jmp }\end{array}$ \\
\hline
\end{tabular}

Table 5.3: The linear Genetic Programming primitives.

sake of simplicity, we stick to a straightforward notation based on arithmetical operators which differs from usual assembler mnemonics. Furthermore, memory addresses like [3], which maps to the second memory cell in local memory in the example given in Figure 5.1, will be expressed as 1[1] instead. Access to global memory will similarly be annotated with the prefix $g$ and writing to or reading from the stack is illustrated with push and pop mnemonics, i. e., [tos] $=[3]$ or [0] $\equiv$ push 1[1] or g[0], for instance.

\subsubsection{On-the-Fly Compiler in the DGPF}

When we discussed the two Standard Genetic Programming methods in Section 5.1 and Section 5.2, we did not elaborate on the way in which programs in these representation are actually executed. The example in Figure 5.2 suggests that interpreting these programs will be a rather complicated process, especially if a division in atomic instructions is considered in order to allow for the simulation of parallelism. Executing linear Genetic Programming code, on the other hand, is rather simple and the programs are already divided into atomic instructions.

The main reason for including linear Genetic Programming in the presented form is to use it as a problem space for the SGP and eSGP approaches. Their tree representations are the genotypes which are processed by the reproduction operations whereas the LGP code corresponds to phenotypes which are evaluated by the objective functions. Therefore, we apply genotype-phenotype mappings $\mathrm{gpm}_{1}:$ SGP $\mapsto$ LGP and $\mathrm{gpm}_{2}:$ eSGP $\mapsto$ LGP which 
translate, i. e., compile, programs in the SGP and the eSGP representation to this pseudoassembler code. ${ }^{6}$

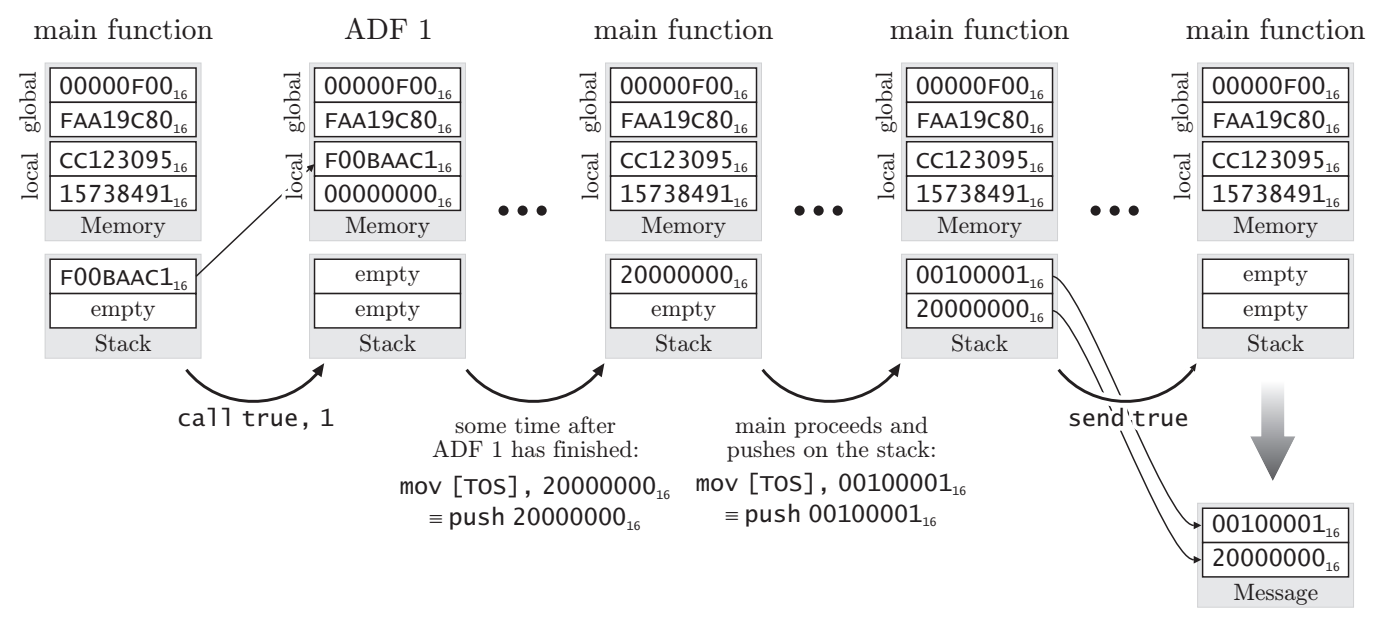

Figure 5.3: The example from Figure 5.2 translated to the LGP approach.

The example in Figure 5.2, for instance, translates to Figure 5.3 where the combinations of different expressions to the send command and its parameters is represented as a simple sequence of instructions. First, the function at index 1, i. e., the second $A D F$, is invoked. call instructions, like jumps and the send primitive, are conditional in this representation and the "true" stands for "execute always" but may be replaced by any other condition listed under jmp in Table 5.3. The behavior of the memory is similar to the (extended) SGP approach and F0OBAAC1 16 shows up in the local memory of the called function. The function stores its return value on its local stack. When it finishes, its local stack is automatically copied to the local stack of the calling function (if it was called by a function and not invoked as interrupt, that is). The main function subsequently stores another value on the stack before executing the send command which broadcasts a message with both values.

\subsection{Fraglets}

\subsubsection{Approach}

In his seminal work, Tschudin [281] introduced Fraglets ${ }^{7}$, a new artificial chemistry suitable for the development and even evolution of network protocols. Fraglets represent an execution model for communication protocols which resembles the chemical reactions in living organisms.

From the theoretical point of view, the Fraglet approach is an instance of Post's string rewriting systems [233] and Gamma systems [13, 15]. Fraglets are symbolic strings of the form $\left[s_{1}: s_{2}: \ldots: s_{n}\right]$. The symbols $s_{i}$ either represent control information or payload. Each node in the network has a Fraglet store which corresponds to a reaction vessel in chemistry. Such vessels usually contain equal molecules multiple times and the same goes for Fraglet stores which can be implemented as multisets keeping track on the multiplicity of the Fraglets they contain.

\footnotetext{
${ }^{6}$ A similar approach has been followed by Juillé and Pollack [151] in order to improve the parallelizability of the program evaluation.

7 See http://www. fraglets.net/ [accessed 2008-05-02] for more information.
} 
Tschudin [281] defines a simple prefix programming language with a fixed instruction set comprising transformation and reaction rules for Fraglets. Transformations like dup and nop modify a single Fraglet whereas reactions such as match and matchP combine two Fraglets.

\subsubsection{Adaptation}

The Fraglet language utilized in our Genetic Programming approaches is specified in Table 5.4. It is a subset of the Fraglet instruction set as of September 2007 defined in http:// ww. fraglets.net/frag-instrset-20070924. txt $^{8}$ with minor extensions. In Table 5.4, we use the syntax $\left[\mathbf{s}_{1}: \mathbf{s}_{2}: \ldots:\right.$ tail] where $\mathbf{s}_{i}$ is a symbol and tail is a possibly empty sequence of symbols.

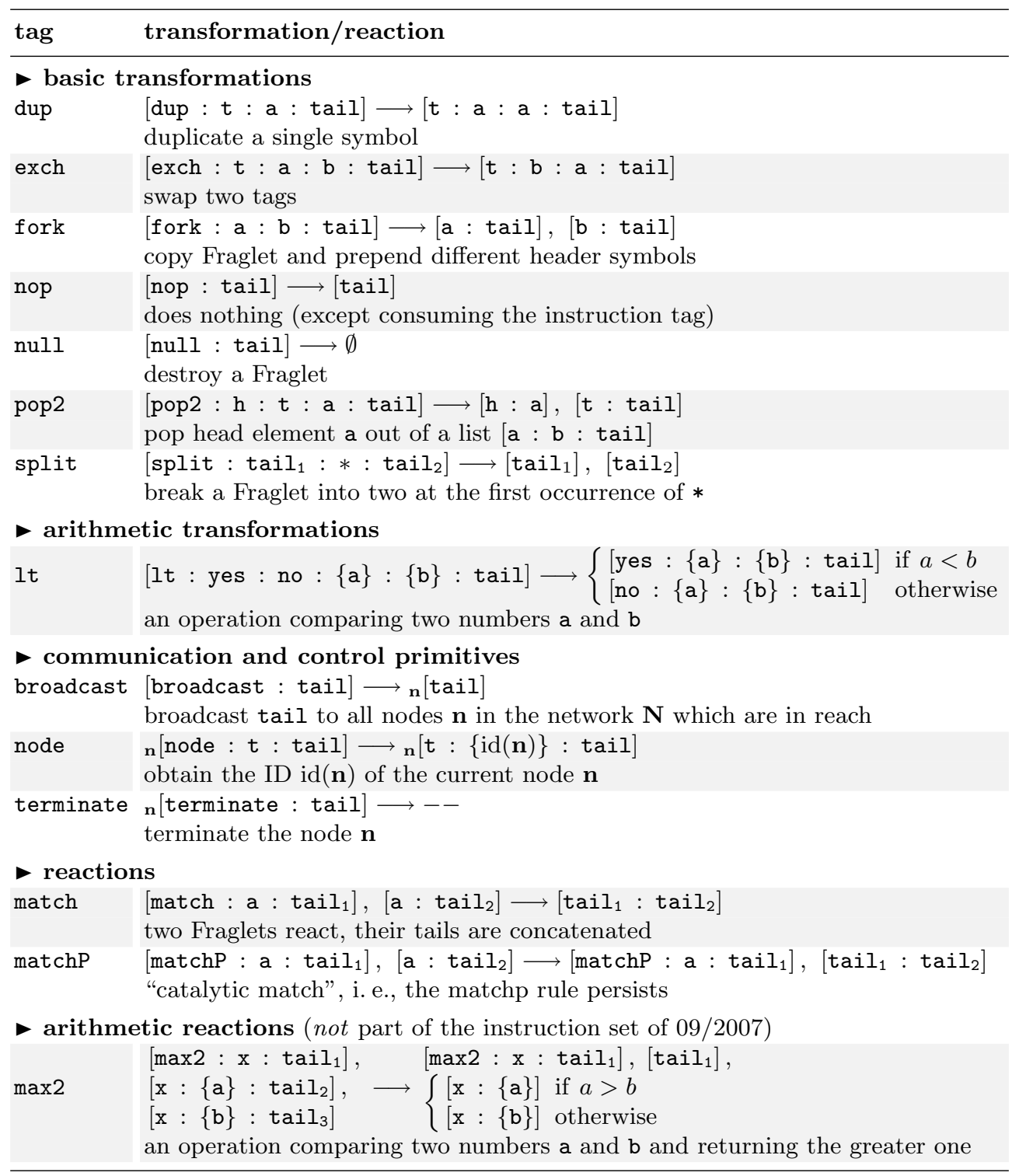

Table 5.4: The Fraglet instructions in our Genetic Programming approach.

\footnotetext{
${ }^{8}$ Accessed 2008-05-02.
} 
Besides Tschudin's work on the topic of evolutionary protocol synthesis, we also summarized the joint research of Yamamoto and Tschudin on online adaptation of protocols in Section 3.2. The utility of the Fraglet representation for this purpose is, at least partly, rooted in its low positional epistasis, since the order of the Fraglets in the Fraglet store plays no role. The order of the single symbols inside a Fraglet, however, is significant.

In the context of this dissertation, we will apply a Genetic Programming approach which encodes each Fraglet symbol as one integer value. A Fraglet program is thus a sequence of integer strings, each standing for one Fraglet rule. Onto such a string, we apply the same genetic operations as in our linear Genetic Programming method. Obviously, the structure of Fraglets is very different from the aforementioned program representations. There are no distinguishable modules or functions, no control flow statements such as jumps or function invocations, and no distinction exists between memory and code. Nevertheless, the Fraglet system is at least as powerful as the SGP approach and there are indications that it is likely Turing-complete [74].

It should be noted that we simulate the execution of Fraglet programs in our system by picking Fraglets with a uniform probability from the Fraglet store in a way that respects their multiplicity. In actual implementations, the Fraglets are selected for execution with probabilities closer to the way they would be in chemical reactions. This difference should have only minor influence on the evolution of programs. We chose our simplified approach for speeding up the simulations and because of its appealing simplicity.

\subsection{Rule-based Genetic Programming [RBGP]}

\subsubsection{Approach}

In Section 4.5 we have argued that epistasis is one of the key problems in Genetic Programming. We gave short outlines of some GP approaches which try to circumvent this problem and discussed, for instance, the Algorithmic Chemistry method by Lasarczyk and Banzhaf [176]. Besides such special representations, there exists one very general class of evolutionary algorithms that elegantly circumvents positional epistasis: the (Learning) Classifier Systems family $[132,130]$ which we briefly introduced based on a simple example in Section 2.6.

In the Pittsburgh Learning Classifier Systems approach associated with Spears and de Jong [267], a population of rule sets is evolved with a genetic algorithm [262]. Each individual in this population consists of multiple classifiers (the rules) that transform input signals into output signals. The evaluation order of the rules in such a classifier system $C$ plays absolutely no role except maybe for rules concerning the same output bits, i. e., $\theta(C) \approx 1.9$

The basic idea behind our Rule-based Genetic Programming approach is to use this knowledge to create a new program representation that retains high $\theta$-values in order to become more robust in terms of reproduction operations [298]. With RBGP, the aforementioned disadvantages (such as non-determinism) of Algorithmic Chemistries and soft assignments are completely circumvented. RBGP may be considered as a high-level classifier systems variant which introduces mightier concepts like mathematical operations. It furthermore exhibits a certain amount of non-uniform neutrality which, as we believe, is likely to increase the chance of finding better solutions.

We illustrate this new Genetic Programming method by using an example in Figure 5.4. Like in Pitt-style Learning Classifier Systems, the depicted programs consist of arbitrary many rules which can be encoded binary. A rule evaluates the values of the symbols in its condition part (left of $\Rightarrow$ ) and, in its action part, assigns a new value to one symbol or

\footnotetext{
${ }^{9} \theta$ as a measure for positional epistasis has been defined in Section 4.5 .2 on page 58 .
} 
may invoke any other procedure provided in its configuration. In its structure, the RBGP language is similar to Dijkstra's Guarded Command Language (GCL) [73]. ${ }^{10}$

\begin{tabular}{c|l} 
Symbol & Encoding \\
\hline 0 & 0000,1100 \\
1 & 0001,1101 \\
start & 0010,1110 \\
id & 0011,1111 \\
netSize & 0100 \\
in & 0101 \\
out & 0110 \\
enterCS & 0111 \\
csLeft & 1000 \\
a & 1001 \\
b & 1010
\end{tabular}

\begin{tabular}{c|c} 
Comp. & Enc. \\
\hline$>$ & 000 \\
$\geq$ & 001 \\
$=$ & 010 \\
$\leq$ & 011 \\
$<$ & 100 \\
$\neq$ & 101 \\
true & 110 \\
false & 111
\end{tabular}

\begin{tabular}{c|c} 
Concat. & Enc. \\
\hline$\wedge$ & 0 \\
$\vee$ & 1
\end{tabular}

\begin{tabular}{c|c} 
Action & Enc. \\
\hline$=\mathrm{x}+\mathrm{y}$ & 00 \\
$=\mathrm{x}-\mathrm{y}$ & 01 \\
$=\mathrm{x}$ & 10 \\
$=1-\mathrm{x}$ & 11
\end{tabular}

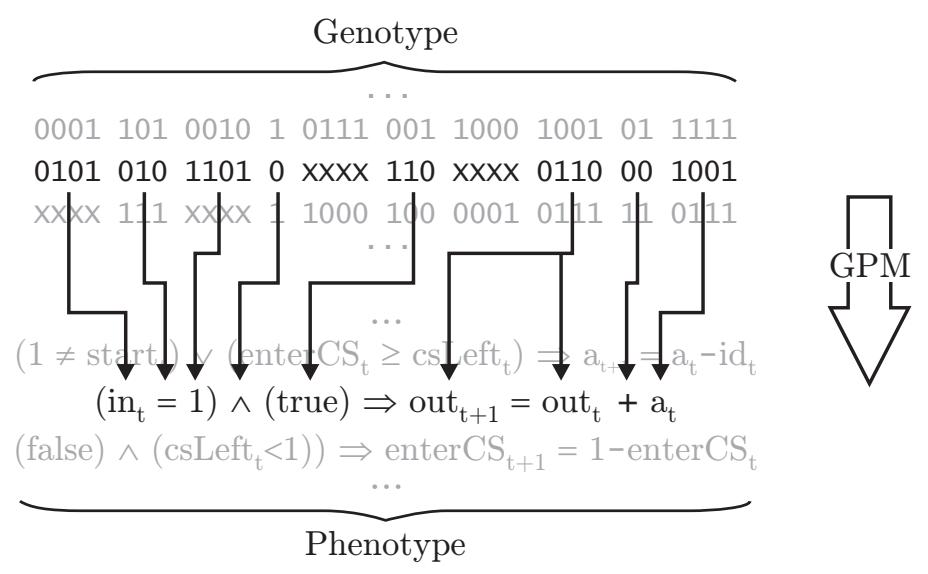

Figure 5.4: Example for a genotype-phenotype mapping in RBGP.

\section{Representation}

Before the evolution in Rule-based Genetic Programming begins, the number of symbols and their properties must be specified as well as the possible actions. Each symbol identifies an integer variable which is either read-only or read-write. Some read-only symbols are defined for constants like 0 and 1 , for instance. The symbol start is only 1 during the first application of the rule set and becomes 0 afterwards (but may be written to by the program). Furthermore, a program can be provided with some general-purpose variables ( $\mathrm{a}$ and $\mathrm{b}$ in the example). Additional symbols with special meanings can be introduced. For evolving distributed algorithms, for instance, an input symbol in where incoming messages will occur and a variable out from which outgoing messages can be transmitted could be added. If messages should be allowed to contain more than one value, multiple such symbols have to be defined. These out symbols may trigger message transmission directly when written to as in Figure 5.4. Alternatively, a message can be sent by a special send action as is the case in this work (see Table 5.5).

An action set containing mathematical operations like addition, subtraction, value assignment, and an equivalent to logical negation ${ }^{11}$ is sufficient for many problems but may

${ }^{10}$ I want to thank David R. White for our discussion at the GECCO'08 regarding that matter.

${ }^{11}$ In order to emulate a logical not, we use the expression 1-x where $\mathrm{x}$ can be an arbitrary symbol. 
be extended arbitrarily. In conjunction with the constants 0 and 1 and the comparison operation, the evolutionary process can build arbitrary complex logical expression.

From the initial symbol and action specifications, the system can determine how many bits are needed to encode a single rule. A binary encoding where this is the size of the genes in variable-length bit string genotypes can then be used. With such simple genotypes, any possible nesting depth of condition statements and all possible logical operations can be encoded. If needed, a tree-like program structure (as in Standard Genetic Programming) can be constructed from the rule sets, since each rule corresponds to a single conditional statement in a normal programming language.

There are similarities between our RBGP and some special types of Learning Classifier Systems, like the abstracted LCS by Browne and Ioannides [40] and the S-expression-based LCS by Lanzi and Perrucci [175]. The two most fundamental differences lie in the semantics of both, the rules and the approach: In RBGP, a rule may directly manipulate symbols and invoke external procedures with (at most) two in/out-arguments. This includes mathematical operations like multiplication and division which do not exist a priori in classifier systems but would have to evolve on basis of binary operations (which is, although possible, very unlikely). Furthermore, the individuals in RBGP are not classifiers but programs. Classifiers are intended to be executed once for a given situation, judge it, and decide upon an optimal output. A program, on the other hand, runs independently, asynchronously performs a computation, and interacts with its environment. Also, the syntax of RBGP is very extensible because the nature of the symbols and actions is not bound to specific data types but can easily be adapted to floating point computation, for instance.

\section{Program Execution and Dimensions of Independence}

The simplest method for executing a rule-based program is to loop over it in a cycle. Although this approach is sufficient for the simulation purposes in our experiments, it would result in a waste of CPU power on a real system. This consumption of computational power (and thus, energy) can be reduced very much if the conditional parts of the rules are only evaluated when one of the symbols that they access changes. In our experiments, one time step in the simulation equals one application of all rules.

\section{Positional Independence}

Changes in the values of the symbols can either be caused by data incoming from the outside, like messages which are received (and stored in the in-symbols in our example) or by the actions invoked by the program itself. In RBGP, actions do not directly modify the values of the symbols but rather write their results to a temporary storage. After all rules have been processed, the temporary storage is committed to the real memory, as sketched in Figure 5.5. The symbols in the condition part and in the computation parts of the actions are annotated with the index $t$ and those in the assignment part of the actions are marked with $t+1$ in order to illustrate this issue.

This approach allows for a great amount of disarray in the rules since the only possible positional dependencies left are those of rules which write to the same symbols. All other rules can be freely permutated without any influence on the behavior of the program. Hence, the positional epistasis in RBGP is very low.

\section{Cardinality Independence}

By excluding any explicit learning features (like the Bucket Brigade Algorithm used in Learning Classifier Systems [126, 127]) from the evolution, we also gain insensitivity in terms of rule cardinality. It is irrelevant whether a rule occurs once, twice, or even more 
1: $\quad j=a$;

2: $p=1$

3: while $(i>0)\{$

4: $\quad p=p * i$;

6: \} $p=p * i$
$i=i-1 ;$

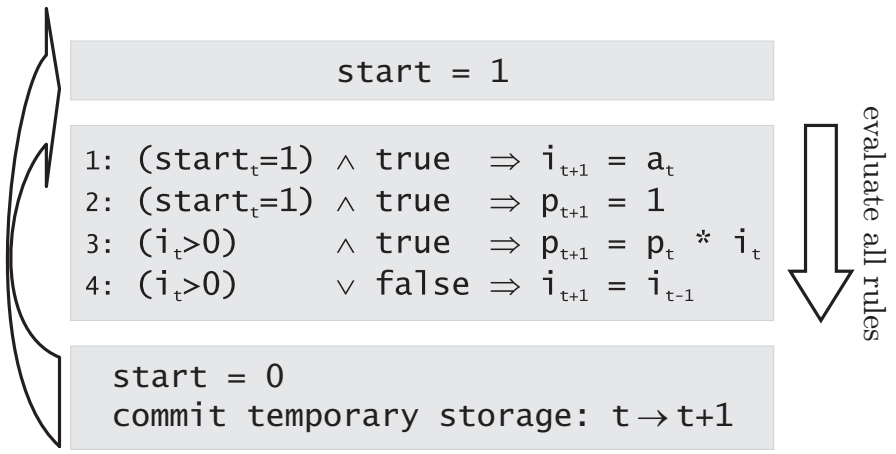

Rule-based Genetic Programming Syntax

Figure 5.5: A program computing the faculty $\mathrm{p}$ of a natural number a in Java and RBGP syntax.

often in a program. If triggered, all occurrences of the rule use the same input data and thus, will write the same values to the temporary variable representing their target symbol. Assuming that an additional objective function which puts pressure into the direction of smaller programs is always imposed, superfluous rules will be wiped out during the course of the evolution anyway.

\section{Neutrality}

The existence of neutral reproduction operations can have a positive as well as negative influence on the evolutionary progress (see Section 4.3.1 on page 52). The positional and cardinality independence are clear examples of phenotypic neutrality and redundancy in RBGP. They allow a useful rule to be replicated arbitrarily often in the same program without decreasing its functional fitness. This is likely to happen during crossover. By doing so, the conditional parts of the rule will (obviously) be copied too. Subsequent mutation operations may now slightly modify the rule and lead to improved behavior, i. e., act as exploitation operations. Based on the discussion of neutrality and our more detailed elaborations in [306, 293], we expect this form of non-uniform redundancy to have a rather positive effect on the evolution.

All these new degrees of freedom are achieved without most of the drawbacks that are inherent in Algorithmic Chemistries. The program flow is fully deterministic and so are its results. Like in Algorithmic Chemistries, it is harder to determine the number of steps needed for program execution, although we can easily detect the termination of local algorithms as the point where an execution does not lead to any change in the symbols.

\section{Complex Statements}

From the previous descriptions, it would seem that rule-based programs are strictly sequential, without branching or loops. This is not the case. Instead, a wide variety of complex computations can be expressed with them. Here we will give some intuitive examples for such program structures in RBGP syntax.

\section{Complex Conditions}

Assume that we have five variables a to e and want to express something like 


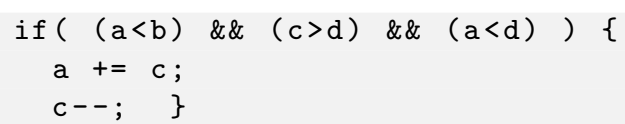

Listing 5.1: A complex conditional statement in a C-like language.

We can do this in RBGP with four rules:

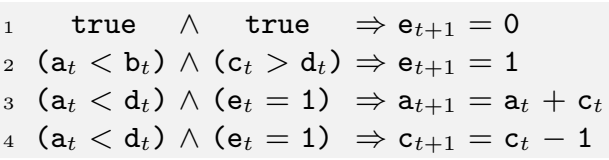

Listing 5.2: The RBGP version of Listing 5.1.

Although this does not look very elegant, it fulfills the task by storing the result of the evaluation of the first part of the condition as logical value in the variable e. e will normally be 0 because of line 1 and is only set to 1 by rule 2 . Since both rules write to the same temporary variable, the then-part of the condition in Listing 5.1 (lines 3 and 4 in Listing 5.2) will be reached in the next round if a<d holds too. Notice that the only positional dependency in Listing 5.2 is that rule 2 must always occur after rule 1 . All rule permutations that obey this statement are equivalent (hence, $\theta=0.5$ ) and so is Listing 5.3:

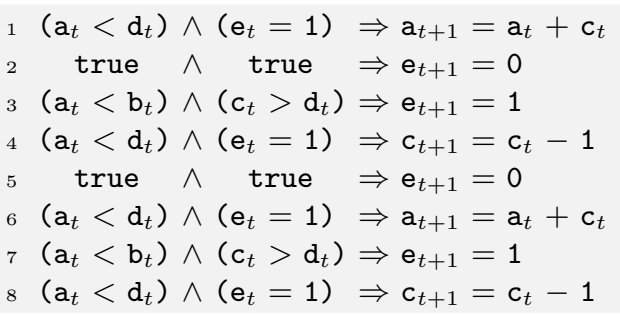

Listing 5.3: An equivalent alternative version of Listing 5.2.

\section{Loops}

Loops in RBGP can be created in the very same fashion:

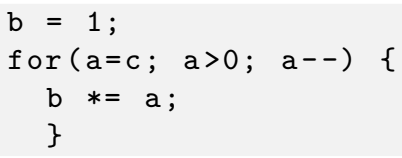

Listing 5.4: A loop in a C-like language.

The loop defined in Listing 5.4 can be expressed in RBGP as outlined in Listing 5.5, where we use the start-symbol (line 1 and 2) to initialize a and b. As its name suggests, start is only 1 at the very beginning of the program's execution and 0 afterwards (unless modified by an action).

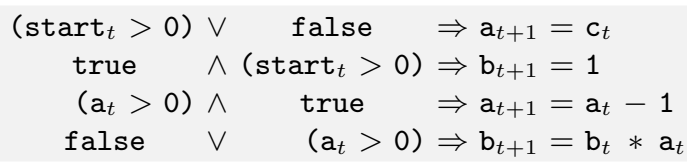

Listing 5.5: The RBGP-version of Listing 5.4.

Here, no positional or cardinality restrictions occur at all, so Listing 5.6 is equivalent to Listing 5.5 and $\theta=1$. 


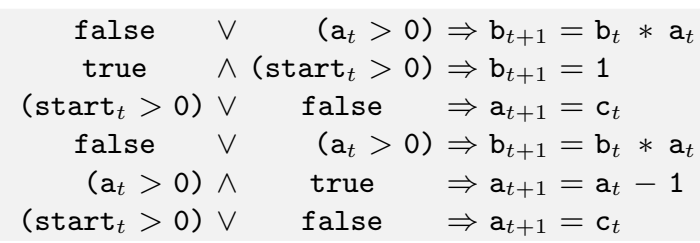

Listing 5.6: An equivalent, alternative version of Listing 5.5.

\subsubsection{Adaptation}

In the previous sections, we laid down how RBGP programs are executed and gave some examples for illustration purposes. The adaptation of this new form of program representation to the evolution of distributed algorithms is rather straightforward and only involves defining a dedicated action and symbol set which is given in Table 5.5.

\begin{tabular}{|c|c|}
\hline Primitive & Meaning \\
\hline \multicolumn{2}{|l|}{ D Actions } \\
\hline $\operatorname{var}_{1, t+1}=\operatorname{var}_{1, t}+\operatorname{var}_{2, t}$ & $\begin{array}{l}\text { (instr) add the current value of } \operatorname{var}_{2} \text { to } \operatorname{var}_{1} \text { and make the result avail- } \\
\text { able in the next iteration }\end{array}$ \\
\hline $\operatorname{var}_{1, t+1}=\operatorname{var}_{1, t}-\operatorname{var}_{2, t}$ & $\begin{array}{l}\text { (instr) subtract the current value of } \operatorname{var}_{2} \text { from } \operatorname{var}_{1} \text { and make the result } \\
\text { available in the next iteration }\end{array}$ \\
\hline $\operatorname{var}_{1, t+1}=\operatorname{var}_{1, t} * \operatorname{var}_{2, t}$ & $\begin{array}{l}\text { (instr) multiply the current value of } \operatorname{var}_{1} \text { with } \operatorname{var}_{2} \text { and make the result } \\
\text { available in the next iteration }\end{array}$ \\
\hline $\operatorname{var}_{1, t+1}=\operatorname{var}_{1, t} / \operatorname{var}_{2, t}$ & $\begin{array}{l}\text { (instr) divide the current value of } \operatorname{var}_{1} \text { by } \operatorname{var}_{2} \text { and make the result } \\
\text { available in the next iteration }\end{array}$ \\
\hline $\operatorname{var}_{1, t+1}=\operatorname{var}_{1, t} \% \quad \operatorname{var}_{2, t}$ & $\begin{array}{l}\text { (instr) compute the modulo of the current value of } \operatorname{var}_{1} \text { to } \operatorname{var}_{2} \text { and } \\
\text { make the result available in the next iteration }\end{array}$ \\
\hline $\operatorname{var}_{1, t+1}=$ not $\operatorname{var}_{1, t}$ & $\begin{array}{l}\text { (instr) set the current value of } \operatorname{var}_{1} \text { to } 1-\text { var }_{1} \text { and make the result } \\
\text { available in the next iteration }\end{array}$ \\
\hline terminate & $\begin{array}{l}\text { (instr) terminate the instance of the program on the current VM without } \\
\text { committing the changes of this iteration }\end{array}$ \\
\hline send & $\begin{array}{l}\text { (instr) broadcast the values in all output registers after this iteration } \\
\text { has ended }\end{array}$ \\
\hline \multicolumn{2}{|c|}{ - Symbols (in correct order, starting at index 0) } \\
\hline$a, b, \ldots$ & (exp) local variables \\
\hline 0 & (exp) the constant zero \\
\hline 1 & (exp) the constant one \\
\hline start & $\begin{array}{l}\text { (exp) the (writable) start symbol (value } 1 \text { in first iteration, then } 0 \text { - unless } \\
\text { programmatically changes) }\end{array}$ \\
\hline out $_{1 . . n}$ & (exp) $n$ symbols into which outgoing messages are written \\
\hline $\operatorname{in}_{1 . . n}$ & (exp) $n$ symbols into which incoming messages are written \\
\hline incomingMsg & (exp) a symbol which is 1 if a new message was received and 0 otherwise \\
\hline ID & (exp) the (writable) symbol holding the current node's ID \\
\hline
\end{tabular}

Table 5.5: The Rule-based Genetic Programming primitives.

\subsection{Extended Rule-based Genetic Programming [eRBGP]}

\subsubsection{Approach}

We have shown that, although looking rather simple, the primitives of Rule-based Genetic Programming are mighty enough to express many of the constructs known from high-level 
programming languages. However, in the original RBGP approach, there are some inherent limitations.

Its most obvious drawback is the lack of Turing completeness which we also observed in SGP. Like in the transition from SGP to eSGP, adding indirect memory access to the programming language forming the basis of Rule-based Genetic Programming would allow the evolution of more complex algorithms. We therefore define the notation $\left[a_{t}\right]_{t}$, which stands for the value of the $\left(\mathrm{a}_{t}\right)$ th symbol at time step $t$ in the ordered list of all symbols. In this, it equals a simple pointer dereferentiation (*a) in the $\mathrm{C}$ language.

With this extension alone, it becomes possible to use the RBGP language for defining list sorting algorithms, for instance. Assume that the following symbols $\left(i_{0}, i_{1}, . ., \dot{i}_{n-1}, 1, a, b\right)$ have been defined. The symbols $i_{0}$ to $i_{n-1}$ constitute the memory which can be used to store the list elements and 1 is initialized with the length of the list, i. e., the number of the i-elements actually used (which has to be smaller or equal to $n$ ). a and $\mathrm{b}$ are multi-purpose variables. In the symbol list, $i_{0}$ is at position 0,1 at position $n$, a at index $n+1$ and so on. With very little effort, Listing 5.7 can be defined which performs a variant of selection sort. Notice that, since writing to variables is not committed before all rules were applied, no explicit temporary variable is required in the third and fourth rule.

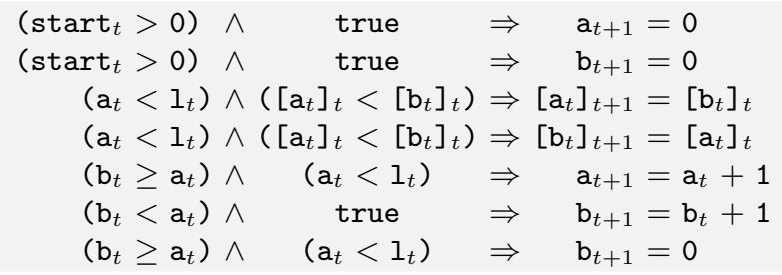

Listing 5.7: A simple selection sort algorithm written in the eRBGP language.

Listing 5.2, one of our previous examples shows another feature of RBGP which might prove troublesome: The condition part of a rule always consists of two single conditions. This is totally unimportant as long as a logical expression to be represented contains only one or two conditions. (If it needs only one, the second condition of the rule may be set to true and concatenated with an $\wedge$-operator.) In Listing 5.2, however, we try to represent the conditional statement from Listing 5.1 which consists of three conditions. In order to do so, we needed to introduce the additional symbol e.

Here we can draw an analogy to the human memory which may be divided into procedural (implicit) memory [110, 251, 315] storing, for instance, motor skills and declarative (explicit) $[83,160]$ memory holding facts and data. In comparison with RBGP, we would find that the expressiveness of the equivalent of the procedural memory in RBGP is rather limited, which needs to be mitigated by using more of it and storing additional information in the declarative memory. We used this approach when translating Listing 5.1 to Listing 5.2, for instance. This issue can be compared to a hypothetical situation in which we were not able to learn the complete motion of lifting a jar to our lips and instead, could only learn how to lift a jar from the table and how to move an already lifted jar to our mouth while needing to explicitly remember that both moves belong together.

Admittedly, this analogy may be a bit farfetched, but it illustrates that Rule-based Genetic Programming could be forced to go through a seemingly complex learning process for building a simple algorithm under some circumstances. We therefore extend its expressiveness by dropping the constraints on the structure of its rules which increases the number of ways that RBGP can be utilized for representing complicated expressions. The ability of using genetic algorithms with fixed-size genes for evolving rule-based programs, however, has to be traded in in order to facilitate this extension. Additionally, this extension might bring back some of the epistasis which we had previously successfully decreased. 
$\left(\left(a_{t}<b_{t}\right) \wedge\left(\left(c_{t}>d_{t}\right) \wedge\left(a_{t}<d_{t}\right)\right)\right) \Rightarrow a_{t+1}=\left(a_{t}+c_{t}\right)$

$2\left(\left(a_{t}<b_{t}\right) \wedge\left(\left(c_{t}>d_{t}\right) \wedge\left(a_{t}<d_{t}\right)\right)\right) \Rightarrow c_{t+1}=\left(c_{t}-1\right)$

Listing 5.8: The eRBGP version of Listing 5.1 and Listing 5.2.

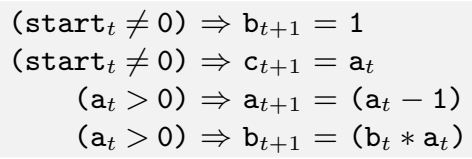

Listing 5.9: The eRBGP version of Listing 5.4 and Listing 5.5.

In Listing 5.8 and Listing 5.9, we repeat the RBGP examples Listing 5.2 and Listing $5.5-$ this time in eRBGP syntax. As already mentioned, we cannot use a simple genetic algorithm to evolve these programs since their structure does not map to a fixed gene size anymore. However, tree-based Genetic Programming as discussed in Section 2.5 can perfectly fulfill this purpose. Listing 5.8, for instance, fits to the tree phenotype depicted in Figure 5.6.

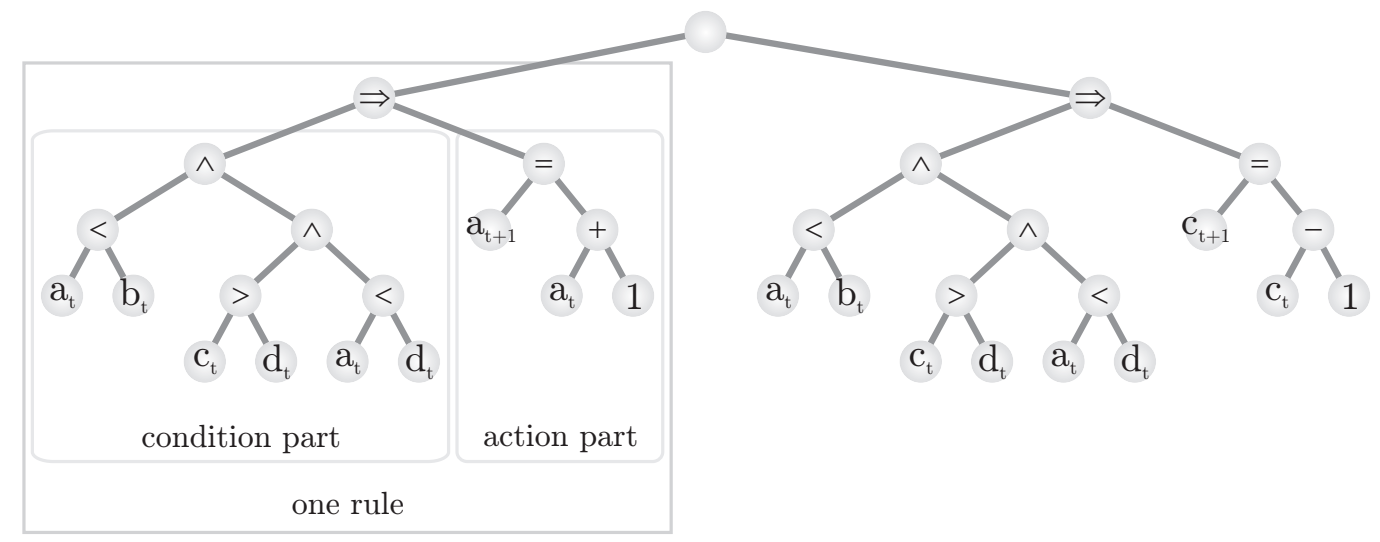

Figure 5.6: The tree phenotype (and genotype) of Listing 5.8.

With these changes, Extended Rule-based Genetic Programming becomes much more powerful in comparison with plain Rule-based Genetic Programming and is now able to evolve arbitrary algorithms and data structures. Also, the proof for Turing completeness of Genetic Programming languages with indexed memory by Teller [275] can easily be adapted to Extended Rule-based Genetic Programming.

\subsubsection{Adaptation}

The eRBGP approach can be used for the evolution of distributed algorithms in the same way as plain Rule-based Genetic Programming. In Table 5.6, we show the primitives available in our experiments ${ }^{12}$ and (in compliance with Section 5.1.2), we annotate instructions with (instr) and expressions with (exp).

\footnotetext{
$\overline{12}$ The modulo operator was only available in the GCD experiment (see Chapter 9).
} 


\begin{tabular}{|c|c|}
\hline Primitive & Meaning \\
\hline \multicolumn{2}{|l|}{ - Actions } \\
\hline $\exp \Rightarrow$ action & execute action if exp evaluates to a non-zero value \\
\hline $\mathrm{a}_{t+1}=\exp$ & $\begin{array}{l}\text { (instr) set the value of symbol to the value of exp and make it available } \\
\text { in the next iteration }\end{array}$ \\
\hline$\left[\mathrm{a}_{t}\right]_{t+1}=\exp$ & $\begin{array}{l}\text { (instr) set the value in the symbol with the number } a_{t} \text { to the value of } \\
\text { exp }\end{array}$ \\
\hline $\exp _{1}+\exp _{2}$ & $(\exp )$ add $\exp _{1}$ and $\exp _{2}$ and return the result \\
\hline $\exp _{1}-\exp _{2}$ & (exp) subtract $\exp _{2}$ from $\exp _{1}$ and return the result \\
\hline $\exp _{1} * \exp _{2}$ & (exp) multiply $\exp _{1}$ by $\exp _{2}$ and return the result \\
\hline $\exp _{1} / \exp _{2}$ & (exp) divide $\exp _{1}$ by $\exp _{2}$ and return the result \\
\hline $\exp _{1} \% \exp _{2}$ & $\begin{array}{l}(\exp ) \text { compute the modulo of the division of } \exp _{1} \text { by } \exp _{2} \text { and return the } \\
\text { result }\end{array}$ \\
\hline $\exp _{1} \wedge \exp _{2}$ & (exp) -1 if both, $\exp _{1}$ and $\exp _{2}$, evaluate to non-zero values, 0 otherwise \\
\hline $\exp _{1} \vee \exp _{2}$ & $\begin{array}{l}\text { (exp) }-1 \text { if at least one of } \exp _{1} \text { or } \exp _{2} \text { evaluate to non-zero values, } 0 \\
\text { otherwise }\end{array}$ \\
\hline not $(\exp )$ & $(\exp )-1$ if $\exp =0,0$ otherwise \\
\hline $\exp _{1} \mathrm{cmp} \exp _{2}$ & $\begin{array}{l}\text { (exp) compare } \exp _{1} \text { and } \exp _{2} \text { according to the operator of } \mathrm{cmp} \text {, which } \\
\text { is either }<, \leq,=, \geq,>, \neq \text { and return }-1 \text { if the condition is met or } 0 \\
\text { otherwise }\end{array}$ \\
\hline send & $\begin{array}{l}\text { (instr) broadcast the values in all output registers after this iteration } \\
\text { has ended }\end{array}$ \\
\hline terminate & terminate the process \\
\hline \multicolumn{2}{|c|}{ - Symbols (in correct order, starting at index 0) } \\
\hline $\mathrm{a}, \mathrm{b}, \ldots$ & (exp) same as in Table 5.5 \\
\hline start & (exp) same as in Table 5.5 \\
\hline out $_{1 . . n}$ & (exp) same as in Table 5.5 \\
\hline$i n_{1 . . n}$ & (exp) same as in Table 5.5 \\
\hline incomingMsg & $(\exp )$ same as in Table 5.5 \\
\hline ID & (exp) same as in Table 5.5 \\
\hline
\end{tabular}

Table 5.6: The Extended Rule-based Genetic Programming primitives. 


\subsection{Summary}

In this chapter, we have introduced six different program representations and Genetic Programming approaches and adapted them to the evolution of distributed algorithms. In Table 5.7 we summarize their search spaces $\mathbb{G}$, their problem spaces $\mathbb{X}$, whether they are Turing-complete (TC) or not, and what their equivalent of one single execution step is.

\begin{tabular}{|c|c|c|c|c|}
\hline & search space $\mathbb{G}$ & problem space $\mathbb{X}$ & $\mathrm{TC}$ & 1 step $\equiv$ \\
\hline SGP & trees & LGP & no & 1 instruction \\
\hline eSGP & trees & LGP & yes & 1 instruction \\
\hline LGP & $\begin{array}{l}\text { variable-length lists of variable-length in- } \\
\text { teger strings, } 1 \text { list } \equiv 1 \text { function, gene size: } \\
4 \text { integers, } 1 \text { gene } \equiv 1 \text { instruction }\end{array}$ & $\begin{array}{l}\equiv \mathbb{G}, \text { direct interpre- } \\
\text { tation }\end{array}$ & yes & 1 instruction \\
\hline Fraglets & $\begin{array}{l}\text { variable-length lists of variable-length in- } \\
\text { teger lists, } 1 \text { integer list } \equiv 1 \text { fraglet string, } \\
1 \text { integer } \equiv 1 \text { fraglet }\end{array}$ & $\begin{array}{l}\equiv \mathbb{G}, \text { direct interpre- } \\
\text { tation }\end{array}$ & $\begin{array}{l}\text { likely } \\
{[74]}\end{array}$ & 1 reaction \\
\hline RBGP & $\begin{array}{l}\text { variable-length bit strings; gene size de- } \\
\text { pends on number of symbols and actions }\end{array}$ & $\begin{array}{l}\text { rule sets; GPM: } 1 \\
\text { gene } \equiv 1 \text { rule }\end{array}$ & no & $\begin{array}{l}1 \text { application } \\
\text { of all rules }\end{array}$ \\
\hline eRBGP & trees & $\equiv \mathbb{G}$ & yes & $\begin{array}{l}1 \text { application } \\
\text { of all rules }\end{array}$ \\
\hline
\end{tabular}

Table 5.7: The six Genetic Programming approaches utilized in this work.

We think that with these approaches, we have covered a wide area of different program representations and genomes. This will enable us to draw meaningful comparisons and conclusions in our experiments. 


\section{Distributed System Model and Simulations}

In this chapter, we will discuss how the features of distributed systems relevant for the Genetic Programming of distributed algorithms can be modeled. In Section 6.1, we clarify the basic concepts of distributed systems and algorithms. A system model is given as foundation for network simulations in Section 6.2. Section 6.3 contains the exact structure and definitions of the simulations which we use to determine the utility of distributed algorithms. The overall system architecture of our Genetic Programming system is outlined in Section 6.4. Section 6.5 provides a short summary on this chapter.

\subsection{Distributed Systems and Algorithms}

Various definitions have been issued for the terms distributed system and distributed algorithms by several researchers such as Lamport [168], Tanenbaum and van Steen [273], Mattern [193] and Coulouris et al. [54]. These definitions most often only differ in minor details and can be summarized as follows.

Definition 6.1 (Distributed System). A distributed system is a set of autonomous systems (nodes) which are connected by a network and communicate via the exchange of messages.

Definition 6.2 (Distributed Algorithm). Distributed algorithms [193, 20] are algorithms which are executed by multiple computers in a distributed system and cooperatively try to solve a given problem.

Distributed algorithms can be distinguished from sequential algorithms because they run on multiple nodes in parallel in order to cooperatively solve one problem. They can be distinguished from mere parallel algorithms since each node in the distributed system executes instances of the same algorithm ${ }^{1}$ with a (usually) different view on the global state [273].

The reason for this lack of a common view on the global state is that each node has only knowledge about the information locally available on it. Information on the other nodes can only be obtained via communication which usually comprises the exchange of messages.

Messages sent over any medium are delayed for a certain amount of time. Of course, with technical effort such as special clocking, any latency effects could be made transparent for the system. In general computer networks, let alone MANETs [279, 278] or sensor networks $[139,42]$, this is not possible. Because of this latency, the nodes cannot have

\footnotetext{
${ }^{1}$ This is not necessarily the case in all forms of distributed algorithms. In the context of this work,
} however, we limit our considerations to distributed algorithms where this holds. 
exactly the same view on the world and it is not possible to have a globally synchronized system time available. Furthermore, networks may induce arbitrary errors into the content of the messages which may even get lost, i. e., have an infinite latency.

Distributed algorithms can provide the following advantages (depending on their design): modularity, flexibility, resource-sharing, no central point of failure, scalability because of decentralization, robustness, high availability, and fault-tolerance. In turn, they may have the following drawbacks, again depending on their design: higher complexity, no common view on the global state, no accurate global time, processes may fail, latency and faults in communication, problems in termination detection, deadlocks, and race conditions [54, 193].

According to Definition 4.5 on page 69 , we refer to distributed algorithms which are able to fulfill their tasks from the viewpoint of an observer as adequate. The non-functional quality of a distributed algorithm is determined by the resources it requires to ensure its functionality such as its communications complexity, i. e., how many messages need to be exchanged in order to fulfill its task, or its time complexity, i. e., how many computational steps need to be performed on the single nodes. Both features depend on how good the advantages of distribution are utilized and on how strongly its drawbacks are present.

\subsection{The Distributed System Model}

One of the key points of our work is that we consider the evolution of distributed algorithms for asynchronous systems [54]. By doing so, the results reflect the applicability of Genetic Programming to distributed systems in real-world scenarios. Our approach can thus clearly be distinguished from the research of Qureshi [235, 237] and Iba [137, 138], who showed that synchronous communication can be helpful for agents in pursuit and rendezvous games, for instance.

\subsubsection{The Node Model}

Figure 6.1 sketches the model which defines the environment for the evaluation of distributed algorithms in our Distributed Genetic Programming Framework (DGPF). This model is very general and only imposes few restrictions on the nodes, which is commanded by the wide variety of Genetic Programming approaches we have applied.

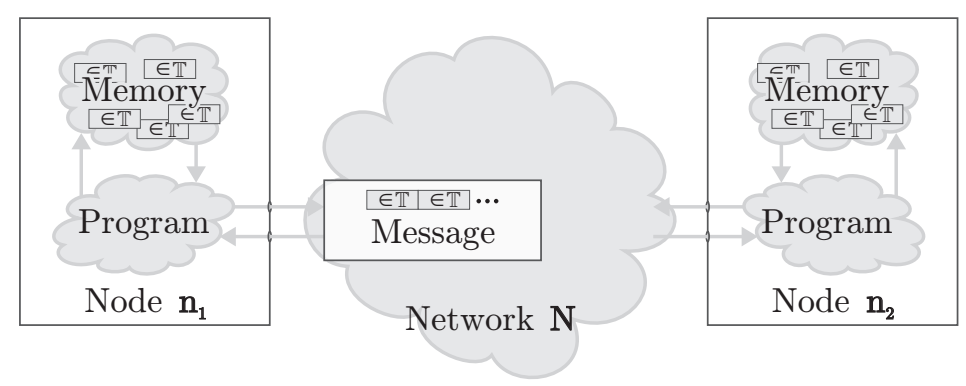

Figure 6.1: A Sketch of the DGPF Network Model.

The memory of the nodes is not bound to any structure and its only limitation is that it consists of elements from the space $\mathbb{T}$. In our work, $\mathbb{T}$ is a subset of the integer numbers: $\mathbb{T} \subset \mathbb{Z}, \forall t \in \mathbb{T} \Rightarrow-2^{31} \leq t<2^{31}$. The way in which variables of this type are arranged in a node depends on the program representation utilized. 
In LGP, SGP, and eSGP, for instance, there is global, local, and stack memory. RBGP, on the other hand, uses a symbolic memory where every cell is addressed via a fixed name. In the Fraglets language, there is no distinction between memory and program code at all since Fraglets represent data and code alike.

The structure of the messages exchanged by the nodes is the same for every GP approach. A message is a linear sequence of elements from $\mathbb{T}$ where each element has one exact position. The length of the messages is either fixed or at least limited with an upper bound. In the Fraglet representation, each message is exactly one Fraglet string.

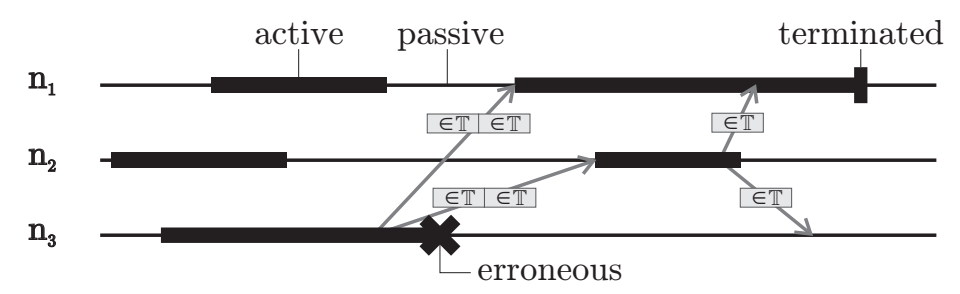

Figure 6.2: A timing diagram annotated with the states of the nodes/processes.

In Figure 6.2, we sketch a general example of how a distributed algorithm could proceed using a notation similar to Tanenbaum and van Steen's in [273]. A node (and the process running on it) in our network model is always in one of the four states illustrated in Figure 6.3:

1. A node is active if it is currently executing instructions of the distributed algorithm. An active node may send or receive messages.

2. A node is passive when it is currently not executing any instruction but has not explicitly terminated itself either. It cannot send messages in this state but may become active again and perform some computations when receiving one.

3. A node is in the state terminated if it has explicitly ended its program execution by invoking a dedicated termination instruction. This state is terminal and a terminated node can neither send nor receive messages and also cannot become active again.

4. A node is erroneous if it has performed an erroneous or forbidden action. The only difference to the state terminated is that erroneous is usually not reached deliberately.

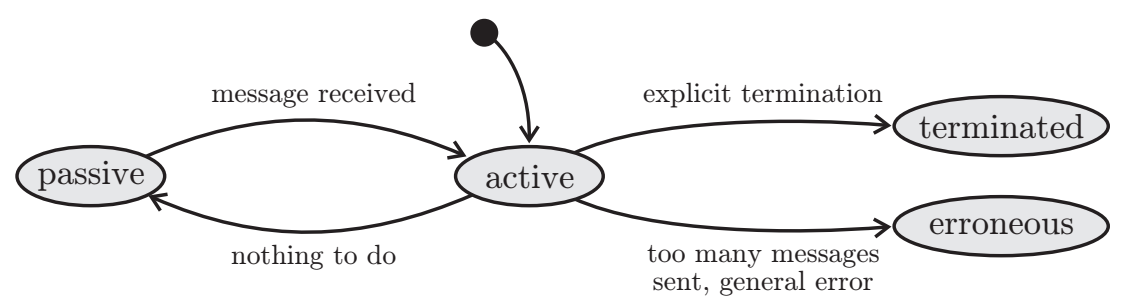

Figure 6.3: The state diagram of the nodes in our simulations.

In the following sections, we will clarify the components of our distributed systems model by defining the way in which the instructions of the evolved algorithms are executed (Section 6.2.2), the way in which the network structure is modeled (Section 6.2.3), and how the latency of the messages exchanged between the nodes is determined (Section 6.2.4). 


\subsubsection{The Parallelism Model}

The processes executing the programs on the nodes of a network all run in parallel. The single processes always carry out instructions in the order prescribed by the program. Apart from this constraint, the global execution sequence of the instructions of all instances of a program in a distributed system is unpredictable if no special synchronization approaches are utilized. If a program consisting of the instructions 1 and 2 is executed on two nodes a and b, any of the following six sequences may result (a1, a2, b1, b2), (a1, b1, a2, b2), $(\mathrm{a} 1, \mathrm{~b} 1, \mathrm{~b} 2, \mathrm{a} 2),(\mathrm{b} 1, \mathrm{~b} 2, \mathrm{a} 1, \mathrm{a} 2),(\mathrm{b} 1, \mathrm{a} 1, \mathrm{~b} 2, \mathrm{a} 2)$, and (b1, a1, a2, b2) In order to simulate distributed algorithms appropriately, a model which allows us to explore this parallel behavior in a formalized fashion is needed.

Modeling time continuously in simulations as it would be physically correct is complicated and, in this context, not really needed. The discretization of time simplifies many aspects of a parallel system. Such an approach can be justified with the fact that time, as a continuum, can be split into infinitely small units. Similar to Poisson processes [264], for time steps $\Delta t$ of such a short length, we make the assumptions that either

1. nothing happens or that

2. exactly one node of the modeled systems performs exactly one atomic action.

With this simplification, all atomic actions in the network model have implicitly been sequenced. We further assume that the transmission latencies of the messages can be determined at the moment they are sent. Therefore, all time steps where "nothing happens" can be disregarded in our further considerations. There are two possible ways in which a distributed system can be modeled based on this approach.

\section{Synchronous Models}

In synchronously running distributed systems, all nodes proceed with exactly the same speed. This can be modeled by executing atomic algorithm steps according to the round robin principle [270]. Then, from the perspective of a single node, all its neighbors are always as fast as it is itself. This becomes clear when visualizing that the time where a node "does nothing", i.e., where none of its atomic instructions are executed, does not exist from the viewpoint of the node. From the global perspective, runtime is assigned to the nodes as illustrated in Figure 6.4.

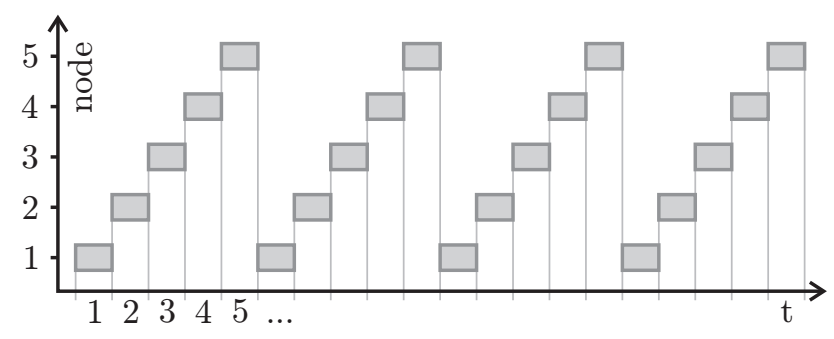

Figure 6.4: Synchronous parallelism in a model of a network of five nodes.

\section{Asynchronous Models}

In some cases, it may be sufficient to consider a distributed system as synchronous but most real networks do not fit to this model. They consist of computers that run asynchronously, possibly at different speeds which may not be constant either. 
Such behavior can be modeled in a surprisingly simple manner. Instead of assigning the runtime to the nodes using the round robin principle, in each time step one node is picked randomly according to the uniform distribution. Thus, every node has exactly the same probability of executing one atomic action. In average over infinite time, all nodes will perform the same amount of algorithm steps. For a given time period however, it is possible that one node can execute five steps while another one just proceeds by two, as sketched in Figure 6.5. It is obvious that in this time period, node one progresses with more than twice the speed of node four. Nevertheless, during the following twenty time units, this ratio may as well be reversed.

This approach thus also allows to model changing execution speeds. Still, all nodes have the same average speed as it would be in a homogeneous network of computers of the same type. In case this is not wanted, the probability mass function determining which the nodes are picked for executing a computation step can be adjusted so that it deviates from the uniform distribution.

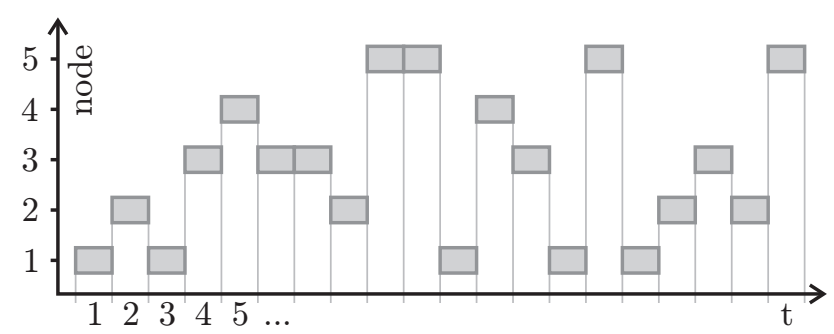

Figure 6.5: Asynchronous parallelism in a model of a network of five nodes.

In the context of this work, we will apply the asynchronous model since it is more realistic [54] and allows for a greater amount of network situations under which the evolved algorithms can be tested. In order to be facilitated in this model, each distributed algorithm subject to analyses needs to be broken up into atomic actions. The way this is done again depends on the program representation which is outlined in Chapter 5 (see especially Table 5.7).

\subsubsection{The Topology and Messaging Model}

Network topology is the study of arrangement of the components of a network. The network layout itself can also be referred to as topology. In the context of this work, the topology is defined by the neighbor relation. For each node $\mathbf{n}$ in a network $\mathbf{N}$, the neighbor set neighbors $(\mathbf{n}) \subseteq \mathbf{N} \backslash \mathbf{n}$ is the set of all other nodes to which $\mathbf{n}$ can send a message. Whenever a node $\mathbf{n}$ transmits a message, it is treated as a multicast to all the nodes in neighbors $(\mathbf{n})$. In the moment where the message is sent, it is split into $\mid$ neighbors $(\mathbf{n}) \mid$ identical transmissions, each targeting exactly one node and having its own latency.

This messaging mode roughly resembles Ethernet broadcasts [269]: All directly connected nodes receive the broadcasted frame, but the times where the contents are actually delivered to the single processes may differ due to a variety of factors.

Many of the features of distributed algorithms depend on the topology in which the nodes executing them are arranged. There are algorithms that only work on specific topologies while others may proceed properly in any partition-free network layout. In many cases however, the network topology has severe impact on the convergence and progression speed of the distributed algorithms [297, 304].

Notice that the topology of the networks in which the evolved algorithms are simulated may also influence the probability of overfitting. Assume that an election algorithm 
(Chapter 7) or data aggregation protocol (Chapter 9) for general networks is to be evolved. Such an algorithm involves data dissemination over multiple hops. In a fully connected topology where all nodes can directly communicate with each other, algorithms merely able to propagate information for exactly one hop may behave adequately. If such a topology is used in the simulations with which the objective values of the functional optimization criteria are determined, it is likely that programs evolve which only work in fully connected networks. This would be considered as overfitting in the case where programs for arbitrary topologies are sought, whereas it is perfectly fine in scenarios solely focused on fully connected networks.

\subsubsection{The Message Latency Model}

Latency is the time difference between the moment where something is initiated and the moment when its effects become observable [54]. Communication usually involves latency. Whenever a process sends a message, the contents are handed down to the operating system or a middleware. From this moment on, the process considers the message as sent. The operating system must then initialize the communication, prepare the message for the transmission medium, and send it to its destination(s).

The laws of physics enforce an additional delay, preventing the message from instantaneously occurring at its target node. This delay is normally negligible. Yet it is observable in satellite communication [327], for example when the host of a news show talks with a reporter on the other side of the globe, or in the communication with space exploration vehicles like Sojourner [322, 202]. It is furthermore the reason why global positioning systems like the NAVSTAR GPS can work [318].

Once a message arrives at the destination node, it is reassembled from the medium and the operating system or middleware passes its contents to the receiving process. From the moment on where the execution of this process is resumed, the message is considered as received.

Since the communication latency in a real network depends on so many factors $[54,7]$, it is hard to estimate it correctly with a simple probability distribution. In principle, we can choose between at least four simplified approximations for this purpose:

1. Many experiments indicate that network latency is roughly exponentially distributed. The latency/probability diagram on the third page in [30], for instance, resembles an exponential distribution. The exponential distribution is often used in models and simulations [292].

2. Since the exact form of the distribution of latency may vary from application to application, it also makes sense to approximate it with a normal distribution. A normally distributed random number can take on zero or negative values with a non-zero probability. Therefore, a cut-off value preventing such physical unsoundness must be applied in models and simulations.

3. Drawing uniformly distributed random numbers is maybe the simplest way to determine message latency. One reason for choosing it is that in our model, the exact distribution of the message delays is not important. The one thing that matters is that messages can be delayed in a way that allows them to outpace each other and that some nodes may receive them earlier than others. These are the most critical scenarios for distributed algorithms and we can create them with the uniform distribution as well as with every other one. Also, the uniform distribution does not give preference to any special configuration or scenario.

4. Another attractive approach to modeling latency is to make it a function of the distance between the sender and the receiver [150]. If we consider a routed network, we can assume a constant delay for each router which is passed by the message on its way. 
Additionally, we could allow for a bit of jitter by adding a small random number to each deterministically computed delay.

In this work, we chose to uniformly distribute the message latency. This choice is based on the fact that with the uniform distribution, the probability of phenomena such as messages taking over each other is very high. Thus, simulations based on this model are more challenging for the algorithms being evaluated and the chances of discovering errors or deficiencies is the highest.

\subsection{The Structure of the Simulations}

In Section 4.8 it became apparent that simulations must be used in order to derive the functional fitness when evolving algorithms. The DGPF utilizes simulations governed by the model defined in the previous section for computing the objective values. As motivated in Section 4.6.3 and Section 4.7.1, using multiple simulation scenarios for evaluating each algorithm helps against overfitting and increases the robustness of the algorithms evolved. In Section 4.4.3, we could even argue that this approach may mitigate the intractability of needle-in-a-haystack problems.

As discussed in Section 4.7.1, we will use the same set of scenarios for all evaluations of solution candidates in the same generation. Doing so is mandatory in order to make the simulation results comparable. Two scenarios $s c e n_{1}$ and $s c e n_{2}$ are identical if and only if every algorithm possible in a given representation shows exactly the same behavior in $s c e n_{1}$ as in $s c e n_{2}$ and thus, also achieves the same objective values in both scenarios. Also, scenarios can only be considered to be identical if they exhibit the same timing and topology even for different algorithms. This means that

1. the network topologies in $s c e n_{1}$ and $s c e n_{2}$ must be identical (see Section 6.2.3),

2. the features of the nodes (such as their IDs) in the networks must be the same,

3. the assignment of the time steps to the nodes in the simulation of asynchronicity (see Section 6.2.2) must be identical, and

4. the message latencies assigned to the single transmissions must be the same (see Section 6.2.4).

5. Identical sequences of messages concerning their sources and the order in which they are sent (but not their contents) must be identically delayed.

6. The assignment of time steps to the simulated processes should be independent from the instructions executed by the algorithms (except for process termination).

The structure of the scenarios which can be constructed for the program evaluation is subject to certain constraints. The performance and memory of the computers on which the actual simulations will run is limited, and thus, the number of network nodes that they can simulate in reasonable time is too. Therefore, we define the following parameters:

1. a maximum number of time steps $m x S$ for which the simulations will run,

2. a minimum $(m n \mathrm{~N})$ and maximum number $(m x \mathrm{~N})$ of nodes allowed in the simulated networks, and

3. a minimal (mnlat) and a maximal (mxlat) message latency.

In the scenarios, random numbers determine every stochastic parameter. The same sequence of numbers can be generated by resetting the seed of the random number generator in each simulation. Multiple generators should be used in order to make the assignment of time slots to nodes and the message delays independent from each other. We decided to 
generate multiple pools of random numbers for each scenario beforehand in order speed up the simulations. The creation of one scenario incorporates

1. determining the number numNodes $(\mathbf{N})$ of nodes to participate in the scenario uniformly distributed between $m n N$ and $m x N$,

2. generating nextPrime $(128 * m x N)$ message latencies uniformly distributed between mnlat and mxlat,

3. generating nextPrime $\left(\left\lfloor(128 * m x N)^{1.02}\right\rfloor\right)$ time step assignments uniformly distributed in $0 . .\left(2^{32}-1\right)$, and

4. generating numNodes(N) unique identifiers uniformly distributed between $m n I D=0$ and $m x I D=2048$.

The pools with the pre-computed random numbers for latency and time step assignments both are accessed like ring buffers, i. e., if all numbers have been exhausted (which happens only rarely), reading will begin at the start of the pools again. In order to decrease the chance of repeating sequences, we ensure that the size of each pool is prime ${ }^{2}$. Based on these definitions, a network simulation proceeds as follows:

1. The remaining latencies of the messages in the network are decreased by one. ${ }^{3}$ The messages which have a remaining latency of zero are delivered to their target. If the destination of a message is in passive state, it becomes active. If it already is terminated or erroneous, the message is discarded.

2. A new random number $r$ from the time step assignment pool is drawn. Amongst the $0<q \leq$ numNodes $(\mathbf{N})$ active nodes, the $(r \bmod q)$ th node is picked and granted one execution step.

3. If now all nodes in the network are either a) terminated, b) erroneous, c) passive and no message is underway, or $d$ ) the total number of time steps to be simulated is exhausted, the network simulation terminates. Otherwise, it continues at point 1.

The number $m x M s g$ of messages that a node is allowed send will be limited to a number in $\mathbf{O}\left(\right.$ numNodes $\left.(\mathbf{N})^{3}\right)$ or $\mathbf{O}\left(\right.$ numNodes $\left.(\mathbf{N})^{2.5}\right)$ in order to prevent the simulation from hanging because of too many messages in the message queue or crashing because of an out-of-memory error. ${ }^{4}$ Hence, if node $\mathbf{n}$ sends a message, the following actions have to be performed

1. First, it is checked whether the node has exhausted the maximum number of messages it is allowed to send. If this is the case, the simulation is terminated and the node is marked as erroneous.

2. The message is cloned for each node in the neighbor set neighbors $(\mathbf{n})$ of $\mathbf{n}$.

3. Each of the clones is assigned to one neighbor and thus, turned into a message with a distinct destination.

4. Each of the messages is attributed with a delay from the latency pool.

5. All messages are enqueued into the message queue of the simulation.

The total number $\#$ scen of possible different scenarios in this framework is thus determined by Equation 6.1. The result of this equation takes on extremely huge values even for small settings of the scenario parameters. Furthermore, \#scen increases sharply with any additional degrees of freedom, such as randomized times for critical sections as in Chapter 8.

\footnotetext{
${ }^{2}$ nextPrime is an operation returning the next prime number larger than its argument.

3 This operation is performed on an efficient queuing date structure.

${ }^{4}$ Notice that if each node can send $\mathbf{O}\left(\operatorname{numNodes}(\mathbf{N})^{2.5}\right)$ messages, this avails to $\mathbf{O}\left(\right.$ numNodes $\left.(\mathbf{N})^{3.5}\right)$ messages in total.
} 
One additional justification for the usage of the uniform distribution in all randomized situations is that it gives all these possible scenarios the same probability, as we have already argued in Section 6.2.4 on page 96.

$$
\# \text { scen }=\sum_{i=m n N}^{m x N}\left[\prod_{j=m x I D}^{m x I D-m n I D-i+1} \prod^{m n I D+1} j * i^{m x S} *(\text { mxlat }- \text { mnlat }+1)^{\min \{m x M s g * i, m x S\}}\right]
$$

\subsection{The System Architecture}

The system which we use for evolving distributed algorithms consists of two separate frame-

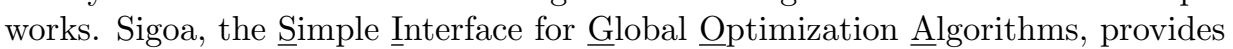

1. the basic evolutionary algorithm functionality,

2. standard genomes $\mathbb{G}$ (trees, bit and integer strings, multidimensional strings),

3. the corresponding search operations,

4. fitness assignment and selection procedures,

5. base classes for simulations and objective functions,

6. an environment for the automated execution of test series, and

7. facilities for logging the results in files nicely sorted in folders in the file system.

Another feature of Sigoa is the transparent parallelization of the simulations in the evaluation phase of the evolutionary algorithms, which can lead to a significant increase in performance on multi-core CPUs. The Sigoa system has been developed iteratively over a time of approximately three years and became the foundation of several other optimization applications some of which are listed in Chapter 11. Formerly, the classes providing the features enumerated above were part of the DGPF (Distributed Genetic Programming Framework) and specialized for Genetic Programming. Step by step, the DGPF became more and more generally applicable so we decided to separate the Genetic Programming functionality from the optimization core. Now the DGPF is placed on top of Sigoa and extends it by introducing

1. the special genomes and phenomes for Genetic Programming defined in Chapter 5,

2. predefined objective functions for these program representations, and

3. simulation environments adhering to the definitions given in the previous sections.

Figure 6.6 gives an overview on the most important classes directly involved in the Genetic Programming process of the DGPF. These classes have intuitive names corresponding to the terms introduced in this and the previous chapter.

The system is centered around the class VirtualMachine which is able to execute an (evolved) VirtualMachineProgram. The different Genetic Programming approaches tested in this thesis correspond to different specializations of this class. A special case are the classes SGPProgram and eSGPProgram. They represent genotypes which are compiled to instances of LGPProgram prior execution.

In order to execute a program, the VirtualMachine needs to instantiate a corresponding memory container (VirtualMachineMemory) since the structure of the memory of the nodes depends on the program representation. Single virtual machines can be used to test the behavior of local algorithms. For evolving distributed algorithms, the class VirtualMachine is extended to NetVirtualMachine and $m x \mathrm{~N}$ instances of it are placed into a Network. ${ }^{5}$ The

\footnotetext{
${ }^{5}$ Depending on the simulation scenario, not all of these nodes are used.
} 


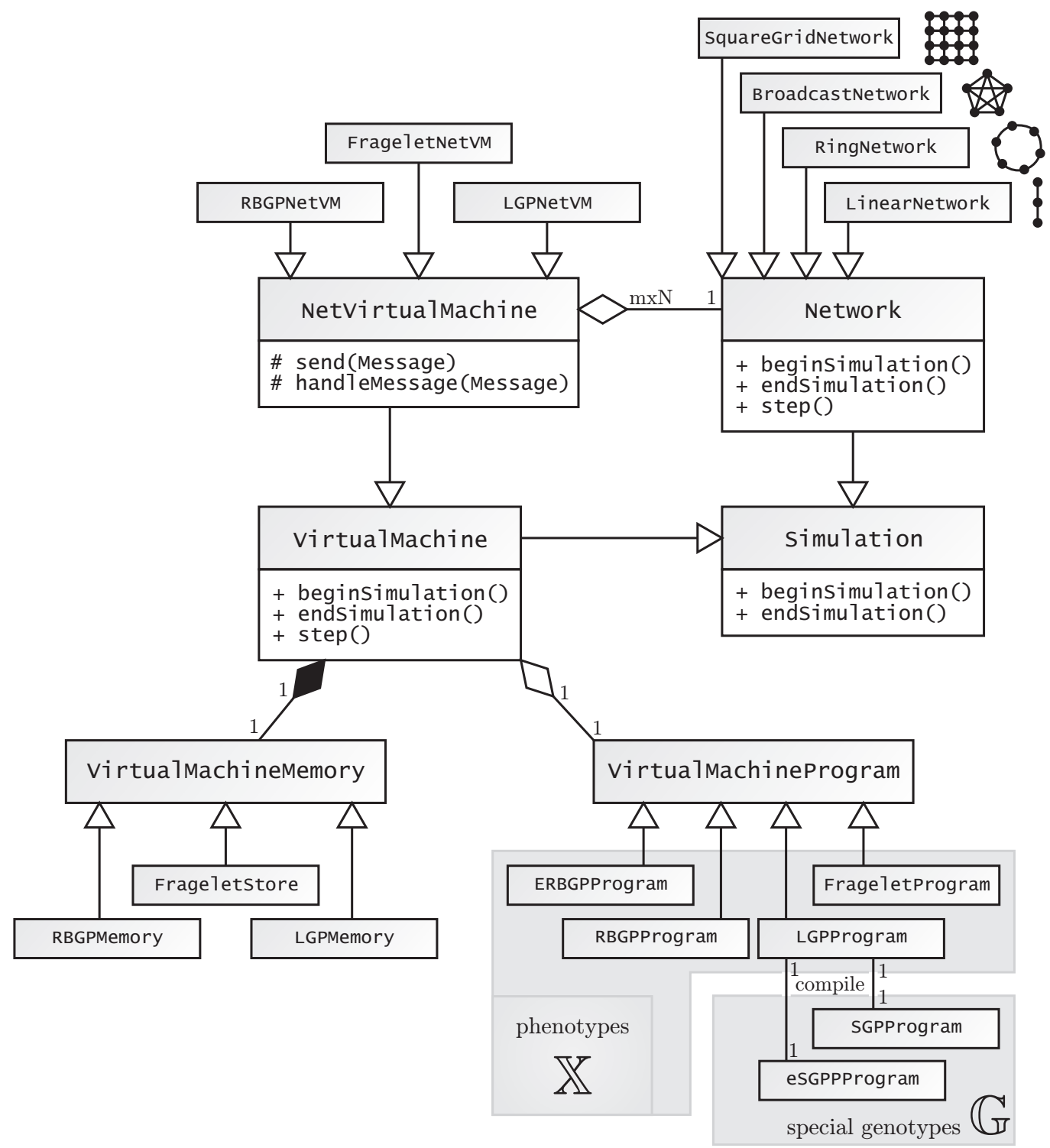

Figure 6.6: The Structure of the DGPF.

DGPF supports the simulations of multiple different topologies, such as square grids, fully connected graphs, rings, and linear arrangements. Small extensions of the NetVirtualMachine class facilitate the requirements of the RBGP, Fraglets, and LGP program representations.

The class Network is derived from Simulation which is part of the objective function evaluation subsystem of the Sigoa framework. ObjectiveFunctions (not illustrated in Figure 6.6) can access the simulation instances which record all relevant information for them. For our experiments, predefined objective functions for the non-functional criteria (such as program size and the number of exchanged messages) are used together with specialized objective functions, measuring the number of violations of a critical section, for instance (see Section 8.2). 


\subsection{Summary}

In this chapter, we first outlined a few general features of distributed systems and algorithms. We then derived a model of the nodes executing the algorithms synthesized with GP and for the network connecting them. Based on this model, a simulation environment was designed which allows us to test the evolved algorithms in close-to-reality conditions. Finally, we gave a short overview on the structure of the Sigoa and DGPF frameworks which form the foundation for implementing the simulations and the Genetic Programming approaches discussed in Chapter 5. 



\section{Evolving Election Algorithms}

Election algorithms have many applications in distributed systems. They are used to determine the coordinators in several routing [119] or group communication protocols [289]. In Windows domains, the master browser is chosen with an election algorithm [75]. Election is part of certain clock synchronization methods [116]. Furthermore, it is also a very common functionality needed in many agent scenarios [56].

According to Le Lann [178], a distributed election algorithm can be initiated by any number of nodes and will reach a terminal configuration in which exactly one node is elected as leader and all nodes agree to this choice. The challenge of such an election procedure is the distributed nature: It must be ensured that all nodes get a consistent view of the election. Le Lann makes the following assumptions about the distributed system in which the election takes place:

1. Every node has a unique name (ID).

2. A total order can be imposed on the IDs.

3. Each node knows the IDs of all other nodes.

4. A node does not know the states of the other nodes and whether they are up and running (active) or down and out (terminated or erroneous).

Many different methods for distributed elections have been developed, such as Le Lann's original approach for ring topologies, the message extinction algorithm by Chang and Roberts [46], the Bully-Algorithm by Garcia-Molina [99], and the probabilistic Las Vegas algorithm for rings of known size by Itai and Rodeh [144, 145]. Efficient election algorithms for arbitrary topologies [163, 164] and special algorithms for wireless sensor networks or mobile ad-hoc networks $[191,288]$ followed a few years later.

In this chapter, we will investigate the ability of our Genetic Programming system to evolve new election algorithms. We will slightly modify the problem definition in order to make the synthesized algorithms applicable in a wide range of scenarios. Then, a very detailed series of experiments is carried out with multiple sets of objective functions. The results of these experiments will be analyzed rigorously.

\subsection{Specification of the Global Behavior}

For this work, we will modify the assumptions of Le Lann [178] about the network $\mathbf{N}$ of nodes $\mathbf{n}$ performing the election as follows:

1. The IDs of the nodes are unique numbers drawn from the natural numbers $\mathbb{N}$ and

2. the order imposed on them is the <-relation in $\mathbb{N}$. 


$$
\begin{aligned}
& \forall \mathbf{n} \in \mathbf{N} \Rightarrow \operatorname{id}(\mathbf{n}) \in \mathbb{N} \\
& \nexists \mathbf{n}_{1}, \mathbf{n}_{2} \in \mathbf{N}:\left(\mathbf{n}_{1} \neq \mathbf{n}_{2}\right) \wedge\left(\operatorname{id}\left(\mathbf{n}_{1}\right)=\operatorname{id}\left(\mathbf{n}_{2}\right)\right)
\end{aligned}
$$

3. The nodes do not know the IDs of the other nodes which makes the problem harder.

4. At startup, a node $\mathbf{n} \in \mathbf{N}$ only knows its own ID $\mathrm{id}(\mathbf{n})$ which is stored in a dedicated variable, symbol, or memory cell.

5. During the election, each node $\mathbf{n}_{1}$ in the network $\mathbf{N}$ will decide for a node $\mathbf{n}_{2} \in \mathbf{N}$ which it thinks has won the election. It will store the ID of this node in another dedicated memory cell $\left(\operatorname{id}\left(\mathbf{n}_{2}\right)=\operatorname{elected}\left(\mathbf{n}_{1}\right)\right)$.

The main difference between the election task defined here and the "standard election problem" is point 3. One of the original goals of this work was to evolve algorithms for sensor networks. In this domain, a possibly large number of nodes are deployed and the assumption that each one knows all IDs or even the exact number of other nodes in the network will generally not hold. Therefore, our definition here is more realistic and complies with the research already available on this case [191, 118, 288].

\subsection{Objective Functions}

With Genetic Programming, we want to find algorithms which solve the election problem. We apply an evolutionary process governed by two objective functions: A functional objective $f_{e l}$ which furthers functional adequacy and a non-functional criterion $f_{p s}$ which minimizes the size of the synthesized programs.

\subsubsection{Functional Objective Functions}

According to the problem definition, all nodes $\mathbf{n}$ which participate in the election should have the same (valid) ID stored in their result variable "elected(n)" after a certain amount of simulated time. Since the nodes run asynchronously in parallel, they will begin executing the program at different points in time. They do not have any information about the execution state of the other nodes unless it is explicitly sent to them. In this scenario, we propose four possible definitions of functional adequacy of an election algorithm:

a) The algorithm is an anytime algorithm ([274], Section 4.8.2) intended to keep the application or the operating system up-to-date on the current guess about who the leader is. If a node thinks that it is not the leader but receives messages for the leader, it would simply propagate them to the node which it assumes to be the elected one. Such an algorithm will not necessarily terminate.

b) The algorithm terminates like in the classical election scenario and returns the ID of the leader to the application or operating system.

c) In a), we did not give any specific restrictions about which node should be elected. We can extend this approach by specifying that the elected node must either have the maximum or minimum ID, as it is the case in some well-known election schemes such as [178, 46], and [99].

d) The same restrictions can also be imposed on the variant b).

\section{Problem Variant a)}

In Algorithm 7.1, we have specified the functional objective function $f_{\text {el.a }}$ which can be used for synthesizing algorithms of the category a). This function will always take on values between zero and one, where 0 is the optimum and 1 is the worst case. In principle, it allows the evolutionary algorithm to learn, step by step, the following information: 
1. It is good if the election programs store values which are valid IDs in their dedicated memory cells, i. e., $\forall \mathbf{n}_{1} \in \mathbf{N} \exists \mathbf{n}_{2} \in \mathbf{N}: \operatorname{id}\left(\mathbf{n}_{2}\right)=\operatorname{elected}\left(\mathbf{n}_{1}\right)$ should hold.

2. It is even better if the number of different votes is less than the number of valid votes, i. e., there is at least some form of partial consensus. In the worst case in this respect, each node would vote for itself.

3. The optimum is reached when the number of different votes becomes one, which means that all nodes (which have issued valid votes) have agreed on one winner.

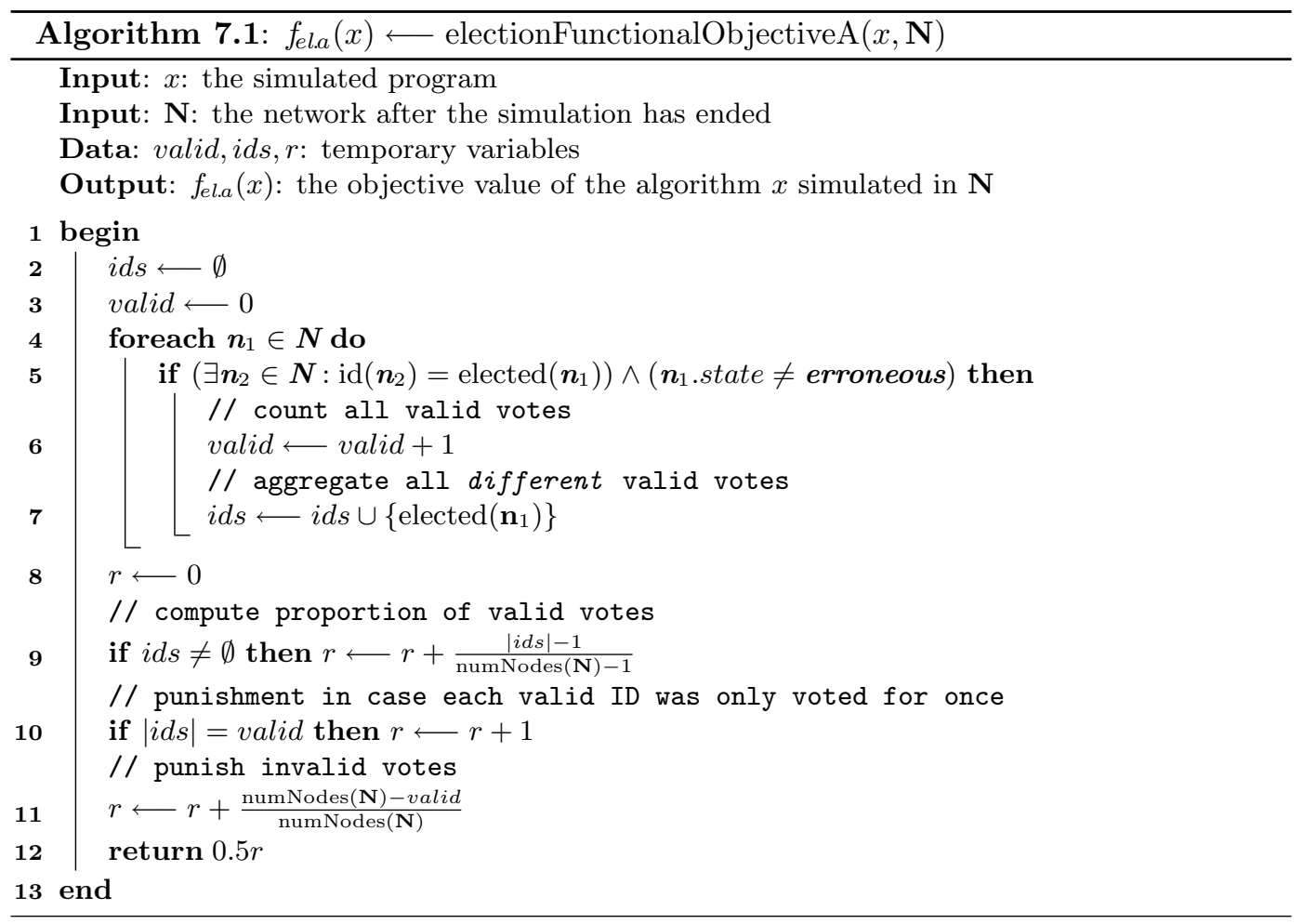

We give a rough sketch of the shape of the objective function $f_{\text {el.a }}$ for a network $\mathbf{N}$ of numNodes $(\mathbf{N})=16$ nodes in Figure 7.1. The values of $f_{\text {el.a }}$ are plotted based on the number of valid votes that were collected from the nodes $\mathbf{n} \in \mathbf{N}$ at the end of the simulation and the number $|i d s|$ of different values amongst them. $f_{e l . a}$ takes on the worst value (1) if no valid vote was casted (valid $=0$ ). Obviously, the set $i d s$ of different IDs is then empty $(|i d s|=0)$. In all other cases, at least one correct vote exists. The best case (with $f_{\text {el.a }}=0$ ) occurs when all 16 votes are valid $($ valid $=16)$ and all nodes have agreed on the same ID, i. e., $|i d s|=1$.

\section{Problem Variant b)}

In Algorithm 7.2 we introduce a penalty term for nodes which have not terminated. The results of $f_{e l . b}$ again are always in the interval $[0,1]$ because $r$ can become 2.5 at most (since a faulty node can never terminate properly) and $0.4 r$ is returned. 


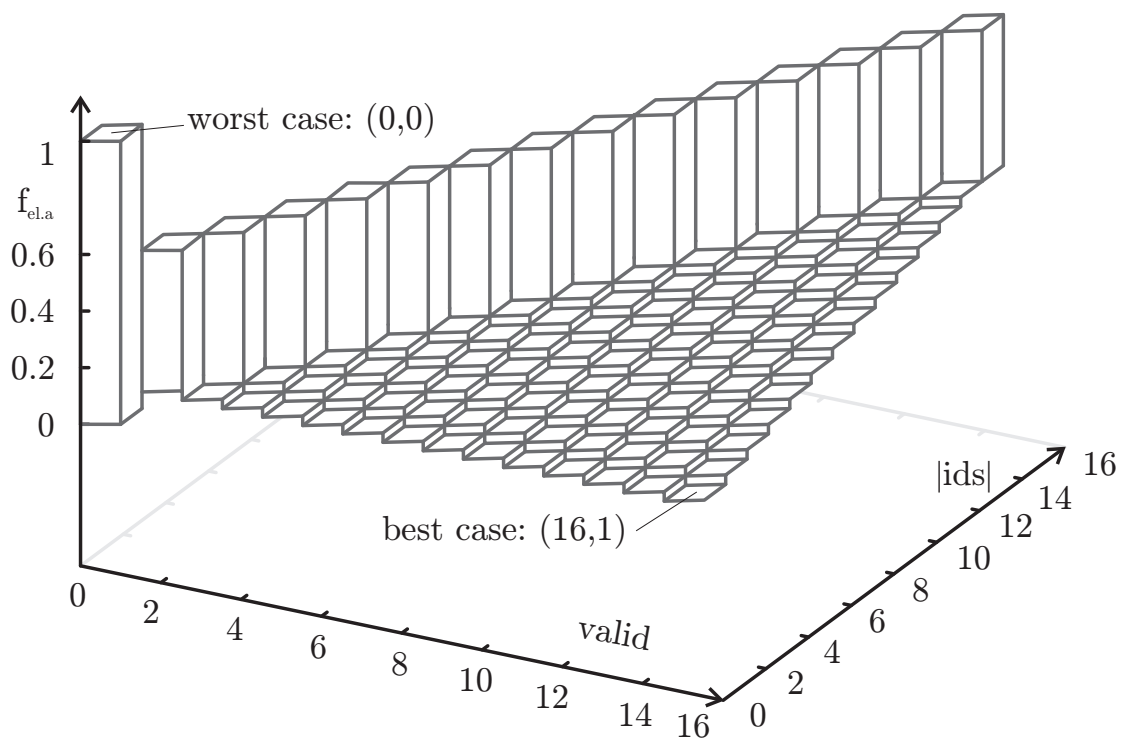

Figure 7.1: The functional objective function $f_{\text {el.a }}$ in the election problem.

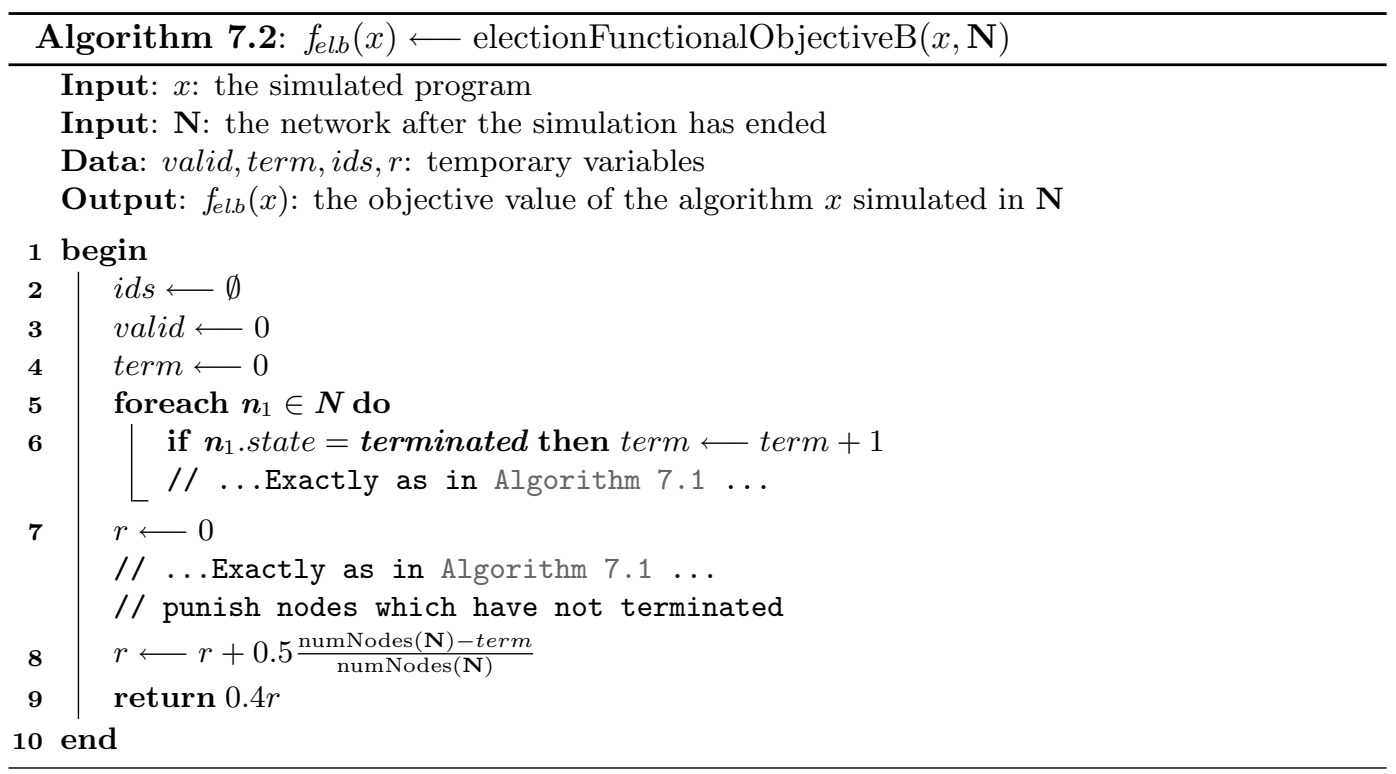

\section{Problem Variant c)}

The objective function $f_{\text {el.c }}$ is computed exactly like $f_{\text {el.a }}$, but adds a penalty of 1 to $r$ if Equation 7.3 does not hold. The result of $f_{\text {el.c }}$ is then $r / 3$ so that again $0 \leq f_{\text {el.c }}(x) \leq 1 \forall x \in \mathbb{X}$.

$$
\left(\max _{i \in i d s} i=\max _{\mathbf{n} \in \mathbf{N}} \operatorname{id}(n)\right) \vee\left(\min _{i \in i d s} i=\min _{\mathbf{n} \in \mathbf{N}} \operatorname{id}(\mathbf{n})\right)
$$




\section{Problem Variant d)}

The fourth possible functional objective function for the election problem, $f_{\text {eld. }}$, is derived from $f_{\text {el. } b}$ in the same way: It adds the same penalty term and returns $r / 3.5$ so the value of $f_{\text {el.d }}$ is always in $[0,1]$ as well.

\subsubsection{Non-Functional Objective}

As sole non-functional criterion $f_{p s}$, we minimize the number of constructs in the program representation. We therefore set $f_{p s}(x)$ to the number of instructions or expressions in $x$. The influence of other non-functional criteria in the evolution of distributed algorithms has been discussed by us in [295, 323], for instance, and will not be considered here.

Using too many objective functions may result in the many-objectives problem [306, 234 - a situation where most of the solution candidates become non-dominated and the search performance decreases significantly. Using more objectives was only feasible in our experiments in $[295,323]$ because we used larger population sizes and ran fewer experiments there.

\subsection{Experimental Settings}

We applied all six Genetic Programming approaches introduced in Chapter 5 to the election problem. For each algorithm evaluation, we used twenty scenarios with networks consisting of between four and twenty virtual machines. To each such simulation, a maximum step number $m x S$ of

1. 20000 for RBGP and eRBGP (because of the higher execution density, see Table 5.7),

2. 40000 for Fraglets and LGP programs, and

3. 120000 for SGP and eSGP programs (because of their usually longer LGP-representation) was granted.

The networks were organized in a linear topology where each node can only communicate with its direct predecessor and successor ${ }^{1}$ since the highest required number of messages for finding the leader is to be expected in such an arrangement. Because of the broadcast nature of the communication, we expected the results of the evolution to be valid for other topologies as well. Each node was allowed to send 1788 messages. If this limit was exceeded, the node was immediately terminated and marked as erroneous. The message sizes were limited to two memory words except in the evolution of Fraglets, where the limit was the maximum Fraglet length.

The LGP, SGP, and eSGP approaches were provided with two cells of global, local, and stack memory each. Nodes executing RBGP or eRBGP programs were equipped with two multi-purpose variables and the length of Fraglets was limited to 15. The ID of the nodes was stored in the first global memory cell (LGP, SGP, eSGP), a dedicated (writable) symbol (RBGP, eRBGP), or available via a special Fraglet (Fraglets).

We repeated the experiments with the four different functional objectives from Section 7.2.1 in order to find out about the "GP hardness" of the different aspects of this problem and how the choice of the target criteria influences the outcome of the evolution. The following optimization problems were defined: $F_{a}=\left(f_{e l . a}, f_{p s}\right), F_{b}=\left(f_{e l . b}, f_{p s}\right)$, $F_{c}=\left(f_{e l . c}, f_{p s}\right)$, and $F_{d}=\left(f_{\text {el. }}, f_{p s}\right)$.

\footnotetext{
${ }^{1}$ The first node has no predecessor and the last node had no successor.
} 
For the EA, we used a population size of 512, tournament selection with five contestants, and the means already discussed in Chapter 4. The complete list of settings of these experiments is provided in Section A.1 on page 167 and in Section A.2 we show that adequate programs for all four election tasks can be defined manually.

\subsection{Evolved Programs}

From our experiments, we obtained twofold results: We were able to collect a fast amount of statistically significant data which will be evaluated in the next section. Here, on the other hand, we will discuss the algorithms which were synthesized by the artificial evolution.

\subsubsection{For Problem Variant a)}

In problem definition $a$ ), the goal of the evolution was to find an anytime election algorithm which is able to name a winner after a certain amount of simulated time steps. All GP approaches were able to solve this problem driven by the set of objective functions $F_{a}$, although differing in the fraction of successful runs. The evolved programs most often belonged to the same, very crude algorithm classes presented in Algorithm 7.3 and Algorithm 7.4. The only exception was the Fraglets approach, where all evolved adequate programs for problem a) belonged to the later class.

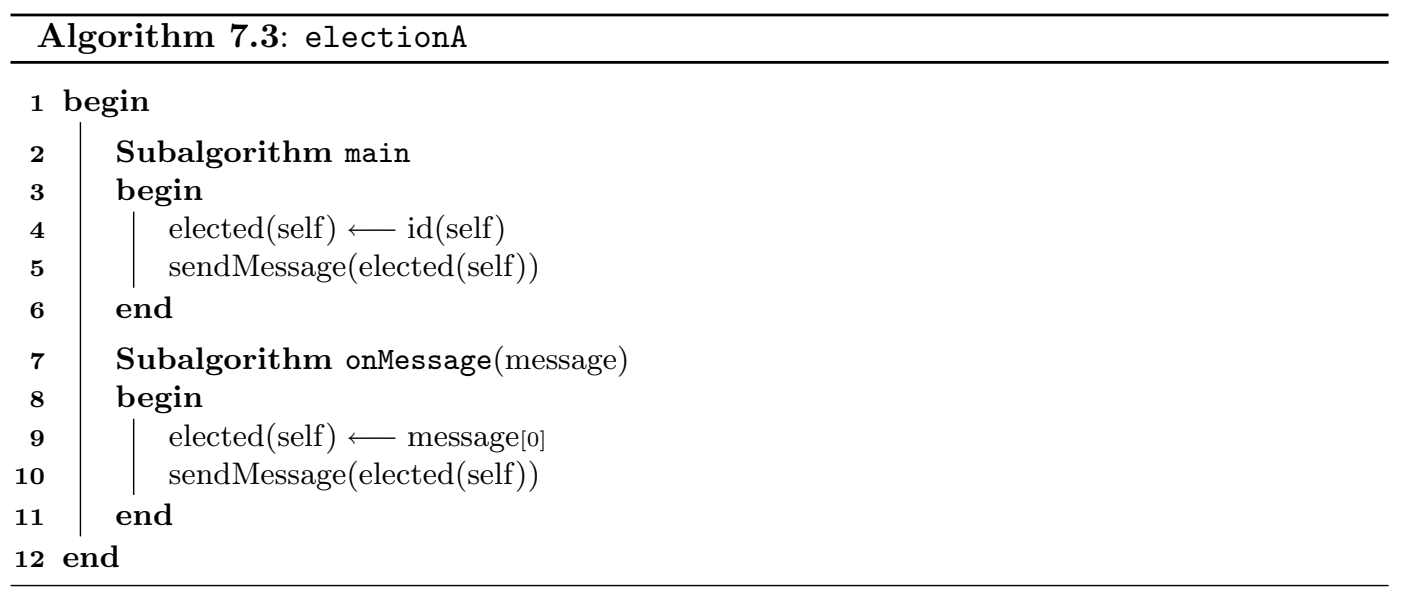

Interestingly, Algorithm 7.3 works in almost all scenarios perfectly well since it relies on the randomness in the message latencies. Regardless of the size of the network $\mathbf{N}$, it will eventually converge to a situation where $\operatorname{elected}\left(\mathbf{n}_{1}\right)=\operatorname{elected}\left(\mathbf{n}_{2}\right) \forall \mathbf{n}_{1}, \mathbf{n}_{2} \in \mathbf{N}$ if the delay in the communication is sufficiently random. In its behavior, Algorithm 7.3 is somehow similar to a Moran process [201], a simple model for dynamics in finite (biological) populations. $^{2}$ Some examples of evolved programs which follow this pattern are given in Listing 7.1 and Listing 7.2.

Notice that the LGP phenotype (Listing 7.3) belonging to the eSGP genotype illustrated in Listing 7.2 is much shorter and more readable. The on-the-fly compiler is able to compact and resolve statements in the SGP and eSGP languages. However, it cannot detect longer useless sequences of code such as the repeated filling and subsequent emptying of the stack by calling an empty function in Listing 7.2. More complex code optimization routines in

\footnotetext{
${ }^{2}$ Thanks to Thomas Meyer for pointing this out.
} 


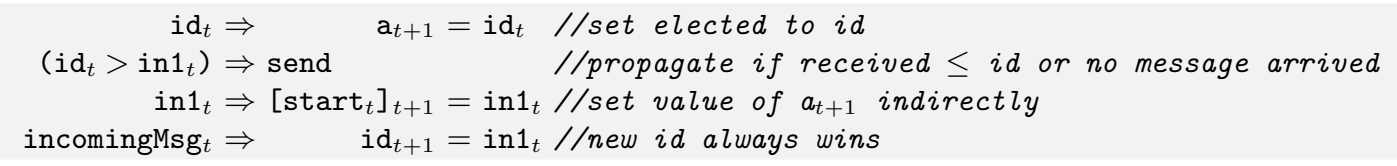

Listing 7.1: One evolved eRBGP version of Algorithm $7.3\left(f_{\text {el.a }}=0, f_{p s}=18\right)$.

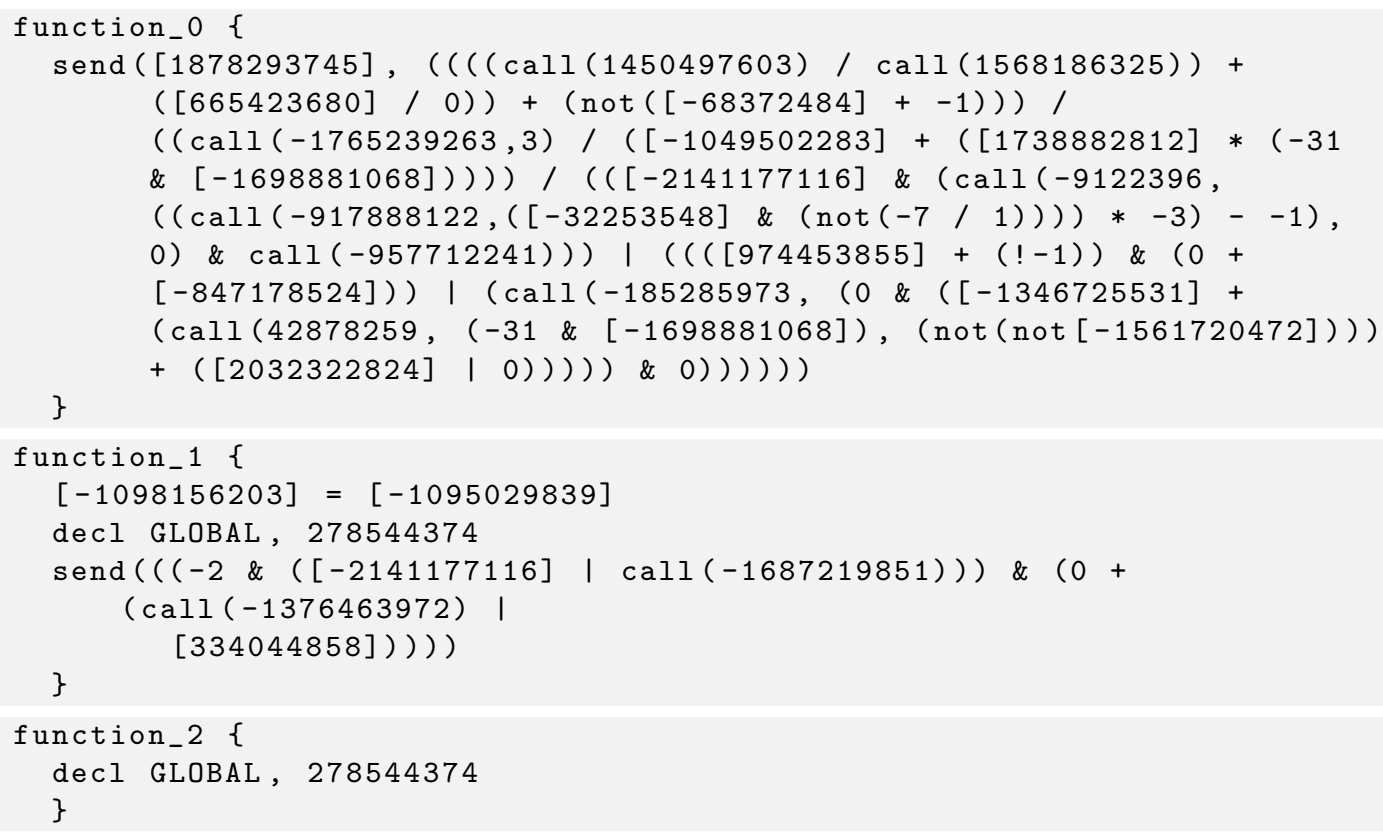

Listing 7.2: One evolved eSGP version of Algorithm 7.3 (Phenotype: see Listing 7.3).

the compiler would increase its time consumption and thus, prolong the runtime of the experiments.

It should not go unnoticed that all objective functions are computed on basis of the phenotype, i. e., the seemingly larger fractions of redundant code in Listing 7.2 which are removed by the compiler have no influence on the $f_{p s}$-value of the program. Thus, there is no selection pressure against them.

\subsubsection{For Problem Variant c)}

With the exception of the SGP approach, all Genetic Programming methods also found solutions to the problem variant $c$ ) - again, with largely differing success rates. The programs for this election problem, where either the maximum or minimum ID has to be voted for, generally followed the scheme defined in Algorithm 7.4.

The RBGP program specified in Listing 7.4 is an example for this algorithm. Such programs do not need to rely on the randomness of the message latencies anymore and will behave adequately in all possible scenarios. Another instance of Algorithm 7.4 is the Fraglets program given in Listing 7.5 which fully utilizes the features of the max2-Fraglet which we introduced in Table 5.4 on page 80 . The Fraglet rule in line 5 not only computes the maximum of two elected-Fraglets holding votes, but also creates a Fraglet which can react with the result and broadcast it to the neighbors. The winner of an election on a Fraglet-driven node is the ID which occurs most often in its Fraglet store. The program in Listing 7.5 


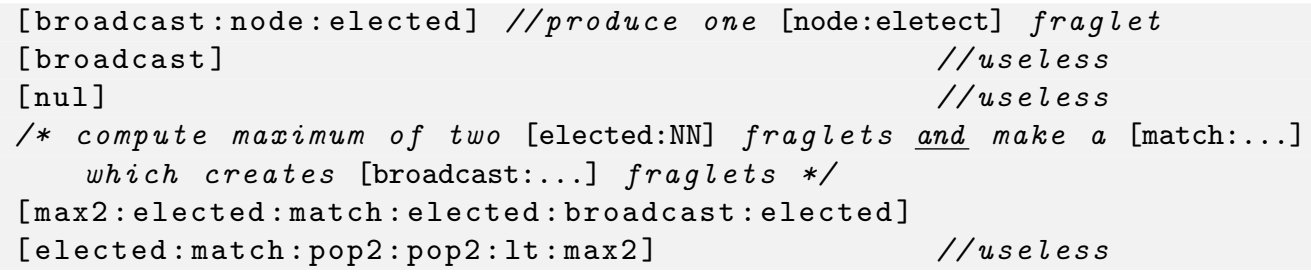

Listing 7.5: A Fraglets program solving $F_{a}$ and $F_{c}\left(f_{e l . a}=0, f_{p s}=17\right)$.

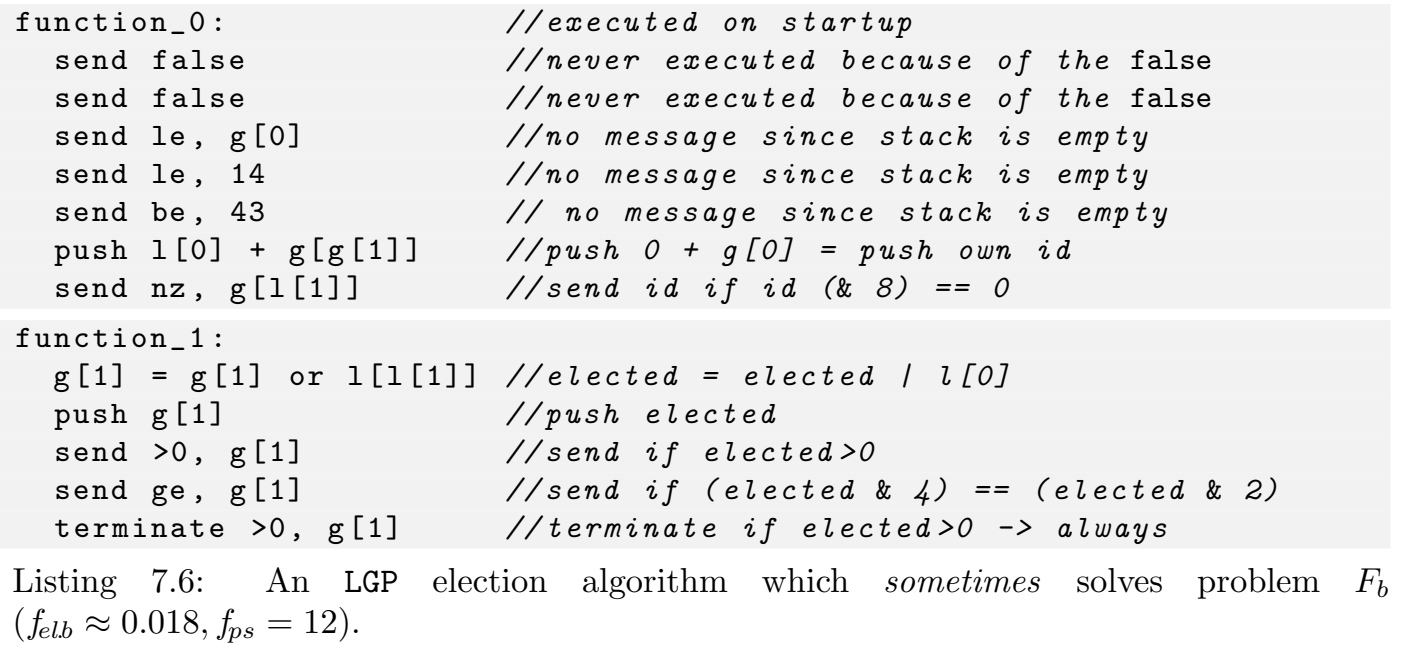

Listing 7.6: An LGP election algorithm which sometimes solves problem $F_{b}$ $\left(f_{\text {el. }} \approx 0.018, f_{p s}=12\right)$.

additionally reduces the number of elected-Fraglets on a node and thus, prevents it from overflowing.

\subsubsection{For Problem Variant b) and d)}

Other than the variants $a$ ) and $c$ ), the problems of type $b$ ) and $d$ ) could not be solved by any experimental run in an adequate manner. It seems to be very hard for the artificial evolution to synthesize valid criteria based on which a distributed (election) program can decide when it should terminate under the harsh resource constraints defined.

However, all approaches again found programs which are instances of either Algorithm 7.3 for $F_{b}$ or Algorithm 7.4 for $F_{d}$. Since these programs do not terminate, they cannot perform better than $f_{\text {el. }}=0.4(0.5 * 1)=0.2$ and $f_{\text {el. }}=(0.5 * 1) / 3.5=\frac{1}{7}$. The only Genetic Programming methods which dipped below these thresholds were LGP, eRBGP, and the Fraglets.

The LGP program in Listing 7.6, for instance, manages to achieve a functional fitness of around $f_{e l . b} \approx 0.02$ in average over 20 randomized scenarios in a very simple manner: Each node votes for the ID it receives, propagates the message, and then immediately terminates. In the main routine called at startup, the own ID is only sent if it has the fourth bit (of value 8) not set. This generally halves the number of IDs which can be voted for. Because of the asynchronicity of the execution environment, some nodes will already have a message in their "incoming" buffer and start with their second ADF. Since we can assume the chance of this being again at least around $50 \%, f_{\text {el.b }}$ will normally be around $0.4 *(0.25 \operatorname{numNodes}(\mathbf{N})-1) / \operatorname{numNodes}(\mathbf{N})$ which evaluates to something between 0.0 and 0.1 in our scenarios with between four and twenty nodes, exactly fitting to the observed results. 


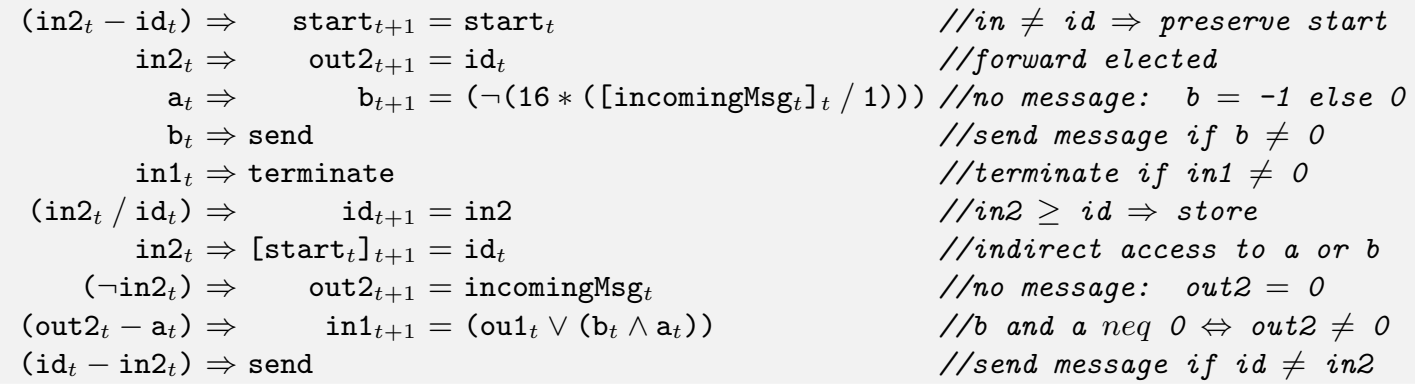

Listing 7.7: One evolved eRBGP program for $F_{b}\left(f_{\text {el. }} \approx 0.021, f_{p s}=57\right)$.

Listing 7.7 is a more interesting approach to the election problem $F_{b}$ which would also work almost adequately for $F_{d}$. This program evolved in eRBGP representation and works as follows. Initially, the start symbol is 1 , so rule 7 stores the own ID of the node $\mathbf{n}$ in variable b. After start is reset for the next rule evaluation, the value of id will always be written to a - the symbol which elected $(\mathbf{n})$ evaluates to. $\mathrm{b}$ is overwritten by rule 3 which sets it to -1 when no message is received and to $\overline{16}$ otherwise. If a and $\mathrm{b}$ have at least one bit of value 1 in common, in1 will be non-zero in the next rule evaluation (rule 9). In this case, the program will be terminated by rule 5 . However, since out1 is never set, i. e., is always 0 , any received message will immediately clear in1. In other words, the algorithm can only terminate if no message was received and if either a message was received in the previous step or $(\operatorname{elected}(\mathbf{n})=\mathrm{a})$ has the fourth bit set. If a message is received, then in2 will be different from 0 and rule 6 stores the ID received in in2 only in id if in2 $\geq$ id (because only then the integer division will have a non-zero result). Since $\mathrm{b}$ is only zero during the first evaluation of all rules, a message is sent in every time step. Initially, these messages have the content $(0,0)$ (rule 8), then $(0,1)$ (rule 8) and, from there on, $(0$, id = elected(n)) (rule 2). The termination of this algorithm depends on the random message delays in the network. In this, it has adopted the mechanism which was used for election in Algorithm 7.3 for termination! Although the program does not achieve 0 in $f_{\text {el.b }}$, i. e., functional adequateness in at least twenty scenarios from the perspective of the objective function, it represents a surprisingly simple and, although inefficient, in many situations functioning approach. If the delays were set properly, such a program could indeed be used for a practical application.

\subsection{Results and Evaluation}

\subsubsection{Overview}

We consider a run as successful if it yielded at least one individual with optimal values in the functional criteria, i.e., in which a program behaving adequately in all tested randomized simulation scenarios of one generation was found. In the evaluation of the results, we take all experimental runs into consideration which completed at least 750 generations or were successful earlier.

In Table 7.1, we show the number $\# s$ and proportion $s / r$ of such successful runs in relation with the number $\# r$ of total runs. Because of the different runtime of the experiments and the way in which the cluster on which they ran was utilized, $\sharp r$ is not the same for all configurations. The measures $\# s$ and $s / r$ clearly show that only problems the variants $a$ ) and c) of the election problem could be solved. In terms of $s / r$, eRBGP performs best, followed by the Standard Genetic Programming approaches. RBGP and Fraglets have the lowest success rates for problem $a$ ) but become better in problem $c$ ). 


\begin{tabular}{|c|c|c|c|c|c|c|c|c|c|c|c|c|c|}
\hline Case & $\# \boldsymbol{r}$ & $\# \boldsymbol{S}$ & $s / r$ & $\check{s t}$ & $\overline{s t}$ & $\widehat{s t}$ & $\overline{f_{e l}}$ & $\overline{f_{e l}}$ & $\widetilde{f_{p s}}$ & $\overline{f_{p s}}$ & $T_{\bar{s}}$ & $T_{s}$ & $T$ \\
\hline$F_{a}$ & 37 & 35 & 0.95 & 6 & 198 & 782 & .000 & 0.052 & 10.0 & 17.8 & $319 \mathrm{~s}$ & $316 \mathrm{~s}$ & $316 \mathrm{~s}$ \\
\hline$F_{b}$ & 32 & 0 & 0.00 & - & - & 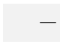 & 200 & .246 & 12.0 & 19.1 & $24 \mathrm{~s}$ & - & $324 \mathrm{~s}$ \\
\hline Øु $F_{c}$ & 32 & 0 & .00 & - & - & - & 216 & .339 & 10.0 & 15.6 & $207 \mathrm{~s}$ & - & $207 \mathrm{~s}$ \\
\hline$F_{d}$ & 31 & 0 & 0.00 & - & - & - & 0.143 & 0.383 & 10.0 & 16.5 & $205 \mathrm{~s}$ & - & $205 \mathrm{~s}$ \\
\hline$F_{a}$ & 55 & 43 & 0.78 & 14 & 354 & 997 & 0 & 147 & 10.0 & 17.3 & $53 \mathrm{~s}$ & $275 \mathrm{~s}$ & $227 \mathrm{~s}$ \\
\hline 의 $F_{b}$ & 55 & 0 & 0.00 & - & - & - & .200 & 0.361 & 10.0 & 17.9 & $250 \mathrm{~s}$ & - & $250 \mathrm{~s}$ \\
\hline की $F_{c}$ & 57 & 1 & 0.02 & 981 & 981 & 981 & 0.000 & 0.310 & 10.0 & 17.2 & $202 \mathrm{~s}$ & $196 \mathrm{~s}$ & $202 \mathrm{~s}$ \\
\hline$F_{d}$ & 49 & 0 & 0.00 & - & - & $\begin{array}{lll}- & \\
\end{array}$ & .144 & 0.460 & 10.0 & 15.5 & $170 \mathrm{~s}$ & 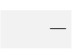 & $170 \mathrm{~s}$ \\
\hline$F_{a}$ & 42 & 21 & 0.50 & 92 & 325 & 675 & .0 & 0.331 & 10.0 & 18.5 & 27 & $82 \mathrm{~s}$ & $55 \mathrm{~s}$ \\
\hline$F_{b}$ & 41 & 0 & .00 & - & - & - & 018 & 09 & 10.0 & 12.0 & $5 \mathrm{~s}$ & & $5 \mathrm{~s}$ \\
\hline$\stackrel{\text { J }}{ } F_{c}$ & 39 & 2 & 0.05 & 488 & 637 & 786 & 0.000 & 0.542 & 10.0 & 10.5 & $65 \mathrm{~s}$ & $93 \mathrm{~s}$ & $67 \mathrm{~s}$ \\
\hline$F_{d}$ & 40 & 0 & م 0 م & - & - & - & 0.200 & .562 & 10.0 & 10.1 & $8 \mathrm{c}$ & 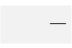 & $8 \mathrm{~s}$ \\
\hline \multirow{4}{*}{ 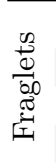 } & 4 & 8 & & 31 & 362 & 514 & & 20 & 15.0 & 31.1 & $393 \mathrm{~s}$ & $901 \mathrm{~s}$ & $485 \mathrm{~s}$ \\
\hline & 52 & 0 & 0.00 & - & - & - & 0.035 & 0.626 & 6.0 & 11.9 & $80 \mathrm{~s}$ & - & $80 \mathrm{~s}$ \\
\hline & 23 & 5 & 0.22 & 479 & 677 & 958 & 0.000 & 0.039 & 12.0 & 35.7 & $395 \mathrm{~s}$ & $478 \mathrm{~s}$ & $413 \mathrm{~s}$ \\
\hline & 52 & 0 & 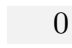 & - & - & - & 0.144 & 0.546 & 3.0 & 14.6 & $51 \mathrm{~s}$ & - & $51 \mathrm{~s}$ \\
\hline$F_{a}$ & 31 & 3 & 0 & 40 & 397 & 615 & 0.000 & 0.768 & 8.0 & 14.2 & $47 \mathrm{~s}$ & $81 \mathrm{~s}$ & $150 \mathrm{~s}$ \\
\hline 의 $F_{b}$ & 51 & 0 & 0. & - & - & 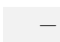 & 0.200 & 0.756 & 8. & 13.1 & $143 \mathrm{~s}$ & - & $143 \mathrm{~s}$ \\
\hline 色 $F_{c}$ & 54 & 10 & 0.1 & 204 & 477 & 648 & 0.000 & 0.501 & 11.0 & 22.4 & $135 \mathrm{~s}$ & $172 \mathrm{~s}$ & $142 \mathrm{~s}$ \\
\hline$F_{d}$ & 43 & 0 & 0.00 & - & - & - & 0.143 & 0.455 & 6.0 & 18.0 & $164 \mathrm{~s}$ & - & $164 \mathrm{~s}$ \\
\hline \multirow{4}{*}{ 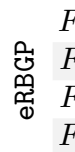 } & 1 & 31 & & 30 & 141 & 742 & 0 & 0.000 & 17.0 & 24.2 & & $225 \mathrm{~s}$ & $225 \mathrm{~s}$ \\
\hline & 40 & 0 & & - & - & - & 0.021 & 0.260 & 16.0 & 34.5 & $115 \mathrm{~s}$ & - & $115 \mathrm{~s}$ \\
\hline & 59 & 59 & 1.0 & 35 & 197 & 950 & 0.000 & 0.000 & 18.0 & 21.6 & 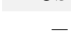 & $226 \mathrm{~s}$ & $226 \mathrm{~s}$ \\
\hline & 36 & 0 & 0.00 & - & - & - & 0.005 & 0.198 & 18.0 & 36.0 & $107 \mathrm{~s}$ & - & $107 \mathrm{~s}$ \\
\hline
\end{tabular}

Table 7.1: The Genetic Programming approaches in direct comparison.

We furthermore distinguish the minimum $(\check{s t})$, mean $(\overline{s t})$, and maximum generation $(\widehat{s t})$ in which the first adequate program was found in the successful runs. eRBGP also has by far the best $\check{s t}$ and $\overline{s t}$ values.

Additionally, Table 7.1 lists the optimal (minimal) and mean values of the two objective functions $\left(\widetilde{f_{e l}}, \overline{f_{e l}}, \widetilde{f_{p s}}, \overline{f_{p s}}\right)$ of the individuals with the best functional objective values in the last generations of the experimental runs. It makes no sense to compare the Genetic Programming approaches in terms of $f_{p s}$, since the structures of the programs evolved with them are too different. In terms of $\overline{f_{e l}}$, however, eRBGP is best in problems $a$ ), c), and $d$ ) and loses to only in SGP in $b$ ). In cases a) and $c$ ), the Fraglets approach reaches the second best mean performance after eRBGP while being not as competitive in the other two election tasks.

The last three columns contain the average time per generation of runs which did not succeed $\left(T_{\bar{s}}\right)$, of those which did $\left(T_{s}\right)$, and of both $(T)$ for each configuration. We will take a closer look on these values in Section 7.5.4.

\subsubsection{Convergence}

Besides the raw numbers describing the final results of the Genetic Programming processes given in Table 7.1, it is interesting to see how the different approaches "got there". We will therefore compare their convergence behavior, too. For each election problem and GP method, we have therefore computed the mean, median (med), and the 5\% and $95 \%$ 
quantiles $^{3}$ (Q.05, Q.95) of the $f_{e l}$ values of the best individual (again in terms of $f_{e l}$ ) in each generation over all runs. These values are illustrated in the convergence diagrams Figure 7.2 and Figure 7.3 for the problems $a$ ) and $b$ ). Similar diagrams for the tasks $c$ ) and $d$ ) are given in Section A.3.1 on page 170 in the appendix.

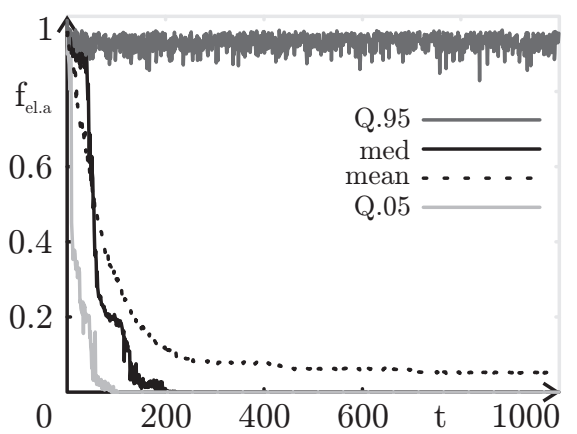

Fig. 7.2.a: Convergence: SGP for $f_{\text {el.a }}$.

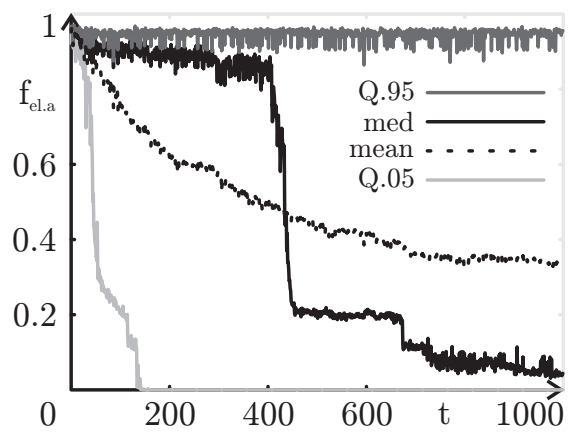

Fig. 7.2.c: Convergence: LGP for $f_{e l . a}$.

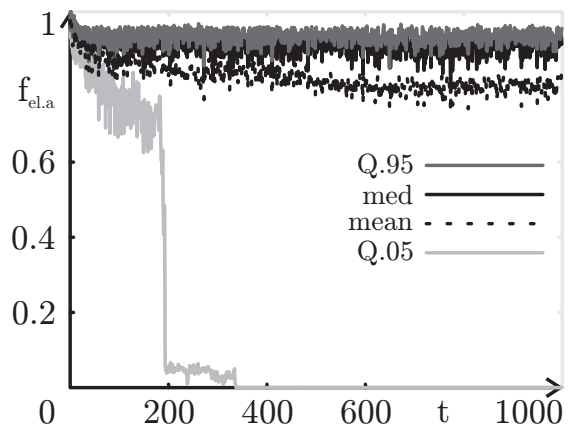

Fig. 7.2.e: Convergence: RBGP for $f_{e l . a}$.

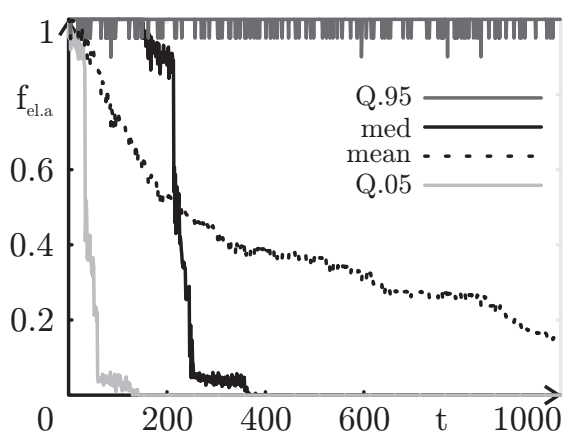

Fig. 7.2.b: Convergence: eSGP for $f_{\text {el.a }}$.

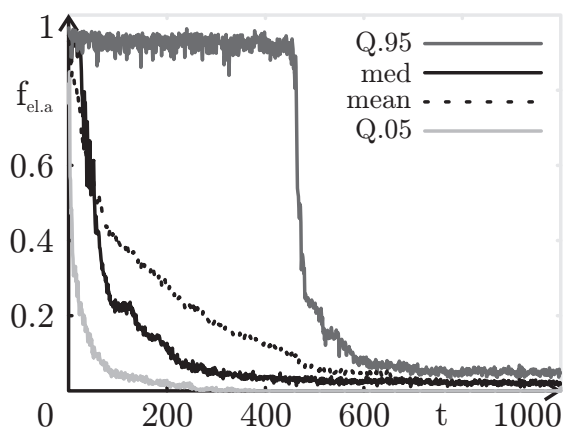

Fig. 7.2.d: Convergence: Fraglets for $f_{\text {el.a. }}$.

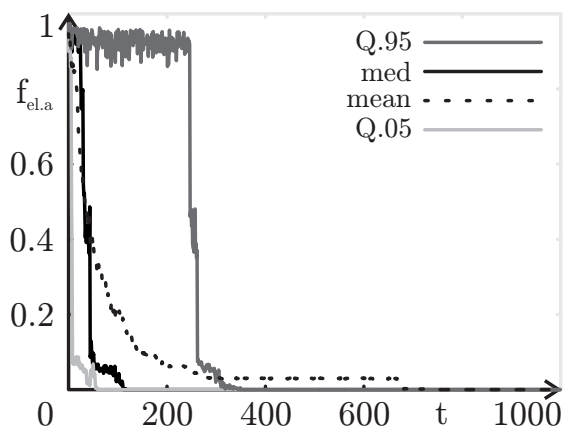

Fig. 7.2.f: Convergence: eRBGP for $f_{\text {el.a }}$.

Figure 7.2: Convergence diagrams for the election problem $f_{\text {el.a }}$.

Figure 7.2 substantiates our previous assumption that eRBGP finds solutions for problem a) the fastest. It reaches $f_{\text {el.a }}=0$ with all of four performance indicators in fewer generations than the other approaches. Its $95 \%$ quantile hits the abscissa at about the same time that the

${ }^{3}$ These two quantiles most often evaluate to the second best and second worst individuals in the experiments here and are thus slightly more stable than just the minimum or maximum results. In the other experiments with more runs, they are even more substantial. 
median of eSGP does, meaning that by the time where around half of the runs with eSGP had found a solution, less than $5 \%$ of the eRBGP experiments were still searching. Interestingly, the Fraglets method is the only approach besides eRBGP where the $95 \%$ quantile dropped below 0.6 in case electionCase $A$ ). In the election problem $b$ ), eRBGP was the only method

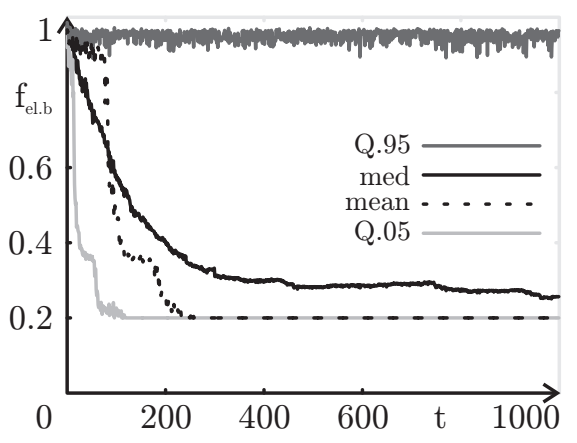

Fig. 7.3.a: Convergence: SGP for $f_{\text {el.b }}$.

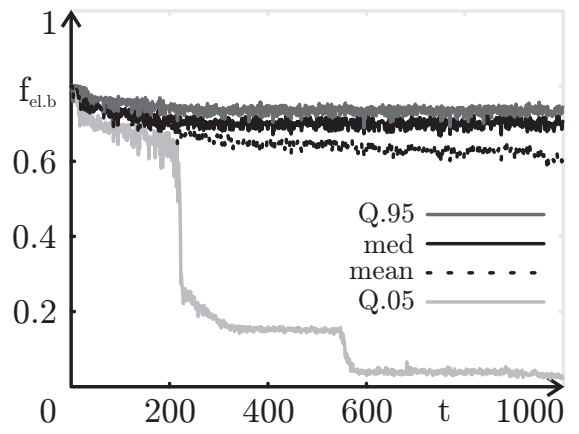

Fig. 7.3.c: Convergence: LGP for $f_{\text {el.b }}$.

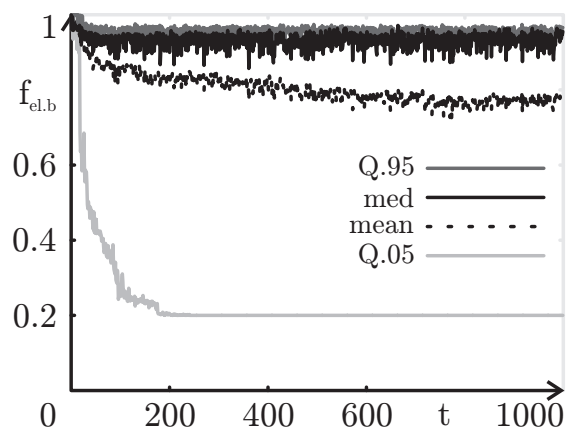

Fig. 7.3.e: Convergence: RBGP for $f_{e l . b}$.

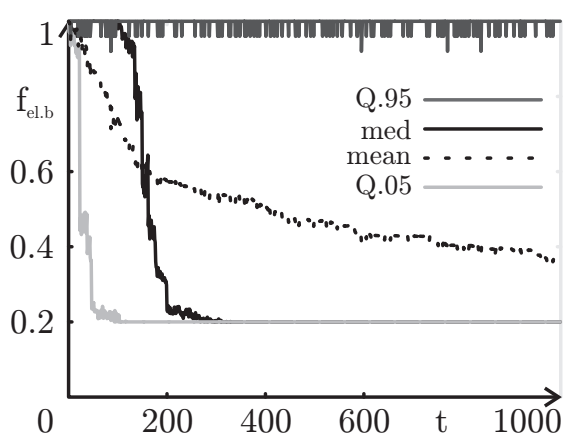

Fig. 7.3.b: Convergence: eSGP for $f_{\text {el. }}$.

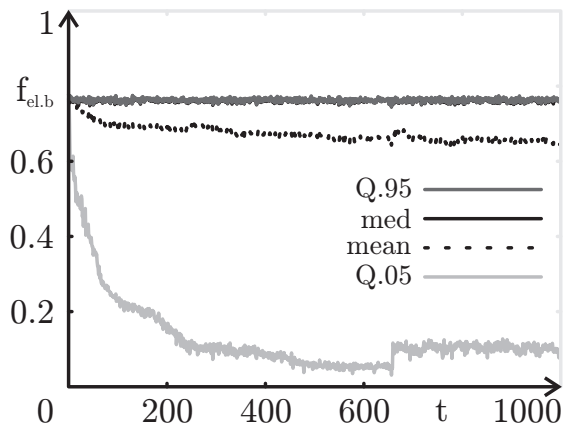

Fig. 7.3.d: Convergence: Fraglets for $f_{\text {el.b. }}$.

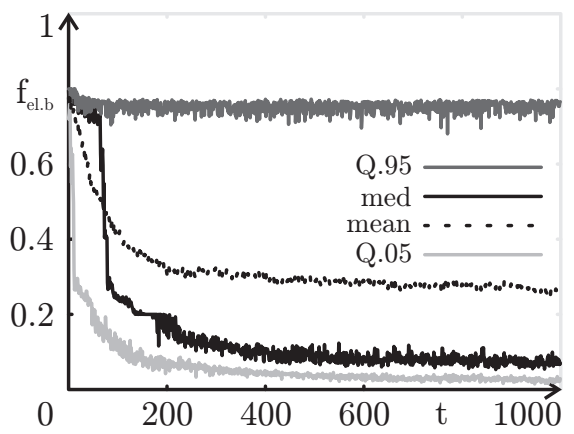

Fig. 7.3.f: Convergence: eRBGP for $f_{\text {el.b. }}$.

Figure 7.3: Convergence diagrams for the election problem $f_{e l . b}$.

where both, the $5 \%$ quantile and the median value of $f_{\text {el. }}$ could reach below 0.2 , as illustrated in Figure 7.3. 


\subsubsection{Comparison of the GP Approaches}

The two previous sections gave a good overview of the results of the election experiments and indicated some tendencies. In this section, we evaluate the results of the experiments thoroughly and apply statistical means in order to verify these assumptions and to reach profound conclusions.

We therefore have partially ordered the six Genetic Programming programming approaches used in this work according to different performance metrics in Figure 7.4. In each of the eight diagrams, an arrow from one approach A to an approach B means that B beats $\mathrm{A}$ in the given problem according to the specified metric in a two-tailed test with a $2 \%$ significance level. In other words, the error probability $\alpha$, i. e., the chance that the arrow does not reflect the real situation, is less than 0.02 . The illustrated relations are transitive.

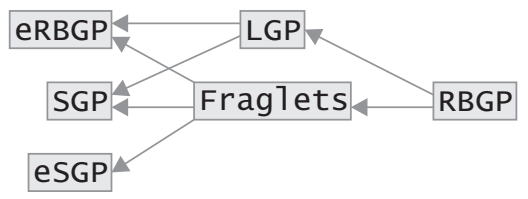

Fig. 7.4.a: According to $f_{\text {el.a }}$ in $F_{a}$.

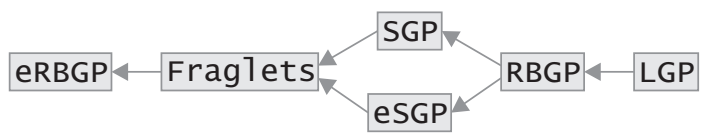

Fig. 7.4.c: According to $f_{\text {el.c }}$ in $F_{c}$.

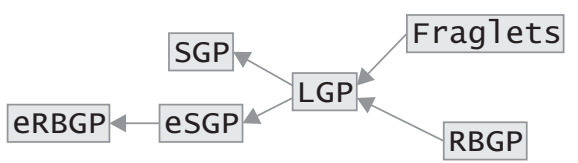

Fig. 7.4.e: According to $s / r$ in $F_{a}$.

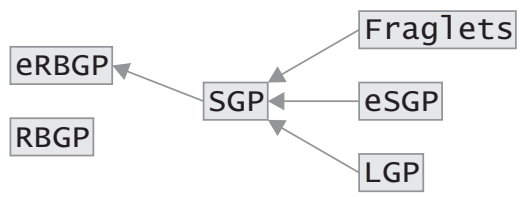

Fig. 7.4.g: According to st in $F_{a}$.

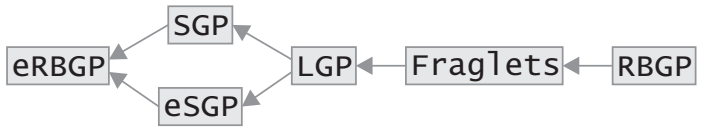

Fig. 7.4.b: According to $f_{\text {el. }}$ in $F_{b}$.

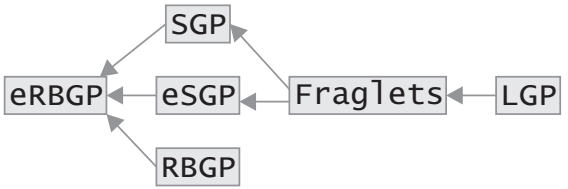

Fig. 7.4.d: According to $f_{\text {el.d }}$ in $F_{d}$.

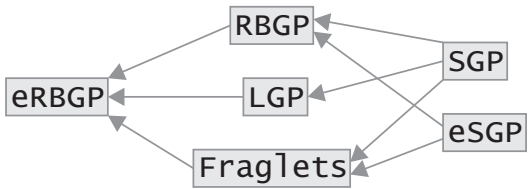

Fig. 7.4.f: According to $s / r$ in $F_{c}$.

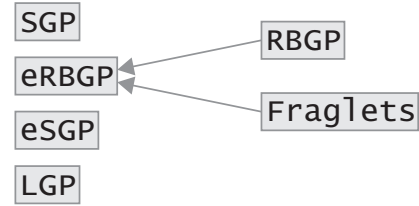

Fig. 7.4.h: According to st in $F_{c}$.

Figure 7.4: Partial orders of the GP approaches according to their performance in the election experiments.

In Fig. 7.4.a to Fig. 7.4.d, we used the Mann-Whitney U Test [192] to compare the best $f_{e l}$-values from the last generation of each single run for the problems $F_{a}$ to $F_{d}$. This test was also applied to the numbers of generations needed to find adequate solutions $(s t)$ in Fig. 7.4.g and Fig. 7.4.h. Obviously, this measure is only available for the problems $F_{a}$ and $F_{c}$, since only these could be solved successfully. In order to find out which of the approaches were the most effective ones in these two problems, we used Fisher's exact test $[88,89]$ to compare the success rates $s / r$ in Fig. 7.4.e and Fig. 7.4.f. Tabular represenations of Figure 7.4 can be found in Section A.3.2 in the appendix. 


\section{Moderateness SGP and eSGP}

In Table 7.1, we found that SGP and eSGP are good in solving $F_{a}$, bad in $F_{c}$, and unable to find any useful program for $F_{b}$ or $F_{d}$. The orderings given in Figure 7.4 show that neither of the two is generally better or worse than the other, but there is a slight advantage for SGP.

\section{LGP: The Baseline}

The linear Genetic Programming approach usually performed not so well and is often beaten by several other GP representations. It is able to solve at least $F_{a}$ reasonably good whilst failing in the other problems. Still, for $F_{b}$ it found the program coming closest to adequate behavior (see Listing 7.6 on page 111) in terms of $f_{\text {el.b. }}$. The overhead of evolving programs in tree form with SGP and eSGP and explicitly compiling them to the linear representation seems to pay off, since both of these methods often win against plain LGP.

\section{Inferiority of RBGP}

Very much to our surprise, our first approach designed to have an especially low epistasis, RBGP, was inferior in almost all categories and could beat SGP, eSGP, and LGP only once each in the statistical tests. Here, the importance of statistically significant experiments and comparative studies became obvious: in our prior experiments of smaller scale, quite the opposite seemed to be the case and the behavior of RBGP appeared to be excellent.

\section{Dominance of eRBGP}

Even more surprising than the weakness of RBGP is the sheer superiority of eRBGP. In the statistical tests, this approach never lost in any performance measure or problem category in the election problem. Instead, it beats the other approaches on a constant basis and finds better solutions faster and more often. The question why the two related methods behave that much different will be subject to a detailed discussion in Section 10.3.1.

\section{Fraglets}

The convergence diagrams and the overview table show that a larger fraction of the Fraglets runs find good solution candidates in problems a) and c) than any other GP method except eRBGP. In the statistical tests, the Fraglets approach has a behavior similar to LGP. This is interesting since both are also based on the same type of phenome (lists of integer strings) but stand for totally different execution models.

\subsubsection{Runtime}

One important aspect of the evolution of distributed algorithms is the runtime. In our experiments, we used computers with AMD 64bit 2.1GHz processors and 4GB RAM. Figure 7.5 shows the arithmetic mean of the time in seconds per generation for the six Genetic Programming approaches applied to the four optimization problems $F_{a}$ to $F_{d}$.

The runtime time spans from five seconds per generation (LGP, $F_{2}$ ) to $901 \mathrm{~s}$ for the Fraglets approach applied to the problem $F_{a}$. In other words, one experimental run took between one and a half hour and ten and a half days, depending on the problem and the program representation chosen. One thousand generations of the eRBGP approach, which has proven to solve the election problem best, take about two days. In total, this experimental series took 2052 CPU days, distributed over between 15 and 30 workstations. 


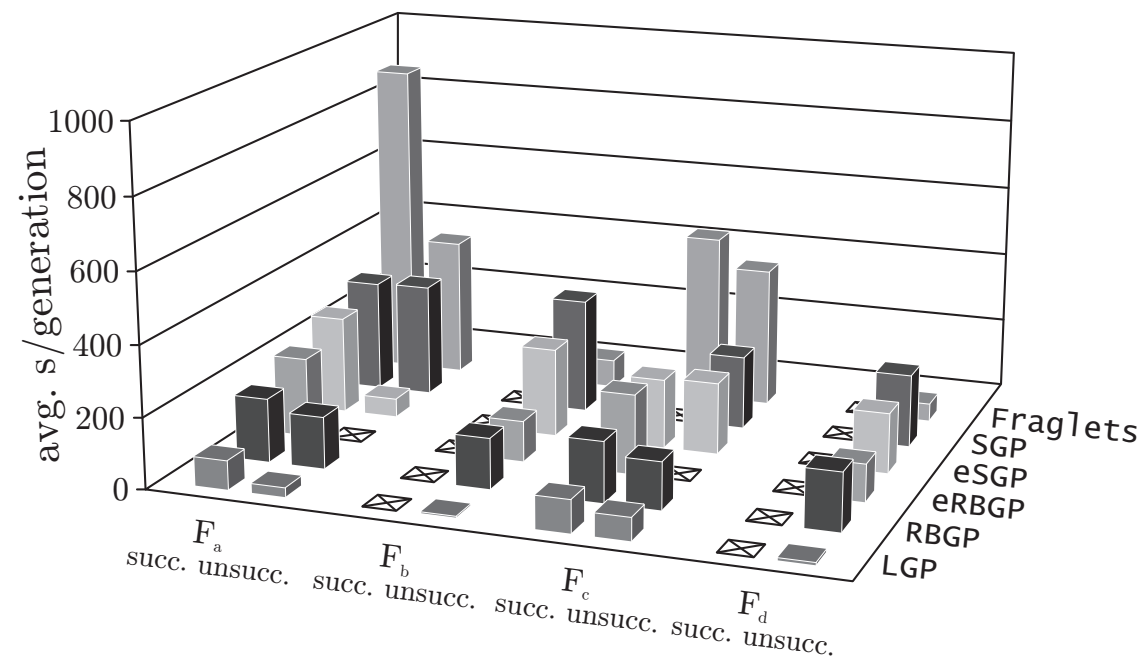

Figure 7.5: The average time per generation of the different approaches in the four problems.

Comparing the utility of the different approaches with each other on basis of the runtime is not advisable, since they have different expressivenesses and were thus granted different numbers of simulation steps. Also, the approaches have different basic simulation complexities.

In Figure 7.5, we computed separate means for runs which were successful and for those which were not. Although for some problems and representations, either all runs failed or succeeded, the figure indicates that successful runs tend to take more time than unsuccessful ones. The reason for this is that if no solution for a problem could be found, a trivial algorithm which is finished after only few or even a single simulation step has a relatively good fitness in comparison with the other solution candidates in the population. In runs where close-to-optimal programs emerged, these perform a "real" distributed computation which needs several steps to decide upon the elected node. Hence, their execution in the simulation takes longer and thus, the runs will do so too.

\subsection{Summary}

This experiment clearly shows that all six Genetic Programming approaches can be utilized to evolve distributed algorithms. It also shows that different program representations lead to different results and success probabilities.

Especially interesting here is the performance of the Rule-based Genetic Programming methods: While eRBGP achieved a high success rate, plain RBGP did not. The former shows that the targeted design of representations can indeed lead to significantly better results. The worse performance of RBGP may have been caused by the tight structural constraints which allow only rules with exactly two conditions in order to allow for a binary encoding of the programs.

None of the Genetic Programming approaches could solve the problems b) and $d$ ) which required termination. This may have two reasons: First, the simulation time chosen may have been not long enough, i. e., some vital algorithm steps are carried out too close before the end of the simulation in some scenarios. Then, there would be insufficient time to detect termination. The number of steps which were granted to the simulations, however, is relatively high and should be sufficient to allow the algorithms to terminate gracefully after electing a leader. 
Second, and more likely, the code needed for termination itself may be too complicated so its coevolution together with the election procedure was too hard in the relatively small populations utilized. After all, Genetic Programming of full-fletched algorithms still has to cope with all the problems listed in Chapter 4. 



\section{Evolving Mutual Exclusion for Critical Sections}

The architecture of computers changed dramatically in the mid-1950s with the invention of larger magnet stores and asynchronously operating peripheral hardware. The concept of interrupts was invented in order to allow processes to run on fast processors while slower periphery devices were operating simultaneously. This concurrency posed completely new problems to the software design and was one of the reasons of the software crisis in the mid-1960s.

Sharing resources in asynchronous systems always holds many dangers. If two or more processes simultaneously access common variables and at least one of them in a modifying manner, phenomena like lost update or other data inconsistencies are bound to happen. Dijkstra realized that software engineers must ensure mutual exclusion, i.e., guarantee that at most one process may access the resource at a time. He also coined the term critical section for the code accessing the resource. The following criteria for correct mutual exclusion algorithms can be extracted from Dijkstra's work [71, 72]:

1. At most one processes is granted access to the shared resource at a time.

2. If one of the processes is stopped well outside its critical section, this is not allowed to lead to potential blocking of the other process. (Deadlock, [71])

3. If the two processes are about to enter their critical sections, it must be impossible to devise for them such finite speeds, that the decision which one of the two is the first to enter its critical section is postponed until eternity. (Lifelocks, [71])

4. The algorithm can be implemented for an arbitrary number of processes.

The first algorithm which solved this problem efficiently was developed by Dekker. This decentralized algorithm uses shared control variables for synchronization. ${ }^{1}$ Developing mutual exclusion algorithms for distributed systems is more cumbersome since usually no globally shared memory exists. Instead, the processes are running concurrently on different nodes and have to communicate by the means of message exchange. Based on the messages sent and received, a process has to decide whether it is allowed to access the shared resource or has to wait. First distributed algorithms for mutual exclusion at a critical section were introduced by Lamport [168] in 1976 and by Ricart and Agrawala [244] in 1981, followed by Maekawa's optimal solution in terms of the number of exchanged messages in 1985 [189].

\subsection{Specification of the Global Behavior}

Like in the election experiment, we envisage a scenario where the resulting algorithms are to be implemented on tiny devices with very little memory and computing power. Again,

\footnotetext{
${ }^{1}$ Centralized approaches, on the other hand, rely on primitives provided by the operating system
} or the programming languages (such as semaphores which were introduced by Dijkstra) instead. 
the actual number of nodes in the networks is unknown and potentially large. Hence, it may not be possible for the devices to store the IDs of all other nodes in their memory. The goal of this experiment is to evolve algorithms which

1. ensure mutual exclusion of the access to a shared resource as good as possible and

2. allow the processes to access this resource as often as possible.

Besides the hardness of creating an adequate algorithm in this scenario, directly checking the validity of point three from Dijkstra's requirements, the absence of lifelocks, is impossible with testing based on randomized simulations. However, due to the inherent randomness of the communication delays in real-world networks, this issue may not be too important.

We want to evolve programs which follow the scheme used by Dijkstra and try to access the critical section in an infinite loop. Therefore, the program representations listed in Chapter 5 are extended with the instruction enterCS. enterCS places the invoking node into passive mode for a randomly selected number $w$ of time steps. This sleeping time is a simulation for accessing the shared resource and doing something useful with it. The node cannot be woken up during these $w$ steps by any other event (such as incoming messages). Afterwards, it will resume execution normally.

Hence, Genetic Programming is to find a way to embed calls to entercs into proper synchronization code. Obviously, a single invocation of enterCS will not achieve a high access rate of the critical section, so some sort of loop should be built around it. This loop may be constructed indirectly based on message sending and processing. Here, the GP system is not bound to any predefined structure.

\subsection{Objective Functions}

In this experiment, two objective functions focusing on functional adequacy are used together with the non-functional program size criterion $f_{p s}$ defined in Section 7.2.2 on page 107. Here we will only discuss the functional aspects - the number of collisions in the critical section and its utilization.

\subsubsection{Protection of the Critical Section}

We define the objective function $f_{c o l}$ as the total number of all collisions caused by a program $x$ in a given simulation scenario. This value is normalized into the range $[0,1]$ and subject to minimization.

As we have previously mentioned, a simulation is executed for $m x S$ time steps. A collision has occurred in a time step $i$ when two processes $A$ and $B$ both have entered the critical section (by calling enterCs) without leaving it yet. In a real-world scenario, this would mean that at this point in time, the two processes may mutually impair their integrity.

Equation 8.1 denotes the number of collisions which occurred in a time step $i \in 1$.. $m x S$. In a collision-free system, the number inCS $(i)$ of processes executing their respective critical section code at the same time is lower than two. One collision results from $\operatorname{inCS}(i)=2$. Three simultaneous accesses to a shared resource lead to three collisions: process $A$ with $B$, $A$ with $C$, and $B$ with $C$.

$$
\operatorname{numColls}(i)=\left\{\begin{array}{cl}
0.5 \operatorname{inCS}(i) *(\operatorname{inCS}(i)-1) & \text { if } \operatorname{inCS}(i)>1 \\
0 & \text { otherwise }
\end{array}\right.
$$

According to this definition, $k$ processes can cause $0.5 k(k-1)$ collisions - the number of connections in a fully connected graph with $k$ nodes - if all of them execute their critical 
sections at once. Therefore, the maximum number maxColls of collision which can possible occur in a network $\mathbf{N}$ during the complete simulated time is

$$
\operatorname{maxColls}=m x S * 0.5 * \operatorname{numNodes}(\mathbf{N}) *(\operatorname{numNodes}(\mathbf{N})-1)
$$

Equation 8.3 finally counts the number of collisions caused by executing program $x$ throughout the simulation and normalizes it with maxColls. If the critical section is never entered, it also returns 1 as penalty. In Figure 8.1, we sketch an example scenario with twenty time steps $i$ in which four nodes execute their critical section codes.

$$
f_{\text {col }}(x)=\left\{\begin{array}{cl}
\frac{1}{\operatorname{maxColls}} \sum_{i=1}^{m x S} \operatorname{numColls}(i) & \text { if } \exists i \in 0 . . m x S: \operatorname{inCS}(i)>0 \\
1 & \text { otherwise }
\end{array}\right.
$$

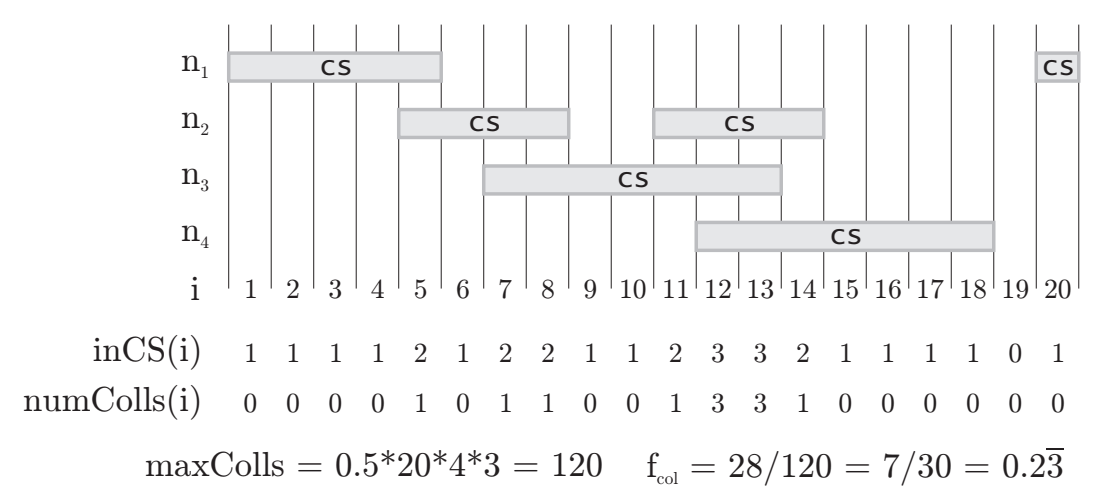

Figure 8.1: Example for the collision-counting objective function $f_{\text {col }}$.

\subsubsection{Utilization of the Critical Section}

The optimal value of $f_{c o l}$ is obviously 0 . It may be reached by negotiating (i. e., electing) a single process which is the only one allowed to utilize the shared resource. Although never accessing the critical section also causes no collisions, such behavior will be rated with $f_{\text {col }}=1$ according to Equation 8.3. Both of these behaviors are certainly no valuable approaches to the critical section problem. With the second functional objective function $f_{\text {use }}$, we want to further a good utilization of the critical section which also excludes the first inadequate mutual exclusion method not yet penalized by $f_{c o l}$.

The value of $f_{u s e}$ is computed with Algorithm 8.1 which examines the observed behavior of a program $x$ in a simulated network $\mathbf{N}$. $f_{\text {use }}$ is a mapping of a numNodes $(\mathbf{N})$-dimensional space to the real interval $[0,1]$. Therefore, a graphical representation as for the election objective in Figure 7.1 is not possible. Instead, we will give some examples for a network with ten nodes in Table 8.1. This table shows how often the nodes $\mathbf{n}$ enter their critical sections (inCS(n)). In its lower half, the values of the variables check and total used in Algorithm 8.1 are listed together with the resulting objective value $f_{\text {use }}$.

$f_{\text {use }}$ penalizes exactly the two non-solutions mentioned above: As it can be seen in the examples $\# 1$ to $\# 3$, trivial programs only achieve objective values very close to 1 . Even if a single process manages to enter its critical section very often (\#3), its utility can never surpass the one of a program which allows two processes to use the shared resource, even if they are permitted to do so only once (\#4): Assume that total $\rightarrow+\infty$ but check $[k]=$ $1 \forall k \in 1 . .5$, then $f_{\text {use }}$ evaluates to $\frac{5 m-5}{5 m-4}$. For a network size $m=\operatorname{numNodes}(\mathbf{N})$ of $4, f_{\text {use }}$ 


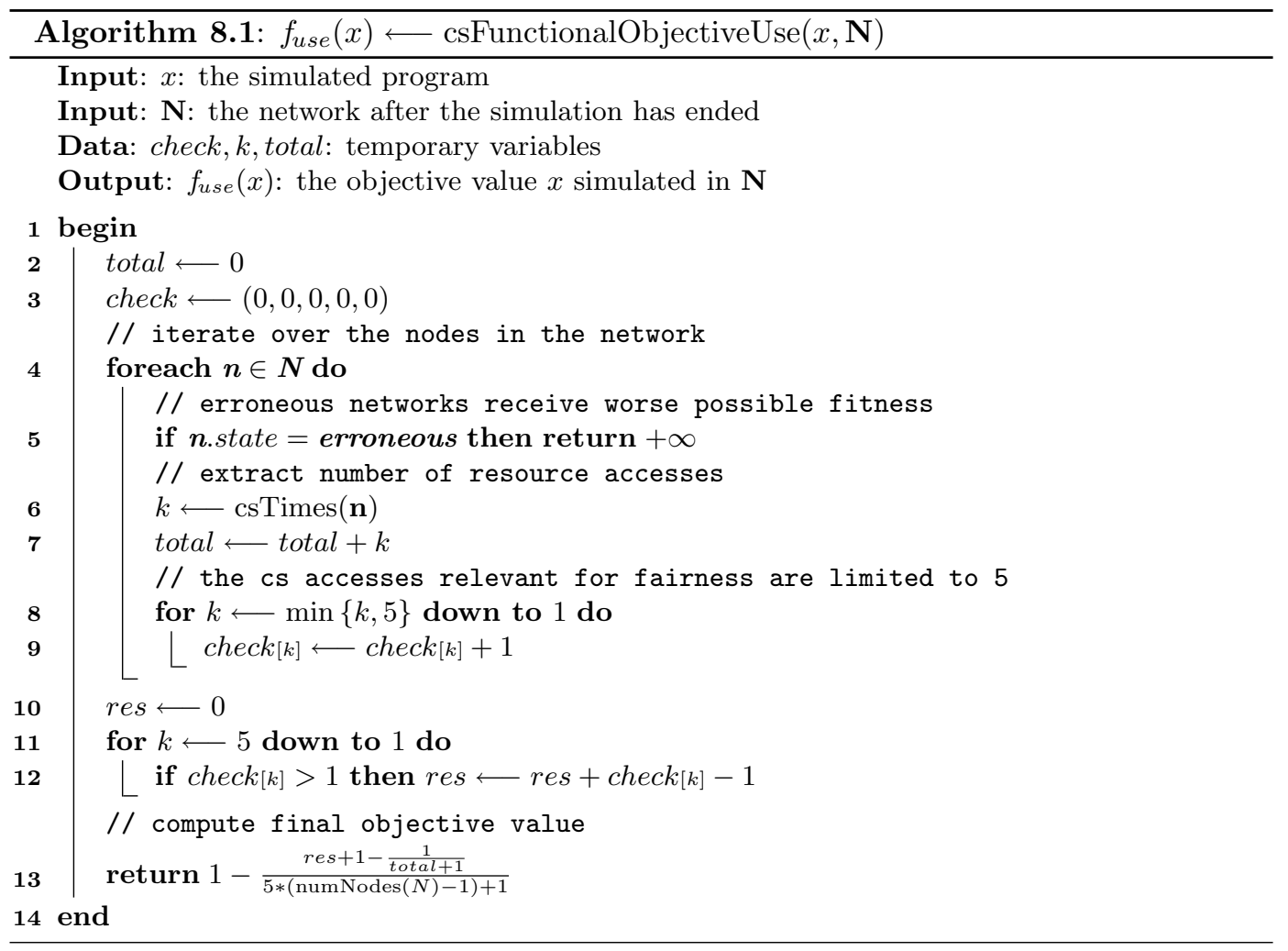

\begin{tabular}{|c|c|c|c|c|c|c|c|c|c|c|c|c|c|}
\hline & \multicolumn{13}{|c|}{$\operatorname{cs} \operatorname{Times}(\mathbf{n})$ values in scenario scen } \\
\hline & $\# 1$ & $\# 2$ & $\# \mathbf{3}$ & $\# 4$ & $\# 5$ & $\# 6$ & $\# 7$ & $\# 8$ & $\# 9$ & \#10 & \#11 & \#12 & \#13 \\
\hline $\mathbf{n}_{1}$ & 0 & 1 & 100 & 1 & 1 & 2 & 2 & 2 & 2 & 3 & 3 & 100 & 2 \\
\hline $\mathbf{n}_{2}$ & 0 & 0 & 0 & 1 & 1 & 1 & 1 & 2 & 2 & 3 & 3 & 100 & 2 \\
\hline $\mathbf{n}_{3}$ & 0 & 0 & 0 & 0 & 1 & 1 & 1 & 1 & 2 & 3 & 3 & 100 & 1 \\
\hline $\mathbf{n}_{4}$ & 0 & 0 & 0 & 0 & 1 & 1 & 1 & 1 & 2 & 3 & 4 & 100 & 1 \\
\hline $\mathbf{n}_{5}$ & 0 & 0 & 0 & 0 & 1 & 1 & 1 & 1 & 2 & 5 & 4 & 100 & - \\
\hline $\mathbf{n}_{6}$ & 0 & 0 & 0 & 0 & 1 & 1 & 1 & 1 & 2 & 5 & 4 & 100 & - \\
\hline $\mathbf{n}_{7}$ & 0 & 0 & 0 & 0 & 1 & 1 & 1 & 1 & 2 & 2 & 5 & 100 & - \\
\hline $\mathbf{n}_{8}$ & 0 & 0 & 0 & 0 & 1 & 1 & 1 & 1 & 2 & 2 & 5 & 100 & - \\
\hline $\mathbf{n}_{9}$ & 0 & 0 & 0 & 0 & 1 & 1 & 1 & 1 & 2 & 4 & 5 & 100 & - \\
\hline $\mathbf{n}_{10}$ & 0 & 0 & 0 & 0 & 1 & 0 & 1 & 0 & 2 & 2 & 5 & 100 & - \\
\hline var & & & & & val & ue of & variak & le $v a r$ & & & & & \\
\hline check[1] & 0 & 1 & 1 & 2 & 10 & 9 & 10 & 9 & 10 & 10 & 10 & 10 & 4 \\
\hline check[2] & 0 & 0 & 1 & 0 & 0 & 1 & 1 & 2 & 10 & 10 & 10 & 10 & 2 \\
\hline check[3] & 0 & 0 & 1 & 0 & 0 & 0 & 0 & 0 & 0 & 7 & 10 & 10 & 0 \\
\hline check[4] & 0 & 0 & 1 & 0 & 0 & 0 & 0 & 0 & 0 & 3 & 7 & 10 & 0 \\
\hline check[5] & 0 & 0 & 1 & 0 & 0 & 0 & 0 & 0 & 0 & 2 & 4 & 10 & 0 \\
\hline total & 0 & 1 & 100 & 2 & 10 & 10 & 11 & 11 & 20 & 32 & 41 & 1000 & 6 \\
\hline$f_{\text {use }}$ & 1 & 0.989 & 0.978 & 0.964 & 0.785 & 0.806 & 0.784 & 0.784 & 0.588 & 0.392 & 0.196 & $\approx 0$ & 0.696 \\
\hline
\end{tabular}

Table 8.1: Thirteen example results of $f_{\text {use }}$ for different scenarios scen. 
becomes $\frac{15}{16}=0.9375$ and for $m=23, f_{\text {use }}=\frac{110}{111}=0 . \overline{990}$. The worst possible fitness value in cases where at least two nodes enter the critical section (total $=2$, res $=1$ ) is $\frac{5 m-5.5}{5 m-4}$ which is $\frac{29}{32}=0.90625$ for a network consisting of $m=4$ nodes and $\frac{73}{74}=0.9 \overline{864}$ for $m=23$ nodes.

The more processes are allowed to execute their critical section, the lower $f_{\text {use }}$ will be (see, for instance, \#10). The examples \#5, \#6, and \#7 illustrate this promotion of fairness: allowing a process to access the shared resource twice will only be beneficial if this additional access is not achieved by "stealing" it away from another node. The last, separated column (\#13) corresponds to the example for the computation of the collision objective value for the four node network given in Figure 8.1.

Notice that the presence of a deadlock or a lifelock would increase the value of $f_{\text {use }}$. Therefore, if a program has an affinity for creating such situations, this is likely to show since multiple scenarios are tested for determining the final objective values. Hence, $f_{\text {use }}$ also minimizes the likeliness of both phenomena, although it cannot prevent their occurrence for obvious reasons.

\subsection{Experimental Settings}

The six Genetic Programming approaches defined in Chapter 5 were applied to the critical section problem under the three objective functions $F=\left\{f_{c o l}, f_{u s e}, f_{p s}\right\}$. They therefore were extended with an enterCS instruction which blocked the invoking nodes for a number of steps uniformly distributed in 69..206 (generated according to the same pattern as the message delays in Section 6.3). The SGP, eSGP, and LGP approaches were additionally provided with a second automatically defined function which is asynchronously called after enterCS returns in order to explore which of the two waiting mechanisms is prefered by Genetic Programming. RBGP and eRBGP received a csLeft symbol set to 1 when the critical section was left and in the Fraglet stores of the Fraglets approach, a symbol [csLeft] was injected in this case.

For each algorithm evaluation, twenty scenarios with networks consisting of between four and 23 virtual machines were executed. Because of this setting, we can define a program $x$ as marginally fair if it reaches $f_{\text {use }}(x) \leq 0.90625$. This threshold is the lowest boundary for a network with four nodes where at least two nodes have accessed the critical section, as shown in the previous section. Since the values of $f_{u s e}$ are the arithmetic mean over twenty randomized training scenarios with networks of at least the size four, we can expect such programs to actually utilize the resource much better. To each such simulation, a maximum step number $m x S$ of

1. 30682 for RBGP and eRBGP,

2. 61364 for Fraglets and LGP programs, and

3. 184092 for SGP and eSGP programs was granted.

We tested the evolution of mutual exclusion algorithms in three different network topologies:

fc) a fully connected network where all nodes can directly communicate with each other,

r) an directed ring topology where each node has a direct successor to which and only to which it can send its messages, and

nc) a network where all messages vanish into thin air immediately after being sent, i.e., where communication is disabled, in order to test whether GP is able to create efficient communication patterns.

Each node was allowed to send 2536 messages. If this limit was exceeded, the corresponding VM was immediately terminated and marked as erroneous. The messages were limited to 
three memory words except in the evolution of Fraglets, where the limit was the maximum Fraglet length.

The LGP, SGP, and eSGP approaches were provided with five cells of global and local memory plus three words of stack. Nodes executing RBGP or eRBGP programs were equipped with five multi-purpose variables and the length of Fraglets was limited to 15. The ID of the nodes was again stored in the first global memory cell (LGP, SGP, eSGP), a dedicated (writable) symbol (RBGP, eRBGP), or available via a special Fraglet (Fraglets). The complete list of settings of these experiments is provided in Section B.1 on page 175 and a manually derived adequate solution for the critical section problem is given in Section B.2.

\subsection{Evolved Programs}

The programs evolved for all three network scenarios are rather similar. The fully connected topology, the case $f c$, was the only experimental setting where programs evolved which were both fair and able to protect the critical section. The eSGP program given in Listing 8.1 and its corresponding phenotype in Listing 8.2 are examples for the way in which Genetic Programming achieved these features.

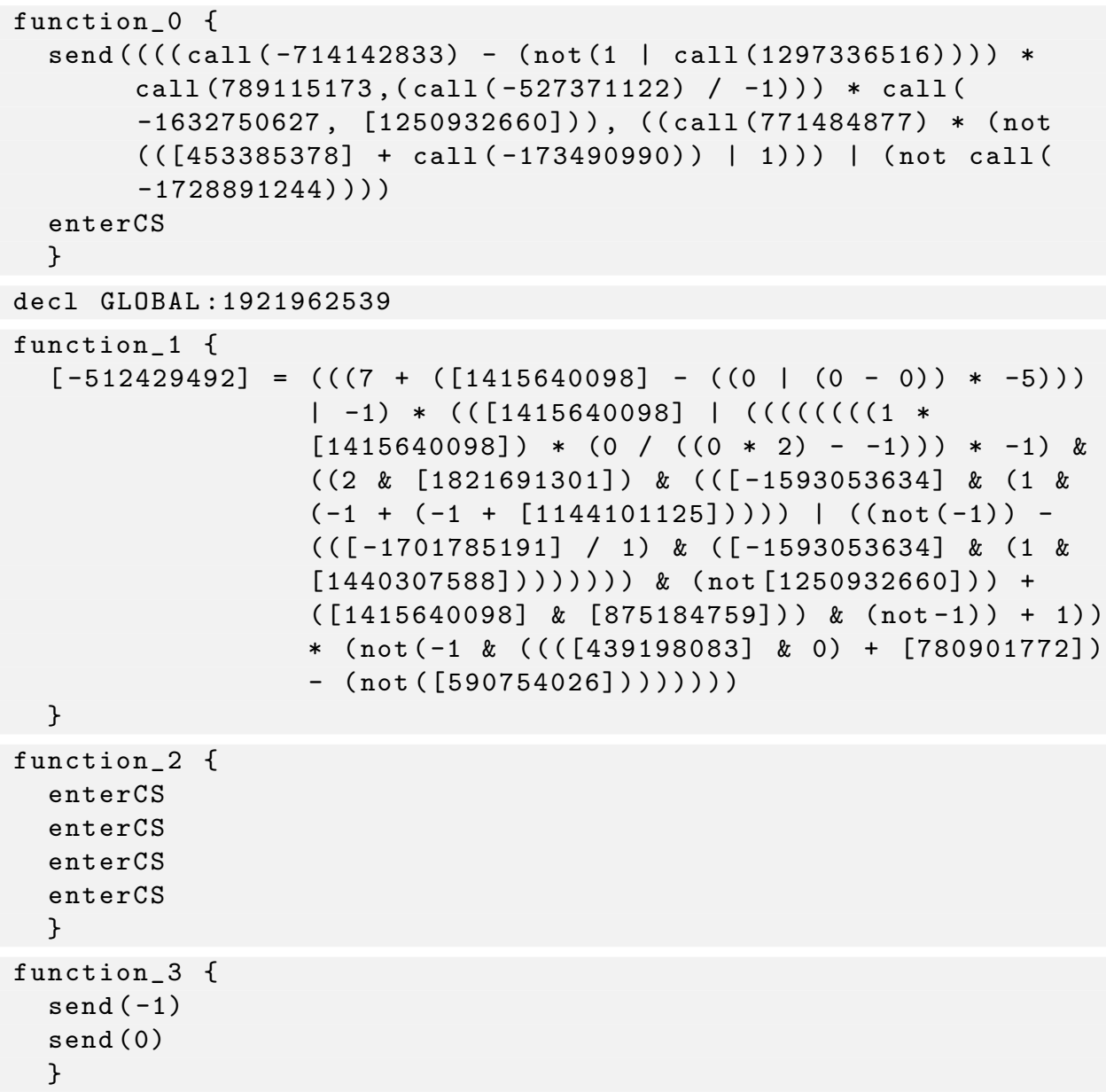

Listing 8.1: The fairest eSGP program for case $f c$ (Phenotype: see Listing 8.2). 


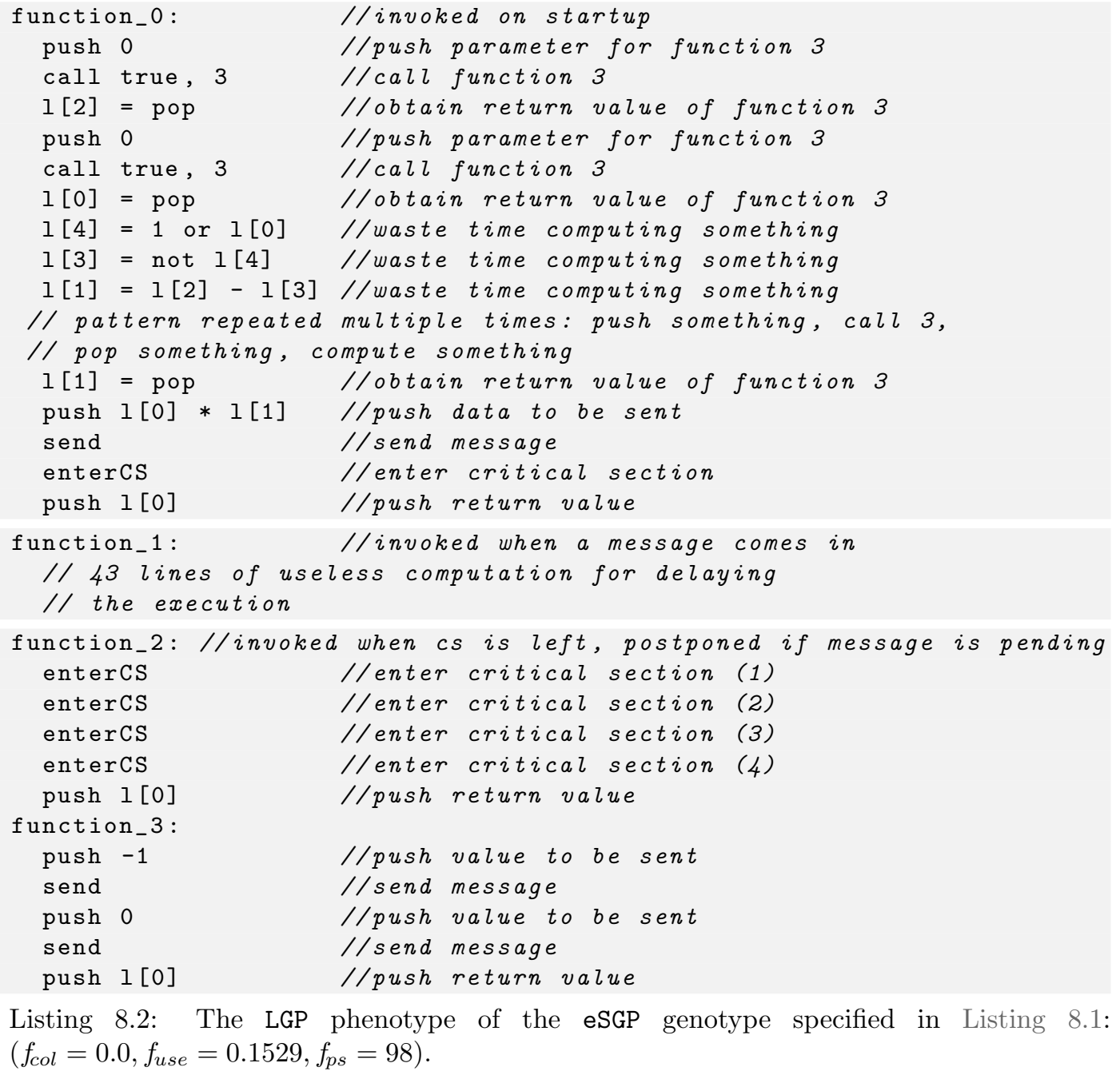

Listing 8.2: The LGP phenotype of the eSGP genotype specified in Listing 8.1: $\left(f_{\text {col }}=0.0, f_{\text {use }}=0.1529, f_{p s}=98\right)$.

The Fraglets program defined in Listing 8.3 has a relatively similar behavior. In order to delay the computation, the network is systematically flooded with as many Fraglets with lots of nop instructions as possible. Because of their high multiplicities in the Fraglet stores and the corresponding high probability of executing the nop and nop-duplicating code, these Fraglets consume most of the runtime of the nodes. Rarely, a node will pick an enterCS Fraglet and enter the critical section. Interestingly, the number of times the critical section is utilized is limited in this program since the enterCS Fraglets are duplicated only a few times and not recreated.

Generally, most of the programs evolved follow the scheme defined in Algorithm 8.2 of mutual stalling and delaying. Here, the communication medium is used as signaling device for synchronization. A node only enters the critical section if it did not receive any message for some time. The other nodes try to prevent this by frequently broadcasting messages. Whether a node can access the "shared resource" therefore depends on the randomness of the message latency and parallelism. In this, these algorithms are similar to those for the election problem el.a (see Section 7.4.1) or the eRBGP program evolved for $F_{b}$ shown in Listing 7.7 on page 112. 

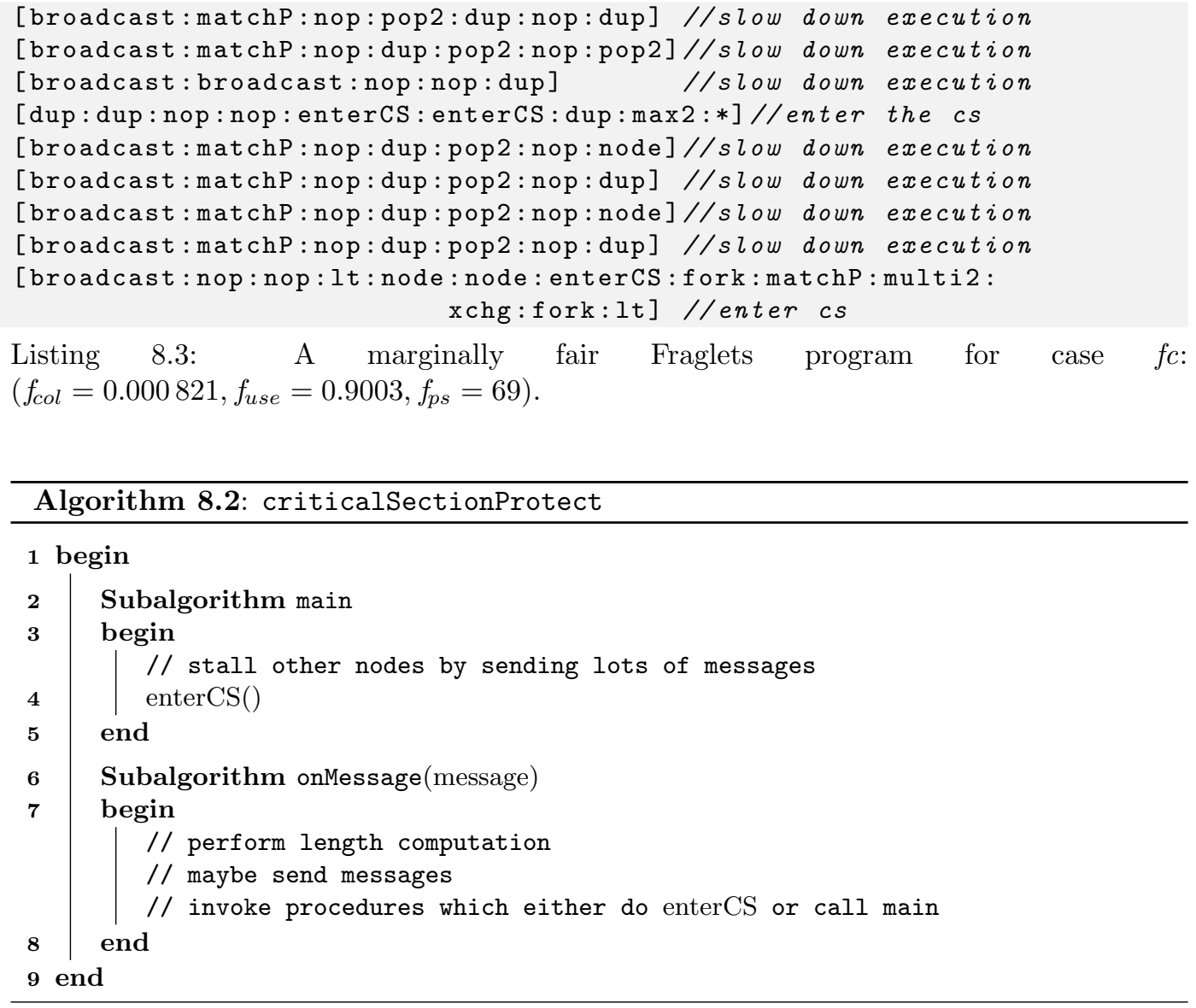

The programs evolved directly in the LGP representation often extend this scheme by terminating the processes running on some of the nodes based on arbitrary criteria. By doing so, the chance of collisions (but also the potential fairness) is reduced.

One of the interesting features of the evolved algorithms in SGP, eSGP, and LGP representation is that they do not involve explicit loops although special language constructs for such structures were available. Even more interesting is that SGP and eSGP most often use code without any conditional branch or function call. Therefore, these programs seem to be trivial. Yet, they are not. Despite of never making use of any sophisticated feature, they achieve full functional adequacy in more than twenty randomly created scenarios, usually over many generations in the EA. Functional adequacy here involves both, proper protection of the critical section and a fair resource utilization - the value $f_{u s e}=0.1529$ for Listing 8.1 is indeed very good when compared to the examples in Table 8.1 on page 124 .

Given the apparent simplicity of the programs evolved in the fully connected topology $f c$, one would assume them to be overfitted. To a certain degree, this may be true. They have, however, not learned a specific type of scenarios. When taking a look at Equation 6.1 on page 99, it becomes clear that they likely are solutions for an incredibly huge number of possible scenarios instead. We can rule out that the evolutionary process may have memorized a subset of these scenarios, because we changed them in every generation and drew new ones with uniform probability, i. e., in a way that gives no precedence to any configuration. Thus, the synthesized programs can indeed be considered as general, at least for the class of critical section scenarios which can be simulated in our DGPF 


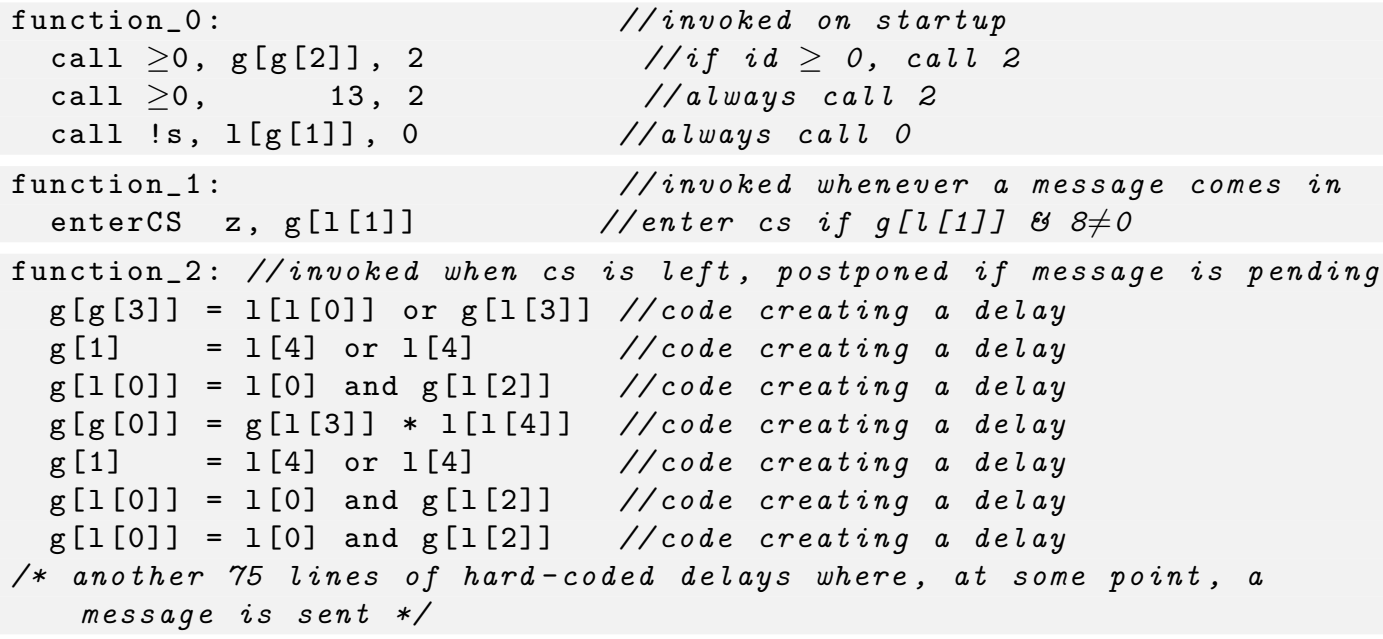

Listing 8.4: An LGP for case $r$ with $\left(f_{c o l}=1 \cdot 10^{-4}, f_{u s e}=0.876, f_{p s}=89\right)$.

The fact that the programs evolved for the other topologies did not achieve the same efficiency in terms of low $f_{c o l}$-values (see Section 8.5.5) furthermore indicates that the programs are not just dumb decision tables. Like the two examples provided in Listing 8.4 and Listing 8.5, Genetic Programming is able to come very close to functional adequacy but cannot fully reach it in the ring network and performs even worse in the network with disabled communication.

Compared to the three GP approaches with LGP phenome, RBGP and eRBGP have a striking disadvantage: They cannot evolve time wasting sequences of instructions. Whereas the previously listed programs primarily relied on such sequences triggered by incoming messages in order to make collisions improbable, the RBGP rule set given in Listing 8.5 is much more complex. In the critical section experiments, the two rule-based approaches created control flows consisting of long chains of rules activating each other. This basically achieves similar effects like the delaying computations in LGP and thus only rarely leads to the invocation of the enterCS action. The synthesis of such rule chains, however, seems to be more complicated. A clear indicator backing this assumption is that similar structures did not evolve in the GP approaches which have the LGP language as problem space. Instead, these preferred the simple instruction sequences not available in the rule-based representations. Like the Fraglets approach, neither RBGP nor eRBGP achieve full functional adequacy in any run although they get very close to it. 


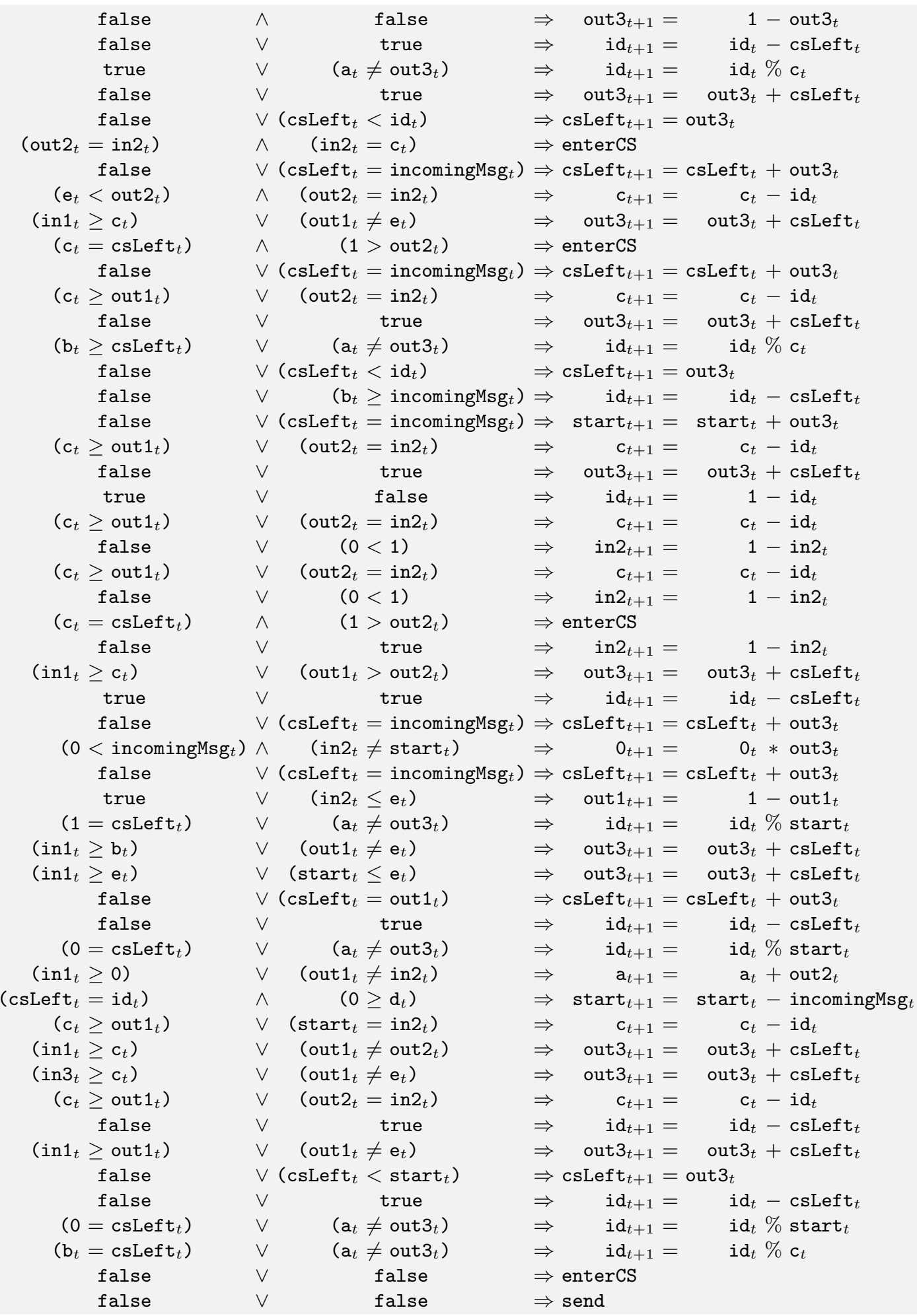

Listing 8.5: An almost adequate and fair RBGP program for networks with disabled communication ( case $n c, f_{\text {col }}=0.0489, f_{\text {use }}=0.7700, f_{p s}=309$ ). 


\subsection{Results and Evaluation}

\subsubsection{Overview}

A run of the critical section experiment can be considered as successful if it yielded at least one individual $x$ with the optimal value in the collision-minimizing objective function and which is at least marginally fair. Here we take all runs into consideration which have finished the full 700 generations.

\begin{tabular}{|c|c|c|c|c|c|c|c|c|c|c|c|}
\hline Case & $\# \boldsymbol{r}$ & $\# \boldsymbol{p}$ & $\boldsymbol{p} / r$ & $\# \boldsymbol{z}$ & $\# \boldsymbol{S}$ & $S / r$ & $\left(\widetilde{f_{\text {col }}}, f_{\text {use }}\right)_{z}$ & {$\left[\overline{f_{\text {col }}}\right]_{z}$} & {$\left[\overline{f_{\text {use }}}\right]_{z}$} & {$\left[\overline{f_{\text {use }}}\right]_{z}$} & $T$ \\
\hline fc & 38 & 38 & 1.00 & 38 & 37 & 0.97 & $(0.000,0.185)$ & $3 \cdot 10^{-10}$ & 0.185 & 0.364 & $286 \mathrm{~s}$ \\
\hline \multirow{2}{*}{ 岕 } & 63 & 0 & 0.00 & 63 & 0 & 0.00 & $\left(8 \cdot 10^{-6}, 0.906\right)$ & $1 \cdot 10^{-4}$ & 0.635 & 0.874 & $332 \mathrm{~s}$ \\
\hline & 21 & 0 & 0.00 & 21 & 0 & 0.00 & $\left(4 \cdot 10^{-4}, 0.825\right)$ & 0.001 & 0.642 & 0.789 & $251 \mathrm{~s}$ \\
\hline \multirow{2}{*}{$\begin{array}{l}D \vec{U} \\
\text { W } \\
0\end{array}$} & 46 & 46 & 1.00 & 46 & 45 & 0.98 & $(0.000,0.153)$ & $1 \cdot 10^{-7}$ & 0.153 & 0.367 & $299 \mathrm{~s}$ \\
\hline & 52 & 0 & 0.00 & 52 & 0 & 0.00 & $\left(6 \cdot 10^{-6}, 0.905\right)$ & $2 \cdot 10^{-4}$ & 0.818 & 0.884 & $337 \mathrm{~s}$ \\
\hline nc & 25 & 0 & 0.00 & 25 & 0 & 0.00 & $\left(5 \cdot 10^{-4}, 0.820\right)$ & 0.001 & 0.726 & 0.793 & $265 \mathrm{~s}$ \\
\hline \multirow{3}{*}{ 荷 } & 32 & 29 & 0.91 & 32 & 8 & 0.25 & $0,0.478)$ & $4 \cdot 10^{-4}$ & 478 & 0.811 & $75 \mathrm{~s}$ \\
\hline & 48 & 0 & 0.00 & 48 & 0 & 0.00 & $\left(1 \cdot 10^{-4}, 0.876\right)$ & 0.002 & 0.764 & 0.887 & $73 \mathrm{~s}$ \\
\hline & 24 & 0 & 0.00 & 24 & 0 & 0.00 & $(0.001,0.898)$ & 0.003 & 0.814 & 0.883 & $72 \mathrm{~s}$ \\
\hline \multirow{3}{*}{ 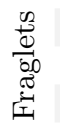 } & 27 & 14 & 0.52 & 27 & 0 & 0.00 & $(0.001,0.900)$ & 0.002 & 0.884 & 0.899 & $446 \mathrm{~s}$ \\
\hline & 75 & 0 & 0.00 & 75 & 0 & 0.00 & $(0.001,0.896)$ & 0.007 & 0.781 & 0.882 & $92 \mathrm{~s}$ \\
\hline & 44 & 0 & 0.00 & 44 & 0 & 0.00 & $(0.003,0.901)$ & 0.006 & 0.831 & 0.879 & $112 \mathrm{~s}$ \\
\hline & 23 & 6 & 0.26 & 23 & 0 & 0.00 & $\left(2 \cdot 10^{-4}, 0.871\right)$ & 0.004 & 0.742 & 0.870 & $424 \mathrm{~s}$ \\
\hline 崩 $r$ & 40 & 0 & 0.00 & 40 & 0 & 0.00 & $\left(5 \cdot 10^{-5}, 0.731\right)$ & 0.003 & 0.731 & 0.883 & $635 \mathrm{~s}$ \\
\hline nc & 21 & 0 & 0.00 & 21 & 0 & 0.00 & $(0.001,0.898)$ & 0.010 & 0.770 & 0.848 & $538 \mathrm{~s}$ \\
\hline \multirow{3}{*}{ 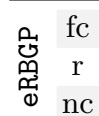 } & 37 & 5 & 0.14 & 37 & 0 & 0.00 & $(0.001,0.887)$ & 0.004 & 0.807 & 0.893 & $321 \mathrm{~s}$ \\
\hline & 27 & 0 & 0.00 & 27 & 0 & 0.00 & $\left(2 \cdot 10^{-4}, 0.891\right)$ & 0.004 & 0.545 & 0.864 & $444 \mathrm{~s}$ \\
\hline & 11 & 0 & 0.00 & 11 & 0 & 0.00 & $(0.007,0.905)$ & 0.015 & 0.784 & 0.865 & $280 \mathrm{~s}$ \\
\hline
\end{tabular}

Table 8.2: The Genetic Programming approaches in direct comparison.

Like in the election problem, we start the evaluation by giving an overview of the most important performance indicators. In Table 8.2, $\sharp r$ is again the number of total runs for each configuration. We further list the number $\sharp p$ (and proportion $p / r$ ) of runs which achieved to evolve individuals which could protect the critical section although not necessarily in a fair way, i. e., $f_{c o l}=0 \wedge f_{\text {use }}=*$. We find that only for the fully connected topology $(f c)$, such programs could evolve. The two Standard Genetic Programming approaches achieved this in all these experiments and LGP has a very high $p / r$ rate. Both Rule-based Genetic Programming methods fall behind the Fraglets approach in this measure.

In contrast to $\# p, \# z$ is the number of runs where programs evolved which utilized the critical section in at least a marginally fair way but not necessarily ensured that only one node accessed it at a time, i. e., $f_{\text {col }}=* \wedge f_{\text {use }} \leq 0.90625$. All approaches found such programs in all of the experimental runs since this just means that each node has to execute its entercs instruction at least once.

$\# s$ is the number and $s / r$ is the proportion of successful runs, i. e., runs where least one individual evolved for which the criteria for both, $p$ and $z$, hold. Such programs could only be found with SGP, eSGP, and LGP and only for the fully connected topology.

In this chapter, we will use the subscript $z$ to annotate values belonging to individuals which posses at least marginal fairness. $\left(\widetilde{f_{c o l}}, f_{u s e}\right)$, for instance, lists the two functional objective values for minimally-fair individuals with the best values of $f_{\text {col }}$ from all runs. 
We determined such tuples for every single run and listed their mean value in the collision objective functions $\left[\overline{f_{c o l}}\right]_{z}$ and the minimum $\left[\overline{f_{\text {use }}}\right]_{z}$ and mean $\left[\overline{f_{\text {use }}}\right]_{z}$ of the fairness-of-use criterion. Again, SGP, eSGP, and LGP perform best in these measures and also come close to finding programs which somehow protect their critical sections in the ring $(r)$ topology and in scenarios with disable communication $(n c)$. Additionally, the mean time per generation over all experiments of a configuration is given in column $T$.

\subsubsection{Pareto Frontiers}

In the election experiments, we always used two objective functions in combination, the functional criterion $f_{e l}$ evaluating the adequacy of the evolved programs and the non-functional criterion $f_{p s}$ rating their size. When investigating the outcomes of these experiments, we only focused on $f_{e l}$, since the values of $f_{p s}$ cannot be compared due to the different program representations utilized.

In the critical section experiment, there are two functional objectives $f_{c o l}$ and $f_{\text {use }}$. Therefore, no single comparison criterion exists and each run always leads to multiple functionally non-dominated solutions. ${ }^{2}$ We have depicted both objective values of the elements of the joint result sets of all runs of the $f c$-experiments in Figure 8.2 and those of the other experiments in Section B.3.1 in the appendix.

These figures indicate a great difference in the performance of the six Genetic Programming approaches. Whereas the SGP, eSGP, and LGP have extremely steep Pareto frontiers (Fig. 8.2.a to Fig. 8.2.c), the Pareto sets found with the other program representations resemble visibly convex curves. In other words, SGP and eSGP were able to find programs which achieve excellent values in both, $f_{c o l}$ and $f_{\text {use }}$, while the rule-based and the Fraglets approaches need to trade-off between the two objectives.

\footnotetext{
$\overline{{ }^{2} \text { See Section }} 2.2 .1$ on page 26 for a discussion of Pareto optimality and the domination relation.
} 


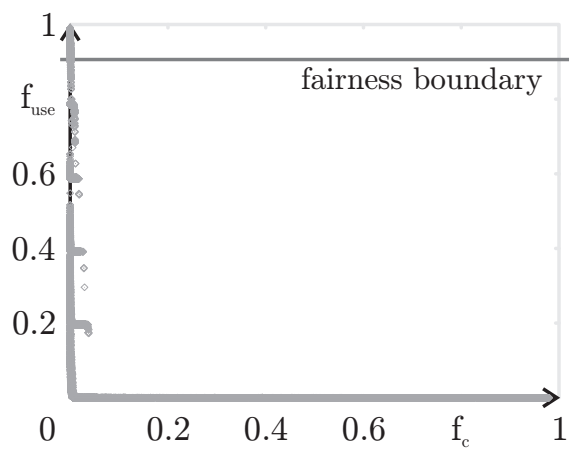

Fig. 8.2.a: SGP

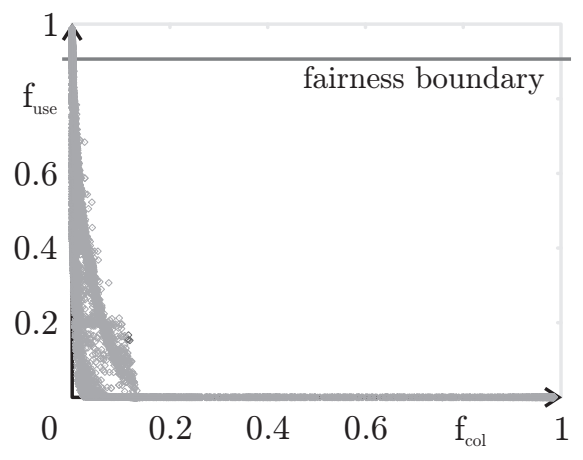

Fig. 8.2.c: LGP

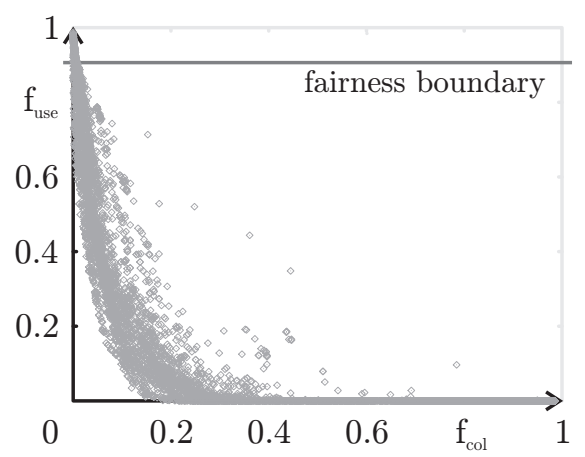

Fig. 8.2.e: RBGP

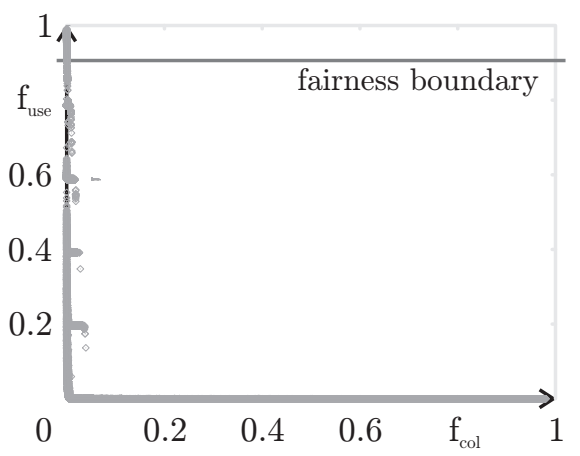

Fig. 8.2.b: eSGP

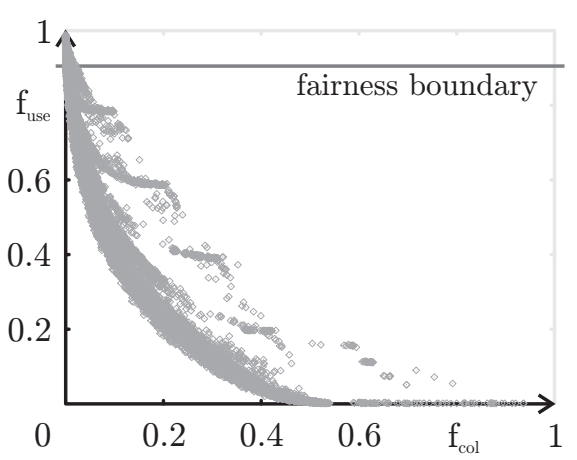

Fig. 8.2.d: Fraglets

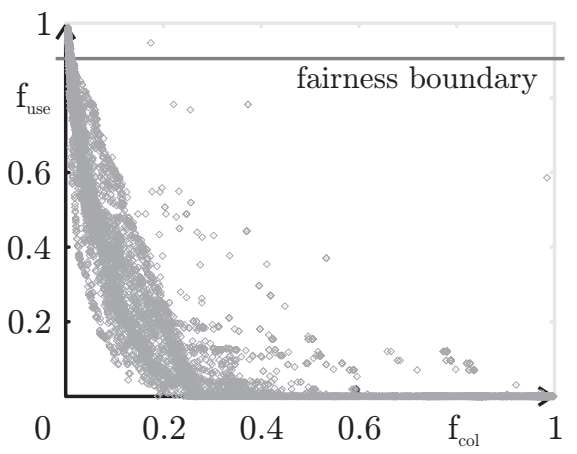

Fig. 8.2.f: eRBGP

Figure 8.2: The joint Pareto sets of all experiments of the GP approaches in the fully connected topology $f c$.

\subsubsection{Convergence}

Let us now analyze the convergence behavior of the Genetic Programming approaches. Like in the election experiments, we computed the mean, median (med), and the $5 \%$ and $95 \%$ quantiles (Q.05, Q.95) of the solution candidates with the best values of the (first) functional objective function over all experimental runs. We juxtapose the resulting curves for $f_{\text {col }}$ with the corresponding curves for $f_{\text {use }}$ of the same individuals.

Figure 8.3 and Figure 8.4 illustrate the convergence behaviors for the fully connected topology. It becomes clear that all GP approaches very quickly find programs which achieve full protection of the critical section, i.e., that the search converges quickly in the dimensions 


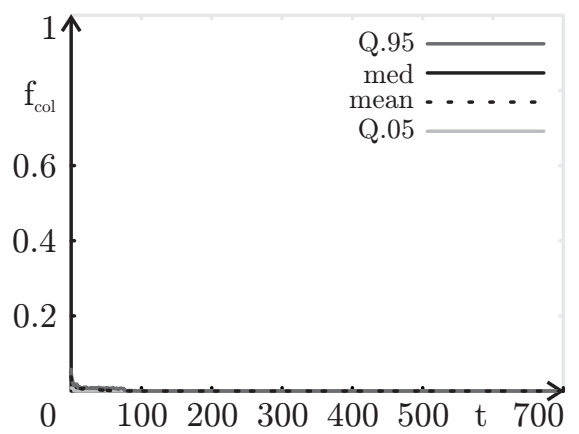

Fig. 8.3.a: Convergence: SGP for $f c$ and $f_{\text {col }}$.

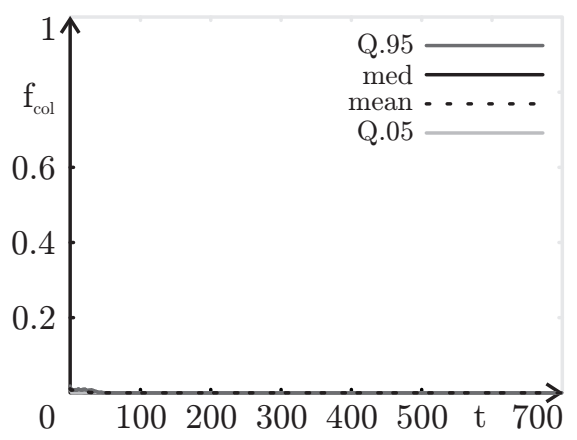

Fig. 8.3.c: Convergence: eSGP for $f c$ and $f_{\text {col }}$.

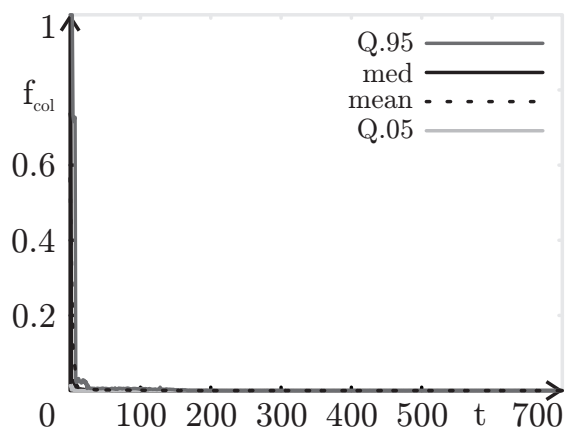

Fig. 8.3.e: Convergence: LGP for $f c$ and $f_{\text {col }}$.

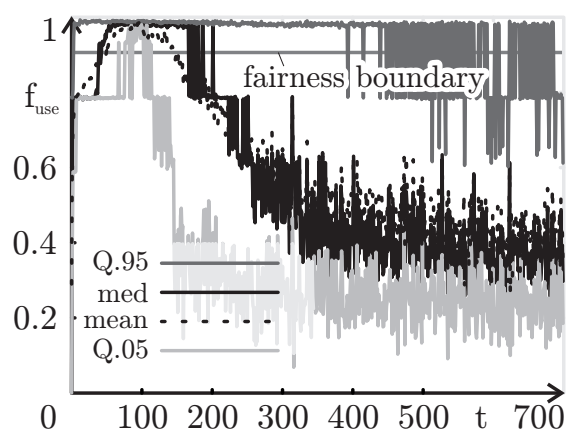

Fig. 8.3.b: Convergence: SGP for $f c$ and $f_{\text {use }}$.

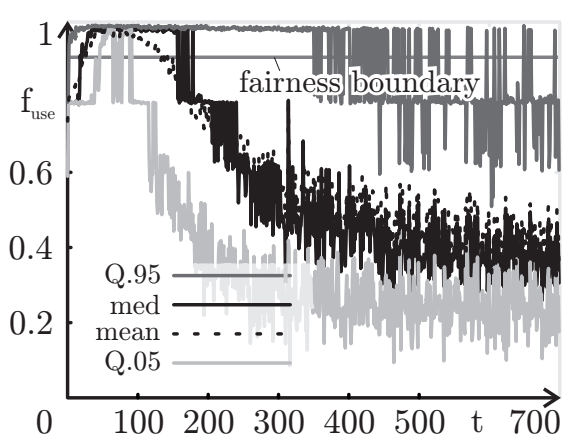

Fig. 8.3.d: Convergence: eSGP for $f c$ and $f_{\text {use. }}$

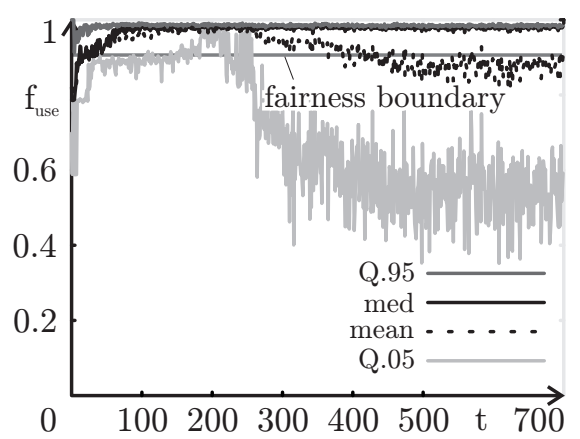

Fig. 8.3.f: Convergence: LGP for $f c$ and $f_{\text {use }}$.

Figure 8.3: Convergence diagrams (I) for the critical section problem case $f c$.

$f_{\text {col }}$. In SGP, eSGP, and LGP, the evolution continues in dimension $f_{u s e}$ for a long period. The other three approaches, however, stagnate well above the fairness boundary without any progress after the first hundred generations. This stagnation can be observed for all six GP methods in the cases $r$ ) and $n c$ ) (see Section B.3.2 in the appendix). 


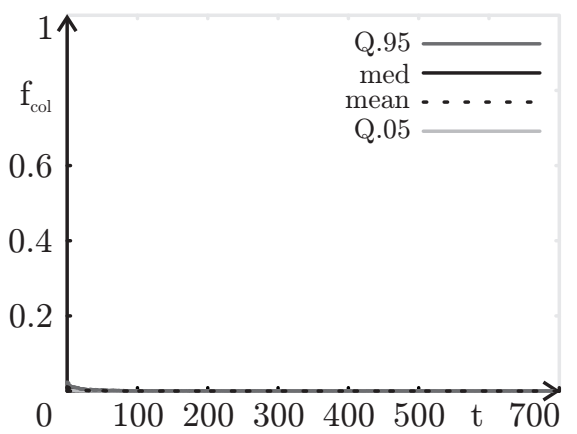

Fig. 8.4.a: Convergence: Fraglets for $f c$ and $f_{c o l}$.

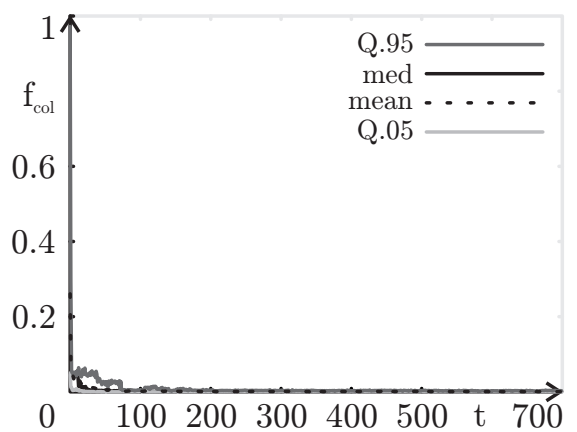

Fig. 8.4.c: Convergence: RBGP for $f c$ and $f_{\text {col }}$.

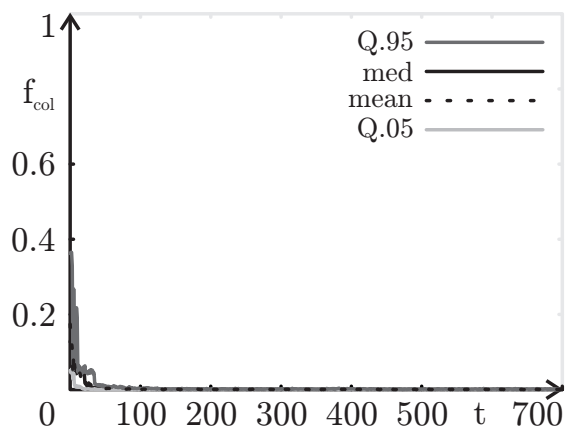

Fig. 8.4.e: Convergence: eRBGP for $f c$ and $f_{\text {col }}$.

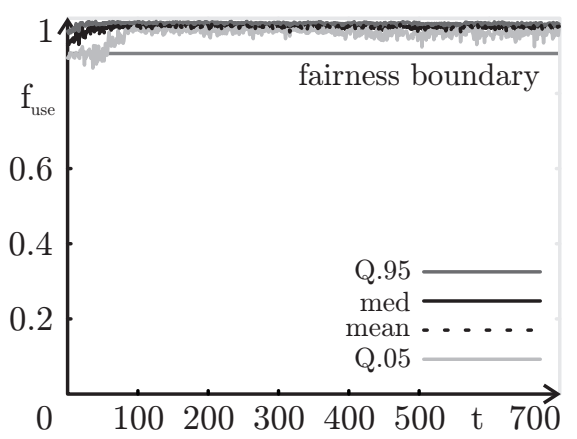

Fig. 8.4.b: Convergence: Fraglets for $f c$ and $f_{\text {use }}$.

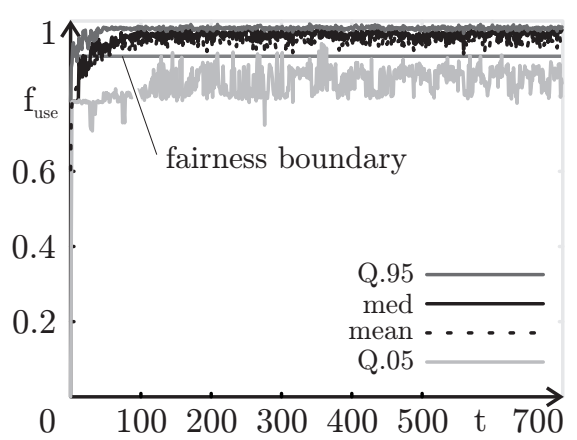

Fig. 8.4.d: Convergence: RBGP for $f c$ and $f_{\text {use. }}$

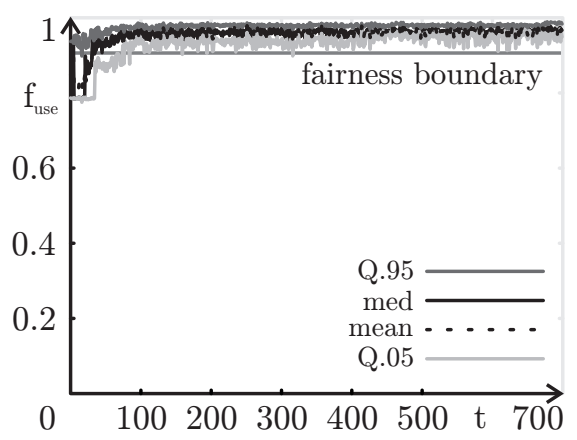

Fig. 8.4.f: Convergence: eRBGP for $f c$ and $f_{u s e}$.

Figure 8.4: Convergence diagrams (II) for the critical section problem case $f c$.

\subsubsection{Comparison of the GP Approaches}

The previous considerations already give strong indications that, in the critical section experiments, the Standard Genetic Programming and linear Genetic Programming approaches were much more successful than the rule-based ones or the Fraglets method. In order to substantiate this assumption, we decided to statistically evaluate two measurements:

1. $\widetilde{f_{c o l}}$, the best value of $f_{\text {col }}$ reached in a run (or the configuration, as in Table 8.2), and

2. $\left[\widetilde{f_{c o l}}\right]_{z}$, the best value of $f_{\text {col }}$ reached by an individual with at least marginal fairness in a run (or the configuration, as in Table 8.2). 


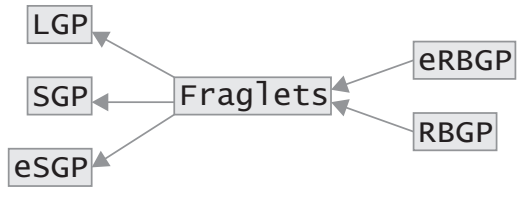

Fig. 8.5.a: According to $\widetilde{f_{c o l}}$ in $f c$.

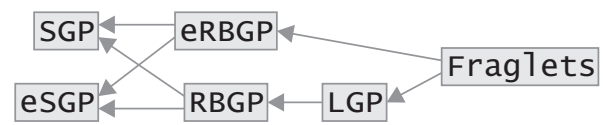

Fig. 8.5.c: According to $\widetilde{f_{\text {col }}}$ in $r$.

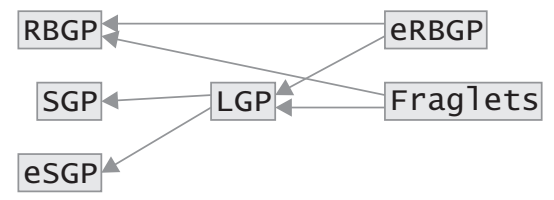

Fig. 8.5.e: According to $\widetilde{f_{c o l}}$ in $n c$.

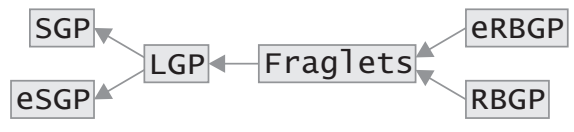

Fig. 8.5.b: According to $\left[\widetilde{f_{c o l}}\right]_{z}$ in $f c$.

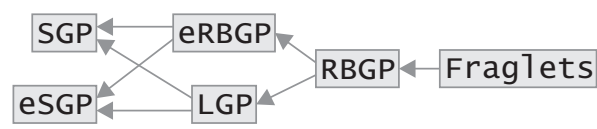

Fig. 8.5.d: According to $\left[\widetilde{f_{c o l}}\right]_{z}$ in $r$.

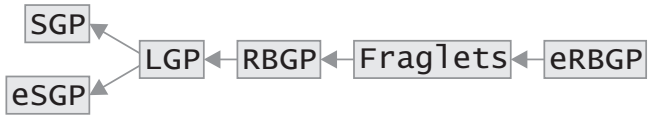

Fig. 8.5.f: According to $\left[\widetilde{f_{c o l}}\right]_{z}$ in $n c$.

Figure 8.5: Partial orders of the GP approaches according to their performance in the critical section experiments.

Similarly to our evaluation in the election experiment, we computed the partial orders of the program representations according to these performance measures. We again used a two-tailed Mann-Whitney U Test [192] with a significance level of $\alpha=2 \%$. Figure 8.5 shows that indeed, SGP and eSGP performed much better than all other GP methods in this experiment. The order of the remaining approaches varies and the only constant is that the Fraglets-evolving GA could never surpass the efficiency of LGP. The table-based representations of Figure 8.5 can be found in Section B.3.3 in the appendix.

\subsubsection{Comparison of the Scenarios}

In the same way in which the Genetic Programming approaches were compared with each other in terms of their performance in the different network configurations, we can compare the network configurations according to how well the GP methods performed in them. The corresponding partial orders in terms of $\widetilde{f_{c o l}}$ and $\left[\widetilde{f_{c o l}}\right]_{z}$, again obtained with two-tailed Mann-Whitney U Tests with a 2\% significance level, are illustrated in Figure 8.6.

In six out of twelve experiments, Genetic Programming yielded more efficient algorithms for the fully connected topology $f c$ than in the ring topology $r$ for which, in turn, better programs evolved than for the scenario without communication $n c$ (see Fig. 8.6.a). In three other experiments, $f c$ is still best but distinguishing between $n c$ and $r$ is not possible at $\alpha=0.02$ (Fig. 8.6.b). Only in the RBGP experiments, better algorithms evolved for the ring topology than for the fully connected one (Fig. 8.6.d). This never happened in the configuration $n c$ with disabled communication.

These results proof that Genetic Programming was able to utilize communication efficiently. They also show that it is harder to synthesize algorithms for the ring topology than for the fully connected case. As expected, since it is not possible to guarantee mutual exclusion in an asynchronous system without any means of communication, GP performed worst in the $n c$ setting. The table-based representations of Figure 8.6 can be found in Section B.3.3 on page 185 . 


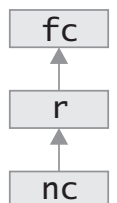

Fig. 8.6.a: According to $\widetilde{f_{\text {col }}}$ (SGP, eSGP, eRBGP) and $\left[\widetilde{f_{\text {col }}}\right]_{z}$ (SGP, eSGP, LGP).

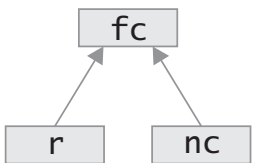

Fig. 8.6.b: According to $\widetilde{f_{\text {col }}}$ (LGP, Fraglets) and $\left[\widetilde{f_{\text {col }}}\right]_{z}$ (Fraglets).

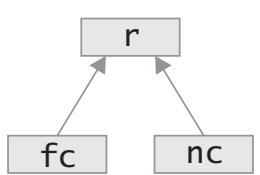

Fig. 8.6.d: According to $\left[\widetilde{f_{c o l}}\right]_{z}$ in RBGP.

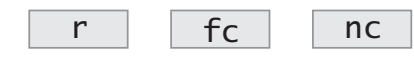

Fig. 8.6.c: According to $\widetilde{f_{\text {col }}}$ in RBGP.

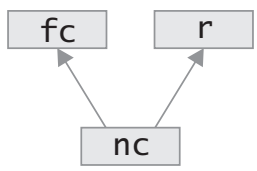

Fig. 8.6.e: According to $\left[\widetilde{f_{\text {col }}}\right]_{z}$ in eRBGP.

Figure 8.6: Partial orders of the critical section scenarios according to the performance of the Genetic Programming approaches in them.

\subsubsection{Runtime}

The complete series of all experiments consumed 1459 processor days on the same hardware and software configurations as specified in Section 7.5.4. Again, the experiments with the Fraglets had the highest runtime requirements due to the complexity of simulating the Fraglet store. The programs natively evolved in the LGP representation were once more executed the fasted.

Nevertheless, even the $485 \mathrm{~s}$ per generation used by the Fraglets approach amount to a mere $24 \mathrm{~ms}$ for a complete network simulation ${ }^{3}$ (4ms for linear Genetic Programming) which is still very fast. Also, in a practical application of GP, the computational load could have been distributed over multiple computers which would decrease the total runtime significantly.

\subsection{Summary}

The outcomes of the critical section experiments were surprising at the first glance, especially in the light of the results of the election experiments. Two interesting observations could be made.

SGP and eSGP have effectively changed places with eRBGP and RBGP and now perform significantly better. The cause for these overtakes is the ability of SGP, eSGP, and LGP to create long sequences of instructions for slowing down the execution. Twelve out of the 16 instructions of the LGP language can be randomly inserted into the code in order to do this. Therefore, the Genetic Programming process only has to adjust their number to the right amount and to incorporate communication accordingly in order to achieve good fitness. RBGP, eRBGP, and to a lesser amount, the Fraglets method, cannot do this. Instead, they have to evolve complex interactions between different rules or Fraglets in order to achieve the same delaying. Hence their worse results.

The second interesting point is that only in the fully connected topology $f c$, where each node can directly communicate with every other node, programs which completely protect

\footnotetext{
${ }^{3}$ Since we used steady-state populations, 1024 individuals have to be evaluated each generation instead of the actual population size $p s=512$.
} 
the critical section could evolve. The reason for this is that the communication medium is used as signaling device. Incoming messages usually trigger delaying computations so that the critical section is only entered if no messages were received for a certain number of time steps. A topology where broadcasts reach all nodes is predestined for such a behavior, whereas a ring $(r)$ requires forwarding of the messages and the evolution of longer waiting periods. If no communication is available at all $(n c)$, the waiting periods have to be further extended and only randomization via indeterministic parallelism and seeding with the node IDs can reduce the chance of collisions. 


\section{Evolving Algorithms for the Distributed Greatest Common Divisor Problem}

In [293], we have used the evolution of algorithms which determine the greatest common divisor (GCD) of two natural numbers as an example problem for the Genetic Programming of mathematical algorithms. Here, we will extend this approach to the cooperative computation of the GCD of a set of numbers distributed in a network.

Definition 9.1 (GCD). For two integer numbers $a, b \in \mathbb{N}$, the greatest common divisor (GCD) is the largest number $c \in \mathbb{N}$ that divides both, $a$ and $b$.

The GCD of two numbers can be computed with the Euclidian algorithm [216]. Mattern used a distributed version of this procedure (here specified as Algorithm 9.1) as example in his foundational book [193]. Each node $\mathbf{n}$ of the network $\mathbf{N}$ starts with an own number

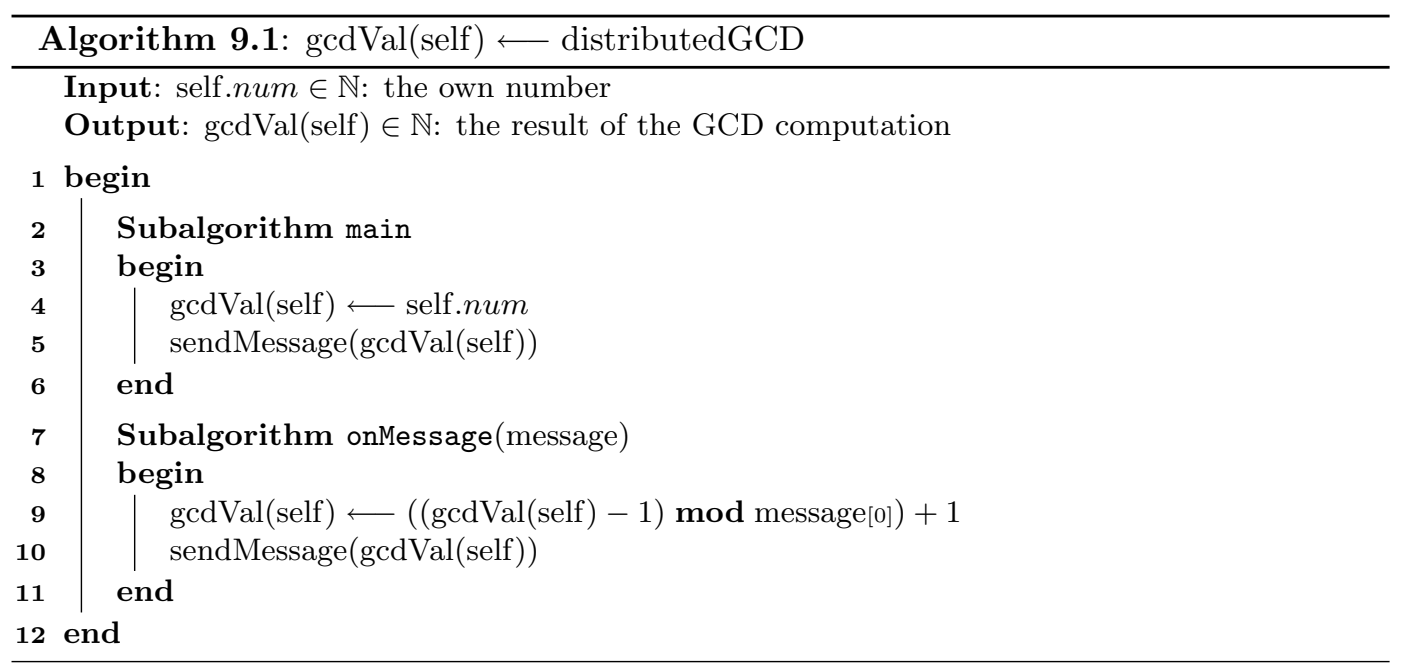

n.num which will be its first guess about what the GCD of all numbers distributed over the network is $(\operatorname{gcdVal}(\mathbf{n})=\mathbf{n}$. num $)$. Initially, it sends this number to its neighbors. Whenever a node receives a message "message" containing the number of one of its neighbors, it will update its own estimation according to line 9. Step by step, the $\operatorname{gcdVal}(\mathbf{n})$ values of all nodes $\mathbf{n} \in \mathbf{N}$ will converge to the real GCD. Notice that Algorithm 9.1 is a perfect example of an anytime algorithm as defined in Section 4.8.2 on page 70 and in [274]. 


\subsection{Specification of the Global Behavior}

The initial situation of a network $\mathbf{N}$ be that each of its nodes $\mathbf{n} \in \mathbf{N}$ knows exactly one number n.num $\in \mathbb{N}$. We wish to evolve programs which, if executed on these nodes, compute the greatest common divisor $\operatorname{corr}=\operatorname{gcd}_{\forall \mathbf{n} \in \mathbf{N}} \mathbf{n}$. num of all the numbers distributed over the network. This number should be stored in a special variable or symbol $\operatorname{gcd} \operatorname{Val}(\mathbf{n})$ on each of the nodes $\mathbf{n}$.

\subsection{Objective Functions}

Our design process for distributed algorithms prescribes a manual transformation of a network behavior description into objective functions. These functions are to rate how close the actual behavior exhibited by a network in a simulation comes to the targeted one. Generally, we can derive multiple objective functions for the same behavior specification. In the critical section experiment, for instance, we could have replaced $f_{\text {use }}$ with a function which returns the arithmetic mean of the number $\operatorname{csTimes}(\mathbf{n})$ of times the nodes $\mathbf{n} \in \mathbf{N}$ have executed their critical sections.

In the distributed greatest common divisor experiments, we want to test how different functional criteria for the same behavior influence the results of the evolution. We therefore define two functional objective functions gcd.1 and gcd.2:

gcd.1 (specified as Algorithm 9.2) rewards programs which decide for return values "gcdVal" divisible by the correct result corr. This reward is the higher, the closer the algorithms get to the real result. All values greater or equal to the minimum initial number $\min _{\forall \mathbf{n} \in \mathbf{N}} \mathbf{n}$. num receive the same default fitness in order to prevent the evolution of algorithms which simply converge to this number.

gcd.2 (specified as Algorithm 9.3) directly provokes the all-or-nothing problem in Genetic Programming by only giving rewards if a node has found the correct GCD. With this objective function, we provide the optimization process only with very little information. It thus should not be able to exceed the performance of a random walk by too much.

Programs which simply assume that the number initially stored on the nodes on which they are executed can achieve a functional fitness of 0.5 in $f_{g c d .1}$ but only score 1.0 in $f_{g c d .2}$. In other words, $f_{g c d .1}$ rewards programs which have at least found the memory cell containing the input data and copy its value to the correct output cell, whereas such first steps into the right direction play no role in $f_{g c d .2}$.

$f_{g c d .2}$ provides not much more information than a Boolean decision criterion about the correctness of a program. According to our discussion in Section 4.4 .3 on page 56, it can, however, already take on numNodes $(\mathbf{N}) * t c+1$ different values where $t c$ is the number of training cases applied during its evaluation. Still, we would expect the objective function $f_{p s}$ to be the driving force of the evolution and that many very small programs should occur. Since all programs solving the GCD problem adequately have a certain minimum size, we set a lowest boundary of 25 for $f_{p s}$ under which it cannot drop. ${ }^{1}$

\footnotetext{
${ }^{1}$ This boundary was determined in the first experimental series $\left(f_{g c d .1}, p s=512\right)$ where it was not
} yet applied. 

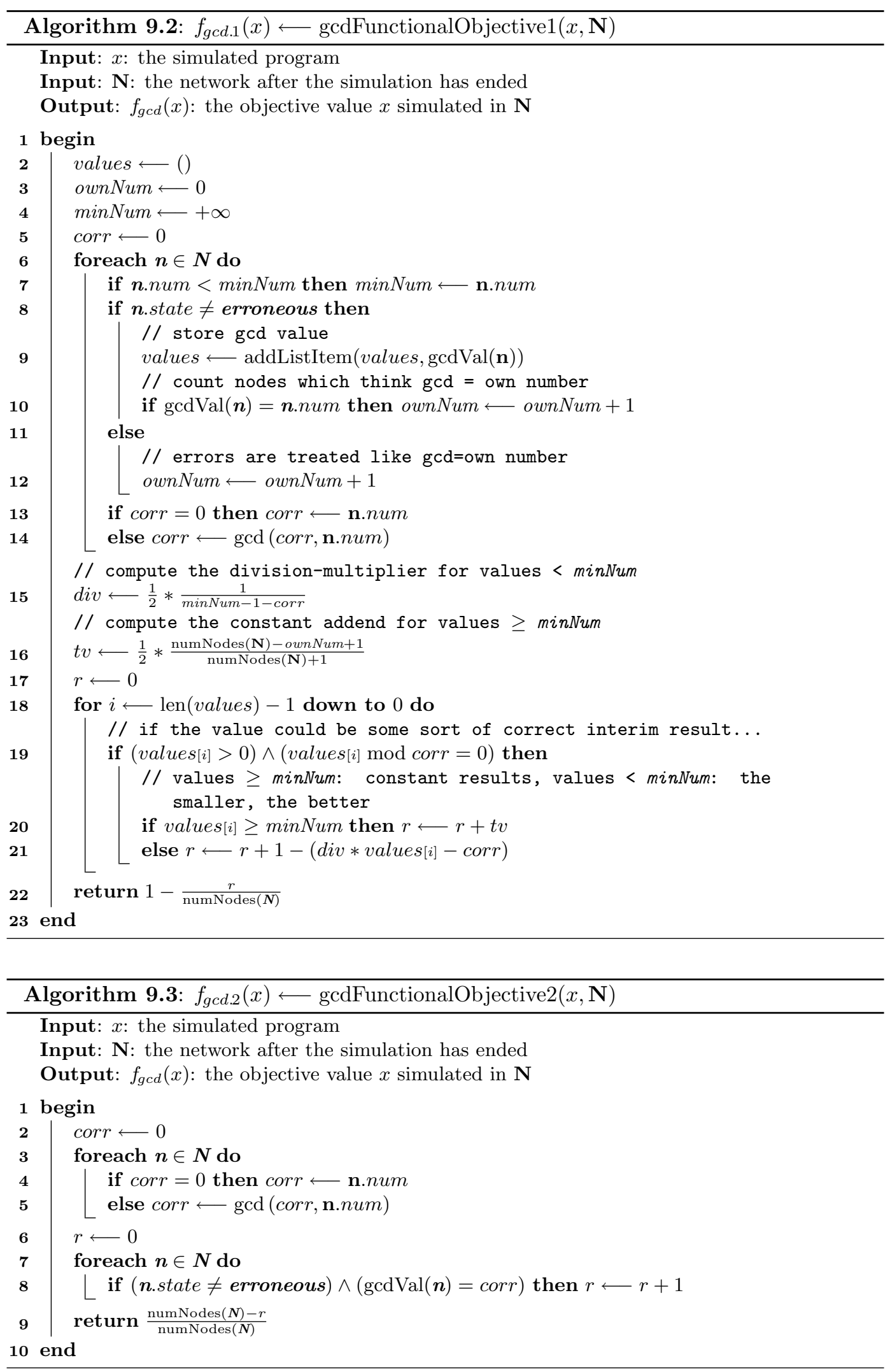


\subsection{Experimental Settings}

We applied all Genetic Programming approaches introduced in Chapter 5 except the Fraglets $^{2}$ method to the distributed GCD problem. We used the same settings as for the election experiment (see Section A.1 on page 167) except that we

1. evolved the algorithms in a rectangular topology where each node had up to four neighbors to communicate with and

2. provided four variables to the RBGP and eRBGP approaches and four global and local memory cells to the SGP, eSGP, and LGP Genetic Programming methods.

We repeated the experiments for the different functional optimization criteria gcd.1 and gcd.2, always using a population size of $p s=512$ individuals. In order to test the influence of increasing the population, we also performed one set of experiments with $g c d .1$ and $p s=$ 1536. The numbers we distributed over the simulated network and for which the GCD was to be computed have the following properties:

1. They are unique, meaning that no two nodes in a network have the same starting number.

2. The final result is not amongst them, i. e., no node starts with the correct GCD.

3. For each scenario, initially a prime number $p \in 103 . .1009$ is picked. The starting number of each single node is then computed by multiplying $p$ with a value uniformly distributed in $10 . .509$.

This way, the numbers have a non-trivial GCD and cannot be guessed by GP. The complete list of settings of these experiments is provided in Section C.1 on page 189.

\subsection{Evolved Programs}

eRBGP was the GP approach that solved the distributed GCD problem most often. One of the adequate programs it was able to synthesize is shown in Listing 9.1. In Mattern's method, the values of $\operatorname{gcdVal}(\mathbf{n})$ were prevented from becoming zero by adding one to results of the modulo divisions used to compute the GCD step by step (line 9 in Algorithm 9.1 on page 139). Like the program in Listing 9.1, most of the algorithms evolved with eRBGP follow a different approach given as Algorithm 9.4. They store the modulo of the current estimate of the GCD and the received value in a temporary variable. This variable is then written back to the estimate if it is not zero.

In Section 4.8.1, we argued that programs evolved with Genetic Programming should always be checked by a human being. We also pointed out that this may sometimes be complicated because of the representation in which the algorithms evolve. The reader will probably have encountered this issue when taking a look at the programs given in SGP or

\footnotetext{
${ }^{2}$ We did not include the Fraglets approach in our experiments because this would have required too much of a deviation from the instruction set given in Table 5.4 on page 80 and used until now.
}

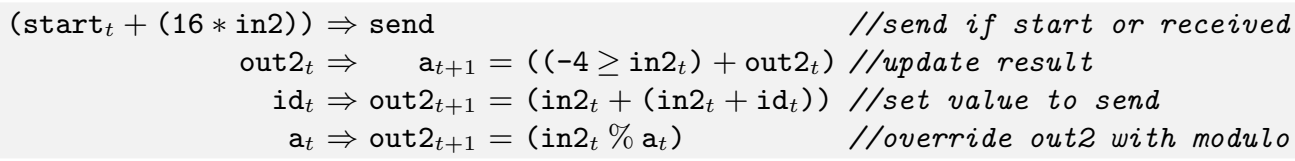

Listing 9.1: One evolved eRBGP program for problem $F_{g c d .1}$ in the 512 individual population $\left(f_{g c d .1}=0, f_{p s}=30\right)$. 


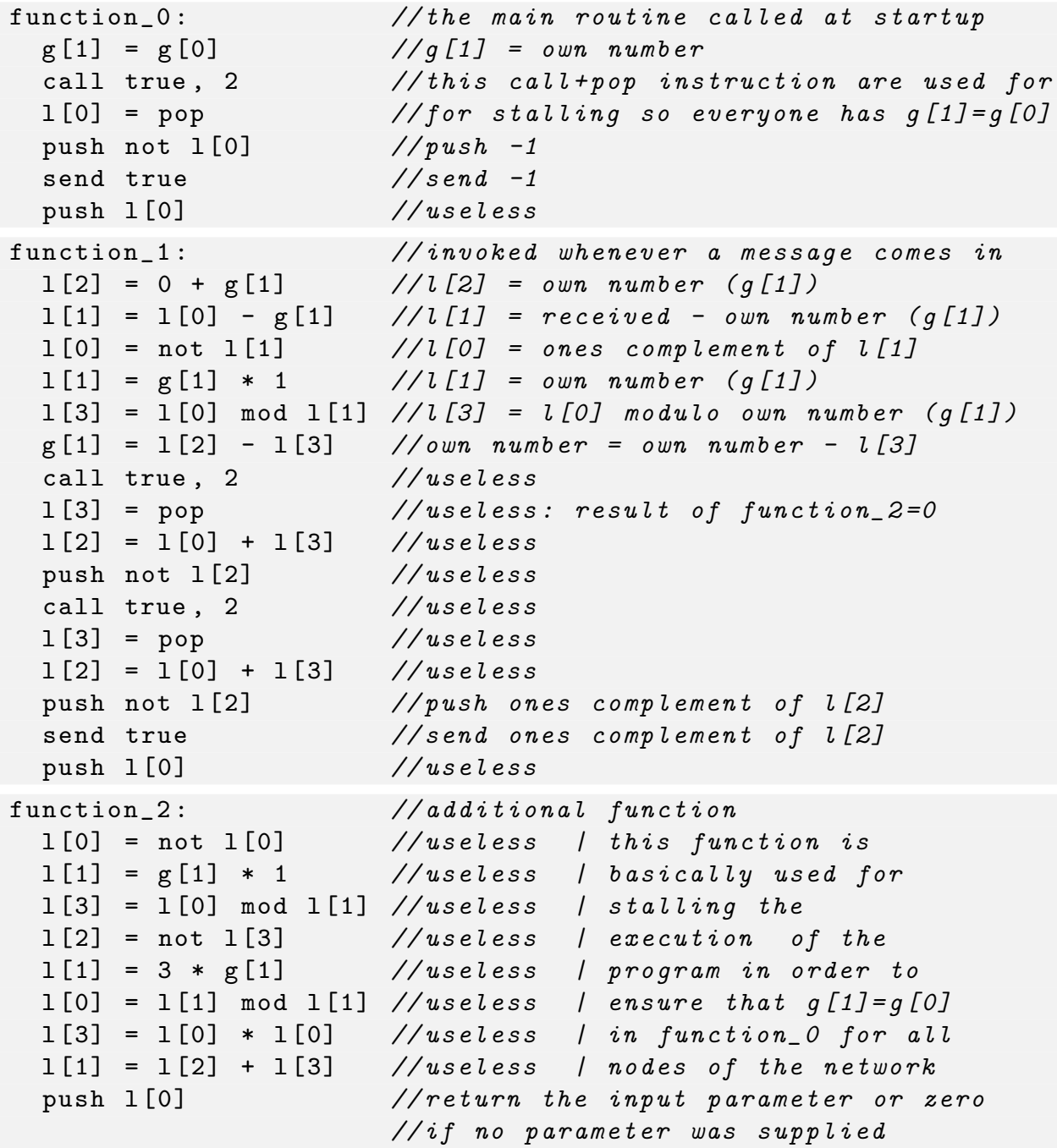

Listing 9.3: The LGP phenotype of the eSGP program given in Listing 9.2.

calculations. Finding out which of the instructions in Listing 9.3 are useful and which are not is actually very complicated.

function_2, for instance, looks like it would play an important role in the GCD computation since it is invoked from multiple locations, receives values extracted from the received messages as parameters, and contains modulo division operations. Instead, it is just used to delay the execution in order to ensure that the $\mathrm{g}[1]=\mathrm{g}[0]$ in line 2 has taken place on all nodes before the real distributed computation begins. ${ }^{3}$ This computation is performed by lines 9 to 14, each of which is important. Furthermore, the GCD estimates are exchanged between the nodes in their ones complements for no particular reason.

Manually analyzing such an evolved program can turn out to be a very cumbersome and thus, a very error-prone process. Therefore, using automated verification tools as suggested in Section 4.8.2 may become unavoidable in practical applications.

${ }^{3}$ This issue makes the program fragile in environments with extremely fast message transmission. The eRBGP program from Listing 9.1 does not have this problem. 


\subsection{Results and Evaluation}

\subsubsection{Overview}

In Table 9.1, we have noted the same measurements as provided for the election experiment one page 113. The number $\# s$ and proportion $s / r$ of successful runs in relation with the number $\# r$ of total runs is very low in all experiments. In case of the population size $p s=512$ and $f_{g c d .1}$, only eSGP and eRBGP have a non-zero success ratio and only eRBGP finds a valid program more than once. After increasing the population size to 1536, SGP becomes able to create solutions too whereas the other three approaches still cannot solve the GCD problem. For the second way of expressing the functional optimization criterion $\left(f_{g c d .2}\right)$, only eRBGP - seemingly accidentally - finds one single solution.

\begin{tabular}{|c|c|c|c|c|c|c|c|c|c|c|c|c|c|c|c|}
\hline & $\mathrm{Ca}$ & $p s$ & $\# \boldsymbol{r}$ & $\# s$ & $s$ & $s t$ & $\overline{s t}$ & $\widehat{s t}$ & $\widetilde{f_{g c d}}$ & $\overline{f_{g c d}}$ & $\widetilde{f_{p s}}$ & $\overline{f_{p s}}$ & $T_{\bar{s}}$ & $T_{s}$ & $T$ \\
\hline \multirow{3}{*}{$\begin{array}{l}\text { 口ि } \\
\text { च }\end{array}$} & $F_{g c d .1}$ & 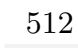 & 30 & 0 & 0.00 & 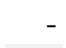 & - & & 96 & 0.500 & 25.0 & 25 . & $167 \mathrm{~s}$ & 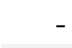 & $167 \mathrm{~s}$ \\
\hline & $F_{g c d .1}$ & 536 & 87 & 2 & 02 & 72 & 424 & 576 & 000 & 0.472 & 25.0 & 26.8 & $492 \mathrm{~s}$ & $4 \mathrm{~s}$ & $498 \mathrm{~s}$ \\
\hline & $F_{g c d .2}$ & 512 & 153 & 0 & 00 & & 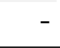 & & 630 & 0.997 & 25.0 & 25.7 & $17.8 \mathrm{~s}$ & 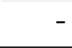 & $17.8 \mathrm{~s}$ \\
\hline \multirow{3}{*}{ 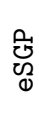 } & $F_{g c d .1}$ & & 53 & 1 & & 2 & 12 & 91 & & 83 & 5.0 & 25.5 & $161 \mathrm{~s}$ & $26 \mathrm{~s}$ & $160 \mathrm{~s}$ \\
\hline & $F_{g c d .1}$ & 36 & 56 & 6 & 0.11 & 5 & 699 & 948 & 000 & 0.434 & 25.0 & 27.3 & $512 \mathrm{~s}$ & $5 \mathrm{~s}$ & $515 \mathrm{~s}$ \\
\hline & $F_{g c d .2}$ & 512 & 52 & 0 & 0.00 & & & & 00 & 1.000 & 25.0 & 25.0 & $17.4 \mathrm{~s}$ & & $17.4 \mathrm{~s}$ \\
\hline & $F_{g c d .1}$ & & & 0 & & & - & & & 0.499 & 0 & 10. & 36 & & $36 \mathrm{~s}$ \\
\hline & $F_{g c d .1}$ & 2 & 6 & 0 & 0.0 & & - & - & 96 & 0.492 & 25.0 & 25.0 & 111 & & $111 \mathrm{~s}$ \\
\hline & $F_{g c d .2}$ & 5 & 61 & 0 & 0.00 & - & - & & 86 & 1.000 & 25.0 & 25.4 & 1 & & $1 \mathrm{~s}$ \\
\hline \multirow{3}{*}{$\begin{array}{l}\text { 号 } \\
\text { 品 }\end{array}$} & & & & 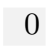 & & - & - & & & 0. & & 5.2 & & & $129 \mathrm{~s}$ \\
\hline & $F_{g c d .1}$ & 1 & 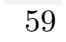 & 0 & & - & - & - & & 0.500 & 25.0 & 26.7 & $304 \mathrm{~s}$ & & $304 \mathrm{~s}$ \\
\hline & $F_{g c d .2}$ & 5 & 149 & 0 & 0.00 & - & - & & 90 & 1.000 & 25.0 & 29.8 & $37 \mathrm{~s}$ & & $37 \mathrm{~s}$ \\
\hline \multirow{3}{*}{ 密 } & & & & 5 & & 230 & 406 & & & 0.4 & 25.0 & 29. & 45 & $226 \mathrm{~s}$ & $57 \mathrm{~s}$ \\
\hline & $F_{g c d .1}$ & 1536 & 77 & 10 & & 81 & 194 & & & & 25.0 & 39.2 & $91 \mathrm{~s}$ & $710 \mathrm{~s}$ & $178 \mathrm{~s}$ \\
\hline & $F_{g c d .2}$ & 512 & 147 & 1 & 0.01 & 880 & 880 & 880 & 0.000 & 0.985 & 25.0 & 28.5 & $19 \mathrm{~s}$ & $53 \mathrm{~s}$ & $20 \mathrm{~s}$ \\
\hline
\end{tabular}

Table 9.1: The Genetic Programming approaches in direct comparison.

In terms of the minimum $(\breve{s t})$, mean $(\overline{s t})$, and maximum generation $(\widehat{s t})$ in which the first adequate program was found in the successful runs, eRBGP is again best. The same goes for the minimum and mean functional objective values $\left(\overline{f_{g c d}}, \overline{f_{g c d}}\right)$ of the individuals with the best functional objective values. Generally, these values are much better for case 1 than for case 2 of the GCD problem.

The corresponding values of the non-functional criteria $\left(\widetilde{f_{p s}}, \overline{f_{p s}}\right)$ are again listed for the sake of completeness. The last three columns of Table 9.1 contain the average time per generation of runs which did not succeed $\left(T_{\bar{s}}\right)$, of those which did $\left(T_{s}\right)$, and the mean runtime of all experiments $(T)$ for each configuration.

\subsubsection{Convergence}

Like in the previous two experiments, we investigated the convergence behavior of the Genetic Programming approaches with diagrams showing the mean, median (med), and the $5 \%$ and $95 \%$ quantile (Q.05, Q.95) of the functional criterion for each generation measured over all experimental runs. In Figure 9.1, we illustrate the progress of $f_{g c d .1}$ for population size $p s=1536$. Similar diagrams for $p s=512$ and $f_{g c d .2}$ can be found in Section C.3.1. 


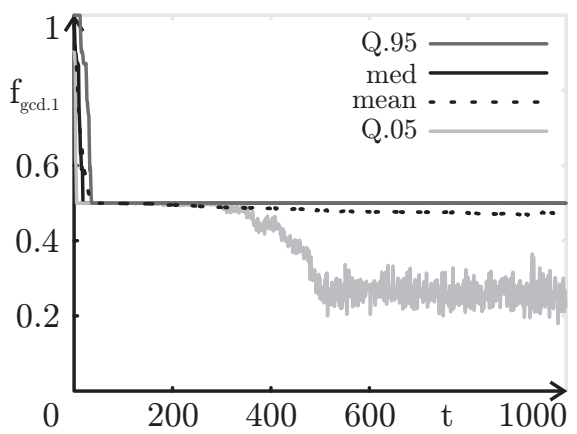

Fig. 9.1.a: Convergence: SGP, $f_{g c d .1}, p s=$ 1536 .

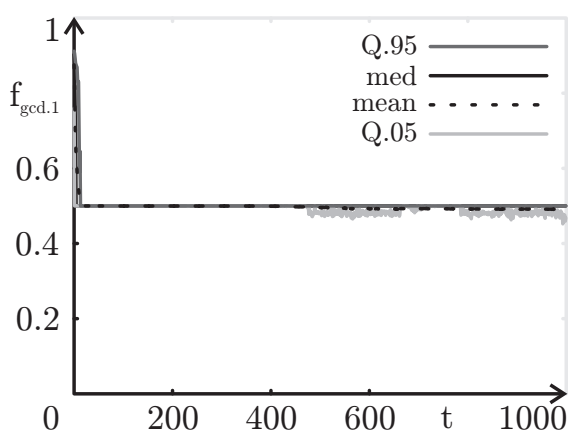

Fig. 9.1.c: Convergence: LGP, $f_{g c d .1}, p s=$ 1536 .

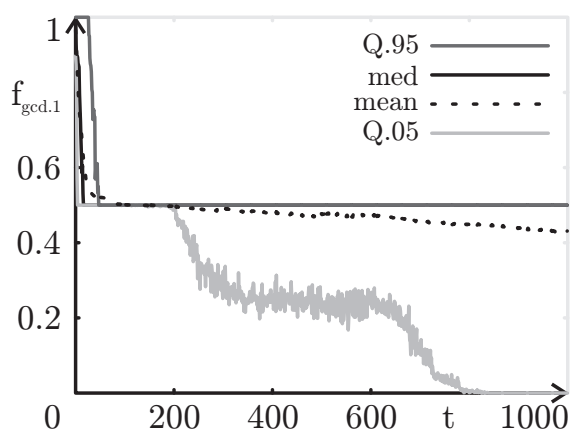

Fig. 9.1.b: Convergence: eSGP, $f_{g c d .1}$, ps $=1536$.

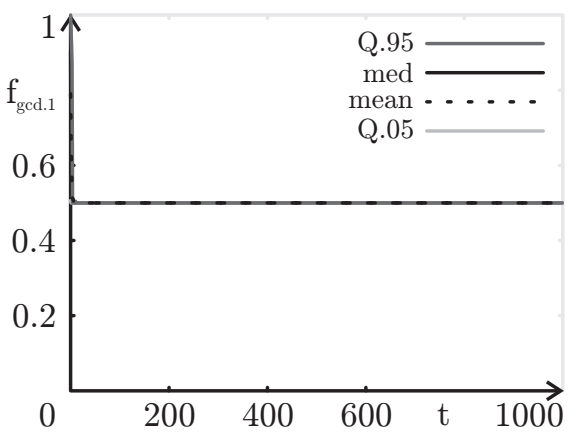

Fig. 9.1.d: Convergence: RBGP, $f_{g c d .1}$, ps $=1536$.

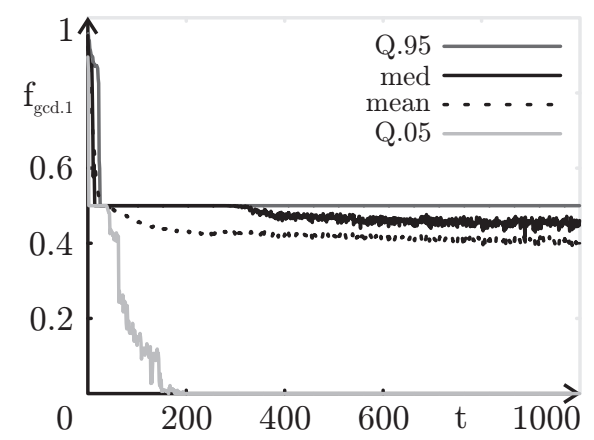

Fig. 9.1.e: Convergence: eRBGP, $f_{g c d .1}$, ps $=1536$.

Figure 9.1: Convergence diagrams for the GCD problem $f_{g c d .1}$ and $p s=1536$.

Because of the low success rates in these experiments, we cannot expect these performance metrics to take on small values. The only two approaches where at least Q.05 reaches the abscissa in Figure 9.1 are eRBGP and eSGP. In eRBGP it does so in about one fourth of the generations required in eSGP.

Remarkable in Figure 9.1 is that only eRBGP can achieve a median result better than 0.5. Even more remarkable is that it even achieves this in the setup with population size 512 (see Fig. C.1.e on page 192). In other words, eRBGP is the only approach of the five tested ones which finds programs which are better than guessing that the GCD is their initial input value in more than $50 \%$ of the runs. 


\subsubsection{Comparison of the GP Approaches}

Like in the election experiment, we have compared the success rates of the different Genetic Programming approaches with Fisher's exact test $[88,89]$ and the best values $\overline{f_{\text {gcd.1 }}}$ of the functional objective functions with the Mann-Whitney U Test [192]. For both tests, we again used the two-tailed variants with a significance level of $\alpha=0.02$. The resulting comparison tables are listed in Section C.3.2 in the appendix and the meaningful partial orders which they represent are illustrated in Figure 9.2. Because of the low success rates of the experiments, most trends turned out to be insignificant and only few, strong relations could be confirmed.

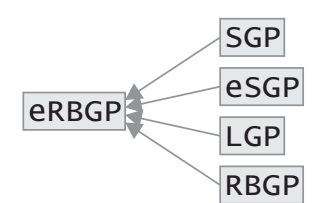

Fig. 9.2.a: According to $\overline{f_{g c d .1}}$ for $F_{g c d .1}$ and both, $p s=512$ and $p s=1536$.

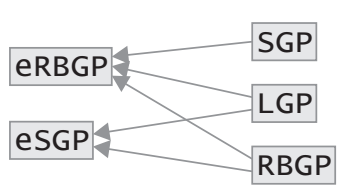

Fig. 9.2.b: According to $s / r$ for $F_{g c d .1}$ and ps $=1536$.

Figure 9.2: Partial orders of the GP approaches according to their performance in the GCD experiments.

\section{GCD Problem Case 1}

Regarding the achieved functional objective values, eRBGP dominates in both experiments. The success rates of the runs, however, could not be declared as different with an error probability of no more than $2 \%$ in the experiments with population size $p s=512$. In the experiments with the larger populations consisting of $p s=1536$ individuals, eRBGP beats all approaches except eSGP significantly in terms of $s / r$.

Table 9.1 indicates that the increase in the population size led to an increase of the success rates of the SGP, eSGP, and eRBGP approaches. We checked this again with statistical tests (see Table C.4 on page 194) but found that this assumption cannot be verified at the prescribed significance level. We also applied the Mann Whitney U test to the results in terms of $\overline{f_{g c d .1}}$ and found that the only GP approach where the bigger population led to significantly better results regarding the functional objective function was eRBGP. From these tests, we may draw two conclusions:

eRBGP is the only Genetic Programming approach which we can deem efficient in this domain. It can solve the distributed GCD problem, although with a low success rate. Putting more computational power behind eRBGP can improve its outcome - if not in terms of the success rate, then at least in how close the approach comes to a solution.

We should consider whether the solutions found by eRBGP may have merely been created by accident. Let us therefore assume that the optimization process backing GP was not able to utilize the information obtained by exploring the search space. We would then expect its behavior to equal a random sampling and its success rate to increase noticeable as the number of samples from the search space triples. This was not the case and instead, as previously mentioned, we could not even detect a significant difference. Therefore, we can assume that eRBGP has a performance different from a random sampling process in the distributed GCD scenario $F_{g c d .1}$. 


\section{GCD Problem Case 2}

In order to further clarify the question whether Genetic Programming indeed utilizes the information gained from sampling the search space efficiently, we specified the second functional criterion $f_{g c d .2}$. This function provokes an all-or-nothing problem and gives only little more information than a Boolean decision about the correctness of a program. Since the value of $f_{p s}$ was bounded below, we would expect the search to behave like a random walk in the space of programs which are not much longer than the specified minimum size and to find solutions only very rarely.

The result of the experiments with $F_{g c d .2}$ fully meet this expectation. Only one solution was found in a total of more than 760 runs, interestingly by the eRBGP approach. We compared the success rates for $F_{g c d .1}$ and $F_{g c d .2}$, both achieved with a population size of 512, and found that there only was a significant difference for eRBGP (see Table C.6 on page 195 in the appendix). This does not necessarily mean that the other approaches perform no better than (bounded) random walks - with more experimental runs, the differences may become significant - but it means that eRBGP definitely does.

\subsubsection{Runtime}

All experiments for the distributed GCD together consumed a runtime of 1849 processor days on the same hardware configurations as the previous experiments. With tripling the population size, the runtime approximately triples too, as illustrated in Table 9.1.

The reduction of valuable information provided by the objective functions caused by the transition from $f_{g c d .1}$ to $f_{g c d .2}$ reduced the runtime of the experiments by between $65 \%$ and $97 \%$. This supports our assumption from Section 7.5.4, where indicators were found that experiments finding close-to-adequate programs take longer than experiments which only produce trivial solution candidates.

\subsection{Summary}

Our experiments targeting the evolution of algorithms for computing the greatest common divisor in a distributed way led us to three conclusions. First, the GCD task seems to be the most complicated one of all three solvable instances of the problems which we have tested. Although at least three GP approaches found solutions for it, the success rates of the experiments are very low. Second, only eRBGP is able to utilize larger population sizes in order to produce better results in this scenario. The other approaches could not improve their solutions significantly when tripling the population size. Third, only eRBGP is significantly better than a (bounded) random walk in this domain.

On one hand, these results reveal the hardness of needle-in-a-haystack problems which we anticipated in Section 4.4 on page 56 and to which the GCD task seems to belong. On the other hand, it is a further indicator for the strength of eRBGP and justifies its design outlined in Section 5.5 and Section 5.6. 
Part III

Conclusions 



\section{Critical Discussion}

In the introduction, we motivated that Genetic Programming could be used as a foundation for a new design approach for distributed algorithms. We have implemented this approach with six different GP methods and applied it to three example problems from distributed computing. In this chapter, we want to discuss our findings critically. We will present arguments that seem to speak against using our design method and we will refute them.

\subsection{Criticism of the Approach}

\subsubsection{Complexity of Realization}

In Section 1.2, we introduced our approach for the automatic synthesis of distributed algorithms based on Genetic Programming. This design process requires the following prerequisites:

1. a search space $\mathbb{G}$ with corresponding search operations, a representation $\mathbb{X}$ for distributed programs, and a translation gpm between them (if $\mathbb{G} \nsubseteq \mathbb{X}$ ),

2. an execution environment for the synthesized programs $x \in \mathbb{X}$ based on

3. a fast and sufficiently realistic network simulation,

4. an optimization framework which allows putting all of them together,

5. a clear specification of the wanted behavior of the distributed system,

6. an adaptation of points 1 through 4 to this specification if necessary, and

7. a set of functional and non-functional optimization criteria to steer the optimization process.

Our Sigoa framework is a versatile optimization engine well-suited for point 4. The DGPF library, on the other hand, covers points 1 through 3. First, it defines various Genetic Programming representations, search operations working on them, and related genotypephenotype mappings. Additional to the GP approaches used in this work, there is, for instance, also a package [297] especially tailored to the evolution of distributed aggregation protocols (see Section 11.1 on page 157). Second, the DGPF provides fast randomized network and node simulations for a variety of topologies and communication situations. Third, the DGPF additionally includes pre-defined objective functions for numerous nonfunctional aspects, such as the function $f_{p s}$ used in our experiments, criteria for minimizing the number of messages sent or the number of computation errors, and functions that further proper termination of the distributed programs.

The only remaining issues are the definition of objective functions targeting functional adequacy and, if required, an adaptation of the DGPF to special necessities which may arise from the global behavior specification. 
Especially the definition of the objective functions has a large impact on the experimental results as shown in the GCD experiment. Since it is often possible to specify a certain functional criterion in many different ways, multiple test runs should be performed before actually starting the experimental series. This requires some effort and learning curve.

In summary, utilizing our algorithm design approach is not trivial. Yet, it is also not too complicated and can be feasible in a variety of scenarios. Furthermore, we have provided software covering most of the requirements for doing so.

\subsubsection{The Evolved Programs}

The most severe objection against the idea of evolving (distributed) algorithms with Genetic Programming is that the results are not necessarily correct. We ourselves raised this issue in Section 4.8 on page 67 and also argued that effects like overfitting or oversimplification can never be ruled out.

We have used the urban legend of The Monkey and the Orange in Section 4.8.1 as an illustrative example that these problems also exists in natural evolution. Another example would be parasitic insects which sneak into the colonies of ants by copying their chemical signaling systems [167]. Because of the low frequency in which such "errors" happen, the species which is taken advantage of can still spread and thrive.

This argumentation does not really carry over to man-made (distributed) systems. Obviously, such systems shall always work adequately from the perspective of the users. However, it is our strong believe that it is impossible to rule out the occurrence of temporarily limited unpredicted and infeasible system behavior in all (biology-inspired) approaches for self-adaption or system synthesis not involving complete automated program verification. If a system is able to explore new behaviors - be it offline as in our GP approach or online as in the work of Yamamoto and Tschudin [319, 320] - their utility as well as the similarity to the wanted behavior is not known in advance. Additionally, even if the system detects adequate behavior, this adequacy cannot be assumed to be general but may only hold for the situations in which it has been observed.

Still, some of the larger-scale network types named in the motivation of our approach tolerate a certain amount of malfunction. In sensor networks, for instance, some of the nodes will inevitably fail due to depleted batteries. In MANETs, temporary network partitions may occur. Especially for networks of these kinds, evolving algorithms may indeed be a useful software design approach. The election algorithms synthesized for the problems $F_{a}$ and $F_{c}$, for instance, will exhibit adequate behavior in virtually all scenarios and may only lead to disagreements in very few situations.

The mutual exclusion algorithms from Section 8.4 are able to achieve this degree of utility as well. Assume that the instructions used for stalling the execution would be translated to calls to a sleep function provided by an operating system so that the CPUs of the involved nodes would not waste energy. Further assume that an upper boundary for the time spent in a critical section of a real system is known or can be approximated. In this case, using the communication medium as signaling device to determine whether or not the critical section can be accessed combined with sleeping delays may indeed perform well. Many of the evolved algorithms for the GCD problem were even correct and the others were vulnerable to rather improbable network scenarios only.

Following our argumentation about the impossibility of preventing temporary inadequacy, there are strong reasons speaking against using GP for creating algorithms for securityor otherwise critical applications. Yet, with further research and a better integration into software design utilities (such as MDD tool chains), our proposed methodology becomes applicable. This holds especially for new forms of wireless or mobile networking - in other words, for the scenarios it was proposed for. 


\subsection{Significance of our Experiments}

\subsubsection{Settings}

Because of the long runtime, we used only one single configuration of the EA for all experiments. We have tested these settings in previous, smaller test series and outlined their motivation in Chapter 4. So, on one hand, the configurations used in the presented experiments result from experience and clearly justified assumptions.

On the other hand, there is a manifold of EA parameters which can be tuned, such as the population size, the steady-state/generational setting, the selection and fitness assignment algorithms, the mutation and crossover rates, and whether or not to use elitism. Better yet, these settings may have different impact depending on the search space $\mathbb{G}$, the problem space $\mathbb{X}$, and the search operations. Using $70 \%$ crossover might be the best choice for treebased Standard Genetic Programming methods but a very bad decision for string genomes, for instance. Our simple convergence prevention algorithm SCP may further the evolution of election algorithms but could complicate finding mutual exclusion algorithms for the distributed critical section.

It remains an open question whether our experimental settings were most suitable for the problems we have applied them to. Finding optimal configurations would require running a number of tests several magnitudes higher than practically feasible. Still, the outcomes of the experiments were significant for these settings. Furthermore, if there indeed were better configurations than those used, the utility of Genetic Programming for evolving distributed algorithms would be even higher than one would assume based on our results.

\section{Population Size}

One important thing to mention is that the population size of 512 individuals, which we chose in most of our experiments, is rather low. In our earlier research, we used much bigger populations: six PCs with up to 8192 individuals each connected by a peer-to-peer network according to the island model in [295], for instance. Then again, we ran only small experimental series with such configurations. Population sizes of 65536 [151] (1996), 100000 [166] (1997), and even up to 1048576 [171, 170] (2007) individuals have been used in various research projects. In other words, we have shown that good results can be achieved even with relatively small populations. This is a strong indicator for the high utility of Genetic Programming in our domain.

In a practical application, much fewer runs will be executed and thus, larger populations can be used. The triplication in the GCD experiment led to a small but significant improvement in the eRBGP setup. An increase to 100000 individuals, approximately 200 times as many as in the population in our experiments, for instance, would likely improve the outcome much more.

\subsubsection{Why Evolutionary Algorithms?}

It is not clear whether using a plain evolutionary algorithm as optimization backend for Genetic Programming is the best choice or not. Generally, many other optimization algorithms such as Simulated Annealing [156, 293], Tabu Search [104, 293], and Memetic Algorithms (a hybridized form of EAs) [204, 293] or even simple greedy searches could be used for this purpose, too.

In [295] and [294], we have shown that the combination of a set of different optimization algorithms connected loosely by a peer-to-peer network according to the island model can lead to better results than the application of a plain EA. We could have used such a heterogeneous approach for our experiments here, too. This, however, would make it even more 
complicated to draw general conclusions. Our intention is to provide a baseline discussion and comparison of different GP methods for evolving distributed algorithms. By focusing on EAs, our results are more general and can better be compared with the work of other researchers.

\subsubsection{Genetic Programming Approaches}

We have tested six different approaches for evolving distributed algorithms which can be considered representative for the most common streams of Genetic Programming. It was not possible to test all these streams and we excluded, for instance, Grammar-guided Genetic Programming methods [293, 194] such as Grammatical Evolution [248] or Tree-Adjoining Grammar-guided Genetic Programming [208, 209], graph-based methods [293] like Cartesian Genetic Programming [199] and Parallel Distributed Genetic Programming [229, 230], or special genotype-phenotype mappings [293] such as Gene Expression Programming [84, 85] and Edge Encoding [187]. Since all these techniques represent totally different approaches to GP, we may have lost a significant amount of insight by not utilizing them. Furthermore, we only used straightforward reproduction operators in the experiments. With more sophisticated search operations, such as jump-target correcting mutation and crossover in LGP, better results may have been obtained.

Nevertheless, we can provide a good overview on what the six selected techniques can and what they cannot do in the context of distributed computing. All the approaches used in our experiments can easily be implemented and tested based on the specifications we have given.

One result of our work is that no Genetic Programming approach was superior in all experiments. The rule-based eRBGP method, especially developed to have low epistasis, had an excellent performance in the election and the GCD problem. The feature which decreased its epistasis, the absence of instruction sequences, turned against it in the critical section domain. Here, our adaptations of the traditional Standard Genetic Programming methods could therefore outperform it.

\subsubsection{Adaptation of the Genetic Programming Approaches}

We gave our Genetic Programming methods slightly different instruction sets. eSGP, for instance, has a native construct for for loops which SGP does not have. In neither RBGP, eRBGP, nor in Fraglets, loops can be declared explicitly. At least three of the approaches were Turing-complete (eRBGP, LGP, eSGP) whereas two were not (SGP, RBGP). Besides the differences in the structure of the approaches, differences in expressive power may have had impact on the outcomes of the experiments.

Another aspect which should be considered is that we granted simulations for programs in the Fraglet and LGP representation twice as much runtime than the rule-based approaches because they have a more fine-grained execution model. SGP and eSGP, in turn, were granted three times the runtime of LGP, because the compiled results of the tree-based programs in average tended to be longer by approximately this factor in preliminary experiments. The different values of the number $m x S$ of simulation steps may have changed the outcome of the objective functions. $f_{\text {col }}$ given in Equation 8.3 on page 123, for instance, depends directly on $m x S$. These values were set by rules of thumb which may not be valid for all experiments, but based on our experiences, we think that they gave the GP approaches approximately equal chances to solve the problems.

\subsubsection{Choice of Scenarios}

In this dissertation, we have applied our methodology only to three problems, the election, the critical section, and the GCD problem. It is hard to draw valid conclusions from such 
a small sample set. The focus of our work presented here was on running large-scale experiments with significant outcomes. We tackled three problems which are different in many

\begin{tabular}{|c|c|c|}
\hline Election & Mutual Exclusion & GCD \\
\hline two objective functions & three objective functions & $\begin{array}{l}\text { two objective functions; two dif- } \\
\text { ferent functional criteria }\end{array}$ \\
\hline $\begin{array}{l}\text { four scenarios, from low to high } \\
\text { difficulty }\end{array}$ & three scenarios of high difficulty & one hard scenario \\
\hline one topology: linear & $\begin{array}{l}\text { three topologies: fully connect- } \\
\text { ed, ring, no communication }\end{array}$ & one topology: square grid \\
\hline population size: 512 & population size: 512 & population size: 512,1536 \\
\hline
\end{tabular}

Table 10.1: Comparison of election, distributed mutual exclusion, and the GCD experiment.

aspects, as outlined in Table 10.1. We tested, for instance, the influence of the choice of the Genetic Programming approach, the topology, the choice of the objective functions for the functional adequacy, and the population size. Together with our research work published elsewhere, these form a solid foundation for discussions about the applicability of Genetic Programming to distributed systems.

Our research is not solely based on the three example domains discussed here. Instead, we tackled multiple other distributed algorithm problems. We evolved, for instance, distributed aggregation functions (see Section 11.1). We also researched GP as Offline Emergence Engineering approach for multi-agent systems and evolved, for example, load balancing algorithms with RBGP (see Section 11.3). The results of these works all complied with our findings discussed here.

\subsection{Quality GP Approach Features}

\subsubsection{Is Rule-based Genetic Programming Good?}

One of the main contributions of this research work is the Rule-based Genetic Programming approach. In this thesis, we have applied RBGP in two flavors: The simple, binary-coded variant RBGP and the tree-based version eRBGP.

In many of the experiments, there was a rather large difference between the performances of both approaches. In the election experiment (Chapter 7), eRBGP dominated all other Genetic Programming approaches whereas RBGP lost in almost all comparisons. Both GP methods could not find adequate algorithms for mutual exclusions at the critical section. In the GCD experiments, eRBGP was again the best approach whereas RBGP was not able to find any solution.

From the experimental results, it is obvious that Rule-based Genetic Programming has a right to exist, supposedly not limited to the evolution of distributed algorithms. At least the eRBGP approach had an excellent performance in two of the three tested problem domains. Hence, the question whether this idea is any good can be answered with yes.

\subsubsection{Is Indexed Memory Good?}

eRBGP often achieved much better results than RBGP. This may either be rooted in the Turing completeness achieved by incorporating indexed memory into this approach or in the higher degrees of freedom for constructing complex expressions. In our experiments, we have tested 
two other GP approaches which are similar and between which the main distinction is also the availability of indexed memory respectively the lack of it: eSGP and SGP. Between these two methods, no significant difference in the performance could be detected in most of the experiments. Therefore, we believe that the versatility provided by the tree genome is the decisive difference between RBGP and eRBGP. From this perspective, our results do not allow us to decide whether the usage of indexed memory is beneficial. However, we could not detect any disadvantage either (although the presence of indexed memory leads to more degrees of freedom and thus, enlarges the search space).

Interestingly, besides the rule-orientation, the major distinction between the rule-based and the Standard Genetic Programming methods is the usage of temporary memory which is committed after the evaluation of all rules. This form of memory is the sole reason why the order of the rules plays no role in RBGP. We strongly assume that introducing it into SGP or eSGP may improve their performance significantly, too. Verifying this hypothesis is left for future work. 


\section{Other Projects}

\subsection{The Evolution of Aggregation Protocols}

The experiments reported in this work are actually only a subset of our work on the subject of evolving distributed algorithms. In [297], [304], and [293], we presented an approach for the automated synthesis of proactive aggregation protocols [149] using Genetic Programming and discussed major decisions in modeling and simulating them. We developed a genotype structure, which is an abstract specification for aggregation protocols. We evolved programs in this representation for various conditions such as static and dynamic input data and again demonstrated the utility of our automated algorithm design approach. In this context, also the efficiency of targeted, application specific design of search and problem spaces became apparent once more.

\subsection{Combining GP and MDD}

During our research, the idea of integrating Genetic Programming into model driven development (MDD, $[1,8]$ ) emerged. Our methodology of transforming descriptions of global behaviors into programs for local execution dovetails nicely with model-driven design. In this dissertation, we focused on using evolutionary algorithms to perform these transformations but did not discuss this combination.

Nevertheless, in [299, 300], and [307], we outlined how this integration can be achieved using MOF [162] models encoded in XMI [143] as output format of the Genetic Programming process. These models can then be fed into any XMI-compliant modeling software where they can be incorporated into existing application models. In the same papers, we also showed how such platform-independent algorithm models resulting from Genetic Programming can easily be transformed to program code in a programming language such as Java or $\mathrm{C}$ using the transformation language MOFScript [241]. This research became possible especially because of the help and expertise of our colleagues Mohammad Ullah-Khan and Michael Zapf. With this joint work, we showed that the usability of Genetic Programming for distributed algorithm design can be increased remarkably with simple means and that its integration in existing software design utilities is possible.

\subsection{Offline Emergence Engineering with GP}

Together with our colleague Michael Zapf, we researched the applicability of Genetic Programming in multi-agent systems (MAS). The ADELFE approach of Bernon et al. [27, 28] allows useful behavior to emerge in MAS at runtime. In contrast to this online approach, 
emergent behavior is often an inherent property of distributed algorithms evolved with Genetic Programming. Michael Zapf and me investigated such an offline emergence engineering $(O E E)$ process based on RBGP and eRBGP in-depth, analyzing scenarios such as load balancing, election, and mutual exclusion at critical sections in agent societies [323, 324, 325, 326]. This work showed that Genetic Programming is not only applicable for the bio-inspired design of distributed algorithms, but is also located at the juncture to research in complex systems. It became clear that emergent cooperation patterns in MAS can be created with our methodology too.

\subsection{Tunable Model for Problematic Fitness Landscapes}

Together with our student Stefan Niemczyk, a tunable model for many of the problematic features of optimization tasks discussed in Chapter 4 has been developed [302, 210, 293]. Amongst the modeled aspects is the affinity for overfitting and oversimplification, epistasis, ruggedness, uniform neutrality, and multi-objectivity. All of these are also eminent facets of the optimization processes involved in the experiments discussed in this dissertation.

The goal of our work with Stefan was to create a benchmark with which different techniques for mitigating the problematic features of such optimization tasks can be assessed. All experimental settings used in this thesis and especially our variety preserving fitness assignment process (Section D.1.2) and the simple convergence prevention algorithm (Section D.2) [293] were assessed with this model beforehand.

\subsection{GAs for Semantic Web Service Composition}

The international Web Service Challenge (WSC) [16] is an annual event providing a platform for researchers in the area of Web Service composition which allows them to compare their systems and to exchange experiences. Each year, new tasks revolving around the composition of semantically annotated web services are introduced.

With Steffen Bleul, we took part in the 2006 WSC in San Francisco with a composition system which could utilize an IDDFS and a heuristic for semantic service composition as well as a genetic algorithm [33]. With this system, we reached the first place, being able to solve all challenges significantly faster than the other contestants. Although we found that the genetic algorithm was much slower than our heuristic approach [301, 34, 296], it was fully functional, solved every task, and outclassed the plain IDDFS algorithm. We expect that the application of genetic algorithms is likely to become advantageous if more complex competition tasks - maybe such involving QoS parameters - are to be solved.

With this work, we proved that important questions from current practice in different areas of distributed systems such as enterprise computing can be answered with evolutionary algorithms.

\subsection{The Electronic Book on Global Optimization}

One of the major foundations of our research work is the electronic book Global Optimization Algorithms - Theory and Application [293] which we began writing in 2006 and steadily extended since then. Although the basic principles of evolutionary algorithms are rather simple, Evolutionary Computation is an extremely wide research area with a plethora of contributions, dependencies, and approaches available.

Writing this summary became our major tool for understanding and systemizing Evolutionary Computation and global optimization in general. The in-depth analysis of possible 
problems in Genetic Programming in Chapter 4, for instance, is a result of this information aggregation process.

\subsection{Sigoa and DGPF}

From the beginning of our research, we implemented all the software required for the evolution of programs on our own (in the Java programming language). Although there are many frameworks for Genetic Programming, this approach improved our understanding of the practical aspects of the matter and also allowed us to experiment with different new fitness assignment or diversity-preserving approaches such as those listed in Chapter D in the appendix.

We developed a framework for Genetic Programming called DGPF which, like our electronic book, became more and more comprehensive and versatile over the three years of our work. In 2007 we decided to divide it into a software bundle for optimization in general called Sigoa and a Genetic Programming package placed on top of it which retained the name DGPF. All experiments reported in this thesis were conducted using the DGPF and Sigoa. 



\section{Outlook and Summary}

\subsection{Outlook}

In this dissertation, we have laid the foundation of a new design methodology for distributed algorithms based on Genetic Programming. We have carried out experiments and obtained representative results indicating the utility of this approach. Our research not only complements the jigsaw puzzle of bio-inspired approaches for distributed computing by another piece, but also creates points for tying up with interesting projects and future work.

\subsubsection{From Theory to Practice}

One of the major drawbacks of our research is that it is not yet practically applicable. Although we provided the necessary optimization and Genetic Programming frameworks, a certain learning curve is still unavoidable. In Section 11.2, we gave pointers to some of our work not directly incorporated into this thesis: the integration of the results of the Genetic Programming process into a model-driven development process.

However, we would not only like to use MDD tools as backend for GP but also as frontend. The utility of our methodology will increase very much if it becomes possible to model the behavior of the anticipated system by defining and combining optimization criteria in a more graphical, straightforward way. We therefore would like to provide the user with abstract building blocks for behavior patterns and options to configure the structure and parameters of the simulation scenarios. Creating such a user interface itself is a large and non-trivial research task.

Once this problem is solved, the internal course of a MDD tool based on our approach is rather simple: From the behavior model specified by a user, a (Java) executable will be generated which can be deployed to a PC or a cluster and carries out the optimization process synthesizing the distributed algorithms. These are then fed back into the tool as discussed in Section 11.2 which allows integrating them into existing application models and automated code generation.

\subsubsection{Investigating the Optimization of Non-Functional Features}

One of the initial objectives of this project was to create a design method for synthesizing distributed algorithms especially suitable for new forms of networks such as MANETs or sensor networks. In these networks, non-functional aspects such as the message complexity and energy consumption play a major role.

In the presented research work, we did not focus on these aspects since we wanted to show the utility of GP to evolve distributed algorithms in general. It is a well-known fact 
that increasing the number of (conflicting) objective functions also increases the number of non-dominated solutions [306, 293]. As the Pareto frontier grows, the number of individuals needed to sample it with a constant density increases, too. Hence, we can either expect the number of runs finding good solution candidates according to one of the objectives (such as functional adequacy) to decline or need to use larger population sizes. In the former case, the success rates of the experiments would go down and we would need to perform more experimental runs in order to obtain significant differences between the results. Then, as well as in the latter case, the total runtime of our experiments would have prolonged to an infeasible level.

In our work, the utility of Genetic Programming for evolving distributed algorithms has been shown with statistical significance and the most efficient GP approaches, eRBGP and depending on the problem, SGP or eSGP, have been determined. The next step should thus be to also incorporate additional objective functions and to research whether the message complexity, for instance, can be optimized along with synthesizing the functionality.

We have already published the results of smaller-scale experiments with higher population sizes targeting this issue in [295]. Although these experiments turned out rather positive, a confirmation with larger scale test series is required in order to determine the reliability of the approach.

\subsubsection{Combining Temporary Memory with other GP Approaches}

As we mentioned in Section 10.3.2, Rule-based Genetic Programming is different from Standard Genetic Programming in two aspects: First, its execution model is based on rules evaluated in parallel and not on instruction sequences and second, it uses a temporary storage committed after all rule evaluations. With our experiments, we cannot be sure which of these two points contributed most to the dominance of eRBGP in many of the experiments or whether it was their combination.

We would therefore very much like to introduce "transactional" memory into SGP, maybe with a special commit instruction. By allowing the Genetic Programming process to decide when to commit the changes to the variables, we would reduce the positional epistasis of the Standard Genetic Programming approaches. With the presented work, we also have plenty of data to compare the performance of such new "transacted" SGP or eSGP methods with.

\subsection{Summary}

The goal of our research was to explore a new method for designing algorithms for distributed systems. This method prescribes a process which starts with the definition of a wanted global behavior of the anticipated system. From this definition, objective functions are derived manually which rate how close a simulated network resembles the target behavior. On each node in these network simulations, the same synthesized programs run which, in turn, are the solution candidates of an evolutionary algorithm. With its genetic operators, the EA modifies and combines the programs in order to achieve optimal objective values. This leads to an iterative translation of a specified global behavior to programs which achieve this behavior when being executed locally on the nodes of a network.

As pointed out in Chapter 3, there exists little related work on the utilization of Genetic Programming for evolving non-trivial distributed programs. One reason for this lack of research may be that such applications of EAs usually exhibit difficult fitness landscapes. Different aspects such as ruggedness and epistasis which contribute to this issue have been discussed in Chapter 4 along with methods to mitigate them to a certain degree.

In order to get a broad view on the utility which GP can achieve in the face of the remaining hardness, we tested six different Genetic Programming approaches (Chapter 5). 
Three of them (SGP, eSGP, and LGP) are extensions of well-researched GP methods, one is a bio-inspired approach (Fraglets), and two representations (RBGP, eRBGP) have been designed with the goal of reducing epistasis, one of the root causes of the difficult facets of GPfitness landscapes. The last method, eRBGP, performed excellent in the election and GCD experiments, supporting the assumptions underlying its design. Interestingly, its worse performance in the critical section experiments is most likely caused by the same design principles.

Besides the search and problem spaces for Genetic Programming, the evolution of distributed programs also asks for network simulations and execution models. In Chapter 6, we introduced these prerequisites and outlined the architecture of the DGPF and Sigoa systems with which we have carried out our experiments.

These experiments encompass three problem domains: the evolution of election algorithms (Chapter 7), of mutual exclusion algorithms for a distributed critical section (Chapter 8), and the synthesis of algorithms for the distributed GCD problem (Chapter 9). The results of our experiments show that distributed algorithms which behave adequately in terms of objective functions for non-trivial problems can be synthesized with Genetic Programming. The programs achieving this adequate behavior, however, may largely differ from man-made solutions and employ unexpected means to achieve their goals. In Chapter 10 we critically discussed our findings before listing starting points for future work in the current chapter. 

Appendices 



\section{The Election Experiments}

\section{A.1 Precise Settings}

With this section, we complement the discussion of the settings of the election experiments in Section 7.3 with a complete list of all parameters and their values.

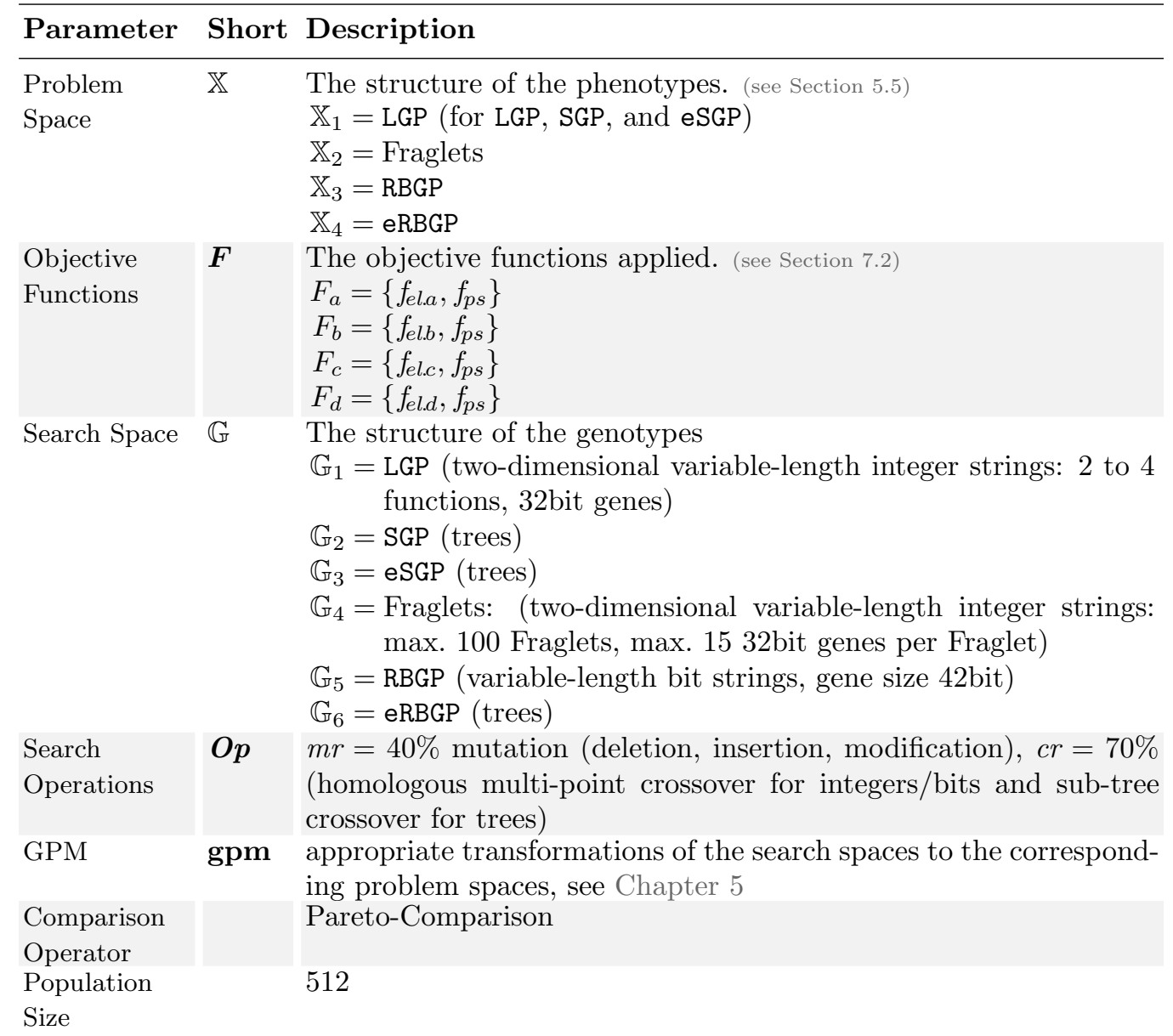




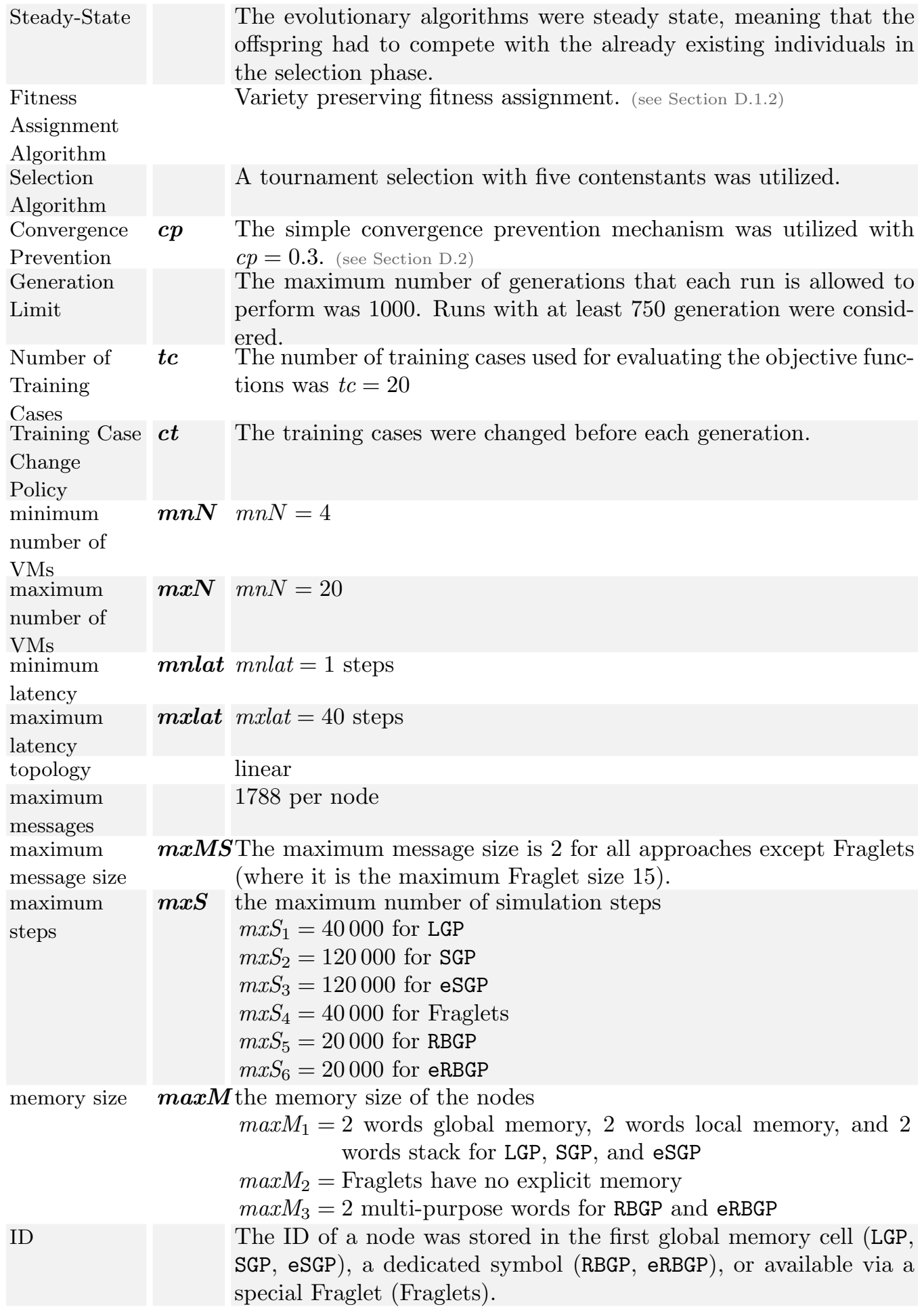


marginal solutions in order to solve problem $c$ ) or $d$ ). From experience, it rather is unlikely that a program as complicated as the one given in Listing A.1 will evolve. The interesting question was rather which of the four problems can be solved adequately, how close Genetic Programming can get to adequate solutions in the other problems, and whether there are significant differences between the six approaches.

\section{A.3 Further Results}

\section{A.3.1 Convergence Diagrams}

In this section, we list the remaining two convergence diagrams for the cases $c$ ) (Figure A.1) and $d$ ) (Figure A.2) not given in Section 7.5.2. They show the mean, median (med), and the $5 \%$ and $95 \%$ quantiles (Q.05, Q.95) of the best values of the functional objective function $f_{e l}$ computed over each generation of all runs in of each configuration of these problems. 


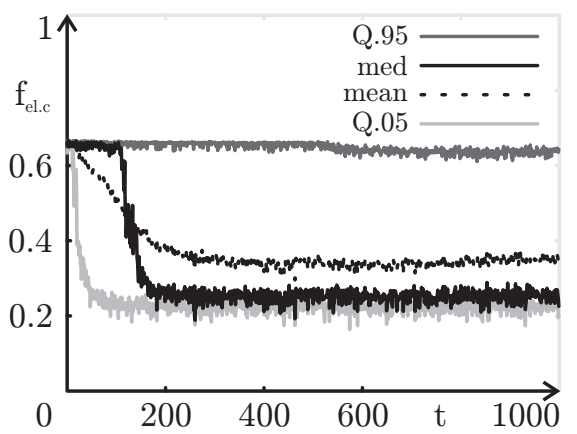

Fig. A.1.a: Convergence: SGP for $f_{\text {el.c. }}$.

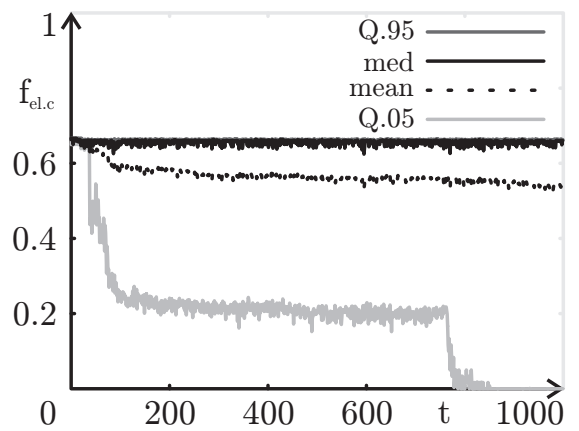

Fig. A.1.c: Convergence: LGP for $f_{e l . c}$.

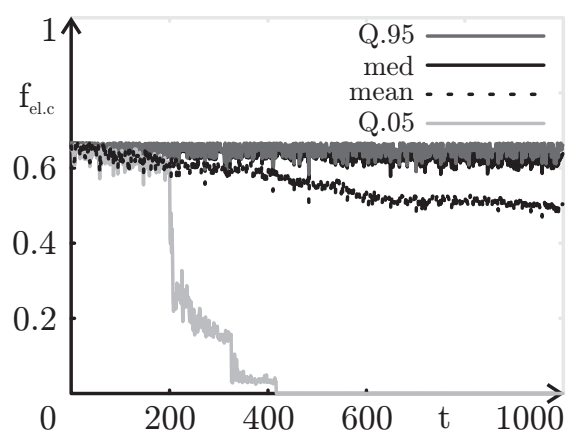

Fig. A.1.e: Convergence: RBGP for $f_{\text {el.c. }}$.

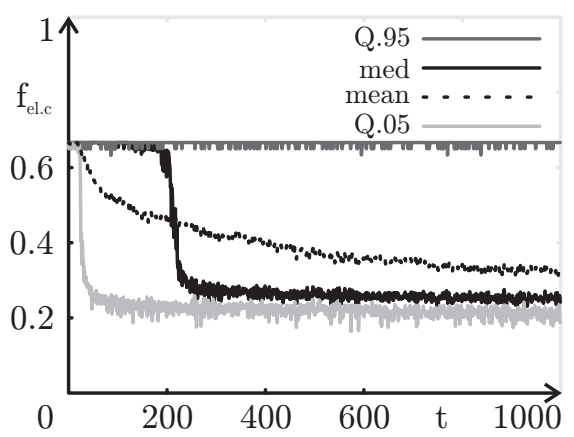

Fig. A.1.b: Convergence: eSGP for $f_{\text {el.c. }}$.

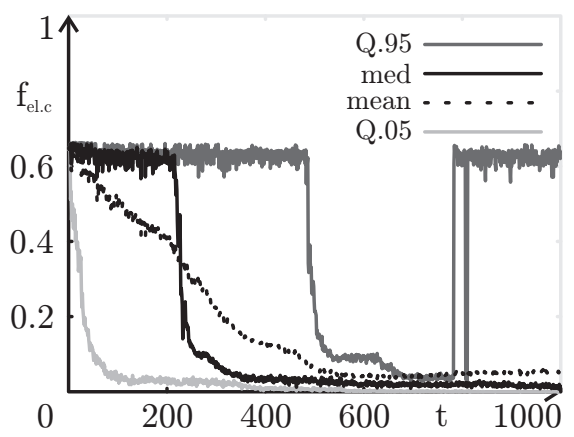

Fig. A.1.d: Convergence: Fraglets for $f_{\text {el.c. }}$.

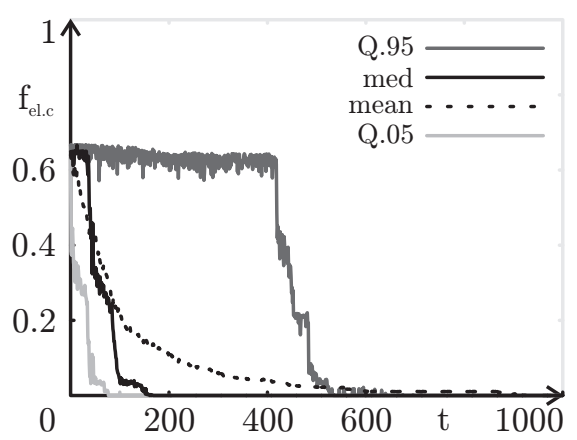

Fig. A.1.f: Convergence: eRBGP for $f_{\text {el.c }}$.

Figure A.1: Convergence diagrams for the election problem $f_{e l . c}$. 


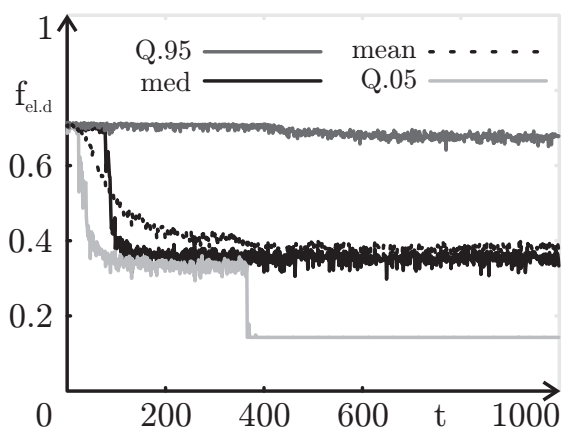

Fig. A.2.a: Convergence: SGP for $f_{e l . d}$.

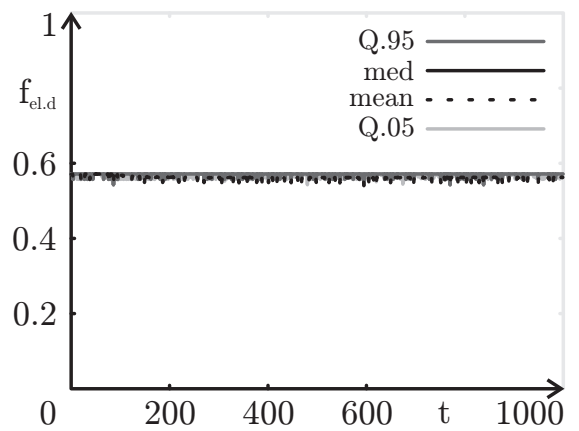

Fig. A.2.c: Convergence: LGP for $f_{\text {el.d }}$.

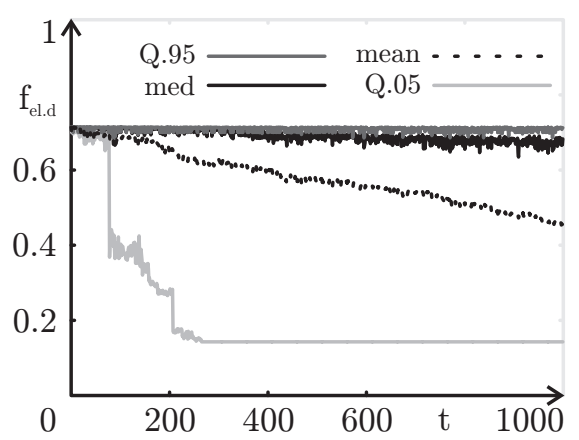

Fig. A.2.e: Convergence: RBGP for $f_{\text {el.d }}$.

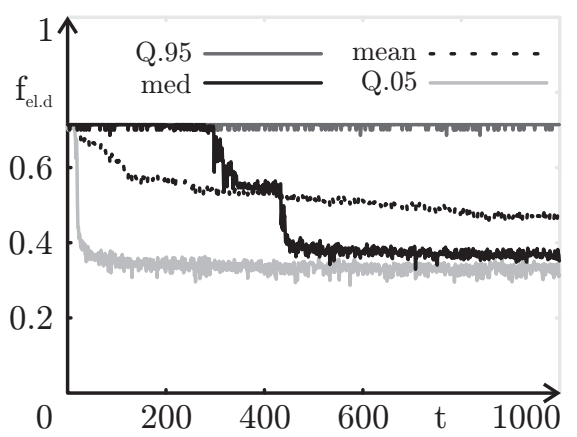

Fig. A.2.b: Convergence: eSGP for $f_{e l . d}$.

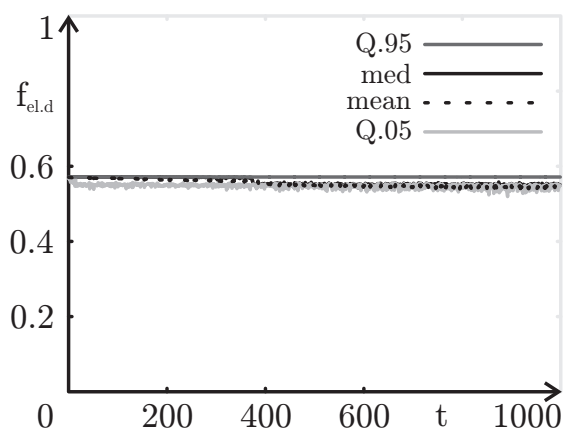

Fig. A.2.d: Convergence: Fraglets for $f_{\text {el.d. }}$.

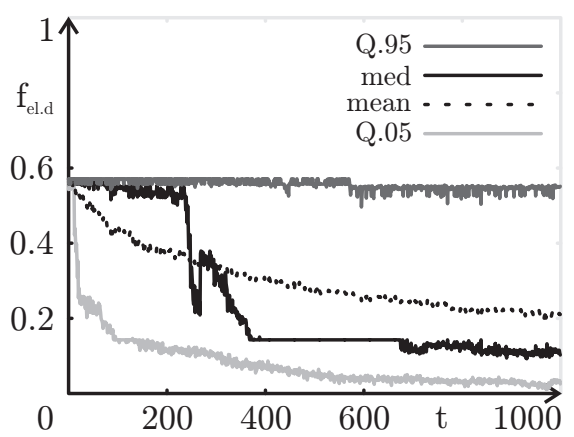

Fig. A.2.f: Convergence: eRBGP for $f_{\text {el.d }}$.

Figure A.2: Convergence diagrams for the election problem $f_{e l . d}$. 


\section{A.3.2 Statistical Evaluation}

In Table A.2 and Table A.3, we have compared the six different approaches according to their success rate $s / r$ (upper triangle) and the number of generations $s t$ (lower triangle) which they need to succeed. A + in a cell means that the GP approach of the corresponding row beats the approach of the column to which the cell belongs with $\alpha=2 \%$ significance in a two-tailed significance test concerning the respective criterion. A - means it loses and if neither one is significantly better than the other, 0 is noted. The success rates were compared with Fisher's exact test $[88,89]$ and for comparing the number of generations st needed by a run to succeed as well as the best values of $f_{e l}$, we used the Mann-Whitney U Test [192] both calculated with the utilities by Lowry $[181,182]$. The graphical representation of the comparison tables can be found in Figure 7.4 on page 116.

\begin{tabular}{r|c|c|c|c|c|c}
\hline $\boldsymbol{F}_{a}$ & SGP & eSGP & LGP & RBGP & eRBGP & Fraglets \\
\hline SGP & & 0 & + & + & 0 & + \\
eSGP & - & & + & + & - & + \\
LGP & - & 0 & & + & - & + \\
RBGP & 0 & 0 & 0 & & - & 0 \\
eRBGP & + & + & + & 0 & & + \\
Fraglets & - & 0 & 0 & 0 & - & \\
\hline
\end{tabular}

Table A.2: Comparison of $s / r$ and generations for $F_{a}$.

\begin{tabular}{r|c|c|c|c|c|c}
\hline $\boldsymbol{F}_{\boldsymbol{c}}$ & SGP & eSGP & LGP & RBGP & eRBGP & Fraglets \\
\hline SGP & & 0 & - & - & - & - \\
eSGP & 0 & & 0 & - & - & - \\
LGP & 0 & 0 & & 0 & - & 0 \\
RBGP & 0 & 0 & 0 & & - & 0 \\
eRBGP & 0 & 0 & 0 & + & & + \\
Fraglets & 0 & 0 & 0 & 0 & - & \\
\hline
\end{tabular}

Table A.3: Comparison of $s / r$ and generations for $F_{c}$.

\begin{tabular}{r|c|c|c|c|c|c}
\hline $\boldsymbol{F}_{\boldsymbol{a}} / \boldsymbol{F}_{\boldsymbol{c}}$ & SGP & eSGP & LGP & RBGP & eRBGP & Fraglets \\
\hline SGP & & 0 & + & + & 0 & + \\
eSGP & 0 & & 0 & + & 0 & + \\
LGP & - & - & & + & - & 0 \\
RBGP & - & - & + & & - & - \\
eRBGP & + & + & + & + & & + \\
Fraglets & + & + & + & + & - & \\
\hline
\end{tabular}

Table A.4: Comparison of best $f_{e l}$ values in $F_{a}$ and $F_{c}$. 


\begin{tabular}{r|c|c|c|c|c|c}
\hline $\boldsymbol{F}_{\boldsymbol{b}} / \boldsymbol{F}_{\boldsymbol{d}}$ & SGP & eSGP & LGP & RBGP & eRBGP & Fraglets \\
\hline SGP & & 0 & + & + & - & + \\
eSGP & 0 & & + & + & - & + \\
LGP & - & - & & + & - & + \\
RBGP & 0 & 0 & 0 & & - & - \\
eRBGP & + & + & + & + & & + \\
Fraglets & - & - & + & 0 & - & \\
\hline
\end{tabular}

Table A.5: Comparison of best $f_{e l}$ values in $F_{b}$ and $F_{d}$. 


\section{The Critical Section Experiments}

\section{B.1 Precise Settings}

With this section, we complement the discussion of the settings of the critical section experiments in Section 8.3 with a complete list of all parameters and their values.

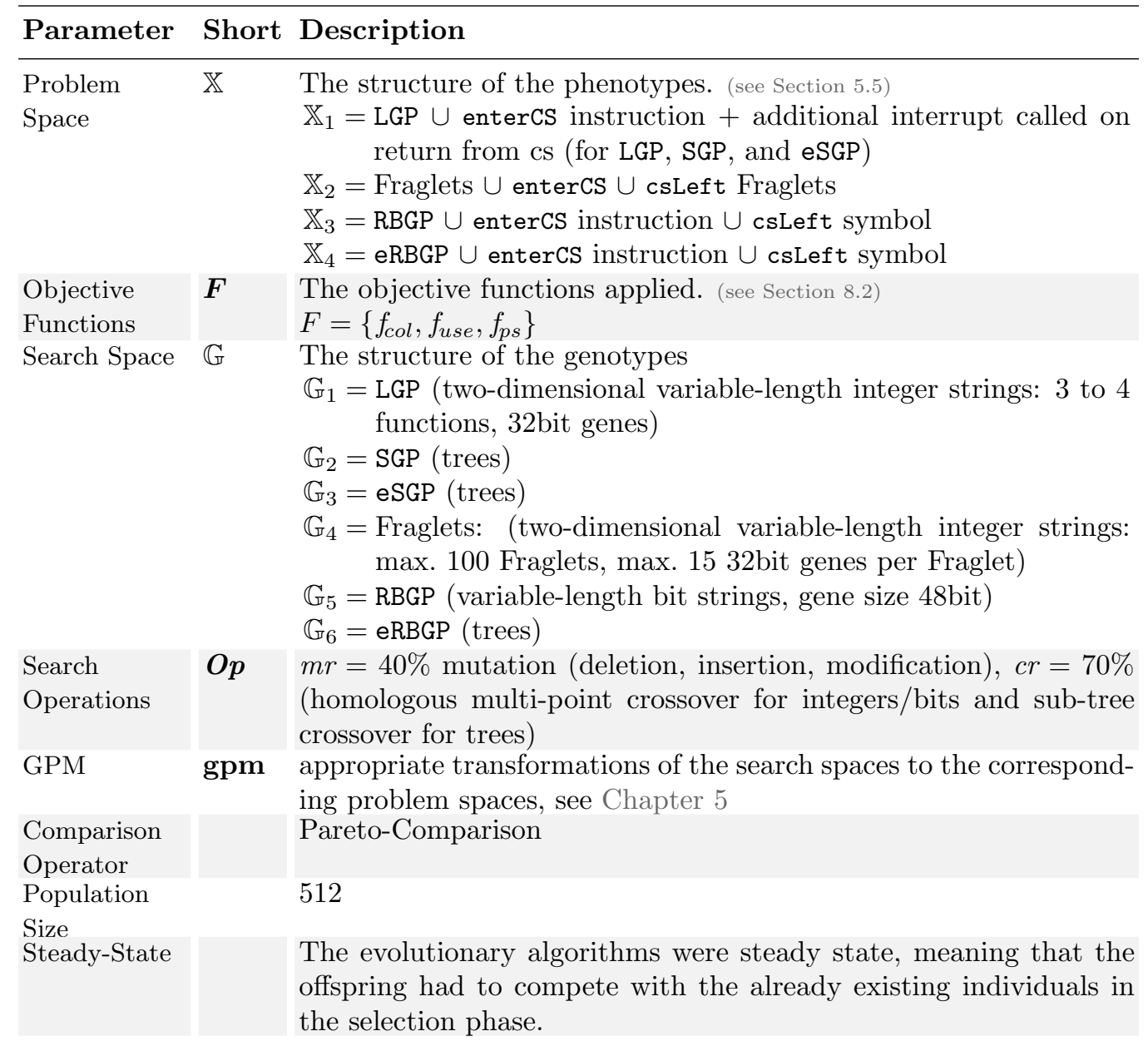


Variety preserving fitness assignment. (see Section D.1.2)

Assignment

Algorithm

Selection

Algorithm

Convergence

Prevention

Generation

Limit

Number of $\quad \boldsymbol{t} \boldsymbol{c}$

Training

Cases

Training Case

Change

Policy

minimum

number of

VMs

maximum

number of

VMs

minimum

latency

maximum

mnlat mnlat $=1$ steps

latency

topology

mxlat $m x l a t=46$ steps

maximum

fully connected mesh

messages

maximum

message size

cs time

cp The simple convergence prevention mechanism was utilized with

$c p=0.3$. (see Section D.2)

The maximum number of generations that each run is allowed to perform was 700 . Runs with at least 500 generation were considered.

$c$ The number of training cases used for evaluating the objective functions was $t c=20$

ct The training cases were changed before each generation.

$m n N \quad m n N=4$

$\boldsymbol{m x} \boldsymbol{N} \quad \operatorname{mn} N=23$

2536 per node

$\boldsymbol{m} \boldsymbol{x} \boldsymbol{M S}$ The maximum message size is 3 for all approaches except Fraglets (where it is the maximum Fraglet size 15).

The time a node spends in its critical section is uniform distributed in $69 . .206$

maximum $\quad \boldsymbol{m x} \boldsymbol{S}$ the maximum number of simulation steps

steps $m x S_{1}=61364$ for LGP

$m x S_{2}=184092$ for SGP

$m x S_{3}=184092$ for eSGP

$m x S_{4}=61364$ for Fraglets

$m x S_{5}=30682$ for RBGP

$m x S_{6}=30682$ for eRBGP

memory size $\boldsymbol{m a x} M$ the memory size of the nodes

$\max _{1}=5$ words global memory, 5 words local memory, and 3 words stack for LGP, SGP, and eSGP

$\max _{2}=$ Fraglets have no explicit memory

$\max _{3}=5$ multi-purpose words for RBGP and eRBGP

ID

The ID of a node was stored in the first global memory cell (LGP, SGP, eSGP), a dedicated symbol (RBGP, eRBGP), or available via a special Fraglet (Fraglets).

System $\quad \boldsymbol{C f g}$ GNU/Linux 2.6.18-92.1.13.el5, x86_64 processor (AMD Opteron ${ }^{\mathrm{TM}}$

Configuration

Processor 248, 2.1GHz), 4GB RAM, Java HotSpot ${ }^{\mathrm{TM}}$ 64-Bit Server VM (build 1.6.0_01-b06, mixed mode)

Table B.1: The settings of the critical section experiments. 


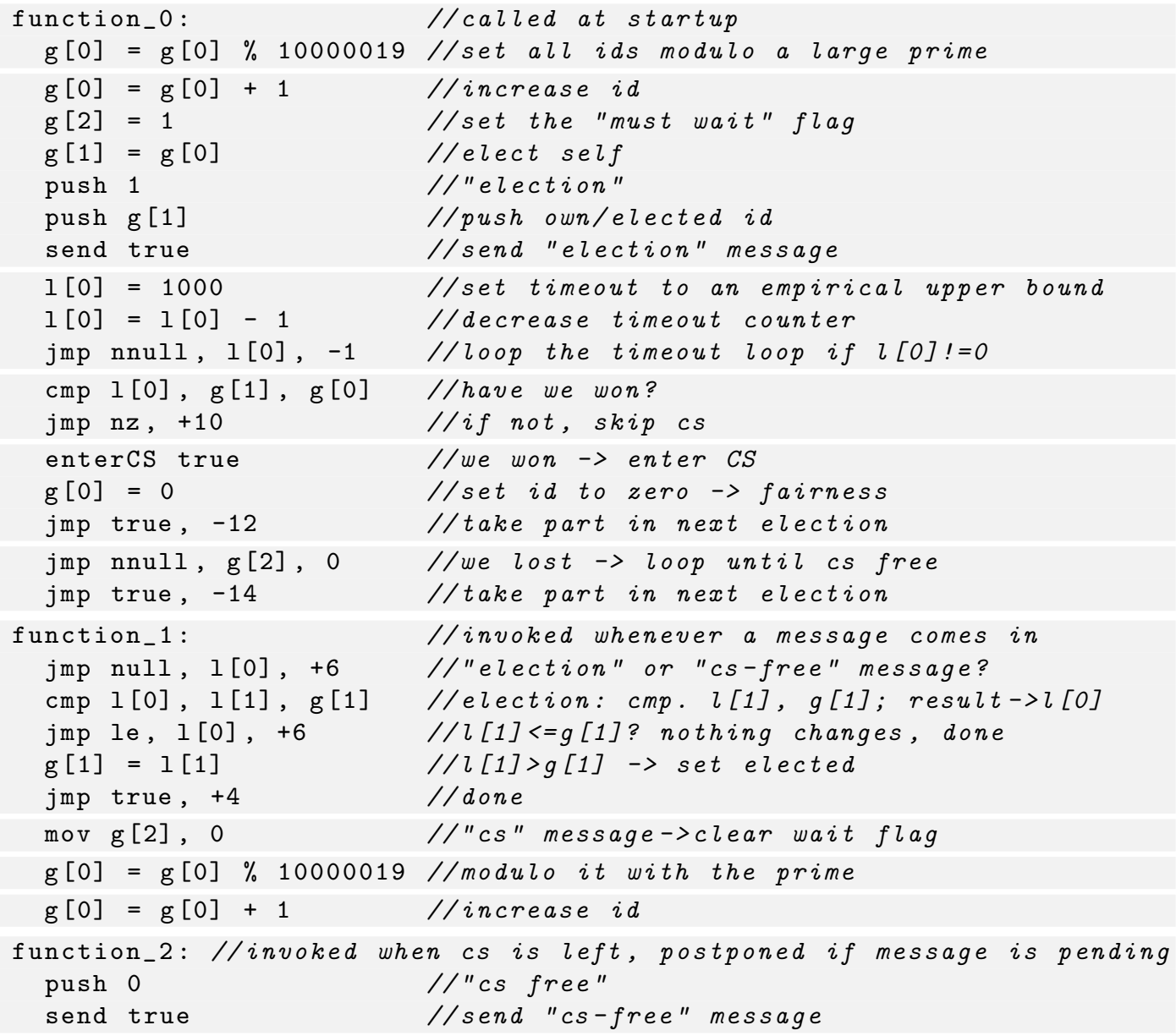

Listing B.1: A simple manually derived solution for the critical section problem in the fully connected topology (case $f c$ ).

\section{B.2 Manual Solution}

Finding a valid solution for the critical section problem with such limited resources is as hard as finding a solution for the election problem type $d$ ). In Listing B.1 we present a solution for the fully connected topology (case $f c$ ). The program basically performs an election for each time the critical section is free. In the election, all nodes compete and the one with the biggest ID wins. While the winner enters its critical section, the losers perform busy waiting in a loop until they receive notification that the critical section is being left. When the winner leaves its critical section, the additional automatically defined function is invoked, broadcasting the message cs-free. This is the notification the other nodes waited for - now they increase their ID by one and can leave the busy waiting loop. Then, all nodes again compete for entry in the critical section. After the second ADF has returned control, the execution resumes with the instructions after enterCS. Then, the node resets its own ID to zero in order to realize fairness.

Again, this solution is not really elegant since it relies on an upper bound for the communication delay and the fact that no ID must be greater than 10000019 (which can actually easily be realized). However, in the given scenario $f c$ ) where broadcasts can reach every node, this algorithm may score well if the upper bound is adjusted carefully. Also, it operates with only three cells of global and two cells of local memory which is less than provided 
by the configuration. We gave more memory, i. e., additional degrees of freedom, to the evolutionary algorithm in order to explore how they are utilized and whether they may help to create useful solutions.

\section{B.3 Further Results}

\section{B.3.1 Remaining Pareto Frontiers}

Here, the remaining Pareto frontiers of the critical section experiments with ring topology $(r$, Figure B.1) and disabled communication ( $n c$, Figure B.2) are plotted. The remaining diagrams for the fully meshed topology $f c$ are given in Section 8.5.2. 


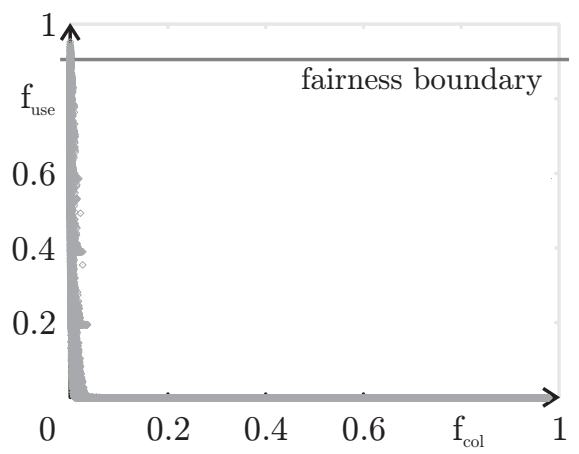

Fig. B.1.a: SGP

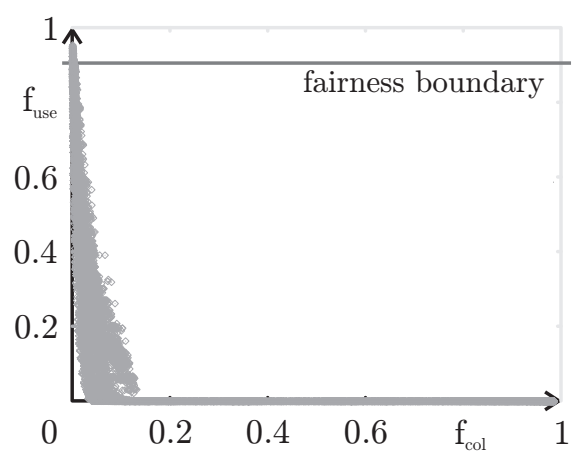

Fig. B.1.c: LGP

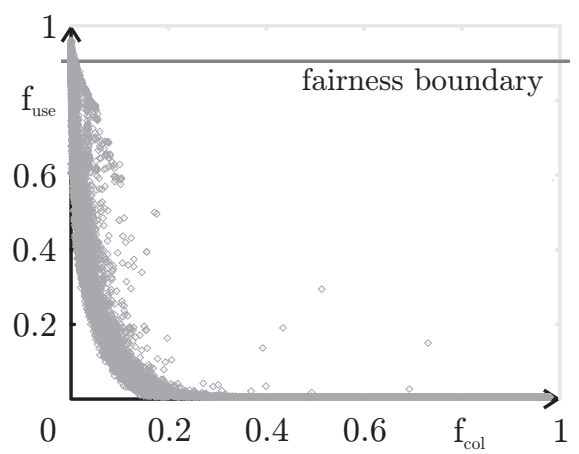

Fig. B.1.e: RBGP

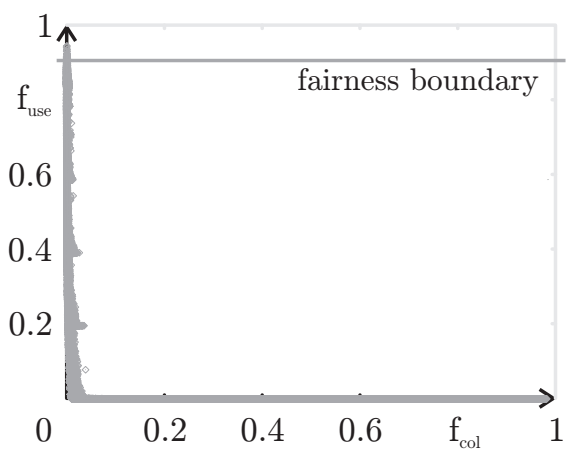

Fig. B.1.b: eSGP

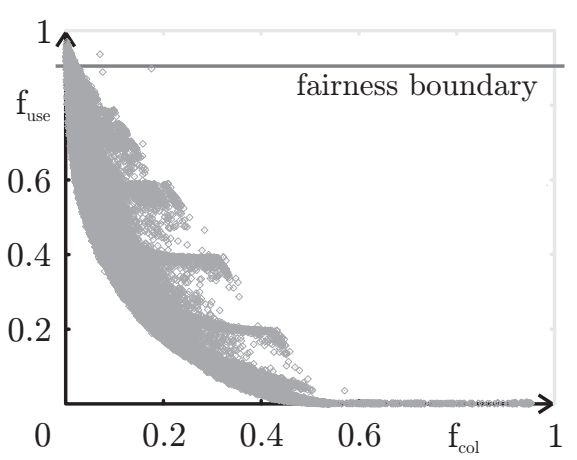

Fig. B.1.d: Fraglets

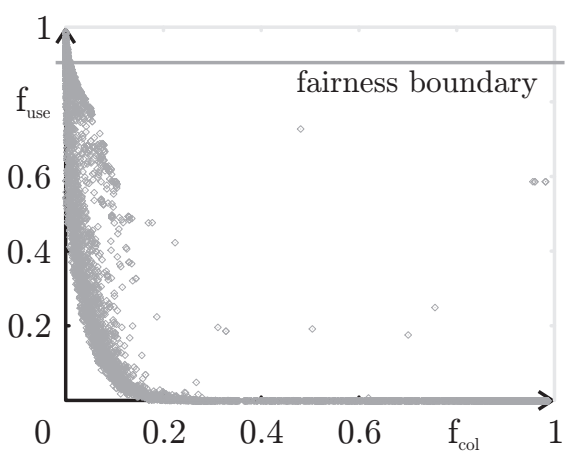

Fig. B.1.f: eRBGP

Figure B.1: The joint Pareto sets of all experiments of the GP approaches on the unidirectional ring $r$ ). 


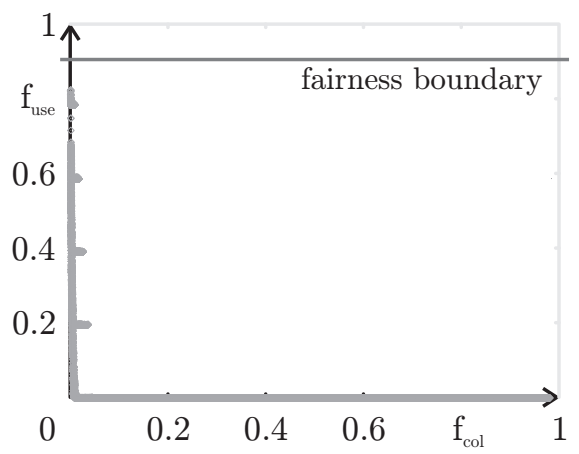

Fig. B.2.a: SGP

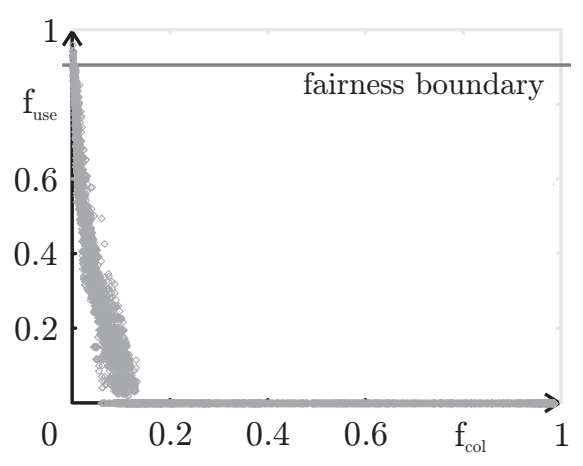

Fig. B.2.c: LGP

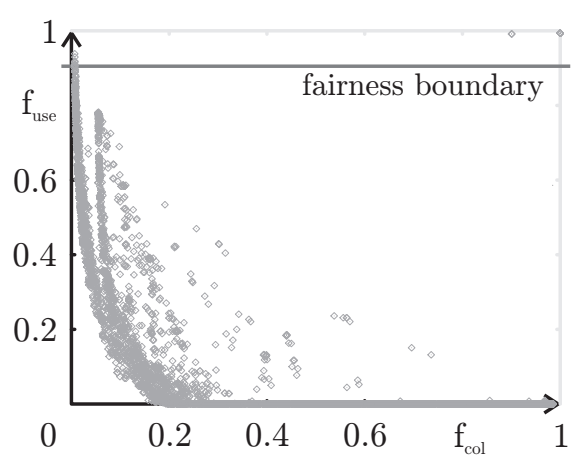

Fig. B.2.e: RBGP

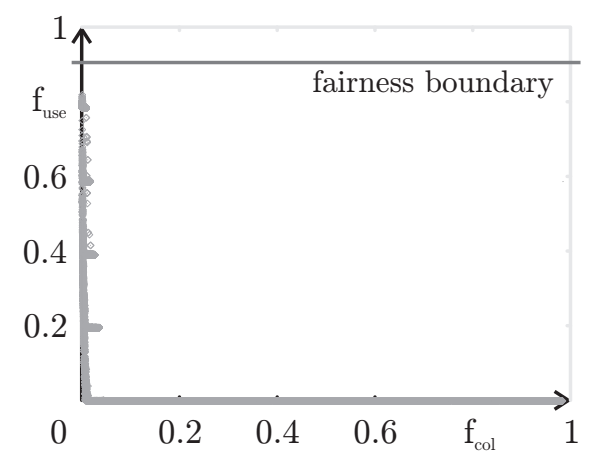

Fig. B.2.b: eSGP

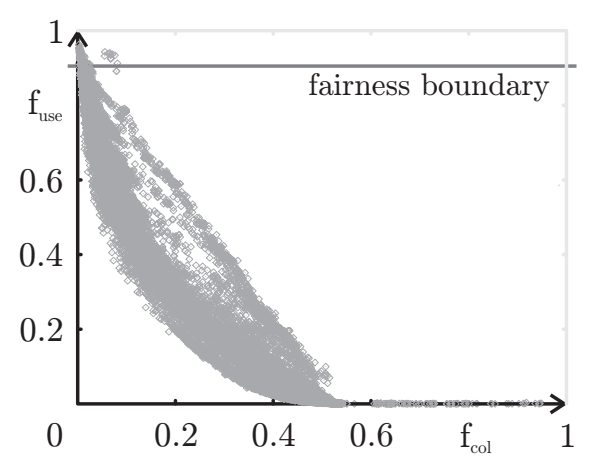

Fig. B.2.d: Fraglets

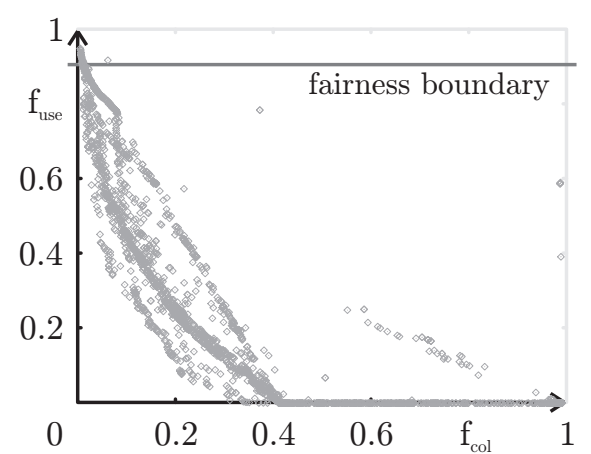

Fig. B.2.f: eRBGP

Figure B.2: The joint Pareto sets of all experiments of the GP approaches with disabled communication $n c)$. 


\section{B.3.2 Convergence Diagrams}

In Figure B.3 to Figure B.6, the remaining four convergence diagrams for the critical section diagrams are given. As described in Section 8.5.3 on page 133, we juxtapose the mean, median (med), and the $5 \%$ and $95 \%$ quantiles (Q.05, Q.95) of the $f_{\text {col }}$ and $f_{\text {use }}$ values of the best individuals in terms of $f_{c o l}$ over all runs for each generation.

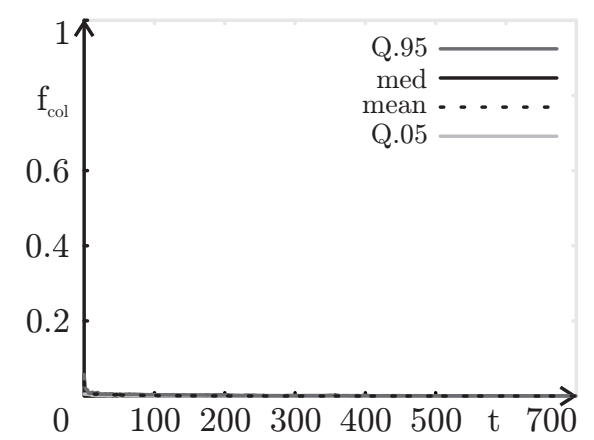

Fig. B.3.a: Convergence: SGP for $r$ and $f_{\text {col }}$.

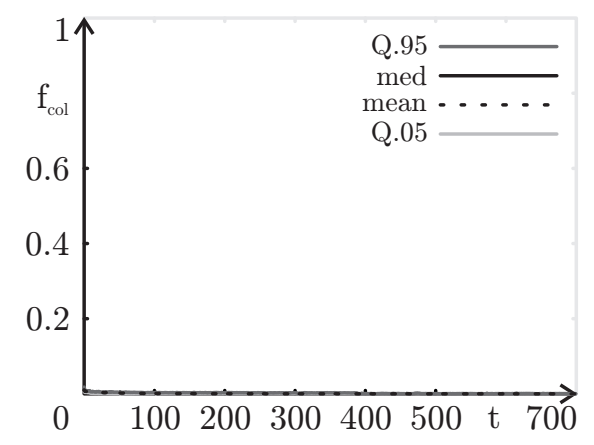

Fig. B.3.c: Convergence: eSGP for $r$ and $f_{\text {col }}$.

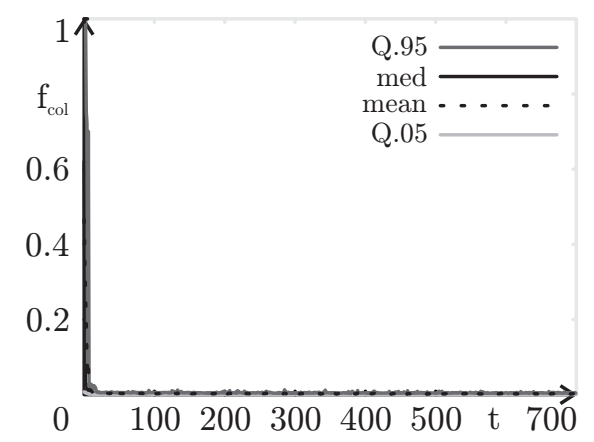

Fig. B.3.e: Convergence: LGP for $r$ and $f_{\text {col }}$.

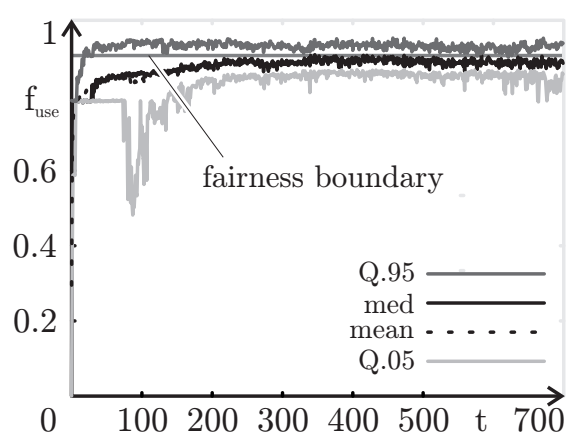

Fig. B.3.b: Convergence: SGP for $r$ and $f_{\text {use. }}$.

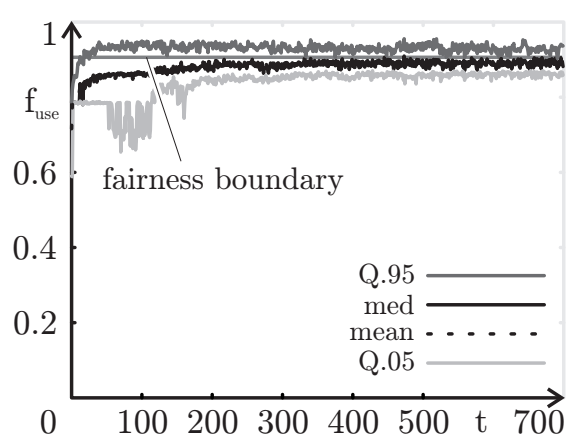

Fig. B.3.d: Convergence: eSGP for $r$ and $f_{\text {use. }}$

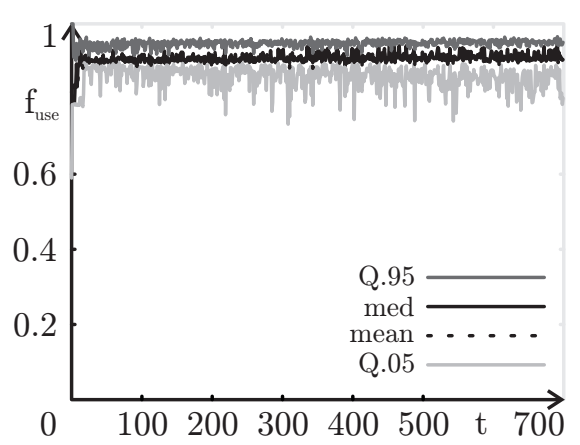

Fig. B.3.f: Convergence: LGP for $r$ and $f_{\text {use }}$.

Figure B.3: Convergence diagrams (I) for the critical section problem case $r$. 


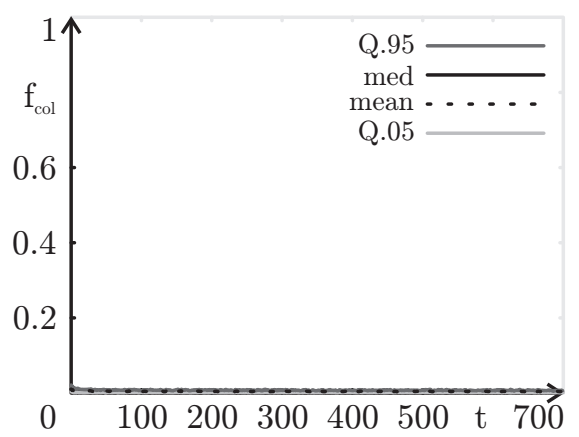

Fig. B.4.a: Convergence: Fraglets for $r$ and $f_{\text {col }}$.

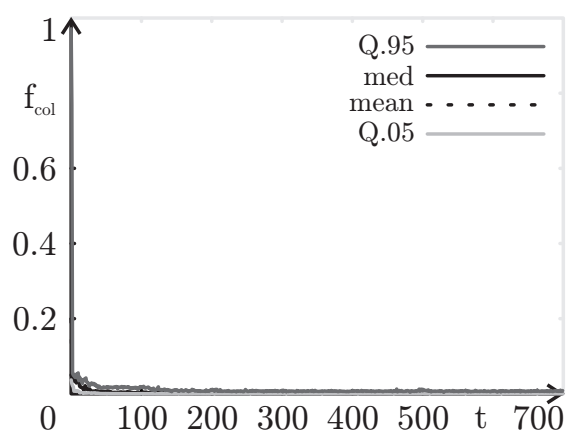

Fig. B.4.c: Convergence: RBGP for $r$ and $f_{\text {col }}$.

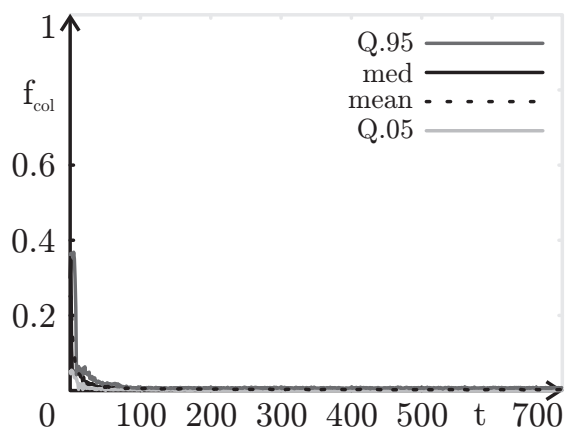

Fig. B.4.e: Convergence: eRBGP for $r$ and $f_{\text {col }}$.

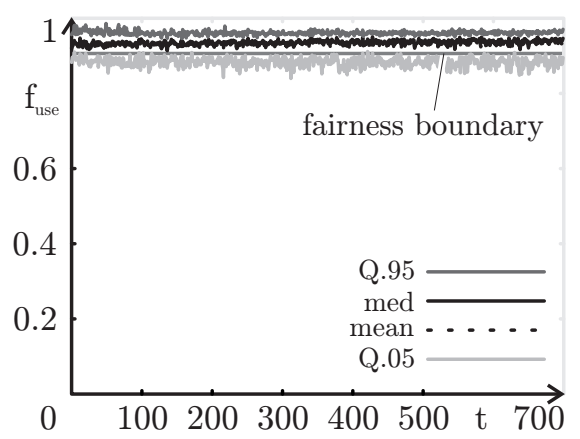

Fig. B.4.b: Convergence: Fraglets for $r$ and $f_{\text {use }}$.

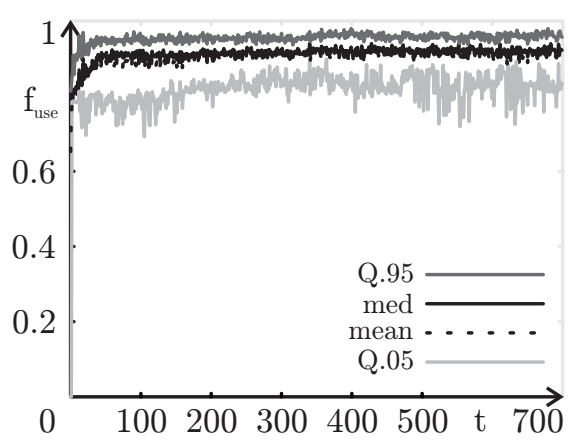

Fig. B.4.d: Convergence: RBGP for $r$ and $f_{\text {use. }}$.

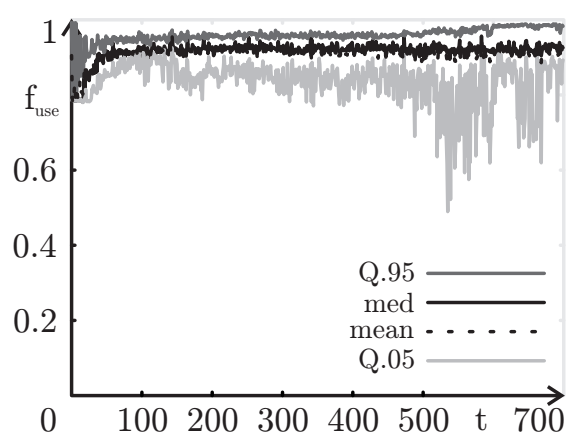

Fig. B.4.f: Convergence: eRBGP for $r$ and $f_{u s e}$.

Figure B.4: Convergence diagrams (II) for the critical section problem case $r$. 


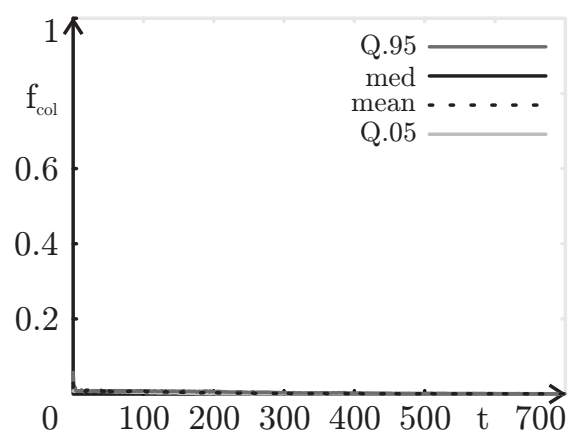

Fig. B.5.a: Convergence: SGP for $n c$ and $f_{\text {col }}$.

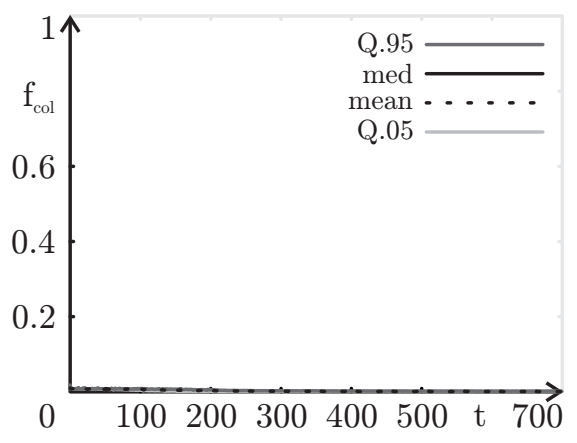

Fig. B.5.c: Convergence: eSGP for $n c$ and $f_{\text {col }}$.

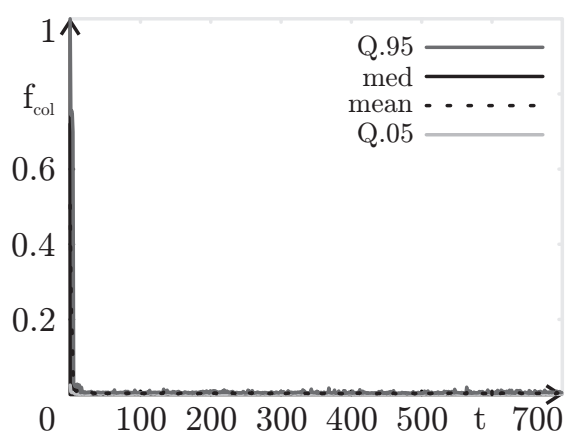

Fig. B.5.e: Convergence: LGP for $n c$ and $f_{\text {col }}$.

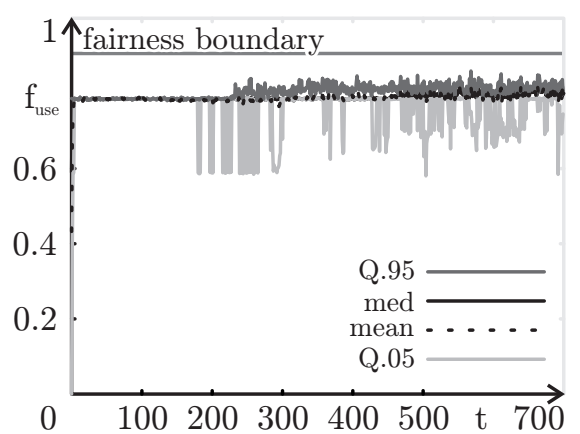

Fig. B.5.b: Convergence: SGP for $n c$ and $f_{\text {use }}$.

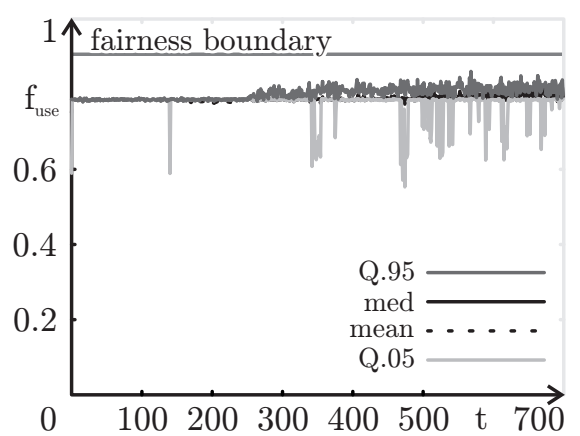

Fig. B.5.d: Convergence: eSGP for $n c$ and $f_{\text {use }}$.

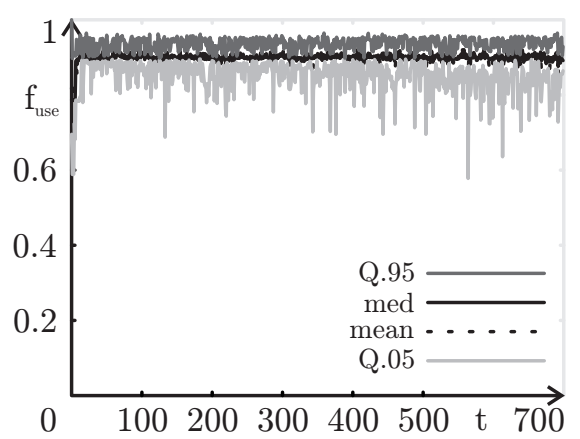

Fig. B.5.f: Convergence: LGP for $n c$ and $f_{\text {use. }}$.

Figure B.5: Convergence diagrams (I) for the critical section problem case $n c$. 


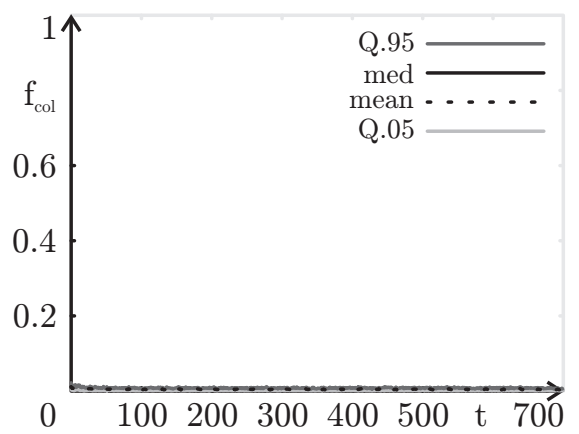

Fig. B.6.a: Convergence: Fraglets for $n c$ and $f_{\text {col }}$.

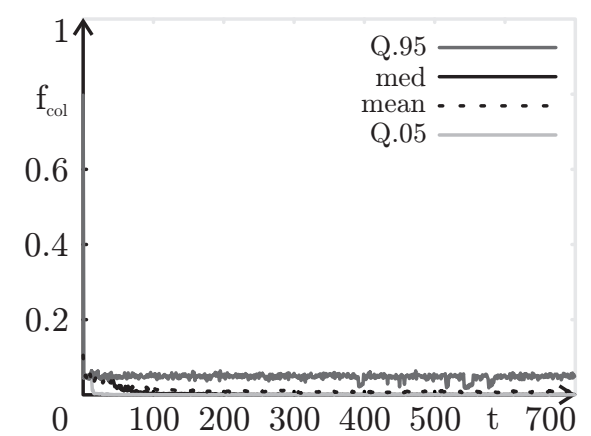

Fig. B.6.c: Convergence: RBGP for $n c$ and $f_{\text {col }}$.

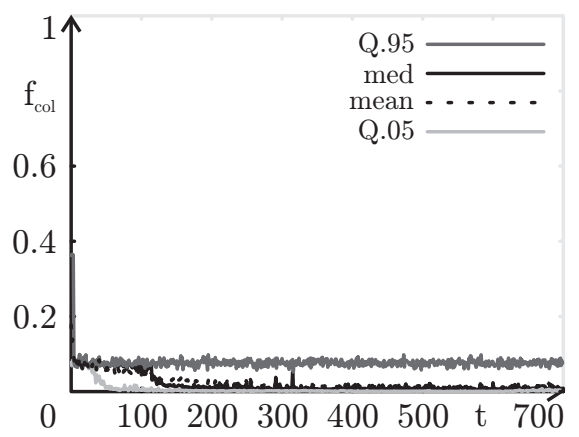

Fig. B.6.e: Convergence: eRBGP for $n c$ and $f_{\text {col }}$.

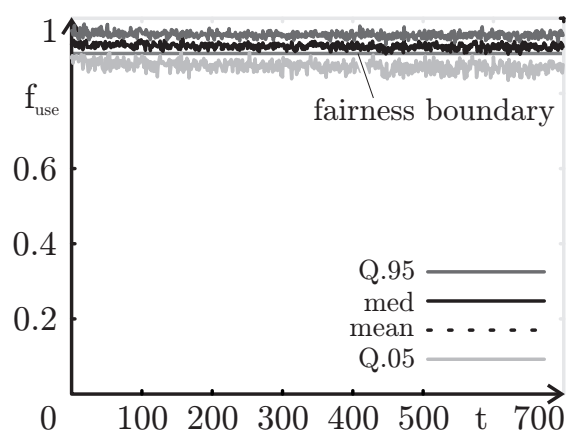

Fig. B.6.b: Convergence: Fraglets for $n c$ and $f_{\text {use }}$.

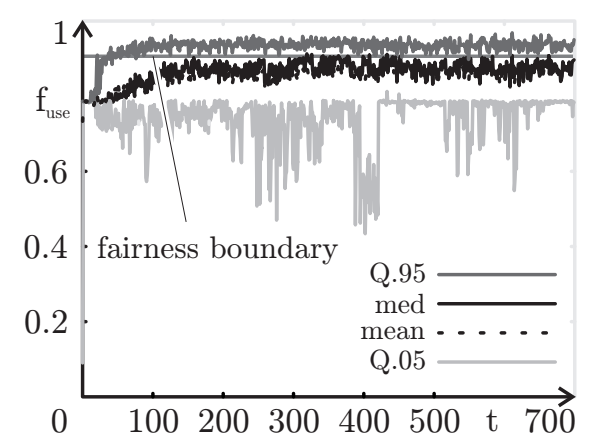

Fig. B.6.d: Convergence: RBGP for $n c$ and $f_{\text {use }}$.

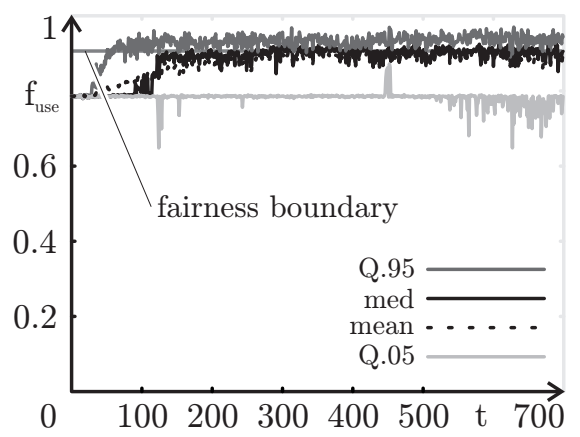

Fig. B.6.f: Convergence: eRBGP for $n c$ and $f_{u s e}$.

Figure B.6: Convergence diagrams (II) for the critical section problem case $n c$. 


\section{B.3.3 Statistical Evaluation}

We compare the ability to solve the critical section problem of the six Genetic Programming approaches according to

1. $\widetilde{f_{c o l}}$ (upper triangle) and $\left[\widetilde{f_{c o l}}\right]_{z}$ (lower triangle) for the fully connected topology $f c$ in Table B.2,

2. $\widetilde{f_{c o l}}$ (upper triangle) and $\left[\widetilde{f_{c o l}}\right]_{z}$ (lower triangle) for the ring topology $r$ in Table B.3, and

3. $\widetilde{f_{c o l}}$ (upper triangle) and $\left[\widetilde{f_{c o l}}\right]_{z}$ (lower triangle) for the scenario with disabled communication $n c$ in Table B.4

by again using a $\alpha=2 \%$ significance two-tailed Mann-Whitney $\mathrm{U}$ Test [192] via the tools provided by Lowry [182]. The graphical representation of these result tables can be found in Figure 8.5 on page 136. In Table B.5 to Table B.10, we furthermore use the two-tailed

\begin{tabular}{r|c|c|c|c|c|c}
\hline Case $f \boldsymbol{c}$ & SGP & eSGP & LGP & RBGP & eRBGP & Fraglets \\
\hline SGP & & 0 & 0 & + & + & + \\
eSGP & 0 & & 0 & + & + & + \\
LGP & - & - & & + & + & + \\
RBGP & - & - & - & & 0 & - \\
eRBGP & - & - & - & 0 & & - \\
Fraglets & - & - & - & + & + & \\
\hline
\end{tabular}

Table B.2: Comparison of $\widetilde{f_{c o l}}$ (upper triangle) and $\left[\widetilde{f_{c o l}}\right]_{z}$ (lower triangle) in the fully connected topology $f c$.

\begin{tabular}{r|c|c|c|c|c|c}
\hline Case $r$ & SGP & eSGP & LGP & RBGP & eRBGP & Fraglets \\
\hline SGP & & 0 & + & + & + & + \\
eSGP & 0 & & + & + & + & + \\
LGP & - & - & & - & 0 & + \\
RBGP & - & - & - & & 0 & + \\
eRBGP & - & - & 0 & + & & + \\
Fraglets & - & - & - & - & - & \\
\hline
\end{tabular}

Table B.3: Comparison of $\widetilde{f_{c o l}}$ (upper triangle) and $\left[\widetilde{f_{c o l}}\right]_{z}$ (lower triangle) in the ring topology $r$.

\begin{tabular}{r|c|c|c|c|c|c}
\hline Case $\boldsymbol{n} \boldsymbol{c}$ & SGP & eSGP & LGP & RBGP & eRBGP & Fraglets \\
\hline SGP & & 0 & + & 0 & + & + \\
eSGP & 0 & & + & 0 & + & + \\
LGP & - & - & & 0 & + & + \\
RBGP & - & - & - & & + & + \\
eRBGP & - & - & - & - & & 0 \\
Fraglets & - & - & - & - & + & \\
\hline
\end{tabular}

Table B.4: Comparison of $\widetilde{f_{c o l}}$ (upper triangle) and $\left[\widetilde{f_{c o l}}\right]_{z}$ (lower triangle) in case of disabled communication $n c$. 
Mann-Whitney $\mathrm{U}$ Test with $\alpha=0.02$ to test the influence of the communication scenario on the results of the evolution. We therefore compared the values of $\widetilde{f_{c o l}}$ (upper triangles) and $\left[\widetilde{f_{c o l}}\right]_{z}$ (lower triangles). The graphical representation of these tables can be found in Figure 8.6 on page 137.

\begin{tabular}{r|rrrr}
\hline SGP & fc & r & nc \\
\hline $\mathbf{f c}$ & & + & + \\
$\mathbf{r}$ & - & & + \\
$\mathbf{n c}$ & - & - & \\
\hline
\end{tabular}

Table B.5: Comparison of SGP in terms of the scenario and $\widetilde{f_{c o l}}$ (upper triangle) and $\left[\widetilde{f_{c o l}}\right]_{z}$ (lower triangle).

\begin{tabular}{r|rrr}
\hline eSGP & fc & $\mathbf{r}$ & $\mathbf{n c}$ \\
\hline $\mathbf{f c}$ & & + & + \\
$\mathbf{r}$ & - & & + \\
$\mathbf{n c}$ & - & - & \\
\hline
\end{tabular}

Table B.6: Comparison of eSGP in terms of the scenario and $\widetilde{f_{\text {col }}}$ (upper triangle) and $\left[\widetilde{f_{\text {col }}}\right]_{z}$ (lower triangle).

\begin{tabular}{r|rrr}
\hline LGP & fc & $\mathbf{r}$ & $\mathbf{n c}$ \\
\hline $\mathbf{f c}$ & & + & + \\
$\mathbf{r}$ & - & & 0 \\
$\mathbf{n c}$ & - & - & \\
\hline
\end{tabular}

Table B.7: Comparison of LGP in terms of the scenario and $\widetilde{f_{c o l}}$ (upper triangle) and $\left[\widetilde{f_{c o l}}\right]_{z}$ (lower triangle).

\begin{tabular}{r|rrr}
\hline Fraglets & fc & r & nc \\
\hline fc & & + & + \\
$\mathbf{r}$ & - & & 0 \\
nc & - & 0 & \\
\hline
\end{tabular}

Table B.8: Comparison of Fraglets in terms of the scenario and $\widetilde{f_{c o l}}$ (upper triangle) and $\left[\widetilde{f_{c o l}}\right]_{z}$ (lower triangle). 


\begin{tabular}{r|rrr}
\hline RBGP & fc & $\mathbf{r}$ & $\mathbf{n c}$ \\
\hline $\mathbf{f c}$ & & 0 & 0 \\
$\mathbf{r}$ & + & & 0 \\
$\mathbf{n c}$ & 0 & - & \\
\hline
\end{tabular}

Table B.9: Comparison of RBGP in terms of the scenario and $\widetilde{f_{\text {col }}}$ (upper triangle) and $\left[\widetilde{f_{\text {col }}}\right]_{z}$ (lower triangle).

\begin{tabular}{r|rrr}
\hline eRBGP & fc & $\mathbf{r}$ & $\mathbf{n c}$ \\
\hline $\mathbf{f c}$ & & + & + \\
$\mathbf{r}$ & 0 & & + \\
$\mathbf{n c}$ & - & - & \\
\hline
\end{tabular}

Table B.10: Comparison of eRBGP in terms of the scenario and $\widetilde{f_{\text {col }}}$ (upper triangle) and $\left[\widetilde{f_{\text {col }}}\right]_{z}$ (lower triangle). 



\section{The Distributed Greatest Common Divisor Experiments}

\section{C.1 Precise Settings}

With this section, we complement the discussion of the settings of the experiments intended to evolve algorithms which compute the greatest common divisor for a set of natural numbers in a distributed fashion given in Section 9.3 with a complete list of all parameters and their values.

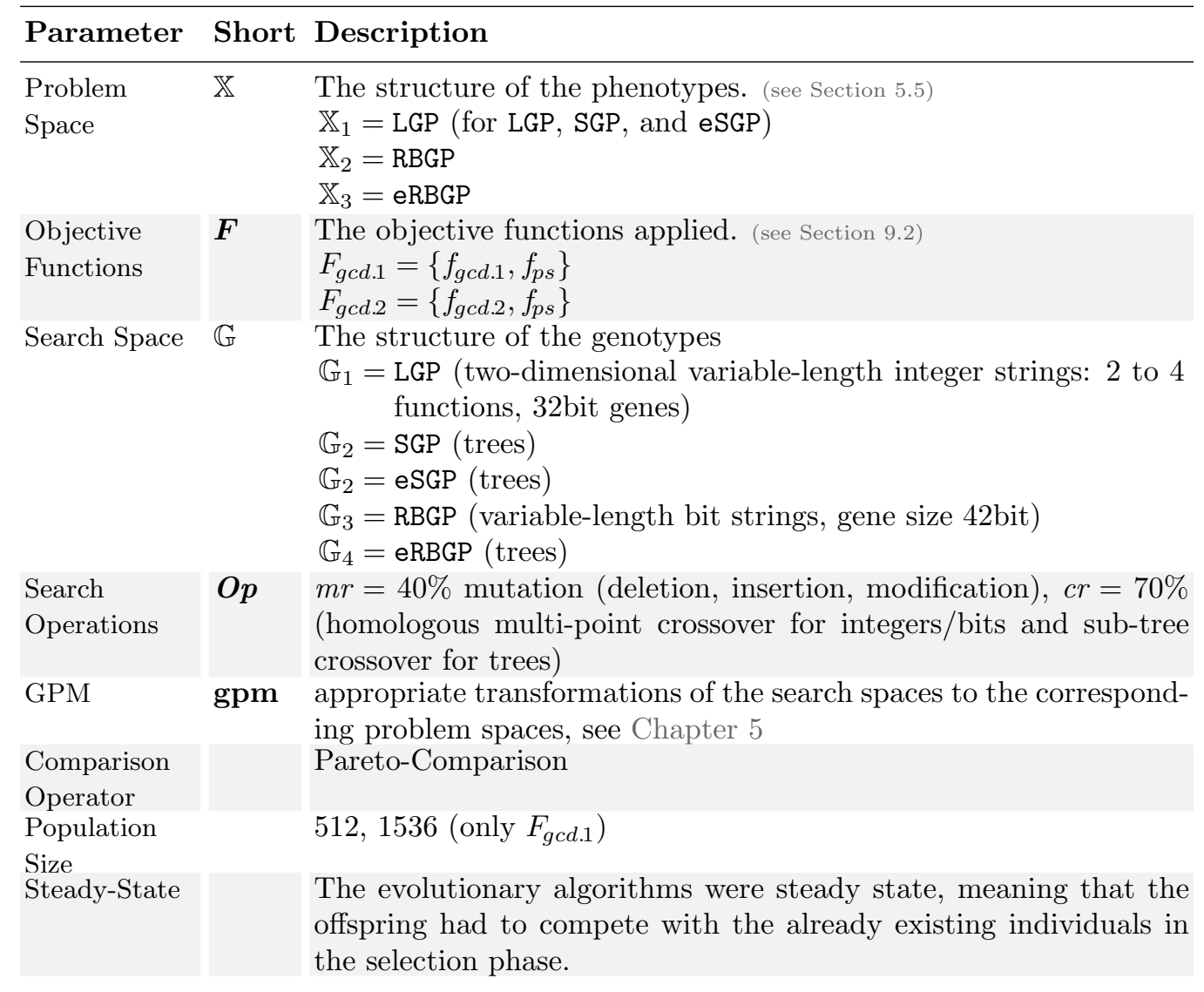


Fitness

Assignment

Algorithm

Selection

Algorithm

Convergence

Prevention

Generation

Limit

Number of $\quad \boldsymbol{t c}$

Training

Cases

Training Case

Change

Policy

minimum

number of

VMs

maximum

number of

VMs

minimum

latency

maximum

latency

topology

maximum

messages

maximum

message size

maximum

steps

\section{. \\ memory size}

n.num

\begin{tabular}{l|l} 
System & $\boldsymbol{C f g}$ \\
Configuration &
\end{tabular}

Configuration
Variety preserving fitness assignment. (see Section D.1.2)

A tournament selection with five contenstants was utilized.

$\boldsymbol{c p} \quad$ The simple convergence prevention mechanism was utilized with $c p=0.3$. (see Section D.2)

The maximum number of generations that each run is allowed to perform was 1000 . Runs with at least 750 generation were considered.

$c$ The number of training cases used for evaluating the objective functions was $t c=20$

ct The training cases were changed before each generation.

$\boldsymbol{m n} \boldsymbol{N} \quad m n N=4$

$\boldsymbol{m x} \boldsymbol{N} \operatorname{mn} N=20$

mnlat mnlat $=1$ steps

mxlat mxlat $=40$ steps

linear

1788 per node

$\boldsymbol{m} \boldsymbol{x} \boldsymbol{M S}$ The maximum message size is 2 for all approaches except Fraglets (where it is the maximum Fraglet size 15).

$\boldsymbol{m x} \boldsymbol{S}$ the maximum number of simulation steps

$m x S_{1}=40000$ for LGP

$m x S_{2}=120000$ for SGP

$m x S_{3}=120000$ for eSGP

$m x S_{4}=40000$ for Fraglets

$m x S_{5}=20000$ for RBGP

$m x S_{6}=20000$ for eRBGP

$\max M$ the memory size of the nodes

$\max _{1}=4$ words global memory, 4 words local memory, and 2 words stack for LGP, SGP, and eSGP

$\max _{2}=4$ multi-purpose words for RBGP and eRBGP

The initial number of a node was stored in the first global memory cell (LGP, SGP, eSGP), a dedicated symbol (RBGP, eRBGP), or available via a special Fraglet (Fraglets).

GNU/Linux 2.6.18-92.1.13.el5, x86_64 processor (AMD Opteron ${ }^{\mathrm{TM}}$ Processor 248, 2.1GHz), 4GB RAM, Java HotSpot ${ }^{\text {TM }}$ 64-Bit Server VM (build 1.6.0_01-b06, mixed mode)

Table C.1: The settings of the distributed greatest common divisor experiments.

\section{C.2 Manual Solution}

Before running the experiments, we again ensured that there exist solutions for the GCD problem which may be found during the evolutionary process. Listing C.1 illustrates how 


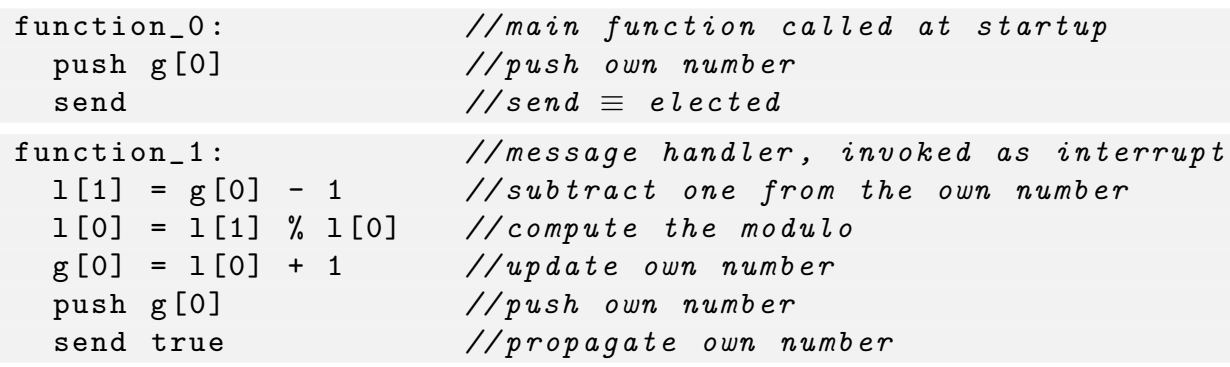

Listing C.1: A LGP version of Mattern's algorithm [193] given in Algorithm 9.1.

Mattern's algorithm [193] can be expressed in the LGP language. It becomes obvious that two global and local memory cells would be more than enough for such an algorithm. In the experiments, we used four cells in order to make the problem harder for Genetic Programming by providing unnecessary degrees of freedom.

\section{C.3 Further Results}

\section{C.3.1 Convergence Diagrams}

The diagrams in Figure C.1 show the mean, median (med) values, and the 5\% and 95\% quantile (Q.05, Q.95) of $f_{g c d .1}$ for each generation measured over all experimental runs for population size $p s=512$ and Figure C.2 provides the same diagrams for $f_{g c d .2}$. 


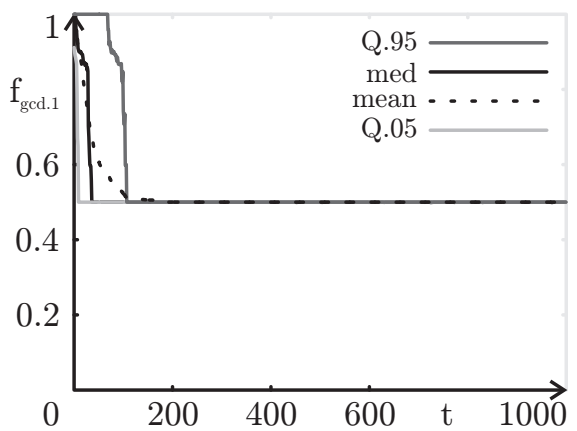

Fig. C.1.a: Convergence: SGP, $f_{g c d .1}$, $p s=512$.

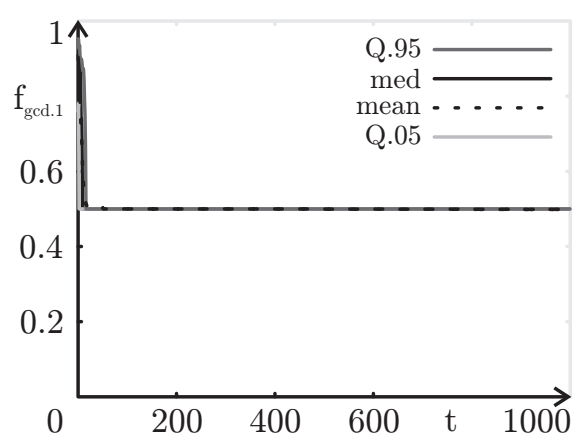

Fig. C.1.c: Convergence: LGP, $f_{g c d .1}$, $p s=512$.

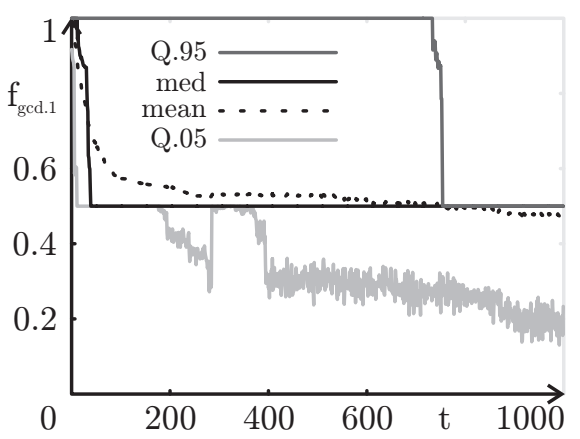

Fig. C.1.b: Convergence: eSGP, $f_{g c d .1}$, $p s=512$.

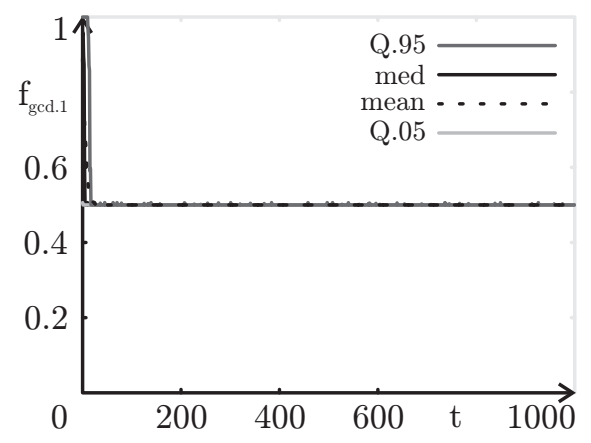

Fig. C.1.d: Convergence: RBGP, $f_{g c d .1}$, $p s=512$.

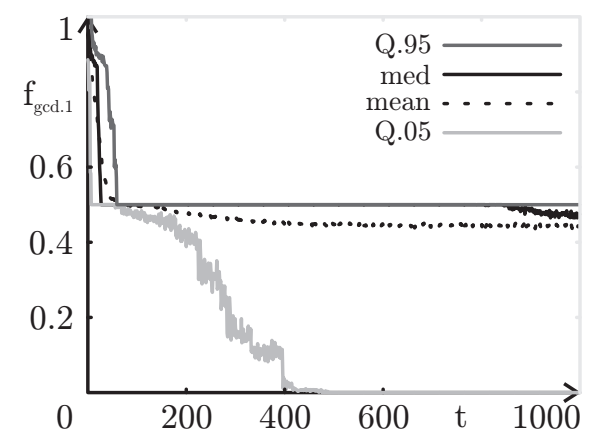

Fig. C.1.e: Convergence: eRBGP, $f_{g c d .1}$, $p s=512$.

Figure C.1: Convergence diagrams for the GCD problem $f_{g c d .1}$ and $p s=512$. 


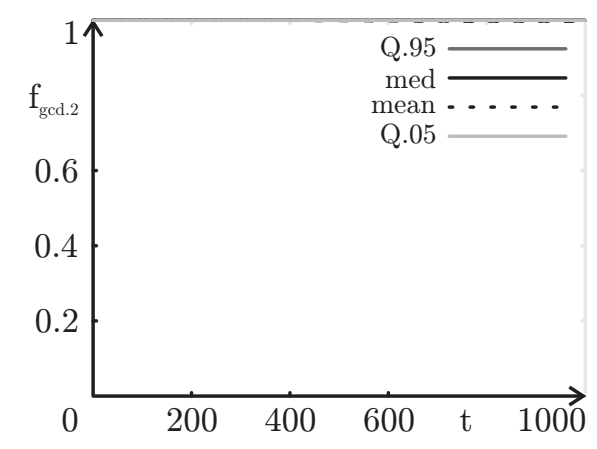

Fig. C.2.a: Convergence: SGP, $f_{g c d .2}$, $p s=512$.

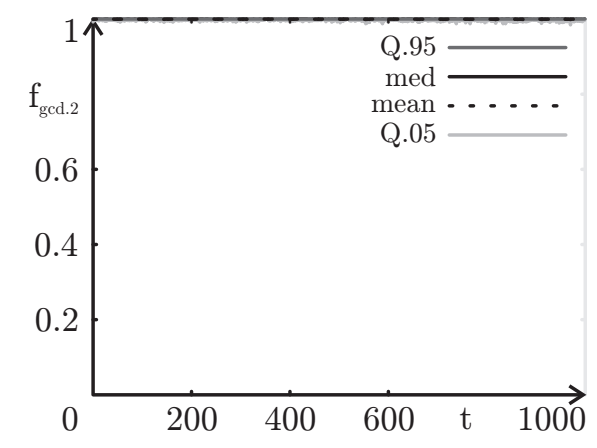

Fig. C.2.c: Convergence: RBGP, $f_{g c d .2}$, $p s=512$.

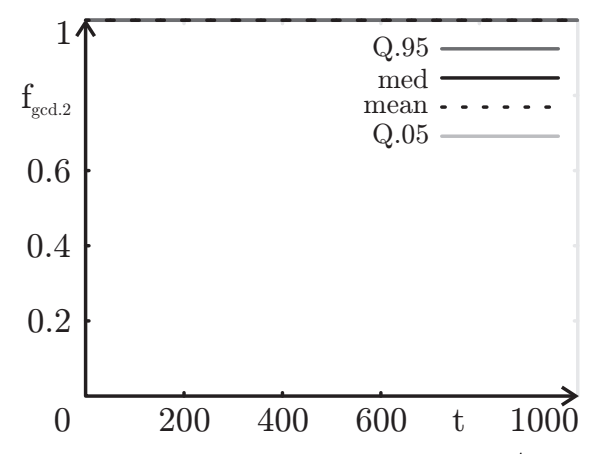

Fig. C.2.b: Convergence: eSGP/LGP, $f_{g c d .2}, p s=512$.

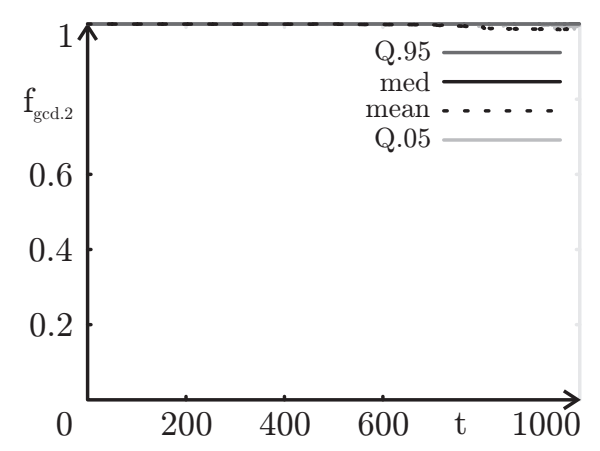

Fig. C.2.d: Convergence: eRBGP, $f_{g c d .2}$, $p s=512$.

Figure C.2: Convergence diagrams for the GCD problem $f_{g c d .2}$ and $p s=512$. 


\section{C.3.2 Statistical Evaluation}

Like in the election experiment, we have compared the success rates of the different Genetic Programming approaches with Fisher's exact test $[88,89]$ and the best values $\overline{f_{g c d .1}}$ of the functional objective functions were compared with the Mann-Whitney U Test [192]. For both tests, we again used the two-tailed variants with a significance level of $\alpha=0.02$. The results of the tests with the first objective function, $f_{g c d .1}$, are illustrated in Table C.2 for population size $p s=512$ and in Table C.3 for $p s=1536$.

\begin{tabular}{r|c|c|c|c|c}
\hline $\boldsymbol{F}_{\boldsymbol{g} \boldsymbol{c} \boldsymbol{d} . \mathbf{1}}$ & SGP & eSGP & LGP & RBGP & eRBGP \\
\hline SGP & & 0 & 0 & 0 & - \\
eSGP & 0 & & 0 & 0 & - \\
LGP & 0 & 0 & & 0 & - \\
RBGP & 0 & 0 & 0 & & - \\
eRBGP & 0 & 0 & 0 & 0 & \\
\hline
\end{tabular}

Table C.2: Comparison of $\overline{f_{g c d .1}}$ (upper triangle) and $s / r$ (lower triangle) for $F_{g c d .1}$ and ps $=512$.

\begin{tabular}{r|c|c|c|c|c}
\hline $\boldsymbol{F}_{\boldsymbol{g} \boldsymbol{c} \boldsymbol{d . 1}}$ & SGP & eSGP & LGP & RBGP & eRBGP \\
\hline SGP & & 0 & 0 & 0 & - \\
eSGP & 0 & & 0 & 0 & - \\
LGP & 0 & - & & 0 & - \\
RBGP & 0 & - & 0 & & - \\
eRBGP & + & 0 & + & + &
\end{tabular}

Table C.3: Comparison of $\overline{f_{g c d .1}}$ (upper triangle) and $s / r$ (lower triangle) for $F_{g c d .1}$ and $p s=1536$.

In Table C.4, we analyze whether increasing the population size from $p s=512$ to $p s=$ 1536 led to an improvement in the success rates of the GP approaches or their results in the functional objective functions. For the required comparisons, we again use Fisher's exact test and the Mann-Whitney $\mathrm{U}$ Test with a significance level of $\alpha=2 \%$.

\begin{tabular}{r|ccccc}
\hline & SGP & eSGP & LGP & RBGP & eRBGP \\
\hline $\boldsymbol{F}_{\boldsymbol{g} \boldsymbol{c d . 1} \mathbf{1}}$ & 0 & 0 & 0 & 0 & + \\
$\boldsymbol{s} / \boldsymbol{r}$ & 0 & 0 & 0 & 0 & 0 \\
\hline
\end{tabular}

Table C.4: Comparison between the two population sizes $p s=512$ and $p s=1536\left(F_{g c d .1}\right)$.

In Table C.5 we have performed the same tests as in Table C.2, this time for the experiments with $f_{g c d .2}$. In Table C.6, the success rates of the Genetic Programming approaches applied to the different objective functions for the distributed greatest common divisor problem are compared. 


\begin{tabular}{r|c|c|c|c|c}
\hline $\boldsymbol{F}_{\boldsymbol{g} \boldsymbol{c d . 2}}$ & SGP & eSGP & LGP & RBGP & eRBGP \\
\hline SGP & & 0 & 0 & 0 & 0 \\
eSGP & 0 & & 0 & 0 & 0 \\
LGP & 0 & 0 & & 0 & 0 \\
RBGP & 0 & 0 & 0 & & 0 \\
eRBGP & 0 & 0 & 0 & 0 & \\
\hline
\end{tabular}

Table C.5: Comparison of $\overline{f_{g c d .2}}$ (upper triangle) and $s / r$ (lower triangle) for $F_{g c d .2}$ and ps $=512$.

\begin{tabular}{l|ccccc}
\hline & SGP & eSGP & LGP & RBGP & eRBGP \\
\hline $\boldsymbol{F}_{\boldsymbol{g} \boldsymbol{c d} \text {.1 }}$ vs. $\boldsymbol{F}_{\boldsymbol{g} \boldsymbol{c d} . \mathbf{2}}$ & 0 & 0 & 0 & 0 & +
\end{tabular}

Table C.6: Comparison of the success rates $s / r$ of the different objective functions. 



\section{Diversity-Preserving Algorithms}

\section{D.1 Fitness Assignment Methods}

\section{D.1.1 Sharing Functions}

Pareto ranking does not incorporate any information about whether the solution candidates in the population reside closely to each other or in regions of the problem space which are only sparsely covered by individuals. Sharing, as a method for including such diversity information into the fitness assignment process, was introduced by Holland [125] and later refined by Deb [65], Deb and Goldberg [68], Goldberg and Richardson [107].

Definition D.1 (Sharing Function). A sharing function Sh : $\mathbb{R}^{+} \mapsto \mathbb{R}^{+}$is a function used to relate two individuals $p_{1}$ and $p_{2}$ to a value that decreases with their distance $d=$ $\operatorname{dist}\left(p_{1}, p_{2}\right)$ in a way that it is 1 for $d=0$ and 0 if the distance exceeds a specified constant $\sigma$.

Sharing functions can be employed in many different ways and are used by a variety of fitness assignment processes [107, 65]. For sharing, the distance of the individuals in the search space $\mathbb{G}$ as well as their distance in the problem space $\mathbb{X}$ or the objective space may be used. Often, the Euclidian distance measure dist $_{\text {eucl }}\left(F\left(p_{1} . x\right), F\left(p_{2} . x\right)\right)$ in the objective space is applied in sharing functions, but of course, any other distance measure could be applied as well.

Definition D.2 (Niche Count). The niche count $\mathrm{m}(p, P)[66,198]$ of an individual $p$ is the sum its sharing values with all individual in a list $P$.

The niche count $\mathrm{m}$ is always greater than zero, since $p \in P$ and, hence, $\operatorname{Sh}_{\sigma}(\operatorname{dist}(p, p))=1$ is computed and added up at least once. The original sharing approach was developed for single-objective optimization where only one objective function $f$ was subject to maximization. In this case, its value was simply divided by the niche count, punishing solutions in crowded regions [198]. The goal of sharing was to distribute the population over a number of different peaks in the fitness landscape, with each peak receiving a fraction of the population proportional to its height [134]. The results of dividing the fitness by the niche counts strongly depends on the height differences of the peaks and thus, on the complexity class of $f$. On $f_{1} \in \mathbf{O}(x)$, for instance, the influence of $\mathrm{m}$ is much bigger than on a $f_{2} \in \mathbf{O}\left(e^{x}\right)$.

\section{D.1.2 Variety Preserving Ranking}

Using sharing and the niche counts naïvely leads to more or less unpredictable effects. Of course, it promotes solutions located in sparsely populated niches but how much their fitness 
will be improved is rather unclear. Using distance measures which are not normalized can lead to strange effects, too. Imagine two objective functions $f_{1}$ and $f_{2}$. If the values of $f_{1}$ span from 0 to 1 for the individuals in the population whereas those of $f_{2}$ range from 0 to 10000 , the components of $f_{1}$ will most often be negligible in the Euclidian distance of two individuals in the objective space. Another problem is that the effect of simple sharing on the pressure into the direction of the Pareto frontier is not obvious either or depends on the sharing approach applied. Some methods simply add a niche count to the Pareto rank, which may cause non-dominated individuals having worse fitness than any others in the population. Other approaches scale the niche count into the interval $[0,1)$ before adding it which not only ensures that non-dominated individuals have the best fitness but also leave the relation between individuals at different ranks intact, which does not further variety very much.

Variety Preserving Ranking is a fitness assignment approach based on Pareto ranking using the domination relation and sharing [293]. We have developed it in order to mitigate all these previously mentioned side effects and balance the evolutionary pressure between optimizing the objective functions and maximizing the variety inside the population. In the following, we will describe the process of Variety Preserving Ranking-based fitness assignment which is defined in Algorithm D.1.

Before this fitness assignment process can begin, it is required that all individuals with infinite objective values must be removed from the population Pop. If such a solution candidate is optimal, i. e., if it has negative infinitely large objectives in a minimization process, for instance, it should receive fitness zero, since fitness is subject to minimization. If the individual is infeasible, on the other hand, its fitness should be set to len $(P o p)+$ $\sqrt{\operatorname{len}(P o p)}+1$, which is one larger than every other fitness values that may be assigned by Algorithm D.1.

In lines 2 to 8 , we create a list ranks which we use to efficiently compute the Pareto rank of every solution candidate in the population. The highest rank encountered in the population is stored in the variable maxRank. This value may be zero if the population contains only non-dominated elements. The lowest rank will always be zero since domination relations are non-circular by definition. We will use maxRank to determine the maximum penalty for solutions in an overly crowded region of the search space later on.

From line 9 to 17, we determine the maximum and the minimum values that each objective function takes on when applied to the individuals in the population. These values are used to store the inverse of their ranges in the array rangeScales, which we will use to scale all distances in each dimension (objective) of the individuals into the interval [0,1]. There are $|F|$ objective functions in $F$ and, hence, the maximum Euclidian distance between two solution candidates in the (scaled) objective space becomes $\sqrt{|F|}$. It occurs if all the distances in the single dimensions are 1.

The most complicated part of the Variety Preserving Ranking algorithm is between line 18 and 32. Here we computed the scaled distance from every individual to each other solution candidate in the objective space and use this distance to aggregate share values (in the array shares). Therefore, again two nested loops are needed (lines 21 and 23). The distance components of two individuals $P o p[i]$ and $P o p[j]$ are scaled and summarized in a variable dist in line 26. The Euclidian distance between them is $\sqrt{\text { dist }}$ which we use to determine a sharing value in 27 . We therefore have decided for exponential sharing with power 16 and $\sigma=\sqrt{|F|}$, as introduced in Equation D.1.

$$
\text { Sh_exp }_{\sigma, p}(d)=\left\{\begin{aligned}
1 & \text { if } d \leq 0 \\
0 & \text { if } d \geq \sigma \\
\frac{e^{-\frac{p d}{\sigma}}-e^{-p}}{1-e^{-p}} & \text { otherwise }
\end{aligned}\right.
$$




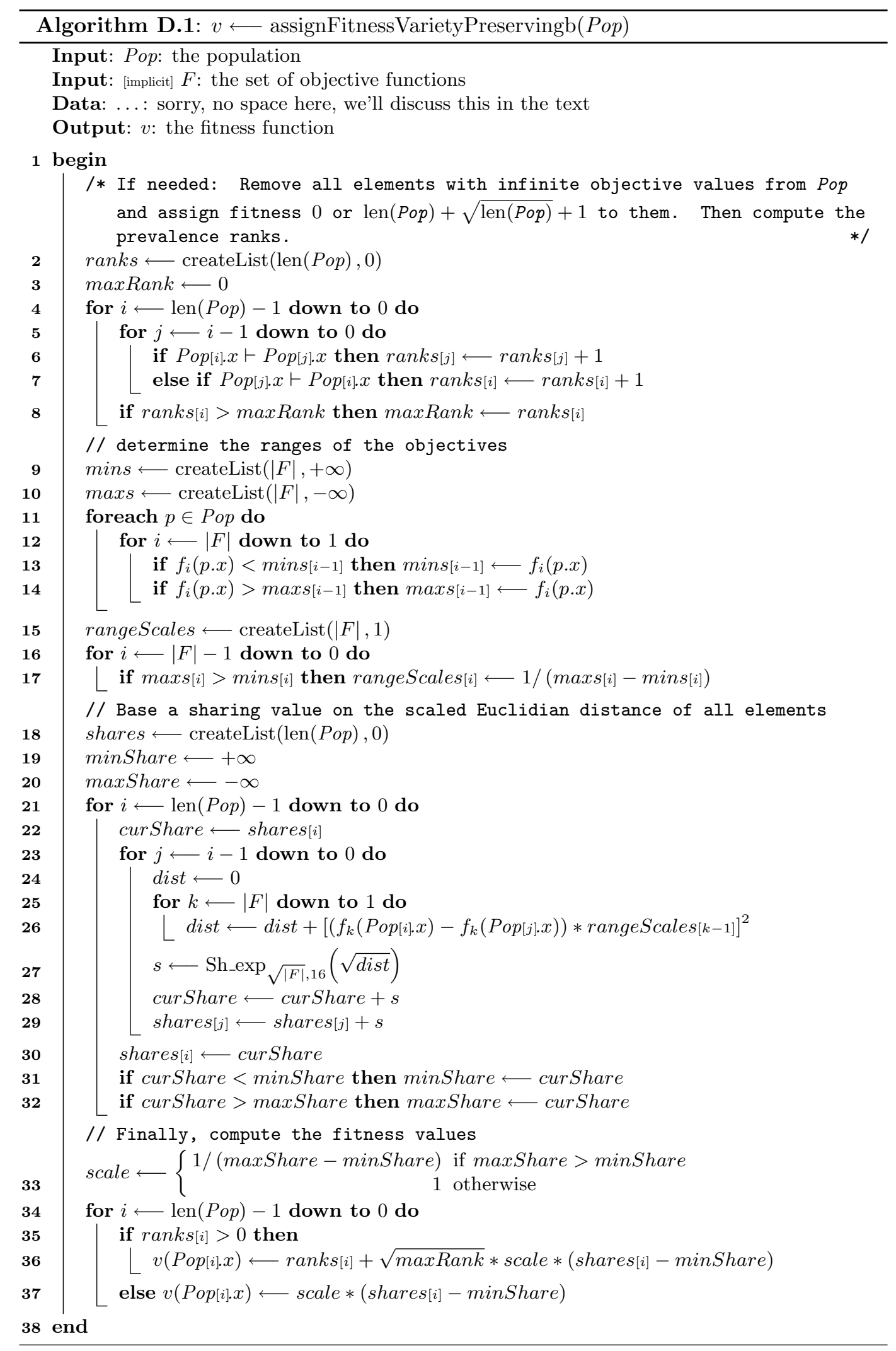


For every individual, we sum up all the shares (see line 29). While doing so, we also determine the minimum and maximum such total share in the variables minShare and max Share in lines 31 and 32.

We will use these variables to scale all sharing values again into the interval $[0,1]$ (line $33)$, so the individual in the most crowded region always has a total share of 1 and the most remote individual always has a share of 0 . So basically, we now know two things about the individuals in Pop:

1. their Pareto ranks, stored in the array ranks, giving information about their relative quality according to the objective values and

2. their sharing values, held in shares, denoting how densely crowded the area around them is.

With this information, we determine the final fitness values of an individual $p$ as follows: If $p$ is non-dominated, i. e., its rank is zero, its fitness is its scaled total share (line 37 ). Otherwise, we multiply the square root of the maximum rank, $\sqrt{\max R a n k}$, with the scaled share and add it to its rank (line 36). By doing so, we preserve the supremacy of nondominated individuals in the population but allow them to compete with each other based on the crowdedness of their location in the objective space. All other solution candidates may degenerate in rank, but at most by the square root of the worst rank.

\section{Example}

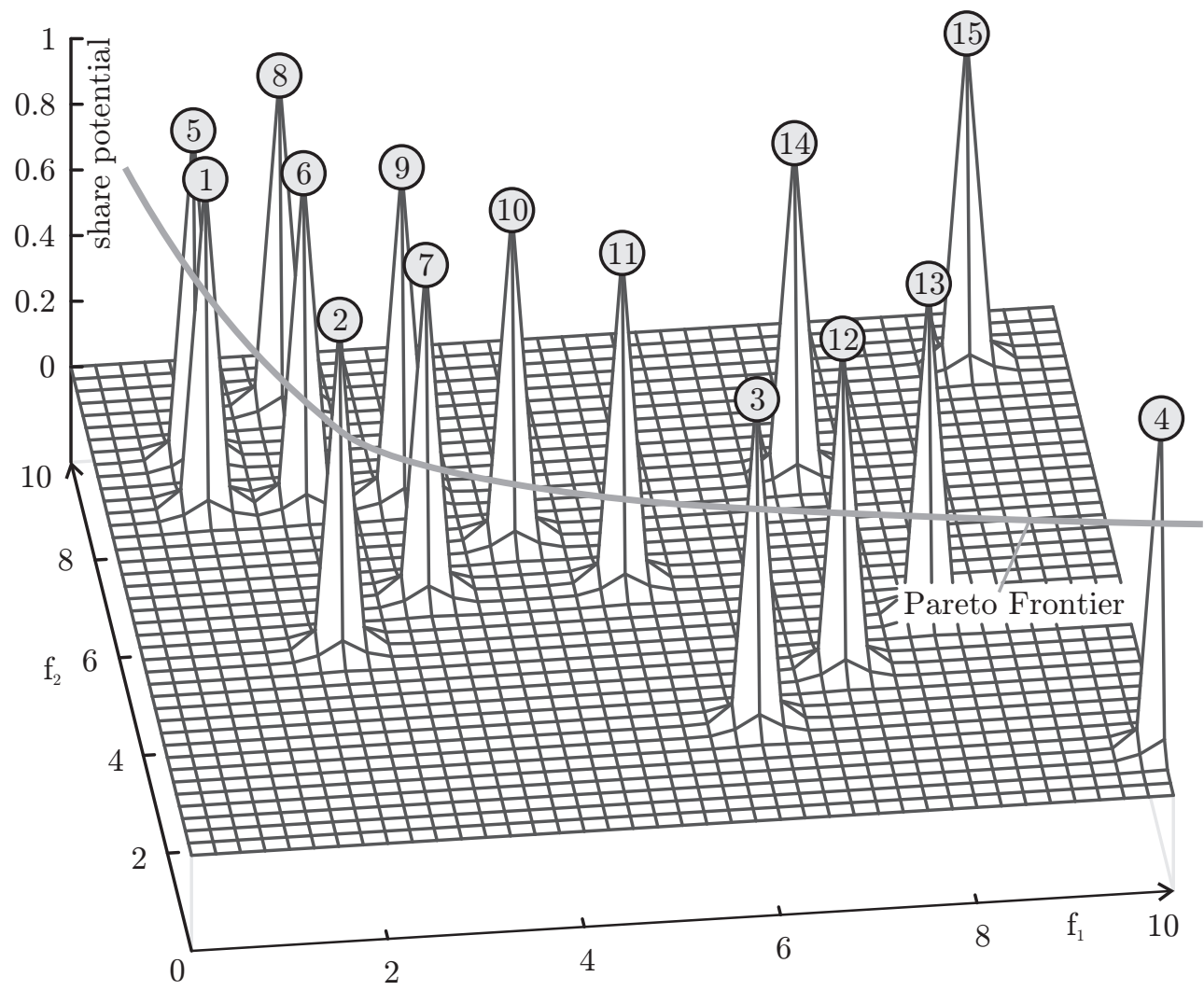

Figure D.1: The sharing potential in the Variety Preserving Ranking example. 
Let us now apply Variety Preserving Ranking to the examples for Pareto ranking from Section 2.2.2. In Table D.1, we again list all the solution candidates from Figure 2.3 on page 27 , this time with their objective values obtained with $f_{1}$ and $f_{2}$ corresponding to their coordinates in the diagram. In the third column, you can find the Pareto ranks of the individuals. The columns share/ $u$ and share/s correspond to the total sharing sums of the individuals, unscaled and scaled into $[0,1]$.

\begin{tabular}{rrrrrrrr}
\hline$x$ & $\boldsymbol{f}_{\mathbf{1}}$ & $\boldsymbol{f}_{\mathbf{2}}$ & rank & share/u & share $/ \mathbf{s}$ & $\boldsymbol{v}(\boldsymbol{x})$ \\
\hline 1 & 1 & 7 & 0 & 0.71 & 0.779 & 0.779 \\
2 & 2 & 4 & 0 & 0.239 & 0.246 & 0.246 \\
3 & 6 & 2 & 0 & 0.201 & 0.202 & 0.202 \\
4 & 10 & 1 & 0 & 0.022 & 0 & 0 \\
5 & 1 & 8 & 1 & 0.622 & 0.679 & 3.446 \\
6 & 2 & 7 & 2 & 0.906 & 1 & 5.606 \\
7 & 3 & 5 & 1 & 0.531 & 0.576 & 3.077 \\
8 & 2 & 9 & 4 & 0.314 & 0.33 & 5.191 \\
9 & 3 & 7 & 4 & 0.719 & 0.789 & 6.845 \\
10 & 4 & 6 & 2 & 0.592 & 0.645 & 4.325 \\
11 & 5 & 5 & 2 & 0.363 & 0.386 & 3.39 \\
12 & 7 & 3 & 1 & 0.346 & 0.366 & 2.321 \\
13 & 8 & 4 & 3 & 0.217 & 0.221 & 3.797 \\
14 & 7 & 7 & 9 & 0.094 & 0.081 & 9.292 \\
15 & 9 & 9 & 13 & 0.025 & 0.004 & 13.01 \\
\hline
\end{tabular}

Table D.1: An example for Variety Preserving Ranking based on Figure 2.3.

As already mentioned, we know the Pareto ranks of the solution candidates from Figure 2.3, so the next step is to determine the ranges of values the objective functions take on for the example population. These can again easily be found out from Figure 2.3. $f_{1}$ spans from 1 to 10 , which leads to rangeScale $[0]=1 / 9$. rangeScale $[1]=1 / 8$ since the maximum of $f_{2}$ is 9 and its minimum is 1 . With this, we now can compute the (dimensionally scaled) distances amongst the solution candidates in the objective space, the values of $\sqrt{\text { dist }}$ in algorithm Algorithm D.1, as well as the corresponding values of the sharing function Sh_exp $\sqrt{|F|}, 16(\sqrt{\text { dist }})$. We have noted these in Table D.2, using the upper triangle of the table for the distances and the lower triangle for the shares.

By summing up the single sharing values for each individual, we obtain the fifth column of Table D.2, the unscaled share values. Their minimum is around 0.022 and the maximum is 0.94 . Therefore, we must subtract 0.022 from each of these values and multiply the result with 1.131. By doing so, we build the column shares/s. Finally, we can compute the fitness values $v(x)$ according to lines 37 and 36 in Algorithm D.1.

The last column of Table D.1 lists these results. All non-dominated individuals have retained a fitness value less than one, lower than those of any other solution candidate in the population. However, amongst these best individuals, solution candidate 4 is strongly preferred, since it is located in a very remote location of the objective space. Individual 1 is the least interesting non-dominated one, because it has the densest neighborhood in Figure 2.3. In this neighborhood, the individuals 5 and 6 with the Pareto ranks 1 and 2 are located. They are strongly penalized by the sharing process and receive the fitness values $v(5)=3.446$ and $v(6)=5.606$. In other words, individual 5 becomes less interesting than solution candidate 7 which has a worse Pareto rank. 6 now is even worse than individual 8 which would have a fitness better by two if strict Pareto ranking was applied.

Based on these fitness values, algorithms like Tournament selection or fitness proportionate approaches will pick elements in a way that preserves the pressure into the direction of 
Upper triangle: distances. Lower triangle: corresponding share values.

\begin{tabular}{rrrrrrrrrrrrrrrrr} 
& $\mathbf{1}$ & $\mathbf{2}$ & $\mathbf{3}$ & $\mathbf{4}$ & $\mathbf{5}$ & $\mathbf{6}$ & $\mathbf{7}$ & $\mathbf{8}$ & $\mathbf{9}$ & $\mathbf{1 0}$ & $\mathbf{1 1}$ & $\mathbf{1 2}$ & $\mathbf{1 3}$ & $\mathbf{1 4}$ & $\mathbf{1 5}$ \\
\hline $\mathbf{1}$ & & 0.391 & 0.836 & 1.25 & 0.125 & 0.111 & 0.334 & 0.274 & 0.222 & 0.356 & 0.51 & 0.833 & 0.863 & 0.667 & 0.923 \\
$\mathbf{2}$ & 0.012 & & 0.51 & 0.965 & 0.512 & 0.375 & 0.167 & 0.625 & 0.391 & 0.334 & 0.356 & 0.569 & 0.667 & 0.67 & 0.998 \\
$\mathbf{3}$ & $7.7 \mathrm{E}-5$ & 0.003 & & 0.462 & 0.933 & 0.767 & 0.502 & 0.981 & 0.708 & 0.547 & 0.391 & 0.167 & 0.334 & 0.635 & 0.936 \\
$\mathbf{4}$ & $6.1 \mathrm{E}-7$ & $1.8 \mathrm{E}-5$ & 0.005 & & 1.329 & 1.163 & 0.925 & 1.338 & 1.08 & 0.914 & 0.747 & 0.417 & 0.436 & 0.821 & 1.006 \\
$\mathbf{5}$ & 0.243 & 0.003 & $2.6 \mathrm{E}-5$ & $1.8 \mathrm{E}-7$ & & 0.167 & 0.436 & 0.167 & 0.255 & 0.417 & 0.582 & 0.914 & 0.925 & 0.678 & 0.898 \\
$\mathbf{6}$ & 0.284 & 0.014 & $1.7 \mathrm{E}-4$ & $1.8 \mathrm{E}-6$ & 0.151 & & 0.274 & 0.25 & 0.111 & 0.255 & 0.417 & 0.747 & 0.765 & 0.556 & 0.817 \\
$\mathbf{7}$ & 0.023 & 0.151 & 0.003 & $2.9 \mathrm{E}-5$ & 0.007 & 0.045 & & 0.512 & 0.25 & 0.167 & 0.222 & 0.51 & 0.569 & 0.51 & 0.833 \\
$\mathbf{8}$ & 0.045 & 0.001 & $1.5 \mathrm{E}-5$ & $1.5 \mathrm{E}-7$ & 0.151 & 0.059 & 0.003 & & 0.274 & 0.436 & 0.601 & 0.933 & 0.914 & 0.609 & 0.778 \\
$\mathbf{9}$ & 0.081 & 0.012 & $3.3 \mathrm{E}-4$ & $4.8 \mathrm{E}-6$ & 0.056 & 0.284 & 0.059 & 0.045 & & 0.167 & 0.334 & 0.669 & 0.67 & 0.444 & 0.712 \\
$\mathbf{1 0}$ & 0.018 & 0.023 & 0.002 & $3.2 \mathrm{E}-5$ & 0.009 & 0.056 & 0.151 & 0.007 & 0.151 & & 0.167 & 0.502 & 0.51 & 0.356 & 0.67 \\
$\mathbf{1 1}$ & 0.003 & 0.018 & 0.012 & $2.1 \mathrm{E}-4$ & 0.001 & 0.009 & 0.081 & 0.001 & 0.023 & 0.151 & & 0.334 & 0.356 & 0.334 & 0.669 \\
$\mathbf{1 2}$ & $8 \mathrm{E}-5$ & 0.002 & 0.151 & 0.009 & $3.2 \mathrm{E}-5$ & $2.1 \mathrm{E}-4$ & 0.003 & $2.6 \mathrm{E}-5$ & 0.001 & 0.003 & 0.023 & & 0.167 & 0.5 & 0.782 \\
$\mathbf{1 3}$ & $5.7 \mathrm{E}-5$ & 0.001 & 0.023 & 0.007 & $2.9 \mathrm{E}-5$ & $1.7 \mathrm{E}-4$ & 0.002 & $3.2 \mathrm{E}-5$ & 0.001 & 0.003 & 0.018 & 0.151 & & 0.391 & 0.635 \\
$\mathbf{1 4}$ & 0.001 & 0.001 & 0.001 & $9.3 \mathrm{E}-5$ & $4.6 \mathrm{E}-4$ & 0.002 & 0.003 & 0.001 & 0.007 & 0.018 & 0.023 & 0.003 & 0.012 & 0.334 \\
$\mathbf{1 5}$ & $2.9 \mathrm{E}-5$ & $1.2 \mathrm{E}-5$ & $2.5 \mathrm{E}-5$ & $1.1 \mathrm{E}-5$ & $3.9 \mathrm{E}-5$ & $9.7 \mathrm{E}-5$ & $8 \mathrm{E}-5$ & $1.5 \mathrm{E}-4$ & $3.1 \mathrm{E}-4$ & 0.001 & 0.001 & $1.4 \mathrm{E}-4$ & 0.001 & 0.023 & \\
\hline
\end{tabular}

Table D.2: The distance and sharing matrix of the example from Table D.1.

the Pareto frontier but also leads to a balanced and sustainable variety in the population. The benefits of this approach have been shown, for instance, in [228, 305]. 


\section{D.2 Clearing and Simple Convergence Prevention (SCP)}

In our experiments not limited to this work, but especially in Genetic Programming and problems with discrete objective functions, we often used a very simple mechanism to prevent premature convergence (see Section 4.1) which we outline in Algorithm D.2. The idea of this $S C P$ method is simple: the more similar individuals we have in the population, the more likely are we converged. We do not know whether we have converged to a global optimum or to a local one. If we got stuck at a local optimum, we should maybe limit the fraction of the population which resides at this spot. In case we have found the global optimum, this approach does not hurt, because in the end, one single point on this optimum suffices.

The first one to apply such an explicit limitation method was Pétrowski [226] who divided the population of an EA into several sub-populations according to a distance measure applied in the genotypic $(\mathbb{G})$ or phenotypic space $(\mathbb{X})$ in each generation. Then, the fitness of all but the $k$ best individuals in such a niche is set to the worst possible value, which effectively prevents that the niche gets too crowded. Sareni and Krähenbühl [249] showed that this method is very promising.

We modified this approach in two respects: We measure similarity not in form of a distance in $\mathbb{G}$ or $\mathbb{X}$, but in the objective space $\mathbb{R}^{|F|}$. All individuals are compared with each other. If two have exactly the same objective values ${ }^{1}$, one of them is thrown away with probability ${ }^{2} c p \in[0,1]$ and does not take part in any further comparisons. This way, we weed out similar individuals without making any assumptions about $\mathbb{G}$ or $\mathbb{X}$ and make room in the population and mating pool for a wider diversity of solution candidates. For $c p=0$, this prevention mechanism is turned off, for $c p=1$, all remaining individuals will have different objective values.

Although this approach is very simple, the results of our experiments were often significantly better with this convergence prevention method turned on than without it [293, 228, 305]. Additionally, in none of our experiments, the outcomes were influenced negatively by this filter, which makes it even more robust than other methods for convergence prevention like sharing or variety preserving. Algorithm D.2, which has to be applied after the evaluation of the objective values of the individuals in the population and before any fitness assignment or selection takes place, specifies how our simple mechanism works.

If an individual $p$ occurs $n$ times in the population or if there are $n$ individuals with exactly the same objective values, Algorithm D.2 cuts down the expected number of their occurrences $S(p)$ to

$$
S(p)=\sum_{i=1}^{n}(1-c p)^{i-1}=\sum_{i=0}^{n-1}(1-c p)^{i}=\frac{(1-c p)^{n}-1}{-c p}=\frac{1-(1-c p)^{n}}{c p}
$$

In Figure D.2, we sketch the expected number of remaining instances of the individual $p$ after this pruning process if it occurred $n$ times in the population before Algorithm D.2 was applied.

From Equation D.2 follows that even a population of infinite size which has fully converged to one single value will probably not contain more than $\frac{1}{c p}$ copies of this individual after the simple convergence prevention has been applied. This threshold is also visible in Figure D.2.

$$
\lim _{n \rightarrow \infty} S(p)=\lim _{n \rightarrow \infty} \frac{1-(1-c p)^{n}}{c p}=\frac{1-0}{c p}=\frac{1}{c p}
$$

\footnotetext{
${ }^{1}$ The exactly-the-same-criterion makes sense in combinatorial optimization and many Genetic Programming problems but may easily be replaced with a limit imposed on the Euclidian distance in real-valued optimization problems, for instance.

${ }^{2}$ instead of defining a fixed threshold $k$
} 

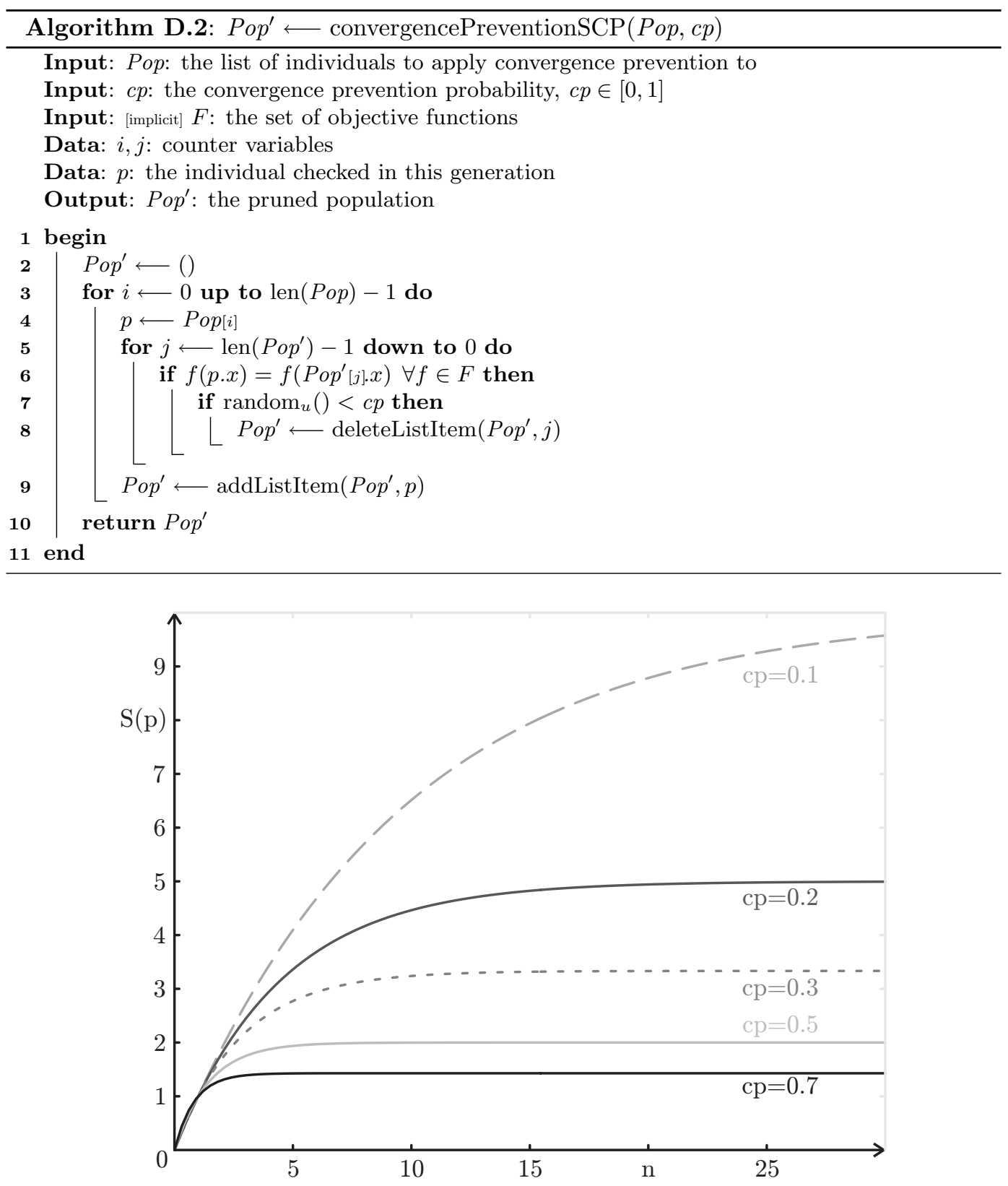

Figure D.2: The expected numbers of occurrences for different values of $n$ and $c p$.

In Pétrowski's clearing approach [226], the maximum number of individuals which can survive in a niche was a fixed constant $k$ and, if less than $k$ individuals resided in a niche, none of them would be affected. Different from that, an expected value of the number of individuals allowed in a niche is specified with the probability $c p$ and may be both, exceeded or undercut. Another difference of the approaches arises from the space in which the distance is computed. Whereas clearing prevents the EA from concentrating too much on a certain area in the search or problem space, SCP stops it from keeping too many individuals with equal utility. The former approach works against premature convergence to a certain solution structure while the latter forces the EA to "keep track" of a trail to 
solution candidates with worse fitness which may later evolve to good individuals with traits different from the currently exploited ones.

Which of the two approaches is better has not yet been tested with comparative experiments and is part of our future work not related to this thesis. At the present moment, we assume that in real-valued search or problem spaces, clearing should be more suitable whereas we know from experiments using our approach only that $S C P$ performs very good in combinatorial problems [228, 305] and Genetic Programming [293]. 



\section{Ausführliche Deutsche Zusammenfassung}

\section{E.1 Grundlagen}

\section{E.1.1 Einleitung}

\section{Motivation}

Verteilte Systeme sind eine der wichtigsten Komponenten der Wirtschaft. Sie bieten Kommunikationsinfrastrukturen sowohl weltweite mit dem Internet als auch lokal, in dem sie zum Beispiel verschiedene elektronische Module von Fahrzeugen miteinander verbinden. Praktisch jedes Computersystem ist entweder bereits Teil eines verteilten Systems oder wird zukünftig einer werden [273].

Während viele Internettechnologien, Protokolle und Applikationen in den letzten Jahren eine hohe Reife erreicht haben, sind kontinuierlich neue Formen verteilter Systeme aufgekommen. Dazu zählen zum Beispiel kabellose Netze, Sensornetze [139], kabellose Sensornetze [42], Smart Homes, ubiquitäre Systeme [82] und experimentelle Ideen wie Amorphous Computing [2].

Die Geräte, die in solchen Netzwerken miteinander interagieren, haben oftmals stark begrenzte Ressourcen wie beispielsweise wenig Speicher, geringe Rechenkraft und niedrige Batteriekapazität. Dazu kommen völlig neue Anforderungen wie Selbstadaption [102] und Selbstorganisation [101]. Der Entwickler eines Sensornetzes, zum Beispiel, muss sich nicht nur auf die funktionalen Aspekte wie Datenaggregation konzentrieren, sondern auch nichtfunktionale Aspekte wie Energieverbrauch während des gesamten Softwareentwurfsprozesses beachten.

Es wäre sicher vorschnell, wegen diesen neuen Herausforderungen direkt neue Programmierparadigmen zu fordern. Dennoch wird niemand abstreiten wollen, dass die Entwicklung von Anwendungen und Algorithmen für die neuen Klassen von verteilten Systemen komplizierter ist als für traditionelle Systeme. Daher kann das Erforschen des Nutzens neuer Programmiermethodiken zum aktuellen Zeitpunkt durchaus als notwendig betrachtet werden. Da Algorithmen die Grundlage aller Rechneranwendungen sind, betrifft dies auch das Erforschen neuer Repräsentationen für verteilte Algorithmen.

Definition E.1 (Entwurf eines verteilten Algorithmus). Wie in Definition 1.1 erklärt, ist der Entwurf eines verteilten Algorithmus die Transformation einer Spezifikation eines gewünschten Verhaltens eines Netzwerks als Ganzen in ein Programm das, wenn es lokal auf den einzelnen Knoten des Netzwerks ausgeführt wird, genau zu diesem globalen Verhalten führt.

In dieser Arbeit werden einige erste Schritte in Richtung der Automatisierung des in Definition E.1 (und Definition 1.1) beschriebenen Prozesses dargestellt. 

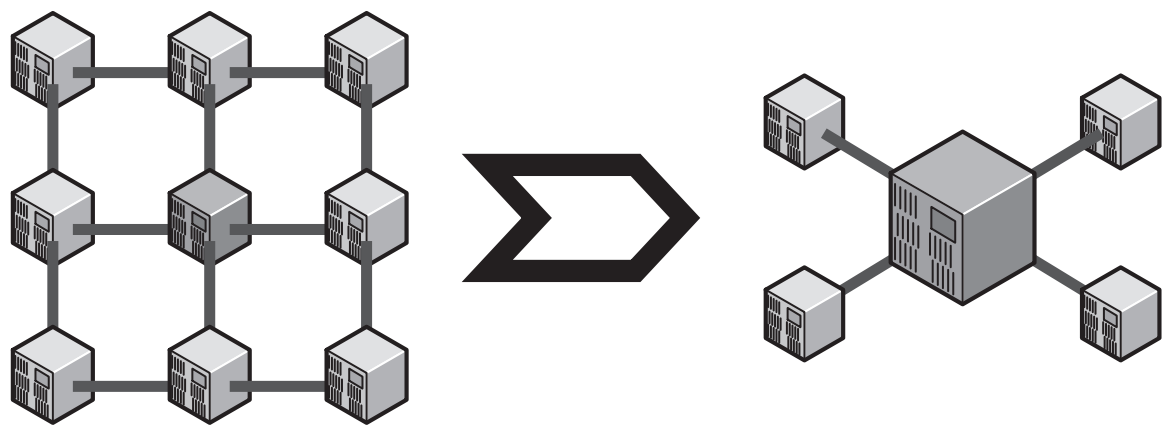

Abbildung E.1: Design von verteilten Algorithmen.

Die Transformation von globalem Systemverhalten zu lokalen Regeln, wie in Abbildung E.1 (bzw. Figure 1.1) dargestellt, ist kein Prozess der ausschließlich den Entwurf verteilter Systeme betrifft. Eine sehr breit erforschte Instanz davon sind zum Beispiel Schwarmverhalten [242] in der Natur, die die Evolution in Millionen von Jahren hervorgebracht hat.

In dem sie sich gemeinsam in einer Konfiguration fortbewegen, die ein gutes Verhältnis von Volumen zu Oberfläche aufweist, können die Individuen einer Spezies ihre Abwehr gegen Jäger stärken, die Wahrscheinlichkeit, Futter und Fortpflanzungspartner zu finden, erhöhen und die Hydro- bzw. Aerodynamik verbessern. Viele verschiedene Arten wie Fische, Vögel und Heuschrecken haben solche Verhaltensformen, wie sie in Abbildung E.2 und Figure 1.2 dargestellt werden, hervorgebracht [219].

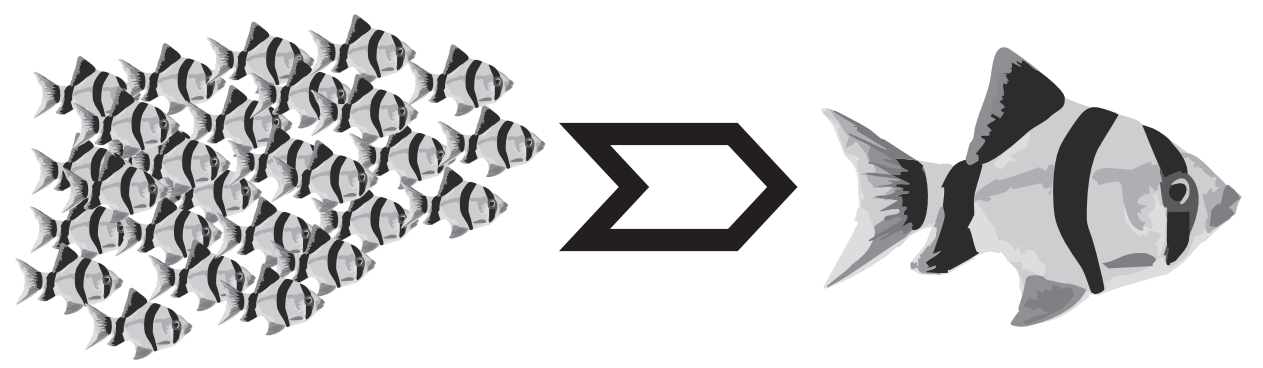

Abbildung E.2: Evolutionäres Design von Verhalten.

\begin{abstract}
Ansatz
Evolutionäre Algorithmen (EAs, engl. evolutionary algorithms) kopieren die natürliche Evolution um schwierige Optimierungsaufgaben zu lösen [9, 293]. In dieser Arbeit nutzen wir ein Genetische Programmierung (GP, engl. Genetic Programming) genanntes Verfahren [232, 293], eine Klasse von evolutionären Algorithmen, um verteilte Algorithmen zu züchten. In anderen Worten, wir nutzen die Genetische Programmierung um Beschreibungen globalen Systemverhaltens in lokale auszuführende Programme zu überführen.

Abbildung E.3 (und Figure 1.3) illustriert diese neuen Entwurfsmethodik für verteilte Algorithmen. Zuerst wird das gewünschte Systemverhalten spezifiziert. Zielfunktionen die bewerten, wie nahe das Verhalten eines (gezüchteten) Programms dem spezifizierten Verhalten kommt, werden manuell von der Spezifikation abgeleitet. Um die Qualität der "gewachsenen" Programmen zu bewerten, werden diese auf den simulierten Knoten eines simulierten
\end{abstract}


Netzwerks ausgeführt. Wir wenden eine Mehrzieloptimierung (multi-objective Genetic Programming) an, da diese es uns ermöglicht, Algorithmen sowohl hinsichtlich Funktionalität als auch nichtfunktionaler Aspekte wie Programmgröße, Speicherverbrauch und minimale Kommunikation zu optimieren.

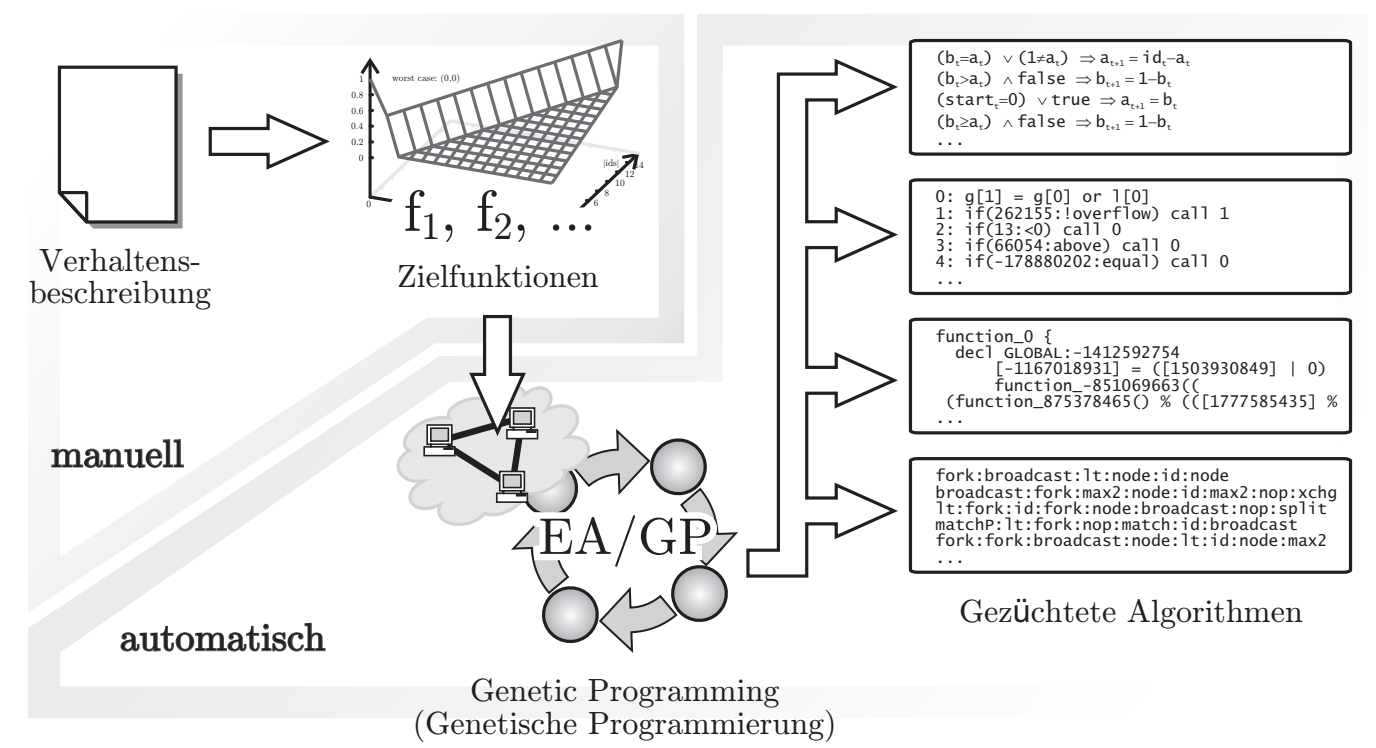

Abbildung E.3: Der in dieser Arbeit verfolgte Ansatz.

Als Teil unserer Forschungsarbeit, wurde ein allgemeines Softwarepaket für Optimierung $\left(\right.$ Sigoa $\left.^{1}\right)$ implementiert und eine Erweiterungsbibliothek für Genetische Programmierung $\left(\mathrm{DGPF}^{2}\right)$ entwickelt. Der Kern dieser Dissertation ist die Evaluierung des soeben vorgestellten Ansatzes mit statistisch signifikanten Experimenten mit Hilfe dieser beiden Softwarepakete.

\section{Wichtigste Ergebnisse}

Die in dieser Dissertation dokumentierte Forschungsarbeit hat zu den folgenden vier wichtigen Ergebnissen geführt:

1. Ein neuer Ansatz für den Entwurf von Programmen für verteilte Systeme wurde auf der Basis von evolutionären Algorithmen und Genetischer Programmierung definiert.

2. Sechs verschiedene Methoden (Programmrepräsentationen), welche für die Umsetzung dieses Ansatz genutzt werden können, wurden an drei verschiedenen Programen getestet.

3. Die Effizienz unseres Ansatzes wird durch eine Vielzahl von Aspekten, welche die Evolution (verteilter) Programme erschweren, bedroht. Wir haben diese Aspekte sehr gründlich diskutiert und eine Menge von Gegenmaßnahmen aufgeführt.

4. Zwei der sechs Programmrepräsentationen (RBGP, eRBGP) wurden speziell unter Berücksichtigung der problematischen Aspekten entwickelt. Einer der beiden Ansätze (eRBGP) zeigt ein auffällig besseres Verhalten im Vergleich zu den anderen Techniken in einem Großteil der Experimente.

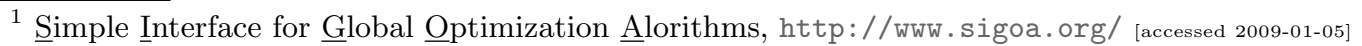

${ }^{2}$ Distributed Genetic Programming Framework, auch verfügbar unter http://www.sigoa.org/ 


\section{Struktur}

Diese Dissertation ist in drei Teile untergliedert. Die Grundlagen des Fachgebiets und der involvierten Techniken werden im ersten Teil diskutiert. Dies beinhaltet Grundlagen von metaheurstischer Optimierung (Chapter 2) und verwandte Arbeiten über deren Anwendung auf verteilte Systeme (Chapter 3). Aus der Analyse der verwandten Arbeiten wird klar, dass bisher nur wenig Forschung betrieben wurde, die sich speziell auf die Evolution verteilter Algorithmen konzentriert, speziell auf dem Gebiet asynchroner Systeme.

Die wichtigsten Ergebnisse unserer Forschung sind in Part II enthalten. In Chapter 4 untersuchen wir Gründe, die gegen das Synthetisieren von verteilten Algorithmen mittels Genetischer Programmierung sprechen: eine Menge von problematischen Aspekten, die die Evolution verteilter Algorithmen besonders schwierig machen. Eines der Ziele unserer Forschung ist es, Programmrepräsentationen zu finden, die von diesen Problemen nicht betroffen sind oder sie zumindest abschwächen können. Darum analysieren wir sechs verschiedene GP-Techniken:

1. das "normale", baumbasierte Standard-GP (SGP, Section 5.1),

2. eine erweiterte Form davon (eSGP, Section 5.2),

3. Lineare Genetische Programmierung (LGP, Section 5.3),

4. Fraglets (Section 5.4),

5. Regelbasierte Genetische Programmierung (RBGP, Section 5.5), und

6. eine erweiterte Form davon (eRBGP, Section 5.6).

SGP und LGP basieren beide auf allgemein bekannte Methoden, die mit drei Formen von Speicher angereichert wurden, um sie für die Evolution verteilter Algorithmen anzupassen. eSGP ist eine Erweiterung von SGP mit indirekt adressierbarem Speicher. Die FragletsMethode stammt von biologisch-inspirierter Forschun. RBGP und eRBGP wurden von uns speziell im Hinblick auf die auf die problematischen Aspekte von Chapter 4 entworfen.

Das Modell für verteilte Systeme, welches in unserer Forschung verwendet wurde, ist in Chapter 6 definiert. Die sechs Ansätze wurden auf drei verschiedene Beispieldomänen angewandt: (verteilte) Auswahlalgorithmen (Chapter 7), wechselseitigen Ausschluss am (verteilten) kritischen Abschnitt (Chapter 8) und die (verteilte) Berechnung des größten gemeinsamen Teilers (GGT, Chapter 9).

In Part III ziehen wir dann Schlussfolgerungen von diesen Experimenten, diskutieren die Nützlichkeit und Anwendbarkeit unserer Idee und geben Ansatzpunkte für weitere Forschungen.

\section{E.2 Die verschiedenen Ansätze der Genetischen Programmierung}

In dieser Arbeit wurden sechs verschiedene GP-Methoden angewendet. Als wir diese ausgewählt und entworfen haben, war es unser Ziel, eine möglichst breite Palette verschiedener Programmrepräsentationen und Genome abzudecken. Dadurch erhofften wir, einen guten Eindruck von der Nützlichkeit der Genetischen Programmierung für das Züchten verteilter Algorithmen zu erhalten.

\section{E.2.1 Standard Genetic Programming [SGP]}

Der grundlegendste Ansatz für Genetische Programmierung ist ein baumbasiertes Genom zu verwenden, wie es Koza in seiner bahnbrechenden Arbeit Genetic Programming: On the Programming of Computers by Means of Natural Selection [165] 1992 getan hat. Daher 
werden solche Ansätze noch heute also Standard Genetic Programming (SGP) bezeichnet. In einem Baum, der ein Programm darstellt, entsprechen die inneren Knoten Funktionen und Operationen wohingegen die Blätter terminal Symbole wie Konstanten oder Variablen sind. In unserer Arbeit haben wir diesen Ansatz speziell für das Züchten verteilter Algorithmen angepasst die folgenden Modifikationen eingeführt:

1. Ein Programm besteht aus mindestens zwei automatisch definierten Funktionen (ADFs).

2. Die erste Funktion wird beim Programmstart ausgeführt, ähnlich der main-Routine in $\mathrm{C}++$-Programmen.

3. Die zweite Funktion ähnelt einer Interrupt Service Routine (ISR), welche asynchron aufgerufen immer dann wird, wenn eine Nachricht vom Knoten auf dem das Programm läuft empfangen wird.

4. Ein Programm kann aus mehr als zwei Funktionen bestehen, wenn der evolutionäre Prozess diese hinzufügt. Diese zusätzlichen Funktionen sind keine ADFs.

5. Der Speicher eines Kotens ist in globalen Speicher, welcher von allen Funktionen aus zugreifbar ist, und lokalen Speicher, der private nur im Gültigkeitsbereich eines Funktionsaufrufs existiert, unterteilt.

6. Alle Parameter einer Funktion werden im lokalen Speicher abgelegt.

7. Der Inhalt eintreffender Nachrichten werden im lokalen Speicher der zweiten ADF abgelegt.

8. Funktionen können vom gezüchteten Kode mit einer beliebigen Anzahl von Parametern aufgerufen werden. Die Gesamtzahl der Parameter einer Funktion wird automatisch als die maximale Anzahl von Parametern mit denen sie aufgerufen wird bestimmt. Wenn sie mit weniger Parametern gerufen wird, werden die ungenutzten Parameter auf Null gesetzt.

9. Eine Funktion kann einen Rückgabewert zurückliefern und der Befehl call kann daher als Ausdruck verwendet (und somit auch in andere Ausdrücke eingebettet) werden.

10. Die Parameter von Funktionen sind Ausdrücke.

11. Der Befehl send erhält zwischen einem und drei Parameter, die für den Inhalt der zu sendenden Nachricht stehen.

\section{E.2.2 Erweitertes Standard GP [eSGP]}

Die beiden verschiedenen Arten von Speicher SGP machen den Ansatz bereits sehr mächtig. Dennoch ist er nicht Turing-vollständig. Um sich dieses Problem vor Augen zu führen, braucht man sich nur die Einschränkung, dass nur primitive typen (wie Integer-Variablen) in einer Programmiersprache wie Java zur Verfügung stehen würden, vorstellen. Dadurch wird es sehr schwierig, Programme die z.B. Listen verarbeiten zu definieren, da auf jedes Element explizit über den dafür zugewiesenen Variablennamen zugegriffen werden müsste. Ein Programm das beliebig lange Listen sortiert kann dann gar nicht definiert werden.

Dieselben Einschränkungen gelten auch für SGP - die Symbole dort entsprechen einfachen Integer-Variablen.

Teller [275, 274], Nordin and Banzhaf [212] und Woodward [316] haben alle gezeigt, dass Turing-Vollständigkeit durch das Hinzufügen von indirekt adressierbaren Speicher erreicht werden kann. Die einfachste Art, eine solche Erweiterung für SGP zu definieren, ist es die Adressierungsarten zu erhöhen. Zu den drei vorhandenen Methoden (lokaler und globaler Speicher sowie ein impliziter Stack) kommt nun der Zugriff auf eine Zelle im

1. globalen Speicher an einer Adresse vom globalen Speicher,

2. globalen Speicher an einer Adresse vom lokalen Speicher, 
3. lokalen Speicher an einer Adresse vom globalen Speicher und

4. lokalen Speicher an einer Adresse vom lokalen Speicher.

Dieser Ansatz hat jedoch den kleinen Nachteil, dass nun dreimal so viele Adressierungsmöglichkeiten bestehen. In einem Szenario mit jeweils zwei Zellen globalem und lokalen Speicher entstehen $2 * 6=12$ verschiedene Adressen. Dadurch verringert sich die Chance, dass Informationen die von einem Befehl an einer bestimmten Stelle im Speicher abgelegt wurden, von einem anderen, zufällig generierten Speicherzugriffsbefehl wiedergefunden werden, auf ein Drittel.

Um dieser Korrosion entgegenzuwirken führen wir das Konzept deklarierter Variablen ein. Wie in den meisten Hochsprachen, müssen Variablen zuerst formell deklariert werden, bevor auf sie zugegriffen werden kann. Diese Deklaration wird vom decl-Befehl vorgenommen, der jeweils für den gesamten Block, in dem er Auftritt, Gültigkeit besitzt. decl weist einer spezifischen Adresse in einem der sechs Modi einen Namen zu. Durch den decl-Befehl steigt die Anzahl möglicher Speicherstellen, an denen Informationen abgelegt werden, nur noch mit den tatsächlich verwendeten Adressen an und ist von der Speichergröße unabhängig (bzw. hat diese lediglich als obere Grenze). Damit ist der eSGP-Ansatz Turing-Vollständig ohne unter einer Degeneration in den Wahrscheinlichkeiten, im Speicher abgelegte Werte wiederzufinden, zu leiden.

\section{E.2.3 Lineare Genetische Programmierung [LGP]}

Bäume sind nicht die einzige Methode, mit der Programme dargestellt werden können. Tatsächlich verarbeiten Computer Programme in Form von Sequenzen einfacher Befehle. Diese Sequenzen können Verzweigungen in Form von Sprüngen zu anderen Stellen im Kode enthalten. Jedes Kontrollflussdiagramm, das das Verhalten eines Programms beschreibt, kann in eine solche Sequenz übersetzt werden. Daher ist nur natürlich, dass der erste Ansatz zur automatischen Programmerzeugung von Friedberg [97] Ende der 1950er Jahre auf Befehlssequenzen fester Länge abzielte [97, 98]. Das Gebiet der Genetischen Programmierung, dass solche Befehlsketten verarbeitet, wird als Lineare Genetische Programmierung (LGP) bezeichnet [293].

Eine der frühesten Beiträge zu diesem Gebiet kommt von Banzhaf [17], der 1993 eine Genotyp-Phänotyp-Abbildung mit speziellen Reparaturmechanismen nutzte, um einen Bitstring in eine Sequenz von einfachen mathematischen Instruktionen zu übersetzen. Das Compiling Genetic Programming System ${ }^{3}$ (CGPS) von Nordin [211], das Automatic Induction of Machine Code with GP-System (AIMGP) von Nordin et al. [215] und die Analysen von Brameier and Banzhaf [37] sind wahrscheinlich die wichtigsten Arbeiten auf dem Gebiet der LGP. Der größte Vorteil, der allen diesen Ansätzen gemeinsam ist, ist die direkte und einfache Art, in der solche Befehlsketten interpretiert und ausgewertet werden können.

In unserem LGP-Ansatz unterscheiden wir wieder zwischen globalem und lokalem Speicher. Außerdem führen wir wieder einen privaten Stack für jede Funktion ein um Rückgabewerte und Parameter übergeben zu können. Alle Programme sind Listen von Integer-Strings, wobei jeder einzelne String einer Funktion entspricht. Die Knoten, die diese Ketten verarbeiten, sind Dreiadressmaschinen, also Computer bei denen arithmetische Befehle bis zu drei Parametern haben: die Zieladresse und zwei Quelladressen. In dieser Hinsicht ist unser LGP-Ansatz der von Lasarczyk and Banzhaf [176] in ihrem Algorithmic Chemistries-Ansatz verwendeten Sprache recht ähnlich.

Jeder Befehl wird von vier Integerwerten repräsentiert. Die erste Zahl ist der Opcode, der den Befehl identifiziert. Die anderen drei Werte sind die Argumente. Jedes Programm besteht wieder aus mindestens zwei ADFs mit exakt der gleichen Semantik wie im SGP und

\footnotetext{
${ }^{3}$ Anders als unser DGPF arbeitet dieses System direkt auf dem Maschinenkode und führt eigentlich
} keine Kompilierung durch. 
eSGP-Ansatz. Tatsächlich werden die Programme von diesen beiden Repräsentationen nach LGP "kompiliert", bevor sie in den Netzwerksimulationen ausgeführt werden.

\section{E.2.4 Fraglets}

In seiner bahnbrechenden Arbeit führte Tschudin [281] Fraglets ${ }^{4}$, eine neue künstliche Chemie für die Entwicklung und sogar Evolution von Netzwerkprotokollen ein. Fraglets stehen für ein Ausführungsmodell für Kommunikationsprotokolle, das chemische Reaktionen in lebenden Organismen nachempfindet.

Vom theoretischen Gesichtspunkt her ist der Fraglet-Ansatz sowohl eine Instanz von Post's String Rewriting Systemen [233] als auch von Gamma Systemen [13, 15]. Fraglets sind Ketten von Symbolen in der Form $\left[\mathbf{s}_{1}: \mathbf{s}_{2}: \ldots: \mathbf{s}_{n}\right]$. Die Symbole $\mathbf{s}_{i}$ stehen entweder für Kommandos oder Protokollnutzlast. Jeder Knoten im Netzwerk hat einen FragletSpeicher der etwa einem Reaktionsgefäß in der Chemie entspricht. Solche Reaktionsgefäße beinhalten oftmals viele gleiche Moleküle und auch die Fraglet-Speicher können beinhalten gleiche Fraglet-Ketten mehrmals beinhalten. Sie werden als Multiset implementiert, das die Vielfachheit dieser Ketten berücksichtigt.

Tschudin [281] definiert eine einfache Prefix-Programmiersprache mit einem festen Befehlssatz bestehend aus Transformationen und Reaktionsregeln für Fraglets. Transformationen wie dup und nop verändern ein einzelnes Fraglet wohingegen Reaktionen wir match und matchP zwei Fraglets kombinieren.

\section{E.2.5 Regelbasierte Genetische Programmierung [RBGP]}

\section{Idee}

Wir haben in unserer Analyse der problematischen Aspekte der Genetischen Programmierung argumentiert, dass Epistase eines der Kernprobleme in dieser Anwendung ist (Section 4.5). Als Beispiel von Ansätzen gegen Epistase haben wir die Algorithmic ChemistryMethode von Lasarczyk and Banzhaf [176] herangeführt. Neben solchen speziellen Repräsentationen für Lösungen gibt es noch eine sehr allgemeine Klasse von evolutionären Algorithmen, die elegant den positionalen Aspekt der Epistase umgehen: die (Learning) Classifier Systems-Familie [132, 130], die kurz in Section 2.6 eingeführt wird.

Im Pittsburgh Learning Classifier Systems von Spears and de Jong [267] wird eine Population von Regelsätzen mit einem Genetischen Algorithmus gezüchtet [262]. Jedes Individuum in dieser Population besteht aus mehreren Klassifikatoren (den Regeln) die eine Menge von Eingabesignalen in Ausgabesignale umwandeln. Die Reihenfolge, in der diese Regeln ausgewertet werden spielt absolut keine Rolle. Daher ist nahezu keine positionale Epistase vorhanden.

Die grundlegende Idee hinter unserem regelbasierten Ansatz zur Genetischen Programmierung (engl. Rule-based Genetic Programming, RBGP) ist es, eine neue Programmrepräsentation mit solch einer niedrigen positionalen Epistase zu erzeugen, um robust gegen negative Ergebnisse von Reproduktionsoperatoren zu werden [298]. Mit RBGP werden gleichzeitig die Nachteile von Algorithmic Chemistries und der Soft Assignment-Methode von McPhee and Poli [195] wie Indeterminismus umgangen. RBGP könnte als eine high-level Variante von classifier systems angesehen werden, die mächtigere Konzepte wie mathematische Operationen einführt.

Wir illustrieren diese neue GP-Methode an dem in Abbildung E.4 (und Figure 5.4) gegebenen Beispiel. Wie Pitt-style Learning Classifier Systems besteht das dargestellte Programm aus einer beliebigen Menge Regeln die binär kodiert werden können. Eine Regel

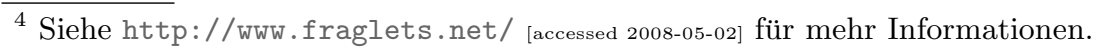


wertet die Symbole in ihrem Konditionsteil aus (links von $\Rightarrow$ ) und weißt ihnen in ihrem Aktionsteil gegebenenfalls neue Werte zu oder ruft andere Prozeduren auf. In ihrer Struktur ist die RBGP-Sprache der Guarded Command Language (GCL) von Dijkstra [73] nicht unähnlich.

\begin{tabular}{c|l} 
Symbol & Kodierung \\
\hline 0 & 0000,1100 \\
1 & 0001,1101 \\
start & 0010,1110 \\
id & 0011,1111 \\
netSize & 0100 \\
in & 0101 \\
out & 0110 \\
enterCS & 0111 \\
csLeft & 1000 \\
a & 1001 \\
b & 1010
\end{tabular}

\begin{tabular}{c|ccc|c} 
Vergl. & Kod. & & Verbind & Kod. \\
\cline { 4 - 5 }$>$ & 000 & & $\wedge$ & 0 \\
$\geq$ & 001 & & $\vee$ & 1 \\
$=$ & 010 & & \\
$\leq$ & 011 & & \\
$<$ & 100 & & \\
$\neq$ & 101 & & Aktion & Kod. \\
true & 110 & & Ax+y & 00 \\
false & 111 & & $=\mathrm{x}-\mathrm{y}$ & 01 \\
& & & $=\mathrm{x}$ & 10 \\
& & & $=1-\mathrm{x}$ & 11
\end{tabular}

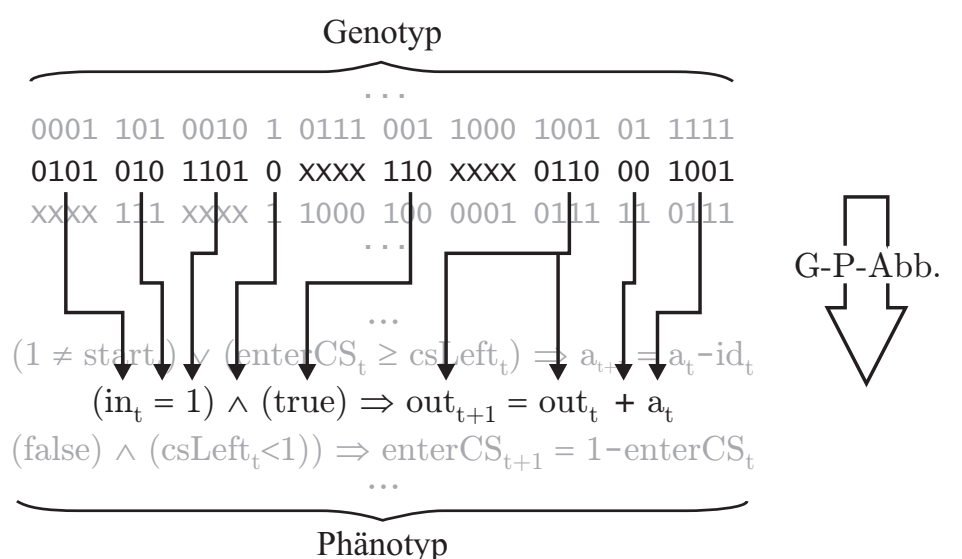

Abbildung E.4: Beispiel für die Genotyp-Phänotyp-Abbildung in RBGP (siehe auch Figure 5.4).

\section{Programmausführung und Ebenen der Unabhängigkeit}

Die einfachste Methode, ein regelbasiertes Programm auszuführen, ist alle Regeln in einer Schleife auszuwerten. Obwohl dieser Ansatz ausreichend für die Simulationszwecke in unseren Experimenten ist, würde er in einer echten Implementierung unnötig Rechenkraft und damit Energie verschwenden. Dieser Energieverbrauch kann signifikant verringert werden, wenn nur die Regeln ausgewertet werden, deren Konditionsteile Symbole enthalten, deren Wert sich geändert hat.

Änderungen in den Werten eines Symbols können entweder von Außen (z.B. durch eintreffenden Nachrichten) oder durch Aktionen vom Programm selbst ausgelöst werden. In RBGP können Aktionen die Werte von Symbolen nicht direkt ändern und schreiben stattdessen in einen temporären Speicher, der nach jeder vollständigen Auswertung aller (auszuwertenden) Regeln in den echten Speicher zurückgeschrieben wird, wie in Abbildung E.5 (und Figure 5.5) dargestellt. Die Symbole in den Konditionsteilen sind daher mit dem Zeitindex $t$ und die Zielsymbole in den Aktionsteilen mit $t+1$ annotiert. 


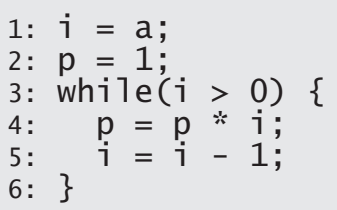

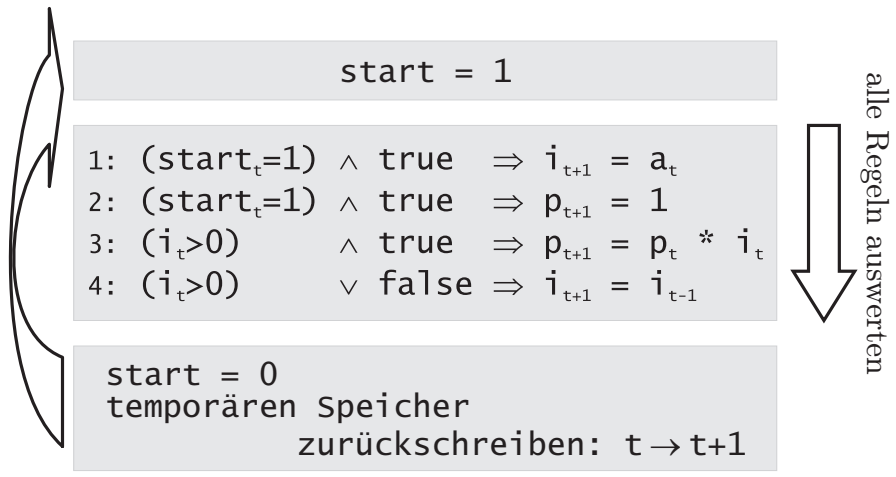

Syntax: Regelbasierte Genetische Programmierung

Abbildung E.5: Ein Programm, dass die Fakultät p einer natürlichen Zahl a berechnet, dargestellt in Java und RBGP-Syntax (siehe auch Figure 5.5).

Dieser Ansatz erlaubt es, die Regeln in einer großen Unordnung zu definieren, da keine positionalen Abhängigkeiten mehr bestehen (außer zwischen Regeln, die auf dasselbe Symbol schreiben). Daher ist die positionale Epistase in RBGP sehr niedrig.

\section{E.2.6 Erweiterte Regelbasierte Genetische Programmierung [eRBGP]}

Obwohl der RBGP-Ansatz recht einfach aussieht, kann man damit bereits viele relativ komplizierte Ausdrücke darstellen. Dennoch hat der originale RBGP-Ansatz einige Einschränkungen. Sein offensichtlichster Nachteil ist, dass er nicht Turing-vollständig ist - genau wie wir das schon bei SGP beobachtet haben. Und genau wie bei der Transition von SGP nach eSGP können wir diesen Nachteil durch das Hinzufügen von indirekt adressierbarem Speicher lösen. Der Ausdruck $\left[\mathrm{b}_{t}\right]_{t}$ (wobei b ein Symbol ist) bedeutet nun, dass zuerst der Wert des Symbols $\mathrm{b}_{t}$ bestimmt wird. Dieser wird dann als Adresse in die Liste der Symbole verwendet auf das so adressierte Symbol wird zugegriffen.

Mit dieser Erweiterung alleine wird es schon möglich, Algorithmen zum Sortieren beliebiger Listen in eRBGP zu definieren. Nehmen wir an, dass die folgenden Symbole spezifiziert wurden: $\left(i_{0}, i_{1}, . ., i_{n-1}, 1, a, b\right)$. Die Symbole $i_{0}$ bis $i_{n-1}$ stellen den Speicher für die Listenelemente dar und 1 wird mit der Länge der Liste, also der Anzahl der i-Elemente, die tatsächlich genutzt werden, initialisiert (diese Zahl ist kleiner oder gleich $n$ ). a und b seien beliebige Variablen. In der Liste der Symbole sei $i_{0}$ an Position 0, 1 an Position $n$, a an Index $n+1$ und so weiter. Mit sehr geringem Aufwand kann nun Listing E.1 (siehe auch Listing 5.7) definiert werden, ein Programm, das eine Art Selektionsort durchführt. Da keine Variable aus dem temporären Speicher zurückgeschrieben wird, bevor nicht alle Regeln ausgewertet wurden, benötigen wir keine weitere Variable, um die Listenelemente beim Sortieren zwischenzuspeichern.

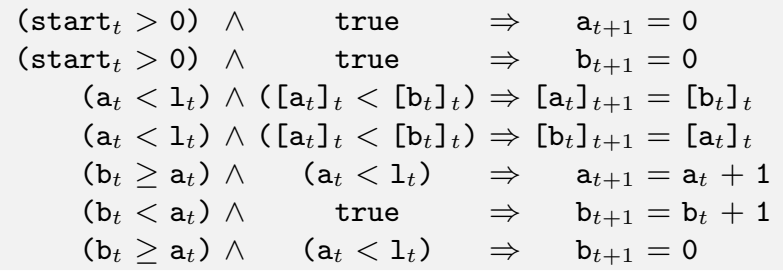

Listing E.1: Ein einfaches Selektionsortprogramm in eRBGP-Syntax. 
In RBGP kann es wegen der binären Kodierung mit der festen Länge kompliziert werden, Regeln, die aus mehr als zwei Konditionen bestehen, zu definieren. Diese müssen dann in mehrere Regeln aufgeteilt werden, die ihre Ergebnisse in extra definierten Variablen zwischenspeichern. Dies kann RBGP dazu zwingen, durch einen relativ komplizierten Aufbauprozess zu gehen, um ein gewünschtes Verhalten zu erzeugen. Wir erhöhen daher in eRBGP die Ausdruckskraft indem wir sämtliche Beschränkungen die Struktur der Regeln betreffend fallenlassen. Dadurch können wir allerdings keinen Genetischen Algorithmus mehr verwenden, um die Regeln zu züchten, da sie nicht mehr direkt binär kodiert werden können: Wenn die Anzahl von Konditionen und die Verschachtelungstiefe von Ausdrücken beliebig wird, ist nicht mehr möglich, diese in einer festen, vordefinierten Anzahl von Bits zu kodieren. Deshalb nutzen wir in eRBGP wiederum ein baumbasiertes Genom ähnlich dem Standard-GP in SGP, um diese Erweiterung zu ermöglichen.

$\left(\left(\mathrm{a}_{t}<\mathrm{b}_{t}\right) \wedge\left(\left(\mathrm{c}_{t}>\mathrm{d}_{t}\right) \wedge\left(\mathrm{a}_{t}<\mathrm{d}_{t}\right)\right)\right) \Rightarrow \mathrm{a}_{t+1}=\left(\mathrm{a}_{t}+\mathrm{c}_{t}\right)$

$2\left(\left(a_{t}<b_{t}\right) \wedge\left(\left(c_{t}>d_{t}\right) \wedge\left(a_{t}<d_{t}\right)\right)\right) \Rightarrow c_{t+1}=\left(c_{t}-1\right)$

Listing E.2: Die eRBGP-Version von Listing 5.1 und Listing 5.2

In Listing E.2 drücken wir nun eine komplizierte Regel die aus drei Konditionen besteht in eRBGP aus (siehe Listing 5.2 und Listing 5.8). Wie bereits erwähnt, können wir nicht mehr einfach einen Genetischen Algorithmus nehmen, um dieses Programm zu kodieren bzw. solche Programme zu züchten. Stattdessen nutzen wir baumbasierte Genetische Programmierung, und Listing E.2 entspricht nun den in Abbildung E.6 (und Figure 5.6) dargestelltem Phänotyp.

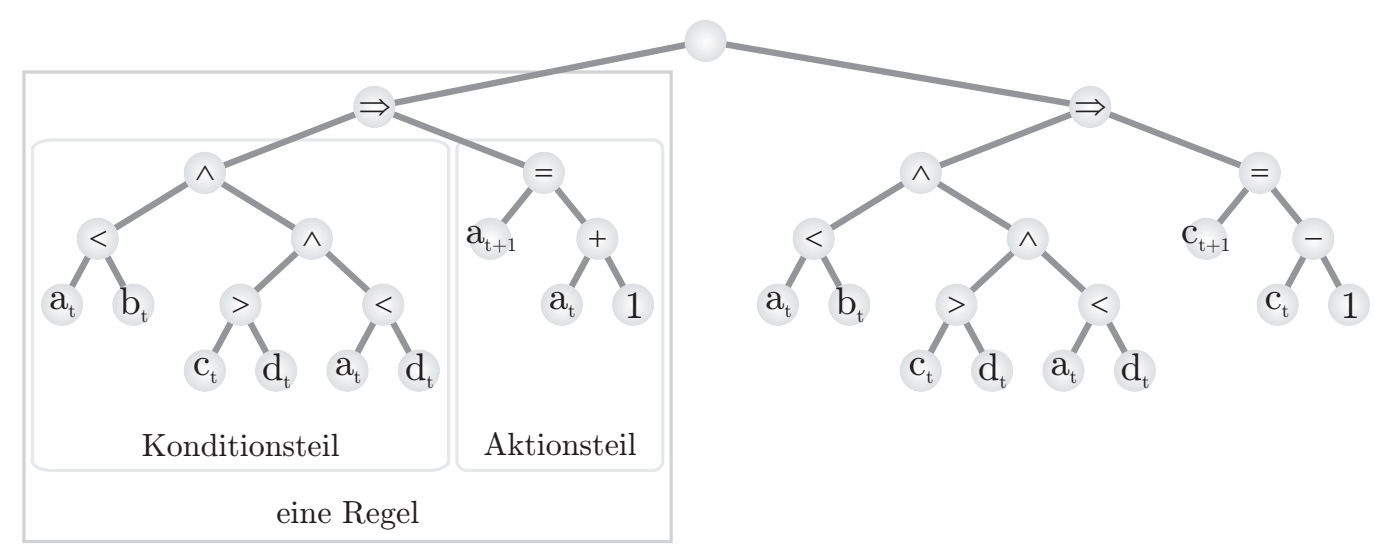

Abbildung E.6: Der baumbasierte Genotyp und Phänotyp von Listing E.2 (und Listing 5.8).

Mit diesen Erweiterungen wird eRBGP sehr mächtig und kann nun beliebige Programme darstellen bzw. zum Züchten beliebiger Programme verwendet werden. Der Beweis für Turing-Vollständigkeit von Sprachen mit indiziertem Speicher von Teller [275] nun leicht auf eRBGP übertragen werden.

\section{E.3 Experimente}

Die sechs im vorigen Abschnitt vorgestellten Ansätze zur Genetischen Programmierung wurden nun auf drei Beispielprobleme vom Gebiet der verteilten Systeme angewandt. 


\section{E.3.1 Auswahlalgorithmen}

Auswahlalgorithmen haben viele Anwendungen in verteilten Systemen. Sie werden benutzt, um Koordinatoren in mehreren Routing- [119] oder Gruppenkommunikationsprotokollen [289] zu bestimmen. In Windowsdomänen wird der Master Browser mit einem Auswahlalgorithmus bestimmt [75]. Auswahlalgorithmen sind auch Teil verschiedener Uhrensynchronisationsmethoden [116] und Agentenszenarios [56].

Nach Le Lann [178], können verteilte Auswahlalgorithmen von beliebigen Knoten gestartet werden und erreichen einen Endzustand in dem genau ein Knoten als Anführer gewählt ist und alle Knoten dieser Wahl zustimmen. Die größte Herausforderung bei der Auswahl ist es, zu sichern das alle Knoten die gleiche Sicht auf die Wahl haben. Le Lann macht die folgenden Annahmen über das verteilte System, in dem die Auswahl stattfindet:

1. Jeder Knoten hat einen eindeutigen Namen (ID).

2. Es kann eine totale Ordnung über diese Namen (IDs) definiert werden.

3. Jeder Knoten kennt die IDs aller anderen Knoten.

4. Ein Knoten kennt nicht die Zustände der anderen Knoten.

Es wurden viele verschiedene Methoden für verteilte Auswahlen entwickelt. Angefangen mit Le Lann's originalem Ansatz für Ringe, den Message Extinction-Algorithmus von Chang and Roberts [46] und dem Bullyalgorithmus von Garcia-Molina [99] bis hin zu dem probabilistischen Las Vegas-Algorithmus von Itai and Rodeh [144, 145]. Effiziente Auswahlalgorithmen für beliebige Topologien $[163,164]$ und spezielle Algorithmen für kabellose Sensornetze und MANETs [191, 288] folgten einige Jahre später.

Wir haben die Fähigkeit der GP-Ansätze, solche Auswahlalgorithmen zu erzeugen studiert. Unsere Experimente haben klar gezeigt, dass alle sechs Ansätze benutzt werden können, um Auswahlalgorithmen zu erzeugen. Sie haben weiterhin gezeigt, dass die verschiedenen Programmrepräsentationen zu verschiedenen Ergebnissen und Erfolgswahrscheinlichkeiten führen.

Besonders interessant ist das Verhalten der Regelbasierten Genetischen Programmierung: Währen eRBGP sehr hohe Erfolgsraten erzielte, konnte das einfache RBGP keine guten Ergebnisse erreichen. Dies zeigt zum Einen, dass unser gezielter Entwurf des regelbasierten Ansatzes eine gute Idee war und zu signifikant besseren Resultaten führte. Zum Anderen lässt es vermuten, dass die feste Struktur von RBGP zu unflexibel ist, um die Evolution komplexerer Programme spürbar zu vereinfachen.

Keiner der GP-Ansätze konnte die zwei der vier Auswahlaufgaben lösen, die explizites Terminieren verlangen. Dies mag daran liegen, dass der Kode zur Terminierung an sich schon so komplex ist, dass er nicht so einfach mit der eigentlichen Funktionalität "nebenher" gezüchtet werden kann. Hier sehen wir die Grenzen der Genetischen Programmierung.

\section{E.3.2 Wechselseitiger Ausschluss am Kritischen Abschnitt}

Die Architektur von Computern hat sich Mitte der 1950er Jahre mit der Erfindung von magnetischen Speichern und asynchron arbeitenden Peripheriegeräten dramatisch geändert. Das Konzept der Interrupts wurde erfunden, um es Prozessen zu ermöglichen, weiterzulaufen während langsame Peripheriegeräte gleichzeitig ihre Aufträge abarbeiten. Diese Nebenläufigkeit führte zu komplett neuen Problemen und machte die Softwareentwicklung schwieriger, was auch mit zur Softwarekriese der 1960er Jahre beitrug.

Ressourcen in asynchronen Systemen gemeinsam zu nutzen führt immer zu Gefahren. Wenn zwei oder mehr Prozesse gleichzeitig auf gemeinsame Variablen zugreifen, welche von wenigstens einem von ihnen verändert werden, so können Phänomene wie lost updates auftreten. Dijkstra erkannte, dass Softwareentwickler den wechselseitigen Ausschluss an solchen 
kritischen Abschnitten erzwingen müssen. Die folgenden Anforderungen an einen Algorithmus für den wechselseitigen Ausschluss wurden aus Dijkstra's Arbeit [71, 72] entnommen:

1. Zu einem Zeitpunkt darf höchstens ein Prozess darf die gemeinsame Ressource benutzen.

2. Wenn ein Prozess außerhalb des kritischen Abschnitts angehalten wurde, dann darf dies nicht zu einer Blockade anderer Prozesse führen (Deadlock, [71])

3. Wollen zwei Prozesse den kritischen Abschnitt betreten, so muss es unmöglich sein, ihnen Ablaufgeschwindigkeiten auf eine Art zuzuweisen, die dazu führt, dass die Entscheidung, wer den kritischen Abschnitt betreten darf, bis in alle Ewigkeit aufgeschoben wird. (Lifelocks, [71])

4. Der Algorithmus kann für eine beliebige Anzahl Prozesse implementiert werden.

Der erste Algorithmus, der dieser Definition entsprach, wurde von Dekker entwickelt. Dieser dezentrale Algorithmus nutzte gemeinsame Steuervariablen zur Synchronisation. Die Entwicklung von Algorithmen zum wechselseitigen Ausschluss in verteilten Systemen ist schwieriger, da keine gemeinsamen Variablen zur Verfügung stehen. Stattdessen laufen die Prozesse asynchron auf verschiedenen Knoten und kommunizieren über latenzbehafteten Nachrichtenaustausch. Erste verteilte Algorithmen für den wechselseitigen Ausschluss unter diesen Bedingungen wurden von Lamport [168] 1976 und Ricart and Agrawala [244] 1981 entwickelt, gefolgt von Maekawa's optimaler Lösung 1985 [189].

Die Ergebnisse unserer Experimente zur Synthese von solchen Algorithmen sind auf den ersten Blick aus zwei Gründen besonders interessant. Erstens haben SGP und eSGP effektiv mit eRBGP die Plätze getauscht und liefern nun die besten Ergebnisse. Schuld daran ist ihre Fähigkeit, lange Sequenzen von Befehlen zu erzeugen, die den Programmablauf verzögern - wohingegen es weder in RBGP, eRBGP, noch in der Fraglets-Repräsentation so etwas wie explizite Sequenzen gibt. Stattdessen müssten hier komplexe Abfolgen von Regeln oder Reaktionen gezüchtet werden, was wiederum wesentlich schwerer zu sein scheint.

Der zweite interessante Punkt ist, dass die Experimente in vollvermaschten Topologie die einzig erfolgreichen sind und das es keinem Ansatz gelang, adäquate Algorithmen für Ringe oder dysfunktionale Kommunikationssysteme zu entwickeln. Speziell für den letzteren Fall wäre das ohnehin nicht möglich. Diese Ergebnisse entsprechen daher weitestgehend unseren Erwartungen und bestätigen, dass die Genetische Programmierung tatsächlich in der Lage ist, Kommunikation effektiv einzusetzen.

\section{E.3.3 GGT}

In [293] haben wir die Evolution von Algorithmen für den größten gemeinsamen Teiler (GGT) als Beispiel für die Anwendung von Genetischer Programmierung auf schwere mathematische Probleme betrachtet. Hier wollen wir diesen Ansatz auf die kooperative Berechnung des GGT von mehreren, über ein Netzwerk verteilten Zahlen betrachten.

Definition E.2 (GGT). Für zwei Zahlen $a, b \in \mathbb{N}$ ist der größte gemeinsame Teiler (GGT) die größte Zahl $c \in \mathbb{N}$ die sowohl $a$ als auch $b$ teilt (siehe auch Definition 9.1).

Der GGT zweier Zahlen kann mit dem Euklidischen Algorithmus [216] berechnet werden. Mattern benutzte die verteilte Variante dieser Prozedur (hier als Algorithmus E.1 und Algorithm 9.1 definiert) als Beispiel in seinem Grundlagenbuch [193]. Jeder Knoten $\mathbf{n}$ des Netzwerks $\mathbf{N}$ beginnt mit der eigenen Zahl $\mathbf{n}$.nummer die auch gleichzeitig seine erste Abschätzung für den GGT ist $(\operatorname{gcdVal}(\mathbf{n})=\mathbf{n}$.nummer $)$. Anfänglich sendet er jetzt diese Zahl an alle seine Nachbarn. Wann immer ein Knoten eine Nachricht "Nachricht" mit der Zahl von einem seiner Nachbarn bekommt, so wird er sofort seine eigene Abschätzung 


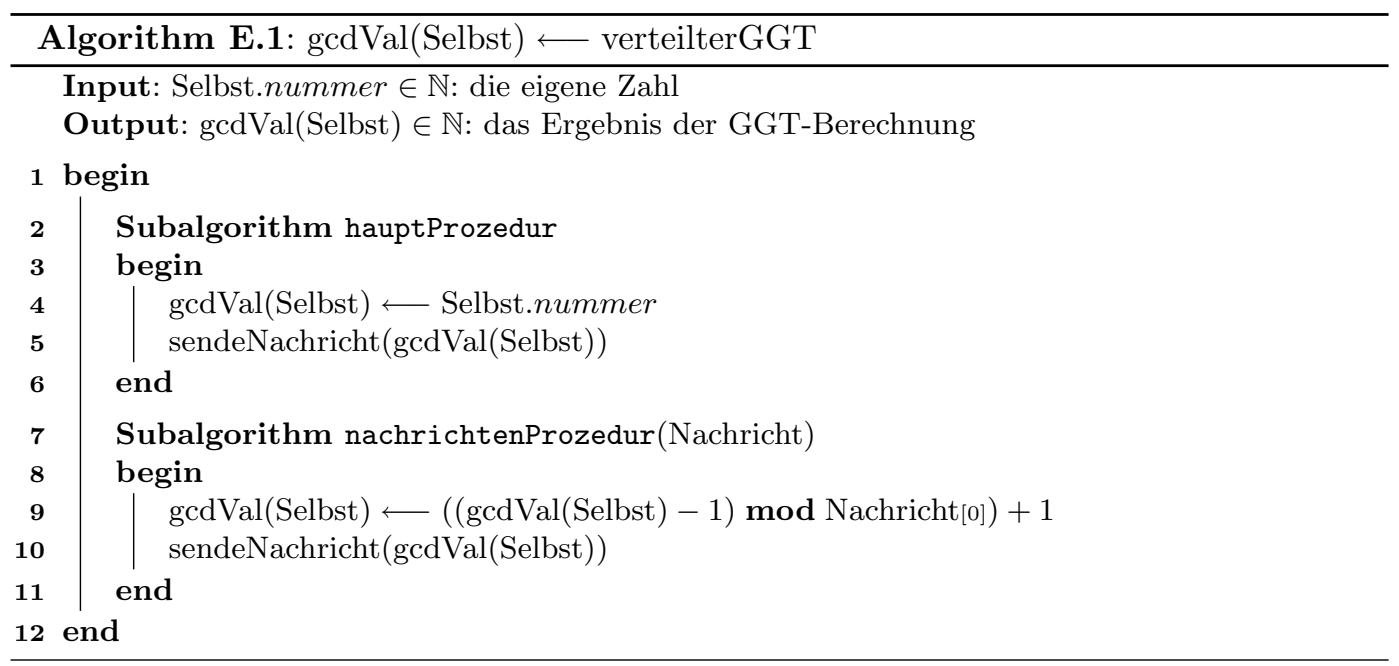

des Endergebnisses gemäß von Zeile 9 erneuern. Schrittweise nähern sich nun die gcdVal(n)Werte aller Knoten $\mathbf{n} \in \mathbf{N}$ dem echten GGT an. Hier fällt auf, dass Algorithmus E.1 (und Algorithm 9.1) perfekte Beispiele von Anytime-Algorithmen sind, wie sie von [274] definiert wurden.

Unsere Experimente mit dem Ziel, solche Algorithmen für den verteilten GGT zu züchten, führten zu drei grundlegenden Erkenntnissen. Erstens erscheint das GGT-Problem die schwerste aller drei (lösbaren) Domänen zu sein, die wir getestet haben. Obwohl mindestens drei Ansätze Lösungen dafür gefunden haben, sind die Erfolgsraten in den Experimenten extrem niedrig. Zweitens haben wir erkannt, dass nur eRBGP in der Lage war, größere Populationen sinnvoll auszunutzen und damit seine Ergebnisse zu verbessern. Drittens haben wir erkannt, dass nur eRBGP signifikant besser als Random Sampling bzw. Random Walks in dieser Domäne ist.

Auf der einen Seite zeigen diese Ergebnisse die große Härte von "Nadel-im-HeuhaufenProblemen", die wir bereits in unserer Analyse vorausgesehen haben und zu denen das GGTProblem wahrscheinlich gehört. Auf der anderen Seite bieten sie eine klare Rechtfertigung für das Design von eRBGP und bestätigen die große Nützlichkeit dieses Ansatzes.

\section{E.4 Kritische Diskussion}

In der Einleitung wurde postuliert, dass die Genetische Programmierung als Grundlage für einen neuen Entwurfsansatz für verteilte Algorithmen dienen kann. Wir haben diesen neuen Ansatz in sechs verschiedenen Varianten implementiert und auf drei Beispielprobleme angewendet. Hier wollen wir nun unsere Ergebnisse kritisch diskutieren und das Für und Wider zu unserer Idee gegeneinander abwägen.

\section{E.4.1 Kritik am Ansatz}

\section{Schwierigkeiten bei der Umsetzung}

In Abschnitt E.1.1 und Section 1.2 haben wir unsere Methode zur automatischen Synthese verteilter Algorithmen auf Basis der Genetischen Programmierung eingeführt. Der vorgestellte Entwurfsprozess hat die folgenden Voraussetzungen:

1. einen Suchraum $\mathbb{G}$ mit dazugehörigen Suchoperationen, eine Repräsentation $\mathbb{X}$ für verteilte Programme und eine Abbildung gpm zwischen beiden (wenn $\mathbb{G} \not \subset \mathbb{X}$ ). 
2. eine Ausführungsumgebung für die erzeugten Programme $x \in \mathbb{X}$ basierend auf

3. einer schnellen und ausreichend realistischen Netzwerksimulation,

4. eine Optimierungssoftware mit der alles das zusammengesetzt werden kann,

5. eine klare Spezifikation des Zielverhaltens des verteilten Systems,

6. eine Anpassung der Punkte 1 bis 4 auf diese Spezifikation (falls notwendig) und

7. eine Menge von funktionellen und nichtfunktionellen Optimierungskriterien, um den Optimierungsprozess zu steuern.

Unser Sigoa-Framework ist eine vielseitige Optimierungssoftware, welche sehr geeignet ist, Punkt 4 zu erfüllen. Auf der anderen Seite deckt die DGPF-Bibliothek die Punkte 1 bis 3 ab. In ihr sind sowohl verschiedene Programmrepräsentationen definiert als auch die dazugehörigen Suchoperationen und die benötigten Genotyp-Phänotyp-Abbildungen [295, 297]. Außerdem stellt das DGPF schnelle zufallsbasierte Netzwerk- und Rechenknotensimulationen zur Verfügung sowie Zielfunktionen für nichtfunktionale Aspekte wie Programmgröße oder Nachrichtenkomplexität.

Die einzig verbliebenen Punkte betreffen also die Definition von Zielfunktionen für funktionelle Adäquatheit und, falls notwendig, eine Anpassung des DGPF an spezielle Gegebenheiten die aus der Spezifikation des gewünschten globalen Verhaltens resultieren.

Speziell die Definition von Zielfunktionen hat einen hohen Einfluss auf die experimentellen Ergebnisse, wie wir beispielsweise im GGT-Experiment gezeigt haben. Da es oftmals möglich ist, eine spezifisches funktionelles Kriterium auf verschiedene Art und Weise zu definieren, kann es notwendig werden, mehrere Testläufe durchzuführen, bevor die eigentliche Experimentserie gestartet wird. Dies erfordert einen gewissen Vorlauf und Einarbeitungsaufwand.

Insgesamt lässt sich sagen, dass das Anwenden unseres Ansatzes nicht trivial ist. Dennoch ist es auch nicht zu kompliziert und kann in einer weiten Menge verschiedener Szenarios durchaus nützlich sein. Nicht zuletzt haben wir eine Software zur Verfügung gestellt, die die meisten gestellten Anforderungen an den Ansatz bereits erfüllt.

\section{Die gezüchteten Programme}

Der schwerste Einwand gegen die Idee, (verteilte) Algorithmen mit Hilfe der Genetischen Programmierung zu züchten, ist der, dass die Ergebnisse nicht notwendigerweise korrekt sind. Wir haben dieses Problem selbst in Section 4.8 aufgezeigt und argumentiert, dass Effekte wie Overfitting oder Übervereinfachung niemals ausgeschlossen werden können.

Wir haben die Geschichte vom Affen und der Orange in Section 4.8.1 genutzt, um zu zeigen, dass ähnliche Probleme auch in der natürlichen Evolution auftreten. Andere Beispiele wären parasitische Insekten die in Kolonien von Ameisen eindringen, in dem sie deren chemische Signale kopieren [167]. Da solche "Fehler" nur mit geringer Häufigkeit auftreten, können die so ausgetricksten Arten dennoch überleben und sich ausbreiten.

Diese Argumentation lässt sich nicht so einfach auf vom Menschen gemachte (verteilte) Systeme übertragen. Offensichtlicherweise sollen diese immer adäquat funktionieren. Dennoch ist es unsere feste Überzeugung, dass es unmöglich ist, dass Auftreten von zeitlich begrenztem, unvorhergesehenem und unerwünschtem Systemverhalten in Systemen auszuschließen, die auf (biologisch inspirierten) Ansätzen für Selbstadaption oder Systemsynthese beruhen und über keine Werkzeuge zur Verifikation verfügen. Ein System, dass in der Lage ist, neue Verhalten auszuprobieren - sei es offline wie in unserem Ansatz oder online wie in der Arbeit von Yamamoto and Tschudin [319, 320] - kann deren Ähnlichkeit zu angestrebten Verhalten im Vorhinein nicht abschätzen. Dazu kommt, dass selbst wenn das System adäquates Verhalten feststellt, diese Adäquatheit nicht als generell betrachtet werden kann, da sie im schlimmsten Falle nur für genau die beobachtete Situation gilt. 
Dennoch tolerieren einige der in der Motivation genannten Netzwerktypen eine gewisse Menge von Fehlverhalten. In Sensornetzwerken, zum Beispiel, ist es unumgänglich, dass früher oder später einzelne Knoten wegen Batterieerschöpfung ausfallen. In mobilen Ad-hoc Netzen (MANETs) können jederzeit temporäre Teilungen des Netzes auftreten. Besonders für Netzwerke mit solchen Charakteristiken kann das Züchten verteilter Algorithmen durchaus eine interessante Möglichkeit sein. Einige der gezüchteten Auswahlalgorithmen, zum Beispiel, funktionieren in praktisch allen Scenarios, in denen die Nachrichtenlatenzen nicht für alle Nachrichten gleich sind. Sie sind damit zwar nicht korrekt, aber fast immer adäquat. Ähnliches gilt für die Algorithmen, die aus den Experimenten für den verteilten wechselseitigen Ausschluss hervorgegangen sind.

Wenn wir also unserer Argumentation hinsichtlich der Nichtausschließbarkeit von Fehlverhalten folgen, so ist unser Ansatz nicht in sicherheitskritischen Systemen einsetzbar. Gerade für neuartige Netztypen ist er jedoch durchaus eine interessante Alternative.

\section{E.4.2 Signifikanz unserer Experimente}

\section{Einstellungen}

Wegen der hohen Laufzeit konnten wir jeweils nur eine einzige Konfiguration des evolutionären Algorithmus für alle Experimente nutzen. Wir haben diese Einstellungen in vorherigen, kleineren Testserien ausprobiert und ihre Motivation in Chapter 4 aus den problematischen Aspekten der Genetischen Programmierung hergeleitet.

Andererseits gibt es eine extreme Vielzahl von Parametern bei evolutionären Algorithmen. Dazu kommt noch, dass diese nicht einzeln betrachtet werden können, sondern sich gegenseitig beeinflussen in ihren Auswirkungen auch vom jeweiligen Optimierungsproblem abhängen. Daher stellt sich die Frage, in wie fern die von uns gewählten Einstellungen für die Problemstellungen gut waren. Das Finden optimaler Einstellungen wäre praktisch nicht möglich bzw. würde eine gigantische Anzahl von Experimenten erfordern.

Gleichgültig ob die von uns gewählten Konfigurationen gut oder schlecht waren, unsere Ergebnisse sind für diese Einstellungen signifikant. Sollte es bessere Einstellungen als unsere geben, so würde das die Nützlichkeit unseres Ansatzes nur noch weiter unterstreichen.

Wir wollen noch erwähnen dass die von uns in den meisten Experimenten verwendete Bevölkerungsgröße von 512 Individuen eher klein ist. In unseren vorhergehenden Forschungen nutzten wir größere Bevölkerungen: sechs über ein Inselmodell verbundene PCs mit bis zu 8192 Individuen pro Rechner in [295], zum Beispiel. Wir konnten allerdings nur kleine Testserien mit solch großen Bevölkerungen fahren. Bevölkerungsgrößen mit 65536 [151] (1996), 100000 [166] (1997) und bis zu 1048576 [171, 170] (2007) Individuen sind in den Arbeiten anderer Forscher durchaus gängig. Das unser Ansatz auch mit viel kleineren Bevölkerungen funktioniert ist ein starker Indikator für seine Nützlichkeit.

\section{Warum evolutionäre Algorithmen}

Es ist auch nicht klar, ob einfache evolutionäre Algorithmen als Optimierungsverfahren für die Gentische Programmierung die beste Wahl ist. Generell hätten wir auch andere Algorithmen wie Simulated Annealing [156, 293], Tabusuche [104, 293] und Memetische Algorithmen (eine hybridisierte Form von EAs) [204, 293] oder auch eine einfache Greedysuche nehmen können.

In [295] und [294] haben wir bereits gezeigt, dass die Kombination von verschiedenen Optimierungsalgorithmen zu besseren Ergebnissen beim Züchten verteilter Algorithmen führen kann. Wir hätten einen solchen heterogenen Ansatz auch hier verfolgen können. Dass hätte aber das Ziehen von allgemeinen Schlussfolgerungen und das Nachvollziehen unserer Experimente erschwert. Unser Ziel war es aber, eine allgemeine Grundlagendiskussion und 
Vergleiche zwischen verschiedenen GP-Ansätzen zu liefern, die gut mit anderen Ergebnissen verglichen werden kann

\section{GP Ansätze}

Wie haben sechs verschiedene Ansätze für das Züchten verteilte Algorithmen getestet, die als repräsentativ für die allgemeinen Strömungen von Genetischer Programmierung angesehen werden können.

Dennoch konnten wir viele andere Ansätze wie Grammar-guided Genetic Programming Methoden [293, 194] wie zum Beispiel Grammatical Evolution [248] oder Tree-Adjoining Grammar-guided Genetic Programming [208, 209], Graphen-basierte Ansätze [293] wie Cartesian Genetic Programming [199] und Parallel Distributed Genetic Programming [229, 230], oder spezielle Genotyp-Phänotyp-Abbildung [293] wie in Gene Expression Programming [84, 85] und Edge Encoding [187]. Da alle diese Techniken ganz verschiedene Ansätze für die Genetische Programmierung darstellen, könnten wir eine signifikante Menge möglicher Erkenntnisse verloren haben, weil wir sie nicht ausprobiert haben. Weiterhin haben wir nur einfache Reproduktionsoperatoren verwendet und mit komplexeren Operationen hätten wir vielleicht bessere Ergebnisse erzielen können.

Dennoch haben wir eine gute Übersicht über die Leistungsfähigkeit der sechs getesteten Ansätze gewonnen. Außerdem können alle unsere Methoden leicht implementiert und nachvollzogen werden. Unser Ergebnis ist, dass keiner der Ansätze generell in allen Experimenten besser war. Die regelbasierte eRBGP-Methode, die speziell entworfen wurde, um eine niedrige Epistase zu erreichen, funktionierte exzellent im Auswahl- und im GGT-Problem. Die Standard-GP-basierte SGP-Variante, auf der anderen Seite, löste das Problem des kritischen Abschnitts am besten.

\section{Anpassung der GP-Ansätze}

Wir haben allen GP-Methoden leicht verschiedene Befehlssätze gegeben. eSGP, zum Beispiel, hat ein natives Konstrukt für for-Schleifen, dass SGP fehlt. Weder in RBGP, eRBGP, noch in Fraglets können Schleifen explizit deklariert werden. Weiterhin sind (mindestens) drei der Ansätze Turing-vollständig (eRBGP, LGP, eSGP) während zwei (RBGP, SGP) es nicht sind. Dieser Sachverhalt hat sicherlich einen Einfluss auf die Ergebnisse der Experimente gehabt.

Neben den Unterschieden in der Struktur der Ansätze haben wir ihnen auch verschieden viele Rechenschritte in den Simulationen zugestanden. Dies basierte auf Erfahrungswerten, die wir in vorhergehenden Experimenten gewonnen hatten. Dies könnte Einfluss auf die Werte der Zielfunktionen, wie zum Beispiel diejenige, die die Kollisionen beim kritischen Abschnitt bewertet, gehabt haben können. Aufgrund unserer Erfahrungen glauben wir aber, dass alle Ansätze etwa gleiche Chancen hatten, die Aufgaben in den Experimenten zu lösen.

\section{Wahl der Szenarien}

Wir konnten unsere Methodologie leider nur auf drei Beispielprobleme, Auswahl, kritischen Abschnitt, und größter gemeinsamer Teiler, anwenden. Es ist schwer, davon gültige Schlussfolgerungen abzuleiten. Der Fokus unserer Arbeit war es, großangelegte Testserien zu fahren um signifikante Ergebnisse zu erzielen. Wir haben die drei Probleme

so gewählt, dass sie sich in vielen Aspekten unterscheiden, wie in Tabelle E.1 (und Table 10.1) dargestellt. Wir haben den Einfluss der Topologie, der Wahl der Zielfunktionen und der Bevölkerungsgröße auf das Ergebnis der Evolution getestet. 


\begin{tabular}{|c|c|c|}
\hline Auswahl & krit. Abschnitt & GGT \\
\hline zwei Zielfunktionen & drei Zielfunktionen & $\begin{array}{l}\text { zwei Zielfunktionen; zwei ver- } \\
\text { schiedene funktionelle Kriterien }\end{array}$ \\
\hline $\begin{array}{l}\text { vier Szenarien von niedriger bis } \\
\text { hoher Schwierigkeit }\end{array}$ & $\begin{array}{l}\text { drei Szenarien hoher Schwierig- } \\
\text { keit }\end{array}$ & ein hartes Szenario \\
\hline eine Topologie: linear & $\begin{array}{l}\text { drei Topologien: vollvermascht, } \\
\text { Ring, ohne Kommunikation }\end{array}$ & $\begin{array}{l}\text { eine Topologie: quadratischer } \\
\text { Grid }\end{array}$ \\
\hline Populationsgröße: 512 & Populationsgröße: 512 & Populationsgröße: 512, 1536 \\
\hline
\end{tabular}

Tabelle E.1: Vergleich zwischen dem Auswahlexperiment, dem kritischen AbschnittExperiment und dem GGT-Experiment (siehe auch Table 10.1).

Unsere Forschung basiert nicht ausschließlich auf den drei vorgestellten Domänen. Wir haben auch einige andere Probleme auf dem Gebiet der verteilten Algorithmen getestet. Zusammen mit diesen andernorts veröffentlichten Arbeiten glauben wir, ein solides Fundament für die Bewertung unserer Methode gelegt zu haben.

\section{E.4.3 Qualität der Eigenschaften der GP-Ansätze}

\section{Ist Rule-based Genetic Programming gut?}

Eine der wichtigsten Ergebnisse unserer Forschung ist der regelbasierte Ansatz zur Genetischen Programmierung (engl. Rule-based Genetic Programming, RBGP). Wir haben RBGP in zwei Varianten angewendet: die simple, binär kodierte Version RBGP und die baumbasierte eRBGP variante.

In vielen unserer Experimente gab es einen großen Unterschied zwischen der Performanz beider Methoden. Im Auswahlalgorithmus-Szenario (Chapter 7) dominierte eRBGP alle anderen Ansätze wohingegen RBGP in fast allen Vergleichen verloren hat. Beide Ansätze konnten keinen adäquaten Algorithmus für den wechselseitigen Ausschluss finden. In der GGT-Domäne war eRBGP wieder die beste Methode wobei RBGP keine einzige Lösung finden konnte.

Aus diesen experimentalen Ergebnissen folgt, dass die regelbasierte Genetische Programmierung durchaus eine Daseinsberechtigung hat die höchstwahrscheinlich nicht auf das Gebiet der verteilten Algorithmen beschränkt ist. Zumindest die eRBGP-Variante hat sich in zwei der drei getesteten Domänen exzellent verhalten. Daher kann die Frage ob dieser Ansatz irgendeinen Nutzen haben könnte klar mit Ja. beantwortet werden.

\section{Ist indirekt adressierbarer Speicher gut?}

eRBGP hat oftmals wesentlich bessere Ergebnisse als RBGP erzielt. Dies mag entweder an der Turing-Vollständigkeit durch den verwendeten indirekt adressierbaren Speicher oder an den höheren Freiheitsgraden durch das Baumgenom gelegen haben. In unseren Experimenten haben wir noch zwei weitere, einander ähnliche, Ansätze getestet, zwischen denen der Hauptunterschied die (vorhandene oder fehlende) Turing-Vollständigkeit durch (vorhandenen oder fehlenden) indirekt adressierbaren Speicher war: eSGP und SGP. Zwischen diesen beiden Ansätzen konnte jedoch meist kein signifikanter Performanzunterschied festgestellt werden.

Daher vermuten wir, dass die durch das baumbasierte Genom zur Verfügung gestellte Vielseitigkeit der entscheidende Unterschied zwischen eRBGP und RBGP ist. Eine Aussage darüber ob indirekt adressierbarer Speicher in Szenarien wo er nicht zwingend erforderlich ist nützlich ist, ist daher nicht möglich. Dennoch führt sein Vorhandensein auch nicht zu 
messbaren Nachteilen - obwohl auch diese zu mehr Freiheitsgraden und einer Vergrößerung des Suchraums führt.

Interessanterweise ist die Verwendung eines temporären Speichers der Hauptunterschied zwischen den beiden regelbasierten GP-Ansätzen und den Standard-GP-Methoden (neben der Regelbasiertheit). Diese Form von Speicher ist der einzige Grund warum die Reihenfolge der Regeln in RBGP keine Rolle Spielt. Wir vermuten dass das Einführen eines temporären Speichers und expliziter Commit- und Rollback-Instruktionen in SGP oder eSGP einen starken positiven Einfluss auf deren Effizienz haben könnte. Diese Hypothese sollte in zukünftigen Forschungsarbeiten unbedingt geklärt werden.

\section{E.5 Fazit}

Das Ziel unserer Forschung war es, eine neue Methode für den Entwurf von Algorithmen für verteilte Systeme zu erforschen. Diese Methode schreibt einen Prozess vor, der mit der Spezifikation des gewünschten globalen Systemverhaltens beginnt. Von dieser Definition werden Zielfunktionen manuell abgeleitet, die bewerten, wie nahe ein in einer Netzwerksimulation beobachtetes Systemverhalten dem gewünschten Verhalten kommt. Auf jedem Knoten des simulierten Systems laufen dieselben Programme, welche wiederum die Lösungskandidaten eines evolutionären Algorithmus sind. Mit seinen Suchoperationen modifiziert und kombiniert der EA diese Programme um neue, immer bessere und schließlich optimale Zielfunktionswerte zu erreichen. Dies führt zu einer iterativen Übersetzung eines gewünschten globalen Systemverhaltens zu Programmen, die lokal auf den Knoten des Systems ausgeführt werden müssen, um dieses Verhalten zu erreichen.

Wie in Chapter 3 dargestellt, gibt es nur wenige verwandte Arbeiten auf diesem Gebiet. Einer der Gründe dafür mag sein, dass das Züchten "echter" Algorithmen ein besonders schweres Problem mit komplizierter Fitnesslandschaft mit Aspekten wie Schroffheit, Neutralität und Epistase darstellt.

Um einen Überblick über die Anwendbarkeit von Genetischer Programmierung auf diese Problemklasse zu gewinnen, haben wir sechs verschiedene GP-Ansätze getestet. Drei von ihnen (SGP, eSGP und LGP) sind Erweiterungen bekannter Methoden, einer (Fraglets) entstammt biologisch-inspirierter Forschung und zwei (RBGP, eRBGP) wurden speziell von uns im Hinblick auf die Fitnesslandschaft entwickelt. Die letzte Methode, eRBGP, zeigte ein exzellentes Verhalten in vielen der Experimente was die ihrem Design zugrundeliegenden Vermutungen bestätigt.

Unsere Experimente umfassten drei Problemdomänen (Auswahl, kritische Abschnitte und den verteilten GGT). Die Ergebnisse der Experimente zeigen, dass verteilte Algorithmen, die sich adäquat hinsichtlich der Zielfunktionen verhalten, für nicht-triviale Probleme mit GP synthetisiert werden können. Die so erzeugten Programme können jedoch merklich von menschengemachten Lösungen abweichen und sind nicht notwendigerweise korrekt. In Abschnitt E.4 (und Chapter 10) haben wir dieses Verhalten ausführlich bewertet und Gebiete aufgezeigt, für die unser Ansatz nützlich sein kann und wo er eine interessante Alternative zu traditionellen Methoden darstellt. 


\section{Bibliography}

[1] Jan Oyvind Aagedal, Jean Bezivin, and Peter F. Linington. Model-Driven Development. In J. Malenfant and Bjarte M. Ostvold, editors, ECOOP 2004 Workshop Reader, Proc. of ECOOP 2004 Workshop, volume 3344 of LNCS. Springer, June 14-18, 2004, Oslo, Norway. ISBN: 3-5402-3988-X.

[2] Hal Abelson, editor. Workshop on Amorphous Computing, September 13-14, 1999, Cambridge, USA. MIT AI Lab. Online available at http://www-swiss.ai.mit.edu/projects/ amorphous/workshop-sept-99/ [accessed 2008-07-27].

[3] Aesop. The Boy and the Filberts, around 600 BC. Online available at http://www. aesopfables.com/cgi/aesop1.cgi?1\&TheBoyandtheFilberts [accessed 2008-11-29].

[4] Enrique Alba and J. Francisco Chicano. Evolutionary Algorithms in Telecommunications. In IEEE Mediterranean Electrotechnical Conference, MELECON 2006, pages 795-798, May 1619, 2006. doi:10.1109/MELCON.2006.1653218. Online available at http://dea.brunel.ac . uk/ugprojects/docs/melecon06.pdf [accessed 2008-08-01].

[5] Lee Altenberg. NK Fitness Landscapes. In Handbook of Evolutionary Computation, chapter B2.7.2. Oxford University Press, November 27, 1996. In collection [10]. Online available at http://dynamics.org/Altenberg/FILES/LeeNKFL.pdf [accessed 2008-10-06].

[6] Peter John Angeline and Jordan Pollack. Evolutionary Module Acquisition. In David B. Fogel and W. Atmar, editors, Proc. of 2nd Annual Conference on Evolutionary Programming, pages 154-163. Evolutionary Programming Society, February 25-26, 1993, La Jolla, USA. Online available at https://eprints.kfupm.edu.sa/38410/ [accessed 2008-10-18].

[7] Grenville Armitage, Mark Claypool, and Philip Branch. Network Latency, Jitter and Loss. In Networking and Online Games: Understanding and Engineering Multiplayer Internet Games, chapter 5, pages 69-82. Wiley \& Sons, 2006. ISBN: 978-0-47001-857-6. doi:10.1002/047003047X.ch.

[8] Uwe Aßmann, Mehmet Aksit, and Arend Rensink, editors. Model Driven Architecture, European MDA Workshops: Foundations and Applications, MDAFA 2003 and MDAFA 2004, Twente, The Netherlands, June 26-27, 2003 and Linköping, Sweden, June 10-11, 2004, Revised Selected Papers, volume 3599 of LNCS, 2005. Springer. ISBN: 3-5402-8240-8.

B

[9] Thomas Bäck. Evolutionary Algorithms in Theory and Practice: Evolution Strategies, Evolutionary Programming, Genetic Algorithms. Oxford University Press, January 1996. ISBN: 0-1950-9971-0. 
[10] Thomas Bäck, David B. Fogel, and Zbigniew Michalewicz, editors. Handbook of Evolutionary Computation. Computational Intelligence Library. Oxford University Press, April 1997. ISBN: 978-0-75030-392-7. Partly online available at http://books.google.de/books? id=n5nuiIZvmpAC [accessed 2008-11-15].

[11] James Mark Baldwin. A New Factor in Evolution. The American Naturalist, 30:441-451, June 1896. ISSN: 0003-0147. Online available at http://www.brocku.ca/MeadProject/Baldwin/ Baldwin_1896_h.html [accessed 2008-09-10]. See also [12].

[12] James Mark Baldwin. A New Factor in Evolution. In Richard K. Belew and Melanie Mitchell, editors, Adaptive individuals in evolving populations: models and algorithms, volume 26 of Santa Fe Institue Studies in the Sciences of Complexity, pages 59-80. Addison-Wesley Longman Publishing, January 15, 1996. ISBN: 978-0-20148-369-7. See also [11].

[13] Jean-Pierre Banâre, Pascal Fradet, and Yann Radenac. Chemical Specification of Autonomic Systems. In Proc. of ISCA 13th International Conference on Intelligent and Adaptive Systems and Software Engineering (IASSE), pages 72-79, July 1-3, 2004, Nice, France. Online available at http://download.scientificcommons.org/140384 [accessed 2009-02-21].

[14] Jean-Pierre Banâtrea, Pascal Fradet, and Yann Radenac. Principles of Chemical Programming. In Slim Abdennadher and Christoph Ringeissen, editors, Proc. of Fifth International Workshop on Rule-Based Programming (RULE 2004), June 1, 2004, Aachen, Germany. Online available at http://pop-art.inrialpes.fr/ fradet/PDFs/RULE04.pdf [accessed 2009-02-21].

[15] Jean-Pierre Banâtrea, Pascal Fradet, and Yann Radenac. Principles of Chemical Programming. Electronic Notes in Theoretical Computer Science, 124(1):133-147, March 2, 2005. ISSN: 1571-0661. doi:doi:10.1016/j.entcs.2004.07.019. Online available at http://dx.doi. org/10.1016/j.entcs.2004.07.019 [accessed 2009-02-21]. See also [14].

[16] Ajay Bansal, M. Brian Blake, Srividya Kona, Steffen Bleul, Thomas Weise, and Michael C. Jaeger. WSC-08: Continuing the Web Service Challenge. In Proc. of IEEE Joint Conference (CEC/EEE 2008) on E-Commerce Technology (Tenth CEC'07) and Enterprise Computing, E-Commerce and E-Services (Fifth EEE'08), pages 351-354. IEEE, July 21-24, 2008, Washington, D.C., USA. ISBN: 978-0-76953-340-7. doi:10.1109/CEC/EEE.2008.67. Online available at http://www . it-weise.de/documents/files/BBKBWJ2008WSC08CTWSC . pdf [accessed 200905-07].

[17] Wolfgang Banzhaf. Genetic Programming for Pedestrians. In Stephanie Forrest, editor, Proc. of 5th International Conference on Genetic Algorithms, ICGA, pages 17-21. Morgan Kaufmann, July 17-21, 1993, University of Illinois at Urbana-Champaign, USA. ISBN: 1-5586-0299-2. Online available at http://www.cs.bham.ac.uk/ wbl/biblio/gp-html/ banzhaf_mrl.html [accessed 2008-09-17].

[18] Wolfgang Banzhaf and Christian W. G. Lasarczyk. Genetic Programming of an Algorithmic Chemistry. In Una-May O'Reilly et al., editor, Genetic Programming Theory and Practice II, Proc. of Genetic Programming Theory Practice 2004 Workshop (GPTP-2004), volume 8 of Genetic Programming Series, pages 175-190. Springer, May 13-15, 2004, Ann Arbor, USA. ISBN: 978-0-38723-253-9. doi:10.1007/0-387-23254-0_11. Online available at http://www.cs . bham.ac.uk/ wbl/biblio/gp-html/banzhaf_2004_GPTP.html [accessed 2008-04-30].

[19] Wolfgang Banzhaf, Peter Nordin, Robert E. Keller, and Frank D. Francone. Genetic Programming: An Introduction - On the Automatic Evolution of Computer Programs and Its Applications. Morgan Kaufmann, November 30, 1997. ISBN: 978-1-55860-510-7. Partly online available at http://books.google.de/books?id=1697qefFdtIC [accessed 2008-09-16].

[20] Valmir C. Barbosa. An Introduction to Distributed Algorithms. The MIT Press, September 1996. ISBN: 978-0-26202-412-9. Partly online available at http://books.google.de/books? id=fPaGr08KFqQC [accessed 2008-11-25].

[21] Lionel Barnett. Tangled Webs: Evolutionary Dynamics on Fitness Landscapes with Neutrality. Master's thesis, School of Cognitive Science, University of East Sussex, Brighton, UK, 1997. Online available at ftp://ftp.cogs.susx.ac.uk/pub/users/inmanh/lionelb/ [accessed 2007-08-13].

[22] Lionel Barnett. Ruggedness and Neutrality - The NKp Family of Fitness Landscapes. In Artificial Life VI: Proc. of sixth international conference on Artificial life, volume 6 of Complex Adaptive Systems, pages 18-27. MIT Press, June 1998, Madison, USA. ISBN: 978-0-26251-099-8. Online available at http://citeseer.ist.psu.edu/ barnett98ruggedness.html [accessed 2008-06-01], online available at http://citeseer.ist.psu. edu/144047.html [accessed 2008-02-27]. 
[23] William Bateson. Mendel's Principles of Heredity. Cambridge University Press, 1909. ISBN: 978-1-42864-819-7. 1930: fourth impression of the 1909 edition.

[24] Boris Beizer. Software Testing Techniques. International Thomson Computer Press, second edition, June 1990. ISBN: 978-1-85032-880-3.

[25] Mordechai Ben-Ari. Principles of Concurrent and Distributed Programming. Prentice-Hall International Series in Computer Science. Addison Wesley / Pearson Education, 2nd edition, March 6, 2006. ISBN: 978-0-32131-283-9. Partly online available at http://books.google. de/books?id=oP-2hpMEdb8C [accessed 2008-10-02].

[26] Mordechai Ben-Ari. Principles of the Spin Model Checker. Springer, January 2008. ISBN: 978-1-84628-769-5.

[27] Carole Bernon, Valérie Camps, Marie-Pierre Gleizes, and Gauthier Picard. Designing Agents' Behaviours within the Framework of ADELFE Methodology. In International Workshop on Engineering Societies in the Agents World (ESAW 2003), volume 3071 of LNCS, pages 311-327. Springer, October 29-31 2003, London, UK. ISBN: 978-3-54022-231-6. doi:10.1007/b98212. Online available at ftp://ftp.irit.fr/pub/IRIT/SMAC/DOCUMENTS/ PUBLIS/ESAW03_Bernon.pdf [accessed 2008-11-19].

[28] Carole Bernon, Valérie Camps, Marie-Pierre Gleizes, and Gauthier Picard. Engineering Adaptive Multi-Agent Systems: The ADELFE Methodology. In Brian Henderson-Sellers and Paolo Giorgini, editors, Agent-Oriented Methodologies, chapter VII, pages 172-202. Idea Group Inc (IGI), June 2005. ISBN: 978-1-59140-581-8. Partly online available at http://books . google . de/books?id=ylGAvAA3dPUC [accessed 2008-11-19]. See also [29].

[29] Carole Bernon, Valérie Camps, Marie-Pierre Gleizes, and Gauthier Picard. Engineering Adaptive Multi-Agent Systems: The ADELFE Methodology. In Vijayan Sugumaran, editor, Intelligent Information Technologies: Concepts, Methodologies, Tools and Applications, chapter 2.9, pages 513-535. Idea Group Inc (IGI), November 2007. ISBN: 978-1-59904-941-0. Partly online available at http://books.google.de/books?id=SYH7f_JjEqYC [accessed 2008-11-19]. See also [28].

[30] Robert Beverly, Karen Sollins, and Arthur Berger. SVM Learning of IP Address Structure for Latency Prediction. In MineNet'06: Proc. of 2006 SIGCOMM workshop on Mining network data, pages 299-304. ACM, September 2006, Pisa, Italy. Online available at http://portal. acm.org/citation. cfm?id=1162678.1162682 [accessed 2007-08-13].

[31] Hans-Georg Beyer. Towards a Theory of 'Evolution Strategies': Some Asymptotical Results from the $(1,+\lambda)$-Theory. Technical Report SYS-5/92, Fachbereich Informatik, Universität Dortmund, Germany, December 1, 1992. See also [32].

[32] Hans-Georg Beyer. Toward a Theory of Evolution Strategies: Some Asymptotical Results from the $(1,+\lambda)$-Theory. Evolutionary Computation, 1(2):165-188, Summer 1993. ISSN: 1063-6560. Online available at http://1s11-www.cs.uni-dortmund.de/ beyer/coll/ Bey93a/Bey93a.ps [accessed 2008-07-19]. See also [31].

[33] Steffen Bleul, Thomas Weise, and Kurt Geihs. Large-Scale Service Composition in Semantic Service Discovery. In Ws-Challenge Part: M. Brian Blake, Andreas Wombacher, Michel C. Jaeger, and William K. Cheung, editors, Proc. of 2006 IEEE Joint Conference on E-Commerce Technology (CEC'06) and Enterprise Computing, E-Commerce and E-Services (EEE'06), pages 427-429. IEEE, June 26-29, 2006, Millbrae, USA. ISBN: 978-0-76952-511-2. 1st place in 2006 WSC. Online available at http://www.it-weise.de/documents/files/ BWG2006WSC.pdf [accessed 2009-05-07]. See 2007 WSC [34] and [296].

[34] Steffen Bleul, Thomas Weise, and Kurt Geihs. Making a Fast Semantic Service Composition System Faster. In Proc. of IEEE Joint Conference (CEC/EEE 2007) on E-Commerce Technology (9th CEC'O7) and Enterprise Computing, E-Commerce and E-Services (4th EEE'07), pages 517-520. IEEE, July 23-26, 2007, Tokyo, Japan. ISBN: 978-0-76952-913-4. 2nd place in 2007 WSC. Online available at http://www.it-weise.de/documents/files/BWG2007WSC . pdf [accessed 2009-05-07]. See 2006 WSC [33] and [296].

[35] Stefan Bleuler, Martin Brack, Lothar Thiele, and Eckart Zitzler. Multiobjective Genetic Programming: Reducing Bloat Using SPEA2. In Proc. of IEEE Congress on Evolutionary Computation, CEC2001, pages 536-543. IEEE, May 27-30, 2001, Seoul, Korea. ISBN: 0-7803-6658-1. Online available at ftp://ftp.tik.ee.ethz.ch/pub/people/zitzler/ BBTZ2001b.ps.gz [accessed 2007-09-07].

[36] Tobias Blickle and Lothar Thiele. Genetic Programming and Redundancy. In J. Hopf, editor, Genetic Algorithms within the Framework of Evolutionary Computation, Workshop at 
KI-94, pages 33-38. Max-Planck-Institut für Informatik (MPI-I-94-241), 1994, Saarbrücken, Germany. Online available at http://www.tik.ee.ethz.ch/ tec/publications/bt94/ GPandRedundancy.ps.gz [accessed 2007-09-07].

[37] Markus F. Brameier and Wolfgang Banzhaf. A Comparison of Linear Genetic Programming and Neural Networks in Medical Data Mining. IEEE Transactions on Evolutionary Computation, 5(1):17-26, 2001. Online available at http://web.cs.mun.ca/ banzhaf/papers/ ieee_taec.pdf [accessed 2007-09-09].

[38] Markus F. Brameier and Wolfgang Banzhaf. Explicit Control of Diversity and Effective Variation Distance in Linear Genetic Programming. In James A. Foster et al., editor, Proc. of 5th European Conference on Genetic Programming, EuroGP 2002, volume 2278/2002 of LNCS, pages 37-49. Springer, April 3-5, 2002, Kinsale, Ireland. ISBN: 3-5404-3378-3. Online available at http://citeseer.ist.psu.edu/552561.html [accessed 2007-09-09].

[39] Jürgen Branke. Creating Robust Solutions by Means of Evolutionary Algorithms. In Ágoston E. Eiben et al., editor, Proc. of 5th International Conference on Parallel Problem Solving from Nature - PPSN V, volume 1498/1998 of LNCS, pages 119-128. Springer, September 27-30, 1998, Amsterdam, The Netherlands. ISBN: 978-3-54065-078-2. doi:10.1007/BFb0056855.

[40] Will N. Browne and Charalambos Ioannides. Investigating Scaling of an Abstracted LCS Utilising Ternary and S-Expression Alphabets. In Dirk Thierens et al., editor, Proc. of Genetic and Evolutionary Computation Conference, GECCO 2007, pages 2759-2764. ACM, July 7-12, 2007, London, UK. ISBN: 978-1-59593-697-4. doi:10.1145/1274000.1274067. Online available at http://portal.acm.org/citation.cfm?id=1274000.1274067 [accessed 2007-08-01].

[41] Edmund K. Burke, Steven Matt Gustafson, Graham Kendall, and Natalio Krasnogor. Is Increasing Diversity in Genetic Programming Beneficial? An Analysis of the Effects on Fitness. In Ruhul Sarker et al., editor, Proc. of IEEE Congress on Evolutionary Computation, CEC2003, pages 1398-1405. IEEE, December 8-12, 2003, Canberra, Australia. ISBN: 0-7803-7804-0. Online available at http://citeseer.ist.psu.edu/700607.html [accessed 200807-22].

C

[42] Edgar H. Callaway, Jr. Wireless Sensor Networks: Architectures and Protocols. AUERBACH, August 26, 2003. ISBN: 978-0-84931-823-8.

[43] Valérie Camps, Marie-Pierre Gelizes, and Pierre Glize. Une théorie des phénomènes globaux fondée sur des interactions locales. In Jean-Paul Barthès, Vincent Chevrier, and Christian Brassac, editors, JFIADSMA'98: Actes des Sixième journées francophones pour l'Intelligence Artificielle Distribuée et les Systèmes Multi-Agents (IADESSMA). Editions Hermès, November 18-20, 1998, Nance, France. ISBN: 978-2-86601-733-0. Online available at ftp://ftp. irit.fr/pub/IRIT/SMAC/DOCUMENTS/PUBLIS/JFIADSMA98.pdf [accessed 2008-10-08].

[44] Erick Cantú-Paz, Martin Pelikan, and David E. Goldberg. Linkage Problem, Distribution Estimation, and Bayesian Networks. Evolutionary Computation, 8(3):311-340, Fall 2000. ISSN: 1063-6560. doi:10.1162/106365600750078808. Online available at http://citeseerx. ist.psu.edu/viewdoc/summary?doi=10.1.1.16.236 [accessed 2008-10-18].

[45] Vinton Cerf, Yogen Dalal, and Carl Sunshine. Specification of Internet Transmission Control Program (December 1974 Version). Request for Comments (RFC) 675, Network Working Group, December 1974. Online available at http://tools.ietf.org/html/rfc675 [accessed 2008-06-13].

[46] Ernest Chang and Rosemary Roberts. An improved Algorithm for decentralized ExtremaFinding in Circular Configurations of Processes. Communications of the ACM, 22(5):281-283, 1979. ISSN: 0001-0782. doi:10.1145/359104.359108. Online available at http://doi.acm.org/ 10.1145/359104.359108 [accessed 2007-10-24].

[47] Alonzo Church. An Unsolvable Problem of Elementary Number Theory. American Journal of Mathematics, 58(2):345-363, April 1936.

[48] Alonzo Church. A Note on the Entscheidungsproblem. Journal of Symbolic Logic, 1(1):40-41, March 1936. 
[49] Edmund M. Clarke and E. Allen Emerson. Design and Synthesis of Synchronization Skeletons Using Branching-Time Temporal Logic. In Dexter Kozen, editor, Logics of Programs, Workshop, volume 131/1982 of LNCS, pages 52-71. Springer, May 1981, New York, USA. ISBN: 978-3-54011-212-9. doi:10.1007/BFb0025774. Published in 1982.

[50] Edmund M. Clarke, Jr., Orna Grumberg, and Doron A. Peled. Model Checking. The MIT Press, January 2000. ISBN: 978-0-26203-270-4. Partly online available at http://books . google .de/books?id=Nmc4wEaLXFEC [accessed 2008-10-02].

[51] Francesc Comellas and G. Giménez. Genetic Programming to Design Communication Algorithms for Parallel Architectures. Parallel Processing Letters (PPL), 8(4):549-560, December 1998. ISSN: 0129-6264. doi:10.1142/S0129626498000547. Online available at http:// citeseer.ist.psu.edu/comellas98genetic.html [accessed 2007-09-14].

[52] David Wolfe Corne, Martin J. Oates, and George D. Smith, editors. Telecommunications Optimization: Heuristic and Adaptive Techniques. Wiley \& Sons, September 2000. ISBN: 978-0-47198-855-7. doi:10.1002/047084163X.

[53] Pablo Cortés Achedad, Luis Onieva Giménez, Jesús Muñuzuri Sanz, and José Guadix Martín. A Revision of Evolutionary Computation Techniques in Telecommunications and An Application for The Network Global Planning Problem. In Ang Yang, Yin Shan, and Lam Thu Bui, editors, Success in Evolutionary Computation, volume 92/2008 of Studies in Computational Intelligence, pages 239-262. Springer, 2008. ISBN: 978-3-54076-285-0. doi:10.1007/978-3-540-76286-7_11.

[54] George F. Coulouris, Jean Dollimore, and Tim Kindberg. Distributed Systems: Concepts and Design. Pearson Education / Addison-Wesley Longman, fourth rev. edition, June 2005. ISBN: 978-0-32126-354-4. Partly online available at http://books.google.de/books? id=d63sQPvBezgC [accessed 2008-11-25].

[55] Steven H. Cousins. Species Diversity Measurement: Choosing the Right Index. Trends in Ecology and Evolution (TREE), 6(6):190-192, June 1991. ISSN: 01695347. doi:10.1016/0169-5347(91)90212-G. Online available at http://dx.doi.org/10.1016/ 0169-5347 (91)90212-G [accessed 2008-11-10].

\section{D}

[56] Shantanu Das, Paola Flocchini, Amiya Nayak, and Nicola Santoro. Effective Elections for Anonymous Mobile Agents. In Algorithms and Computation, volume 4288/2006 of LNCS, chapter Session 9B: Distributed Computing and Cryptography, pages 732-743. Springer, 2006. ISBN: 978-3-54049-694-6. doi:10.1007/11940128 and 10.1007/11940128_73.

[57] Yuval Davidor. Epistasis Variance: A Viewpoint on GA-Hardness. In Bruce M. Spatz and Gregory J. E. Rawlins, editors, Proc. of First Workshop on Foundations of Genetic Algorithms (FOGA), pages 23-35. Morgan Kaufmann, July 15-18, 1990, Indiana, USA. ISBN: 1-5586-0170-8.

[58] Martin Davis. Engines of Logic. W.W. Norton \& Company, 2000. ISBN: 0-3933-2229-7.

[59] Richard Dawkins. The Evolution of Evolvability. In Christopher G. Langdon, editor, Artificial Life: The Proc. of an Interdisciplinary Workshop on the Synthesis and Simulation of Living Systems, volume 6 of Santa Fe Institute Studies in the Science of Complexity, pages 201-220. Addisson-Wesley Publishing Company, September 1987, Los Alamos, USA. ISBN: 978-0-20109-356-8.

[60] Sérgio Granato de Araújo, Aloysio de Castro Pinto Pedroza, and Antônio Carneiro de Mesquita Filho. Evolutionary Synthesis of Communication Protocols. In Proc. of 10th International Conference on Telecommunications (ICT'03), volume 2, pages 986-993, February 23-March 1, 2003, Tahiti, French Polynesia. doi:10.1109/ICTEL.2003.1191573. Online available at http:// www.gta.ufrj.br/ftp/gta/TechReports/AMP03a.pdf [accessed 2008-06-21]. See also [61, 62] for Portuguese versions.

[61] Sérgio Granato de Araújo, Aloysio de Castro Pinto Pedroza, and Antônio Carneiro de Mesquita Filho. Uma Metodologia de Projeto de Protocolos de Comunicação Baseada em Técnicas Evolutivas. In XX Simpósio Brasileiro de Telecomunicações (SBT'03), October 5-8, 2003, 
Rio de Janeiro, Brasil. Online available at http://www.gta.ufrj.br/ftp/gta/TechReports/ AMP03e.pdf [accessed 2008-06-21]. See also [62] and the English version [60].

[62] Sérgio Granato de Araújo, Antônio Carneiro de Mesquita Filho, and Aloysio de Castro Pinto Pedroza. Síntese de Circuitos Digitais Otimizados via Programação Genética. In $X X X$ Seminário Integrado de Software e Hardware (SEMISH'03) in Proc. of XXIII Congresso da Sociedade Brasileira de Computação, volume III, pages 273-285, August 4-5, 2003, Campinas, Brasil. Online available at http://www.gta.ufrj.br/ftp/gta/TechReports/AMP03d. pdf [accessed 2008-06-21]. See also [61] and the English version [60].

[63] Edwin D. de Jong, Richard A. Watson, and Jordan B. Pollack. Reducing Bloat and Promoting Diversity using Multi-Objective Methods. In Lee Spector et al., editor, Proc. of Genetic and Evolutionary Computation Conference (GECCO'01), pages 11-18. Morgan Kaufmann, July 7-11, 2001, San Francisco, USA. ISBN: 978-1-55860-774-3. Online available at http:// www.cs.bham.ac.uk/ wbl/biblio/gp-html/jong_2001_gecco.html [accessed 2008-07-20].

[64] Alfonso Ortega de la Puente, Marina de la Cruz Echeandía, and Manuel Alfonseca. Christiansen Grammar Evolution: Grammatical Evolution With Semantics. IEEE Transactions on Evolutionary Computation, 11(1):77-90, February 2007. ISSN: 1089-778X. Online available at http://arantxa.ii.uam.es/ alfonsec/artint/ieeetec.pdf [accessed 2007-09-09].

[65] Kalyanmoy Deb. Genetic Algorithms for Multimodal Function Optimization. Master's thesis, The Clearinghouse for Genetic Algorithms, University of Alabama, Tuscaloosa, 1989. TCGA Report No. 89002.

[66] Kalyanmoy Deb. An Introduction to Genetic Algorithms. Sadhana, 24(4-5):293-315, 1999. Online available at http://www.iitk.ac.in/kangal/papers/sadhana.ps.gz [accessed 2007-0728].

[67] Kalyanmoy Deb. Genetic Algorithms for Optimization. KanGAL Report 2001002, Kanpur Genetic Algorithms Laboratory (KanGAL), Kanpur, India, 2001. Online available at http:// www.iitk.ac.in/kangal/papers/isna.ps.gz [accessed 2007-07-28].

[68] Kalyanmoy Deb and David E. Goldberg. An Investigation of Niche and Species Formation in Genetic Function Optimization. In J. David Schaffer, editor, Proc. of 3rd International Conference on Genetic Algorithms, pages 42-50. Morgan Kaufmann, June 1989, Fairfax, USA. ISBN: 1-5586-0066-3.

[69] Tom Dietterich. Overfitting and Undercomputing in Machine Learning. ACM Computing Surveys (CSUR), 27(3):326-327, 1995. ISSN: 0360-0300. doi:10.1145/212094.212114. Online available at http://citeseer.ist.psu.edu/dietterich95overfitting.html [accessed 2007-0913].

[70] Edsger Wybe Dijkstra. A Note on Two Problems in Connexion with Graphs. Numerische Mathematik, 1:269-271, 1959. Online available at http://www-m3.ma.tum.de/twiki/pub/ MN0506/WebHome/dijkstra.pdf [accessed 2008-06-24].

[71] Edsger Wybe Dijkstra. Cooperating Sequential Processes. EWD 123, Techniche Hogeschool Eindhoven, 1965. Online available at http://www.cs.utexas.edu/users/EWD/ transcriptions/EWD01xx/EWD123.html [accessed 2008-11-16]. Discusses Theodorus Jozef Dekker's algorithm. See also [72].

[72] Edsger Wybe Dijkstra. Cooperating Sequential Processes. In F. Genuys, editor, Programming languages (Electronic computers), pages 43-112. Academic Press, 1968. ISBN: 978-0-12279-750-7. See also [71]. Book contains the lectures given at a three week summer school held in Villard-de-Lans sponsored by the NATO Science Committee under the 1966 Advanced Study Institute Programme and organized with the co-operation of the University of Grenoble.

[73] Edsger Wybe Dijkstra. Guarded Commands, Nondeterminacy and Formal Derivation of Programs. Communications of the ACM, 18(8):453-458, August 1975. ISSN: 00010782. doi:10.1145/360933.360975. Online available at http://doi . acm.org/10.1145/360933. 360975 [accessed 2008-07-23].

[74] Paolo Dini. Section 1: Science, New Paradigms. Subsection 1: A Scientific Foundation for Digital Ecosystems. In Francesco Nachira et al., editor, Digital Business Ecosystems. The European Commission in association and with the support of the FP6 projects DBE and OPAALS, 2007. ISBN: 9-2790-1817-5. Online available at http://www.digital-ecosystems . org/book/Section1.pdf [accessed 2008-11-09]. 
[75] Robert Eckstein, David Collier-Brown, and Peter Kelly. Using Samba. O'Reilly \& Associates, 1st edition, November 1999. ISBN: 1-5659-2449-5. Online available at http://www.oreilly . com/catalog/samba/chapter/book/ [accessed 2007-10-24].

[76] Ágoston E. Eiben and C. A. Schippers. On Evolutionary Exploration and Exploitation. Fundamenta Informaticae, 35(1-4):35-50, 1998. Online available at http://citeseer.ist. psu.edu/eiben98evolutionary.html [accessed 2007-08-22].

[77] Anikó Ekárt and Sándor Zoltán Németh. Selection Based on the Pareto Nondomination Criterion for Controlling Code Growth in Genetic Programming. Genetic Programming and Evolvable Machines, 2(1):61-73, March 2001. ISSN: 1389-2576. doi:10.1023/A:1010070616149. Online available at http://www.springerlink.com/content/j70v0303523v6x65/fulltext. pdf [accessed 2008-05-04].

[78] Niles Eldredge and Stephen Jay Gould. Punctuated equilibria: The Tempo and Mode of Evolution Reconsidered. Paleobiology, 3(2):115-151, April 1, 1977. Online available at http:// www.nileseldredge.com/pdf_files/Punctuated_Equilibria_Gould_Eldredge_1977.pdf [accessed 2008-11-09].

[79] Nils Eldredge and Stephen Jay Gould. Punctuated Equilibria: an Alternative to Phyletic Gradualism. In Thomas J. M. Schopf, editor, Models in Paleobiology, chapter 5, pages 82-115. Freeman, Cooper \& Co / DoubleDay, January 1972. ISBN: 978-0-38503-024-3. Online available at http://www.blackwellpublishing.com/ridley/classictexts/eldredge.pdf [accessed 2008-07-01].

[80] Larry J. Eshelman and J. David Schaffer. Preventing Premature Convergence in Genetic Algorithms by Preventing Incest. In Richard K. Belew and Lashon Bernard Booker, editors, Proc. of 4th International Conference on Genetic Algorithms, pages 115-122. Morgan Kaufmann, July 1991, San Diego, USA. ISBN: 1-5586-0208-9.

[81] Altannar Chinchuluun et al., editor. Pareto Optimality, Game Theory and Equilibria, volume 17 of Springer Optimization and Its Applications. Springer, 2008. ISBN: 978-0-38777-246-2. doi:10.1007/978-0-387-77247-9. Partly online available at http://books . google.de/books?id=kNqHxU3Tc2YC [accessed 2009-02-20].

[82] John Krumm et al., editor. UbiComp 200\%: Ubiquitous Computing. Proc. of 9th International Conference, volume 4717 of LNCS, September 16-19, 2007, Innsbruck, Austria. Springer. ISBN: 978-3-54074-852-6. doi:10.1007/978-3-540-74853-3.

[83] Kim Kirsner et al., editor. Implicit and Explicit Mental Processes. Lawrence Erlbaum Associates, 1998. ISBN: 978-0-80581-359-3. Partly online available at http://books.google.de/ books? id=3o3mFVUtkjkC [accessed 2008-12-01].

\section{F}

[84] Cândida Ferreira. Gene Expression Programming: A New Adaptive Algorithm for Solving Problems. Complex Systems, 13:87, 2001. Online available at http://www. gene-expression-programming.com/GEPBiblio. asp [accessed 2007-08-23].

[85] Cândida Ferreira. Mutation, Transposition, and Recombination: An Analysis of the Evolutionary Dynamics. In H. John Caulfield et al., editor, Proc. of 6th Joint Conference on Information Science (JCIS), pages 614-617. JCIS / Association for Intelligent Machinery, March 2002, Research Triangle Park, USA. ISBN: 0-9707-8901-7. Online available at http://www. gene-expression-programming.com/GEPBiblio. asp [accessed 2007-08-23].

[86] Mark Fewster and Dorothy Graham. Software Test Automation. Addison-Wesley Professional, August 25, 1999. ISBN: 0-2013-3140-3.

[87] Sir Ronald Aylmer Fisher. The Correlations between Relatives on the Supposition of Mendelian Inheritance. Philosophical Transactions of the Royal Society of Edinburgh, 52:399-433, 1918. See also http://en.wikipedia.org/wiki/ The_Correlation_Between_Relatives_on_the_Supposition_of_Mendelian_Inheritance [accessed 2008-03-02]. 
[88] Sir Ronald Aylmer Fisher. On the Interpretation of $\chi^{2}$ from Contingency Tables, and the Calculation of P. Journal of the Royal Statistical Society, 85:87-94, 1922. Online available at http://hdl. handle.net/2440/15173 [accessed 2008-12-08].

[89] Sir Ronald Aylmer Fisher. Statistical Methods for Research Workers. Oliver and Boyd, 1925, 1970. ISBN: 978-0-05002-170-5. Online available at http://psychclassics.yorku.ca/ Fisher/Methods/ [accessed 2008-12-08].

[90] J. Michael Fitzpatrick and John J. Grefenstette. Genetic Algorithms in Noisy Environments. Machine Learning, 3(2-3):101-120, October 1988. ISSN: 0885-6125. doi:10.1007/BF00113893. Online available at http://www.springerlink.com/content/n6w328441t63q374/fulltext. pdf [accessed 2008-07-19].

[91] David B. Fogel, editor. Evolutionary Computation: The Fossil Record. Wiley-IEEE, May 1, 1998. ISBN: 978-0-78033-481-6.

[92] Carlos M. Fonseca and Peter J. Fleming. Multiobjective Optimization and Multiple Constraint Handling with Evolutionary Algorithms - Part I: A Unified Formulation. IEEE Transactions on Systems, Man, and Cybernetics, Part A: Systems and Humans, 28(1):26-37, 1998. Online available at http://citeseer.ist.psu.edu/fonseca98multiobjective.html [accessed 2007-07-29]. See also [93].

[93] Carlos M. Fonseca and Peter J. Fleming. Multiobjective Optimization and Multiple Constraint Handling with Evolutionary Algorithms - Part II: Application example. IEEE Transactions on Systems, Man, and Cybernetics, Part A: Systems and Humans, 28(1):38-47, 1998. Online available at http://citeseer.ist.psu.edu/27937.html [accessed 2007-09-19]. See also $[92]$.

[94] Walter Fontana. Algorithmic Chemistry. In Christopher G. Langton et al., editor, Artificial Life II: Proc. of Workshop on Artificial Life, volume X of Santa Fe Institute Studies in the Science of Complexity, pages 159-210. Addison-Wesley, February 1990, Santa Fe, USA. ISBN: 978-0-20152-571-7.

[95] Christian V. Forst, Christian Reidys, and Jacqueline Weber. Evolutionary Dynamics and Optimization: Neutral Networks as Model-Landscapes for RNA Secondary-Structure FoldingLandscapes. In Federico Morán, Alvaro Moreno, Juan J. Merelo Guervós, and Pablo Chacón, editors, Advances in Artificial Life, Proc. of Third European Conference on Artificial Life, volume 929 of $L N C S$, pages 128-147. Springer, June 4-6, 1995, Granada, Spain. ISBN: 3-5405-9496-5. Online available at http://citeseer.ist.psu.edu/8900.html [accessed 2008-0601].

[96] Frank D. Francone, Markus Conrads, Wolfgang Banzhaf, and Peter Nordin. Homologous Crossover in Genetic Programming. In Proc. of Genetic and Evolutionary Computation Conference, volume 2, pages 1021-1026, 1999. Online available at http://www.cs.bham.ac.uk/ $\sim$ wbl/biblio/gecco1999/GP-463.pdf [accessed 2008-06-16].

[97] Richard M. Friedberg. A Learning Machine: Part I. IBM Journal of Research and Development, 2:2-13, November 1958. Online available at http://www.research.ibm.com/journal/ rd/021/ibmrd0201B.pdf [accessed 2007-09-06]. See also [98].

[98] Richard M. Friedberg, B. Dunham, and J. H. North. A Learning Machine: Part II. IBM Journal of Research and Development, 3(3):282-287, March 1959. Online available at http:// www.research.ibm.com/journal/rd/033/ibmrd0303H.pdf [accessed 2007-09-06]. See also [97].

\section{G}

[99] Hector Garcia-Molina. Elections in a Distributed Computing System. IEEE Transactions on Computers, C-31(1):47-59, January 1982. ISSN: 0018-9340. doi:10.1109/TC.1982.1675885.

[100] Sergey Gavrilets. Fitness Landscapes and the Origin of Species. Number MPB-41 in Monographs in Population Biology. Princeton University Press, July 2004. ISBN: 0-6911-1983-X.

[101] Kurt Geihs. Mega-Trend Selbstorganisation. Praxis der Informationsverarbeitung und Kommunikation (PIK) - Fachzeitschrift für den Einsatz von Informationssystemen, 28(4):188, October-December 2005. ISSN: 0930-5157. doi:10.1515/PIKO.2005.188. 
[102] Kurt Geihs. Selbst-Adaptive Software. Informatik-Spektrum, 31(2):133-145, April 2008. ISSN: 0170-6012. doi:10.1007/s00287-007-0198-9. Online available at http://www.springerlink. com/content/q33m18044164jn25/fulltext.pdf [accessed 2008-11-25].

[103] Marie-Pierre Gleizes, Valérie Camps, and Pierre Glize. A Theory of Emergent Computation based on Cooperative Self-Organization for Adaptive Artificial Systems. In Fourth European Congress of Systems Science. National Ministry of Education Online Cooperation Center for Artificial Intelligence, September 20-24, 1999, Valencia, Spain. Online available at http:// www.irit.fr/ACTIVITES/EQ_SMI/SMAC/Publications.html [accessed 2008-10-08].

[104] Fred Glover. Future Paths for Integer Programming and Links to Artificial Intelligence. Computers \& Operations Research, 13(5):533-549, 1986. ISSN: 03050548. doi:10.1016/0305-0548(86)90048-1. Online available at http://dx.doi.org/10.1016/ 0305-0548 (86) 90048-1 [accessed 2008-03-27].

[105] Fred Glover and Gary A. Kochenberger, editors. Handbook of Metaheuristics, volume 57 of International Series in Operations Research \& Management Science. Kluwer Academic Publishers, 2003. ISBN: 978-1-40207-263-5. doi:10.1007/b101874.

[106] David E. Goldberg. Genetic Algorithms in Search, Optimization, and Machine Learning. Addison-Wesley Longman Publishing, January 1989. ISBN: 0-2011-5767-5.

[107] David E. Goldberg and Jon Richardson. Genetic Algorithms with Sharing for Multimodal Function Optimization. In John J. Grefenstette, editor, Genetic Algorithms and Their Applications: Proc. of Second International Conference on Genetic Algorithms and their Applications, pages 41-49. American Association for Artificial Intelligence, Lawrence Erlbaum Associates, July 28-31, 1987, Cambridge, USA. ISBN: 0-8058-0158-8.

[108] David E. Goldberg, Kalyanmoy Deb, and Bradley Korb. Messy Genetic Algorithms: Motivation, Analysis, and First Results. Complex Systems, 3(5):493-530, 1989. ISSN: 0891-2513.

[109] David E. Goldberg, Kalyanmoy Deb, and Bradley Korb. Messy Genetic Algorithms Revisited: Studies in Mixed Size and Scale. Complex Systems, 4(4):415-444, 1990. ISSN: 0891-2513.

[110] Peter Graf and Daniel L. Schacter. Implicit and Explicit Memory for new Associations in Normal and Amnesic Subjects. Journal of Experimental Psychology: Learning, Memory, and Cognition, 11(3):501-518, July 1985. ISSN: 0278-7393.

[111] Garrison W. Greenwood, Xiaobo Sharon Hu, and Joseph G. D'Ambrosio. Fitness Functions for Multiple Objective Optimization Problems: Combining Preferences with Pareto Rankings. In Richard K. Belew and Michael D. Vose, editors, Proc. of 4th Workshop on Foundations of Genetic Algorithms (FOGA), pages 437-455. Morgan Kaufmann, August 5, 1996, San Diego, USA. ISBN: 1-5586-0460-X.

[112] Horst Greiner. Robust Filter Design by Stochastic Optimization. Proc. of SPIE (The International Society for Optical Engineering), 2253:150-161, November 4, 1994 . ISBN: 978-0-81941-562-2. doi:10.1117/12.192107. Advances in Design and Design Techniques: Posters, June 5, 1994, Grenoble, France, Optical Interference Coatings, Florin Abeles, ed.

[113] Horst Greiner. Robust Optical Coating Design with Evolutionary Strategies. Applied Optics, 35(28):5477-5483, October 1, 1996.

[114] Frédéric Gruau and L. Darrell Whitley. Adding Learning to the Cellular Development of Neural Networks: Evolution and the Baldwin Effect. Evolutionary Computation, 1(3):213233, 1993. ISSN: 1063-6560. doi:10.1162/evco.1993.1.3.213. Online available at http:// citeseer.ist.psu.edu/gruau93adding.html [accessed 2008-09-12].

[115] L. S. Gurin and Leonard A. Rastrigin. Convergence of the Random Search Method in the Presence of Noise. Automation and Remote Control, 26:1505-1511, 1965.

[116] Riccardo Gusella and Stefano Zatti. An Election Algorithm for a Distributed Clock Synchronization Program. Technical Report UCB/CSD-86-275, EECS Department, University of California, Berkeley, 1986. Online available at http://www.eecs.berkeley.edu/Pubs/ TechRpts/1986/CSD-86-275 .pdf [accessed 2007-10-24].

[117] Steven Matt Gustafson, Anikó Ekárt, Edmund K. Burke, and Graham Kendall. Problem Difficulty and Code Growth in Genetic Programming. Genetic Programming and Evolvable Machines, 5(3):271-290, September 2004. ISSN: 1389-2576. doi:10.1023/B:GENP.0000030194.98244.e3. Online available at http://citeseer.ist.psu. edu/730858.html [accessed 2008-07-21]. 
[118] Kostas P. Hatzis, George P. Pentaris, Paul G. Spirakis, Vasilis T. Tampakas, and Richard B. Tan. Fundamental Control Algorithms in Mobile Networks. In SPAA'99: Proc. of eleventh annual ACM symposium on Parallel algorithms and architectures, pages 251-260. ACM, June 27-30, 1999, Saint Malo, France. ISBN: 1-5811-3124-0. doi:10.1145/305619.305649. Online available at http://courses . csail .mit.edu/6.885/spring06/papers/Hatzis-etal . pdf [accessed 2008-11-16].

[119] Wendi Rabiner Heinzelman, Anantha Chandrakasan, and Hari Balakrishnan. Energy-Efficient Communication Protocol for Wireless Microsensor Networks. In HICSS'00: Proc. of 33rd Hawaii International Conference on System Sciences, volume 2. IEEE, January 4-7, 2000. ISBN: 0-7695-0493-0. Online available at https://eprints.kfupm.edu.sa/37623/1/37623. pdf [accessed 2008-11-16].

[120] Jörg Heitkötter and David Beasley, editors. Hitch-Hiker's Guide to Evolutionary Computation: A List of Frequently Asked Questions (FAQ). ENCORE (The EvolutioNary Computation REpository Network), 1998. USENET: comp.ai.genetic. Online available at http:// alife.santafe.edu/ joke/encore/www/ [accessed 2007-07-03].

[121] David Hilbert and Wilhelm Friedrich Ackermann. Grundzüge der theoretischen Logik, volume XXVII of Die Grundlehren der mathematischen Wissenschaften in Einzeldarstellungen mit besonderer Berücksichtigung der Anwendungsgebiete. W. Blaschke, R. Rammel, E. Hopf, et al., Julius Springer, August 1928. ISBN: 3-5400-5843-5. English version in [122].

[122] David Hilbert and Wilhelm Friedrich Ackermann. Principles of Mathematical Logic. American Mathematical Society (AMS) Chelsea Publishing, 1950. ISBN: 978-0-82182-024-7. Partly online available at http://books.google.com/books?id=7E34DeXok9gC [accessed 2008-03-25]. Original German version: [121].

[123] Geoffrey E. Hinton and Steven J. Nowlan. How Learning Can Guide Evolution. Complex Systems, 1:495-502, 1987. See also [124]. Online available at http://htpprints.yorku.ca/ archive/00000172/ [accessed 2008-09-10].

[124] Geoffrey E. Hinton and Steven J. Nowlan. How Learning Can Guide Evolution. In Richard K. Belew and Melanie Mitchell, editors, Adaptive individuals in evolving populations: models and algorithms, volume 26 of Santa Fe Institue Studies in the Sciences of Complexity, pages 447454. Addison-Wesley Longman Publishing, January 15, 1996. ISBN: 978-0-20148-369-7. See also [123].

[125] John Henry Holland. Adaptation in Natural and Artificial Systems: An Introductory Analysis with Applications to Biology, Control, and Artificial Intelligence. The University of Michigan Press, 1975. ISBN: 978-0-47208-460-9. Reprinted by MIT Press, April 1992, NetLibrary, Inc.

[126] John Henry Holland. Properties of the Bucket Brigade Algorithm. In John J. Grefenstette, editor, Proc. of 1st International Conference on Genetic Algorithms and their Applications, pages 1-7. Lawrence Erlbaum Associates, July 24-26, 1985, Pittsburgh, USA. ISBN: 0-8058-0426-9.

[127] John Henry Holland. Escaping Brittleness: The Possibilities of General-Purpose Learning Algorithms Applied to Parallel Rule-Based Systems. In R. S. Michalski, J. G. Carbonell, and T. M. Mitchell, editors, Machine Learning: An Artificial Intelligence Approach: Volume II, pages 593-623. Kaufmann, 1986. See also [128].

[128] John Henry Holland. Escaping Brittleness: The Possibilities of General-Purpose Learning Algorithms Applied to Parallel Rule-Based Systems. Computation E Intelligence: Collected Readings, pages 275-304, 1995. ISBN: 0-2626-2101-0. See also [127].

[129] John Henry Holland. Genetic Algorithms. Scientific American, 267(1):44-50, July 1992. Online available at http://www.cc.gatech.edu/ turk/bio_sim/articles/ genetic_algorithm.pdf [accessed 2007-08-29].

[130] John Henry Holland and Arthur W. Burks. Adaptive Computing System Capable of Learning and Discovery. Number 619349 filed on 1984-06-11 in United States Patent. United States Patent and Trademark Office, 1987. Online available at http://www.freepatentsonline. com/4697242.html [accessed 2008-04-01].

[131] John Henry Holland and Judith S. Reitman. Cognitive Systems based on Adaptive Algorithms. ACM SIGART Bulletin, 63:49, June 1977. ISSN: 0163-5719. Online available at http://doi.acm.org/10.1145/1045343.1045373 [accessed 2007-08-18]. See also [132]. 
[132] John Henry Holland and Judith S. Reitman. Cognitive Systems based on Adaptive Algorithms. In D. A. Waterman and F. Hayes-Roth, editors, Pattern directed inference systems, pages 313-329. Academic Press, 1978. Reprinted in [91]. See also [131].

[133] Gerald J. Holzmann. The Spin Model Checker - Primer and Reference Manual. AddisonWesley Professional/Longman, September 4, 2003. ISBN: 978-0-32122-862-8. Partly online available at http://books.google.de/books?id=NpBdZKmOHO8C [accessed 2008-10-02].

[134] Jeffrey Horn, Nicholas Nafpliotis, and David E. Goldberg. A Niched Pareto Genetic Algorithm for Multiobjective Optimization. In Zbigniew Michalewicz et al., editor, Proc. of First IEEE Conference on Evolutionary Computation, IEEE World Congress on Computational Intelligence, volume 1, pages 82-87. IEEE, June 27-29, 1994, Orlando, USA. ISBN: 0-7803-1899-4. doi:10.1109/ICEC.1994.350037. Online available at http://www.lania.mx/ ccoello/EMOO/ horn2.ps.gz [accessed 2007-08-28].

[135] Helmut Hörner. A C++ Class Library for GP: Vienna University of Economics Genetic Programming Kernel (Release 1.0, operating instructions). Technical Report, Vienna University of Economics, May 29, 1996. Online available at http://citeseer.ist.psu.edu/253491. html [accessed 2007-09-09].

[136] Marijn A. Huynen, Peter F. Stadler, and Walter Fontana. Smoothness within Ruggedness: The Role of Neutrality in Adaptation. Proc. of National Academy of Science, USA, 93:397401, January 1996. Evolution. Online available at http://citeseer.ist.psu.edu/91710. html [accessed 2008-02-27].

[137] Hitoshi Iba. Emergent Cooperation for Multiple Agents Using Genetic Programming. In HansMichael Voigt et al., editor, Parallel Problem Solving from Nature - PPSN IV, International Conference on Evolutionary Computation. Proc. of 4 th International Conference on Parallel Problem Solving from Nature, volume 1141/1996 of LNCS, pages 32-41. Springer, September 22-24, 1996, Berlin, Germany. ISBN: 3-5406-1723-X. doi:10.1007/3-540-61723-X_967.

[138] Hitoshi Iba. Evolutionary Learning of Communicating Agents. Information Sciences - Informatics and Computer Science: An International Journal, 108(1-4):181-205, July 1998. ISSN: 0020-0255. doi:10.1016/S0020-0255(97)10055-X. Online available at http://dx.doi.org/10 . 1016/S0020-0255 (97) 10055-X [accessed 2008-07-28].

[139] Mohammad Ilyas and Imad Mahgoub, editors. Handbook of Sensor Networks: Compact Wireless and Wired Sensing Systems. CRC, July 16, 2004. ISBN: 978-0-84931-968-6.

[140] Information Sciences Institute, University of Southern California, 4676 Admiralty Way, Marina del Rey, California 90291, USA. Internet Protocol, Darpa Internet Program Protocol Specification. RFC 791, Defense Advanced Research Projects Agency, Information Processing Techniques Office, 1400 Wilson Boulevard, Arlington, Virginia 22209, USA, September 1981. Online available at http://tools.ietf.org/html/rfc791 [accessed 2008-06-13].

[141] Lester Ingber. Adaptive Simulated Annealing (ASA): Lessons learned. Control and Cybernetics, 25(1):33-54, 1996. Online available at http://citeseer.ist.psu.edu/592447.html [accessed 2008-03-27].

[142] Intel Corporation. Intel Architecture Software Developer's Manual - Volume 1: Basic Architecture, 1997. Online available at http://developer.intel.com/design/pentium/manuals/ 24319001.pdf [accessed 2008-11-08].

[143] International Organization for Standardization. ISO/IEC 19503:2005-11, 2005.

[144] Alon Itai and Michael Rodeh. Symmetry Breaking in Distributive Networks. In Proc. of 22nd Annual Symposium on Foundations of Computer Science, FOCS, pages 150-158. IEEE, October 28-30, 1981, Nashville, USA. See also [145].

[145] Alon Itai and Michael Rodeh. Symmetry Breaking in Distributed Networks. Information and Computation, 88(1):60-87, 1990. See also [144]. 
[146] David Jackson. Parsing and Translation of Expressions by Genetic Programming. In HansGeorg Beyer et al., editor, Proc. of Genetic and Evolutionary Computation Conference, GECCO 2005, pages 1681-1688. ACM, June 25-29, 2005, Washington, D.C., USA. ISBN: 1-5959-3010-8. doi:10.1145/1068009.1068291. Online available at http://doi.acm.org/10. 1145/1068009.1068291 [accessed 2008-05-04].

[147] Jiri Jaroš and Vaclav Dvořák. An Evolutionary Design Technique for Collective Communications on Optimal Diameter-Degree Networks. In Maarten Keijzer et al., editor, Proc. of Genetic and Evolutionary Computation Conference, GECCO 2008. ACM, July 12-16, 2008, Atlanta, USA. ISBN: 978-1-60558-131-6.

[148] Chevalier de Lamarck Jean-Baptiste Pierre Antoine de Monet. Philosophie zoologique - ou Exposition des considérations relatives à l'histoire naturelle des Animaux; à la diversité de leur organisation et des facultés qu'ils en obtiennent; aux causes physiques qui maintiennent en eux la vie et donnent lieu aux mouvements qu'ils exécutant; enfin, à celles qui produisent les unes le sentiment, et les autres l'intelligence de ceux qui en sont doués. Dentu / J. B. Baillière Liberaire, 1809, 1830. ISBN: 978-1-41211-646-6. Online available at http://books . google.fr/books?id=L6qAG6ZPgj4C [accessed 2008-09-10].

[149] Márk Jelasity, Alberto Montresor, and Özalp Babaoğlu. Gossip-based Aggregation in Large Dynamic Networks. ACM Transactions on Computer Systems, 23(3):219-252, 2005. ISSN: 0734-2071. Online available at http://portal.acm.org/citation.cfm?id=1082470 [accessed 2007-08-01].

[150] Jesper M. Johansson, Salvatore T. March, and J. David Naumann. Modeling Network Latency and Parallel Processing in Distributed Database Design. Decision Sciences, 34(4):677706, November 2003. Online available at http://findarticles .com/p/articles/mi_qa3713/ is_200310/ai_n9253628 [accessed 2007-08-13].

[151] Hugues Juillé and Jordan B. Pollack. Massively Parallel Genetic Programming. In Peter J. Angeline and Kenneth E. Kinnear, Jr, editors, Advances in Genetic Programming, volume 2 of Complex Adaptive Systems, pages 339-358. MIT Press, October 26, 1996. ISBN: 978-0-26201-158-7. Online available at http://citeseerx.ist.psu.edu/viewdoc/summary? doi=10.1 1.49.26 [accessed 2008-12-24].

\section{K}

[152] Peter Kampstra. Evolutionary Computing in Telecommunications - A likely EC success story. Master's thesis, Vrije Universiteit, Amsterdam, August 2005. Online available at http://www . few.vu.nl/stagebureau/werkstuk/werkstukken/werkstuk-kampstra.pdf [accessed 2008-09-04].

[153] Peter Kampstra, Rob D. van der Mei, and Ágoston E. Eiben. Evolutionary Computing in Telecommunication Network Design: A Survey, 2006. Online available at http://www.few. vu.nl/ mei/articles/2006/kampstra/art.pdf [accessed 2008-09-04].

[154] Michael J. Kearns, Yishay Mansour, Andrew Y. Ng, and Dana Ron. An Experimental and Theoretical Comparison of Model Selection Methods. In COLT'95: Proc. of eighth annual conference on Computational learning theory, pages 21-30. ACM, 1995, Santa Cruz, USA. ISBN: 0-8979-1723-5. doi:10.1145/225298.225301. Online available at http://doi.acm.org/ 10.1145/225298.225301 [accessed 2008-03-02]. See also [155].

[155] Michael J. Kearns, Yishay Mansour, Andrew Y. Ng, and Dana Ron. An Experimental and Theoretical Comparison of Model Selection Methods. Machine Learning, 27(1):7-50, 1997. See also [154].

[156] Scott Kirkpatrick, C. Daniel Gelatt, Jr., and Mario P. Vecchi. Optimization by Simulated Annealing. Science, 220(4598):671-680, May 13, 1983. doi:10.1126/science.220.4598.671. Online available at http://citeseer.ist.psu.edu/kirkpatrick83optimization.html [accessed 2008-03-26]. 
[157] I. M. A. Kirkwood, S. H. Shami, and Mark C. Sinclair. Discovering Simple Fault-Tolerant Routing Rules using Genetic Programming. In George D. Smith, Nigel C. Steele, and Rudolf F. Albrecht, editors, Proc. of International Conference on Artificial Neural Nets and Genetic Algorithms, ICANNGA. Springer, April 1-4, 1997, Norwich, U.K. ISBN: 978-3-21183-087-1. Online available at http://citeseer.ist.psu.edu/120285.html [accessed 2008-06-24]. See also $[254]$.

[158] Marc Kirschner and John Gerhart. Evolvability. Proc. of National Academy of Science of the USA (PNAS), 95(15):8420-8427, July 21, 1998. ISSN: 0027-8424. Online available at http://www . pnas.org/cgi/content/full/95/15/8420 [accessed 2007-08-05].

[159] Ralf Klasing, Burkhard Monien, Regine Peine, and Elena Stöhr. Broadcasting in Butterfly and de Bruijn Networks. In Alain Finkel and Matthias Jantzen, editors, Proc. of 9th Annual Symposium on Theoretical Aspects of Computer Science (STACS'92), volume 577 of LNCS, pages 351-362. Springer, February 13-15, 1992, Cachan, France. ISBN: 978-3-54055-210-9.

[160] Barbara J. Knowlton and Larry R. Squire. Remembering and Knowing: Two different Expressions of declarative Memory. Journal of Experimental Psychology Learning, Memory and Cognition, 21(3):699-710, May 1995. ISSN: 0278-7393.

[161] Krasimir Kolarov. Landscape Ruggedness in Evolutionary Algorithms. In Thomas Bäck, Zbigniew Michalewicz, and Xin Yao, editors, IEEE International Conference on Evolutionary Computation, CEC9\%, pages 19-24. IEEE, April 13-16, 1997, Indianapolis, USA. ISBN: 0-7803-3949-5. Online available at http://de.scientificcommons.org/27191 [accessed 2007-0819].

[162] Dimitrios S. Kolovos, Richard F. Paige, and Fiona A.C. Polack. Model Comparison: A Foundation for Model Composition and Model Transformation Testing. In GaMMa'06: Proc. of 2006 international workshop on Global integrated model management, pages 13-20. ACM, 2006, Shanghai, China. ISBN: 1-5959-3410-3. Online available at http://doi.acm.org/10. 1145/1138304.1138308 [accessed 2007-09-17].

[163] Ephraim Korach, Shay Kutten, and Shlomo Moran. A Modular Technique for the Design of Efficient Distributed Leader Finding Algorithms. In Proc. of Fourth Annual ACM Symposium on Princiles of Distributed Computing, PODC, pages 163-174. Association for Computing Machinery (ACM), August 5-7, 1985, Minaki, Canada. ISBN: 0-8979-1167-9. Online available at http://www.cs.technion.ac.il/ moran/r/PS/kkm.pdf [accessed 2008-12-01]. See also [164].

[164] Ephraim Korach, Shay Kutten, and Shlomo Moran. A Modular Technique for the Design of Efficient Distributed Leader Finding Algorithms. ACM Transactions on Programming Languages and Systems (TOPLAS), 12(1):84-101, January 1990. ISSN: 0164-0925. doi:10.1145/77606.77610. Online available at http://doi .acm.org/10.1145/77606.77610 [accessed 2008-12-01]. See also [163].

[165] John R. Koza. Genetic Programming: On the Programming of Computers by Means of Natural Selection. A Bradford Book. The MIT Press, 1992. ISBN: 978-0-26211-170-6. Partly online available at http://books.google.de/books?id=Bhtxo60BVOEC [accessed 2008-08-16].

[166] John R. Koza, Forrest H. Bennett, Jeffrey L. Hutchings, Stephen L. Bade, Martin A. Keane, and David Andre. Evolving Sorting Networks using Genetic Programming and the Rapidly Reconfigurable Xilinx 6216 Field-Programmable Gate Array. In Conference Record of the Thirty-First Asilomar Conference on Signals, Systems \& Computers, volume 1, pages 404-410, November 2-5 1997, Pacific Grove, USA. ISBN: 0-8186-8316-3. doi:10.1109/ACSSC.1997.680275. Online available at http://www.cs.bham.ac.uk/ wbl/ biblio/gp-html/koza_1997_ASILIMOAR.html [accessed 2008-12-24].

\section{L}

[167] Duccio Lambardi, Francesca R. Dani, Stefano Turillazzi, and Jacobus J. Boomsma. Chemical Mimicry in an Incipient Leaf-Cutting Ant Social Parasite. Behavioral Ecology and Sociobiology, 61(6):843-851, April 2007. ISSN: 0340-5443. doi:10.1007/s00265-006-0313-y. Online available at http://www. springerlink. com/content/c3500k0333144k68/ [accessed 2009-01-05]. 
[168] Leslie Lamport. Time, Clocks, and the Ordering of Events in a Distributed System. Communications of the ACM, 21(7):558-565, July 1978. ISSN: 0001-0782. doi:10.1145/359545.359563. Online available at http://portal . acm.org/citation. cfm?id=359563 [accessed 2007-08-01].

[169] William B. Langdon. The Halting Probability in von Neumann Architectures. Technical Report CSM-456, Computer Science, University of Essex, UK, July 2006. 2 page summary of [173]. Online available at http://www.cs.bham.ac.uk/ wbl/biblio/gp-html/ langdon_2006_eurogp2p.html [accessed 2008-11-09].

[170] William B. Langdon. A SIMD Interpreter for Genetic Programming on GPU Graphics Cards. Computer Science Technical Report CSM-470, Departments of Mathematical and Biological Sciences, University of Essex, UK, July 3, 2007. Online available at http://cswww.essex. ac.uk/technical-reports/2007/csm_470.pdf [accessed 2008-12-24].

[171] William B. Langdon and Wolfgang Banzhaf. A SIMD Interpreter for Genetic Programming on GPU Graphics Cards. In Michael O'Neill et al., editor, Genetic Programming - Proc. of 11th European Conference on Genetic Programming, EuroGP 2008, volume 4971/2008 of LNCS, pages 73-85. Springer, March 26-28 2008, Naples, Italy. ISBN: 978-3-54078-670-2. doi:10.1007/978-3-540-78671-9. Online available at http://www. cs . bham . ac.uk/ wbl/biblio/gp-html/langdon_2008_eurogp.html [accessed 2008-12-24]. See also [170].

[172] William B. Langdon and Riccardo Poli. Fitness Causes Bloat: Mutation. In Wolfgang Banzhaf et al., editor, Proccedings of the First European Workshop on Genetic Programming, EuroGP'98, volume 1391/1998 of LNCS, pages 37-48. Springer, April 14-15, 1998, Paris, France. ISBN: 3-5406-4360-5. doi:10.1007/BFb0055923, 978-3-540-64360-9. Online available at ftp:// ftp.cwi.nl/pub/W.B.Langdon/papers/WBL . euro98_bloatm.ps.gz [accessed 2007-09-09].

[173] William B. Langdon and Riccardo Poli. The Halting Probability in von Neumann Architectures. In Pierre Collet et al., editor, Proc. of 9th European Conference Genetic Programming, EuroGP 2006, volume 3905/2006 of LNCS, pages 225-237. Springer, April 10-12, 2006, Budapest, Hungary. ISBN: 3-5403-3143-3. Online available at http://www.cs.bham.ac.uk/ wbl/biblio/gp-html/langdon_2006_eurogp.html [accessed 2008-11-09]. See also [169].

[174] William B. Langdon, Terry Soule, Riccardo Poli, and James A. Foster. The Evolution of Size and Shape. In Lee Spector et al., editor, Advances in Genetic Programming, volume 3 of Complex Adaptive Systems, chapter 8, pages 163-190. MIT Press, July 16, 1999. ISBN: 978-0-26219-423-5. Online available at http://citeseer.ist.psu.edu/ langdon99evolution.html [accessed 2007-09-07].

[175] Pier Luca Lanzi and Alessandro Perrucci. Extending the Representation of Classifier Conditions Part II: From Messy Coding to S-Expressions. In Wolfgang Banzhaf et al., editor, Proc. of Genetic and Evolutionary Computation Conference (GECCO 1999), pages 345-352. Morgan Kaufmann, July 13-17, 1999, Orlando, USA. ISBN: 1-5586-0611-4. Online available at http://webspace.elet.polimi.it/lanzi/papers//lanzi1999GECCOgp.pdf [accessed 2007-0801].

[176] Christian W. G. Lasarczyk and Wolfgang Banzhaf. An Algorithmic Chemistry for Genetic Programming. In Maarten Keijzer et al., editor, Proc. of 8th European Conference on Genetic Programming, EuroGP2005, volume 3447/2005 of LNCS, pages 1-12. Springer, March 30April 1, 2005, Lausanne, Switzerland. ISBN: 3-5402-5436-6. Online available at http://www . cs.bham.ac.uk/ wbl/biblio/gp-html/eurogp_LasarczykB05.html [accessed 2008-04-30].

[177] Christian W. G. Lasarczyk and Wolfgang Banzhaf. Total Synthesis of Algorithmic Chemistries. In Hans-Georg Beyer and Una-May O'Reilly, editors, Workshop Proc. of Genetic and Evolutionary Computation Conference, GECCO 2005, pages 1635-1640. ACM, June 25-29, 2005, Washington, D.C, USA. Online available at http://doi.acm.org/10 . 1145/1068009.1068285 [accessed 2008-04-02].

[178] Gérard Le Lann. Distributed Systems - Towards a Formal Approach. In Bruce Gilchrist, editor, Information Processing, Proc. of International Federation for Information Processing World Computer Congress, IFIP Congress 77, pages 155-160. North Holland / Elsevier Science, August 8-12, 1977, Toronto, Canada. ISBN: 978-0-72040-755-6.

[179] Jack Yiu-Bun Lee and P. C. Wong. The Effect of Function Noise on GP Efficiency. In Xin Yao, editor, Progress in Evolutionary Computation, Selected Papers of the AI'93 and AI'94 Workshops on Evolutionary Computation Melbourne, Victoria, Australia, November 16, 1993 and Armidale, NSW, Australia, November 21-22, 1994, volume 956/1995 of LNCS, pages 1-16. Springer, May 1995. ISBN: 978-3-54060-154-8. doi:10.1007/3-540-60154-6_43. 
[180] Richard Lowry. Concepts and Applications of Inferential Statistics. Vassar College, 2006. Companion site of [183]. Online available at http://faculty.vassar. edu/lowry/webtext. html [accessed 2008-12-08].

[181] Richard Lowry. VassarStats 2x2 Contingency Table Tests. In VassarStats: Web Site for Statistical Computing, chapter 8 in [180]. Vassar College, March 30, 2006. In collection [183]. Online available at http://faculty.vassar. edu/lowry/tab2x2.html [accessed 2008-12-08].

[182] Richard Lowry. VassarStats Mann-Whitney Test. In VassarStats: Web Site for Statistical Computing, chapter 11a in [180]. Vassar College, September 22, 2006. In collection [183]. Online available at http://faculty.vassar.edu/lowry/utest.html [accessed 2008-12-07].

[183] Richard Lowry. VassarStats: Web Site for Statistical Computing. Vassar College, 2006. See also [180]. Online available at http://faculty • vassar . edu/lowry/VassarStats . html [accessed 2008-12-08].

[184] Haiming Lu. State-of-the-art Multiobjective Evolutionary Algorithms - Pareto Ranking, Density Estimation and Dynamic Population. PhD thesis, Faculty of the Graduate College of the Oklahoma State University, Stillwater, Oklahoma, August 2002. Online available at http:// www.lania.mx/ ccoello/EMOO/thesis_lu.pdf.gz [accessed 2007-08-25].

[185] Sean Luke. Code Growth is Not Caused by Introns. In L. Darrell Whitley, editor, Late Breaking Papers at Genetic and Evolutionary Computation Conference (GECCO'00), pages 228-235, July 8-12, 2000, Las Vegas, USA. Online available at http://www.cs.gmu.edu/ $\sim$ sean/papers/intronpaper.pdf [accessed 2007-09-07].

[186] Sean Luke and Liviu Panait. A Comparison of Bloat Control Methods for Genetic Programming. Evolutionary Computation, 14(3):309-344, 2006. ISSN: 1063-6560.

[187] Sean Luke and Lee Spector. Evolving Graphs and Networks with Edge Encoding: A Preliminary Report. In John R. Koza, editor, Late Breaking Papers at the First Annual Conference Genetic Programming (GP-96). Stanford Bookstore, July 28-31, 1996, Stanford University, USA. ISBN: 0-1820-1031-7. Online available at http://cs.gmu.edu/ sean/papers/ graph-paper.pdf [accessed 2008-08-02].

[188] Jay L. Lush. Progeny Test and Individual Performance as Indicators of an Animal's Breeding Value. Journal of Dairy Science, 18(1):1-19, January 1935. Online available at http://jds . fass.org/cgi/reprint/18/1/1 [accessed 2007-11-27].

\section{M}

[189] Mamoru Maekawa. A $\sqrt{N}$ Algorithm for Mutual Exclusion in Decentralized Systems. $A C M$ Transactions on Computer Systems (ACM TOCS), 3(2):145-159, May 1985. ISSN: 07342071. doi:10.1145/214438.214445. Online available at http://doi . acm.org/10.1145/214438 . 214445 [accessed 2008-11-16].

[190] Anne E. Magurran. Biological Diversity. Current Biology Magazine, 15(4):R116-R118, February 22, 2005. doi:10.1016/j.cub.2005.02.006. Online available at http://dx.doi.org/10. 1016/j.cub.2005.02.006 [accessed 2008-11-10].

[191] Navneet Malpani, Jennifer L. Welch, and Nitin Vaidya. Leader Election Algorithms for Mobile Ad Hoc Networks. In DIALM'00: Proc. of 4th international workshop on Discrete algorithms and methods for mobile computing and communications, pages 96-103. ACM, 2000, Boston, USA. ISBN: 1-5811-3301-4. doi:10.1145/345848.345871. Online available at http://courses . csail.mit.edu/6.885/spring06/papers/MalpaniWV-dialm00.pdf [accessed 2008-11-16].

[192] Henry B. Mann and Donald R. Whitney. On a Test of Whether one of Two Random Variables is Stochastically Larger than the Other. The Annals of Mathematical Statistics, 18(1):50-60, March 1947. doi:10.1214/aoms/1177730491. Online available at http://projecteuclid.org/ euclid.aoms/1177730491 [accessed 2008-10-24].

[193] Friedemann Mattern. Verteilte Basisalgorithmen, volume 226 of Informatik-Fachberichte (IFB). W. Bauer im Auftrag der Gesellschaft für Informatik (GI), Springer, 1989. ISBN: 978-3-54051-835-8.

[194] Robert Ian McKay, Xuan Hoai Nguyen, Peter Alexander Whigham, and Yin Shan. Grammars in Genetic Programming: A Brief Review. In L. Kang, Z. Cai, and Y. Yan, editors, Progress in Intelligence Computation and Intelligence: Proc. of International Symposium on Intelligence, 
Computation and Applications, pages 3-18. China University of Geosciences Press, April 2005. Online available at http://sc.snu.ac.kr/PAPERS/isica05.pdf [accessed 2007-08-15].

[195] Nicholas Freitag McPhee and Ricardo Poli. Memory with Memory: Soft Assignment in Genetic Programming. In Maarten Keijzer et al., editor, Proc. of Genetic and Evolutionary Computation Conference, GECCO 2008, pages 1235-1242. ACM, July 12-16, 2008, Atlanta, USA. ISBN: 978-1-60558-131-6.

[196] Brad L. Miller and David E. Goldberg. Genetic Algorithms, Tournament Selection, and the Effects of Noise. IlliGAL Report 95006, Illinois Genetic Algorithms Laboratory, Department of General Engineering, University of Illinois, Urbana-Champaign, USA, July 1995. See also [197]. Online available at http://www.illigal.uiuc.edu/pub/papers/IlliGALs/95006.ps. Z [accessed 2007-07-28].

[197] Brad L. Miller and David E. Goldberg. Genetic Algorithms, Selection Schemes, and the Varying Effects of Noise. Evolutionary Computation, 4(2):113-131, Summer 1996. ISSN: 1063-6560. doi:10.1162/evco.1996.4.2.113. See also [196]. Online available at http://citeseerx.ist. psu. edu/viewdoc/download; jsessionid=4225507A21F8083F025E28499309D1FB?doi=10 . 1 . 1.31.3449\&rep=rep1\&type=pdf [accessed 2008-07-21].

[198] Brad L. Miller and Michael J. Shaw. Genetic Algorithms with Dynamic Niche Sharing for Multimodal Function Optimization. IlliGAL Report 95010, Department of General Engineering, University of Illinois at Urbana-Champaign, 117 Transportation Building, 104 South Mathews Avenue, Urbana, IL 61801, USA, December 1, 1995. Online available at http://citeseer. comp.nus.edu.sg/5466.html [accessed http://www.illigal.uiuc.edu/pub/papers/IlliGALs/95010.ps.Z]2008-0315.

[199] Julian Francis Miller and Peter Thomson. Aspects of Digital Evolution: Geometry and Learning. In Evolvable Systems: From Biology to Hardware, volume 1478/1998 of LNCS, pages 25-35. Springer, 1998. ISBN: 978-3-54064-954-0. doi:10.1007/BFb0057601. Online available at http://www. elec.york.ac.uk/intsys/users/jfm7/ices1998-miller-thomson. pdf [accessed 2007-11-03].

[200] Daniele Miorandi, Paolo Dini, Eitan Altman, and Hisao Kameda. WP 2.2 - Paradigm Applications and Mapping, D2.2.2 Framework for Distributed On-line Evolution of Protocols and Services. BIOlogically inspired NETwork and Services (BIONETS), $2^{\text {nd }}$ edition, June 14, 2007. Editor: Lidia A. R. Yamamoto. Online available at http://www.bionets.eu/docs/ BIONETS_D2_2_2.pdf [accessed 2008-06-23].

[201] Patrick Alfred Pierce Moran. Random Processes in Genetics. (Mathematical) Proc. of Cambridge Philosophical Society, 54(01):60-71, January 1958. ISSN: 0305-0041. doi:10.1017/S0305004100033193.

[202] Jack C. Morrison and Tam T. Nguyen. On-Board Software for the Mars Pathfinder Microrover. In Proc. of Second IAA International Conference on Low-Cost Planetary Missions, April 1619, 1996, Laurel, USA. Online available at http://hdl.handle.net/2014/31458 [accessed 200812-01].

[203] Ronald W. Morrison and Kenneth Alan de Jong. Measurement of Population Diversity. In Pierre Collet et al., editor, Selected Papers of the 5th International Conference on Artificial Evolution, Evolution Artificielle, EA 2001, volume 2310 of LNCS, pages 1047-1074. Springer, October 29-31, 2001, Le Creusot, France. ISBN: 3-5404-3544-1. doi:10.1007/3-540-46033-0_3. Online available at http://citeseerx.ist.psu.edu/viewdoc/ summary?doi=10.1.1.108.1903 [accessed 2008-11-10].

[204] Pablo Moscato. On Evolution, Search, Optimization, Genetic Algorithms and Martial Arts: Towards Memetic Algorithms. Technical Report C3P 826, Caltech Concurrent Computation Program 158-79, California Institute of Technology, Pasadena, CA 91125, USA, Pasadena, CA, 1989. Online available at http://www.densis.fee.unicamp.br/ moscato/papers/ bigone.ps [accessed 2007-08-18].

[205] Markus Müller-Olm, David A. Schmidt, and Bernhard Steffen. Model Checking: A Tutorial Introduction. In Agostino Cortesi and Gilberto Filé, editors, Proc. of 6th Static Analysis Symposium SAS'99, volume 1694 of LNCS, pages 330-354. Springer, September 22-24 1999 , Venice, Italy. ISBN: 978-3-54066-459-8. doi:10.1007/3-540-48294-6_22. Online available at http://people.cis.ksu.edu/ schmidt/papers/sas99.ps.gz [accessed 2008-10-02]. 
[206] Bart Naudts and Alain Verschoren. Epistasis On Finite And Infinite Spaces. In Proc. of 8th International Conference on Systems Research, Informatics and Cybernetics, pages 19-23, 1996. Online available at http://citeseer.ist.psu.edu/142750.html [accessed 2007-08-13].

[207] Xuan Hoai Nguyen. A Flexible Representation for Genetic Programming: Lessons from Natural Language Processing. PhD thesis, School of Information Technology and Electrical Engineering University College, University of New South Wales, Australian Defence Force Academy, December 2004. Online available at http://www.cs.ucl.ac.uk/staff/W.Langdon/ftp/ papers/hoai_thesis.tar.gz [accessed 2007-07-15].

[208] Xuan Hoai Nguyen and Robert Ian McKay. A Framework for Tree-adjunct Grammar Guided Genetic Programming. In Proc. of Post-graduate ADFA Conference on Computer Science (PACCS'01), volume 1 of ADFA Monographs in Computer Science Series, pages 93-100, July 14, 2001, Canberra ACT, Australia. ISBN: 0-7317-0507-6. Online available at http:// sc.snu.ac.kr/PAPERS/TAG3P.pdf [accessed 2007-09-09].

[209] Xuan Hoai Nguyen, Robert Ian McKay, Daryl Leslie Essam, and R. Chau. Solving the Symbolic Regression Problem with Tree-Adjunct Grammar Guided Genetic Programming: The Comparative Results. In David B. Fogel et al., editor, Proc. of IEEE Congress on Evolutionary Computation, CEC2002, pages 1326-1331. IEEE, May 12-17, 2002, Honolulu, USA. ISBN: 0-7803-7278-6. Online available at http://sc.snu.ac.kr/PAPERS/MCEC2002 .pdf [accessed 2007-09-09].

[210] Stefan Niemczyk. Ein Modellproblem mit einstellbarer Schwierigkeit zur Evaluierung Evolutionärer Algorithmen. Diplom i arbeit (equiv. bachelor thesis), Universität Kassel, May 5, 2008. Online available at http://www.it-weise.de/documents/files/N2008MPMES.pdf [accessed 2009-05-07].

[211] Peter Nordin. A Compiling Genetic Programming System that Directly Manipulates the Machine Code. In Kenneth E. Kinnear, Jr., editor, Advances in Genetic Programming, volume 1 of Complex Adaptive Systems, chapter 14, pages 311-331. MIT Press, April 7, 1994. ISBN: 978-0-26211-188-1. Machine code GP Sun Spark and i868.

[212] Peter Nordin and Wolfgang Banzhaf. Evolving Turing-Complete Programs for a Register Machine with Self-modifying Code. In Larry J. Eshelman, editor, Proc. of Sixth International Conference on Genetic Algorithms, pages 318-325. Morgan Kaufmann, July 15-19, 1995, Pittsburgh, USA. ISBN: 1-5586-0370-0. Online available at http://citeseerx.ist.psu. edu/viewdoc/summary?doi=10.1.1.34.4526 [accessed 2008-09-16].

[213] Peter Nordin, Frank Francone, and Wolfgang Banzhaf. Explicitly Defined Introns and Destructive Crossover in Genetic Programming. In Justinian P. Rosca, editor, Proc. of Workshop on Genetic Programming: From Theory to Real-World Applications, pages 6-22. Morgan Kaufmann, July 9, 1995, Tahoe City, USA. Online available at http://www.cs.bham.ac.uk/ $\sim$ wbl/biblio/gp-html/nordin_1995_introns.html [accessed 2007-09-07]. See also [214].

[214] Peter Nordin, Frank Francone, and Wolfgang Banzhaf. Explicitly Defined Introns and Destructive Crossover in Genetic Programming. In Peter J. Angeline and Kenneth E. Kinnear, Jr, editors, Advances in Genetic Programming, volume 2 of Complex Adaptive Systems, pages 111-134. MIT Press, October 26, 1996. ISBN: 978-0-26201-158-7. See also [213]. Online available at http://www.cs.bham.ac.uk/ wbl/biblio/gp-html/nordin_1995_introns.html [accessed 2008-09-17], ftp://lumpi.informatik.uni-dortmund.de/pub/biocomp/papers/ML95.ps . gz [accessed 2007-08-17].

[215] Peter Nordin, Wolfgang Banzhaf, and Frank D. Francone. Efficient Evolution of Machine Code for CISC Architectures using Instruction Blocks and Homologous Crossover. In Lee Spector et al., editor, Advances in Genetic Programming, volume 3 of Complex Adaptive Systems, chapter 12, pages 275-299. MIT Press, July 16, 1999. ISBN: 978-0-26219-423-5. Online available at http://www.cs.bham.ac.uk/ wbl/aigp3/ch12.pdf [accessed 2008-09-16]. 
[216] Euclid of Alexandria. Stoicheia (Elements). Alexandria, 300 BC. Online available at http:// Www.gutenberg.org/etext/21076 [accessed 2007-10-05] (in english).

\section{$\mathbf{P}$}

[217] Ingo Paenke, Jürgen Branke, and Yaochu Jin. On the Influence of Phenotype Plasticity on Genotype Diversity. In Jerry M. Mendel, Takashi Omari, and Xin Yao, editors, The First IEEE Symposium on Foundations of Computational Intelligence (FOCI'07), pages 33-40. IEEE, April 1-5, 2007, Honolulu, USA. ISBN: 978-1-42440-703-3. doi:10.1109/FOCI.2007.372144. Online available at http://www.honda-ri.org/intern/ Publications/PN\%2052-06 [accessed 2008-11-10].

[218] Hui Pan, Ling Wang, and Bo Liu. Particle Swarm Optimization for Function Optimization in Noisy Environment. Applied Mathematics and Computation, 181(2):908-919, October 15, 2006. ISSN: 0096-3003. doi:10.1016/j.amc.2006.01.066.

[219] Julia K. Parrish and William M. Hamner, editors. Animal Groups in Three Dimensions: How Species Aggregate. Cambridge University Press, December 1997. ISBN: 978-0-52146-024-8. Partly online available at http://books.google.de/books?id=zj4I4HNZSROC [accessed 2008-1125].

[220] Norman R. Paterson. Genetic Programming with Context-Sensitive Grammars. PhD thesis, Saint Andrew's University, September 2002. Online available at ftp://ftp.dcs.st-and.ac. uk/pub/norman/GPwCSG.ps.gz [accessed 2007-09-09].

[221] Norman R. Paterson and Mike Livesey. Distinguishing Genotype and Phenotype in Genetic Programming. In John R. Koza, editor, Late Breaking Papers at the First Annual Conference Genetic Programming (GP-96), pages 141-150. Stanford Bookstore, July 28-31, 1996, Stanford University, USA. ISBN: 0-1820-1031-7. Online available at ftp://ftp.dcs.st-and.ac . uk/pub/norman/GADS .ps.gz [accessed 2007-09-07].

[222] Witold Pedrycz and Athanasios V. Vasilakos. Computational Intelligence: A Development Environment for Telecommunications Networks. In Witold Pedrycz and Athanasios V. Vasilakos, editors, Computational Intelligence in Telecommunications Networks, chapter 1, pages 1-27. CRC Press, September 15, 2000. ISBN: 978-0-84931-075-1.

[223] Witold Pedrycz and Athanasios V. Vasilakos, editors. Computational Intelligence in Telecommunications Networks. CRC Press, September 15, 2000. ISBN: 978-0-84931-075-1. Partly online available at http://books.google.de/books?id=dE6d-jPoDEEC [accessed 2008-09-03].

[224] Radek Pelánek. BEEM: Benchmarks for Explicit Model Checkers. In Dragan Bošnački and Stefan Edelkamp, editors, Proc. of SPIN 2007, the 14th International SPIN Workshop on Model Checking Software, volume 4595/2007 of LNCS, pages 263-267. Springer, July 1-3, 2007, Berlin, Germany. ISBN: 978-3-54073-369-0. doi:10.1007/978-3-540-73370-6_17. Online available at http://spinroot.com/spin/Workshops/ws07/Pelanek.pdf [accessed 2008-10-02].

[225] Martin Pelikan, David E. Goldberg, and Erick Cantú-Paz. BOA: The Bayesian Optimization Algorithm. In Wolfgang Banzhaf et al., editor, Proc. of Genetic and Evolutionary Computation Conference (GECCO 1999), pages 525-532. Morgan Kaufmann, July 13-17, 1999, Orlando, USA. ISBN: 1-5586-0611-4. Online available at https://eprints.kfupm.edu.sa/ 28537/ [accessed 2008-10-18].

[226] Alan Pétrowski. A Clearing Procedure as a Niching Method for Genetic Algorithms. In Proc. of IEEE International Conference on Evolutionary Computation, CEC96, pages 798-803. IEEE, May 20-22, 1996, Nagoya, Japan. doi:10.1109/ICEC.1996.542703. Online available at http://citeseerx.ist.psu.edu/viewdoc/summary?doi=10.1.1.33.8027 [accessed 2008-12-16].

[227] Patrick C. Phillips. The Language of Gene Interaction. Genetics, 149(3):1167-1171, July 7, 1998. ISSN: 0016-6731. Online available at http://www.genetics.org/cgi/content/full/ 149/3/1167 [accessed 2008-03-02].

[228] Alexander Podlich. Intelligente Planung und Optimierung des Güterverkehrs auf Straße und Schiene mit evolutionären Algorithmen. Master's thesis, University of Kassel, March 2009.

[229] Riccardo Poli. Parallel Distributed Genetic Programming. Technical Report CSRP-96-15, School of Computer Science, The University of Birmingham, Birmingham, UK, September 1996. Online available at http://citeseer.ist.psu.edu/86223.html [accessed 2007-11-04]. 
[230] Riccardo Poli. Some Steps Towards a Form of Parallel Distributed Genetic Programming. In The 1st Online Workshop on Soft Computing (WSC1). Nagoya University, Japan, August 1930, 1996. Online available at http://citeseer.ist.psu.edu/156274.html [accessed 2007-11-04].

[231] Riccardo Poli. A Simple but Theoretically-Motivated Method to Control Bloat in Genetic Programming. In Conor Ryan et al., editor, Proc. of 6th European Conference on Genetic Programming, EuroGP 2003, volume 2610/2003 of LNCS, pages 204-217. Springer, April 1416, 2003, Essex, UK. ISBN: 3-5400-0971-X.

[232] Riccardo Poli, William B. Langdon, and Nicholas Freitag McPhee. A Field Guide to Genetic Programming. http://lulu.com, 2008. ISBN: 978-1-40920-073-4. Online available at http:// www.gp-field-guide.org.uk/ [accessed 2008-03-27]. (With contributions by John R. Koza).

[233] Emil Leon Post. Formal Reductions of the General Combinatorial Decision Problem. American Journal of Mathematics, 65(2):197-215, 1943, Baltimore, USA. ISSN: 0002-9327. doi: $10.2307 / 2371809$.

[234] Robin Charles Purshouse. On the Evolutionary Optimisation of Many Objectives. PhD thesis, Department of Automatic Control and Systems Engineering, The University of Sheffield, September 2003. Online available at http://citeseerx.ist.psu.edu/viewdoc/summary? doi=10 - 1 1 1 10.8816 [accessed 2008-12-06].

\section{Q}

[235] Mohammad Adil Qureshi. Evolving Agents. In John R. Koza et al., editor, Proc. of First Annual Conference Genetic Programming (GP-96), pages 369-374. MIT Press, July 28-31, 1996, Stanford University, USA. See also [236]. Online available at http://www.cs.ucl.ac. uk/staff/W. Langdon/ftp/papers/AQ.gp96.ps.gz [accessed 2007-09-17].

[236] Mohammad Adil Qureshi. Evolving Agents. Research Note RN/96/4, UCL, London, UK, January 1996. Online available at http://www.cs.bham.ac.uk/ wbl/biblio/gp-html/ qureshi_1996_eaRN.html [accessed 2008-09-02]. See also [235].

[237] Mohammad Adil Qureshi. The Evolution of Agents. PhD thesis, University College, London, London, UK, July 2001. Online available at http://citeseer.ist.psu.edu/759376.html [accessed 2007-09-14].

\section{R}

[238] Nicholas J. Radcliffe. Non-Linear Genetic Representations. In Reinhard Männer and Bernard Manderick, editors, Proc. of Parallel Problem Solving from Nature 2, PPSN II, pages 259268. Elsevier, September 28-30, 1992, Brussels, Belgium. Online available at http://users . breathe.com/njr/papers/ppsn92.pdf [accessed 2007-09-05].

[239] Ingo Rechenberg. Evolutionsstrategie: Optimierung Technischer Systeme nach Prinzipien der biologischen Evolution. Frommann-Holzboog Verlag, 1973. ISBN: 978-3-77280-374-1.

[240] Christian M. Reidys and Peter F. Stadler. Neutrality in Fitness Landscapes. Applied Mathematics and Computation, 117(2-3):321-350, January 25, 2001. ISSN: 0096-3003. doi:10.1016/S0096-3003(99)00166-6. Online available at http://citeseer.ist.psu.edu/ reidys98neutrality.html [accessed 2008-06-01].

[241] Arend Rensink and Jos Warmer, editors. Proc. of Second European Conference on Model Driven Architecture - Foundations and Applications, ECMDA-FA 2006, volume 4066 of LNCS, July 10-13, 2006, Bilbao, Spain. Springer. ISBN: 3-5403-5909-5.

[242] Craig W. Reynolds. Flocks, Herds and Schools: A Distributed Behavioral Model. SIGGRAPH Computer Graphics, 4(10):25-34, July 1987. ISSN: 0097-8930. Online available at http:// www. cs.toronto.edu/ dt/siggraph97-course/cwr87/ [accessed 2008-07-27]. See also [243]. 
[243] Craig W. Reynolds. Flocks, Herds and Schools: A Distributed Behavioral Model. In SIGGRAPH'87, Proc. of 14th annual conference on Computer graphics and interactive techniques, pages 25-34. Association for Computing Machinery (ACM), July 27-31, 1987, Anaheim, USA. ISBN: 0-8979-1227-6. doi:10.1145/37401.37406. Online available at http://doi .acm.org/10. 1145/37401.37406 [accessed 2008-07-27]. See also [242].

[244] Glenn Ricart and Ashok K. Agrawala. An Optimal Algorithm for Mutual Exclusion in Computer Networks. Communications of the ACM, 24(1):9-17, January 1981. ISSN: 00010782. doi:10.1145/358527.358537. Online available at http://portal.acm.org/citation. cfm?id=358537 [accessed 2008-11-16].

[245] Simon Ronald. Robust Encodings in Genetic Algorithms: A Survey of Encoding Issues. In Thomas Bäck, Zbigniew Michalewicz, and Xin Yao, editors, IEEE International Conference on Evolutionary Computation, CEC97, pages 43-48. IEEE, April 13-16, 1997, Indianapolis, USA. ISBN: 0-7803-3949-5. doi:10.1109/ICEC.1997.592265.

[246] Justinian P. Rosca and Dana H. Ballard. Causality in Genetic Programming. In Larry J. Eshelman, editor, Proc. of Sixth International Conference on Genetic Algorithms, pages 256263. Morgan Kaufmann, July 15-19, 1995, Pittsburgh, USA. ISBN: 1-5586-0370-0. Online available at http://citeseer.ist.psu.edu/rosca95causality.html [accessed 2007-08-12].

[247] R. D. Routledge. Diversity Indices: Which ones are admissible? Journal of Theoretical Biology, 76(4):503-515, February 21, 1979. doi:10.1016/0022-5193(79)90015-8. Online available at http://dx.doi.org/10.1016/0022-5193(79)90015-8 [accessed 2008-11-10].

[248] Conor Ryan, Michael O'Neill, and J. J. Collins. Grammatical Evolution: Solving Trigonometric Identities. In Proc. of 4th International Mendel Conference on Genetic Algorithms, Optimisation Problems, Fuzzy Logic, Neural Networks, Rough Sets, pages 111-119, June 2426, 1998, Brno, Czech Republic. ISBN: 8-0214-1131-7. Online available at http://citeseer. ist.psu.edu/360445.html [accessed 2007-11-12].

\section{S}

[249] Bruno Sareni and Laurent Krähenbühl. Fitness Sharing and Niching Methods Revisited. IEEE Transactions on Evolutionary Computation, 2(3):97-106, September 1998. ISSN: 1089-778X. doi:10.1109/4235.735432.

[250] Warren Sarle. What is overfitting and how can I avoid it? Usenet FAQs: comp.ai.neural-nets FAQ, 3: Generalization(3), May 13, 2007. Online available at http://www.faqs.org/faqs/ ai-faq/neural-nets/part3/section-3.html [accessed 2008-02-29].

[251] Daniel L. Schacter. Critical Review - Implicit Memory: History and Current Status. Journal of Experimental Psychology: Learning, Memory, and Cognition, 13(3):501-518, July 1987. ISSN: 0278-7393. Online available at http://pages.pomona.edu/ rt004747/1gcs11read/ Schacter87.pdf [accessed 2008-12-01].

[252] J. David Schaffer, Larry J. Eshelman, and Daniel Offutt. Spurious Correlations and Premature Convergence in Genetic Algorithms. In Bruce M. Spatz and Gregory J. E. Rawlins, editors, Proc. of First Workshop on Foundations of Genetic Algorithms (FOGA), pages 102-112. Morgan Kaufmann, July 15-18, 1990, Indiana, USA. ISBN: 1-5586-0170-8.

[253] Y. Ahmet Şekercioğlu, Andreas Pitsilides, and Athanasios V. Vasilakos. Computational Intelligence in Management of ATM Networks: A Survey of Current State of Research. In ESIT'99, European Symposium on Intelligent Techniques, June 3-4, 1999, Chania-Crete, Greece. Online available at http://www.cs.ucy.ac.cy/networksgroup/pubs/published/ 1999/CI-ATM-London99.pdf [accessed 2008-09-03].

[254] S. H. Shami, I. M. A. Kirkwood, and Mark C. Sinclair. Evolving simple fault-tolerant Routing Rules using Genetic Programming. Electronics Letters, 33(17):1440-1441, August 14, 1997. ISSN: 0013-5194. doi:10.1049/el:19970996. See also [157].

[255] J. Shekel. Test Functions for Multimodal Search Techniques. In Proc. of Fifth Annual Princeton Conference on Information Science and Systems, pages 354-359. Princeton University Press, 1971, Princeton, USA. See also http://en.wikipedia.org/wiki/ Shekel_function [accessed 2007-11-06] and http://www-optima.amp.i.kyoto-u.ac.jp/member/ student/hedar/Hedar_files/TestGO_files/Page2354.htm [accessed 2007-11-06]. 
[256] Mark C. Sinclair. Evolutionary Telecommunications: A Summary. In Proc. of Evolutionary Telecommunications: Past, Present and Future - A Bird-of-a-feather Workshop at GECCO 99, 1999. In proceedings [257]. Online available at http://citeseerx.ist.psu.edu/viewdoc/ summary?doi=10.1.1.38.6797 [accessed 2008-08-01], presentation online available at http://uk. geocities.com/markcsinclair/ps/etppf_sin_slides.ps.gz [accessed 2008-08-01].

[257] Mark C. Sinclair, David Corne, and George D. Smith, editors. Proc. of Evolutionary Telecommunications: Past, Present and Future - A Bird-of-a-feather Workshop at GECCO 99, July 13, 1999, Orlando, USA. Online available at http://uk.geocities.com/markcsinclair/ etppf.html [accessed 2008-06-25].

[258] Michael Sipser. Introduction to the Theory of Computation. PWS Publishing, second edition, 2006. ISBN: 0-5349-4728-X.

[259] Murray Smith. Neural Networks for Statistical Modeling. Wiley \& Sons, April 1993/1996. ISBN: 978-1-85032-842-1.

[260] Peter W. H. Smith and Kim Harries. Code Growth, Explicitly Defined Introns, and Alternative Selection Schemes. Evolutionary Computation, 6(4):339-360, Winter 1998. ISSN: 1063-6560. doi:10.1162/evco.1998.6.4.339. Online available at http://citeseer.ist.psu . edu/harries 98code.html [accessed 2008-07-22].

[261] Shana Shiang-Fong Smith. Using multiple Genetic Operators to Reduce Premature Convergence in Genetic Assembly Planning. Computers in Industry, 54(1):35-49, May 2004. ISSN: 0166-3615. doi:10.1016/j.compind.2003.08.001.

[262] Stephen Frederick Smith. A Learning System based on Genetic Adaptive Algorithms. PhD thesis, University of Pittsburgh, Pittsburgh, USA, 1980. University Microfilms No. 81-12638.

[263] Tom Smith, Phil Husbands, Paul Layzell, and Michael O'Shea. Fitness Landscapes and Evolvability. Evolutionary Computation, 10(1):1-34, Spring 2002. ISSN: 1063-6560. doi:10.1162/106365602317301754. Online available at http://wotan.liu.edu/docis/dbl/ evocom/2002_10_1_1_FLAE.html [accessed 2007-07-28].

[264] Donald L. Snyder and Michael I. Miller. Random Point Processes in Time and Space. Springer, second edition, June 19, 1991. ISBN: 978-0-38797-577-1.

[265] Terence Soule and James A. Foster. Removal Bias: a New Cause of Code Growth in Tree Based Evolutionary Programming. In The 1998 IEEE International Conference on Evolutionary Computation, Proc. of IEEE World Congress on Computational Intelligence, CEC98, pages 781-186. IEEE, May 4-9, 1998, Anchorage, USA. Online available at http://citeseer.ist. psu.edu/313655.html [accessed 2007-09-07].

[266] Bruce M. Spatz and Gregory J. E. Rawlins, editors. Proc. of First Workshop on Foundations of Genetic Algorithms (FOGA), July 15-18, 1990, Indiana, USA. Morgan Kaufmann. ISBN: 1-5586-0170-8. Published July 1, 1991. Partly online available at http://books.google.de/ books?id=Df12yLrlUZYC [accessed 2008-05-29].

[267] William M. Spears and Kenneth Alan de Jong. Using Genetic Algorithms for Supervised Concept Learning. In Proc. of 2nd International IEEE Conference on Tools for Artificial Intelligence, pages 335-341. IEEE, 6-9 1990, Herndon, USA. Online available at http://www. cs. uwyo. edu/ wspears/papers/ieee90.pdf [accessed 2007-09-12].

[268] Lee Spector. Automatic Quantum Computer Programming - A Genetic Programming Approach. Genetic Programming. Kluwer Academic Publishers, paperback edition 2007 edition, June 2004. ISBN: 978-0-38736-496-4. Partly online available at http://books.google.de/ books?id=mEKf vtxmn9MC [accessed 2008-05-04].

[269] Charles E. Spurgeon. Ethernet - The Definitive Guide. O'Reilly, February 2000. ISBN: 978-1-56592-660-8. Partly online available at http://books.google.de/books? id=VbTvnxBKzUgC [accessed 2008-11-17].

[270] William Stallings. Operating Systems: Internals and Design Principles. GOAL (Gradience Online Accelerated Learning) Series. Pearson/Prentice Hall, 2004/2008. ISBN: 978-0-13147-954-8. Partly online available at http://books.google.de/books? id=dBQFXs5NPEYC [accessed 2009-01-04].

[271] Terry Stewart. Extrema Selection: Accelerated Evolution on Neutral Networks. In Proc. of IEEE Congress on Evolutionary Computation, CEC2001, volume 1. IEEE, May 27-30, 2001, Seoul, Korea. ISBN: 0-7803-6658-1. doi:10.1109/CEC.2001.934366. Online available at http://citeseer. ist.psu.edu/654440.html [accessed 2008-06-01]. 
[272] Walter Alden Tackett. Recombination, Selection, and the Genetic Construction of Computer Programs. PhD thesis, Faculty of the Graduate School of the University of Southern California, Los Angeles, CA, USA, April 17, 1994. Online available at http://www.cs.ucl.ac.uk/staff/ W.Langdon/ftp/ftp.io.com/papers/watphd.tar.Z [accessed 2007-09-07].

[273] Andrew S. Tanenbaum and Maarten van Steen. Distributed Systems. Principles and Paradigms. Pearson/Prentice Hall International, March 2003. ISBN: 978-0-13239-227-3.

[274] Astro Teller. Genetic Programming, Indexed Memory, the Halting Problem, and other Curiosities. In Proc. of 7 th annual Florida Artificial Intelligence Research Symposium, pages 270-274. IEEE, May 1994. Online available at http://citeseer.ist.psu.edu/256509.html [accessed 2008-06-16].

[275] Astro Teller. Turing Completeness in the Language of Genetic Programming with Indexed Memory. In Zbigniew Michalewicz et al., editor, Proc. of First IEEE Conference on Evolutionary Computation, IEEE World Congress on Computational Intelligence, pages 136-141. IEEE, June 27-29, 1994, Orlando, USA. ISBN: 0-7803-1899-4. Online available at http:// www. astroteller.net/work/pdfs/Turing.pdf [accessed 2007-09-17].

[276] The SPIN Project. BEEM Database Combined Results - The BEEM Database - Tool Performance, March 31, 2007. The SPIN performance in the BEEM example problems. Online available at http://spinroot.com/spin/beem.html [accessed 2008-10-02]. Database online available at http://anna.fi.muni.cz/models/ [accessed 2008-10-02], see also [224].

[277] Guy Théraulaz and Eric Bonabeau. Swarm Smarts. Scientific American, pages 72-79, March 2000. Online available at http://www.santafe.edu/ vince/press/SciAm-SwarmSmarts.pdf [accessed 2008-06-12].

[278] Chai K. Toh. Ad Hoc Mobile Wireless Networks: Protocols and Systems. Prentice Hall PTR, December 2001. ISBN: 978-0-13007-817-9.

[279] Ozan K. Tonguz and Gianluigi Ferrari. Ad Hoc Wireless Networks: A CommunicationTheoretic Perspective. Wiley \& Sons, March 2006. ISBN: 978-0-47009-110-4.

[280] Marc Toussaint and Christian Igel. Neutrality: A Necessity for Self-Adaptation. In David B. Fogel et al., editor, Proc. of IEEE Congress on Evolutionary Computation, CEC2002, pages 1354-1359. IEEE, May 12-17, 2002, Honolulu, USA. ISBN: 0-7803-7278-6. Online available at http://citeseer.ist.psu.edu/toussaint02neutrality.html [accessed 2007-07-28].

[281] Christian F. Tschudin. Fraglets - a Metabolistic Execution Model for Communication Protocols. In Proc. of 2nd Annual Symposium on Autonomous Intelligent Networks and Systems (AINS), June 30-July 3, 2003, Menlo Park, USA. Online available at http://path. berkeley.edu/ains/final/007\%20-\%2022-tschudin.pdf [accessed 2008-05-02]. See also http:// ww. fraglets.net/ [accessed 2007-09-17].

[282] Christian F. Tschudin and Lidia A. R. Yamamoto. A Metabolic Approach to Protocol Resilience. In Autonomic Communication - Revised Selected Papers from the First International IFIP Workshop, WAC 2004, volume 3457/2005 of LNCS, pages 191-206. Springer, October 18-19, 2004, Berlin, Germany. ISBN: 978-3-54027-417-9. doi:10.1007/11520184_15. Online available at http://cn.cs . unibas.ch/pub/doc/2004-wac-lncs . pdf [accessed 2008-06-20], presentation online available at http://www.autonomic-communication.org/wac/wac2004/ program/presentations/wac2004-tschudin-yamamoto-slides .pdf [accessed 2008-06-20].

[283] Alan Mathison Turing. On Computable Numbers, with an Application to the Entscheidungsproblem. Proc. of London Mathematical Society, 42:230-265, 1936. Online available at http://www . abelard.org/turpap2/tp2-ie.asp [accessed 2007-08-11].

[284] Alan Mathison Turing. On Computable Numbers, with an Application to the Entscheidungsproblem, Errata. Proc. of London Mathematical Society, 43:544-546, 1937.

\section{$\mathbf{U}$}

[285] Jamie Uys. Animals are Beautiful People. Warner Brothers, 1974, South Africa. 
[286] Erik van Nimwegen, James P. Crutchfield, and Melanie Mitchell. Statistical Dynamics of the Royal Road Genetic Algorithm. Theoretical Computer Science, 229(1-2):41-102, November 6, 1999. ISSN: 0304-3975. doi:10.1016/S0304-3975(99)00119-X. Online available at http:// citeseer.ist.psu.edu/nimwegen98statistical.html [accessed 2008-06-01].

[287] Athanasios V. Vasilakos, Kostas G. Anagnostakis, and Witold Pedrycz. Application of Computational Intelligence Techniques in Active Networks. Soft Computing - A Fusion of Foundations, Methodologies and Applications, 5(4):264-271, August 2001. ISSN: 1432-7643. doi:10.1007/s005000100100. Online available at http://www.springerlink.com/content/ xxuyux0wgmnt73gy/fulltext.pdf [accessed 2008-09-03].

[288] Sudarshan Vasudevan, Jim Kurose, and Don Towsley. Design and Analysis of a Leader Election Algorithm for Mobile Ad Hoc Networks. In Proc. of 12th IEEE International Conference on Network Protocols, IEEE ICNP 2004, pages 350-360. IEEE, October 5-8, 2004, Berlin, Germany. ISBN: 0-7695-2161-4. doi:10.1109/ICNP.2004.1348124. Online available at ftp:// gaia.cs.umass.edu/pub/Vasu03_LdrElec.ps.gz [accessed 2008-11-16]. See also [289].

[289] Sudarshan Vasudevan, Jim Kurose, and Don Towsley. Design and Analysis of a Leader Election Algorithm for Mobile Ad Hoc Networks. UMass Computer Science Techincal Report 03-20, Computer networks and performance evaluation research group. Computer Science Department, University of Massachusetts, 2004. Online available at ftp://gaia.cs.umass . edu/pub/Vasu03_LdrElec.pdf [accessed 2007-10-24]. See also [288].

\section{W}

[290] Andreas Wagner. Robustness and Evolvability in Living Systems. Princeton Studies in Complexity. Princeton University Press, August 2005. ISBN: 0-6911-2240-7.

[291] Andreas Wagner. Robustness, Evolvability, and Neutrality. FEBS Letters, 579(8):1772-1778, March 21, 2005. ISSN: 0014-5793. doi:10.1016/j.febslet.2005.01.063. Online available at http://dx.doi.org/10.1016/j.febslet.2005.01.063 [accessed 2007-08-05].

[292] James Z. Wang and Matti Vanninen. Self-Configuration Protocols for Small-Scale P2P Networks. In Proc. of 10th Network Operations and Management Symposium, NOMS 2006, pages 1-4. IEEE/IFIP, April 2006, Vancouver, Cannada. ISBN: 1-4244-0142-9. Online available at http://www.cs.clemson. edu/ jzwang/pub/nomshort.pdf [accessed 2007-08-19].

[293] Thomas Weise. Global Optimization Algorithms - Theory and Application. Self-Published, second edition, 2009. Online available at http://www.it-weise.de/ [accessed 2009-05-07].

[294] Thomas Weise and Kurt Geihs. Genetic Programming Techniques for Sensor Networks. In Proc. of 5. GI/ITG KuVS Fachgespräch "Drahtlose Sensornetze", pages 21-25, July 1718, 2006, Stuttgart, Germany. Online available at http://elib.uni-stuttgart.de/opus/ volltexte/2006/2838/ [accessed 2007-11-07].

[295] Thomas Weise and Kurt Geihs. DGPF - An Adaptable Framework for Distributed MultiObjective Search Algorithms Applied to the Genetic Programming of Sensor Networks. In Bogdan Filipič and Jurij Šilc, editors, Proc. of Second International Conference on Bioinspired Optimization Methods and their Applications (BIOMA 2006), pages 157-166. Jožef Stefan Institute, October 9-10, 2006, Ljubljana, Slovenia. ISBN: 978-9-61630-381-1. Online available at http://www.it-weise.de/documents/files/W2006DGPFc.pdf [accessed 2009-05-07].

[296] Thomas Weise, Steffen Bleul, and Kurt Geihs. Web Service Composition Systems for the Web Service Challenge - A Detailed Review. Kasseler Informatikschriften (KIS) 2007, 7, University of Kassel, November 19, 2007. Online available at http://www.it-weise.de/ documents/files/WBG2007WSCb.pdf [accessed 2007-11-20]. See also [33, 34].

[297] Thomas Weise, Kurt Geihs, and Philipp Andreas Baer. Genetic Programming for Proactive Aggregation Protocols. In Bartłomiej Beliczyński, Andrzej Dzieliński, Marcin Iwanowski, and Bernardete Ribeiro, editors, Proc. of Adaptive and Natural Computing Algorithms, 8th International Conference, ICANNGA 2007, Part I, volume 4431/2007 of LNCS, pages 
167-173. Springer Berlin Heidelberg New York, April 11-14, 2007, Warsaw, Poland. ISBN: 978-3-54071-589-4. doi:10.1007/978-3-540-71618-1. Online available at http://www. it-weise.de/documents/files/W2007DGPFb.pdf [accessed 2009-05-07].

[298] Thomas Weise, Michael Zapf, and Kurt Geihs. Rule-based Genetic Programming. In Proc. of BIONETICS 2007, 2nd International Conference on Bio-Inspired Models of Network, Information, and Computing Systems. Institute for Computer Sciences, Social-Informatics and Telecommunications Engineering (ICST), IEEE, ACM, December 10-13, 2007, Budapest, Hungary. ISBN: 978-9-63979-905-9. Online available at http://www.it-weise.de/ documents/files/WZG2007RBGP.pdf [accessed 2009-05-07].

[299] Thomas Weise, Michael Zapf, Mohammad Ullah Khan, and Kurt Geihs. Genetic Programming meets Model-Driven Development. Kasseler Informatikschriften (KIS) 2007, 2, University of Kassel, July 2, 2007. Online available at http://kobra.bibliothek. uni-kassel.de/handle/ urn:nbn: de:hebis:34-2007070218786 [accessed 2007-09-17].

[300] Thomas Weise, Michael Zapf, Mohammad Ullah Khan, and Kurt Geihs. Genetic Programming meets Model-Driven Development. In Andreas König, Mario Köppen, Ajith Abraham, Christian Igel, and Nikola Kasabov, editors, 7th International Conference on Hybrid Intelligent Systems (HIS 2007). IEEE, September 17-19, 2007, Kaiserslautern, Germany. ISBN: 0-7695-2946-1. Online available at http://www . it-weise.de/documents/files/ WZKG2007DGPFg • pdf [accessed 2009-05-07].

[301] Thomas Weise, Steffen Bleul, Diana Comes, and Kurt Geihs. Different Approaches to Semantic Web Service Composition. In Abdelhamid Mellouk, Jun Bi, Guadalupe Ortiz, Dickson K. W. Chiu, and Manuela Popescu, editors, Proc. of Third International Conference on Internet and Web Applications and Services, ICIW 2008, pages 90-96. IEEE, June 8-13, 2008, Athens, Greece. ISBN: 978-0-76953-163-2. Online available at http://www.it-weise.de/ documents/files/WBCG2008ICIW . pdf [accessed 2009-05-07].

[302] Thomas Weise, Stefan Niemczyk, Hendrik Skubch, Roland Reichle, and Kurt Geihs. A Tunable Model for Multi-Objective, Epistatic, Rugged, and Neutral Fitness Landscapes. In Maarten Keijzer et al., editor, Proc. of Genetic and Evolutionary Computation Conference, GECCO 2008, pages 795-802. ACM, July 12-16, 2008, Atlanta, USA. ISBN: 978-1-60558-131-6. Online available at http://www.it-weise.de/documents/files/ WNSRG2008GECCO.pdf [accessed 2009-05-07].

[303] Thomas Weise, Hendrik Skubch, Michael Zapf, and Kurt Geihs. Global Optimization Algorithms and their Application to Distributed Systems. Kasseler Informatikschriften (KIS) 2008, 3, University of Kassel, University of Kassel, October 14, 2008. Online available at http://ww. it-weise.de/documents/files/WSTG2008GOAATATDS . pdf [accessed 2008-10-17].

[304] Thomas Weise, Michael Zapf, and Kurt Geihs. Evolving Proactive Aggregation Protocols. In Michael O'Neill et al., editor, Genetic Programming - Proc. of 11th European Conference on Genetic Programming, EuroGP 2008, volume 4971/2008 of LNCS, pages 254-265. Springer, March 26-28 2008, Naples, Italy. ISBN: 978-3-54078-670-2. doi:10.1007/978-3-540-78671-9_22. Online available at http://dx.doi.org/10.1007/978-3-540-78671-9_22 [accessed 2009-05-07].

[305] Thomas Weise, Alexander Podlich, Kai Reinhard, Christian Gorldt, and Kurt Geihs. Evolutionary Freight Transportation Planning. In EvoTRANSLOG, 3rd European Workshop on Evolutionary Computation in Transportation and Logistics, April 15-17, 2009, Tübingen, Germany. to appear.

[306] Thomas Weise, Michael Zapf, Raymond Chiong, and Antonio Jesús Nebro Urbaneja. Why Is Optimization Difficult? In Raymond Chiong, editor, Nature-Inspired Algorithms for Optimisation, volume 193 of Studies in Computational Intelligence, chapter 1. Springer, April 2009. ISBN: 978-3-64200-266-3.

[307] Thomas Weise, Michael Zapf, Mohammad Ullah Khan, and Kurt Geihs. Combining Genetic Programming and Model-Driven Development. International Journal of Computational Intelligence and Applications (IJCIA), March 2009. ISSN: 1469-0268. Accepted for publication. Online available at http://www.it-weise.de/documents/ [accessed 2009-05-07].

[308] L. Darrell Whitley, V. Scott Gordon, and Keith E. Mathias. Lamarckian Evolution, The Baldwin Effect and Function Optimization. In Yuval Davidor, Hans-Paul Schwefel, and Reinhard Männer, editors, Parallel Problem Solving from Nature - PPSN III, International Conference on Evolutionary Computation. Proc. of Third Conference on Parallel Problem Solving from Nature, volume 866/1994 of LNCS, pages 6-15. Springer, October 9-14, 1994, Jerusalem, 
Israel. ISBN: 978-3-54058-484-1. doi:10.1007/3-540-58484-6. Online available at http:// citeseerx.ist.psu.edu/viewdoc/summary?doi=10.1.1.18.2428 [accessed 2008-09-11].

[309] Dirk Wiesmann, Ulrich Hammel, and Thomas Bäck. Robust Design of Multilayer Optical Coatings by Means of Evolutionary Algorithms. IEEE Transactions on Evolutionary Computation, 2(4):162-167, November 1998. ISSN: 1089-778X. doi:10.1109/4235.738986. See also [310].

[310] Dirk Wiesmann, Ulrich Hammel, and Thomas Bäck. Robust Design of Multilayer Optical Coatings by Means of Evolutionary Strategies. Sonderforschungsbereich (sfb) 531, Universität Dortmund, March 31 1998. Online available at http://citeseer.ist.psu.edu/325801.html [accessed 2008-07-19]. See also [309].

[311] Wikipedia. Wikipedia - The Free Encyclopedia, 2008. Online available at http://en. wikipedia.org/ [accessed 2009-05-07].

[312] Claus O. Wilke. Adaptive Evolution on Neutral Networks. Bulletin of Mathematical Biology, 63(4):715-730, July 2001. ISSN: 0092-8240. Online available at http://arxiv.org/abs/ physics/0101021v1 [accessed 2008-07-02].

[313] George C. Williams. Pleiotropy, Natural Selection, and the Evolution of Senescence. Evolution, 11(4):398-411, December 1957. doi:10.2307/2406060. See also [314].

[314] George C. Williams. Pleiotropy, Natural Selection, and the Evolution of Senescence. SAGE KE, Science of Aging Knowledge Environment, 2001(1), October 3, 2001. See also [313].

[315] Daniel B. Willingham and Laura Preuss. The Death of Implicit Memory. Psyche, 2(15), October 1995. Online available at http://psyche.cs.monash.edu.au/v2/ psyche-2-15-willingham.html [accessed 2008-12-01].

[316] John R. Woodward. Evolving Turing Complete Representations. In Ruhul Sarker et al., editor, Proc. of IEEE Congress on Evolutionary Computation, CEC2003, volume 2, pages 830-837. IEEE, December 8-12, 2003, Canberra, Australia. ISBN: 0-7803-7804-0. doi:10.1109/CEC.2003.1299753. Online available at http://www.cs.bham.ac.uk/ wbl/ biblio/gp-html/Woodward_2003_Etcr.html [accessed 2008-11-08].

[317] Sewall Wright. The Roles of Mutation, Inbreeding, Crossbreeding and Selection in Evolution. In D. F. Jones, editor, Proc. of Sixth Annual Congress of Genetics, volume 1, pages 356-366, 1932. Online available at http://www.blackwellpublishing.com/ridley/classictexts/ wright.pdf [accessed 2007-08-11].

\section{$\mathrm{X}$}

[318] Guochang Xu. GPS: Theory, Algorithms and Applications. Springer, 2003, 2007. ISBN: 978-3-54067-812-0. Partly online available at http://books.google.com/books? id=aRKPAXBt174C [accessed 2008-12-02].

[319] Lidia A. R. Yamamoto and Christian F. Tschudin. Experiments on the Automatic Evolution of Protocols Using Genetic Programming. In Autonomic Communication - Revised Selected Papers from the Second International IFIP Workshop, WAC 2005, volume $3854 / 2006$ of $L N C S$, pages 13-28. Springer, October 2-5 2005, Athens, Greece. ISBN: 978-3-54032-992-3. doi:10.1007/11687818_2. Online available at http://cn.cs.unibas.ch/ people/ly/doc/wac2005-lyct.pdf [accessed 2008-06-20]. See also [320].

[320] Lidia A. R. Yamamoto and Christian F. Tschudin. Experiments on the Automatic Evolution of Protocols Using Genetic Programming. Technical Report CS-2005-002, University of Basel, April 21, 2005. Online available at http://cn.cs.unibas.ch/people/ly/doc/ wac2005tr-lyct.pdf [accessed 2008-06-20]. See also [319]. 
[321] Lidia A. R. Yamamoto and Christian F. Tschudin. Genetic Evolution of Protocol Implementations and Configurations. In IFIP/IEEE International workshop on Self-Managed Systems and Services (SelfMan 2005), May 19, 2005, Nice, France. Online available at http://cn.cs . unibas.ch/pub/doc/2005-selfman.pdf [accessed 2007-09-17].

\section{$\mathrm{Z}$}

[322] Michael Zapf. Typisierung autonomer Softwareagenten. PhD thesis, Fachbereich Biologie und Informatik, Johann Wolfgang Goethe-Universität, Frankfurt am Main, Germany, dissertation.de - Verlag im Internt GmbH, October 2001. ISBN: 978-3-89825-402-1. Online available at http://www.vs.uni-kassel.de/files/mzapfdiss.pdf [accessed 2008-11-25].

[323] Michael Zapf and Thomas Weise. Offline Emergence Engineering For Agent Societies. In Proc. of Fifth European Workshop on Multi-Agent Systems (EUMAS'07), December 14, 2007, Hammamet, Tunesia. Also presented at the co-located Fifth Technical Forum Group (TFG5). Online available at http://www.it-weise.de/documents/files/ZW2007EUMASTR .pdf [accessed 2009-05-07]. See also [324].

[324] Michael Zapf and Thomas Weise. Offline Emergence Engineering For Agent Societies. Kasseler Informatikschriften (KIS) 2007, 8, University of Kassel, December 7, 2007. Online available at http://www.it-weise.de/documents/files/ZW2007EUMASTR.pdf [accessed 2007-11-20], see also [323].

[325] Michael Zapf and Thomas Weise. Applicability of Emergence Engineering to Distributed Systems Scenarios. In Sixth European Workshop on Multi-Agent Systems (EUMAS'08), December 18-19 2008, Bath, UK. Online available at http://www.it-weise.de/documents/ files/ZW2008AOEETDSS . pdf [accessed 2009-05-07].

[326] Michael Zapf and Thomas Weise. Can Solutions Emerge? In Karin Anna Hummel and James P. G. Sterbenz, editors, The third International Workshop on Self-Organizing Systems (IWSOS'08), volume 5343/2008 of LNCS, pages 299-304. Springer, December 10-12, 2008, Vienna, Austria. ISBN: 978-3-54092-156-1. doi:10.1007/978-3-540-92157-8_29. Online available at http://www.it-weise.de/documents/WZG2008CSE.pdf [accessed 2009-05-07].

[327] Yongguang Zhang, Dante De Lucia, Bo Ryu, and Son K. Dao. Satellite Communications in the Global Internet: Issues, Pitfalls, and Potential. In INET 97 - The Internet: The Global Frontier, The Seventh Annual Conference of the Internet Society, June 24-27, 1997, Kuala Lumpur, Malaysia. Online available at http://www.isoc.org/inet97/proceedings/ F5/F5_1.HTM [accessed 2008-12-01]. 


\section{Index}

Übervereinfachung, 220

AAB, 39

AAS, 39

Abschnitt

kritischer, 210, 217

Acquisition

module, 60

Action, 33

Adaptation

self, 19

ADELFE, 157

Adequacy

functional, 69

ADF, 30, 31, 211, 212

Adäquatheit, 220

funktionelle, 220

Affe, 220

Aggregation, 157

AIMGP, 76, 212

Aktionsteil, 214

Algorithm

anytime, 70, 104

distributed, 91

evolutionary, 27

optimization

baysian, 60

Algorithmic Chemistry, 60

All-Or-Nothing, 42, 56, 140, 148

Allele, 25

Ameise, 220

Anführer, 217

Anytime Algorithm, 70, 104

Assignment

soft, 62,213

Ausschluss wechselseitig, 210

wechselseitiger, 217

Auswahlalgorithmen, 210

Auswalalgorithmus, 217

Automatically Defined Functions, 30

Batteriekapazität, 207

Bayesian Optimization Algorithm, 60

Bee, 32

Behavior

swarm, 20

Bevölkerung, 221

Bevölkerungsgröße, 221, 222

BIONETS, 43

Bird, 32

Black-Box, 23

BOA, 60

\section{Candidate}

solution, 23

Causality, 51

CC, 39

CGPS, 76, 212

Checking

model, 69

Chemie

künstlich, 213

Chemistry

algorithmic, 60

Chromosomes

tree, 29

Classifier, 33

Classifier Systems, 31

learning, 32

Communication

collective, 39 
Compiler, 78

Compiling, 78

Computing

amorphous, 19, 207

high-performance, 39

ubiquitous, 19, 207

Condition, 33

Connection Register, 61

Convergence, 47

premature, 47

prevention, 204

CPU, 39, 61, 99

Critical Section, 121

Crossover, 29, 30

homologous, 29, 42, 77

sticky, 77

tree, 30

CS, 31

Deadlock, 121, 218

DGCD, 139, 140

DGPF, 76, 99, 100, 159, 212

Dijkstra algorithm, 37

Distributed algorithms, 91

Diversity, 49, 55

Divisor

greatest common, 139, 218

DNA, 77

Domination, 26

Don't Care, 33

Dreiadressmaschine, 212

EA, 20, 27, 208

eBook, 158

Efficiency

Pareto, 26

Election, 103

Emergence Engineering

offline, 157

Energie, 214

Energieverbrauch, 207

Entscheidungsproblem, 67

Epistacy, 57

Epistase, 213, 222, 224

positional, 213, 215

Epistasis, 57

in GPMs, 58

positional, 58

semantic, 58

Equilibrium

punctuated, 53

eRBGP, 86, 88, 109, 112, 117, 142, 155, 223

eSGP, 74, 109, 126, 143, 211

Evolution, 20, 208

Evolutionary Algorithm, 20, 27 cycle, 27

Evolutionärer Algorithmus, 208

Evolvability, 53
EvoWeb, 35

Exclusion

mutual, 121

Exploitation, 50

Exploration, 50

Finite State Machine, 40

Fitness, 26

Fitness Assignment

Pareto ranking, 27

Prevalence ranking, 27

Fitness Landscape, 158

rugged, 51

Fitnesslandschaft, 224

Fly, 32

Fraglets, 42, 79, 111, 117, 128, 210, 213 evolution of, 42

FSM, 40

Function

ADF, 30

automatically defined, 30

objective, 20, 25

Funktion

automatisch definiert, 211

GA, 28

Gads, 58

Gads 2, 75

Gamma System, 79, 213

GCD, 139, 218

GCL, 82, 214

GE, 58

Gene, 25

Genetic Algorithm, 28

Genetic Programming, 20, 29, 208

compiling system, 76, 212

crossover

homologous, 77

linear, 76, 212

rule-based, 81, 213

extended, 86, 215

Standard, 71, 74

standard, 210, 211

tree-based, 29

Genetic Programming Kernel, 41

Genetische Programmierung, 208, 222

lineare, 210, 212

regelbasiert, 213, 223

erweitert, 215

standard, 210, 211

Genome, 23

Genomes

tree, 29

Genotyp-Phänotyp-Abbildung, 212, 220

Genotype, 25

Genotype-Phenotype Mapping, 25

genotype-phenotype mapping, 78

Gentische Programmierung, 221

regelbasiert, 210 
GGGP, 58

GGT, 210, 218

GP, 20, 29, 208

GPK, 41

GPM, 25, 78

epistasis, 58

Grammatical Evolution, 58

Christiansen, 58

Greatest Common Divisor, 139, 218

Greedysuche, 221

Gruppenkommunikation, 217

Größter Gemeinsamer Teiler, 218

Guarded Command Language, 82, 214

Halting Problem, 68

Homologous Crossover, 29, 77

Homology, 77

Individual, 26

Insekt, 220

Inselmodell, 221

Interrupt

service routine, 71,211

Intron, 53

Inviable Code, 55

IP, 39

Isolated, 56

ISR, 71, 73, 211

Klassifikator, 213

Kolonie, 220

Konditionsteil, 214

Kritischer Abschnitt, 210, 217

Lamarck, 56

Language

guarded command, 82, 214

Latency, 96

Laufzeit, 221

LCS, 32

Learning Classifer Systems, 32

LGP, 76, 110, 111, 117, 127, 129, 144, 210, 212

Lifelock, 121, 218

Lineare Genetische Programmierung, 212

LISP, 37

Locality, 51

Machine

finite state, 40

MANET, 91, 152, 161, 221

Mapping

genotype-phenotype, 25

MAS, 157

MDD, 157

Mehrzieloptimierung, 209

Memetische Algorithmen, 221

Memory

indexed, 75, 88, 155, 223

with memory, 62
Message, 32

Metaheuristics, 23

Metaheuristiken, 210

Model

checking, 69

Model Checking, 69

Model Driven Development, 157

Module

acquisition, 60

MOEA, 26

MOF, 157

MOFScript, 157

Monkey, 152

Multi-objective, 55

multi-objective, 26

Multimodality, 49

Mutation, 28-30

tree, 29

Mutual Exclusion, 121

Nadel-im-Heuhaufen-Problem, 219

NAVSTAR GPS, 96

Nebenläufigkeit, 217

Needle-In-A-Haystack, 56, 148, 219

Network

on chip, 39

sensor, 19

wireless, 19

wireless, 19

Network Topology, 95

Neutrality, 54

Neutralität, 224

NIAH, 56, 148, 219

Niche Count, 197

NoC, 39

Noise, 63

Non-functional, 67

OAB, 39

OAS, 39

Objective Function, 20, 25

OEE, 157, 158

Optimality

Pareto, 26

Optimization, 23

multi-objective, 26

baysian, 60

multi-objective, 26, 55

Pareto, 26

Optimum

isolated, 56

Orange, 152, 220

Order

partial, 26

Organization self, 19

Overfitting, 55, 65, 220

Overgeneralization, 66

Oversimplification, 66 
Pareto, 26

frontier, 26, 132

optimal, 26

set, 26

Partial order, 26

Phenome, 23

Phenotype, 23

Pleiotropy, 57

Poisson, 94

Population, 26, 27

Population Size, 153

Premature Convergence, 47

Prevention

convergence, 204

Problem Space, 23

Programmgröße, 209

Programmrepräsentation, 209, 210, 213, 220

Promela, 69

Protocol, 39

QoS, 158

RBGP, 81-85, 109, 117, 130, 155, 210, 213-216, 223

extended, 86

Recombination

tree, 30

Regel, 213

Register

connection, 61

Representation state-based, 41

Reproduction

binary, 30

unary, 29

Repräsentation, 213, 219

Programm, 213

Robustness, 63, 64

Routing, 37, 217

Ruggedness, 51

Rule-based Genetic Programming, 81 extended, 86

Runtime, 117, 137, 148

SBR, 41

Scenario, 154

Schroffheit, 224

Schwarmverhalten, 208

SCP, 203

Search Operations, 25

Search Space, 23

Section

critical, 121

Selbstadaption, 207, 220

Selbstorganisation, 207

Self-Adaptation, 19

Self-Modification, 42

Self-Organization, 19

Semantics, 158
Sensor Network, 19, 152

Sensornetze, 207

Sensornetzwerk, 221

Service Routine

interrupt, 71, 211

SGP, 29, 71, 109, 210, 211

extended, 74

Sharing

Variety Preserving, 197

Sharing Function, 197

Sigoa, 99, 100, 159

Simulated Annealing, 221

Smart Home, 19, 207

Soft Assignment, 62, 213

Software engineering, 67

Software Testing, 67

Solution Candidate, 23

Sortieren, 215

Space

problem, 23

search, 23

Speicher, 207, 215

indirekt adressierbar, 223

Speicherverbrauch, 209

SPIN, 69

Standard Genetic Programming, 210, 211

Sticky Crossover, 77

Suchraum, 219

Swarm, 20

System

gamma, 79, 213

string rewriting, 79, 213

Szenario, 222

Tabusuche, 221

TCP, 39

Teiler

größter gemeinsamer, 210, 218

Ternary System, 33

Topologie, 222

Topology, 95

network, 95

Tree Genomes, 29

Ubiquitous Computing, 19, 207

UML, 72, 157

Variety Preserving, 197

Verifikation, 220

Wasp, 32

Web Service, 158

composition, 158

Wechselseitiger Ausschluss, 217

Wildcard, 33

WSC, 158

XMI, 157

Zielfunktion, 208, 220, 222 


\section{List of Figures}

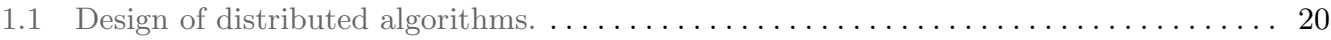

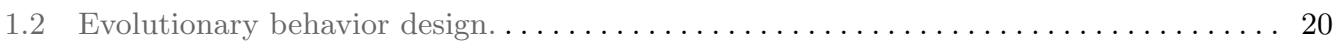

1.3 The general solution approach pursued by this work $\ldots \ldots \ldots \ldots \ldots \ldots \ldots \ldots \ldots$

2.1 Spaces, Sets, and Elements involved in an optimization process. . . . . . . . . . 24

2.2 The relation of genome, genes, and the problem space $\ldots \ldots \ldots \ldots \ldots \ldots \ldots \ldots$

2.3 An example scenario for Pareto ranking. $\ldots \ldots \ldots \ldots \ldots \ldots \ldots \ldots \ldots \ldots \ldots \ldots \ldots \ldots \ldots \ldots \ldots \ldots \ldots$

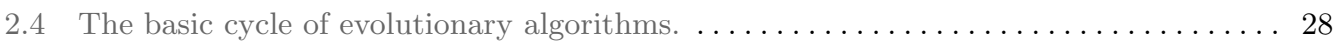

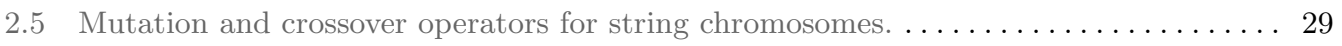

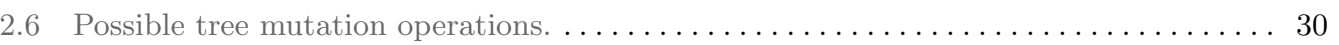

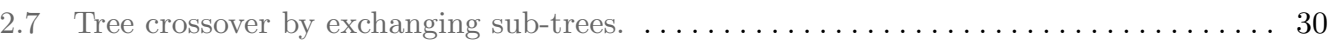

2.8 A concrete example for automatically defined functions. . . . . . . . . . . . 31

2.9 One possible encoding of messages for a frog classifier system. . . . . . . . . . . . 33

3.1 The number of papers studied in $[303,293]$ per year. . . . . . . . . . . . 36

3.2 The number of papers analyzed, broken down to application area and optimization

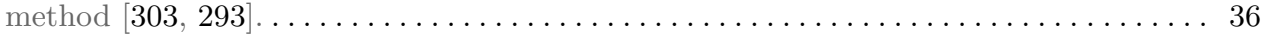

3.3 An evolved routing rule set (left) with target node 0 for the specified network (right). 37

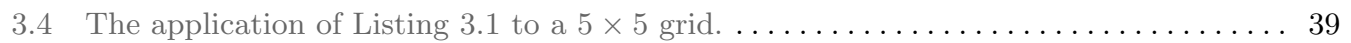

3.5 The scenario for protocol synthesis as used in $[60] \ldots \ldots \ldots \ldots \ldots \ldots \ldots \ldots \ldots$

3.6 The best individual from the experiment of de Araújo et al. [60] . . . . . . . . . . 41

4.1 Different possible properties of fitness landscapes (minimization) $\ldots \ldots \ldots \ldots \ldots \ldots 48$

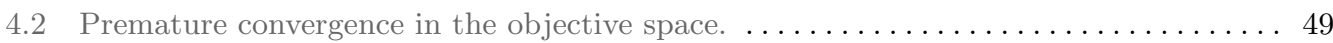

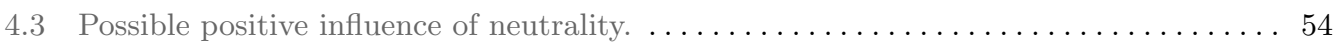

4.4 The influence of epistasis on the fitness landscape. $\ldots \ldots \ldots \ldots \ldots \ldots \ldots \ldots \ldots . \ldots \ldots$

4.5 Positional epistasis in Genetic Programming. . . . . . . . . . . . . . . . . . . . 59

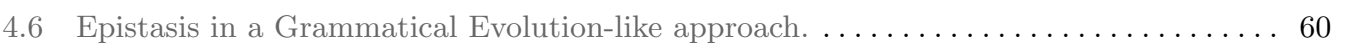

4.7 The difference between linear Genetic Programming and Algorithmic Chemistries. .. 61

4.8 A robust local optimum vs. a "unstable" global optimum. . . . . . . . . . . . 64

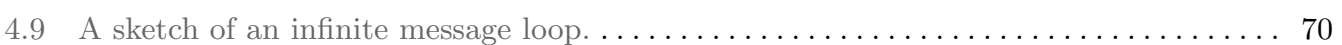

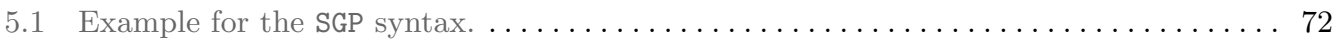

5.2 An example for the use of memory in the SGP approach based on the program

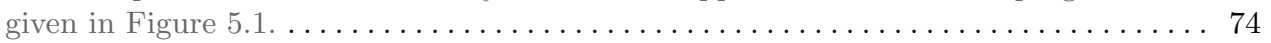


5.3 The example from Figure 5.2 translated to the LGP approach. . . . . . . . . . . 79

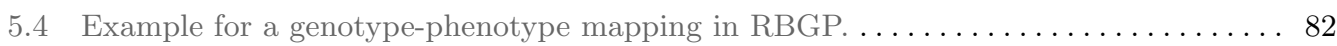

5.5 A program computing the faculty $\mathrm{p}$ of a natural number a in Java and RBGP syntax. 84

5.6 The tree phenotype (and genotype) of Listing $5.8 \ldots \ldots \ldots \ldots \ldots \ldots \ldots \ldots \ldots$

6.1 A Sketch of the DGPF Network Model. . . . . . . . . . . . . . . . . . . . . 92

6.2 A timing diagram annotated with the states of the nodes/processes. . . . . . . . 93

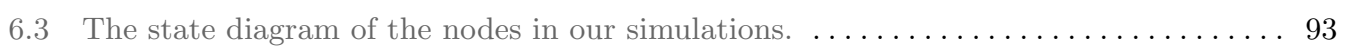

6.4 Synchronous parallelism in a model of a network of five nodes. . . . . . . . . . . 94

6.5 Asynchronous parallelism in a model of a network of five nodes. . . . . . . . . . 95

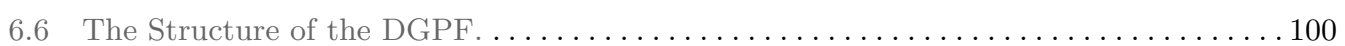

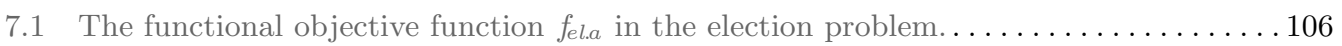

7.2 Convergence diagrams for the election problem $f_{e l . a} \ldots \ldots \ldots \ldots \ldots \ldots \ldots \ldots \ldots \ldots$

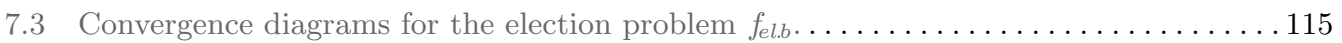

7.4 Partial orders of the GP approaches according to their performance in the election

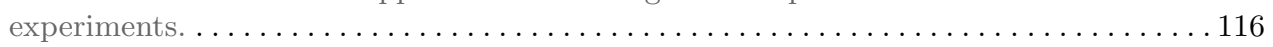

7.5 The average time per generation of the different approaches in the four problems. . . 118

8.1 Example for the collision-counting objective function $f_{\text {col }} \ldots \ldots \ldots \ldots \ldots \ldots \ldots \ldots 123$

8.2 The joint Pareto sets of all experiments of the GP approaches in the fully connected

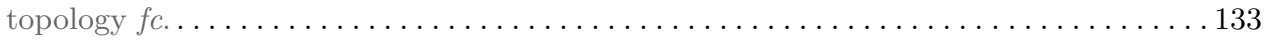

8.3 Convergence diagrams (I) for the critical section problem case $f c \ldots \ldots \ldots \ldots \ldots \ldots 134$

8.4 Convergence diagrams (II) for the critical section problem case $f c \ldots \ldots \ldots \ldots \ldots \ldots 135$

8.5 Partial orders of the GP approaches according to their performance in the critical

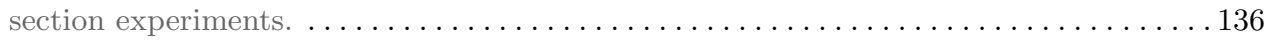

8.6 Partial orders of the critical section scenarios according to the performance of the Genetic Programming approaches in them. ......................... 137

9.1 Convergence diagrams for the GCD problem $f_{g c d .1}$ and $p s=1536 \ldots \ldots \ldots \ldots \ldots$

9.2 Partial orders of the GP approaches according to their performance in the GCD experiments. . . . . . . . . . 147

A.1 Convergence diagrams for the election problem $f_{e l . c} \ldots \ldots \ldots \ldots \ldots \ldots \ldots \ldots \ldots 17$

A.2 Convergence diagrams for the election problem $f_{e l . d} \ldots \ldots \ldots \ldots \ldots \ldots \ldots \ldots \ldots \ldots$

B.1 The joint Pareto sets of all experiments of the GP approaches on the uni-directional

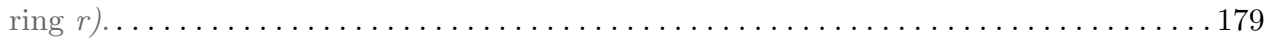

B.2 The joint Pareto sets of all experiments of the GP approaches with disabled

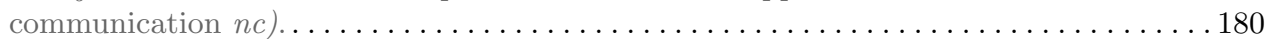

B.3 Convergence diagrams (I) for the critical section problem case $r \ldots \ldots \ldots \ldots \ldots \ldots 181$

B.4 Convergence diagrams (II) for the critical section problem case $r \ldots \ldots \ldots \ldots \ldots \ldots 182$

B.5 Convergence diagrams (I) for the critical section problem case $n c \ldots \ldots \ldots \ldots \ldots . \ldots 183$

B.6 Convergence diagrams (II) for the critical section problem case $n c \ldots \ldots \ldots \ldots \ldots .184$

C.1 Convergence diagrams for the GCD problem $f_{g c d .1}$ and $p s=512 \ldots \ldots \ldots \ldots \ldots \ldots$

C.2 Convergence diagrams for the GCD problem $f_{g c d .2}$ and $p s=512 \ldots \ldots \ldots \ldots \ldots$

D.1 The sharing potential in the Variety Preserving Ranking example. . . . . . . . . . 200

D.2 The expected numbers of occurrences for different values of $n$ and $c p \ldots \ldots \ldots \ldots 204$ 
E.1 Design von verteilten Algorithmen. . . . . . . . . . . . . . . . . . . . . . . 208

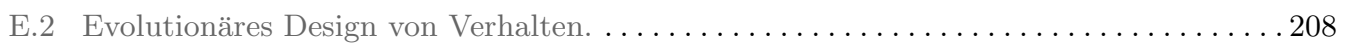

E.3 Der in dieser Arbeit verfolgte Ansatz. . . . . . . . . . . . . . . . . . . . . . . . . . . 209

E.4 Beispiel für die Genotyp-Phänotyp-Abbildung in RBGP (siehe auch Figure 5.4). . . 214

E.5 Ein Programm, dass die Fakultät $\mathrm{p}$ einer natürlichen Zahl a berechnet, dargestellt

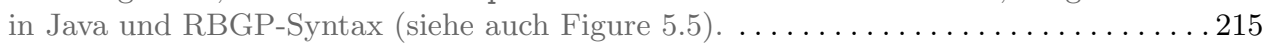

E.6 Der baumbasierte Genotyp und Phänotyp von Listing E.2 (und Listing 5.8) . . . . . 216 



\section{List of Tables}

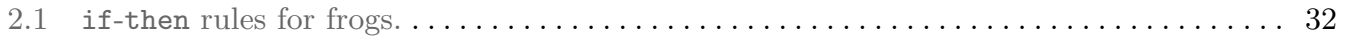

2.2 The encoded form of the if-then rules for frogs from Table $2.1 \ldots \ldots \ldots \ldots \ldots \ldots 34$

5.1 The Standard Genetic Programming primitives. . . . . . . . . . . . . . . 73

5.2 The extended Standard Genetic Programming primitives. . . . . . . . . . . . . 76

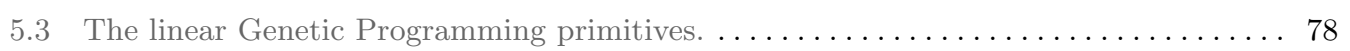

5.4 The Fraglet instructions in our Genetic Programming approach. . . . . . . . . 80

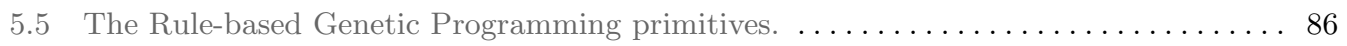

5.6 The Extended Rule-based Genetic Programming primitives. . . . . . . . . . . . . 89

5.7 The six Genetic Programming approaches utilized in this work. . . . . . . . . . 90

7.1 The Genetic Programming approaches in direct comparison. . . . . . . . . . . 113

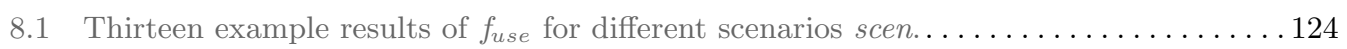

8.2 The Genetic Programming approaches in direct comparison. . . . . . . . . . . . . . 131

9.1 The Genetic Programming approaches in direct comparison. . . . . . . . . . . 145

10.1 Comparison of election, distributed mutual exclusion, and the GCD experiment.....155

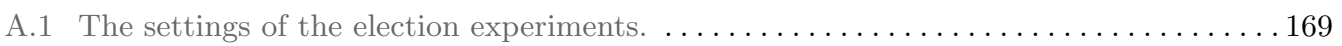

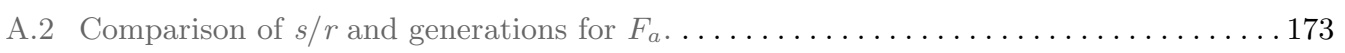

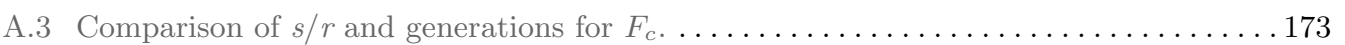

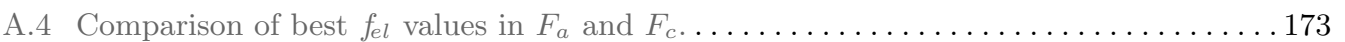

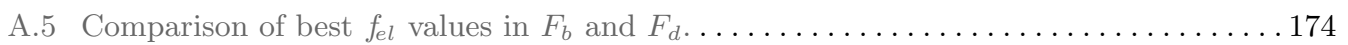

B.1 The settings of the critical section experiments. $\ldots \ldots \ldots \ldots \ldots \ldots \ldots \ldots \ldots \ldots$

B.2 Comparison of $\widetilde{f_{c o l}}$ (upper triangle) and $\left[\widetilde{f_{c o l}}\right]_{z}$ (lower triangle) in the fully connected

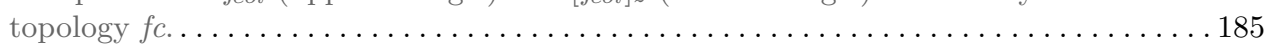

B.3 Comparison of $\widetilde{f_{c o l}}$ (upper triangle) and $\left[\widetilde{f_{c o l}}\right]_{z}$ (lower triangle) in the ring topology $r .185$

B.4 Comparison of $\widetilde{f_{c o l}}$ (upper triangle) and $\left[\widetilde{f_{c o l}}\right]_{z}$ (lower triangle) in case of disabled

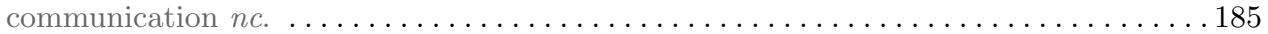

B.5 Comparison of SGP in terms of the scenario and $\widetilde{f_{\text {col }}}$ (upper triangle) and $\left[\widetilde{f_{c o l}}\right]_{z}$

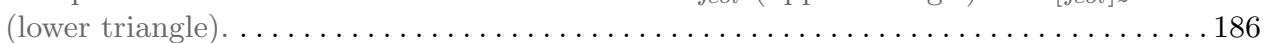

B.6 Comparison of eSGP in terms of the scenario and $\widetilde{f_{c o l}}$ (upper triangle) and $\left[\widetilde{f_{c o l}}\right]_{z}$

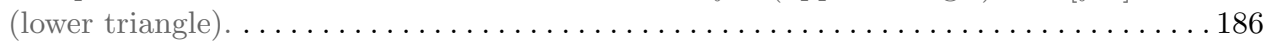


B.7 Comparison of LGP in terms of the scenario and $\widetilde{f_{\text {col }}}$ (upper triangle) and $\left[\tilde{f}_{\text {col }}\right]_{z}$ (lower triangle).

B.8 Comparison of Fraglets in terms of the scenario and $\widetilde{f_{\text {col }}}$ (upper triangle) and $\left[\tilde{f}_{\text {col }}\right]_{z}$

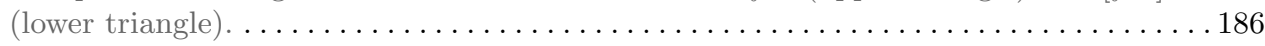

B.9 Comparison of RBGP in terms of the scenario and $\widetilde{f_{c o l}}$ (upper triangle) and $\left[\widetilde{f_{c o l}}\right]_{z}$

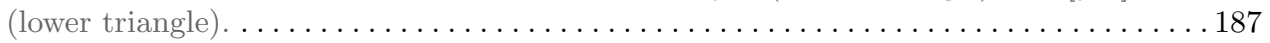

B.10 Comparison of eRBGP in terms of the scenario and $\widetilde{f_{c o l}}$ (upper triangle) and $\left[\widetilde{f_{c o l}}\right] z$

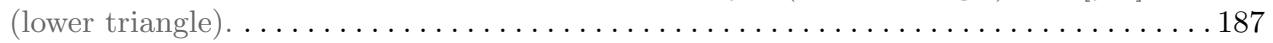

C.1 The settings of the distributed greatest common divisor experiments. . . . . . . . . 190

C.2 Comparison of $\overline{f_{g c d .1}}$ (upper triangle) and $s / r$ (lower triangle) for $F_{g c d .1}$ and $p s=512.194$

C.3 Comparison of $\overline{f_{g c d .1}}$ (upper triangle) and $s / r$ (lower triangle) for $F_{g c d .1}$ and $p s=1536.194$

C.4 Comparison between the two population sizes $p s=512$ and $p s=1536\left(F_{g c d .1}\right) \ldots \ldots 194$

C.5 Comparison of $\overline{f_{g c d .2}}$ (upper triangle) and $s / r$ (lower triangle) for $F_{g c d .2}$ and ps $=512.195$

C.6 Comparison of the success rates $s / r$ of the different objective functions. . . . . . . 195

D.1 An example for Variety Preserving Ranking based on Figure 2.3. . . . . . . . . . . . 201

D.2 The distance and sharing matrix of the example from Table D.1. . . . . . . . . . . 202

E.1 Vergleich zwischen dem Auswahlexperiment, dem kritischen Abschnitt-Experiment und dem GGT-Experiment (siehe auch Table 10.1) . . . . . . . . . . . . . 2223 


\section{List of Algorithms}

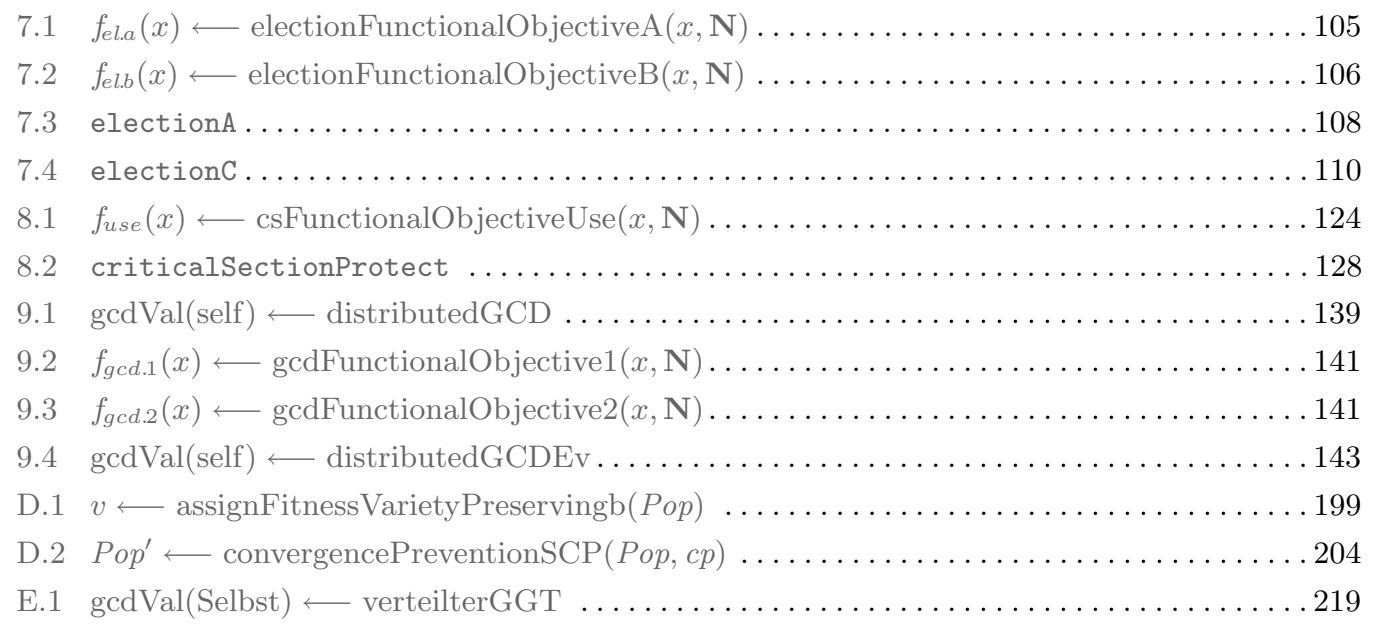





\section{List of Listings}

3.1 The optimal broadcasting scheme evolved for 2-dimensional, directed grids. . . . . . 38

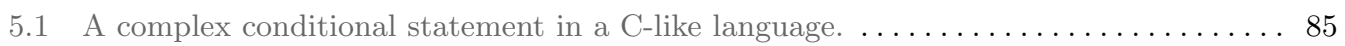

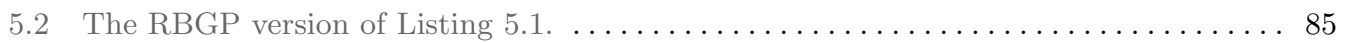

5.3 An equivalent alternative version of Listing $5.2 \ldots \ldots \ldots \ldots \ldots \ldots \ldots \ldots \ldots \ldots$

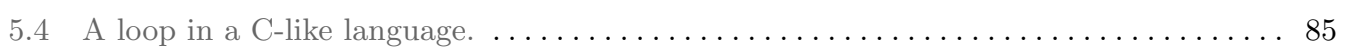

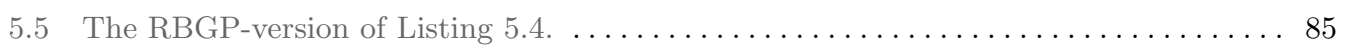

5.6 An equivalent, alternative version of Listing $5.5 \ldots \ldots \ldots \ldots \ldots \ldots \ldots \ldots \ldots \ldots$

5.7 A simple selection sort algorithm written in the eRBGP language. $\ldots \ldots \ldots \ldots \ldots .87$

5.8 The eRBGP version of Listing 5.1 and Listing $5.2 \ldots \ldots \ldots \ldots \ldots \ldots \ldots \ldots \ldots$

5.9 The eRBGP version of Listing 5.4 and Listing $5.5 \ldots \ldots \ldots \ldots \ldots \ldots \ldots \ldots \ldots$

7.1 One evolved eRBGP version of Algorithm $7.3\left(f_{e l . a}=0, f_{p s}=18\right) \ldots \ldots \ldots \ldots \ldots 109$

7.2 One evolved eSGP version of Algorithm 7.3 (Phenotype: see Listing 7.3) . . . . . . . . 109

7.3 The LGP phenotype of the eSGP program given in Listing $7.2\left(f_{e l . a}=0, f_{p s}=21\right) \ldots \ldots 110$

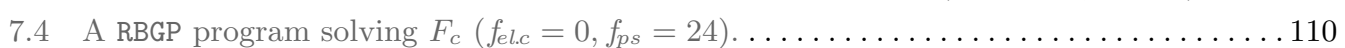

7.5 A Fraglets program solving $F_{a}$ and $F_{c}\left(f_{e l . a}=0, f_{p s}=17\right) \ldots \ldots \ldots \ldots \ldots \ldots \ldots 11$

7.6 An LGP election algorithm which sometimes solves problem $F_{b}\left(f_{\text {el. }} \approx 0.018, f_{p s}=12\right) .111$

7.7 One evolved eRBGP program for $F_{b}\left(f_{e l . b} \approx 0.021, f_{p s}=57\right) \ldots \ldots \ldots \ldots \ldots \ldots \ldots 112$

8.1 The fairest eSGP program for case $f_{c}$ (Phenotype: see Listing 8.2 ) . . . . . . . . 126

8.2 The LGP phenotype of the eSGP genotype specified in Listing 8.1:

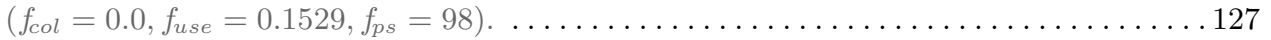

8.3 A marginally fair Fraglets program for case $f_{c}$ :

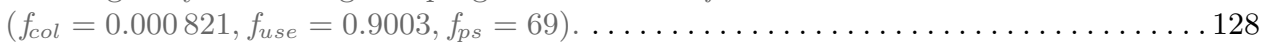

8.4 An LGP for case $r$ with $\left(f_{\text {col }}=1 \cdot 10^{-4}, f_{\text {use }}=0.876, f_{p s}=89\right) \ldots \ldots \ldots \ldots \ldots \ldots 129$

8.5 An almost adequate and fair RBGP program for networks with disabled

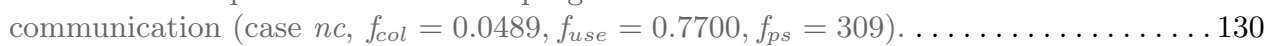

9.1 One evolved eRBGP program for problem $F_{g c d .1}$ in the 512 individual population

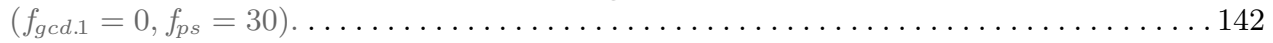

9.2 The only eSGP program for problem $F_{g c d .1}$ in the 512 individual population

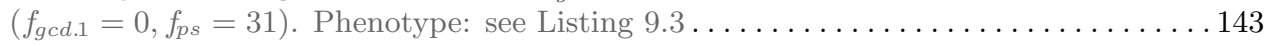

9.3 The LGP phenotype of the eSGP program given in Listing $9.2 \ldots \ldots \ldots \ldots \ldots \ldots \ldots 144$

A.1 A simple manually derived solution for problem definition $d . \ldots \ldots \ldots \ldots \ldots \ldots \ldots 9$

B.1 A simple manually derived solution for the critical section problem in the fully

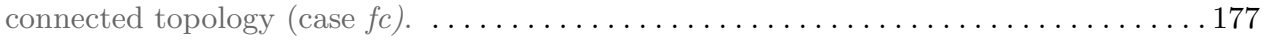

C.1 A LGP version of Mattern's algorithm [193] given in Algorithm 9.1............. 191

E.1 Ein einfaches Selektionsortprogramm in eRBGP-Syntax. . . . . . . . . . . . . . 215 
264 LIST OF LISTINGS

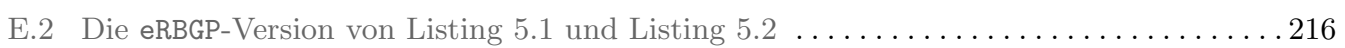

